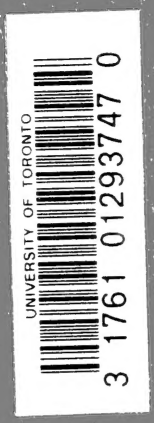

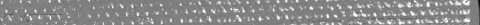


Digitized by the Internet Archive in 2008 with funding from Microsoft Corporation 


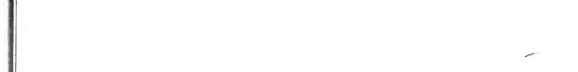





\section{A CATALOGUE}

OF

\section{BRITISH}

\section{FOSSIL VERTEBRATA.}

BY

ARTHUR SMITH WOODWARD, F.G.S.

AND

CHARLES DAVIES SHERBORN F.G.S.

\section{LONDON :}

DULAU \& CO., 37 SOHO SQUARE, W.

January 1890. 


$$
\begin{gathered}
, 3538 \\
, 21619 \\
4 E \\
8 \\
6
\end{gathered}
$$

LONDON:

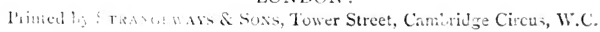




\section{TABLE OF CONTENTS.}

INTRODUCTION ...

DATES OF PUBLICATION OF-

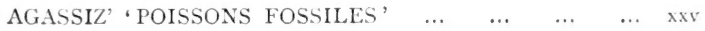

OWEN'S 'ODONTOGRAPHY"

TABLE OF THE STRATIGRAPHICAL DISTRIBUTION OF $\begin{array}{lllllll}\text { BRITISH FOSSIL VERTEBRATA } & \ldots & \ldots & \ldots & \ldots & \mathrm{x} \times \mathrm{x}\end{array}$

CATALOGUE OF BRITISH FOSSIL VERTEBRATA :-

\begin{tabular}{|c|c|c|c|c|c|c|c|c|}
\hline PISCES & $\cdots$ & $\cdots$ & $\cdots$ & $\cdots$ & $\cdots$ & $\cdots$ & $\cdots$ & $\cdots$ \\
\hline AMPHIBIA & $\cdots$ & $\cdots$ & $\cdots$ & $\cdots$ & $\cdots$ & $\cdots$ & $\cdots$ & $\ldots$ \\
\hline REPTILIA & $\cdots$ & $\cdots$ & $\cdots$ & $\cdots$ & $\cdots$ & $\cdots$ & $\cdots$ & $\cdots$ \\
\hline ICHNITES & $\cdots$ & $\cdots$ & $\cdots$ & $\cdots$ & $\ldots$ & $\ldots$ & $\cdots$ & $\ldots$ \\
\hline AVES $\ldots$ & $\cdots$ & $\cdots$ & $\cdots$ & $\ldots$ & $\cdots$ & $\ldots$ & $\cdots$ & $\cdots$ \\
\hline MAMMALIA & $\ldots$ & $\ldots$ & $\ldots$ & $\ldots$ & $\ldots$ & $\ldots$ & $\ldots$ & $\ldots$ \\
\hline
\end{tabular}

$\begin{array}{lllllllll}\text { ADDENDA } & \text { ET CORRIGENDA } & \ldots & \ldots & \ldots & \ldots & \ldots & 395\end{array}$ 



\section{INTRODUCTION.}

T T may appear somewhat unphilosophical at first sight 1 to adopt insignificant topographical features of the present era as the boundaries of a region of the earth to be treated from a Palæontological point of view, since many of the specific types met with among the extinct faunæ represented in British rocks are well known to occur in the corresponding beds of other parts of the world. From the vastness of the subject, however, it is necessary to assign. definite narrow limits to the area under consideration when attempting to record the actual state of knowledge of any great group of extinct animals; and in this work, which deals exclusively with the Fossil Vertebrata, it has therefore been deemed advisable to refer only to forms occurring in Britain. The restriction docs not detract so seriously from the value of the result as might be supposed; for British Palæontology is an epitome of that of the whole world, and, although in some groups the British fossils are comparatively fragmentary, there is no other area of equal extent in which more variety is displayed. Almost every type of importance seems to have some representative in our rocks.

The earliest work of reference of a similar character is the Synoptical Table of Britisle Organic Remains, by Samuel Woodward, published in 1830 . At this date two pages sufficed for the enumeration of the known British Fossil Vertebrata; and the progress of the next twenty years is well illustrated by Professor John Morris' Catalogue of British Fossils, which first appeared in 1843, and comprised thirty pages devoted to the same group. In $\underset{b}{\mathbf{s}}{ }_{4} 8-50$ 
Professor H. G. Bronn's great work, the Index Palcontologicus, provided still more numerous references, incorporating the notices of British forms with those of similar fossils occurring in other parts of the world; and in 1854 a second edition of Professor Morris' Catalogue was published, still further extending the list, and including all genera and species at that time known. Except in the Palæozoic groups, which are treated by Mr. R. Etheridge in his Fossils of the Britis/ Islands, Part i (I888), the considerable advances in British Vertebrate Palæontology during the last thirty-five years have not hitherto been systematically catalogued. The scattered form, however, in which the numerous works and memoirs have appeared, renders such a record almost essential for further progress; and the object of the present volume is to supply this deficiency in the literature of the subject.

Vertebrate fossils have been found in British strata of as early a date as any known in other parts of the world; unless, indeed, the ichthyolites from Ohio described by Professor E. W. Claypole are referable to a slightly lower horizon than the English Ludlow, as already maintained. The ventral shield of a Pteraspidian fish (Scaphaspis ludensis), now in the Museum of Practical Geology, was long ago discovered in the Lower Ludlow of Leintwardine, by the late Mr. John Edward Lee, F.G.S. The Upper Ludlow Bone-bed has furnished undoubted fish remains (e.g., Onchus and Cyathaspis), although some of the fossils in Sir R. I. Murchison's collection, originally described as such, may have been truly referable to invertebrate animals, as remarked by M'Coy. All the Silurian Vertebrata described and figured in the 'Silurian System' unfortunately seem to have been lost.*

The Downton Sandstone, in the neighbourhood of Ludlow, has also yielded shields of Pteraspidian fishes, due chiefly to the exertions of the late Messrs. R. W.

* R. I. Murchison, Qualt. Journ. Geol. Soc., vol. ix, 1853, p. 16. 
Banks and R. Lishtbody of Ludlow, whose collections are now preserved in the Ludlow Museum, the British Museum, and the Museum of the Owens College, Manchester. The well-known Lower Old Red Sandstone Passage Beds of Ledbury, Herefordshire, have proved equally rich in Cephalaspidian fishes, large excavations having been made in these rocks at the Ledbury railway station. The late Dr. Grindrod, of Malvern, accumulated an extensive collection of specimens, principally discovered by the late Henry Brookes, of Ledbury, during the making of the railway, and the collection now occupies a case in the New University Museum, Oxford, having been purchased by the University in I 882 . The most systematic examination of the beds, however, has been made by Mr. George $\mathrm{H}$. Piper, F.G.S., of Ledbury, who has determined the precise stratigraphical position of the various species, and considerably extended the known range of some forms, besides making an unique collection of specimens. Through the generosity of Mr. Piper the finest of these fossils are now placed in the British Museum, and others, from the same donor, are also exhibited in the Hereford Museum.

The Lower Old Red Cornstones of Herefordshire, Worcestershire, and Monmouthshire, have long been noted for their Cephalaspidian and Pteraspidian fossils, and numerous specimens may be seen in the Museums of Hereford, Ludlow, Worcester, and Oxford, the British Muscum, and the Museum of Practical Geology. They seem to have been first collected by the late Rev. T. T. Lewis, of Aymestry, and Dr. Lloyd, of Ludlow, who, through Sir R. I. Murchison, submitted to Agassiz the specimens first described by that ichthyologist. Mr. J. B. Gill, formerly occupied in quarrying near Cradley, five miles from Malvern, carefully preserved a large number of valuable specimens, and disposed of them to various collectors, through whom they have finally reached the public muscums. The late Rev. W. S. Symonds, F.G.S., of 
Pendock, not only seized every opportunity of rescuing isolated discoverics from destruction and oblivion, but also, by his numcrous writings, instigated others to pursue the same course. The late Dr. D. M. McCullough, of Abergavenny, obtained a large series of Pteraspidian shields from that neighbourhood, presenting all the finer examples to the British Museum; and Monmouthshire was also systematically explored by the late Mr. J. E. Lee, F.G.S., who likewise added all his discoveries to the National Collection.

The Upper Old Red Sandstone of Shropshire does not yield many fossils, but a species of Bothriolepis was obtained from these beds in I 862 by Messrs. Baxter and Weaver Jones; and these specimens, with the scalcs and teeth of an undescribed Rhizodont ganoid from the same formation, are now preserved in the British Museum.

The fish-bearing character of the Lower Old Red Sandstone of the Scottish Highlands seems to have been first discovered by the late Hugh Miller, of Cromarty, and Robert Dick, of Thurso, whose combined collections are now, for the most part, preserved in the Museum of Science and Art, Edinburgh. The late Lady Gordon Cumming made extensive cxcavations at Lethen Bar, Nairnshire, providing many specimens for description by Agassiz, and forming the fine collection at present in the Forres Museum. An early worker in the flagstones of Orkney was Dr. T. S. Traill, whose collection furnished many types of species described by Agassiz, and was sold on the founder's death to various collectors; several of the missing types have been recognised by the authors of the present work among Orkney fossils that have finally reached the British Museum, but many important specimens still remain to be re-discovered. About twenty years ago the late Mr. Charles W. Peach obtained a large series of specimens from the flagstones of Wick and John o' Groats, at present forming part of the collections in the British Museum, the 
Museum of Practical Geology, and the Museum of Science and Art, Edinburgh; and, in I\$61, the Rev. J. M. Joass, LL.D., of Golspie, discovered a new fish-bearing horizon at Edderton, near Tain, Ross-shire. Within the last few years, Mr. Jex, collector to Mr. Damon, of Weymouth, has made successful excavations in the well-known nodule beds of Lethen Bar (Nairn), Tynet Burn (Banff), and Gamric (Banff), and thu greatly enriched the British Museum and the Museum of Scicnce and Art, Edinburgh ; and the Worcester Muscum owes a small but fine series of Thurso fossils to the generosity of Dr. Erlin Clarke. The Lower Old Red Sandstone of Forfarshire has been explored by Mr. James Powrie, F.G.S., of Reswallie, whose fine collection contains the types of several new gencra and species described by himself and l'rofessor Ray Lankester, F.R.S.; the late Rev. Hugh Mitchell also obtained scveral specimens from the same beds; and Mr. Jex has more recently collected a fine series, now preserved in the British Museum and the Museum of Science and Art, Edinburgh.

The Upper Old Red Sandstone of Elgin and Nairn yields many fragmentary remains of fossil fishes, and the early collections of Robertson, Malcolinson, and Patrick Duff, furnished several of the type specimens described by Agassiz. The Robertson Collection was dispersed by auction in London, about 1854 , and some of the specimens were purchased by the British Museum, while others ultimately reached the Geological Society; several of the types, however, are missing. The Malcolmson Collection forms a small series in the Forres Nuseum, and that of the late $P$. Duff is now for the most part incorporated with the collection of Mr. Powric, a few specimens being also in the Geological Society's Muscum. About forty years ago, Najor Lambert Brickenden collected a fine scries of Holoptychian and Astcrolepid remains from Scat Craig, still preserved in his private cabinet; and quite recently. Dr. R. H. Traquair has secured for the Edinburgh Nuseum 
many unique specimens of Astcrolcpis from the Nairn quarries. The well-known fish-bearing horizon of Dura Den, Fifeshire, was first cxhaustively explored under the direction of the Rev. John Anderson, D.D. (author of Dura Den, I 859 ), and the principal specimens obtained are now in the British Muscum, the Museum of Practical Geology, and the University Museum, St. Andrews. Numerous isolated discoveries in the Upper Old Red Sandstone of the South of Scotland, now enriching the museums of London and Edinburgh, were also made early in the century by the Rev. James Noble, the Rev. Professor J. Fleming, and others.

In the South of Scotland both the Upper and Lower Carboniferous formations furnish abundant remains of Fishes and Amplibia, which have been known for a long period, and are well represented in the Museums of Glasgow, Edinburgh, and London. Among the earliest collectors were Professor Fleming (the discoverer of Megalichthys), Dr. S. Hibbert (the explorer of the Burdiehouse Limestone), Lord Greenock (who investigated the Wardie nodules), and Dr. Rankin, of Carluke (who especially studied the Coal-Mcasures). Of these, the collections of Fleming and Lord Greenock are now in the Museum of Science and Art, Edinburgh; that of Rankin, in the Hunterian Museum, Glasgow; and that of Hibbert, unfortunately not traceable. Mr. James Thomson, F.G.S., and Mr. John Young, F.G.S., of Glasgow, have made large collections of Fishes and Amphibia from the Coal-Measures of Lanarkshire; Mr. R. Craig, of Beith, has explored the Carboniferous Limestone of Ayrshire, and obtained many Sclachian tecth; the Geological Survey of Scotland has made an important collection of fishes from the Calciferous Sandstones of Eskdale, Dumfrics; and the British Museum is indebted to Mr. Damon's experienced collector, Mr. Jex, for a very fine series from the same formation and locality. Dr. Hunter, of Braidwood, also has a large collection of 
fish-remains from the Scottish Carboniferous; the Edinburgh Museum has been greatly enriched during recent years by the labours of Dr. R. H. Traquair, F.R.S., in that district; and numerous specimens have been obtained from the same prolific field by Messrs. T. Stock, W. T. Kinnear, and others. In England, the late Mr. Thomas Atthey made the unique collection of Amphibia and Fishes from the Northumberland Coal-field now in the Newcastleupon. Tyne Museum; and for many years Mr. T. P. Barkas, F.G.S., systematically collected from the same area, presenting some specimens to the British Museum, others to the Newcastle Museum, and retaining others in his private collection. Mr. John Simm and Mr. T. Cragges, of IVest Cramlington, and Mr. Joseph Taylor, of Shire Moor, have also done much in their respective districts; and to Mr. Cragges is due the series of microscopical sections of teeth from the Coal-Measures, described by Sir Richard Owen in I867, and now in the British MIuscum. The finest collection of English Coal-Mcasure Fishes, however, is that of Mr. John IVard, F.G.S., of Longton, who, by donation and exchange, has supplied specimens to most of the principal Museums in the country. Mr. Ward's collection comprises a large number of type specimens described by Egerton, Young, and Traquair, and is also unique from the circumstance that the precise horizon of each fossil is recorded.* The Coal-Measures of Yorkshire have been especially investigated by Mr. James W. Davis, F.G.S., of Halifax, who has a large collection, comprising the types of species made known in various papers. The Lower Carboniferous of England and Wales yiclds only fragmentary fish-rcmains, chicfly the tceth and spines of sharks, well represented in the British Museum and the Museums of Bristol and York. The Yoredale Rocks of Richmond and Wensleydale, Yorkshire, have becn cxplored

* A complete catalogue is published by J. Ward, Trants. N. Stuffs. Inst. Mining Engineers, vol. x, 1889, with plates. 
by the late Mr. E. IVood, F.G.S., of Richmond, and by Mr. William Horne, F.G.S., of Leyburn, whose collections are now in the York Museum; and a series of teeth entirely detached from matrix has been obtained from South Derbyshire by Mr. Edward Wilson, F.G.S., this being now in the British Museum. The limestone of Oreton, Shropshire, was explored by the late Messrs. T. Baugh, of Bewdley, and Weaver Jones, of Cleobury Mortimer, whose collections are also in the British Museum; and the Bristol limestone is especially well represented in the Museum of that city, where many of the types described by Agassiz are preserved. In the Carboniferous Limestone of North Wales, Mr. G. H. Morton, F.G.S., has discovered several Selachian teeth; and in the North of Ireland, the late Admiral Jones and the Earl of Enniskillen made the unique collection of Selachian teeth and spines now in the British Museum and the Museum of the Geological Society of London.

The Permian fishes of Durham scem to have been first collected by Mr. Henry Witham, of Lartington, whose fossils form the subject of the plates accompanying Sedgwick's classic memoir on the Magnesian Limestone. A large series is preserved in the Newcastleupon-Tyne Museum, as aiso in the private collection of Mr. Nilliam Dinning, of Newcastle; and there is a good typical series in the British Museum. The Newcastle Museum is largely indebted to the labours of Mr. R. Howse* and Mr. James WV. Kirkby, the former of whom, in conjunction with the late Mr. Albany Hancock, was the first to make known the discovery of Reptilia and Amphibia in the English Permian.

British Triassic Vertebrata are very rare, and chiefly represcnted in the Museums of Warwick, Shrewsbury,

* R. Howse, Guide to the Collections of Local Fossils in the Muscum of the Natural History Society, Barras Bridge, Neacasticution-Tyne, i889. 
Bristol, and Elgin, the British Muscum, Museum of Practical Geology, and the Edinburgh Muscum of Science and Art. Fishes are few and scattered, one prolific stratum only having been discovered near Nottingham.* Labyrinthodonts constitute a leading feature of the Warwick Museum; while the Reptilian skeletons met with in the Elgin Sandstones are most nearly complete, and have been principally obtained through the rescarches of the Rev. George Gordon, LL.D., of Birnie. The only known specimens of Telerpeton, except the type, were discovered by Mr. James Grant, of Lossicmouth, in whose collection they are still preserved.

British Rhætic Fishes and Reptiles occur only in a very fragmentary state, and are well represented in the British. Museum and the Museums of Bath, Bristol, and Leicester. The E. T. Higgins Collection of Aust Cliff fossils at Bristol comprises an unique scries of tecth of Ceratodus; and the Charles Moore Collection at Bath, from the Rhretic fissure near Frome, contains the tecth of the British Rhretic Mammal, Microlestes moorei.

Both Fishes and Reptiles are met with in certain horizons of the English Lias in several localitics. The late Mr. Thomas Hawkins collected the Liassic Ichthyosauria and Plesiosauria forming the greater part of the series in the British Muscum; and the late Mr. James Harrison, of Charmouth, discovered and disentombed the fine skeleton of Scelidosanrus in the same collection. Miss Mary Anning was for several years a well-known collector at Lyme Regis; Miss Philpot made a large collection of Lyme Regis fossils, afterwards presented to the Oxford Museum; and Messrs. James and Henry Marder, for a long period, supplied many Muscums and private cabinets from the same locality. The combined collections of the late Earl of Enniskillen and Sir Philip

* E. Wilson, 'Notes on the Triassic Beds at Colwick Wuod, near Nottingham,' Quart. Joum. Geol. Soc, vol. xliii, ISS7, p. $5+2$. 
Egerton, now in the British Museum, form the most cxtensive series of Fossil Fishes from the English Lias hitherto obtained, and include many type specimens described by Agassiz and Egerton. The Warwick Museum has a noteworthy collection from the Lias of the district; the Leicester Museum possesses a typical series of Fishes and Reptiles from Barrow-on-Soar, lately much extended by the researches of the curator, Mr. Montagu Browne, F.G.S.; and the fine collection of the late Mr. William Lee, made in the same locality, is now in the Dublin Museum of Science and Art. The collection of the late Mr. Charles Moore, F.G.S., in the Bath Museum, comprises an extensive series of similar fossils from Somersctshire and Gloucestershire, all in an unusually fine state of preservation. The Upper Lias of Whitby is well represented in the Muscums of that town, Malton, and York, as also in the British Museum. Foremost among the collectors may be mentioned the late Messrs. Ripley and Brown Marshall, of Whitby; and, as the result of researches pursued for a period of fifty ycars, Mr. Martin Simpson, curator of the IVhitby Museum, has been able to define the stratigraphical horizons in which the remains occur, besides adding many specimens to the local collection.*

The most extensive series of remains from the Lower Oolites are those in the Oxford Museum, the British Muscum, and the private collections of Mr. James Parker, F.G.S., of Oxford, and Mr. Thomas Jesson, F.G.S., of Northampton. The Middle and Upper Oolites are especially represented in the British Museum, the Nuseums of Cambridge and Oxford, and the private collections of Mr. Marshall Fisher, of Ely, and Mr. Alfred N. Leeds, of Eyebury, near Peterborough. Mr. Fisher has obtained a large series of remains, especially marine Reptilia, from the Kimeridge Clay of Ely; and

* M. Simpson, The Fossils of the Yorkshire L.ius, I855 (and ed. 2, $\left.1 \delta S_{4}\right)$. 
with the exception of the great Omosanrus, due to the generosity of the Directors of the Swindon Brick and Tile Company,* the British Museum owes all its finer Reptilian fossils from the Kimeridge Clay of more southern areas to the rescarches and liberality of Mr. J. C. ManselPleydell, F.G.S., who has also presented a Pliosaurian paddle to the Dorchester Museum. The late Dr. Henry Porter, of Peterborough, obtained various Teleosaurian, Ichthyosaurian, and Plesiosaurian fossils from the Oxford Clay of that neighbourhood, now for the most part in the Woodwardian Museum, Cambridge; and the unrivalled collection of Mr. Alfred N. Leeds, made in the same locality, comprises not only Dinosauria and numerous nearly complete skeletons of Teleosauria, Ichthyosauria, and Plesiosauria, but also many fish-remains.

The Purbeck Vertebrata of Sivanage seem to have been first systematically collected by the late Messrs. W. R. Brodie and C. IVilcox, and the formation is well represented in the Museums of London, Cambridge, and Dorchester. The most extensive scries of Mammalian fossils and the remains of dwarf Crocodiles, now in the British Museum, were discovered by Mr. S. H. Beckles, F.R.S.; and, as the result of systematic purchases, the late Earl of Enniskillen and Sir l'hilip Egerton accumulated the unique collection of Fossil Fishes from the same horizon, now also in the British Museum. Fossil Fishes likewise occur in the Purbeck Beds of the Vale of Wardour, Wiltshire, and were collected many years ago by the Rev. P. B. Brodie, F.G.S., and more recently by the Rev. W. R. Andrews, F.G.S., of Teffont, in whose private collections the majority are still preserved.

The Vertebrata of the Wealden were first collected in the classic neighbourhoods of Cuclifield and Hastings, in

* W. Davies, 'On the Exhumation and 1)evelopment of a large Reptile (Omosaurus armatus, Owen), from the Kimeridge Clay, Swindon, Wilts,' Ecol. Ilag. [2] vol. iii, I876, p. I93, pls. vii, viii. 
Sussex, usually referred to as 'Tilgate Forest.' Dr. Gideon A. Mantell, F.R.S., the discoverer of Iguanodon and Hylaosaumus, Mr. G. B. Holmes, of Horsham, and Mr. S. H. Beckles, F.R.S., of St. Leonard's, obtained most of the Reptilian fossils described in the early memoirs of Mantell, Melville, and Owen, while Major Lambert Brickenden also added a few noteworthy specimens. The Mantellian Collection was purchased by the British Museum in 1838 and $\mathrm{I} 853$; that of the late Mr. Holmes has recently been acquired by the Brighton Muscum; and that of Mr. Beckles. is still at the residence of its founder at St. Leonard's. The Rev. William Fox, of Brixton, collected numerous remains of Dinosaurs from the Wealden cliffs on the south coast of the Isle of Wight, discovering the fine examples of Ornithopsis, Polacanthus, Hypsilophodon, and other genera now in the British Museum; while Mr. J. W. Hulke, F.R.S., has obtained an important series of bones from the same locality, including the type skeleton of Iguanodon seelyi and other specimens described in his own memoirs. During recent years, Mr. Charles Dawson, F.G.S., has greatly extended the collection of the British Museum, by adding numerous discoveries from Sussex localities, including the type specimens of three new species of Iguanodon.

Except in the Potton Bone-bed, which chiefly consists of remanic fossils from the Kimeridge Clay, Vertebrata of Lower Greensand age are rare in Britain, the only unique specimens being the type skeleton of Iguanodon mantelli and the group of Dinosaurian bones from Hythe (Dinodocus mackesoni), discovered by Mr. H. B. Mackeson, F.G.S., and presented by him to the British Museum. The Upper Cretaceous formations, however, have proved more prolific, the Cambridge Greensand consisting largely of reptilian bones and teeth, and the Gault and various divisions of the Chalk furnishing numerous specimens both of Reptiles and Fishes. The Cambridge Greensand is naturally best represented in the Woodwardian Muscum, Cam- 
bridge ; but there are numerous fine fragments also in the British Museum, Museum of Practical Geology, York Museum, and the private cabinet of Mr. Thomas Jesson, F.G.S., of Northampton; and few collections of importance are destitute of a typical series of spccimens. The Vertebrate fossils of the Gault are more satisfactorily preserved than those of the Cambridge Greensand, and are represented in the British Museum, Museum of Practical Geology, and the Woodwardian Museum, to which they have been chiefly supplied, either directly or indirectly, by the experienced collector, Mr. John Griffiths, of Folkestone. Chalk fossils are seen in most Museums, and the Fishes usually occur in an exquisite state of preservation. The late Dr. Gideon A. Mantell, F.R.S., as in the case of the Wealden Vertebrata, was a pioneer in the collection of those of the Chalk of Sussex; and the numerous fine examples of his patient work of extrication now in the British Museum are described and figured both by Agassiz and by himself. Mr. Frederic Dixon, of Worthing, author of the well-known Geology and Fossils of Sussex, $\uparrow$ the late Dr. J. S. Bowerbank, F.R.S., and Mrs. Smith, of Tunbridge Wells, also obtained the type specimens of many of the species of Fossil Fishes and Reptiles discovered in the Chalk of Sussex, Kent, and Surrey, at present, with rare exceptions, preserved in the British Museum; and for a long period Mr. Henry Willett, F.G.S., of Brighton, has been engaged in collecting the unrivalled series of Chalk fossils, now presented to the Brighton Museum. $\neq$ In more recent

* H. G. Seeley, Index to the Fossil Remains of Aves, Ornithosauria, and Reptilia .... in the Woodwardian Museum, 1869; also Ornithosauria, 1870.

+ This work is dated 1850 , but was not published until after the author's death, and, according to Morris and Mr. William Davies (in litt.), was not issued before 1852 .

$\ddagger$ In the first edition of Dixon's Geology of Silssex, and in some of the memoirs of Owen and Egerton, Mr. Willett is referred to under his earlier surname of Catt. 
years Mr. F. Harford has prepared for study a large number of Fossil Fishes from the Chalk of Kent, lately acquired by the British Museum; and Mr. S. J. Hawkins, F.G.S., has a private collection of similar specimens beautifully extricated from the matrix. Many collectors and museums are indebted to Mr. Joseph Wood for the discovery of fine specimens in the Chalk of Kent.

Eocene Vertebrata are abundant both in the London and Hampshire Basins, and most Museums have representative series. In the early part of the century, Bowerbank collected from the London Clay of Sheppey, and Dixon from the Bracklesham Beds of Sussex and the Barton Clay of Hampshire; Mr. Searles V. Wood, F.G.S., author of the Crag Mollusca, explored the cliffs of Hordwell; the late Barbara, Marchioness of Hastings, chiefly with the help of Mr. Henry Keeping, obtained a large series of Reptilian and Mammalian fossils from the Upjer Eocene of Hordwell and the Hempstead Beds of the Isle of Wight; and the late Earl of Enniskillen and Sir Philip Egerton accumulated an extensive collection of fish-remains from various formations and localities in both basins. All these collections, which comprise the types of most species of British Eocene Vertebrata hitherto described, have been finally acquired by the British Museum, that of Mr. Searles Wood being presented and the others obtained by purchase. To Mr. W. H. Shrubsole, F.G.S., of Sheerness, palæontologists are mainly indebted for the numerous discoveries of Vertebrata in the London Clay of Sheppey during the last twenty years, and all his principal specimens are also now in the British Museum.

The Crag Deposits of Suffolk and Norfolk yicld the remains of Pliocene Vertebrata mingled with those of Miocene and Eocene age, derived from earlier strata. Since the opening of the phosphate diggings in I 840 , large collections have been made by the late Messrs. James Baker and W. Whincopp, of Woodbridge, these being acquired by 
Mr. Edward Charlesworth, F.G.S., and afterwards purchased by the British Muscum, Museum of Practical Geology, Woodwardian Museum, and the York Museum. The collection of the Rer. H. Canham is now in the Ipswich Muscum; Mr. IV. Colchester, F.G.S., has obtained many specimens, still in his private cabinet; and the unique serics at York is due to the generosity of Mr. IVilliam Reed, F.G.S., who has for many years made special efforts to render the collection complete. The only supposed British Pliocene Vertebrate discovered elsewhere than in the Eastern counties is the Elephas meridionalis from Dewlish, Dorset, of which a tooth was presented to the British Museum by Mr. J. C. Mansel-Pleydell, F.G.S., in I 888.

The Mammalian and other fossils of the Norfolk Forest Bed have long been collected, and among the principal explorers of this deposit may be mentioned the late Mr. Samuel Woodward, the late Revs. C. Green, J. Layton, and S. W. King, the Rev. John Gunn, F.G.S., Mr. Randall Johnson, Mr. R. Fitch, F.G.S., Mr. A. Savin, of Cromer, Mr. J. J. Colman, of Corton, Mr. James Backhouse, F.G.S., of York, and Mr. Clement Reid, F.G.S., of the Geological Survey. The Green and Layton Collections are now in the British Museum; that of the Rev. S. W. King, in the Museum of Practical Geology; and those of the late Samuel Woodward and the Rev. John Gunn, in the Norwich Museum. The collections of Messrs. Fitch, Savin, and Backhouse are still in the possession of their respective founders, and that of Mr. Randall Johnson is now for the most part in the cabinct of Mr. J. J. Colman, M.I'., of Corton.

Pleistocene Mammalia occur in all Muscums, and a general sketch of their distribution, so far ascertained to the year 1869 , is given by Professor Boyd Dawkins, F.R.S.* The large collections of the late Mr. John Brown, of Stanway, from Esscx; of Mr. J. J. Owles, of Great

* W. Boyd Dawkins, 'On the Distribution of the British Postglacial Mammals,' Quart. Journ. Geol. Soc., vol. xxv, 1869, p. 192. 
Yarmouth, from the Dogger Bank; ${ }^{*}$ and of Sir Antonio Brady, from the Thames Valley, $\dagger$ are now in the British Museum. The collection of Dr. Spurrell and Mr. Flaxman C. J. Spurrell, F.G.S., made in the neighbourhood of Crayford, is still in their possession at Belvedere, and comprises several specimens described by Falconer, Dawkins, and Sanford. The fine series of remains obtained by Mr. Henry Keeping from the river gravels of Barrington, near Cambridge, is for the most part in the Woodwardian Museum and in the British Museum; Dr. Blackmore's discoveries in the river gravels of Fisherton, near Salisbury, are preserved in the Salisbury Museum; and most of the early isolated discoveries by private collectors, noticed in Owen's British Fossil Hammals and Birds, have finally reached the British Museum. The most extensive series of Mammalian remains from the Turbary of Walthamstow, Essex, is also in the British Museum, having been collected by Mr. Joseph Wood.

The largest collections of Pleistocene Mammalia have been obtained from caverns, $\$$ in the exploration of which Mr. Whidbey (or Whitby) and Dr. Buckland were the pioneers in Britain. At the instigation of Sir Joseph Banks, Mr. Whidbey explored the cave of Oreston, near Plymouth, and obtained the remains described by Sir Everard Home and Mr. W. Clift in the Philosoplical Transactions for 1817 and 1823 . The researches of the Oxford professor in the cave of Kirkdale, Yorkshire,\| led to the discovery of a large series of remains, now in the Museum of the University; and the somewhat later investigations

* IV. Davies, 'On a Collection of Pleistocene Mammals dredged off the Eastern Coast,' Geol. Mlag. [2] vol. v, i 878, p. 97.

+ IV. Davies, A Catalogue of the Pleistocene Vertebrata in the Collection of Sir Antonio Brady, I874.

$\pm \mathrm{H}$. Woodward, "The Freshwater Deposits of the Lea Valley, Gool. Llag., vol. vi, I869, p. 385.

$\S$ W. Boyd Dawkins, Car'e Hunting, 1874.

IV. Buckland, Reliquice Diluwiance, I822. 
of the Rev. J. MacEnery in Kent's Hole, near Torquay, were rewarded by the unearthing of numcrous similar specimens, which were sold on that gentleman's death, and chiefly reached the British Museum and the Museum of the Geological Socicty.* The Caverns of Brixham and Kent's Hole have been systematically explored by Committees of the Royal Society and the British Association, under the superintendence of Mr. WV. Pengelly, F.R.S.; the principal specimens met with being presented to the British Museum, while a representative serics is preserved in the Museum of the Natural History Society of Torquay. The Caverns of the Mendip Hills were explored by the Rev. D. Williams and Messrs. W. Beard, IV. Ayshford Sanford, F.G.S., and Professor Boyd Dawkins, F.R.S., whose discoveries now enrich the Taunton Museum; and the well-known Cresswell Caves, near Worksop, Derby. shire, have furnished to the Rev. J. M. Mello, F.G.S., Professor Boyd Dawkins, F.R.S., and Mr. T. Heath, the fine series of remains at present in the Museum of the Owens College, Manchester. The Victoria Cave, near Settle, was investigated by a Committee of the British Association, the resulting collection being now in the Museum of the Giggleswick Grammar School. From a cave in Teesdale Mr. James Backhouse, F.G.S., has made an important collection, now in his private Museum at York; $\dagger$ and the contents of the fissures of Windy Knoll, Derbyshire, and Raygill, Yorkshire, are at present respectively in the Museums of Manchester and Leeds. The latest discoveries of Messrs. E. Bouverie Luxmoore, F.G.S., and Dr. Henry Hicks, F.R.S., in the caves of the Vale of Clwyd, near St. Asaph, have recently been presented to the British Museum. All the principal collections of Verte-

* J. MacEnery, Cavern Researches, with numerous plates, edited by $\mathrm{E}$. Vivian, $\mathbf{1} 859$.

† J. Backhouse and W. Davies, Geol. Mag. [2] vol. vii, I8So, p. 346 , pls. xi, xii. 
brata from the caves and superficial deposits of Ireland are now in the Dublin Museum of Science and Art.*

While doubtless far from complete, the foregoing enumeration of the sources from which the investigator of Vertebrate Palæontology in Britain may glean materials, suffices to show the ample character of the means for pursuing the subject. Most of the collections mentioned have been consulted during the progress of the present work; and though the magnitude of the task prevents its being a critical resumé throughout, it is hoped that no labour has been spared in attempting to record the correct interpretation of doubtful points. Though all references are given at sufficient length to be understood without the aid of an index, the information has been condensed as far as possible, and the following statement will explain the general plan of the work :-

(i) The genera and species are arranged alphabetically under their respective classes.

(ii) All generic and specific names at present accepted are printed in antique roman type, while the crossreferences to synonyms, and all undefined names, are printed in bold itclics.

(iii) Under each genus the synonyms are printed in SMaLL CAPITALS, and under each species in italics.

(iv) When ascertainable, the nature and present whereabouts of the type specimen of each accepted species are stated in square brackets after the record of the locality; and in the case of synonyms, the types are similarly noticed immediately after the references. When the species at present exists, or when the type is a foreign specimen, the nature of the evidence on which that species is recorded as a British fossil is printed in italics.

(v) The type species of every extinct genus, whether valid or not, is marked by an antique $\mathbf{T}$.

* V. Ball, 'On the Collection of the Fossil Mammalia of Ireland in the Science and Art Museum, Dublin,' Trans. Roy. Dublin Soc. [2] vol. iii (1885), pp. $333-350$, pl. xi. 
In attempting the execution of this plan, considerable difficulties have been met with in all departments, and the writers would claim indulgence for the numerous imperfections which critical studies must soon reveal. The publication of undefined names, for instance, has been a source of much inconvenience, especially among the Reptilia, in which a single University Museum is responsible for no less than seventy meaningless terms; and the numerous hasty publications upon the same subject, which have appeared within recent years, have tended in no small degree to add to the confusion.

While wholly responsible, however, for whatever imperfections the work may contain, the writers are deeply indebted to the kindness of numerous friends and specialists in various departments, who have freely given advice, and, in many cases, read the proofs. To Mr. William Davies thanks are especially due for continual help, he having read the whole of the proofs, and given the writers the benefit of his long experience in connexion with the Fossil Vertebrata in the British Museum. To Dr. R. H. Traquair, F.R.S., Mr. James W. Davis, and Mr. John Ward, the writers are cspecially indebted for information concerning Palæozoic Fishes; and the accepted synonymy of the Coal-Measure Amphibia of Northumberland has been contributed by Mr. William Dinning, of Newcastle. The proofs of the Dinosaurian Reptiles have been read by $\mathrm{Mr}$. J. W. Hulke, F.R.S.; and Mr. R. Lydekker has favoured the writers with continual advice concerning the higher Vertebrata, besides kindly permitting reference to numerous unpublished proof sheets. The section on Aves has been read by Mr. R. Bowdler Sharpe and Mr. E. T. Newton, to the former of whom is due the accepted nomenclature of the recent species. To Mr. G. A. Boulenger the writers are indebted for the revision of the nomenclature of recent Amphibia and Reptilia, in addition to numerous other notes; and $\mathrm{Mr}$. Oldfield Thomas has kindly advised on all 
doubtful points concerning the synonymy and nomenclature of the Mammalia. Professor W. Boyd Dawkins, F.R.S., and Mr. E. T. Newton have also read the proofs of the Mammalian section, and pointed out many emendations resulting from their own special researches; while Mr. Alfred Beli has contributed a series of notes and references, from which a few important omissions have been supplied.

For much information concerning type specimens and old collections the writers are indebted to the Rev. George Gordon, LL.D., of Birnie, Mr. James Powrie, of Reswallie, Mr: Edward Wilson, of Bristol, Mr. H. M. Platnauer, of York, Mr. W. Rupert Jones, of the Geological Society, and many others; and thanks are especially due to Mr. B. B. Woodward, of the Natural History Library of the British Museum, as also to Mr. J. Saunders, for much valuable help in bibliographical researches. Finally, the writers would tender their acknowledgments to Mr. F. Justen, of Messrs. Dulau \& Co., to whose kindly interest is due the publication of the work in its present form.

LONDON,

December 3 Ist, I889. 


\title{
Dates of publication of
}

'Recherches sur les Poissons fossiles'. . par L. Agassiz.

\author{
(Text) Tome I. $-V . \quad$ (Atlas) Tome I.-V.
}

4to. Neuchâte! et Solcure, $18_{33}-43$ [44.]

[Contributed by W. H. Brown.*]

Tome I (Text), pp. i-xlix; I-I $\$ 8$, I $833-44$.

Pp. i-xii, I-16 (I), I 833 .

I7-40 (II), 1834 .

i-xxxii, I-I88 (xvil, xviII), I 844 (a note was published at this time cancelling the previous parts of the text).

xxxii-xlix were separately published, as 'Tableau général des Poissons fossiles,' in 1840 .

Tome I (Atlas), 1833-44.

Tab. A-G (I), I 833 .

$\mathrm{H}, \mathrm{J}, \mathrm{K}(\mathrm{XVIII}), \mathrm{I} 844$.

Tome II (Text pt. I), pp. i-xii, I-310, I $833-44$.

Pp. i-xii (xvil, xviII), $18+4$.

I-48 (I), 1833 .

49-84 (II), I 834 .

$85-200(\mathrm{v}), 1835$.

105, IO5 (carton)(XVII, XVIII), $\mathrm{I} S 44$.

$20 \mathrm{I}-224$ (VI), I 836 .

225-264 (VIII, IX), I 837 .

249,250 (carton) (XVII, XVIII), 1844.

265-306 (XVII, XVIII), I844.

Tome il (Text pt. 2), pp. I-336, $1843-44$.

I-72( $\mathrm{XV}, \mathrm{XVI}), \mathrm{I} 843$.

73-336 (XV1I, XVIII), I844.
Tome II (Pt. I, 2), (Atlas), I 833-44.

Tab. A, I3 (I), I 833 .

$\mathrm{B}^{\prime}, \mathrm{B}^{\prime \prime}(\mathrm{XV}, \mathrm{XVI}), \mathrm{I} 843$.

C (I), 1833 .

$\mathrm{C} a(\mathrm{XV}, \mathrm{XVI}), \mathrm{I} 843$.

D (VI), I 836 .

$\mathrm{E}$ (XVII), I 844 .

F (II), I834.

$\mathrm{G}(\mathrm{XV}, \mathrm{XVI}) \mathrm{I} 843$.

$\mathrm{H}$ (XVII), I 844 .

$\mathrm{J}$ (XVIII), I844.

I (1), 1833 .

I $a, b$ (VIII, IX), I 837 .

$1 i-e(11,1836$.

2 (I), 1833.

$2 a(11), 1836$.

$2 b-t(\mathrm{vi}), \mathrm{1} 836$.

$3,+4(\mathrm{I}), 1833$.

$4 a$ was not published.

$4 b, c(\mathrm{rI}), 1836$.

5-7 (I), I 833 .

$8(\mathrm{VI}), 1836$.

9 (III), I 834 .

IO, IO a (II), I $\$_{3} 6$.

$106(111), 18 j 6$.

Io $c$ (VI), $18 j 6$.

I I, 12 (Tab. I , Palaconiscus Freieslebini, Ag., is erroneously lettered as belonging to Tome III), (II), 1834 .

* Mr. Brown having lately been occupied in assisting in the preparation of the geological slips for the Library Catalogue of the British Miseum (Nitural History), has had special faciities for the compilation of this list, and the writers would express their thanks to him for the valuable contribution. It is based partly upon some original unbound fascicules of Agassiz' work, and partly upon notices in the Neues Jahrbuch. The roman numeral in round brackets immediately preceding the date in each entry is the number of the livraison (see p. xxix). The 'carton' refers to a correction of the original text. 


\section{Tome II (continued) -}

Tab. I3, I4 (III), I834. I4 $a$ (VII), 1836 . ${ }_{14} b, c(V 1),{ }_{3}{ }_{3} 6$. I 5,16 (II), I 834 . I7 (XVIII), I 844 . I8 (XVII), I $8+4$. 19 (XVIII), 1844. 20 was not published. 2 I (v) 1835 .

$2 \mathrm{I} a$ (VH1), $\mathrm{I} 836$. 22 (II), 1834. $23(\mathrm{v}), 1835$. $23 a$ (XVIII), I 844. $23 b-c(x V, x V I), I S+3$. $23 d(\mathrm{VI}), 1836$.

$23 d$ (bis) (Tetragonolepis Leachii, Ag. ; erroneously lettered as $23 e)(\mathrm{x}, \mathrm{X} I \mathrm{I})$, 1839 .

$23 e$ (XVII), 1844 .

24 (IV), I835.

$25,25 a, b(\mathrm{VI}), 1836$.

$25 c(\mathrm{~V}), \mathrm{I} 835$.

$25 d, e(\mathrm{VI}), \mathrm{I} 836$.

26 (II), 1834.

$26 a$ (VII), 1836 .

27 (II), 1834.

$27 a$ (XVIII), I 844 .

28,29 (II), I 834 .

$29 a$ (VII), I 836 .

$29 b, c$ (XVIII), I 84 .

$30(11), 1834$.

$30 a-c(\mathrm{v}), 1835$.

$3 \mathrm{I}(\mathrm{XV}, \mathrm{XV1}), 1 \mathrm{~s}+3$.

32,33 (II), 1834 .

$33 a(\mathrm{XV}, \mathrm{XVI}), \mathrm{I} s_{+3}$.

$33 b(\mathrm{x}, \mathrm{XII}), \mathrm{I}_{3}$ 9.

34 (III), I834.

$34 a$ (XVIII), I 844 .

$35(\mathrm{v}), 1835$.

$36-38$ (xv, xvi), 1843 .

39 (Pholidophoms liechei, Ag., Ph. onychizs, Ag.; erroneously lettered as 49) (VIII, IX), I 837.

40 (II), I 834 .
Tome II (continued) -

Tab. $4 \mathrm{I}(\mathrm{X}, \mathrm{XII}), \mathrm{I} 839$.

$42(\mathrm{XI}), 1838$.

$42 a(\mathrm{XV}, \mathrm{xVI}), \mathrm{I} 843$.

43 (Pholiatophorus latimamus, Ag. ; erroneously lettered as 42) (XI), I838.

$44(\mathrm{XV}, \mathrm{XVI}), \mathrm{I} 843$.

45-47 (XIV), I 842 .

$47 a$ (XVIII), 1844.

48 (VIII, IX), I837.

49 (IV), I 835.

50, $5 \mathrm{I}(\mathrm{X}, \mathrm{XII}), \mathrm{I} 839$.

$5 \mathrm{I} a(\mathrm{Xiv}), \mathrm{I} 842$.

$52,53,53 a(\mathrm{X}, \mathrm{XII}), 1839$.

54,55 (IV), I 835 .

$55 a$ (XVIII), 1844 .

$56(\mathrm{X}, \mathrm{XII}), \mathrm{I} 839$.

$56 a$ (XIV), I842.

57 (X, XII), 1839.

$57 a-b(\mathrm{X} 1 \mathrm{~V}), \mathrm{I} 842$.

$58,58$ «( $\mathrm{X}, \mathrm{XI1}), 1839$.

58 b (XVIII), $18+4$.

$59(\mathrm{X}, \mathrm{XII}), 1 \$ 39$.

$59 a(\mathrm{XIV}), \mathrm{I} 842$.

60 (IV), 1835 .

$6 \mathrm{I}, 6 \mathrm{I} a, 62,63(\mathrm{x}, \mathrm{XII}), \mathrm{I} 839$.

$63 a$ (XVIII), I 844 .

$64(\mathrm{X}, \mathrm{XII}), 1839$.

$65(\mathrm{~V}), \mathrm{I} \&_{35}$.

$65 a$ (XVII), 1844 .

65 a (bis) (lucropoma Mantelli, Ag., erroneously lettered as 65a), 65b-d (VIII, IX), I 837 .

66 (Thrissops intormedius, $v$. Münst, erroneously lettered as 65) (X, XII), 1839. $66 a$ (VIII, IX), 1837 .

67-69, 69 a (Phyllodus, Gyronchus, Gyrodus ; erroneously lettered as 60 a) (X, X1I), I 839.

$69 b$ (XI11), 1839 .

$69 c(x I V), 18+2$.

$70-72,72 a, 73,74(\mathrm{x}, \mathrm{xII})$, 1839 .

75 (XIV), I $8+2$. 
Tome III (Text), pp. i-viii, I-390, I-32 (Table), I 837-44.

Title (XVII, XVIII), I 844 .

Pp. i-viii, I-72 (VIII, IX), I837.

I $3^{-1} 4$ (carton) (XVII, XVIII), 1844 .

$73^{-140}(\mathrm{XI}), 1838$.

I $41-156(\mathrm{X}, \mathrm{XII}), \mathrm{I} 839$.

I $57-\left(382^{*}, 382^{* *}\right) 390$, and

Table des Matières, etc.,

I-32 (XV, XVI), 1843 .

207,208 (carton) (XVII, XVIII), 1844 .

Tome III (Atlas), i 835-47.

Tab. A (XVII), I844.

B, C (VIII, IX), I837.

D (IV), I 835 .

E-H (v), I 835 .

$\mathrm{J}-\mathrm{M}, \mathrm{M}^{\prime}, \mathrm{M}^{\prime \prime}, \mathrm{N}-\mathrm{Q}$ (XVII), 1844 .

R, S (XVIII), I 844 .

I $(\mathrm{XV}, \mathrm{XVI}), \mathrm{I} 843$.

$\mathrm{I} a(\mathrm{XI}), \mathrm{I} 838$.

2-8 (VII), 1836 .

$8 a, b(\mathrm{XI}), \mathrm{I} 838$.

9 (X, XII), I 839 .

Io (VII), I836.

Iо $a, b$ (VHI, IX), I 837 .

II, 12 (VII), 1836 .

I3, 14 (VIII, IX), I8 37 .

I 5 (XI), 1838 .

I6 (VIII, IX), I 837 .

17 (XI), 1838 .

I 8 (Xv, xvi), I 843 .

19, $20(\mathrm{XI}), \mathrm{I} 838$.

2 I (VHII, IX), I 837 .

$22,22 a, b$ (Xv, XVI), I 843 .

23 (X, XII), I 839 .

24 (XI), 1838 .

25 (X, XII), I 839 .

$25 a$ (V11I, IX), 1837 .

$25 b(\mathrm{xI}), \mathrm{i} \delta_{3} \mathrm{~S}$.

$26(\mathrm{v}), \mathrm{I} 835$.

$26 a(\mathrm{xv}, \mathrm{xvI}), \mathrm{I} 843$.

$27-29(v), 1835$.
Tome III (continued)-

Tab. $30(\mathrm{XI}), 1838$.

$30 a(\mathrm{X}, \mathrm{XII}), \mathrm{I} 839$.

$31-35(\mathrm{XI}), \mathrm{I} 838$.

$36,37,37 a(\mathrm{XVII}), \mathrm{I} \$ 44$.

38 ( $\mathrm{XV}, \mathrm{XVI}), \mathrm{I} 843$.

$39,40(\mathrm{XI}), \mathrm{I} 838$.

to a (VIII, IX), 1837 .

$40 b-d(\mathrm{XV}, \mathrm{XVI}), \mathrm{I} S_{43}$.

4I-44 (VII), I 836 .

$45(\mathrm{XV}, \mathrm{XVI}), \mathrm{I} 843$.

46 (IV), I 835 .

$47(\mathrm{XV}, \mathrm{XVI}), 1843$.

Tome IV (Text), pp. i-xvi, I-269, I-22 (Table), I833-44.

Title (xvII, XVIII), 1844 .

Pp. i-xvi, I-I6, I6* (X, XII), I 838 .

I5, I6 (carton) (XVII, XVIII), I 844 .

I 7-32 (I), I 833 .

33-52 (IV), I 835 .

53-108 (VI), I 836 .

IO9-204 (XIII), 1839.

205-29I (I-22, Table des

Matières) (XIV), I842.

292-296 (XVII, XVIII), I $\$ 44$.

Tome IV (Atlas), I833-34.

Tab. A (1), I 833 .

B (III), I 834 .

C, D (VIII, IX), I 837 .

$\mathrm{E}$ (II1), 1834 .

$\mathrm{F}$ (VIII, Ix), I837.

G (III), 1834 .

$\mathrm{H}$ (VIII, IX), I $\$ 37$.

$\mathrm{J}(\mathrm{v}), 1835$.

$\mathrm{K}, \mathrm{L}$ (VIII, IX), IS37.

I, 2 (I), I 833 .

3-6 (II), I 834 .

7 (III), I834.

\&, 9 (VIII, IX), I $\$ 37$.

IO, II (III), I 834 .

I I $a($ VIII, IX), I 837 .

I2 (III), I 834 .

I 3 (VIII, IX), I 837 .

I4 (III), I 834 . 
Tome IV (continued)-

Tab. $14 a-c$ (VIII, IX), I 837 . 15-17 (XIII), 1339 . $16 a$ and 18 were not published. I9, 20 (XVIII), I $8+4$. 21,22 (III), I834. 23 (IV), 1835 .

$23 a$ was not published. $23 b(\mathrm{XV}, \mathrm{XVI}), \mathrm{I} 8+3$. 24,25 were not published. 26-36 (xI1), I 839 . $37(\mathrm{v}), 1837$.

$37 a(\mathrm{XII}), \mathrm{I} 839$.

38 (IV), I 835 .

39 (XIII), 1839.

40 (r), 1835 .

4I, 4 I $a(X 111)$, IS39.

$42,43(\mathrm{v}), 1835$.

44 (XIII), I 839 .

$44 a$ was not published.

Tome I" (Text pt. I), pp. i-xii, I-I22, 1833-44.

Title, pp. i-xii, I-I6/h (XviI, XVIII), I $8+4$.

Pp. I7-24 (I), I833.

25-32 (II), I 834 .

31-32 (carton), 33-I22 (Xvil, XiIII), IS+H.

Tome 1 (Text pt. 2), pp. I-I60, $1838-4 ;$.

I'i. I-56 ( $\mathrm{x}, \mathrm{x} 1 \mathrm{i}), \mathrm{I} 839$.

$3^{-4}$ (carton) (xiII, XVIII), I $8+4$.

$57-8+(\mathrm{Xr}, \mathrm{XVI}), \mathrm{I} 8+3$.

$85-160$ (XVI1, XVIII), I $8+4$.

Tome I' (Atlas),

Tial). A (I), 1833.

H, C (Xv, XVI $), 1843$.

L) (VIH, IX), I 837 .

$\mathrm{E}(\mathrm{XY}, \mathrm{XVI}), \mathrm{I} 8+3$.

Fi (1V), 1835 .

G $(V I 1,1 X), I 837$.

H, J, K (xv, XVI), 1843 .

L I I. I 835 .

M (XI:1), I $8+2$.

\section{Tome V (continued)-}

Tab. I, 2 (1), I 833. 3-6 (II), I 834 . 7,8 (III), I834. 9, $10(\mathrm{XV}, \mathrm{XVI}), \mathrm{I} 843$. I I (III), I 834 . II $a($ IV), 1835 .

I2, I 3 (III), 1834 .

I4 (V), I835.

I5 (XIII), 1839 .

I6-18 (XIV), I842.

$19(v), 1835$.

$20(\mathrm{IV}), 1835$.

21, 2 I a (Nemoptery $x$ elongatus, Ag. ; erroneously lettered as $2 \mathrm{I}$ ), 22 (XIV), 1842.

$23(\mathrm{~V}), 1835$.

24 (IV), I 835.

25 (V), 1835 .

$25 a-c$ (VIII, IX), I 837.

26 (XIII), I 839.

27 (V), 1835 .

$2 S, 29(\mathrm{XV}, \mathrm{XVI}), \mathrm{I} 8+3$.

30, 3 I (XIII), I 839 .

$32-34, \quad 34 a, 35-37, \quad 37 a$ (XIV), 1842 .

$37 b$ (Engraulis exolans, Ag., Clupea macropoma,

Ag. ; erroneously lettered as 37) (XV, XVI), I 843 . 38-42 (X, XII), I839. 43 (XIII), I839.

44-48 (XV, XVI), I 843 . 49 (XII), I 839. $50-51(\mathrm{NV}), \mathrm{I} 835$. 5 I $a-c$ (XVIII), I 844 . $52(\mathrm{v}), \mathrm{I} 835$

$53(\mathrm{x}, \mathrm{xII}), 1839$. 54-59 (IV), 1835. $60(\mathrm{XI}), 1838$. $60 a-c($ VIII, IX), I 837 . $60 d(\mathrm{x}, \mathrm{xII}), \mathrm{x} 839$. 61 (Xi1), 1839. 62-64 (XIV), I839. 
Supplemental Sheets were also issued to the work, under the title, 'Feuilleton additionel su. les Recherches sur les Poissons fossiles.'

Pp. I-20 (II), I 834 . $21-64$ (IV), 1835 . $65-74(\mathrm{v}), \mathrm{I} 835$. $75-86(\mathrm{VI}), \mathrm{I} 836$. $87-98$ (VII), I 836 .
Pp. 99-106 (VIII, IX), 1837. $107-126(\mathrm{X}-\mathrm{XII}), 1838-39$.

127-130 (XIII), I 839 . I3I-I 38 (XIV), I 842 . I39-I 46 (XV, XVI), I 843 .

\section{DATES OF PUBLICATION OF THE LIVRAISONS.}

Livr. I, I 833 .

II, 1834 .

III, I834.

IV, 1835 .

$\mathrm{V}, \mathbf{1} 835$.

VI, I 836.
Livr. VII, 1836 .

VIII \& IX (united), I 837.

X \& XII

(united), I 839 .

$\mathrm{XI}, \mathrm{I} 838$.
Livr. XIII, 1839 .

XIV, I842.

$\mathrm{XV} \& \mathrm{XVI}$

(united), 1843 .

XVII, I844.

XVIII, I 844 .

In a letter to the Neues Jahrbuch, I $\$ 43$ (pp. 198, I99), L. Agassiz states that the following work is to form a Supplement to the 'Recherches sur les Poissons fossiles,' etc. :-

Monographic des Poissons fossiles du Vieux Grìs Rouge ou Systène Dévonien (Old Red Sandstone) des Iles Brittaniques et de Russie. Par L. Agassiz.

Text, pp. xxxvi, I71. 4to. Neuchâtel \& Solcure, I844-45.

Atlas, 43 pls., fol., Neuchâtel, 1844-45.

(Text),

Pp. i-viii, I-72 (I, II), I 844 . ix-xxxvi, 73-17 I (III), I 845 . (Atlas),

Tab. A-D (I, II), I844.

E, F (III), 1845 .

I-I 8 (I, II), I844.
Tab. I0 (III), 1845 .

2O, 2 I (I, II), I 844 .

$2 \mathrm{I} a$ (III), 1845.

22 (I, II), 1844.

23,24 (III), I 845 .

$25(\mathrm{I}-\mathrm{II}), \mathrm{I} 8+4$.

$26-33(111), 1845$.

Dates of publication of 'Odontographyy', by' R. Oiven.

We also append a note of the dates of publication of Richard Owen's Odontography; or a Treatisi on the Comparative Anatomy of the Teeth, etc. (8vo., London, I840-1845), as some confusion seems to have occurred in quotation:-

Part i : I 840 ; pp. I-I I2, pls. I-50.

Part ii : I84I ; pp. i-xl, 1 I3-288, pls. 5I-89 A (excl. 62 A).

Part iii : 1845; Title page, pp. xli-lxxiv, 289-655, pls. i, ii, 62 A, 90-I 50 .

(Some of the plates in part iii bear the date 1844 at the bottom.) 


\section{A TABLE \\ Showing the Stratigraplical Distribution of the Genera of British Fossil Vericbrata.}

[In this table some minor horizons have been separately entered on account of the special character of their Vertebrate Fauna. A few generic names are placed in square brackets in lists from beds from which they are not recorded in the Catalogue; most of these occurrences being hitherto unpublished.]

UPPER SILURIAN.

PrSCES.-Auchenaspis, Cephalaspis, Cyathaspis, Eukeraspis, Onchus, Scaphaspis, Sphagodus, Thelodus.

LOWER OLD RED SANDSTONE (PASSAGE BEDS).

PISCES.-Auchenaspis, Cephalaspis, Onchus.

LOWER OLD RED SANDSTONE.

Pisces.--Acanthodes, Iyssacanthus, Cephalaspis, Cephalopterus, Cheiracanthus, Cheirolepis, Climatius, Coccosteus, (?) Cyathaspis, Didymaspis, Diplacanthus, Diplopterus, Dipterus, Glyptolepis, Gyroptychius, Holaspis, Homosteus, Ischnacanthus, Kallostrakon, Microbrachius, Onchus, Onychodus, Osteolepis, Parexus, Pteraspis, Pterichthys, Scaphaspis, Thursius, Tristicopterus.

UPIER OLD RED SANDSTONE.

Pisces.-Actinolepis, Asterolepis, Bothriolepis, Conchodus, Cosmacanthus, Cricodus, Dendrodus, Glyptolamus, Glyptopomus, Holoptychius, Phaneropleuron, Phyllolepis.

1)EVONIAN.

PISCES.-Phyllolepis, Steganodictyum.

\section{I,OVER CARBONIFEROUS.}

PISCES.-Acanthodes, Acondylacanthus, Acrolepis, Aganacanthus, Archichthys, Asteroptychius, Callopristodus, Canobius, Chalazacanthus, Cheirodopsis, Cheirodus, Chomatodus, Chondrenchelys, Cladodus, Cochliodus, Coelacanthus, Colonodus, Copodus, Cosmacanthus, Cosmoptychius, Cryphiolepis, Ctenacanthus, Ctenodus, Ctenoptychius, Cycloptychius, Cynopodius, Cyrtonodus, Deltodus, Deltoptychius, Dicentrodus, Diclitodus, Dicrenodus, Diplacodus, Diplodus, Dipriacanthus, Echinodus, Elonichthys, Erismacanthus, Euctenius, Eurynotus, Fissodus, Ganopristodus, Glossodus, Glyphanodus, Gnathacanthus, Gomphacanthus, Gonatodus, Gyracanthus, Harpacanthus, Helodus, Holurus, Homacanthus, Isodus, Janassa, Lispacanthus, Megalichthys, Mesolophodus, Nematoptychius, Oracanthus, Orodus, Osteoplax, Petalodus, Petalorhynchus, Petrodus, Phanerosteon, Physonemus, Platysomus, P'leuracanthus, Pleuroplax, l'ocilodus, Polyrhizodus, Pristodus, Psammodus Psammosteus, 
Psephodus, Rhadinichthys, Rhamphodus, Rhizodus, Sandalodus, Sphenacanthus, Stichacanthus, Streblodus, Strepsodus, Tarrasius, Tomodus, Tristychius, Uronemus, Venustodus, Wardichthys, Xystrodus.

Aмphibia.-Loxomma, Pholidogaster, and Genus non det. (\%. MisceLLANEOUS AMIPHIBIA).

UPPER CARBONIFEROUS.

Pisces.-Acanthodes, Acanthodopsis, Archichthys, Callopristodus, Cheirodus, Colacanthus, Ctenodus, Ctenoptychius, Cycloptychius, Diplodus, Elonichthys, Euctenius, Gonatodus, Gyracanthus, $\mathrm{He}-$ lodus, Hoplonchus, Hybodopsis, Janassa, Lepracanthus, Megalichthys, Mesolepis, Ostracacanthus, Petalodus, Platysomus, P'leuracanthus, Pleuroplax, Rhadinichthys, Rhizodopsis, Sphenacanthus, Stemmatodus, Strepsodus.

Aмrpibia.-Anthracosaurus, Anthrakerpeton, Batrachiderpeton, Dolichosoma, Erpetocephalus, Ichthyerpeton, Keraterpeton, Lepterpeton, Loxomma, Megalerpeton, Ophiderpeton, Pholiderpeton, Pteroplax, Urocordylus.

PERMIAN.

Pisces.-Acentrophorus, Acrolepis, Cuelacanthus, Dorypterus, Globulodus, Janassa, Palieoniscus, Platysomus, Pygopterus, Wodnika.

Amphibia.-Dasyceps, Lepidotosaurus.

REPTILIA.-Protorosaurus.

TRIAS.

PISCES.-Acrodus, Ceratodus, Dictyopyge, Diplodus, Dipteronotus, Semionotus.

AMPHIB1A.-Dasygnathus, Diadetognathus, Labyrinthodon, Mastodonsaurus, Rhombopholis.

REPTILIA.-Cladyodon, '?) Dicynodon, Hyperodapedon, Palæosaurus, Rhynchosaurus, Stagonolepis, Telerpeton, Thecodontosaurus, (?) Zanclodon.

RH.ETIC.

PISCES.-Acrodus, Ceratodus, Gyrolepia, Hybodus, Legnonotus, Nemacanthus, Palixospinax, Pholidophorus, Sargodon, Saurichthys, [Semionotus].

Amphimia.-(?) Metopias.

RePtilia.-(?) Ichthyosaurus, Palæosaurus, Plesiosaurus, Psephoderma, Rysosteus, (?) Zanclodon.

Mammalia.-Microlestes.

LIAS.

Pisces.-Acrodus, Arthropterus, Belonorhynchus, Browneichthy's, Centrolepis, Chondrosteus, Cosmolepis, Cyclarthrus, Dapedius, Endactis, Eugnathus, Gyrosteus, Harpactira, Holophagus, Hybodus, Isocolum, Lepidotus, Leptolepis, Mesodon, Myriacanthus, Nothosomus, Osteorhachis, Oxygnathus, Pachycormus, Palæospinax, Pholidophorus, Platysiagum, Ptycholepis, Squaloraja, Tetragonolepis, Thrissonotus.

REPTILIA.-Dimorphodon, Eretmosaurus, Ichthyosaurus, Pelagosaurus, Plesiosaurus, (?) Scaphognathus, Scelidosaurus, Steneosaurus, Teleosaurus, Thaumatosaurus, (?) Zanclodon 


\section{OOLITES.}

PIsCES.-Acrodus, Aspidorhynchus, Asteracanthus, Belonostomus, Caturu;, Ceratodus, Cestracion, Coccoderma, Ctenolepis, Ditaxiodus, Eurycormus, Ganodus, Gyrodus, Hybodus, Hypsocormus, Ischyodus, Leedsichthys, Lepidotus, Leptacanthus, Leptolepis, Macrosemius, Mesodon, Microdon, Nemacanthus, Notidanus, Ophiopsis, Orthacodus, Pachycormus, Pholidophorus, Pristacanthus, Rhinobatus, Semionotus, Strobilodus, Strophodus, Thrissops.

RePtilia.-Bothriospondylus, Camptosaurus, Cardiodon, Cetiosaurus, Cimoliosaurus, Cryptodraco, Dacosaurus, Gigantosaurus, Ichthyosaurus, Machimosaurus, Marmarosaurus, Megalosaurus, Metriorhynchus, Omosaurus, Ophthalmosaurus, Ornithopsis, Pelobatochelys, Peloneustes, (?) Plesiosaurus, Pleurosternum, Pliosaurus, Protochelys, Pterodactylus, Rhamphocephalus, Stegochelys, Steneosaurus, Teleosaurus, Thalassemys, Thaumatosaurus, Tropidemys.

Mammalia.-Amphilestes, Amphitherium, Amphitylus, Phascolotherium, Stereognathus.

\section{PURBECK.}

PIsCEs.-Aspidorhynchus, Asteracanthus, Ceramurus, Coccolepis, Histionotus, Hybodus, Lepidotus, Leptolepis, Megalurus, [Mesodon,] Microdon, Ophiopsis, Oxygonius, Pholidophorus, Pleuropholis, Strobilodus.

RePTILIA.-Chelone, Doratorhynchus, Echinodon, Goniopholis, Hylæeochelys, Iguanodon, Macellodus, Nannosuchus, Niuthetes, Oweniasuchus, Petrosuchus, (?) Platychelys, Plesiochelys, Pleurosternum, Thalassemys, Theriosuchus, Tretosternum.

Mamiaria.-Achyrodon, Amblotherium, Bolodon, Kurtodon, Peralestes, Peramus, Plagiaulax, Spalacotherimn, Stylodon, Triconodon.

\section{WEALDEN}

Pisces.-Acrodus, Asteracanthus, Cœlodus, Hybodus, Lepidotus.

Reptilja.-Archrochelys, Aristosuchus, Calamospondylus, Camptosaurus, Cimoliosaurus, Coulurus, Goniopholis, Heterosuchus, Hoplosaurus, Hylæochampsa, Hyleochelys, Hylæosaurus, Hypsilophodon, Iguanodon, Megalosaurus, Morosaurus, Ornithocheirus, Ornithopsis, Pelorosaurus, I'holidosaurus, Plesiochelys, Pleurocolus, Polacanthus, Regnosaurus, Sphenospondylus, Suchosaurus, Thecospondylus, Tretosternum, Vectisaurus.

(?) Aves.-Ornithodesmus.

\section{POTTON BONE-BED.}

Pisces.--[Asteracanthus, Hybodus], Ischyodus, Lepidotus.

ReptrliA.-Cimoliosaurus, Craterosaurus, Dacosaurus, Iguanodon Megalosaurus, Pliosaurus.

\section{LOWER GREENSAND.}

PISCES.-Edaphodon, Hybodus, Scapanorhynchus, Synechodus.

RePtilia.-Dinodocus, Iguanodon, Polyptychodon, Protemys. 


\section{CANBRIDGE GREENSAND.}

PISCES.-Corax, Edaphodon, Hybodus, Ischyodus, Lamna, Notidanus, Oxyrhina, Plethodus, Scapanorhynchus.

ReptiliA.-Acanthophelis, Anoplosaurus, Cetarthrosaurus, Chelone, Cimoliosaurus, Eucercosaurus. Ichthyosaurus, (?) Lytoloma, Macrurosaurus, (?) Ophthalmosaurus, Ornithocheirus, Ornithostoma, Patricosaurus, Polyptychodon, Priodontognathus, Protemys, Protostega, Rhinochelys, Syngonosaurus, Trachodon.

Aves.-Enaliornis.

\section{GAULT AND UPPER GREENSAND.}

Pisces.-Acrodus, Cestracion, Cœlodus, Corax, Edaphodon, Eurypoma, Ischyodus, Lamna, Notidanus, Oxyrhina, Pachyrhizodus, Plethodus, Portheus, Scapanorhynchus, Squatina, Synechodus, Thrissopater.

RePTilia.-Cimoliosaurus, Hylæochelys, Ichthyosaurus, Ornithocheirus, Polyptychodon, Rhinochelys.

\section{CHALK.}

PISCES.-(?) Acrodus, Acrognathus, Ancistrodon, Aulolepis, Belonostomus, Berycopsis, Beryx, Calamopleurus, Cantioscyllium, Cestracion, Cimolichthys, Cladocyclus, Caelodus, Coelorhynchus, Corax, Dercetis, Edaphodon, Elasmodectes, Enchodus, Gomphodus, Gyrodus, Homonotus, Hoplopteryx, Ichthyodectes, Lamna, Lophiostomus, Macropoma, Microdon, Neorhombolepis, Notidanus, Osmeroides, Oxyrhina, Pachyrhizodus, Pholidurus, (?) Platax, Plethodus, Plinthophorus, Pomognathus, Portheus, Prionolepis, Protosphyræna, Ptychodus, Saurocephalus, Scapanorhynchus, Scyllium, Squatina, Stenostoma, Stratodus, Synechodus, Tomognathus, Xenodolamia.

RePtilia.-Acanthopholis, Chelone, Cimoliochelys, Cimoliosaurus, Coniasaurus, Dolichosaurus, Ichthyosaurus, Leiodon, Lytoloma, Mosasaurus, Ornithocheirus, (?) Platecarpus, Polyptychodon, Protostega, Saurospondylus.

\section{EOCENE.}

PIsces. - Acanthias, Acipenser, Aetobatis, Arius, Bucklandium, Carcharias, Carcharodon, Cestracion, Cœlorhynchus, Cybium, Dictyodus, Diplomystus, Edaphodon, Egertonia, Elasmodus, Galeocerdo, Galeus, Lamna, Lepidosteus, Megalops, Myliobatis, Myripristis, Notidanus, Odontaspis, Phyllodus, Pisodus, Platylæmus, Pristis, Psaliodus, Pycnodus, Rhinoptera, Sciænurus, Squatina, Teratichthys, Tetrapturus.

RePtilia.-Anosteira, Argillochelys, Chrysemys, Crocodilus, Dacochelys, Diplocynodon, (?) Emys, Eosphargis, (?) Gavialis, Iguana, (?) Lacerta, Lytoloma, Ocadia, Palæophis, Paleryx, Placosaurus, Podocnemis, Psephophorus, Pseudotrionyx, Thalassochelys, Trachyaspis, Trionyx.

Aves. - Argillornis, Dasornis, Gastornis, Halcyornis, Lithornis, Macrornis, Odontopteryx, Ptenornis. 
Mammatia. - Acotherulum, Adapis, Anchilophus, An plotherium, Anthracotherium, Argillotherium, (?) Balienoptera, Chœeropotamus, Coryphodon, Dacrytherium, Dichodon, Didelphys, Diplopus, Elotherium, Hyænodon, Hyopotamus, Hyracotherium, Lophiodon, Microchœrus, Neurogymnurus, Palæotherium, Platychoerops, Spalacodon, Theridomys, Viverra, Zeuglodon.

PISCES.-Acipenser. LOWER MIOCENE,

REPTILIA.-Diplocynodon.

Mammala.-Hyopotamus.

PLIOCENE.

Pisces.-Acipenser, Carcharodon, Cetorhinus, [Chrysophrys, Cœlorhynchus, ] (?) Gadus, Labrus, Myliobatis, Notidanus, Oxyrhina, Phyllodus, Platax, [Pristis,] Raja, Rhinoptera, Squatina.

Aves.-Diomedea, Uria.

Mammalia.-Ailurus, Balæna, Balienodon, Balænoptera, Bos, Canis, Castor, Cervulus, Cervus, Cetotherium, Choneziphius, Delphinus, Elephas, Equus, Eucetus, Felis, Gazella, Globicephalus, Halitherium, Herpetocetus, Hipparion, Hoplocetus, Hyæna, Hyænarctos, Hyperoodon, (?) Kogia, Lutra, Mastodon, Megaptera, Mesoplodon, Microtus, Orca, Physeterula, Physodon, Rhinoceros, Squalodon, Sus, Tapirus, Trichechus, Trogontherium, Ursus, Xiplodon.

\section{FOREST BED SERIES.}

Pisces. - Abramis, Acanthias, Acerina, Acipenser, Barbus, Esox, Gadus, Galeus, Leuciscus, Perca, Platax, Platessa, Raja, Tinca.

Amphibia.-Bufo, Molge, Rana.

RePtilia.-Emys, Tropidonotus, Vipera.

Aves.-Anas, Anser, Bubo, Colymbus, Phalacrocorax, Spatula.

Mammalia.-Alces, Balienoptera, Bison, Canis, Capreolus, Caprovis, Castor, Cervus, Delphinapterus, Delphinus, Elephas, Equus, (?) Felis, Gulo, Hippopotamus, Hyæna, Lutra, Machærodus, Microtus, Monodon, Mus, Mustela, Myogale, Ovibos, Phoca, Phocæna, Physeter, Rhinoceros, (?) Sciurus, Sorex, Spermophilus, Sus, Talpa, Trichechus, Trogontherium, Ursus.

PISCES.-Esox.

PLEISTOCENE.

RePTILIA.-Emys.

Aves.-Alca*, Anas*, Anser*, Cygnus, Haliaetus*, CEdemia*, Somateria*.

MammaliA.-Alces, Balænoptera*, Bison, Bos, Canis, Capreolus, Castor, Cervus, Delphinapterus*, Elephas, Equus, Eschrichtius*, Felis, (?) Globicephalus, Hippopotamus, Hyæna, Hyperoodon*, Lepus, Lutra, (?) Macacus, Machærodus, Megaptera*, Meles, Microtus, Monodon*, Mus, Mustela, Myodes*, Orca*, Ovibos, (?) Ovis, Phoca*, Rangifer, Rhinoceros, Sorex*, Spermophilus, Sus, Trichechus, Ursus, (?) Tursiops.

[Those marked with an asterisk may be only Prehistoric or Historic.] 
PREHISTORIC AND HISTORIC.

Aves.-Alca, Anser, Corvus, Pelecanus, Phalacrocorax, Sula.

Mammalia.-Alces, Balæna, Balænoptera, Bos, Canis, Capra, Capreolus, Castor, Cervus, Equus, Felis, Lepus, Lutra, Meles, Microtus, Mus, Mustela, (?) Ovis, Pseudorca, Rangifer, Sus, Ursus.

\section{CAVES}

(Includine Pleistocene, Prehistoric, and Historic).

Aves._Æx, Alauda, Anser, Bernicla, Columba, Colymbus, Corvus, Cygnus, Falco, Lagopus, Perdix, Scolopax, Somateria, Sturnus.

Mammalia.-Alces, Bison, Bos, Canis, Capra, Capreolus, Castor, Cervus, Cricetus, Elephas, Equus, Erinaceus, Felis, Gulo, Hippopotamus, Hyæna, Lagomys, Lepus, Lutra, Lycaon, Machærodus, Meles, Microtus, Mus, Mustela, Myodes, (?) Ovis, Rangifer, Rhinoceros, Rhinolophus, Sorex, Spermophilus, Sus, Ursus, (?) Vesperugo, 



\section{BRITISH FOSSIL VERTEBRATA.}

\section{PISCES.}

AgraAris, Cuvier, Rène Animal, vol. ii, I817, p. I94.

brama, Cuvier, ibid., p. I94; E. T. Newton, Vert. Forest Bed (Mem. Geol. Surv, I882), p. 126, pl. xviii, f. 19, 20.

Cyprinus brama, Linnæus, Syst. Nat., ed. 12, vol. i, 1756, p. 531. LOC. Forest-Bed Series (U. Freshwater Bed); West Runton, Norfolk. [Pharyngeal teeth; Mus. Pract. Geol.]

ACANTHALEPIS, F. M'Coy, Synopsis Silur. Foss. Ireland, I846, p. 7 .

jamesii, F. M'Coy, ibia., p. 7, pl. i, f. 1,2. [CYSTIDEAN.]

ACANTIIA3, J. A. Risso, Hist. Nat. Prod. Europe Mérid., vol. iii, I 826, p. 13 I.

orplensis : Notidanus orpiensis, T. C. Winkler, Archiv. Mus. Teyler, vol. iv, 1876, p. 12, pl. i, f. I3-17 ; A. Rutot, Ann. Soc. Roy. Malacol. Belg., vol. xi, 1876, p. 29, pl. i, f. 3.

Carcharius (Scoliodon) orpiensis, A. S. Woodward, Cat. Foss. Fishes B. M., pt. i, I889, p. 436.

Loc. Woolwich and Reading Beds; Kent. [Tecth; Brit. Mus.]

vulgaris, J. A. Risso, Hist. Nat. Prod. Europe Mérid., vol. ii, I826, p. I 3 I ; E. T. Newton, Vert. Forest Hed (Mem. Geol. Surv., 1882), p. 131, pl. xix, f. 8.

LOC. Weybourn Crag; East Runton, Norfolk. [Teeth; Mus. Pract. Geol.]

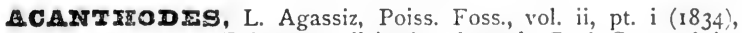
pp. 3, 19; F. Roemer, Zeitschr. deutsch. Geol. Ges., vol. ix, I8 37, p. 65.

Acanthoessus, L. Agassiz, Neues Jahro., 1832, p. 149.

Mesacanthus, R. H. Traquair, Geol. Mag. [3] vol. v, 1888, p. 5 II.

antiquus, Egerton. $\%$. Acanthodes mitchelli. 
ACANWFODIS (continued)-

bronni, Agassiz. Recorded (? in error) from Millstone Grit and L. Coal-Meas. by R. Etheridge, Foss. Brit. Islands, pt. i, I 888 , p. 319.

coriaceus, Egerton. v. Acanthodes peachi.

mitchell, P. M. G. Egerton, Rep. Brit. Assoc., I860 (186I), Sect., p. 77, and Figs. and Descript. Brit. Urganic Remains, dec. $x$ (Mem. Geol. Surv., 186i), p. 6r, pl. vii ; J. Powrie, Quart. Journ. Geol. Soc., vol. xx, 1864, p. 419, and Trans. Edinb. Geol. Soc., vol. i, 1870, p. 288, pl. x, f. 1.

Acanthodes antiquus, P. M. G. Egerton, Rep. Brit. Assoc., I859 (1860), Sect., p. I16 (name only).

Mesacanthus mitchelli, R. H. Traquair, Geol. Mag. [3] vol. v, I 888 , p. 512.

LOC. L. Uld Red Sandst.; Forfarshire. [Fish ; Brit. Mus.]

peachi, P. M. G. Egerton, Figs. and Descript. Brit. Organic Remains, dec. x (Mem. Geol. Surv., I86I), p. 57, pl. vi, f. I, 2.

Acanthodes coriaceus, P. M. G. Egerton, ibid., p. 59, pl. vi, f. 3-5. [Imperfect fish ; Mus. Pract. Geol.]

Mesacanthus peachi, R. H. Traquair, Geol. Mag. [3] vol. v, 1888, p. 5 I2.

LOC. L. Old Red Sandst. ; Caithness. [Fish; Mus. Pract. Geol.]

pustllus, L. Agassiz, Poiss. Foss. V. G. R. (1844), p. 36, pl. xxviii, f. 8-10; J. Morris, Cat. Brit. Foss., I854, p. 315.

Mesacanthus pusillus, R. H. Traquair, Geol. Mag. [3] vol. v, 1888 , p. 512

LOC. L. Old Red Sandst.; Banffshire. [Fishes; Forres Mus.]

sulcatus, L. Agassiz, Poiss. Foss., vol. ii, pt. i (1835), p. 125, pl. ic, f. I, 2 ; J. Morris, Cat. Brit. Foss., 1854, p. 315.

LOC. Calcif. Sandst.; Wardie, near Edinburgh. [Scales; Oxford Mus.]

wardi, P. M. G. Egerton, Quart. Journ. Geol. Soc., vol. xxii, I866, p. 468, pl. xxiii ; [-ii] J. Thomson, Trans. Geol. Soc. Glasgow, vol. iv (1871), p. 57, pl. iv; J. Ward, [Proc.] N. Staffs. Nat. Field Club, 1875, p. 24I ; J. W. Davis, Quart. Journ. Geol. Soc., vol. xxxii, 1876, p. 335.

LOC. Coal-Meas. ; N. Staffordshire, Yorkshire, and Lanarkshire. [Fish; J. Ward Coll.]

ACAMTrZODOPSIS, A. Hancock and T. Atthey, Ann. Mag. Nat. Hist. [4] vol. i, 1868, p. 364, and Nat. Hist. Trans. Northumb. and Durham, vol. iii (1870), p. 103; R. H. Traquair, Proc. Roy. Phys. Soc. Edinb., vol.v (1880), p. I17.

egertoni, Hancock and Atthey. $v$. Acanthodopsis wardi.

ward1, A. Hancock and T. Atthey (non Acanthodes wardi, Egerton), Ann. Mag. Nat. Hist. [4] vol. i, 1868, p. 364, pl. xv, f. 6, and Nat. Hist. Trans. Northumb. and Durham, vol. iii (1870), p. 103, pl. ii, f. 6. 
ACANTIXODOPSIS wardi (continued)-

Acanthodopsis egertoni, A. Hancock and T. Atthey, ibid., I868, p. 367, and ibid., 1870, p. 107. [Jaws, etc.; Newcastle-uponTyne Mus.]

LOc. Coal-Meas.; Newsham, near Newcastle-upon-Tyne. [Jaw; Newcastle-upon-Tyne Mus.]

T.

sp., R. H. Traquair, Proc. Roy. Phys. Soc. Edinb., vol. v (I880), p. 117 .

LOC. Coal-Meas.; South Scotland. [Jaws; Edinburgh Mus.]

ACENT及OPHoæ Us, R. H. Traquair, Quart. Journ. Geol. Soc., vol. xxxiii, 1877, p. 565 .

abbsi (Kirkby), R. H. Traquair, ibid., p. 565.

Palaoniscus abbsii, J. W. Kirkby, Ann. Mag. Nat. Hist. [3] vol. ix, I862, p. 268, and Quart. Journ. Geol. Soc., vol. xx, 1864, p. 355, pl, xviii, f. 3 .

Loc. Magnesian Limest.; Fulwell, Durham. [Fish; Newcastleupon-Tyne Mus.]

altus (Kirkby), R. H. Traquair, Quart. Journ. Geol. Soc., vol. xxxiii, 1877, p. 565.

Palconiscus latus, J. W. Kirkby (non Redfield), Ann. Mag. Nat. Hist. [3] vol. ix, 1862, p. 268.

Palconiscus altus, J. W. Kirkby, Quart. Journ. Geol. Soc., vol. $\mathrm{xx}, \mathrm{1} 864$, p. 356 , pl. xviii, f. I.

LOC. Magnesian Limest.; Fulwell. [Fish; Newcastle-uponTyne Mus.]

glaphyrus (Agassiz), R. H. Traquair, Quart. Journ. Geol. Soc., vol. xxxiii, 1877, p. 562.

Palaoniscus glaphyrus, L. Agassiz, Poiss. Foss., vol. ii, pt. i (1835), p. 98, pl. xc, f. I, 2 ; W. King, Permian Foss. (Pal. Soc., 1850 ), p. 224 , pl. xxii, f. 3,4 ; J. Morris, Cat. Brit. Foss., 1854, p. 337 ; F. M.Coy, Brit. Palæoz. Foss. (1855), p. 607.

LOC. Marl-Slate; Whitley and Midderidge. [Fish; York Mus.]

varians (Kirkby), R. H. Traquair, Quart. Journ. Geol. Soc., vol. xxxiii, 1877, p. 565.

Palconiscus varians, J. W. Kirkby, Ann. Mag. Nat. Hist. [3] vol. ix, 1862, p. 267, and Quart. Journ. Geol. Soc., vol. xx, I 864 , p. 353 , pl. xviii, f. 2.

Loc. Magnesian Limest.; Fulwell. [Fish ; Newcastle-uponTyne Mus.]

$\boldsymbol{r}$.

ACrris A, Cuvier, Règne Animal, vol. ii, 1817, p. 283.

? vulgaris, Cuvier and Valenciennes, Hist. Nat. Poiss., vol, iii (1829), p. 4, pl. xli ; E. T. Newton, Vert. Forest Bed (Mem. Geol. Surv., I882), p. I2 I, pl. xviii, f. 9.

LOC. Forest-Bed Series (U. Freshwater Bed); West Runton, Norfolk. [Otolith; Mus. Pract. Geol.] 
ACESTRE'S, L. Agassiz, Rep. Brit. Assoc., I $84+$ (1845), p. 308 (name only).

ornatıs, L. Agassiz, ibid., p. 308 (name only).

Loc. London Clay; Sheppey. [Cianium; Brit. Mus.]

I.

ACIPINSER, Linnæus, Syst. Nat., ed. 12, vol. i, 1\%66, p. 403.

toliapicus, L. Agassiz, Poiss. Foss., vol. ii, pt. ii (1843), p. 280 (name only); J. Morris, Cat. Brit. Foss., 1854, p. 316 ; A. S. Woodward, Proc. Geol. Assoc., vol. xi (1889), p. 28, pl. i, f. I.

Loc. London Clay; Sheppey. [Scutes; Brit. Mus.]

sp., E. T. Newton, Vert. Forest Bed (Mem. Geol. Surv., 1882), p. I29, pl. xix, f. 6 .

LOC. Forest-Bed Series; Norfolk. [Cephalic plate; Mus. Pract. Geol.]

sp., A. S. Woodward, Proc. Geol. Assoc., vol. xi (I889), p. 28, pl. i, f. 3. Loc. Red Crag ; Suffolk. [Pectoral spines; York Mus.]

sp., A. S. Woodward, Proc. Geol. Assoc., vol. xi (1889), p.28, pl.i,f. 2, LOC. Hempstead Beds; Isle of Wight. [Scute; Brit. Mus., no. P. 5283.]

sp., A. S.Woodward, Proc. Geol. Assoc., vol. xi(1889), p. 28, pl.i, f. 3 b. LOC. U. Eocene (Oligocene); Hampshire Basin. [Pectoral spine ; Brit. Mus., no. P. 1533a.]

ACONDYIACARTrUS, O. St. John and A. H. Worthen, Pal. Illinois, vol. vi, 1875 , p. 432 .

attenuatus, J. W. Davis, Trans. Roy. Dublin Soc. [2] vol, i (1883), p. 352, pl. xlvi, f. 3 .

Loc. L. Carb. Limest. ; Armagh. [Spine ; Woodwardian Mus.]

cole1, J. W. Davis, Trans. Roy. Dublin Soc. [2] vol. I (1883), p. 347 , pl. xlv, f. 7 , pl. xlvi, f. 1 .

LOC. L. Carb. Limest. ; Armagh. [Spine ; Brit. Mus.]

distans (M'Coy), J. W. Davis, Trans. Roy. Dublin Soc. [2] vol. i (1883), p. 349, pl. xlvi, f. 5.

Ctenacanthus distans, F. M'Coy, Ann. Mag. Nat. Hist. [2] vol. ii, 1848 , p. 116 , and Brit. Palæoz. Foss. (1855), p. 625, pl. iii $\mathrm{k}$, f. 15 ; J. Morris, Cat. Brit. Foss., 1854, p. 323 .

LOC. I. Carb. Limest.; Armagh. Carb. Limest. ; Denbighshire. [Spine; Woodwardian Mus.]

jeakinsonl ( $\mathrm{M}^{\prime} \mathrm{C}$ oy), O. St. John and A. H. Worthen, Pal. Illinois, vol. vi, 1875 , p. 433 ; J. W. Davis, Trans. Roy. Dublin Soc. [2] vol. i (1883), p. 35 I, pl. xlvi, f. 2.

Leptacanthus jenkinsoni, F. M'Coy, Brit. Palæoz. Foss. (1855), p. 633, pl. iii G, f. 14-16.

LOC. Carb. Limest. ; Lowick, Northumberland. [Spine ; Woodwardian Mus.) 
ACONDYSACATTYZS (continued)-

Junceus ( $\left.\mathrm{M}^{\circ} \mathrm{C} n y\right)$, O. St. John and A. H. Worthen, Pal. Illinois, vol. vi, 1875 , p. 433 ; J. W. Davis, Trans. Roy. Dublin Soc. [2] vol. i ( 1883 ), p. 350 , pl. xlvi, f. 6 .

Leptacanthus junceus, F. M'Coy, Ann. Mag. Nat. Hist. [2] vol. ii, 1845, p. 122, and Brit. Palreoz. Foss. (1855), p. 633, pl. iii G, f. 13 ; J. Morris, Cat. Brit. Foss., 1854, p. 332.

Loc. Carb. Limest.; Derbyshire. [Spine; Woodwardian Mus.] tenuistriatus, J. W. Lavis, Trans. Roy. Dublin Soc. [2] vol. i (1883), p. 350 , pl. xlv, f. 8.

LOC. L. Carb. Limest. ; Armagh. [Spine ; Brit. Mus.]

tuberculatus, J. W. Davis, Trans. Roy. Dublin Soc. [2] vol. i (1883), p. $3+8$, pl. xlvi, f. 4.

LOC. L. Carb. Limest. ; Armagh. [Spine ; Brit. Mus.]

sp.: A specimen from the U. Carb. Limest. of Richmond, Yorkshire, probably indicating a new form. [Brit. Mus., no. 45652.]

ACrodus, L. Agassiz, Poiss. Foss., vol. iii (1838), p. 139 ; E. C. H. Day, Geol. Mag., vol, i, I864, p. 57 ; A. S. Woodward, Cat. Foss. Fishes B. M., pt. i, I889, p. 279.

Sphenonchus, L. Agassiz, Poiss. Foss., vol. iii (1843), p. 201 (pars).

Thictodus, H. v. Meyer and T. Plieninger, Beitr. Pal. Württembergs, 1844, p. 116.

ann!ngiæe, L. Agassiz, Poiss. Foss., vol. iii (1839), p. 175, pl. xxii, f. 4 : J. Morris, Cat. Brit. Foss., 1854, p. 316 ; E. C. H. Day, Geol. Mag., vol. i, 1864, p. 57, pls. iii, iv ; A. S. Woodward, Cat. Foss. Fishes B. M., pt. i, 1889, p. 289, pl. xiv, f. 4, pl. xv, f. I.

Acrodus undulatus, L. Agassiz, Poiss. Foss., vol. iii (1839), pp. I44, 175 ; J. Morris, Cat. Brit. Foss., 1854, p. 316.

Hybodus curtus, L. Agassiz, Poiss. Foss., vol. iii (1837), p. 49, pl. viii $b$, f. 4-6 (pars); J. Morris, Cat. Brit. Foss., 1854, p. 329. [Spines ; Brit. MIUs.]

LOC. L. Lias; Lyme Regis. [Teeth; Brit. Mus.]

cretaceus, Dixon. $\%$ Cestracion canaliculatus.

gibberulus, Agassiz. v. Acrodus nobilis.

h1rudo, L. Agassiz, Poiss. Foss., vol. iii (1839), p. 148, pl. xxii, f. 27 ; J. Morris, Cat. Brit. Foss., 1854, p. 316 ; A. S. Woodward, Geol. Mag. [3] vol. iv, 1887, p. 102, and Cat. Foss. Fishes B. M., pt. i, 1889, p. 296, pl. xiii, f. 9.

soc. Wealden; Sussex. [Tooth ; Brit. Mus.]

? lllingworthl, F. Dixon, Geol. Sussex, 1850, p. 364, pl. xxx, f. 11, 12, pl. xxxii, f. 9 ; J. Morris. Cat. Brit. Foss., 1854, p. 316 ; A. S. Woodward, Geol. Mag. [3] vol. iv, 1887, p. IOf, and Proc. Geol. Assoc., vol. x (I888), p. 290, and Cat. Fost. Fishes B. M., pt. i, 1880, p. 297.

LOC. Chalk ; Sussex, Surrey, and Kent. [Teeth; Brit. Mus.] 
ACRODUS (continued) -

? keuperinus (Murchison and Strickland), A. S. Woodward, Cat. Foss. Fishes B. M., pt. i, 1889, p. 28 I, pl. xiii, f. I, 2, and Ann. Mag. Nat. Hist. [6] vol. iii, I889, p. 297, pl. xiv, f. I-3.

Hybodus keuperinus, R. I. Murchison and H. E. Strickland, Trans. Geol. Soc. [2] vol. v (1840), p. 338 , pl. xxviii, f. 3 ; J. Morris, Cat. Brit. Foss., I 854 , p. 330.

'Tooth of Hybodus,' Murchison and Strickland, ibid., f. 3 *.

Lophodus, W. S. Symonds, Old Bones, 1861, p. 103.

Nemacanthus, S. J. Mackie, Geologist, vol. vi, 1863, p. 243.

LOC. Keuper; Worcestershire and Warwickshire. [Spine and tooth ; Geol. Soc. London.]

latus, Agassiz. \% Acrodus nobilis.

lelodus, L. Agassiz, Poiss. Foss., vol. i (I844), p. xxxviii (name only); A. S. Woodward, Geol. Mag. [3] vol. iv, I887, p. 10I, woodcut, and Cat. Foss. Fishes B. M., pt. i, 1889, p. 295, pl. xiii, f. 5-7.

LOC. Gt. Oolite (incl. Stonesfield Slate); Gloucestershire and Oxfordshire. Forest Marble; Somersetshire. [Teeth; Brit. Mus.]

leiopleurus, L. Agassiz, Poiss. Foss., vol. iii (1839), p. 145, pl. xxii, f. 5 ; J. Morris, Cat. Brit. Foss., I854, p. 316 ; A. S. Woodward, Geol. Mag. [3] vol. iv, 1887, p. 102, and Cat. Foss. Fishes B. M., pt. i, I889, p. 295, pl. xiii, f. 8.

LOC. Gt. Oolite ; Gloucestershire. [T ooth ; Bristol Mus.]

levis, A. S. Woodward, Geol. Mag. [3] vol. iv, 1887, p. 103, woodcuts 2, 3, and Cat. Foss. Fishes B. M., pt. i, 1889, p. 296, pl. xiv, f. 5-7.

LOC. Gault ; Folkestone. [Teeth ; Brit. Mus.]

mintmus, L. Agassiz, Poiss. Foss., vol. iii (1839), p. 145, pl. xxii, f. 6-12; J. Morris, Cat. Brit. Foss., 1854, p. 316 ; R. Etheridge, Proc. Cardiff Naturalists' Soc., vol. iii (1872), pl. ii, f. $15-17$; A. S. Woodward, Cat. Foss. Fishes B. M., pt. i, 1889 , p. 282, and Trans. Leicester Lit. and Phil. Soc., n.s., pt. xi, I889, p. 19.

LOC. Rhætic; S. W. England, and ? Linksfield, near Elgin. [Teeth ; Bristol Mus. (pars).]

nobilis, W. Buckland (ex Agassiz, MS.), Geol. and Min., ed. 2, I 837, vol. ii, p. 47, pl. xxvii $e$ (name and fig. only); L. Agassiz, Poiss. Fuss., vol. iii (1838), p. I40, pl. xxi ; J. Morris, Cat. Brit. Foss., 1854, p. 316 ; R. Owen, Rep. Brit. Assoc., 1838 (1839), Sect., p. 138, and Odontogr. (I840), p. 54 , pls. xiv, xv ; A. S. Woodward, Cat. Foss. Fishes B. M., pt. i, 1889 , p. 283 , pl. xiii, f. 3,4 , and pl. xiv, f. $1-3$.

á imaculum corrugosum, Luidius, Lithophyl. Brit., I760, p. 75, pl rv f. I503, 1504 . 
ACRODUS noblis (continued) -

Acrodus latus, L. Agassiz, Poiss. Foss., vol. iii (1839), p. I44, pl. xxii, f. I ; J. Morris, Cat. Brit. Foss., 1854, p. 316. [Teeth; Oxford Mus.]

Acrodus gibberulus, L. Agassiz, Poiss. Foss., vol. iii (1839), p. 144, pl. xxii, f. I-3; J. Morris, Cat. Brit. Foss., 1854, p. 316. [Teeth; Oxford Mus.]

Loc. L. Lias; Lyme Regis, and near Bath. [Dentition; Bristol Mus.]

T.

ornatus, A. S. Woodward, Cat. Foss. Fishes B. M., pt. i, I889, p. 296, pl. xiii, f. 10.

LOC. Wealden; Brixton, Isle of Wight. [Tooth; Brit. Mus.]

rugosus, Agassiz. v. Cestracion rugosus.

transversus, L. Agassiz, Poiss. Foss., vol. iii (1843), pl. x b, f. 4, 5 (figs. only); J. Morris, Cat. Brit. Foss., 1854, p. 316. [Indeterminable fossil.]

Loc. Chalk; Lewes.

undulatus, Agassiz. v. Acrodus anningiæ.

sp., P. Duff, Geol. Moray, I842, p. 63, pl. iv, f. 12, I3.

LOC. Rhætic or Lias; Linksfield, near Elgin. [Teeth ; olim J. Martin and P. Duff Colls.]

ACROGNATIUS, L. Agassiz, Poiss. Foss., vol. v, pt. ii (I843), p. 108 ; A. Günther, Introd. Study of Fishes, 1880, p. 631 .

boops, L. Agassiz, Poiss. Foss., vol.v, pt. ii (1843), p. 108, pl. lx $a$, f. I-4; J. Morris, Cat. Brit. Foss., I 854, p. 316; A. S. Woodward, Proc. Geol. Assoc., vol. x (1888), p. 323.

LOC. U. Chalk; S. E. England. [Portion of fish; Brit. Mus.]

ACRoIrPIS, L. Agassiz, Poiss. Foss., vol. ii, pt. i (1833), p. II, and ibid., pt. ii (1843), p. 79 .

exsoulptus, G.v. Münster, Beitr. Petref., pt.v, I 842 , p. 42 , pl.vi, f. 2. Recorded from Marl-Slate of Fulwell and Midderidge by R. Howse, Guide to Coll. Local Fossils Mus. Nat. Hist. Soc. Newcastle-upon-Tyne, 1889 , p. 24. [Fish; Nerucastleupon-Tyne M/us.]

hopkinst, F. M'Coy, Brit. Palrez. Foss. (1855), p. 6o9, pl. iii G, f. 10 .

Holoptychizs hopkinsii, F. M'Coy, Ann. Mag. Nat. Hist. [2] vol. ii, I848, p. 2 ; J. Morris, Cat. Brit. Foss., I854, p. 329.

LOC. U. Carb. Limest. ; Derbyshire. Yoredale Rocks ; Hebden Bridge, Yorkshire. [Scales; Woodwardian Mus.]

kirkbyi, R. Howse, Nat. Hist. Trans. Northumb. and Durham, vol. vii (1878), p. 17 I.

Acrolepis sp., J. W. Kirkby, Quart. Journ. Geol. Soc., vol. xx, 1864 , p. 350. 
ACROTrgPa lrirkbyi (continued) -

Acrolepis sedgwickii, J. W. Kirkby (non Agassiz), Ann. Mag. Nat. Hist. [3] vol. ix, 1862 , p. 26 9.

I.OC. Magnesian Limest.; Marsden Bay and Fulwell Hill. [Imperfect fish ; Newcastle-upon-Tyne Mus.]

rankinel, R. H. Traquair, Quart. Journ. Geol. Soc., vol. xxxiii, 1877 , p. 569.

? Gyrolepis rankinei, L. Agassiz, Poiss. Foss., vol. ii, pt. i (1843), p. 303 ; J. Morris, Cat. Brit. Foss., 1854, p. 328. [Type unknown.]

LOC. L. Carb. ; Carluke and Braidwood. [Scales, etc. ; Hunterian Mus., Glasgow, and Hunter Coll.]

sedgrwlcix, [-ii] L. Agassiz, Poiss. Foss., vol. ii, pt. i (1833), p. II, and ibid., pt. ii (1843), p. 8o, pl. lii ; [-ii] W. King, Permian Foss. (Pal. Soc., 1850), p. 234, pl. xxv, f. I. [See also $A$. kirkbyi.]

'Fossil Fish,' A. Sedgwick, Trans. Geol. Soc. [2] vol. iii (1829), pl. viii, f. 3,4 .

LOC. Permian; Durham. [Fish, wanting head.]

wllsoni, R. H. Traquair (ex J. Ward, MS.), Geol. Mag. [3] vol.v, I 888 , p. 254.

LOC. Yoredile Rocks; Turnditch, near Belper. [Scales; Brit. Mus.]

sp., Aitken. $v$. Elonichthys aitkeni.

sp., Kirkby. \%. Acrolepis kirkbyi.

ACROTEIINUS, Agassiz. v. Colodus.

faba, Agassiz. v. Colodus faba.

ACTrisorgers, L. Agassiz, Poiss. Foss. V. G. R. (1845), p. I4I. [? Asterolepis.]

tuberculatus, L. Agassiz, ibid., p. 141, pl. xxxi, f. 15-18, pl. xxxi $a$, f. 28 ; J. Morris, Cat. Brit. Foss., 1854 , p. 316. [? Asterolepis.]

Loc. U. Old Red Sandst.; Elgin. [Dermal plate ; olim Malcolmson Coll.]

ECHMODUS, Egerton. v. Dapedius.

angulifer (Agassiz), Egerton. $v$. Dapedius angulifer.

confuens (Agassiz), Egerton. v. Dapediús politus.

arsalis (Agassiz), Egerton. v. Dapedius dorsalis.

heteroderma (Agassiz), Egerton. v. Dapedius heteroderma.

leachi (Agassiz), Egerton. v. Dapedius leachi.

leiosomus (Agassiz), Egerton. v. Dapedius leiosomus.

mastodonteus (Agassiz), Egerton. v. Lepidotus mantelli. 
AECHIIODUS (continued)-

orbicularis, Morris. v. Dapedius orbicularis.

ovalis (Agassiz), Egerton. $\%$. Dapedius ovalis.

pholidotus (Agassiz), Egerton. v. Dapedius pholidotus.

pustulctus (Agassiz), Egerton. v. Dapedius granulatus.

radiatus (Agassiz), Egerton. \%. Dapedius radiatus.

speciosus (Agassiz), Egerton. v. Dapedius punctatus.

AzTORATIS, J. Miiller and F. G. Henle, Syst. Beschreib. Plagiostom., 1841, p. 179. [Atnbates, auct.]

Goniobatis, H. Le Hon, Prélim. Mém. Poiss. 'Tert. Belg., I87I, p. 10.

convexus, F. Dixon, Geol. Sussex, 1850, p. 200, pl. xi, f. 5; J. Morris, Cat. Brit. Foss., I854, p. 317 ; A. S. Woodward, Cat. Foss. Fishes B. M., pt. i, 1889 , p. 130.

I.OC. Bracklesham Beds; Sussex. [Lower teeth.]

1rregularis, L. Agassiz, Poiss. Foss., vol. iii (1843), p. 327, pl. xlvii, f. 3-5; F. Dixon, Geol. Sussex, 1850, p. 200, pl. x, f. 6-8, pl. xi, f. 2-4 ; J. Morris, Cat. Brit. Foss., 1854, p. 317 ; A. S. Woodward, Cat. Foss. Fishes B. M., pt. i, 1889, p. 128.

Actobatis rectus, F. Dixon, Geol. Sussex, I850, p. 201 , pl. xi, f. 8 ; J. Morris, Cat. Brit. Foss., 1854, p. 317. [Tooth; Brit. Mus.] LOC. London Clay; Sheppey. Bracklesham Beds; Sussex. Barton Clay ; Hampshire. [Upper teeth ; Brit. Mus.]

marginalis, F. Dixon, Geol. Sussex, 1850, p. 20I, pl. xii, f. I ; J. Morris, Cat. Brit. Foss., 1854, p. 317 ; A. S. Woodward, Cat. Foss. Fishes B. M., pt. i, 1889, p. 129.

LOC. Bracklesham Beds; Sussex. [Upper teeth; Brit. Mus.] rectus, Dixon. v. Aetobatis irregularis.

subarcuatus, L. Agassiz, Poiss. Foss., vol. iii (1843), p. 328 ; F. Dixon, Geology Sussex, 1850, pp. III, 201, pl. xi, f. 7 ; J. Morris, Cat. Brit. Foss., 1854, p. 317 ; A. S. Woodward, Cat. Foss. Fishes B. M., pt. i, I889, p. I 30.

Loc. Bracklesham Beds; Sussex. [Lower tooth; Brit. Mus.]

subconvexus, F. Dixon, Geol. Sussex, 1850, p. 200, pl. xi, f. 6 ; J. Morris, Cat. Brit. Foss., 1854. p. 317 ; A. S. Woodward, Cat. Foss. Fishes B. M., pt. i, 1889, p. 130.

LOC. Bracklesham Beds; Sussex. [Tooth; Brit. Mus.]

AGANACANTIZUS, R. H. Traquair, Geol. Mag. [3] vol. i, I884, p. 64 .

striatulus, R. H. Traquair, itid, p. 64.

LOC. M. Carb. Limest.; Borough Lee, near Edinburgh. [Spine; Edinburgh Mus.] 
AGANODUS, Owen. \%. Diplodus.

apicalis, Owen. $v$. Diplodus gibbosus.

undatus, Owen. v. Diplodus gibbosus.

AGELEODUS, Owen. v. Callopristodus.

dialema, Owen. $v$. Callopristodus pectinatus.

AMBLIPTERUS, L. Agassiz. [No known British species according to R. H. Traquair, Quart. Journ. Geol. Soc., vol. xxxiii, 1877 , p. 552.]

anconocechmodus, Walker. $\%$. Gonatodus punctatus.

nemopterus, Agassiz. v. Elonichthys nemopterus.

portlocki, Egerton. v. Elonichthys portlocki.

punctatus, Agassiz. v. Gonatodus punctatus and Elonichthys intermedius.

stvictus, Agassiz. $v$. Cosmoptyclius striatus.

AMBLIURUS, L. Agassiz. \%. Dapedius.

macrostomus, L. Agassiz, Poiss. Foss., vol. ii, pt. i (1837), p. 220, pl. xxve ; J. Morris, Cat. Brit. Foss., 1854, p. 317 . Founded upon a vertically crushed specimen of Dapcdius (A. S. Woodward, Ann. Mag. Nat. Hist. [6] vol. i, 1888, p. 356).

LOC. L. Lias; Lyme Regis. [Crushed fishes; Oxford Mus.]

T.

Amic lewesiensis, Mantell. \% Macropoma mantelli.

AMPHERISTES, C. König, Icones Foss. Sect., I825, pl. xv, f. 190 (name only).

toliapicus, C. König, ibid., f. I90 (name and fig. only); J. Morris, Cat. Brit. Foss., I854, p. 317.

LOC. London Clay ; Sheppey. [Portion of skull ; Brit. Mus.]

AMPIICENTRUM, Young. v. Cheirodus.

granulosum, Young. $v$. Cheirodus granulosus.

striatum, Hancock and Atthey. \%. Cheirodus striatus.

AaCISTRODON, F. Rnemer (ex Debey, MS.), Kreidebild. Texas, I 852, p. 30 ; W. Dames, Zeitschr. deutsch. geol. Ges., vol. xxxv, 1883, p. 655 .

sp., A. S. Woodward, Proc. Geol. Assoc., vol. x (1888), p. 330, pl. i, f. 10.

LOC. Chalk; Charing, Kent, and Lewes, Sussex. [Teeth; Brit. Mus.]

ANODONTACANTIIUS, Davis. v. Pleuracanthus.

acutus, Davis. $\%$. Pleuracanthus cylindricus.

fastigiatus, Davis. z'. Pleuracanthus lævissimus.

obtusus, Davis. ข. Pleuracanthus lævissimus. 
ANTLIODUS, Newberry and Worthen. $\%$. Petalodus.

Anthropodontoides brilesii, T. P. Barkas, Coal-Meas. Palront., 1873 , p. 45 , f. I6I, 162. Probably jaw of Phyllopod Crustacean, Dithyrocaris (R. Howse, in litteris).

ARCAIC FTrYYS, A. Hancock and T. Atthey, Ann. Mag. Nat. Hist. [4] vol. v, I870, p. 266, and ibid. [4] vol. vii, I871, p. 79, and Nat. Hist. 'Trans. Northumb. and Durham, vol. iv (1872), p. 201; W. J. Barkas, Monthly Rev. Dental Surgery, vol. v $(1876)$, p. 7.

[? Strepsodus.]

portlocki, [-ii] R. H. Traquair, Proc. Roy. Soc. Edinb., vol. ix, (1878), p. 657, and Trans. Roy. Soc. Ldinb., vol. xxx (1881), p. 18.

Holoptychius portlockii, L. Agassiz, Poiss. Foss., vol. i (1844), p. xxxvi (name only); J. E. Portlock, Geol. Londonderry, 1 843 , p. 464, pl. xiii, f. I-9; J. Morris, Cat. Brit. Foss., I854, p. 329 .

Rhizodus hibberti ?, F. M'Coy, Brit. Palæoz. Foss. (I855), p. 612. Rhizodus portlockii, R. Etheridge, Foss. Brit. Islands, pt. i, 1888, p. 34 I.

LOC. L. Carb.; Derry, Tyrone, Fermanagh, and Antrim. Calcif. Sandst. ; Liddesdale, Dumfriesshire, and Abden, Fifeshire. [Teeth and scales; Mus. Pract. Geol.]

sulcidens, A. Hancork and T. Atthey, Ann. Mag. Nat. Hist. [4] vol. vii, 1871, p. 79, and Nat. Fist. Trans. Northumb. and Durham, vol. iv (1872), pp. 201, 393.

Rhizodus, A. Hancock and T. Atthey (errore), Ann. Mag. Nat. Hist. [4] vol. v, 1870 , p. 266, and Nat. Hist. Trans. Northumb. and Durham, vol. iii (1870), p. 8 I.

LOC. Coal-Meas. ; Northumberland. [Mandib. ramus; Newcastle-upon-Tyne Mus.]

T.

ARIUs, Cuvier and Valenciennes, Hist. Nat. Poiss., vol. xv, r840, p. 53 .

? bartonensis, A. S. Woodward, Geol. Mag. [3] vol. iv, I887, p. 306, woodcut.

LOC. Barton Clay ; Hampshire. [Dorsal spine; Brit. Mus.]

crassus (Koken), E. T. Newton, Proc. Zool. Soc., 1889, p. 206, pl. xxi, f. I-3.

Otolithus (incertce sedis) crassus, E. Koken, Zeitschr. deutsch. geol. Gesell., vol. xxxvi, 1884, p. 559, pl. xii, f. 13.

LOC. Barton Clay; Hampshire. ? Headon Beds ; I. of Wight. [Otolith.]

egertoni (Dixon), A. S. Woodward, Geol. Mag. [3] vol. iv, 1887, p. 304, woodcuts I, 2; E. T. Newton, Proc. Zuol. Soc., 1889, p. 206. 
ARIUD egertori (continued)-

Silurus egertoni, F. Dixon, Geol. Sussex, i 850 , pp. I12, 204 , pl. xi, f. II-13 ; J. Morris, Cat. Brit. Foss., I8j4, p. 344.

LOC. Bracklesham Beds and Barton Clay ; Sussex and Hampshire. [Pectoral arch and spine; Brit. Mus.]

sp., E. T. Newton, Proc. Znol. Soc., r 889 , p. 205 , pl. xxi, f. 4-6 ; A. S. Woodward, Cat. Foss. Fishes B. M., pt. i, 1889, pl. iv, f. 4,5 .

Raja similis, A. S. Wnodward, Cat. Foss. Fishes B. M., pt. i, 1889 , p. 86 (errore).

LOC. Barton Clay; Hampshire. [Otoliths; Brit. Mus., no. 28094.]

ARTyROPTERTS, L. Agassiz, Poiss. Foss., vol. iii (1 843), p. 379.

rilez1, L. Agassiz, ibiil., p. 379 ; J. Morris, Cat. Brit. Foss., 1854, p. 317.

Loc. Lias ; Bristol. [Portion of fin ; olim Bristol Mus.]

I.

ASPIDODUS, Newberry and Worthen. \%. Psephodus.

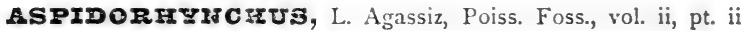
(18+3), p. 135.

? anglicus, L. Agrassiz, ibid.. p. 139 (name only) ; J. Morris, Cat. Brit. Foss., 1854 , p. $31 \%$

LOC. 'U. Lias; Whitby.' [Probably dentary bone of $E \boldsymbol{u}$ gnathus: Brit. Mus.]

euodus, P. M. G. Egerton, Quart. Journ. Geol. Soc., vol. i, 1845, p. 231 ; J. Morris, Cat. Brit. Foss., 1854 , p. 317.

Loc. Oxford Clay ; Christian Malford. [Jaws? Brit. Mus.]

fisheri, P. M. G. Egerton, Figs. and Descript. Brit. Organic Remains, aec. viii (Mem. Geol. Surv., 1855), no. 6, pl. vi ; J. Morris, Cat. Brit. Foss., I854, p. 317.

LOC. Purbeck Bed;; Swanage. [Fish; Dorchester Mus.]

sp., A. S. Woodward, Ann. Mag. Nat. Hist. [6] vol. i, ISSS, p. 355.

Pholidophorus minor?, J. Phillips (non Agassiz), Geol. Oxford, I $87 \mathrm{I}$, p. I 80 , woodcut $\mathrm{xl}$, f. 5,6 . [Mandib. ramus; Oxford Mus.

LOC. Stonesfield Slate. [Skulls and jaws; Brit. Mus.]

ASTrRACANTruUs, L. Agassiz, Poiss. Foss., vol. iii (i 837 ), p. $3 I$; A. S. Woodward, Ann. Mag. Nat. Hist. [6] vol. ii, 1888 , p. 336.

Strophodus, L. Agassiz, Poiss. Foss., vol. iii (1838), p. 116.

Curtodus, H. E. Sauvage, Cat. Poiss. Form. Second. Boulonnais (Méın. Soc. Acad. Boulogne-sur-Mer, vol. ii, 1867), p. 53.

Sphenonchus, L. Agassiz, Poiss. Foss., vol. iii $(18+3)$, p. 20 I (pars). 
ASTMRACANTMUS (continucd)-

acutus, L. Agassiz, Poiss. Foss., vol. iii (1837), p. 33, pl. viii $a$, f. $1-3$; J. Morris, Cat. Brit. Foss., 1854 , p. 317; A. S. Woodward, Cat. Foss. Fishes B. M., pt. i, 1889, p. 313.

Loc. Gt. Oolite (incl. Stonesfield Slate); Oxfordshire. Cornbrash; Northamptonshire. ? Forest Marble; Dorsetshire. [Spine ; Bedford Mus.]

granulosug, P. M. G. Egerton, Ann. Mag. Nat. Hist. [2] vol. xiii, 1854 , p. 434 , and Figs. and Descript. Brit. Organic Remains, dec. viii (Mem. (jeol. Surv., 1855), no. I, pl. i; J. Morris, Cat. Brit. Foss., I854. p. 317 ; A. S. Woodward, Cat. Foss. Fishes B. M., pt. i, 1889, p. 314 .

LOC. Wealden: Tilgate Forest. [Spine; Brit. Mus.]

minor, L. Agassiz, Poiss. Foss., vol. iii (1837), p. 33, pl. viii $a$, f. 4-6 ; J. Morris, Cat. Brit. Foss., I 854 , p. 317.

LOC. Unknown, but recorded by Morris as Oolite, England. [Spine.]

ornatissimus, L. Agassiz, Poiss. Foss., vol. iii (1837), p. 31, pl. viii ; J. Morris, Cat. Brit. Foss., 1854, p. 317 ; R. Damon, Geol. Weymouth, ISSo, Suppl., pl. x, f. 2 ; A. S. Woodward, Cat. Foss. Fishes B. M., pt. i, I889, p. 307, pl. xv, f. 14 .

Siliquastrum gibbosum, Luidius, Lithophyl. Brit., 1760, p. 74, pl. xvi, f. 1474.

Strophodus reticulatus, L. Agassiz, Poiss. Foss., vol. iii (1 838 ), p. 123, pl. xvii (lettered Psammodus); J. Morris, Cat. Brit. Foss., 1854, p. 345. [Associated teeth ; Brit. Mus.]

Strophodus subreticulatus, L. Agassiz, ibid., p. 125, pl. xviii, f. 5-10 ; J. Morris, Cat. I3rit. Foss., 1854, p. 345. [Teeth; Bristol, Oxford, and Brit. Mus.]

Asteracanthus ornatissimus, var. fettonensis, A. S. Woodward, Ann. Mag. Nat. Hist. [6] vol. ii, 1888, p. 341, pl. xii. [Portions of cartilage, teeth, and spines; A. N. Leeds Coll.]

'Snout of some animal of the fish tribe,' H. Baker, Phil. Trans., 1753, p. II 8 , pl. vi.

LOC. Oxford Clay to Kim. Clay; South England. [Spine; Paris Mus.]

T.

semisulcatus, L. Agassiz, Poiss. Foss., vol. iii (1837), p. 34, pl. viii $a$, f. 7, 9, Io (? f. 8); J. Morris, Cat. Brit. Foss., 1854, p. 317 ; A. S. Woodward, Cat. Foss. Fishes B. M., pt. 1, 1889 , p. 312.

Asteracanthus tenuistriatus, J. Phillips, Geol. Oxford, I87I, p. 178, woodcut xxxviii, f. 7. [Spine; Oxford Mus.]

LOC. Stonesfield Slate. [Spines; Brit. Mus.] 


\section{ASTERACANTMUS (continued)-}

gemiverrucosus, P. M. G. Egerton, Ann. Mag. Nat. Hist. [2] vol. xiii, I854, p. 434, and Figs. and Descript. Brit. Organic Remains, dec. viii (Mem. Geol. Surv., 1855), no. 3, pl. iii ; J. Morris, Cat. Brit. Foss., 1854, p. 317.

Loc. Purbeck Beds; Swanage. [Spine; Dorchester Mus.]

stutchburyi, L. Agassiz, Poiss. Foss., vol. iii (1843), p. 177 (name only). [See A. S. Woodward, Cat. Foss. Fishes B. M., pt. i, I 889, p. 3I3.]

LOC. L. Lias; Charmouth, Dorset. [Spine.]

tenuistriatus, Phillips. \%. Asteracanthus semisulcatus.

verrucosus, P. M. G. Egerton, Ann. Mag. Nat. Hist. [2] vol, xiii, I854, p. 433, and Figs. and Descript. Brit. Organic Remains, dec. viii (Mem. Geol. Surv., I855), no. 2, pl. ii ; J. Morris, Cat. Brit. Foss., 1854, p. 318 ; A. S. Woodward, Cat. Foss. Fishes B. M., pt. i, 1889, p. 313.

LOC. Purbeck Beds ; Swanage. [Spine ; Dorchester Mus.]

ASTrRo OIRIS, E. von Eichwald, Bull. Sci. St. Pétersbourg (1840), p. 79 (Astrolepis); R. H. Traquair, Ann. Mag. Nat. Hist. [6] vol. ii, 1888, p. 492. [Non Hugh Miller, Footprints of Creator, 1849, p. 70 ; v. Homosteus.]

Chelonichthys, L. Agassiz, Poiss. Foss., vol. i (1844), p. xxxiii (name only).

asmusii (Agassiz), Morris. v. Homosteus milleri.

major, Eichwald. v. Bothriolepis major.

malcolmsont, L. Agassiz, Poiss. Foss. V. G. R. (1 845), p. 147. pl. $\mathrm{xxx} a$, f. 16 ; J. Morris, Cat. Brit. Foss., 1854, p. 318.

LOC. U. Old Red Sandst. ; Scat Craig, Elgin. [Dermal plate ; olim Malcolmson Coll.]

maximus (Agassiz), R. H. Traquair, Geol. Mag. [3] vol. v, I888, p. 508, and Ann. Mag. Nat. Hist. [6] vol. ii, 1888, p. 495, pl. xviii, f. i, 2.

Coccosteus maximus, L. Agassiz, Poiss. Foss. V. G. R. (1845), p. 137, pl. $\operatorname{xxx} a$, f. 17,18 .

Asterolepis, C. H. Pander, Placoderm. devon. Syst., 1857, p. 17.

Pterichthys major, H. Miller (non Agassiz), Quart. Journ. Geol. Soc., vol. iv, I 848, p. 3 II.

LOC. U. Old Red Sandst.; Nairnshire. [Anterior median dorsal plate; Geol. Soc. London.]

minor, L. Agassiz, Poiss. Foss. V. G. R. (I 844), p. 94, pl. xxviii a. pl. xxx, f. I I, pl. xxxi $a$, f. 29, 30 ; J. Morris, Cat. Brit. Foss., I854, p. 318.

Chelonichthys minor, L. Agassiz, Poiss. Foss., vol. i (1844), p. xxxiii (name only).

LOC. U. Old Red Sandst. ; Elgin. [Dermal plate ; unknown.] 
ASTEROIEPIS (continucd)-

verrucosus, [-a] F. M'Coy, Ann. Mag. Nat. Hist. [2] vol. ii, I848, p. 9; J. Morris, Cat. Brit. Foss., 1854, p. 318.

? Oracanthus, J. WV. Davis, Trans. Roy. Dublin Soc. [2] vol. i (1883). p. 525.

LOC. L. Carb. Limest. ; Armagh. [Dermal plate; Geol. Soc. London.]

AstrRoptychius, F. M'Coy (ex Agassiz, MS.), Ann. Mag. Nat. Hist. [2] vol. ii, I $8+8$, p. 118.

ornatus, L. Agassiz, Poiss. Foss., vol. iii (I 843), p. 176 (name only) ; F. M'Coy, Érit. Palæoz. Foss. (1855), p. 6I5, pl. iii K, f. 23,24 ; J. W. Davis, Trans. Roy. Dublin Soc. [2] vol. i (1883), p. 353, pl. xlvi, f. 7-9.

Asteroptychius semiornatus, F. M'Coy, Ann. Mag. Nat. Hist. [2] vol. ii, 1848, p. 118 , and Brit. Palæoz. Foss. (1855), p. 616, pl. iii K, f. 22 ; J. Morris, Cat. Brit. Foss., 1854 , p. 318. [Spine; Woodwardian Mus.]

LOC. L. Carb. Limest.; Armagh. [Spine; Woodwardian Mus.]

$\mathbf{x}$.

portlocki, [-ii] L. Agassiz, Poiss. Foss., vol. iii (1843), p. 176 (name only).

LOC. L. Carb. Limest.; Armagh. [Spine.]

semiornatus, $\mathrm{M}^{\prime} \mathrm{Coy}$. v. Asteroptychius ornatus.

ASTRABODUS, Davis. v. Psammodus.

expansus, Davis. v. Psammodus expansus.

A Ucyrasas, P. M. G. Egerton, Quart. Journ. Geol. Soc., vol. xiii, 1857, p. 286.

egertoni, E. R. Lankester, Fishes O. R. Sandst., pt. i (Pal. Soc., 1870), p. 57, pl. xiii, f. 3-5, and woodcut 30.

LOc. L. Old Red Sandst. Passage Beds; Ledbury, Herefordshire. [Head-shields; Oxford Mus.]

salter1, P. M. G. Egerton, Quart. Journ. Geol. Soc., vol. xiii, 1857 , p. 286, pl. ix ; E. R. Lankester, Fishes O. R. Sandst., pt. i (Pal. Soc., 1870), p. 56, pl. xiii, f. 7, 8, and woodcut 29.

LOC. Ludlow Tilestones; Herefordshire. [Head-shield; Brit. Mus.]

T.

AUCHENILABRTS, L. Agassiz, Rep. Brit. Assoc., 1844 (1845), p. 308 (name only).

frontalis, L. Agassiz, ibid., p. 308 (name only).

LOC. London Clay; Sheppey. [Portion of skull; Brit. Mus.] 
AULODUS, Dixon. \%. Ptychodus.

agassizi, F. Dixon, Geol. Sussex, 1850, p. 366, pl. xxxii, f. 6 ; J. Morris, Cat. Brit. Foss., 1854, p. 318 . Abraded tooth of Ptychodus, A. S. IVoodward, Proc. Geol. Assoc., vol. $x$ (1888), p. 298.

LOC. Chalk; Sussex. [Tooth; Brighton Mus.]

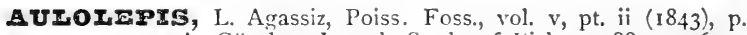
I09; A. Günther, Introd. Study of Fishes, 1880, p. 631 ; A. S. Woodward, Proc. Geol. Assoc., vol. x (1888), p. 323.

typus, L. Agassiz, Poiss. Foss., vol. v, pt. ii (1843), p. ıо9, pl. lx a, f. 5-8; J. Morris, Cat. Brit. Foss., 1854 , p. 318 ; A. S. Woodward, Proc. Geol. Assoc., vol. x (1858), p. 324.

LOC. Chalk ; S.E. England. [Imperfect fish ; Brit. Mus.]

zBATEVI, Cuvier, Règne Animal, vol. ii, 1817, p. 192.

? vulgaris, J. Fleming, Brit. Animals, I828, p. I 85 ; E. T. Newton, Vert. Forest Bed (Mcm. Geol. Surv., I 882), p. I24, pl. xviii, f. 15,16 .

LOC. Forest-Bed Series (U. Freshwater Bed); West Runton. [Pharyngeals; A. Suvin Coll.]

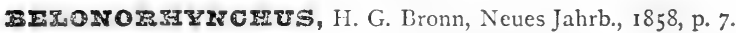

acutus (Agassiz), K. A. von Zittel, Handb. Palæont., vol. iii (1887), p. 222; M. Browne, Vert. Animals Leicestershire, I 889, p. 197.

Belonostomus acutus, L. Agassiz, Poiss. Foss., vol. ii, pt. ii (1843), p. 142, pl. xlvii $a$, f. 3,4 ; J. Morris, Cat. Brit. Foss., 1854 , p. 318 ; F. A. Quenstedt, Der Jura, 1858, p. 234, pl. xxix, f. 8 ; J. F. Blake, in Tate and Blake, Yorkshire Lias, 1876, p. 259.

Belonostomus anningice, L. Agassiz, Poiss. Foss., vol. ii, pt. ii (1843), p. 143 (name only) ; J. Morris, Cat. Brit. Foss., 1854, p. 318. [Head; Brit. Mus.]

Belonostomus tenellus, L. Agassiz, Poiss. Foss., vol. ii, pt. ii (1843), p. I 43 (name only). [Mandib. symphysis ; Brit. Mus.]

Belonorhynchus anningix, A. S. Woodward, Ann. Mag. Nat. Hist. [6] vol. i, I888, p. 355, and Quart. Journ. Geol. Soc., vol. xliv, 1888, p. 147, pl. vii, f. 14 .

LOC. L. Lias; Dorsetshire. (The so-called Leicestershire specimen in the Leicester Mus. is probably from Lyme Regis.) [Fragments of head ; Brit. Mus.]

anningice, Woodward. 2 . Belonorhynchus acutus.

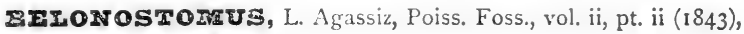
p. 140 .

acutus, Agassiz. v. Belonorhynchus acutus.

anningice, Agassiz. 2\% Belonorhynchus acutus. 
BมxONOSTOMU: (continued) -

attenuatus, F. Dixon, Geol. Sussex, 1850, p. 368, pl. xxxv, f. $4,4^{*}$; J. Morris, Cat. Brit. Fuss., 1854 , p. 318.

LOC. Chalk; Sussex, [Portion of mandible; Brighton Mus.]

cinctus, L. Agassiz, Poiss. Foss., vol. ii, pt. ii (18+3), p. 142, pl. lxvi a, f. 10-13; F. I)ixon, (ieul. Sussex, 1850, p. 357, pl. xxxv, f. 3, 3*; J. Morris, Cat. Biit. Huss., 1854 , p. 318; A. S. Woodward, (2uart. Journ. Geol. Soc., vol. xliv, 1888, p. 145, pl. vii, f. 7-13, and Proc. Geol. Assoc., vol. x (1888), p. 305.

LOC. Chalk ; S. E. England. [Portions of presymphysial bone; Brit. Mus.]

flexuosus: B. Acxuous, J. Phillips, Geol. Oxford, IS71, p. ISo, woodcut xi, f. 3 (name and fig. only). [? Genus.] LOC. Stonesfield Slate. [Mandib. ramus; Oxford Mus.]

? leptosteus, L. Agassiz, Poiss. Foss., vol. ii, pt. ii (1843), p. I43 (name only); J. Morris, Cat. Brit. Foss., 1854, p. 318 ; J. Phillips, Geol. Oxford, I871, p. I8o, woodcut xl, f. 4 (name and fig. only); A. S. Woodward, Ann. Mag. Nat. Hist. [6] vol. i, 1888, p. 355 .

LOC. Stonesficld Slate. [Mandib. symphysis; Oxford Mus.]

tenellus, Agassiz. v. Belonorhynchus acutus.

BrRyCOPSIS, F. I)ixon (cx Agassiz, MS.), Geol. Sussex, i 850 , p. 372 ; A. S. Woodward, Proc. Geol. Assoc., vol. x (I888), p. 328 .

elegans, F. Dixon, Geol. Sussex, I850, p. 372, pl. xxxv, f. 8 ; J. Morris, Cat. Brit. Foss, 1854 , p. 318 ; A. S. Woodward Proc. Geol. Assoc., vol. x (1888), p. 328.

LOC. Chalk; Sussex. [Imperfect fish; Brighton Mus.]

I.

BמRYX, Cuvier, Règne Animal, ed. 2, vol. ii, I 829, p. I 5 I.

lewesieusis (Mantell). \%. Hoplopteryx lewesiensis.

? microcephalus, L. Agassiz, Poiss. Foss., vol. iv (1839), p. I 19, pl. xiv h, f. 3-6, pl. xiv c, f. 10 ; F. Dixon, Geol. Sussex, 1850, p. 372 , pl. xxxiv, f. 3 ; J. Morris, Cat. Brit. Foss., 1854 , p. 318 ; A. S. Woodward, Proc. Geol. Assoc., vol. x (1888), p. 327 .

Loc. Chalk; S. E. England. [Imperfect fish ; Brit. Mus.]

ornutus, Agassiz. $v$. Hoplopteryx lewesiensis.

radians, L. Agassiz, Poiss. Foss., vol, iv (I839), p. I1 8 , pl. xiv $b$, f. 7, pl. xiv $c$, f. 7-9; F. Dixon, Geol. Sussex, 1850, p. 371, pl. xxxvi, f. 4 ; J. Morris, Cat. Brit. Foss., I854. p. 318 ; A. s. Woodward, Proc. Geol. Assoc., vol. x (1888), p. 326, pl. i, f. 9.

Loc. Chalk; S. E. Engiand. [Imperfect fish; Brit. Mus.]

superbus, Dixon. v. Hoplopteryx superbus. 
BOTrRIorgxis, E. von Eichwald, Bull. Sci. St. Pétersbourg, vol. vii (1840), p. 79.

Glyptosteus, L. Agassiz, Poiss. Foss, vol. i (I844), p. xxxiv (name only).

Pamphractus, L. Agassiz, Poiss. Foss. T. G. R. (1844), p. 20. Homothorax, L. Agassiz, ibid., p. 134.

Placothorax, L. Agassiz, ibid., p. I34.

farosus, Agassiz. $\%$. Cricodus favosus.

giganteus, R. H. Traquair, Geol. Mag. [3] vol. v, I 888, p. 510.

Glyplosteus reticulatus, L. Agassiz, Poiss. Foss., vol. i (1844), p. xxxiv (pars, name only).

Bothriolepis ornatus, L. Agassiz (pars, non Eichwald), Poiss. Foss. V. G. R. (I 845 ), p. 99, pl. xxix, f. $3^{-5}$; J. Morris, Cat. Brit. Fosj., I 854 , p. 319 .

LoC. U. Old Red Sandst.; Alves, Elgin. [Dermal plates; Brit. Mus.]

hydrophilus (Agassiz), R. H. Traquair, Geol. Mag. [3] vol. v, I 888 , p. 510 , and Amn. Mag. Nat. Hist. [6] vol. ii, 1888, p. 510.

Pamphractus hydrophiius, L. Agassiz, Poiss. Foss. V. G. R. (18+4), p. 21, pl.iv, f. 4-7.

Pamphractus andersoni, L. Agassiz, ibid., p. 21 ; J. Morris, Cat. Brit. Foss., 1854, p. 337 ; J. Anderson, Dura Den, 1859, pp. 49,52, pl. i, f. I.

Homothorax flemingiz, L. Agassiz, Poiss. Foss. V. G. R. (I $\$+4)$, p. 134 , pl. xxxi, f. 6 ; J. Morris, Cat. Brit. Foss., 1854, p. 329. [Imperfect individual ; olim Fleming Coll.]

Pterichthys hydrophilus, H. Miller and P. M. G. Egerton, Quart. Journ. Geol. Soc., vol, iv, I848, p. 3 I 4 ; J. Morris, Cat. Brit. Foss., I 854, p. 341 ; J. Anderson, Dura Den, I859, pp. 49,52 , pl. i, f. I ; J. Powrie, Quart. Journ. Geol. Soc., vol. xviii, I 862 , p. 435 .

LOC. U. Old Red Sandst.; Dura Den, Fifeshire. [Imperfect individuals; Mus. Pract. Geol.]

macrocephalus (Egerton), R. H. Traquair, Geol. Mag. [3] vol. v, I888, p. 510 , and Ann. Mag. Nat. Hist. [6] vol. ii, I88s, p. 50 I.

Pterichthys macrocephalus, P. M. G. Egerton, Quart. Journ. Geol. Soc., vol. xviii, IS62, p. I03, woodcuts 1-3, pl. iii, f. $7-9$.

LOC. U. Old Red Sandst.; Farlow, Shropshire. [Imperfect individuals; Brit. Mus.]

major (Agassiz), J. Lahusen, Verhandl. russ.-kais. Mineral. Gesell. [2] vol. xi, ISSo, p. I36; R. H. Traquair, Geol. Mas. [3] vol. r, is\&s, p. 510 , and Ann. Mag. Nat. Hist. [6] vol, ii, isSs, p. 501 . 
BOTHRIOIEPIS major (continued)-

Pterichthys major, L. Agassiz, Poiss. Foss. V. G. R. (1844), pp. 5, 19, I33, pl. xxxi, f. I-3 ; J. Morris, Cat. Brit. Foss., $18 ;+$ p. $3+1$.

Placothorax paradoxus, L. Agassiz, ibid., p. I 34 , pl. xxx $a$, f. 2023 ; J. Morris, Cat. Brit. Foss., I854, p. 338 . [Portions of pectoral appendages ; J. Powrie Coll.]

Asterolepis major, E. von Eichwald, Leth. Rossica, 1860, vol. i, p. 1 jII.

Loc. U. Old Red Sandst.; Scat Craig, near Elgin; also Heads of Ayr and Siccar Point. [Proximal plates of pectoral appendage; Geol. Soc. London.]

obesus, R. H. Traquair, Geol. Mag. [3] vol. v, ISSS, p. $j$ Io.

LOC. U. Old Red Sandst.; Rule Water, near Jedburgh. [Dermal plates; Edinburgh IIus.]

omuatus, Agassiz (nom Eichwald. 2'. Bothriolepis giganteus.

BOTHROSTECs, L. Agassiz, Rep. Brit. Assoc., I34t (I $4+5$ ), p. 307 (name only).

brecifions, L. Agassiz, ibid., p. $\hat{j}^{\circ} \mathrm{j}$ (name only).

LOC. London Clay; Sheppey: [Stull.]

latus, L. Agassiz, ibid., p. 30 - (name only).

LOc. London Clay; Sheppey.

minor, L. Agassiz, ibid., p. jo7 (name only).

LOC. London Clay; Sheppey. [Imperfect skull; Brit. .Ius.] $\mathbf{T}$

BRACHYACANTHCS, Egerton. \%. Cimatius.

scutiger, Egerton. $\because$. Climatius scutiger.

BRACHFDEIRLS, A. von Koenen. ¿' Coccosteus.

milleri (Egerton), Koenen. $\because$. Coccosteus decipiens.

pusillus (M'Coy), Koenen. ${ }^{\prime}$ '. Coccosteus decipiens.

BRACHIGNATIIS. L. Agassiz, Poiss. Foss., vol. v, pt. ii $(\mathrm{I} \delta+3)$, p. 139 (name only).

tenuiceps. L. Agassiz, ibid., pp. 139, 142, and Rep. Brit. Assoc., I $\delta_{+4}\left(\mathrm{I} S_{45}\right)$, p. 307 (name only).

Loc. London Clay; Sheppey. [Imperfect skull; Brit. Mus.]

I.

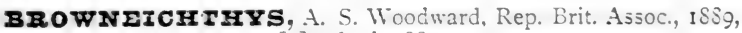
and Geol. Miag. [3] vol. vi, ISS9, p. 455 .

ornatus, A. S. Woodward, ibici., P. 455.

LOC. L. Lias; Barrow-on-Soar. [Imperfect skeleton; Leicester Mus.] 
BRy CrexUs, L. Agassiz, Rep. Brit. Assoc., I 844 (1845), p. 308 (name only).

muelleri, L. Agassiz, ibid., p. 308 (name only).

LOC. London Clay; Sheppey. [Imperfect skull; Brit. Mus.] T.

BUCx I ANDIUM, C. König, Icon. Foss. Sectiles, I825, p. 4 ; A. S. Woodward, Proc. Zool. Soc., 1889, p. 208.

dlluvil, C. König, Icon. Foss. Sectiles, I825, p. 4, pl. viii, no. 9I ; A. S. Woodward, Rep. Brit. Assoc., I888 (1889), p. 679, and Proc. Zool. Soc., 1889, p. 208, pl. xxii.

'? Ephippus, Cuvier,' J. Morris, Cat. Brit. Foss., 1854, p. 3 I9. LOC. London Clay; Sheppey. [Cranium, etc.; Brit. Mus.] $\mathbf{T}$.

ZYSSACANTrus, L. Agassiz, Poiss. Foss. V. G. R. (I844), pp. III, 116.

arcuatus, L. Agassiz, ibil., p. I I ; J. Morris, Cat. Brit. Foss., 1854, p. 319.

Onchus arcuatus, L. Agassiz, Poiss. Foss., vol. iii (1837), p. 7, pl. i, f. 3-5; R. I. Murchison, Silur. Syst., 1839, p. 596, pl.ii, f. IO, I I, and Siluria, I 554 , pl. xxxvi, f. 7,8 .

LOC. Devonian; Bromyard. [? Spine ; olim Murchison Coll.]

CALACANTHUS, auct. = Cœlacanthus.

CALODUS, auct. = Cœlodus.

CAIAMO PIEURUS, L. Agassiz, Poiss. Foss., vol. v, pt. i (1843), p. 122 (name only); F. Dixon, Geol. Sussex, 1850, p. 375 ; A. S. Woodward, Proc. Geol. Assoc., vol. x (1888), p. 324 .

anglicus, F. Dixon, Geol. Sussex, 1850, p. 375, pl. xxxii, f. 12 ; J. Morris, Cat. Brit. Foss., 1854, p. 319 ; A. S. Woodward, Proc. Geol. Assoc., vol. x (1888), p. 324.

LOC. Chalk; S. E. England. [Imperfect fish; Brit. Mus.] T.

CAIIOPRISTODUS, R. H. Traquair, Geol. Mag. [3] vol. v, I888, p. 85.

Ageleodus, R. Owen, Trans. Odontol. Soc., vol. v, I867, p. 340 (insufficiently defined).

Crenoptychius, L. Agassiz, Poiss. Foss., vol. iii (1838), p. 99 (pars).

? aciculatus (W. J. Barkas), A. S. Woodward, Cat. Foss. Fishes B. M., pt. i, 1889, p. 56.

Ctenoptychius aciculatus, W. J. Barkas, Monthly Rev. Dental Surgery, vol. ii, I874, p. 533, f. xxiv-xxvi.

LOC. Coal-Meas.; Newsham, Northumberland. [Tooth; W. J. Barkas Coll.] 
CAIIOPRISTODUS (continued)-

pectinatus (Agassiz), R. H. Traquair, Geol. Mag. [3] vol. v, 1888, p. 85 ; A. S. Woodward, Cat. Foss. Fishes B. M., pt. i, 1889, p. 55 .

Ctenoptychius pectinatus, L. Agassiz, Poiss. Foss., vol. iii (1838), p. IGo, pl. xix, f. 2-4; E. W. Binney, Trans. Manchester Geol. Soc., vol. i (1841), p. 169, pl. v, f. 20, 21 ; J. Morris, Cat. Brit. Foss., 1854, p. 324; T. P. Barkas, Coal-Meas. Palæont., I873, p. I8, f. 17-19; W. J. Barkas, Monthly Rev. Dental Surgery, vol. ii, I874, p. 440, f. xiii, xviii, xix; T. Stock, Ann. MIag. Nat. Hist. [5] vol. ix, 1882, p. 256, pl. viii, f. 5-17.

Ctenoptychius denticulatus, L. Agassiz, Poiss. Foss., vol. iii (1838), p. 10I, pl. xix, f. 5-7; J. Morris, Cat. Brit. Foss., 1854 , p. 324 ; IV. J. Barkas, Monthly Rev. Dental Surgery, vol. ii, 1874, p. 441. [Tooth; Edinburgh Mus.]

Ageleodus diadema, R. Owen, Trans. Odontol. Soc., vol.v, I867, p. 340, pl. iv. [Micro. section of tooth ; Brit. Mus.]

Ctenoptychizus obtusus, T. P. Barkas, English Mechanic, vol. xii (I871), p. 469, woodcut. [Tooth: T. P. Barkas Coll.]

LOC. L. Carb.; Scotch Coalfield. Coal-Meas.; Northumberland, Yorkshire, Lancashire, and Staffordshire. [Teeth ; oiim Roy. Soc. Edinburgh and S. Hibbert Coll.]

$\mathbf{T}$

CALOPOMUS, L. Agassiz, Rep. Brit. Assoc., 1844 (1845), p. 307 (name only).

porosus, L. Agassiz, ibil., p. 307 (name only).

LOC. London Clay; Sheppey. [Imperfect skull ; Brit. Mus.] T.

CAMPYLOTLELION, Huxley. v. Ctenodus.

CANOBIUS, R. H. Traquair, Trans. Roy. Soc. Edinb., vol. xxx (1881), p. 46.

elegantulus, R. H. Traquair, ibid., p. 49, pl. v, f. 5-8.

LOC. Calcif. Sandst. (Cement-stone Group); Glencartholm, Eskdale. [Fish ; Geol. Surv, Scotland, Edinburgh.]

obscurus, R. H. Traquair, ibid., p. 68.

LOC. Calcif. Sandst. (Cement-stone Group); Blackadder Water. [Imperfect fishes; Geol. Surv. Scotland.]

politus, R. H. Traquair, ibid., p. 53, pl. v, f. I4-I6.

LOC. Calcif. Sandst. (Cement-stone Group); Glencartholm, Eskdale. [Fish, wanting tail ; Geol. Surv. Scotland.]

pulchellus, R. H. Traquair, ibid., p. 51, pl. v, f. 9-13.

LOC. Calcif. Sandst. (Cement-stone Group); Glencartholm, Eskdale. [trish; Gecl. Surv. Scotland.]

ramsayi, R. H. Traquair, ioid., p. 47, pl. v, f. I-4.

Loc. Calcif. Sandst. (Cement-stone Group); Glencartholm, Eskdale. [Fish ; Geol. Surv. Scotland.] 
CARrIOSCyuIrUm, A. S. Woodward, Cat. Foss. Fishes B. M., pt. i, 1889 , p. 347 .

Scylliodus, L. Agassiz, Poiss. Foss., vol. iii (1 843 ), p. 377 (pars).

decipiens, A. S. Woodward, Cat. Foss. Fishes B. M., pt. i, 1889, p. 347.

Scylliodus antiquus, L. Agassiz (errore), Poiss. Foss., vol. iii (1843), p. 378 , pl. xxxviii, f. 2.

LOC. L. Chalk ; Burham, Kent. [Remains of head, vertebræ, etc. ; Brit. Mus.]

CARcrarras, Cuvier, Règne Animal, vol. ii, I817, p. 125.

Glyphis, L. Agassiz, Poiss. Foss., vol. iii (I843), p. 243.

(Scollodon) eocænus, A. S. Woodward, Cat. Foss. Fishes B. M., pt. i, i889, p. 436 .

LOC. London Clay; Highgate. [Teeth; Brit. Mus.]

(Scoliodon) orpiensis (Vinkler). \% Acanthias orpiensis.

(rrypoprion) sp., A. S. Woodward, Cat. Foss. Fishes B. M., pt. i, 1889, p. 439.

LOC. Woolwich and Reading Rzds ; Chislehurst. [Teeth ; Brit. Mus., no. P. 4 Io+a.]

(Prionodon) glaucus (Linnæus) : recorded in error from the Pleistocene of Crayford, Kent, by A. S. Woodward, Geol. Mag. [3] vol. v, 1888, p. 528 (W. Whitaker, Geol. London, vol. i (Mem. Geol. Surv., I889), p. 536).

(Prionodon) hastalis (Agassiz), A. S. Woodward, Cat. Foss. Fishes B. M., pt. i, I889, p. 44 I.

Glyphis hastalis, L. Agassiz, Poiss. Foss., vol. iii (1843), p. 244, pl. xxxvi, f. 10-1 3 ; J. Morris, Cat. Brit. Foss., 1854, p. 327.

LOC. 'London Clay ; Sheppey.' ['Tooth ; olim Bristol Mus.]

megalodon, Charlesworth. \% Carcharodon megalodon.

Carcharodon, J. Müller and F. G. Henle, Syst. Beschreib. Plagiostom., 1841, p. 70.

ungustidens, Agassiz. \%. Carcharodon auriculatus.

auriculatus (Blainville), A. S. Woodward, Cat. Foss. Fishes B. M., pt. i, 1889 , p. 4 II.

Squalus auriculatus, H. D. de Blainville, Nouv. Dict. d'Hist. Nat., vol. xxvii, 1818, p. 384 .

Carcharodon angustidens, L. Agassiz, Poiss. Foss., vol. iii (1843), p. 255 , pl. xxviii, f. 20-25, pl. xxx, f. 3 ; J. Morris, Cat. Biit. Foss., i8 54 , p. 319.

Carcharodon turgidus, L. Agassiz, ibid., p. 256, pl. xxx a, f. 8, 9. Carcharodon lenceolatus, L. Agassiz, ibid., p. 257, pl. xxx, f. I. Carcharodon toliupicus, L. Agassiz, ibid., p. 257, pl. $\operatorname{xxx} a$, f. 14. 
CARCHARODON auriculatus (continucd)-

Carcharodon heterodon, L. Agassiz, ibid., p. 258, pl. xxviii, f. 11-16 ; F. Dixon, Geol. Sussex, 1850, p. 204, pl. xi, f. 10, 19. [Tecth; Brit. Muss.]

Carcharodon megalotis, L. Agassiz, Poiss. Foss., vol. iii (1843), p. 258, pl. xxviii, f. 8-10.

Carcharodon disauris, L. Agassiz, ibid., p. 259, pl. xxviii, f. 7 .

LOC. M. and U. Eocene; London and Hampshire Basins. [Teeth; Brit. Mus.]

heterodon, Agassiz. \%. Carcharodon auriculatus.

megalodon, L. Agassiz, Poiss. Foss., vol. iii (1843), p. 247, pl. xxix ; J. S. Bowerbank, Ann. Mag. Nat. Hist. [2] vol. ix, 1852, p. 120; R. Owen, Cat. Foss. Rept. and Pisces Mus. R. Coll. Surgeons, I854, p. 124 ; J. Morris, Cat. Brit. Foss., 1854, p. 319 ; A. S. Woodward, Cat. Foss. Fishes B. M., pt. i, 1889 , p. 415 .

Carcharias megulodon, E. Charlesworth (ex Agassiz, MS.), Mag. Nat. Hist., n.s., vol. i, I837, p. 225, woodcut 24; R. Owen, Rep. Brit. Assoc., I 838 (1839), Sect., p. I4 I.

Carcharodon rectidens, L. Agassiz, Poiss. Foss., vol. iii (1843), p. 250, pl. $x \times x$ al, f. 10.

Carcharodon subauriculatus, L. Agassiz, ibid., p. $25 \mathrm{I}, \mathrm{pl} . \operatorname{xxx} a$, f. II-I3.

Carcharodon productus, L. Agassiz, ibid., p. 251, pl. xxx, f. 2, 4, $6,7,8$.

Carcharodon polygyrus, L. Agassiz, ibid., p. 253, pl. xxx, f. 9-12. LOC. Red Crag ; Suffolk. [Tecth; Ipswich Mits.]

obliqua (Agassiz), Noetling. \% Lamna obliqua.

rondeleti, J. Müller and F. G. Henle, Syst. Beschreib. Plagiostom., I84I, p. 70; [-ii] A. S. Woodward, Cat. Foss. Fishes B. M., pt. i, 1889, p 420.

Carcharodon sulcidens, L. Agassiz, Poiss. Foss., vol. iii (i 843 ), p. 254, pl. $x \times x a$, f. $3-7$.

Loc. Red and White Crags ; Suffolk. [Tecth; Brit. IIus.] T.

subserratus, L. Agassiz, Poiss. Foss., vol. iii (1843), p. 25o, pl. xxxvi, f. I4, 15 ; J. Morris, Cat. Brit. Foss., I854, p. 319.

Carcharodon escheri, L. Agassiz, ibid., p. 260, pl. xxxvi, f. $16-21$.

LOC. London Clay ; Sheppey. [Tooth ; Brit. Mus.]

sulcidens, Agassiz. \% Carcharodon rondeleti.

toliapicus, Agassiz. $\%$. Carcharodon auriculatus.

CARCHAROPSTS, Agassiz. \% Dicrenodus.

colei, Davis. $v$. Dicrenodus dentatus.

portlocki, R. Etheridge, Foss. Brit. Islands, pt. i, I888, p. 32 I. Misprint for Asteroptychius portlocki. 
CARCHAROPSIS (continued)-

prototypus, Agassiz. $v$. Dicrenodus dentatus:

semiornatws, R. Etheridge, Foss. Brit. Islands, pt. i, 1888, p. 321.

Misprint for Asteroptychius semiornatus.

CATOPTERUS, Agassiz. \%. Dipterus.

analis, Agassiz. $\%$ Dipterus valenciennesi.

CATURUS, L. Agassiz, Poiss. Foss., vol. ii, pt. ii (1843), p. II 5.

angustus, L. Agassiz, ibid., p. I18.

LOC. Portlandian; Garsington, near Oxford. [Fish, wanting head ; Worcester Mus.]

buclitandi, L. Agassiz, ibid., p. 119 (name only).

LOC. L. Lias ; Lyme Regis.

pleiodus, L. Agassiz, ibid., p. i I (name only).

LOC. Stonesfield Slate.

similis, L. Agassiz, ibid., p. I \& 8 , pl. lxvi a, f. 9 ; J. Morris, Cat. Brit. Foss., I $\delta_{54}$, p. 319. Indeterminable genus (A. S. Woodward, Proc. Geol. Assoc., vol. x (1888), p. 335).

LOc. Chalk; Lewes. [Fragment of jaw ; Brit. Mus.]

CENTRODUS, M'Coy (non Giebel). 2'. Megalichthys.

striatulus, M'Coy. \%. Megalichthys hibberti.

CrNTrougra, P. M. G. Egerton, Figs. and Descript. Brit. Organic Remains, dec. ix (Mem. Geol. Surv., 1858), no. 5.

asper, L. Agassiz, Poiss. Foss., vol. ii, pt. i (1843), p. 304 (name only); J. Morris, Cat. Brit. Foss., I854, p. 319; P. M. G. Egerton, Figs. and Descript. Brit. Organic Remains, dec. ix (Mem. Geol. Surv., I 858), no. 5, pl. v.

LOC. L. Lias; Lyme Regis. [Anterior half of fish ; Brit. Mus.] T.

CrPIraIASPIS, L. Agassiz, Poiss. Foss., vol. ii, pt. i (1835), p. I 35 ; T. H. Huxley, Quart. Journ. Geol. Soc., vol. xiv, I 858 , p. 267 ; J. Powrie, Geologist, vol. iv, I86I, p. I37 ; E. R. Lankester, Fishes O. R. Sandst., pt. i (Pal. Soc., I870), p. 43. Including subgenera Eucephalaspis, Lankester, ibid., p. 43, Hemicyclaspis, Lankester (ibid.), and Zenaspis, Lankester (ibil.).

(Fucephalaspis) agassizi, [-ii] E. R. Lankester, Fishes O. R. Sandst., pt. i (Pal. Soc., I870), p. 46, pl. ix, f. 2, 3, 6, and woodcut 18 .

Cephalaspis lyellii, L. Agassiz (pars), Poiss. Foss., vol. ii, pt. i (1835), p. 142, pl. i 6, f. 3. [Head-shield.]

LOC. L. Old Red Sandst. (Cornstones); Herefordshire and Worcestershire. [Head-shields; Oxford Mus.] 
CEPHAIASTIS (continued)-

(Eucephalaspis) asper, E. R. Lankester, Fishes O. R. Sandst pt. i (Pal. Soc., 1870), p. 50, pl. x, f. 5, woodcut 23.

LOC. L. Old Red Sandst. ; Perthshire and Forfarshire. [Portion. of head-shield and scales; J. Powrie Coll.]

(esterolepis, Harley. ${ }^{\prime}$. Cephalaspis (Zenaspis) salweyi.

levisi, Agassiz. \%. Scaphaspis lewisi.

lightbodii, E. R. Lankester, Fishes O. R. Sandst., pt. i (Pal. Soc., 1870), p. 55, pl. xiii, f. 19.

LOC. Ludlow Tilestones; Herefordshire. [Inferior rim of headshield; Brit. Mus.]

lloydi, Agassiz. \%. Scaphaspis lewisi.

(aucephalaspis) lyelli, [-ii] L. Agassiz, Poiss. Foss., vol. ii, pt. i (1835), p. I42, pl. i $a$, f. 2 (non, pl. i $a$, f. I, pl. I b, f. I, 3); J. Morris, Cat. Brit. Foss., 1854 , p. 320 ; [-ii] E. R. Lankester, Fishes O. R. Sandst., pt. i (Pal. Soc., I 870), p. 43, pl. viii, f. I, pl. xi, f. I, 2. [Sce also C. agassizi and C. pozuriei.]

Loc. L. Old Red Sandst.; Glammis, Perthshire, and Arbroath, Forfarshire. [Fish; Brit. Mus.]

T.

(Femicyclaspis) murchisoni, P. M. C. Egerton, Quart. Journ. Geol. Soc., vol. xiii, 1857 , p. 284 , pl. ix, f. 1 ; E. R. Lankester, Fishes O R. Sandst., pt. i (Yal. Soc., I870), p. 5 I, pl. viii, f. 6, pl. ix, f. т, pl. xti, f. 3, 4, woocicuts $24,25$.

Cephalespis ornatus, P. M. G. Egrerton, Quart. Journ. Geol. Soc., vol. xiii, I 857 , p. 285 , pl. ix, 1. 2 , 3. [Imperfect head-shield; Brit. Mus.]

LOC. Ludlow Tilestones and L. Old Red Sandst. Passage Beds; Herefordshire. [Head-shield.]

ornutus, Egerton. ¿'. Cephalaspis (Hemicyclaspis) murchisoni.

(Eucephalaspis) pagei, E. R. Lankester, Fishes O. R. Sandst., pt. i (Yal. Soc., 1870), p. 49, pl. x, f. 3, 4, pl. xi, f. 4, woodcuts $2 \mathrm{I}, 23$.

LOC. L. Old Red Sandst.; Forfarshire. [Imperfect fishes; J. Powrie Coll.]

(Eucephalaspls) powrlei, E. R. Lankester, Fishes O. R. Sandst., pt. i (Pdl. Soc., I870), p. 47, pl. ix, f. 5, pl. x, f. I, woodcuts 19, 20.

? Cephaluspis lyeliii, L. Agassiz (pars), Hoiss. Foss., vol. ii, pt. i (1835), p. 142, pl. 1 a, f. 1, pl. I b, f. 1. [Fish ; Brit. Mus.]

LOC. L. Old Red Sandst.; Ley's Mill, near Arbroath, Forfarshire, and Craig, near Muntrose. [Fish ; J. Powrie Coll.]

rostrutus, Agrassiz. T'. Pteraspis ro-tratus.

(Zenaspis) salweyi, P. M. G. L. erton, Quart. Journ. Geol. Soc., vol. xiii, 1857, p. 283 , pl. x, t. 1 ; J. Harley, (Quart. Journ. Geol. Suc., vol. xv, 1859, p. 504; E. R. Lankester, Fishes O. R. Sandst., pt. i (Pal. Soc., 1870), p. 52, pl. xii, f. 2, 5, 6, pl. viii, f. $2,3,4$, woodcu:s $26-28$. 
CPPFAIASPIS (Zenaspis) salweyi (continued)-

Cephalaspis asterolepis, J. Harley, Quart. Journ. Geol. Soc., vol. xv, 1859, p. 503 ; J. IV. Salter, Proc. Woolhope Nat. Field Club, I868, p. 240, with photo. ; H. Woodward, Geol. Mag. [2] vol. viii, p. I9.3, pl vi. [Head-shield; Brit. Mus.]

LOC. L. Old Red Sandst. (Cornstones); Herefordshire. [Portion of head-shield; Ludlow Mus.]

CrPfaxopterus, J. Powrie, Trans. Edinb. Geol. Soc., vol. i (I870), p. 297.

pagei, J. Powrie, ibid., p. 298, pl. xiv, f. I6.

LOC. L. Old Red Sandst.; 'Turin Hill, Forfar. [Imperfect fish; J. Powrie Coll.]

CFrAmurus, P. M. G. Egerton, in P. B. Brodie's Foss. Insects, I 845 , p. 17 .

macrocephalus, P. M. G. Egerton, ibid., p. 17, pl. i, f. 2 ; J. Morris, Cat. Brit. Foss., 1854, p. 320.

LOC. Purbeck Beds; Vale of Wardour. [Fish; P. B. Brodie Coll.]

Crratodus, L. Agassiz, Poiss. Foss., vol. iii (1838), p. 129.

rltels, Agassiz. \%. Ceratodus latissimus.

auglicus, Beyrich. $\%$ Ceratodus latissimus.

bicornis, Agassiz. $\%$. Ceratodus disauris.

cum.s, Agassiz. \%. Ceratodus latissimus.

dcelaleus, Agassiz. \%. Ceratodus latissimus.

disauris, L. Agassiz, ibid., p. 135, pl. xix, f. 19 (C. bicormis on plate); J. Mlorris, Cat. Brit. Foss., 1854, p. 320 ; L. C. Miall, Siren. and Crossopt. Ganoids, pt. i (Pal. Soc., 1878), p. 31 . [? Ceratodus parines.]

Loc. Rhatic ; Aust Cliff. [Tooth; Brit. Mus.]

emarginatus, Agassiz. \%. Ceratodus latissimus.

gibbus, Agassiz. \%. Ceratodus latissimus.

lævissimus. L. C. Miall, Siren. and Crossopt. Ganoids, pt. i

(Pal. Soc., 1878), p. 32, pl. v, f. 2. [? Ceratodus kaupi, Agassiz.]

LOC. U. Keuper; Ripple, Worcestershire. [Tooth ; Brit. Mus.]

latissimus, L. Agassiz, Poiss. Foss., vol. iii (1838), p. I3I, pl. xx, f. 8, 9; J. Morris*, Cat. Brit. Fuss., 1854, p. 320 ; A. S. Woodward, Trans. Leicester Lit. and Phil. Suc., n.s., pt. xi, (1889), p. 2 I.

* Morris also accepts as valid all the undermentioned Agassizian synonyms.

Ceratodus currus, L. Agassiz, Poiss. Foss., vol. iii (1838), p. 13i, pl. xx, f. 10. ['Tooth ; Bristol Mus.] 
CERATODUS latissimus (continued)-

Ceratodus planus, L. Agassiz, ibid., p. 132, pl. xx, f. 6, 7. [Tooth ; Bristol Mus.]

Ceratodus emarginatus, L. Agassiz, ibid., p. 133, pl. xx, f. II-13. [Tooth; Bristol Mus.]

Ceratodus gibbus, L. Agassiz, ibid., p. 133, pl. xx, f. 14, 15. [Tooth ; Bristol Mus.]

Ceratodus dadalens, L. Agassiz, ibid., p. 133, pl. xx, f. 16. [Tooth ; olim Cumberland Coll.]

Ceratodus altus, L. Agassiz, ibid., p. 134, pl. xviii, f. I, 2, pl. xx, f. 2-5. [Tooth ; Bristol Mus.]

Ceratodus anglicus, E. Beyrich, Zeitschr. deutsch. geol. Ges., vol. ii, 1850 , p. 159.

Ceratodus polymorphus, L. C. Miall, Siren. and Crossopt. Ganoids, pt. i (Pal. Soc., 1878), p. 28, pl. ii, f. I-13, pl. iii, f. $1,2,5$, pl. iv, f. I-II, pl. v, f. $1-5$.

LOC. Rhætic; Gloucestershire and Leicestershire. [Lower tooth ; Bristol Mus.]

obtusus, Agassiz. \%. Ceratodus parvus.

parvus, L. Agassiz, Poiss. Foss., vol. iii ( 1838$)$, p. I32, pl. xx, f. I ; J. Morris, Cat. Brit. Foss., I 854, p. 320 ; L. C. Miall, Siren. and Crossopt. Ganoids, pt. i (Pal. Soc., 1878), p. 29, pl. v, f. $3,4,6-10$.

? Ceratodus obtusus, L. Agassiz, Poiss. Foss., vol. iii (1838), p. 134 , pl. xix, f. 20 ; J. Morris, Cat. Brit. Foss., I 854 , p. 320. [Tooth; Brit. Mus.]

LOC. Rhretic; Aust Cliff. [Tooth; Bristol Mus.]

phllipsi, L. Agassiz, Poiss. Foss., vol. iii (1838), p. 135, pl. xix, f. 17 (C.philippsii); J. Morris, Cat. Brit. Foss., 1854, p. 320.

[? Non Ceratodus phillipsii, L. C. Miall, Siren. and Crossopt. Ganoids, pt. i (Pal. Soc., I878), p. 32, pl. v, f. 13. (Tooth; Brit. Mus.)]

LOC. Stonesfield Slate. [Tooth; unknown.]

plamus, Agassiz. \%. Ceratodus latissimus.

polymorphus, Miall. \%. Ceratodus latissimus.

Cestracion, Cuvier, Rìgne Animal, vol. ii, i 817, p. 129.

DRf.PANePhorus, P. M. G. Egerton, Figs. and Descript. Brit. Organic Remains, dec. xiii (Mem. Cieol. Surv., IS72), no. 9.

canaliculatus, P. M. G. Egerton, in Dixon's Geol. Sussex, I8jo, p. 365 , pl. xxxii*, f. 8 , and Quart. Journ. Geol. Soc., vol. ix, 1853 , p. 282 ; J. Morris, Cat. Brit. Foss., 1854 , p. 320 ; C. Hasse, Neues Jahrb., $188_{3}$, vol. ii, p. 66 ; A. S. Wioodward, Cat. Foss. Fishes B. II., pt. i, 1889, p. 33t, pl. xi, f. 24-27. 


\section{CrSTractor canaliculatus (continued)-}

Acrodus cretaceus, F. Dixon, Geol. Sussex, I850, p. 364, pl. xxx, f. I3 ; J. Morris, Cat. Brit. Foss., I854, p. 316. [Tooth.]

Drepanephorus cunaliculatus, P. M. G. Egerton, Figs. and

Descript. Brit. Organic Remains, dec. xiii (Mem. Geol.

Surv., 1872), no. 9, pl. ix ; A. S. Woodward, Geol. Mag. [3]

vol. iv, 1887, p. 105, and P'roc. Geol. Assoc., vol. x (1888), p. 290.

T. (Drepanephorus.)

LOC. U. Chalk; Sussex, Kent, and Surrey. [Teeth; olim Marquis of Northampton Coll.]

rugosus (Agassiz), A. S. Woodward, Cat. Foss. Fishes B. M., pt. i, 1889, p. 335, pl. xi, f. 29.

Acrodus rugosus, L. Agassiz, Poiss. Foss., vol. iii (1839), p. 148, pl. xxii, f. $28,29$.

Drepanephorus, A. S. Woodward, Geol. Mag. [3] vol. iv, I887, p. 103 , and Proc. Geol. Assoc., vol. x (1888), p. 290.

LOC. U. Chalk; Charing, Kent, and Lewes, Sussex. [Teeth; Brit. llus.

sulcatus, A. S. Woodward, Cat. Foss. Fishes B. M., pt. i, 1889, p. 333, pl. xiii, f. 1 I, 12.

Strophodus sulcatus, L. Agassiz, Poiss. Foss., vol. iii (1843),

p. I76 (name only); J. Morris, Cat. Brit. Foss., I 854 , p. 345.

Loc. U. Greensand; Maidstone. [Teeth ; Brit. Mus.]

sp., A. S. Woodward, Cat. Foss. Fishes B. M., pt. i, I889, p. 336, pl. xi, f. 28.

LOC. Gault ; Folkestone. [Tooth ; Brit. Mus., no. 47293a.]

sp., A. S. Woodward, ibid., p. 336.

LOC. London Clay ; Highgate Archway. [Tooth ; Brit. Mus., no. 43136.$]$

? sp., A. S. Woodward, ibid., p. 333.

LOC, Kimmeridge Clay; Weymouth. [Fin-spines; Brit. Ius., nos. $41878,43287,43567$.]

? sp., A. S. Woodward, Geol. Mag. [3] vol. vi, 1889, p. 352.

Loc. Coral Rag; Malton, Yorkshire. [Fin-spine; Malton Mus.]

Cgrormin us, H. 1). de Blainville, Bull. Soc. Philom. Paris, I8I6, P. I2I.

Selache, Cuvier, Règne Animal, vol. ii, 1817, p. 129.

Hannovera, P. J. van Beneden, Bull. Acad. Roy. Sci. Belg. [2] vol. xxxi (1871), p. 504 .

maximus (Linnæus), A. S. Woodward, Cat. Foss. Fishes B. M., pt. i, I 889 , p. 430.

Squalus maximus, Linnacus, Syst. Nat., ed. 12, vol. i, 1766, p. 400 .

LOC. Red Crag; Suffolk. [Spines of claspers of male; Brit. Mus.] 
Cratazacantruts, J. IV. Davis, Trans. Roy. Dublin Soc. [2] vol. 1 (1883), p. 370.

verrucosus, J. W. Davis, ibid., p. 37 I, pl. xlviii, f. 13.

Loc. L. Carb. Limest.; Bristol. [Spine ; Bristol Mus.]

I.

CHARACODUS, Owen. \% Rhizodopsis.

confertus, Owen. $v$ Rhizodopsis sauroides.

CHARACODES, Davis. v. Copodus.

angulatus, Davis. $\%$. Copodus angulatus.

cumeatus, Davis. $v$. Copodus cuneatus.

minimus, J. W. Davis, Geol. Mag. [3] vol. iii, 1886, p. 155, woodcut 8. [? Copodus.]

LOC. Carb. Limest.; Chapel-en-le-Frith, Derbyshire. [Tooth; T. Parker Coll.]

Crmiracantrivs, L. Agassiz, Poiss. Foss., vol. ii, pt. i (r835), p. 125 .

grandispinus, F. M'Coy, Ann. Mag. Nat. Hist. [2] vol. ii, I848, p. 300 , and Brit. Palæoz. Foss. (1855), p. 582, pl. ii B, f. I ; J. Morris, Cat. Brit. Foss., I 854 , p. 320 ; R. H. Traquair, Geol. Mag. [3] vol. v, I888, p. 512.

LOC. L. Old Red Sandst.; Orkney Isles. [Imperfect fish; Woodwardian Mus.]

lateralis, $\mathrm{M}^{\prime} \mathrm{Coy} . \quad v$. Cheiracanthus murchisoni.

latus, P. M. G. Egerton, Figs. and Descript. Brit. Organic Remains, dec. $x$ (Mem. Geol. Surv., 1861), p. 73, pl. x ; R. H. Traquair, Geol. Mag. [3] vol. v, 1888, p. 512.

LOC. L. Old Red Sandst. ; Banffshire. [Fish; olim Duke of Richmond Coll.]

microlepidotus, Agassiz. \%. Cheiracanthus murchisoni.

minor, Agassiz. $v$. Cheiracanthus murchiscni.

murchisoni, L. Agassiz, Poiss. Foss., vol. ii, pt. i (1835), p. 126, pl. i $c$, f. 3,4 ; J. Morris, Cat. Brit. Foss., 1854, p. 321 ; F. M'Coy, Brit. Palæoz. Foss. (I855), p. 583 ; R. H. Traquair, Geol. Mag. [3] vol. v, 1888, p. 512.

Cheiracanthus minor, L. Agassiz, Poiss. Foss., vol. ii, pt. i (1835), p. 127 , pl. i $c$, f. 5 ; J. Morris, Cat. Brit. Foss., 1854, p. 321 ; F. M'Coy, Brit. Palæoz. Foss. $(1855)$, p. 583 . [Fish; olim T. S. Traill Coll.]

Cheiracanthus microlepidotus, L. Agassiz, Poiss. Foss. V. G. R. (1844), p. 38, pl. xv, f. I-3; F. M'Coy, Brit. Palzoz. Foss. (1855), p. 583 ; J. Morris, Cat. Brit. Foss., 1854, p. 32 I.

Cheiracanthus lateralis, F. M'Coy, Ann. Mag. Nat. Hist. [2] vol. ii, 1848, p. 300, and Brit. Palmoz. Foss. (1855), p. 582. [Fish; Woodwardian Mus.] 


\section{CHEIrACANTrus murchisoni (continued)-}

Cheiracanthus pulverulentus, F. M'Coy, Ann. Mag. Nat. Hist. [2] vol. ii, 1848, p. 299, and Brit. Palæez. Foss. (1855), p. 583, pl. ii B, f. 2 ; J. Morris, Cat. Brit. Foss., I 854, p. 32 I. [Fish; Woodwardian Mus.]

LOC. L. Old Red Sandst.; Banffshire, Moray, Caithness, and Orkney Isles. [Imperfect fish ; olim Murchison Coll.] T.

pulverulentus, $\mathrm{H}^{\prime} \mathrm{Coy}$. $\%$ Cheiracanthus murchisoni.

CIrgrzoDorsIs, R. H. Traquair, Trans. Roy. Soc. Edinb., vol. $\mathrm{xxx}$ (188I), p. 56.

geikiei, R. H. Traquair, ibid., p. 56, pl. v, f. 17-19.

LOc. Calcif. Sandst. (Cement-stone Group); Glencartholm, Eskdale. [Fish; Geol. Surv. Scotland.]

Crrgr vol. ii, I848, p. I3I, and Proc. Camb. Phil. Soc., vol. i (1848), p. 66 (Chirodus) ; R. H. Traquair, Ann. Mag. Nat. Hist. [5] vol. ii, 1878 , p. I5.

Amphicentrum, J. Young, Quart. Journ. Geol. Soc., vol. xxii, I 866, p. 306.

Hemiciadodus, J. W. Davis, Quart. Journ. Geol. Soc., vol. xl, I 884, p. 620.

granulosus (Young), R. H. Traquair, Ann. Mag. Nat. Hist. [5] vol. ii, 1878, p. 17, and Trans. Roy. Soc. Edinb., vol. xxix, 1879, p. 363 , pl. v ; J. Ward, Trans. N. Staffs. Inst. Mining Engineers, vol. $x, 1889$, pl. vii, f. I-7.

Amphicentrum gramulosum, J. Young (ex Huxley), Quart. Journ. Geol. Soc., vol. xxii, I866, p. 306, pl. xx; R. H. Traquair, Ann. Mag. Nat. Hist. [4] vol. xvi, 1875, p. 273, pl. ix ; J. Ward, [Proc.] N. Staffs. Nat. Field Club, 1875, p. 236.

T. (Amphicentrum.)

IOC. Coal-Meas.; N. Staffordshire, Northumberland, and Lanarkshire. [Fish; J. Ward Coll.]

pes-ranæe, F. M'Coy, Ann. Mag. Nat. Hist. [2] vol. ii, 1848 p. 13I, and Brit. Palroz. Foss. (1855), p. 616, pl. iii G, f. 9 ; J. Morris, Cat. Brit. Foss., 1854, p. 32 I ; R. H. Traquair, Ann. Mag. Nat. Hist. [5] vol. ii, 1878, p. I7 ; J. W. Davis, Trans. Roy. Dublin Soc. [2] vol. i (1883), p. 523, pl. lxiii, f. 5 .

LOC. U. Carb. Limest. ; Derbyshire. [Splenial bone; Woodwardian Mus.]

striatus (Hancock and Atthey), R. H. Traquair, Trans. Roy. Soc. Edinb., vol. xxix (1879), p. 364.

Amphicentrum striatum, A. Hancock and T. Atthey, Ann. Mag. Nat. Hist. [4] vol. ix, 1872, p. 255 , and Nat. Hist. Trans. Northumb. and Durham, vol. iv (1872), p. 414.

LOC. Coal-Meas.; Northumberland. [Imperfect fish ; Newcastle-upon-Tyne Mus.] 
CHIFIRODUs (continued)-

sp., A. S. Woodward, Cat. Foss. Fishes B. M., pt. i, 1889, p. 460. Amphicentrum granulosum, W. J. Barkas (non Young), Geol. Mag. [2] vol i, I874, p. 43I.

Hemicladodus unicuspidatus, J. W. Davis, Quart. Journ. Geol. Soc., vol. xl, 1884 , p. 620 , pl. xxvii, f. 24 . [Splenial or pterygoid bone; York Mus.] T. (Hemicladodus.)

Loc. U. Carb. Limest. (incl. Yoredale Rocks); N. Yorkshire. [Splenial bone; W. J. Barkas Coll.]

CFrirormprs, L. Agassiz, Poiss. Foss., vol. ii, pt. i (1835), p. 128 ; J. Powrie, Geol. Mag., vol. iv, I867, p. 147; R. H. Traquair, Ann. Mag. Nat. Hist. [4] vol. xv, I875, p. 237.

cummingice, Agassiz. v. Cheirolepis trailli.

curtus, M'Coy. \%. Cheirolepis trailli.

macrocephalıs, M'Coy. v. Cheirolepis trailli.

traili, [-ii]. L. Agassiz, Poiss. Foss., vol. ii, pt. i (1835), p. I 30, pl. i $d$, pl. i $e$, f. 4 ; J. Morris, Cat. Brit. Foss., 1854, p. $321^{*}$; [-ii] F. M'Coy, Brit. Palæoz. Foss. (1855), p. 581 ; J. Powrie, Geol. Mag., vol. iv, 1867, p. I50; R. H. 'Traquair, Geol. Mag. [3] vol. v, 1888, p. 517.

* Morris also admits as valid the undermentioned types.

Cheirolepis uragus, L. Agassiz, Poiss. Foss., vol. ii, pt. i (1835), p. I 32 , pl. i $e$, f. I-3; F. M'Coy, Brit. Palæoz. Foss. (1855), p. 581. [Fishes; olim Murchison and Knight Colls.]

Cheirolepis cummingice, L. Agassiz, Poiss. Foss., vol. ii, pt. i (I 843), p. 3or (name only), and Poiss. Foss. V.G. R. (1844), p. 45, pl. xii ; R. H. Traquair, Ann. Mag. Nat. Hist. [4] vol. xv, 1875, p. 240, pl. xvii. [Fish ; Forres Mus.]

Cheirolepis curtus, F. M'Coy, Ann. Mag. Nat. Hist. [2] vol. ii, I 848, p. 302, and Brit. Palæoz. Foss. (1855), p. 580 , pl. ii D, f. I. [Fish; Woodwardian Mus.]

Cheirolepis macrocephalus, F. M'Coy, Ann. Mag. Nat. Hist. [2] vol. ii, 1848 , p. 303 , and Brit. Palæoz. Foss. (1855), p. 580, pl. ii D, f. 3. [Fish; Woodwardian Mus.]

Cheirolepis velox, F. M'Coy, Ann. Mag. Nat. Hist. [2] vol. ii, 1848 , p. 302 , and Brit. Palæoz. Foss. (I855), p. 581, pl. ii D, f. 2. [Fish; Woodwardian Mus.]

Loc. L. Old Red Sandst.; Banffshire, Morayshire, Cromarty, and Orkney. [Fishes; olim T. S. Traill Coll.]

T.

uragus, Agassiz. $v$. Cheirolepis trailli.

velox, $\mathrm{M}^{\prime} \mathrm{Coy}$. $v$. Cheirolepis trailli.

CHELONTCHTHIS, Agassiz. v. Asterolepis. 


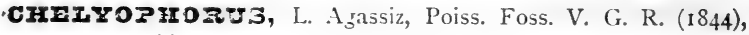
p. 135 .

griffithi, [-ii] F. M'Coy, Ann. Mag. Nat. Hist. [2] vol. ii, I848, p. 8 ; J. Morris, ( at. Brit. Foss., I 854 , p. 321 . Genus non det., C. H. Pander, Placoderm. devon. Syst., 1857, p. 87.

LOC. L. Carb.; Cultra. [Dermal plate ; olim R. Griffith Coll.]

CHIM.TR.1. Linneus, Syst. Nat., ed. 12, vol. i, 1766, p. 401.

agassizi, Buckland. \%. Edaphodon agassizi.

berumonti, Agassiz. v. Ischyodus beaumonti.

brevirostris, Egerton. \% Ischyodus thurmanni.

bucklamali, Agassiz. \%. Ischyodus bucklandi.

colei, Agassiz. \%. Ischyodus colei.

curidens, Egerton. \%. Ganodus curvidens.

egertoni, Buckland. $\%$ Ischyodus egertoni.

falcata, Egerton. \%. Ganodus falcatus.

mantelli, Buckland. \%. Edaphodon mantelli.

neglecta, Egerton. थ. Ganodus neglectus.

overi, Agassiz. v. Ganodus oweni.

psittacina, Egerton. \%. Ganodus psittacinus.

mugulosa, Egerton. \%. Ganodus rugulosus.

sedguickii, Agassiz. \%. Edaphodon sedgwicki.

townsendi, Buckland. \% Ischyodus townsendi.

CHIRACANTHUS, M ${ }^{6} \mathrm{Coy} .=$ Cheiracanthus.

CHIRODOPSIS, MCoy. = Cheirodopsis.

CHIRODUS, $\mathrm{M}^{\circ} \mathrm{Coy} .=$ Cheirodus.

Cromatodus, L. Agassiz, Poiss. Foss., vol. iii (1838), p. 107 (pars). [See also Petalodus.]

[A term provisionally retained, in a restricted sense, for detached teeth of undetermined Cochliodont sharks (A. S. Woodward, Cat. Foss. Fishes B. M., pt. i, I889, p. 218).]

acuminatus, Agassiz. \%. Petalodus acuminatus.

acutus, Davis. ข. Petalodus acutus.

cinctus, L. Agassiz, Poiss. Foss., vol. iii (1838), p. ro7, pl. xv, f. I 3-21 (pars); J. E. Portlock, Rep. Geol. Londonderry, 1843 , p. 467 , pl. xiv $a$, f. 9 ; J. Morris, Cat. Brit. Foss., 1854, p. 321 . [See also Helodus crassus.]

Chnmatodus (Helodus) cinctus, F. M'Coy, Brit. Palæoz. Foss. (1855), p. 617.

Psammodus cinctus, L. Agassiz, Poiss. Foss., vol. iii (1838), lettering of $\mathrm{pl}, \mathrm{x} \mathrm{v}$.

[? = Helodus turgidus, $/ 1$. crassus, and H. tenuis, pars. $]$

LOC. L. Carb. Limest. ; Bristol. [Teeth; Brit. Mus. and Bristol Mus.] 
CHOMATODUS (continued)-

clavatus, M'Coy. $v$. Janassa clavata.

denticulatus, $\mathrm{M}^{\prime} \mathrm{Coy}$. $v$. Venustodus denticulatus.

lamelliformis, Davis. $\%$. Petalodus lamelliformis.

linearis, Agassiz. v. Petalodus linearis and Helodus expansus.

obliqu

trumcatus, M'Coy. v. Janassa clavata.

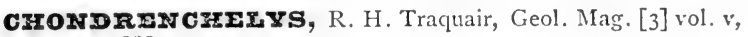
I888, p. 103 .

problematica, R. H. Traquair, ibid., p. I03; A. S. Woodward, Geol. Mag. [3] vol. v, 1888, p. 424, and Cat. Foss. Fishes B. MI., pt. i, I889, p. I6.

LOC. Calcif. Sandst.; Eskdale, Dumfriesshire. [Imperfect skeleton; Edinburgh Mus.]

T.

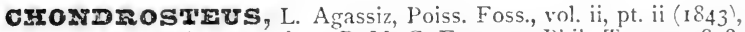
p. 280 (name only); P. M. G. Egerton, Phil. Trans., I 858 , p. 871 ; J. Young, Quart. Journ. Geol. Soc., vol. xxii, I S66, p. 596.

acipenseroides, L. Agassiz, Poiss. Foss., vol. ii, pt. ii (1843), p. 280 ; J. Morris, Cat. Brit. Foss., 1854, P. 321 ; P. M. G. Egerton, Phil. Trans., I 858 , p. 87 I, pls. lxvii, lxviii, f. 2 , lxix ; R. H. Traquair, Geol. Mag. [3] vol. iv, I887, p. 248, woodcuts I-5, and Proc. Roy. Phys. Soc. Edinb., vol. ix, I887, p. 349, woodcuts; J. IV. Davis, Quart. Journ. Geol. Soc., vol. xliii, 1887, p. 605 , pl. xxiii ; A. S. Woodward, Proc. Geol. Assoc., vol xi (1889), p. 36, woodcuts 8-12; M. Browne, Trans. Leicester Lit. and Phil. Soc., n. s., vol. ii, pt. i, I 889, p. I7, pls. i, ii.

Chondrosteus crassior; P. M. G. Egerton, Phil. Trans., 1858, p. 883 , pl. lxviii, f. I, pl. 1xx. [Head, etc. ; Brit. Mus.]

LOC. L. Lias; Lyme Regis and ? Barrow-on-Soar. [Tail and other portions of skeleton : Brit. Mus.]

T.

crassiov, Egerton. v. Chondrosteus acipenseroides.

pachyurus, P. M. G. Egerton, Phil. Trans., I858, p. 883.

LOC. L. Lias; Lyme Regis. [Tail; Mus. Pract. Geol.]

Crmox cłrryz:s, J. Leidy, Trans. Amer. Phil. Soc., vol. xi (1857), p. 95.

levestensis, J. Leidy, ibid., p. 95 ; E. T. Newton, Quart. Journ. Geol. Soc., vol. xxxiv, 1878, p. 792 ; A. S. Woodward, Proc. Geol. Assoc., vol. x (1888), p. 316.

Saurodon leanus, L. Agassiz (non Hays), Poiss. Foss., Feuill., p. 55 (1835), and vol. v, pt. i (1843), p. IO2, pl. xxvc, f. 30, 3 I F. Dixon, Geol. Sussex, 1850, p. 373, pl. xxx, f. 28,29 , pl. xxxii", f. 10 ; J. Morris, Cat. Brit. Foss., 1854, p. 344.

LOC. Chalk; S. E. England. [Portion of palato-pterygoid arch; Brit. Mus.] 
CLADACANTHUS, Agassiz. \% Erismacanthus. paradoxus (Agassiz), Davis. \% Erismacanthus jonesi.

CIADOCycrus, L. Agassiz, Poiss. Foss., vol. v, pt. i (1843), p. 103; A. S. Woodward, Proc. Geol. Assoc., vol. x (1888), p. 325 .

lewesiensis, L. Agassiz, Poiss. Foss., vol. v, pt. i (I843), p. I03 ; J. Morris, Cat. Brit. Foss., 1854 , p. 321 ; A. S. Woodward, Proc. Geol. Assoc., vol. x (1888), p. 326 (C. levesiensis).

Hypsodon lezvesiensis, L. Agassiz, Poiss. Foss., vol. v, pt. i (1843), pl. $\operatorname{xxv} a$, f. 5,6 .

LOC. Chalk ; S. E. England. [Scales; Brit. Mus.]

T.

CIADODUS, L. Agassiz, Poiss. Foss., vol. iii (I843), p. I96.

ccutus, Agassiz. v. Cladodus marginatus.

basalis, Daris. $\approx$. Cladodus mirabilis.

bicuspidatus, Traquair. v. Dicentrodus bicuspidatus.

conicus, L. Agassiz, Poiss. Foss., vol. iii (1843), p. 199, pl. xxii b, f. 24 ; J. Morris, Cat. Brit. Foss., 1854 , p. 321 ; J. W. Davis, Trans. Roy. Dublin Soc. [2] vol. i (1883), p. 378 (non pl. xlix, f. 6). [See Cladodus marginatus.]

LoC. L. Carb. Limest. ; Bristol. [Tooth ; olim Bristol Mus.]

curtus, J. W. Davis, Quart. Journ. Geol. Soc., vol. xxxvii, x88I, p. 424, pl. xxii, f. 9. Spine of Hybodont Shark, i.e., Sphenonchus (A. S. Woodward, Cat. Foss. Fishes B. M., pt. i, 1889, p. 26). [Fragmentary cephalic spine; J. W. Davis Coll.]

curtus, Davis, 1883. \%. Cladodus striatus.

curvus, J. W. Davis, Trans. Roy. Dublin Soc. [2] vol. i (1883), p. 376, pl. xlix, f. I4; A. S. Woodward, Cat. Foss. Fishes B. M., pt. i, I889, p. 20.

LOC. L. Carb. Limest. ; Armagh. [Tooth ; Brit. Mus.]

(lestructor, Davis. $\because$. Cladodus mirabilis.

elegaus, T. P. Barkas. \%. Sphenacanthus hybodoides.

elongatus, Davis. *. Cladodus striatus.

? hibberti, L. Agassiz, Poiss. Foss., vol. iii (1843), p. 200, pl. xxii b, f. 25 ; J, Morris, Cat. Brit. Foss., I854, p. 32 I.

LOC. Calcif. Sandst.; Burdiehouse, near Edinburgh. [Tooth ; olim S. Hibbert Coll.]

hornei, Davis. $v$. Cladodus striatus.

lavis, $\mathrm{M}^{\prime} \mathrm{Coy}$. . Cladodus marginatus.

marginatus, L. Agassiz, Poiss. Foss., vol. iii (1843), p. 198, pl. xxii $b$, f. I8-20; J. Morris, Cat. Brit. Foss., I854, p. 321 ; J. WV. Davis, Trans. Roy. Dublin Soc. [2] vol. i (I883), p. 373 , pl. xlix, f. 7-9; A. S. Woodward, Cat. Foss. Fishes B. M., pt. i, 1889, p. I 8 . 
CIADODUS marginatus (continued) -

Cladodus autus, L. Agrassiz, Poiss. Foss., vol. iii (1843), p. I99, pl. xxii b, f. 2 I ; J. Norris, Cat. Brit. Foss., 1854, p. 32 I ; J. W. Davis, Trans. Roy. I ubl'n Soc. [2] vol. i (1883), p. 377, pl. xlix, f. I7. [Tooth; Geol. S. c. London.]

Cludodus lavis, F. MCoy, Ann. Magr. Nat. Hist. [2] vol. ii, 1848, p. I33, and Is it. Palicoz. Foss. $(1855)$, p. 619, pl. iii $\mathrm{K}$, f. $5 ;$ J. Morris, Cat. Brit. Foss., 1854, p. 321. [Tooth; Woodwardian Mus.]

Cladodus mirabilis, F. $\mathbf{M}^{\circ} \mathrm{Coy}$ (non Agassiz), Brit. Palæoz. Foss. (1855), p. 619 (pars).

Cladodus conicus, J. IV. Davis, Trans. Roy. Dublin Soc. [2] vol. i (1883), p. 564, pl. xlix, f. 6. [Tooth ; Bristol Mus.] (See also Cludodus ionicus.)

LOC. L. Carb. Limest. ; Armagh and ? Bristol. [Tooth ; Geol. Soc. London.]

milleri, Agassiz. \%. Cladodus mirabilis.

mirabilis, L. Agassiz, Poiss. Foss., vol. iii (1843), p. 197, pl. xxii b, f. 9-13; J. Morris, Cat. Brit. Foss., 1854, p. 32 I ; F. Ml'Coy, Brit. Palæoz. Foss. (1855), p. 6Iy (pars); J. W. Davis, Trans. Roy. Dublin Soc. [2] vol. i (1883), p. 372 , pl. xlix, f. I-5; R. H. Traquair, Geol. Mag. [3] vol. v, I 888 , p. $8 \mathrm{I}$; A. S. Woodward, Cat. Foss. Fishes B. M., pt. i, 1889 , p. 16.

Cladodus milleri, L. Agassiz, Poiss. Foss., vol. iii (1843), p. 199, pl. xxii b, f. 22, 23 ; J. Morris, Cat. Brit. Foss., I 854 , p. 321 ; J. W. I) avis, l'rans. Roy. Dublin Soc. [2] vol. i (1883), p. 378 , pl. xlix, f. 16. [Tooth; Bristol Mus.]

Cludodus, J. Morris and (i. E. Roberts, Quart. Journ. Geol. Soc., vol. xviil, 1862, p. 105, pl. iii, f. 6. [TTooth ; Brit. Mus.]

Cladodus destructor, J. WV. Davis, Trans. Roy. Dublin Soc. [2] vol, i (1883), p. 376, pl. xlix, f. 15. [Tooth ; Brit. Mus.]

Cladodus mucronotus, J. W. Davis, ibid., p. 380 , pl. xlix, f. 21 , and Quart. Journ. Geol. Soc., vol. xl, io84, p. 619, pl. xxvii, f. 10. [Tooth; York Mus.]

Cladodus basalis, J. E. Portlock (cr Agassiz, MS., I 843), Rep. Geol. Londonderry, I843, p. $46 \mathrm{I}$ (name only); J. Morris, Cat. Brit. Foss., I 854 , p. 321 ; J. W. Davis, Trans. Roy. Dublin Soc. [2] vol. i (IS83), p. 379, pl. xlix, f. IS. [Tooth; Geol. Soc. London.]

LOC. L. Carb. Limest.; Armagh and Wexford, Ireland; Gloucestershire, Shropshire, and Derbyshire, England. U. Carb. Limest.; Derbyshire and Yorkshire. [Teeth; Geol. Soc. London.1

T.

mucromatus, Davis. \%. Cladodus mirabilis.

? parrus, L. Agassiz, Poiss. Foss., vol. iii (1843), p. 2co, pl. xxii $b$, f. 26,27 .

LOC. Calcif. Sandst. ; Burdiehouse, near Edinburgh. [Teeth ; olim S. Hibbert Coll.] 
CIADODUS (continued) -

striatus, L. Agassiz, Poiss. Foss., vol. iii (18+3), p. 197, pl. xxii $b$, f. I4-17; J. Morris, Cat. Brit. Foss., I 854, p. 322 ; F. MI'Coy, Brit. Palxoz. Foss. (1855), p. 620; J. W. Davis, Trans. Roy. Dublin Soc. [2] vol. i (1883), p. 375, pl. xlix, f. 12, 13, and Quart. Journ. Geol. Soc., vol. xl, I884, p.619; R. H. Traquair, Geol. Mag. [3] vol, v, 1888 , p. 81 ; A. S. Woodward, Cat. Foss. Fishes B. M., pt. i, I889, p. 19.

Cladodus elongatus, J. W. Davis, Trans. Roy. Dublin Soc. [2] vol. i (1883), p. 374, pl. xlix, f. Io, I1. [Tooth; York Mus.]

Cladodus curtus, J. W. Davis (non C. curtus, J. W. Davis, I881, q.v.), ibid., p. 379, pl. xlix, f. 19. [Tooth ; York Mus.]

Cladodus hornei, J. W. Davis, ibid., p. 380 , pl. xlix, f. 20, and Quart. Journ. Geol. Soc., vol. xl, 1884, p. 619, pl. xxvii, f. 1 I. [Tooth; York Mus.]

LOC. L. Carb. Limest.; Armagh. U. Carb. Limest.; Northumberland, Westmoreland, Yorkshire, Derbyshire, and Shropshire. [Teeth ; Geol. Soc. London.]

sp., R. H. Traquair, Geol. Mag. [3] vol. v., I888, p. 82.

LOC. Carb. Limest.; East Kilbride, Lanarkshire. [Anterior portion of fish ; J. Neilson Coll.]

Czxmatrus, L. Agassiz, Poiss. Foss. V. G. R. (1844), p. 119; P. M. G. Egerton, Figs. and Descript. Brit. Organic Remains, dec. x (Mem. Geol. Surv., I86I), p. 65.

Brachyacanthus, P. M. G. Egerton, Rep. Brit. Assoc., I859 (1860), Sect., p. I16.

Euthacanthus, J. Powrie, Quart. Journ. Geol. Soc., vol. xx, I 864, p. 425.

gracilis : Euthacanthus gracilis, J. Powrie, Trans. Edinb. Geol. Soc., vol. i (1870), p. 291, pl. xi, f. 4.

? Euthacanthus clegans, J. Powrie, ibid., p. 292, pl. xii, f. 5. [Impression of fish ; J. Powrie Coll.]

LOC. L. Old Red Sandst.; Farnell, Forfarshire. [Fish, wanting head ; J. Powrie Coll.]

grandis: Euthacanthus grandis, J. Powrie, Trans. Edinb. Geol. Soc., vol. i (1870), p. 292, pl. xii, f. 6.

LOC. L. Old Red Sandst.; Turin Hill, Forfarshire. [Fragments; J. Powrie Coll.]

macnicoli: Euthacanthus mcnicoli, J. Powrie, Quart. Journ. Geol. Soc., vol. xx, 1864, p. 425, pl. xx, f. 2, and Trans. Edinb. Geol. Soc., vol. i (1870), p. 290 , pl. xi, f. 3 (E. minicoli).

T. (Euthacanthus.)

LOC. L. Old Red Sandst.; Forfarshire. [Fish ; J. Powrie Coll.]

reticulatus, L. Agassiz, Poiss. Foss. V. G. R. (1844), p. 120, pl. xxxiii, f. 26 ; J. Morris, Cat. Brit. Foss., 1854, p. 322 ; P. M. G. Egerton, Figs. and Descript. Brit. Organic Re- 
CIIMATIUS reticulatus (continued) -

mains, dec. x (Mem. Geol. Surv., I86I), p. 68, pl. viii, f. III3; J. Powrie, Quart. Journ. Geol. Soc., vol. xx, 1864, p. 421, and Trans. Edinb. Geol. Soc., vol. i (1870), p. 295, pl. xiii, f. Io.

LOC. L. Old Red Sandst. ; Forfarshire. [Fin-spine ; olim Webster Coll.]

T.

scutiger, P. M. G. Egerton, Figs. and Descript. Brit. Organic Remains, dec. x (Mem. Geol. Surv., 1861), p. 65, pl. viii, f. I-IO ; J. Powrie, Quart. Journ. Geol. Soc., vol. xx, 1864, p. 423, and Trans. Edinb. Geol. Soc., vol. i (1870), p. 296, pl. xiv, f. 12, 13 .

Brachyacanthus scutiger, P. M. G. Egerton, Rep. Brit. Assoc., 1859 (I 860), p. I I6.

Loc. L. Old Red Sandst.; Forfarshire. [Fish ; Brit. Mus.]

uncinatus, $\mathrm{J}$. Powrie ( $e x$ Egerton, MS.), Quart. Journ. Geol. Soc, vol. xx, 1854 , p. 422, and Trans. Edinb. Geol. Soc., vol. i (1870), p. 296, pl. xiv, f. II.

LOC. L. Old Red Sandst.; Forfarshire. [Fish; J. Powrie Coll.]

CLIMAXODUS, M'Coy. $\%$ Janassa.

imbricatus, $\mathrm{M}^{\prime} \mathrm{C}$ oy. $v$. Janassa imbricata.

lingureformis, Atthey. $\%$ Janassa linguæformis.

ovatus, T. P. Barkas. \%. Janassa linguxformis.

vermiformis, T. P. Barkas. \% Janassa linguxformis.

CLUPE.1, Linnæus, Syst. Nat., ed. 12, vol. i, I766, p. 522.

vectensis, Newton. $\%$ Diplomystus vectensis.

Coccodarifa, F. A. Quenstedt, Der Jura, i 858, p. Sio (Fokko(lerma); U. Reis, Palicontographica, vol. xxxv (I888), p. 5 I.

substriolatum (Huxley), O. Reis, ibid., p. 5 I.

Macropoma substriolatum, T. H. Huxley, Figs, and Descript. Brit. Organic Remains, dec. xii (Mem. Geol. Surv., 1866), p. 39, pls. ix, x.

LOC. Kim. Clay; Cottenham. [Skull and portion of trunk ; Woodwardian Mus.]

COCCormprs, L. Agassiz, Poiss. Foss., vol. ii, pt. i (1843), p. 300. andrewsi, R. H. Traquair, MS.

LOC. Purbeck; Teffont, near Salisbury. [Fish; Mus. Pract. Geol.]

coccostrus, L. Agassiz, Rep. Brit. Assoc., I $842\langle 18+3\rangle$, p. 85 , and Poiss. Foss. V. G. R. (1844), p. 22.

BRACHYDEIRUS, A. von Koenen, Zeitschr. deutsch. geol. Ges., vol. xxxii, 1880, p. 675 . 


\section{Coccostrus (continued)-}

? carbonarius, F. MIC Coy, Ann. Mag. Nat. Hist. [2] vol. ii, 18.48, p. 9 ; J. Morris, Cat. Brit. Foss., 1854, p. 322. ? Oracanthus (J. W. Davis, Trans. Roy. Dublin Soc. [2] vol. i (1883), p. 525).

Loc. L. Carb. Limest.; Armagh. [Dermal plate; Geol. Soc. London.]

cuspidatus, Agassiz. \%. Coccosteus decipiens.

decipiens, L. Agassiz, Poiss. Foss. V. G. R. (I 844), pp. 26, I 37 , pl. B, f. 2, 3, pls. vii-x, pl. xxx $a$, f. I9; J. Morris, Cat. Brit. Foss., 1854, p. $322^{*}$; IV. H. Baily, Figs. Charact. Brit. Foss., 1875 , pl. xxxiii, f. 3 ; R. H. Traquair, Geol. Mag. [3] vol. v, 1888, p. 511 , and ibid., vol. vi, 1889, p. 4, pl. i, f. 2.

* Morris also admits as valid the undermentioned types, except $C$. latus and C: milleri.

Trionyx; A. Sedgwick and R. I. Murchison, Trans. Geol. Soc. [2] vol. iii (1829), p. 144, pl. xvi, f. 6.

Coccosteus latus, L. Agassiz, Rep. Brit. Assoc., 1842 (1843), p. 87 (name only); F. M'Coy, Brit. Palreoz. Foss. (I 855 ), p. 602.

Coccosteus, H. Miller (ex Agassiz, MS.), Old Red Sandst., I $8+$ I, pl. iii.

Coctosteus oblongus, L. Agassiz, Poiss. Foss. V. G. R. (1844), p. 28 , pl. xi, pl. xxx a, f. $2 ;$ F. M'Coy, Brit. Palæoz. Foss. (1855), p. 603. [Imperfect skeleton; Brit. Mus.]

Coccosteus cuspidatus, L. Agassiz, Poiss. Foss. V. G. R. (1844), pp. 28,137, pl. xxxi, f. 4 ; P. Duff, Geol. Moray, 1842, p. 69, pl. viii, f. i. [Median dorsal plate; Edinburgh Mus.]

Cociosteus microspondylus, F. M'Coy, Ann. Mag. Nat. Hist. [2] vol. ii, I848, p. 298, and Brit. Palceoz. Foss. (1855), p. 602, pl. ii C, f. 4. [Imperfect skeleton; Woodwardian Mus.]

Coccosteus ? trigonaspis, F. $\mathrm{M}^{\prime} \mathrm{Coy}$, ibid. (1848), p. 299, and ibic. (1855), p. 603, pl. ii c, f. 6 . [Imperfect skeleton; Woodwardian Mus.)

Coccostcus pusillus, F. M'Coy, ibid. (1848), p. 298, and ibid. (1855), p. 603, pl. ii C, f. 5. [Imperfect skeleton; Woodwardian Mus.]

Coccosteus milleri, P. M. G. Egerton, Quart. Journ. Geol. Soc., vol. xvi, I860, p. 135. [Imperfect skeleton; Edinburgh Mus.]

Brachydcirus milleri, A. von Koenen, Zeitschr. deutsch. geol. Ges., vol. xxxii, I880, p. 675, and Abh. k. Ges. Wiss. Göttingen, vol. xxx, $188_{3}$, p. 20.

Brachydeirus pusillus, A. von Koenen, ibid. (1883), p. 20.

Loc. L. Old Red Sandst.; Banffshire, Nairnshire, Cromarty, Caithness, and Orkney. [Imperfect skeletons ; Brit. Mus.]

latus, Agassiz. ${ }^{\prime}$. Coccosteus decipiens.

T.

maximus, Agassiz. v. Asterolepis maximus. 
Coccostrus (continued)-

microspondylus, $\mathrm{I}^{\circ} \mathrm{Coy}$. v. Coccosteus decipiens.

milleri, Egerton. $v$. Coccosteus decipiens.

minor, H. Miller, Cruise of the Betsy, 1858, p. 396 ; R. H. Traquair, Geol. Mag. [3] vol. v, 1888, p. 511, and ibid., vol. vi, 1889, p. 8, pl. i, f. 3 .

LOC. L. Old Red Sandst.; Thurso. [Portions of skeletons ; Edinburgh Mus.]

oblongus, Agassiz. v. Coccosteus decipiens.

pusillus, M'Coy. $v$. Coccosteus decipiens.

trigonaspis, $\mathrm{I}^{\circ} \mathrm{Coy}$. v. Coccosteus decipiens.

Cocrimodus, L. Agassiz, Poiss. Foss., vol. iii (1838), p. 113.

acutus, Agassiz. $\%$. Deltoptychius acutus.

angustus, Agassiz. ข. Xystrodus angustus.

compactus, Owen. v. Deltoptychius acutus.

contortus, L. Agassiz, Poiss. Foss., vol. iii (1838), p. 115, pl.xix, f. 14 (non pl. xiv, f. 16-33); R. Owen, Odontogr. (1840), p. 62, pl. xxii, f. i ; J. Morris, Cat. Brit. Foss., 1854, p. 322 ; F. M'Coy, Brit. Palæoz. Foss. (1855), p. 622; R. Owen, Geol. Mag., vol. iv, 1867, p. 59, pl. iii, f. I, 2, 4, 5 ; J. W. Davis, Trans. Roy. Dublin Soc. [2] vol. i (1883), p. 42 I, pl. lii, f. I-6; A. S. Woodward, Cat. Foss. Fishes B. M., pt. i, I889, p. 206, pl. vi, f. 24, woodcut 8. [See also Deltoptychins gibberulus and Tomodus convexus.]

Tomodus conzexus, R. Owen (errore), Geol. Mag., vol. iv, I867, p. 62, pl. iv, f. 2-5. [Jaw ; Woodwardian Mus.]

LOC. L. Carb. Limest.; Armagh. ? Yoredale Rocks; Wensleydale, Yorkshire. [Jaw ; Brit. Mus.]

T.

magmus (Agassiz), M'Coy. \%. Psephodus magnus.

oblongus (Agassiz), M'Coy. \%. Streblodus oblongus.

striatus (Agassiz), $\mathrm{M}^{\circ} \mathrm{C}$ oy. $\tau^{\prime}$. Xystrodus striatus.

Cogncantrus, L. Agassiz, Poiss. Foss., Feuill., p. 83 (I 836 ), and vol. ii, pt. ii $(18+3)$, p. 170 ; T. H. Huxley, Figs. and Descript. Brit. Organic Remains, dec. xii (Mem. Geol. Surv., I 866), p. 8.

Hoplopygus, L. Agassiz, Poiss. Foss., vol. ii, pt. ii (1843), p. 178.

Rhabdoderna, O. Reis, Palæontographica, vol. xxxv (1888), p. $7 \mathrm{I}$.

caudalis, P. M. G. Egerton, in Kine's Permian Foss. (Pal. Soc., I 850 ), p. 236, pl. xxvii, f. 2 ; J. Morris, Cat. Brit. Foss., I854, p. 322 ; T. H. Huxley, Figs. and Descript. Brit. Organic Remains, dec. xii (Mem. Geol. Surv., I866), pp. 14, 21, pl. v, f. 5. [? Immature Calacanthus granulosus.]

Loc. Marl-Slate; Ferry Hill, Durham. [Small fish; Brit. Mus.] 
Cogracantrivs (continued)-

elongatus, T. H. Huxley, Figs. and Descript. Brit. Organic Remains, dec. xii (Mem. Geol. Surv., I 866), p. 23, pl.v, f. 6, 7. Loc. Coal-Meas.; Ballyhedy, co. Cork. [Fish; Geol. Surv. Ireland.]

granulosus, L. Agassiz, Poiss. Foss., vol. ii, pt. ii (1843), p. 172, pl. Ixii (plate issued in 1839, and lettered C. granulatus); P. M. G. Egerton, in King s Permian Foss. (Pal. Soc., I850), p. 235, pl. xxviii* (C. gramulatus); J. Morris, Cat. Brit. Foss., 1854, p. 322.

LOC. Marl-Slate; Midderidge, Ferry Hill, and East Thickley. [Caudal region; olim Ii itham Coll.]

huxleyi, R. H. Traquair, Trans. Roy. Soc. Edinb., vol. xxx, I881, p. 20 , pl. i, f. $1-4$.

Rhabdoderma huxleyi, O. Reis, Palreontographica, vol. xxxv (1888), p. $7 \mathrm{I}$.

LOC. Calcif. Sandst.; Eskdale, Dumfriesshire. [Fishes; Geol. Surv. Scotland.]

lepturus, L. Agassiz, Poiss. Foss., vol. ii, pt. ii (1843), p. 173 (name only); J. Morris, Cat. Brit. Foss., 1854, p. 322 ; T. H. Huxley, Figs. and Descript. Brit. Organic Remains, dec. xii (Mem. Geol. Surv., I866), p. 16, pl. ii, f. I-4, pl. iii, f. I-3, pl. iv, f. I-6; A. Hancock and T. Atthey, Ann. Mag. Nat. Hist. [4] vol. ix, 1872 , p. 256 , pl. xvii, f. 4 , and Nat. Hist. Trans. Northumb. and Durham, vol. iv (1872), p. 416, pl. xv, f. 4; J. Ward, [Proc.] N. Staffs. Nat. Field Club, 1875 , p. 242, and Trans. N. Staffs. Inst. Mining Engineers, vol. x, 1889, pl. v. f. I, 3 ; J. W. Davis, Quart. Journ. Geol. Soc., vol. xxxii, 1876, p. 339.

Hoplopy'gus binneyi, L. Agassiz, Pniss. Foss., vol. ii, pt. ii (1843), p. 178 (undefined). [Fish; Manchester Mus.]

T. (Hoplopygus.)

LOC. Coal-Meas. ; Yorkshire, Northumberland, Lancashire, Staffordshire, and Leicestershire. [Fishes; Mus. Pract. Geol.]

phillipsi, [-ii] L. Agassiz, P'oiss. Foss., vol, ii, pt. ii (1 843$)$, p. 173. LOC. L. Coal-Meas.; Halifax. [Tail; Halifax Mus.]

striatus, Traquair. $\%$. Cryphiolepis striatus.

tingleyensis, J. W. Davis, Trans. Limn. Soc. [2] Zool, vol. ii (1884), p. 427, pls. xlvi-xlix.

Rhabdoderma tingleyense, O. Reis, Palieontographica, vol. $\mathrm{xxxv}$ (1888), p. 72.

LOC. M. Coal-Meas. ; Tingley, Yorkshire. [Portions of fishes ; J. IV. Davis Coll.]

sp., T. M. Hall, Geol. Mag. [2] vol. iii, I 377 , p. 4 Io.

LOC. L. Carb. ; Instow, North Devon. [Fish; Brit. Mus.]

sp. (cf. lepturus), J. W. Davis, Trans. Roy. Dublin Soc. [2] vol. i (1883), p. 524, pl. lxiii, f. 7-12.

LOC. L. Carb. Limest. ; Armagh. [Head-bones, etc. ; Brit. Mus.] 
CAELOCEPHALES, L. Agassiz, Poiss. Foss., vol. v, pt. ii (i 843), p. 139, and Rep. Brit. Assoc., $1844(18+5)$, p. 308 (name only).

salmoneus, L. Agassiz, ibid. (1843), p. 139, and ibid.(1845), p. 308 (name only).

LOC. London Clay ; Sheppey. [Imperfect skull; Brit. Mus.]

GarIODus, J. J. Heckel, Denkschr. k. k. Akad. Wiss. Wien, Math. naturw. $\mathrm{Cl}$., vol. xi, $1 \delta_{5} 6$, p. 202 . [Name universally adopted, but preoccupied by Calodon, Lund, I S39.]

? Acrotemus, L. Agassiz, Poiss. Foss., vol, ii, pt. ii (1843), p. 202.

angustus (Agassiz), K. A. von Zittel, Handb. Palæent., vol. iii (1887), p. 249: A. S. Woodward, Proc. Geol. Assoc., vol. x (1888), p. 307.

Gyrodus angrustus, L. Agassiz, Poiss. Foss., vol. ii, pt. ii (I843), pp. 235,246 , pl. lxvi a, f. 14, I 5 ; J. Morris, Cat. Brit. Foss., I 854 , p. 327 ; F. Dixon, Geol. Sussex, I850, p. 370, pl. xxx, f. 14 , pl. xxxiii, f. I.

Pycnodus angustus, L. Agassiz, Poiss. Foss., vol, ii, pt. ii (1843), p. 199 (name only); J. Morris, Cat. Brit. Foss., I854, p. 342 .

Pycnodus elongatus, L. Agassiz, Poiss. Foss., vol. ii, pt. ii (1843), p. 199.

Loc. Chalk; Sussex and Kent. [Series of teeth; Brit. Mus. (pars).]

cretaceus (Agassiz), K. A. von Zittel, Handb. Palceont., vol. iii (1887), p. 249 ; A. S. Woodwatr, Proc. Geol. Assoc., vol. x (1888), p. 308.

Pycnodus cretaceus, L. Agassiz, Poiss. Foss., vol. ii, pt. ii (18+3), p. 198, pl. Ixxii a, f. 60 ; J. Morris, Cat. Brit. Foss., I 854 , p. 342 .

Loc. L. Chalk; Kent. [Mandib. teeth; olim Cumberland Coll.]

ellpticus, P. M. G. Egerton, Geol. Mag. [2] vol. iv, 187\%, p. 49, pl. iii, f. I.

LOC. Gault ; Folkestone. [R. mandib. dentition; Brit. Mus.]

? faba (Agassiz), K. A. von Zittel, Handb. Palieont., vol. iii (1887), p. 249 ; A. S. Woodward, P'roc. Geol. Assoc., vol. x (I888), p. 308.

Acrotemmus faba, L. Agassiz, Poiss. Foss., vol. ii, pt. ii (1843), p. 203, pl. Ixvi a, f. 16-18; J. Morris, Cat. Brit. Foss., 1854 , p. $316 . \quad$ I. (Acrotemnus.)

Loc. Chalk; Sussex. [Associated teeth ; Brit. Mus.]

gyrodoides, P. M. G. Egerton, Geol. Mag. [2] vol. ir, 1877, p. 52, pl. iv, f. 3 .

LOC. U. Grcensand; Pinney Bay, Dorsetshire. [Vomer; Brit. Mus.] 
CCEIODUS (continued) -

mantell (Agassiz), J. J. Heckel, Denkšchr. k. k. Akad. Wiss. $\mathrm{W}$ ien, Math.-naturw. Cl., vol, xi, 1856, p. 203.

'Palate of an unknown fish,' G. A. Mantell, Illustr. Geol. Sussex, 1827 , p. 58, pl. xvii, f. $26,27$.

Pycnodus mantellii, L. Agassiz, Poiss. Foss., vol. ii, pt. ii (1843), p. 196, pl. Ixxii «l, f. 6-14 ; J. Morris, Cat. Brit. Foss., 1854, p. 342 .

Gyrodus mantellii, L. Agnssiz, ibid., p. 234, pl. 1xix a, f. I8 ; J. Morris, Cat. Brit. Foss., 1854, p. 328. [Left mandib. dentition; Brit. Mus.]

Pycnodus microdon, L. Agassiz, ibid., pt. i (1834), p. 17.

I.OC. Wealden; Tilgate Forest. [Jaws; Brit. Mus.]

parallelus (Dixon), A. S. Woodward, Proc. Geol. Assoc., vol. $x$ (1888), p. 308.

Pycnodus parallelus, F. Dixon, Geol. Sussex, 1850, p. 369, pl. xxxiii, f. 3 ; J. Morris, Cat. Brit. Foss., 1854, p. 342.

Loc. Chalk ; Sussex. [Mandib. dentition; Brighton Mus.]

COELOPERCA, L. Agassiz, Rep. Brit. Assoc., I 844 (1845), p. 307 (name only).

latifrons, L. Agassiz, ibid., p. 307 (name only).

Loc. London Clay; Sheppey. [Imperfect skull ; Brit. Mus.]

COELOI'OM. L. Agassiz, Poiss. Foss., vol. v, pt. ii (1843), p. 139, and Rep. Brit. Assoc., $18+4$ (18+5), p. 307 (name only).

colei, L. Agassiz, ibid. (1843), p. 139, and ibid.(1844), p. 307 (name only).

laeve, L. Agassiz, irid. (1843), p. 139, and ibid. (1844), p. 307 (name only).

LOC. London Clay; Sheppey. [Imperfect skulls; Brit. Mus.]

Corormunchus, L. Agassiz, Poiss. Foss., vol. v, pt. i (18+3), p. 92 ; W. C. Williamson, Phil. Trans., I849, p. 47 I, pl. xliii, f. 35-37, and ibid., I85 I, p. 667 ; A. S. Woodward, Proc. Geol. Assoc., vol. x (1888), p. 329, and Ann. Mag. Nat. Hist. [6] vol. ii, I888, p. 223.

cretaceus, F. Dixon, Geol. Sussex, 1850, descr. to pl. xxxii, f. Io ; J. Moris, Cat. Brit. Foss., 1854, p. 323 ; A. S. Woodward, Proc. Geol. Assoc., vol. x (1888), p. 330, and Ann. Mag. Nat. Hist. [6] vol. ii, 1888, p. 223.

Loc. Chalk ; S. E. England. [Spine; Brighton Mus.]

roctus, L. Agassiz, Poiss. Fos:3, vol. v, pt. i (1843), p. 92 (name only); J. Morris, Cat. Brit. Foss., 1854 , p. 323.

Colorhynchus, F. Dixon, Geol. Sussex, 1850, p. 112, pl. x, f. $14-17$, pl. xi, f. 26 .

LOC. London Clay; Highgate. Bracklesham Beds; Sussex. [Portions of spines; Brit. Mus.] 
COIIORIXNCHUS (continued)-

simurtus, L. Agassiz, Poiss. Foss., vol. v, pt. i (1843), p. 92 (nameonly) ; J. Morris, Cat. Brit. Foss., 1854, p. 323.

I.OC. Bracklesham Beds. [Fragment of spine.]

sp., F. Dixon, Geol. Sussex, I850, p. 205.

LOC. London Clay; Sheppey. Bracklesham Beds.

Cozonodus, F. M'Coy, Ann. Mag. Nat. Hist. [2] vol. ii, $18+8$, p. 5, and Proc. Camb. Phil. Soc., vol. i (1848), p. 65.

longidens, F. M'Coy, Ann. Mag. Nat. Hist. [2] vol ii, I848, p. 5; J. Morris, Cat. Brit. Foss., 1854, p. 323 : J. W. Davis, Trans. Roy. Dublin Soc. [2] vol. i (1883), p. 523, pl. 1 xiii, f. 6 .

T. Loc. L. Carb. Limest.; Armagh. ['Tooth ; Geol. Soc. London.]

COMPSACANTHES, Newberry. ¿'. Pleuracanthus.

carinatus, J. IV. Davis, Trans. Roy. Dublin Soc. [2] vol. i (I883), p. 354 , pl. xlvi, f. Io. [Spine of doubtful genus.]

LOC. L. Carb. Limest.; Armagh. [Fortion of spine; olim Enniskillen Coll.]

major, Davis. $\%$. Pleuracanthus levissimus.

triangularis. Davis. \%. Pleuracanthus robustus.

CONCrODus, F. M'Coy, Ann. Mag. Nat. Hist. [2] vol. ii, I 848 , p. 312, and Brit. Palieoz. Foss. (185;), p. 593 .

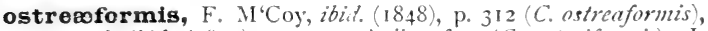
and ibi.t. (1855), p. 593, pl. iic, f. 7 (C. ostreiformis); J. Morris, Cat. Brit. Foss., 1854 , p. 323.

LOc. U Old Red Sandst.; Scat Craig, Elgin. [Tooth; Woodwardian Mus.]

T.

CONODCS, Agassiz. \%. Eugnathus.

ferox, Agassiz. $\%$ Eugnathus chirotes.

Copodus, J. W. Davis (ex Agassiz, MS.), Trans. Roy. Dublin Soc. [2] vol. i (1883), p. 464 .

Labodus, J. W. I)avis (ex Agassiz, MS.), ihid., p. 468.

Mesogonphus, J. W. Davis (p.r. Agassiz, MS.), ibict., p. 470.

Pleurogomphus, J. W. Davis (e.r Agassiz, MS.), ibid., p. $47 \mathrm{I}$.

Rhymodus, J. W. Davis (P Agassiz, MS.), ibid., p. 473.

Characonus, J. W. Davis (ג A rassi\%, MS.), ibit., p. 474.

PInacodus, J. IV. I) avis (er Agrasiz. MS.), ibiul., p. 476.

Mvidax, J. W. Davis (e.r Agassiz, MS.), ibid., p. 479.

Mrlacodus, J. W. Davis (er Agassiz, MS.), ibid., p. 480.

angulatus (Davis), A. S. Woodward, Cat. Foss. Fishes B. M., pt. i, 1889, p. 98. 
COPODUs angulatus (continued) -

Characodus angulatus, J. Morris and G. E. Roberts, Quart. Journ. Geol. Soc., vol. xviii, I862, p. 99 (name only); J. W. Davis, Trans. Roy. Dublin Soc. [2] vol. i (1883), p. 475, pl. Iviii, f. 19, 20.

? Pinacodus gelasimus, J. W. Davis, Trans. Roy. Dublin Soc. [2] vol. i (1883), p. 477, pl. lviii, f. 23 .

LOC. L. Carb. Limest.; Armagh. [Teeth ; Brit. Mus.]

auriculatus (Davis), A. S. Woodward, Cat. Foss. Fishes B. M., pt. i, I889, p. 95.

Pleurogomphus curiculatus, J. Morris and G. E. Roberts (ex Agassiz, MS.), Quart. Journ. Geol. Soc., vol. xviii, 1862, p. IOI (name only); J. W. Davis, Trans. Roy. Dublin Soc. [2] vol. i (1883), p. 472 , pl. lviii, f. 15. T. (Pleurogomphus.)

LOC. L. Carb. Limest.; Armagh. [Tooth; Brit. Mus.]

convexus (Davis), A. S. Woodward, Cat. Foss. Fishes B. M., pt. i, 1889, p. 99.

Rhymodus convexus, J. W. Davis, Geol. Mag. [3] vol. iii, I886, p. 155 , woodcut 7 .

LOC. Carb. Limest.; Chapel-en-le-Frith, Derbyshire. [Tooth ; T. Parker Cull.]

cornutus, J. Morris and G. E. Roberts (ex Agassiz, MS.), Quart. Journ. Geol. Soc., vol. xviii, 1862, p. 100 (name only); J. W. Davis, Trans. Koy. Dublin Soc. [2] vol. i (1883), p. 464, pl. lviii, f. 2-5 (non f. I); A. S. Woodward, Cat. Foss. Fishes 13. M , pt. i, I889, p. 92. [See also Copodus spatulatus.]

Psammodus commotus, L. Agassiz, Poiss. Foss., vol. iii (1843), p. 174 (name only); ?J. E. Portlock, Rep. Geol. Londonderry, $18+3$, p. 466 , pl. xiv $a$, f. 3 ; J. Morris, Cat. Brit. Foss., I854, p. 340 ; ? F. M'Coy, Brit. Palæoz. Foss. (1855), p. 643.

Copodus lumulatus, J. Morris and G. E. Roberts (ex Agassiz, MS.), Quart. Journ. Geol. Soc., vol. xviii, I862, p. Ioo (name only).

? Pinacodus gonophax, J. WV. Davis (ex Agassiz, MS.), Trans. Roy. Dublin Suc. [2] vol. i (1883), p. 477, pl. lviii, f. 22. [Tooth; Brit. Mus.]

T. (Pinacodus.)

LOC. L. Carb. Limest.; Armagh. U. Carb. Limest.; Yorkshire. [Teeth; Brit. Mus.]

T.

? cuneatus (1)avis), A. S. Woodward, Cat. Foss. Fishes B. MI., pt. i, 1889, p. 98.

Characodus cuneatus, J. Norris and G. E. Roberts (ex Agassiz, MS.), (Quart. Journ. Geol. Soc., vol. xviii, 1862, p. 99 (name only); J. W. Lavis, Trans. Roy. Dublin Soc. [2] vol. i (1883), p. 475 , pl. Iviii, f. 2 I.

I.OC. L. Carb. Limest. ; Armagh. [Tooth ; Brit. Mus.]

falcutus, J. Morris and G. E. Roberts (? ex Agassiz, MS.), Quart. Journ. Geol. Soc., vol. xviii, I862, p. 100 (name only), probably misprint for $C$. furcatus, q.v. 
COPODT $\mathbf{S}$ (continued) -

furcatus, $J$. W. Davis (ex Agassiz, MS.), Trans. Roy. Dublin Soc. [2] vol. i (1883), p. 466, pl. lviii, f. 16 ; A. S. Woodward, Cat. Foss. Fishes B. M., pt. i, 1889, p. 94.

LOC. L. Carb. Limest.; Armagh. [Tooth ; Brit. Mus.]

lingua (Davis), A. S. Woodward, Cat. Foss. Fishes B. M., pt. i, 1889, p. 94.

Mesogomphus lingua, J. Morris and G. E. Roberts (ex Agassiz, MS.), Quart. Journ. Geol. Soc., vol. xviii, I862, p. Ior (name only); J. W. Davis, Trans. Roy. Dublin Soc. [2] vol. i (I883), p. 47 I, pl. Iviii, f. I6.

T. (Mesogomphus.)

Loc. L. Carb. Limest ; Armagh. [Tooth; Brit. Mus.]

lumulatus, Agassiz, MS. \%. Copodus cornutus.

minimus, J. W. Davis, Trans. Roy. Dublin Soc. [2] vol. i (1883), p. 467, pl. lviii, f. 8 ; A. S. Woodward, Cat. Foss. Fishes B. M., pt. i, I889, p. 95.

LOC. U. Carb. Limest.; Richmond,Yorkshire. [Tooth ; Brit. Mus.]

oblongus (Davis), A. S. Woodward, Cat. Foss. Fishes B. M., pt. i, 1884, p. 96.

Rhymodus oblongus, J. W. Davis, Trans. Roy. Dublin Soc. [2] vol i (I883), p. 473 , pl. lviii, f. 18 .

Mylar batoides, J. Morris and G. E. Roberts (er Agassiz, MS.), Quart. Journ. Geol. Soc., vol. xviii, I862, p. Ior (name only); J. W. Davis, Trans. Roy. Dublin Soc. [2] vol. i (1883), p. 479, pl. lviii, f. 25, 26. [Teeth; Brit. Mus.] T'. (Mylax.)

LOC. L. Carb. Limest.; Armagh. [Tooth ; Brit. Mus.]

planus (Davis), A. S. Woodward, Cat. Foss. Fishes B. M., pt. i, I889, p. 96.

Labodus planus, J. Morris and G. E. Roberts (ex Agassiz, MS.), Quart. Journ. Geol. Soc., vol. xviii, 1862, p. Ior (name only); J. W. Davis, Trans. Roy. Dublin Soc. [2] vol. i (1883), p. 470, pl. lviii, f. I2-I4.

LOC. L. Carb. Limest.; Armagh. [Teeth ; Brit. Mus.]

prototypus (Davis), A. S. Woodward, Cat. Foss. Fishes B. M., pt. i (1889), p. 97.

Labodus prototypus, J. Morris and G. E. Roberts (ex Agassiz, MS.), Quart. Journ. Geol. Soc., vol. xviii, I862, p. Ior (name only); J. W. Davis, Trans. Roy. Dublin Soc. [2] vol. i (1883), p. 468, pl. Iviii, f. 9-I I.

T. (Labodus.)

Rhymodus transversus, J. W. Davis, ibid., p. 473, pl. Iviii, f. 17. [Tooth ; Brit. Mus.]

Loc. L. Carb. Limest.; Armagh. [Teeth; Brit. Mus.]

spatulatus, J. Morris and G. E. Roberts (ex Agassiz, MS.), Quart. Journ. Geol. Soc., vol. xviii, I862, p. I00 (name only); J. W. Davis, Trans. Roy. Dublin Soc. [2] vol. i (1883), p. 467, pl. lviii, f. 7 ; A. S. Woodward, Cat. Foss. Fishes B. M., pt. i, 1889, p. 93, pl. i, f. 14, I 5. 
COPODus spatulatus (continued) -

Copodus comutus. J. W. Davis (errore), Trans, Roy. Dublin Soc. [2] vol. i (1883), p. 582 , pl. Iviii, f. I, and Quart. Journ. Geol. Soc., vol. xl, 1884, p. 623, pl. xxvii, f. 22. [Tooth; Brit. Mus.]

Mylacodus quadratus, J. IV. Davis, Trans. Roy. Dublin Soc. [2] vol. i (1883), p. 480, pl. lviii, f. 27, 28. [Teeth; Brit. Mus.]

T. (.Mylacodus.)

? Mviacodus sesamini, J. W. Davis, ibid., p. 48I, pl. Iviii, f. 29. [Tooth; Brit. Mus.]

LOC. I.. Carb. Limest.; Armagh. U. Carb. Limest.; Yorkshire and Derbyshire. [Teeth ; Brit. Mus.]

variabilis (Davis), A. S. Woodward, Cat. Foss. Fishes B. M., pt. i, 1889 , p. 99.

Mylacodus variatilis, J. W. Davis, Geol. Mag. [3] vol. iii, I886, p. 154 , woodcuts 5, 6. [? Psammodus.]

LOC. Carb. Limest.; Chapel-en-le-Frith, Derbyshire. [Teeth ; T. Parker Coll.]

CORAx, L. Agassiz, Poiss. Foss., vol. iii (1843), p. 224.

affinis, L. Agassiz, ibid., p. 227, pl. xxvi, f. 2, pl. xxvi $a$, f. 2I-24; A. S. Woodward, Cat. Foss. Fishes B. M., pt. i, 1889, p. 427.

Corax appendiculatus, L. Agassiz (pars), Poiss. Foss., vol. iii (1843), pl. xxvi, f. 3 .

Corax planus, L. Agassiz, ibid., p. 229, pl. xxvi a, f. 51-57.

Corax falcatus, A. S. Woodward, Proc Geol. Assoc., vol. x (1888), p. 293 (pars).

LOC. U. Chalk; Norfok. [Teeth; Brit. Mus.]

"ppendiculatus, Agassiz. $v$. Corax affinis and C. pristodontus.

falcatus, L. Agassiz, Poiss. Foss., vol. iii (I843), p. 226, pl. xxvi, f. 14, pl. xxvi a, f. I-15; F. Dixon, Geol. Sussex, I850, descr. to pl. xxx, f. 18 ; J. Morris, Cat. Brit. Foss., I854, p. 323 ; A. S. Woodward, Proc. Geol. Assoc., vol. x (1888), p. $293($ pars $)$, and Cat. Foss. Fishes B. M., pt. i, I889, p. 424. [Sce also Corax affinis.]

Squalus galeus?, G. A. Mantell (non Linnzeus), Foss. S. Downs, I 822 , p. 227 , pl. xxxii, f. $12-16$.

Galeus pristodontus, L. Agassiz, Poiss. Foss., Feuill., p. 54 ( 1835 ).

Corax heterodon, A. E. Reuss, Verstein. böhm. Kreideform., pt. i, 1845, p. 3, pl. iii, f. 49-71.

? Corax maximus, F. Dixon, Geol. Sussex, I 850 , p. 366, pl. xxx, f. I7 ; J. Norris, Cat. Brit. Foss., I 854 , p. 323. [Tooth; Brit. Mus.]

LOC. U. Greensand; Wiltshire. Cambridge Greensand. Chalk ; Sussex, Surrey, Kent, and Essex. [Teeth; Brit. Mus.]

heterodon, Reuss. $\%$ Corax falcatus.

maximus, Dixon. $v$. Corax falcatus. 
CORAX (continued)-

planus, Agassiz. v. Corax affinis.

pristodontus, L. Agassiz, Poiss. Foss., vol. iii (1843), p. 224, pl. xxvi, f. 9-13 (lettered Galeus pristodontus); A. S. Woodward, Proc. Geol. Assoc., vol. x (1888), p. 293, and Cat. Foss. Fishes B. M., pt. i, 1889, p. 423.

? Corax appendiulatus, L. Agassiz (pars), Poiss. Foss., vol. iii (1843). p. 227, pl. xxvi a, f. 16-20.

Loc. U. Chalk; Norfolk. [Teeth; Brit. Mus.]

T.

Cosmacantraus, L. Agassiz, Poiss. Foss. V. G. R. (1844), p. 120.

Geisacanthus, O. St. John and A. H. Worthen, Pal, Illinois, vol. vi, 1875 , p. 440 .*

carbonarius, F. M'Coy, Ann. Mag. Nat. Hist. [2] vol. ii, I 8.48, p. I19; J. Morris, Cat. Brit. Foss., I854, p. 323 ; J. IV. Davis, Trans. Roy. Dublin Soc. [2] vol. i (1883), p. 357.

Loc. L. Carb. Limest. ; Armagh. [Spines; olim Capt. Jones Coll.]

carinatus, J. IV. Davis, 'Trans. Roy. Dublin Soc. [2] vol. i (1883), p. 356, pl. xlviii, f. 4 .

LOC. L. Carb. Limest. ; Armagh. [Spine ; Brit. Mus.]

malcolmsoni, L. Agassiz, Poiss. Foss. V. G. R. (1844), pp. I I I, 12 I, pl. xxxiii, f. 28 ; J. Morris, Cat. Brit. Foss., 1854, p. 323. [Fragment of Placoderm armour according to C. H. Pander, Placoderm. devon. Sy'st., 1857, p. I 8.]

LOC. U. Old Red Sandst. ; Scat Craig, Elgin. [? Spine or fragment of dermal plate ; olim Malcolmson Coll.]

$\mathbf{x}$.

marginalis, J. W. Davis, Trans. Roy. Dublin Soc. [2] vol. i (I883), p. 355 , pl. xlviii, f. 3 .

LOC. L. Carb. Limest. ; Armagh. [Spine ; Brit. Mus.]

priscus (M'Coy), J. W. Davis, Trans. Roy. Dublin Soc. [2] vol. i (1883), p. 358, pl. xlviii, f. I, 2.

Leptacanthus priscus, L. Agassiz, Poiss. Foss., vol. iii (1843), p. 176 (name only); J. Morris, Cat. Brit. Foss., 1854, p. 332.

Nemacanthus priscus, F. M'Coy, Ann. Mag. Nat. Hist. [2] vol. ii, I848, p. 120; J. Morris, Cat. Brit. Foss., I854, p. 334.

LOC. L. Carb. Limest. ; Armagh. [Spine; Geol. Soc. London.]

Cosmormpis, P. M. G. Egerton, Figs. and Descript. Brit. Organic Remains, dec. ix (Mem. Geol. Surv., I858), no. I.

egertoni, P. M. G. Egerton (ex Agassiz, MS.), ibid., no. I, pl. i.

LOC. L. Lias; Barrow-on-Soar, Leicestershire. [Fish; Brit. Mus.]

T.

* If, as supposed by Pander, the type of Cosmacanthus malcolmsoni be a fragment of Placoderm armour, this name must be adopted for the Carboniferous spines. 


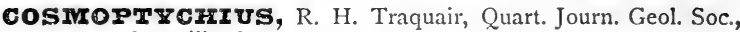
vol. xxxiii, 1877, p. 553 .

striatus (Agassiz), R. H. Traquair, ibid., p. 553, and Ganoid Fishes Brit. Carb. Form. (Pal. Soc., 1877), p. 43, pl, iii, f. I-8.

Amblypterus striatus, L. Agassiz, Poiss. Foss., vol. ii, pt. i (1835), p. III, pl. ivb, f. 3-6; Paterson, Edinb. New Phil. Journ., vol. xxiii, I 837 , p. 153 ; J. Morris, Cat. Brit. Foss., 1854, p. 317.

LOC. Calcif. Sandst.; Wardie, near Edinburgh. [Portions of fishes; Edinburgh Mus.]

CRICACANTIICS, L. Agassiz, Poiss. Foss., vol. iii (1843), p. 176 (name only).

jonesii, L. Agassiz, ibid., p. I76 (name only).

LOC. L. Carb. Limest. ; Armagh. [Spine ; olim Capt. Jones Coll.]

T.

[CRICACANTIIUS.] The following species are entered under this genus by R. Etheridge, Foss. Brit. Islands, pt. i, I888, pp. 324, 325, by error. They are referable to Ctenacanthus :C. crenulatus; dubius; lavis; limaformis; plicatus; pustulatus; rectus; salopiensis; sulcatus.

CRICOdus, L. Agassiz, Poiss. Foss., vol. ii, pt. ii (1843), p. 156, pl. H, f. 9-12 (teeth).

Polyplocodus, C. H. Pander, Saurodipt., Dendrodont., etc., devon. Syst., I 860 , p. 28.

? favosus (Agassiz), R. H. Traquair, Geol. Mag. [3] vol. v, I888, p. 515 .

Bothriolepis favosus, L. Agassiz, Poiss. Foss. V. G. R. (1844), p. I00, pl. xxvii, f. 7, pl. xxviii, f. 12, I3 ; J. Morris, Cat. Brit. Foss., 1854, p. 318.

LOC. U. Old Red Sandst. ; Clashbennie, Perthshire, and Elgin. [Portions of jaws ; unknown.]

incurvus (Duff), L. Agassiz, Poiss. Foss., vol. ii, pt. ii (I843), p. I62 (name only), and Poiss. Foss. V. G. R. (1844), p. 88, pl. xxviii, f. 4,5 .

Dendrodus incurvus, P. Duff, Geol. Moray, I842, p. 68, pl. vi, f. II ; J. Morris, Cat. Brit. Foss., I854, p. 324.

LOC. U. Old Red Sandst. ; Scat Craig, Elgin. [Tooth ; ?]. Powrie Coll.]

CRY PH EIOLEPIS, R. Etheridge, Foss. Brit. Islands, pt. i, I 888 , p. 462. Misprint for Cryphiolepis.

CRYPIIOxשPIS, R. H. Traquair, Geol. Mag. [2] vol. viii, I88I, p. 491 .

striatus, R. H. Traquair, ibid., p. 49I.

Colacanthus striatus, R. H. Traquair, ibid., p. 37.

LOC. M. Carb. Limest. (Blackband Ironstone); Borough Lee, near Edinburgh. [Scales; Edinburgh Mus.] 
CTrisacantrus, L. Agassiz, Poiss. Foss., vol ii (1837), p. 10. [Probably founded on dorsal fin-spines of Orodus, and, if so, Orodus becomes a synonym.]

aequistriatus, Davis. $\%$. Sphenacanthus æquistriatus.

areuatus, L. Agassiz, Poiss. Foss., vol. iii (1843), p. 177 (name only).

LOC. L. Carb. Limest. ; Armagh. [Spine ; unknown.]

brevis, L. Agassiz, Poiss. Foss., vol. iii (1837), p. II, pl. ii, f. 2 ; J. Morris, Cat. Brit. Foss., 1854, p. 323 ; J. W. Davis, Trans. Roy. Dublin Soc. [2] vol. i (1883), p. 337, pl. xliii, f. 3.

Ctenacanthus limaformis, J. W. Davis, ibid., p. 339, pl. xliv, f. 5. [Much-broken spine ; Brit. Mus.]

LOC. L. Carb. Limest; Bristol (non 'Armagh'). [Spine; Bristol Mus.]

costellatus, Traquair. $v$. Sphenacanthus costellatus.

crenatus, F. $\mathrm{I}^{\prime} \mathrm{C}$ oy, Brit. Palæoz. Foss. (1855), p.624, pl. iii I, f. $3 \mathrm{I}$.

Ctenca anthus cremulatus, L. Agassiz, Poiss. Foss., vol. iii (I843), p. 177 (name only); J. W. Davis, Trans. Roy. Dublin Soc. [2] vol. i (1883), p. 345, pl. xlv, f. 6.

Loc. L. Carb. Limest. ; Armagh. [Spine ; Woodwardian Mus.] crenulatus, Agassiz. v. Ctenacanthus crenatus.

denticulatus, F. M'Coy, Ann. Mag. Nat. Hist. [2] rol. ii, I848, p. I16, and Brit. Palroz. Foss. (1855), p. 625, pl. iii k, f. 16 ; J. Morris, Cat. Brit. Foss., I854, p. 323; J. W. Davis, Trans. Roy. Dublin Soc. [2] vol. i (1883), p. 338, pl. xliv, f. 4 .

LOC. L. Carb. ; Monaduff, Drumlish. L. Carb. Limest. ; Armagh and Shropshire. [Spine; Woodwardian Mus.]

distans, $\mathrm{I}^{\circ} \mathrm{Coy} . \quad$. Acondylacanthus distans.

heterogyrus, L. Agassiz, Poiss. Foss., vol. iii (I843), p. 177 (name only); F. M 'Coy, Brit. Palæoz. Foss. (1855), p. 625, pl. iii I, f. 32 ; J. W. Davis, Trans. Roy. Dublin Soc. [2] vol. i (1883), p. 336, pl. xliv, f. I-3.

Loc. L. Carb. Limest. ; Armagh. [Spine ; Woodwardian Mus.]

hybodoides, Egerton. $v$. Sphenacanthus hybodoides.

Iævis, J. W. Davis, Trans. Roy. Dublin Soc. [2] vol. i (I883), p. $34 \mathrm{I}$, pl. xlv, f. I. [? Acondylucanthus.]

Loc. L. Carb. Limest. ; Armagh. [Spine ; Brit. Mus.]

limaformis, Davis. $v$. Ctenacanthus brevis.

major, L. Agassiz, Poiss. Foss., vol. iii (I837), p. ro, pl. iv ; J. Morris, Cat. Brit. Foss., 1854, p. 323 ; J. W. Davis, Trans. Roy. Dublin Soc. [2] vol. i (1883), p. 334, pl. xlii, f. 1, 2.

Ctenacanthus tenuistriatus, L. Agassiz, Poiss. Foss., vol. iii (1837), p. II, pl. iii, f. 7-II ; J. Morris, Cat. Brit. Foss., 1854 , p. 323 ; J. W. Davis, Trans. Roy. Dublin Soc. [2] vol. i (1883), p. 335, pl. xliii, f. 1, 2. [Spine ; Bristol Mus., and Brit. Mus.] 
OTgNACANTrES major (continued)-

Ctenacanthus salopiensis, J. W. Davis, Trans. Roy. Dublin Soc.

[2] vol. i (1883), p. 339, pl. xliv, f. 6. [Spine ; Brit. Mus.]

LOC. L. Carb. Limest.; Gloucestershire and Shropshire. [Spine; Bristol Mus.]

$\mathbf{s .}$

major, Thomson (non Agassiz). 2'. Sphenacanthus hybodoides.

minor, Davis. $\%$ Sphenacanthus minor.

nodosus, Egerton. $\%$. Sphenacanthus hybodoides.

ornatus, L. Agassiz, Poiss. Foss., vol. iii (1837), p. 12, pl. ii, f. I, and Poiss. Foss., V. G. R. (I845), pp. I I I, I19; J. Morris, Cat. Brit. Foss., 1854, p. 323. [Genus non det.]

Loc. Old Red Sandst.; Sapey, Worcestershire, and Wales. [Fragment of spine.]

plicatus, J. W. Davis, Trans. Roy. Dublin Soc. [2] vol i (1883), p. 342 , pl. xlv, f. 4 .

Onchus plicatus, L. Agassiz, Poiss. Foss., vol, iii (1843), p. 177 (name only).

LOC. L. Carb. Limest. ; Armagh. [Spine ; Geol. Soc. London.]

? pustulatus, J. W. Davis, Trans. Roy. Dublin Soc. [2] vol. i (1883), p. 344 , pl. xlv, f. 2.

LOC. L. Carb. Limest. ; Armagh. [Spine ; Brit. Mus.]

rectus, J. W. Davis, Trans. Roy. Dublin Soc. [2] vol. i (1883), p. 345 , pl. xlv, f. 5 .

Onchus rectus, L. Agassiz, Poiss. Foss., vol. iii (1843), p. 177 (name only).

LOC. L. Carb. Limest. ; Armagh. [Spine ; Geol. Soc. London.] salopiensis, Davis. $v$. Ctenacanthus major.

semulatus, Traquair. $\%$ Sphenacanthus serrulatus.

sulcatus (Agassiz), J. W. Davis, Trans. Roy. Dublin Soc. [2] vol. i (1883), p. 343, pl. xlv, f. 3 .

Oncluus sulcatus, L. Agassiz, Poiss. Foss., vol. iii (1837), p. 8, pl. i, f. 6; J. Morris, Cat. Brit. Foss., 1854, p. 334.

LOC. L. Carb. Limest.; Gloucestershire, Shropshire, and ? Armagh. [Spine; Bristol Mus.]

temuistriatus, Agassiz. v. Ctenacanthus major.

CтraOdus, L. Agassiz, Poiss. Foss., vol. iii (1 838), p. 137 ; A. Hancock and T. Atthey, Ann. Mag. Nat. Hist. [4] vol. vii, I87 I, p. I90, and Nat. Hist. Trans. Northumb. and Durham, vol. iv (1872), p. 397 ; A. Fritsch, Fauna der Gaskohle, vol. ii, pt. 3,1888 .

Campylopleuron, T. H. Huxley and E. P. Wright, Trans. Rny. Irish Acad., vol. xxiv, I 866, p. 353.

SAGE:iodus, R. Owen, Trans. Odontol. Soc., vol. v, 1867, p. 365.

Pet 1 loinopsis, W. J. Barkas, Monthly Rev. Dental Surgery, vol. $\mathrm{ii}, 1874$, p. 538 . 
CTrNODUS (continued)-

alutus, L. Agassiz, Poiss. Foss., vo'. iii $(18+3)$, p. $17+$ (name only). LOC. U. Coal-Meas. ; Ardwick, Manchester. [Tooth ; unknown.]

angustulus, R. H. Traquair, Geol. Mag. [2] vol. viii, 188r, p. 36. LOC. M. Carb. Limest. (Blackhand Ironstone); Boroush Lee, near Edinburgit. [ [uoth ; Edinburgh Mus.]

caudatus, W. J. Barkas, Proc. Roy. Soc. N. S. Wales, vol. x, I877, p. 109, pl., f. 9.

Loc. Coal-Meas.; Northumberland. [Tooth ; W. J. Barkas Coll.]

concreus, T. P. Barkas. \% Ctenodus cristatus.

corrugatus, T. Atthey, Ann. Mag. Nat. Hist. [4] vol. i, I868, p. 84; A. Hancock and T. Atthey, Nat. Hist. Trans. Northumb. and Durham, vol. iii (I870), p. 62; W. J. Barkas, Proc. Roy. Soc. N. S. Wales, vol. x, 1877 , p. 106.

Loc. Coal-Meas. ; Newsham. [Tooth ; Newcastle-upon-Tyne Mus.]

cristatus, L. Agassiz, Poiss. Foss., vol. iii (1838), p. 137, pl. xix, f. I6; J. Morris, Cat. Brit. Foss., I854, p. 323 ; '1'. Atthey, Ann. Mag. Nat. Hist. [4] vol. i, 1868, p. 83; A. Hancock and T. Atthey, Nat. Hist. Trans. Northumb. and Durham, vol. iii (1870), pp. 6I, 92 ; J. IVard, [Proc.] N. Staffs Nat. Field Club, 1875 , p. 244 , f. I, 2, and Trans. N. Staffs. Inst. Mining Engineers, vol. $x$, 1889 , pl. iii, f. 3 ; L. C. Miall, Quart. Journ. Geol. Soc., vol. xxx, 1874, p. 772, pl. xlvii, and Ann. Mag. Nat. Hist. [4] vol. xv, 1875 , p. 436; W. J. Barkas, Proc. Roy. Soc. N. S. Wales, vol. x, 1877, p. 102.

‘Palate,' J. De C. Sowerby, Zool. Journ., vol. ii, 1826, p. 23, pl. ii, f. 2.

Ctenodus tuberculatus, T. Atthey, Ann. Mag Nat. Hist. [4] vol, i, 1868, p. 83 ; A. Hancock and T. Atthey, Nat. Hist. Trans. Northumb. and Durham, vol. iii (1870), p. 6r, and ibid., vol. iv (1872), pl. xiv ; 'T. P. Barkas and H. Woodward, Geol. Mag., vol. vi, I869, p. 3I7, pl. ix ; T. P. Barkas, Coal Meas. Palieont., 1873 , p. 28, f. 83, 84, 92 ; J. Ward, [Proc.] N. Staffs. Nat. Field Club, 1875 , p. 246 ; IV. J. Barkas, Proc. Roy. Soc. N. S. Wales, vol. x, I877, p. I04, pl., f. I, IO, I I, 23. [Teeth; Newcastle-upon-Tyne Mus.]

Ctenodus owatus, T. P. Barkas, Scientific Opinion, vol. ii, 1869, p. 557, and Coal Mleas. Palcont., 1873 , p. 28, f. 89 ; W. J. l3arkas, Proc. Roy. Soc. N.S. IVales, vol. x, 1877, p. 108, pl., f. 8. [Upper tooth ; T. P. Barkas Coll.]

Ctenodus concavus, T. P. Barkas, Coal Meas. Palæont., I873, p. 28 , f. 88 ; W. J. Barkas, Proc. Roy. Soc. N. S. Wales, vol. x, 1877 , p. I06, pl., f. 4. [Abraded upper tooth; T. P. Barkas Coll.]

LOC. Coal-Meas. ; Yorkshire, Staffordshire, Derby: hire, Northumberland, Midlothian, and Lanarkshire. [Tooth; Leeds Mus. 


\section{CTrNODUS (continued)--}

elegans, Atthey. v. Ctenodus obliquus.

ellipticus, Atthey. $\approx$. Ctenodus obliquus.

imbricatus, Atthey. \%. Ctenodus obliquus.

Interruptus, T. P. Barkas, Scientific Opinion, vol. ii, 1869, p. 113 ; W. J. Barkas, Proc. Roy. Soc. N. S. Wales, vol. x, I 877 , p. 108.

Loc. Coal-Meas. ; Northumberland. [Tooth ; York Mus.]

monoceros, T. P. Barkas. $\%$. Ctenodus obliquus.

murchisoni, L. Agassiz, Poiss. Foss., vol. i (1844), p. xxxv (name only); described and figured in forthcoming Cat. Foss. Fishes B. M., pt. ii, pl. iv, f. 4.

LOC. U. Coal-Meas. (Spirorbis Limest.); Shropshire. [Tooth ; Brit. Mus.]

obliquus, T. Atthey, Ann. Mag. Nat. Hist. [4] vol. i, 1868, p. 84 ; A. Hancock and T. Atthey, Nat. Hist. Trans. Northumb. and Durham, vol. iii (1870), p. 63, and ibid., vol. iv (1872), pl. xiii, f. 2 ; T. P. Barkas, Coal Meas. Palæont., 1873, p. 28, f. 85,90 ; J. Ward, [Proc.] N. Staffs. Nat. Field Club, 1875 , p. 247 , and Trans. N.Staffs. Inst. Mining Engin., vol. x, 1889 , pl. iii, f. 5 ; T. Atthey, Ann. Mag. Nat. Hist. [4] vol. xv, 1875, p. $309, \mathrm{pl}$. xix, and Nat. Hist. Trans. Northumb. and Durham, vol. v (1877), p. 227 , pl. v ; W. J. Barkas, Proc. Roy. Soc. N. S. Wales, vol. x, I877, p. 105, pl., f. 2, 21 ; L. C. Miall, Proc. Yorksh. Geol. and Polyt. Soc., vol. vii (1881), p. 291, woodcut 4; A. Fritsch, Fauna der Gaskohle, vol. ii (1888), p. 66, pls. lxxi-lxxix, pl. lxxx, f. 5-12, and woodcuts.

Ctenodus elegans, T. Atthey, Ann. Mag. Nat. Hist. [4] vol. i, 1868 , p. 86 ; A. Hancock and T. Atthey, Nat. Hist. Trans. Northumb. and Durham, vol. iii (1870), p. 64, and ibid., vol. iv (1872), p. 407, pl. xiii, f. 3 ; T. P. Barkas, Coal Meas. Palæont., 1873 , p. 28, f. 86 ; W. J. Barkas, Proc. Roy. Soc. N. S. Wales, vol. $x, 1877$, p. 105, pl., f. 3, 12, 13. [Teeth; Newcastle-upon-Tyne Mus.]

Ctenodus imbricatus, T. Atthey, Ann. Mag. Nat. Hist. [4] vol. i, 1868 , p. 86 ; A. Hancock and T. Atthey, Nat. Hist. Trans. Northumb. and Durham, vol. iii (1870), p. 65 ; and ibid., vol. iv (1872), p. 407 , pl. xiii, f. i ; J. Ward, [Proc.] N. Staffs. Nat. Field Club, I875, p. 247 ; W. J. Barkas, Proc. Roy. Soc. N. S. Wales, vol. x, 1877, p. 107, pl., f. 6, 20. [Teeth; Newcastle-upon-Tyne Mus.]

Ctenodus ellipticus, T. Atthey, Ann. Mag. Nat. Hist. [4] vol. i, 1868, p. 87 ; A. Hancock and T. Atthey, Nat. Hist. Trans. Northumb. and Durham, vol. iii (1870), p. 66; J. Ward, [Proc.] N. Staffs. Nat. Field Club, 1875, p. 246, and Trans. N. Staffs. Inst. Mining Engin., vol. x, I889, pl. ii, f.6; W. J. Barkas, Proc. Roy. Soc. N. S. Wales, vol. x, 1877, p. 107. [Teeth; Newcastle-upon-Tyne Mus.] 
Ctenodus monocros, T. P. Barkas, Coal Mieas. Palæont., 1873, p. 28 , f. 87 ; W. J. Barkas, Proc. Roy. Soc. N. S. Wales, vol. $x, 1877$, p. 107, pl., f. 5. [Tooth; T. P. Barkas Coll.]

Ctenodus quadratus, T. P. Barkas, English Mechanic, vol. xviii, (1873), p. 68, woodcuts I, 2 ; W. J. Barkas, Proc. Roy. Soc. N. S. Wales, vol. $x, 1877$, p. 108, pl., f. 7 . [Tooth; T. P. Barkas Coll.]

Petalodopsis mirabilis, W. J. Barkas, Monthly Rev. Dental Surgery, vol. ii, 1874 , p. 538, f. xxx-xxxii, anci ibid., vol. iii, 1875 , p. 4 , f. xxxiii-xxxv.

Vomerine teeth of Ctenodus, T. Atthey, Ann. Mag. Nat. Hist. [4] vol. xv, 1875 , p. 310 , pl. xix, f. 4 ; W. J. Barkas, Proc. Roy. Soc. N. S. Wales, vol. $x$, i 877 , p. I1 5 , f. I4-19.

? Sagenodus incequalis, R. Owen, Trans. Odontol. Soc., vol. v, I 867 , p. 365 , pl. xii. [Micro. sect. of tooth ; Brit. Mus.]

T. (Sagenodus.)

LOC. Coal-Neas.; Northumberland, Yorkshire, Staffordshire, and Lanarkshite. [Teeth; Newcastle-upon-Tyne Mus.]

obliquus, war. quinquecostatus, R. H. Traquair, Geol. Mag. [2] vol. x, 1883 , p. 543 .

Loc. M. Carb. Limest. (Blackband Ironstone); Borough Lee, near Edinburgh. [Tooth; Edinburgh Mus.]

obtusus, W. J. Barkas (ex T. P. Barkas), Proc. Roy. Soc. N. S. Wales, vol. $x, 1877$, p. 108.

Loc. Coal-Meas.; Northumberland. [Tooth; T.P. Barkas Coll.]

octodorsalis, 'T. P. Barkas, Scientific Opinion, vol. ii, 1869, p. 480 ; W. J. Barkas, Proc. Roy. Soc. N. S. Wales, vol. x, 1877 , p. 106.

Loc. Coal-Meas.; Northumberland. [Tooth; T.P. Barkas Coll.]

ovatus, T. P. Barkas. $\%$. Ctenodus cristatus.

quadratus, T. P. Barkas. \%. Ctenodus obliquus.

robertsoni, L. Agassiz, Poiss. Foss., vol. iii (1843), p. 174 (name only).

LOC. L. Carb. Limest.; Burdiehouse. [Tooth.]

tuberculatus, Atthey. \%. Ctenodus cristatus.

(skeleton of.) See especially Hancock and Atthey, Ann. Mag. Nat. Hist. [4] vol. vii, 1871, p. 190, and ibict. [4] vol. ix, 1872, p. $257, \mathrm{pl}$. xviii, and Nat. Hist. Trans. Northumb. and Durham, vol. iv (1S72), pp. 397, 417 ; T. P. Barkas, Coal Meas. Palieont, 1873 ; L. C. Miall, ()uart. Journ. Geol. Soc., vol. xxx, 1874, p. 772 , pl. xlvii, and Proc. Yorksh. Geol. and Polyt. Soc., n.s., vol. vii (1881), p. 289, woodcuts; IV. J. Barkas, Proc. Roy. Soc. N.S. Wales, vol. $x$, I877, p. I I5, pl., f. I4-19. and ibid., vol. xi, 1878, pp. 51, 58 ; and $A$. Fritsch, Fauna der Gaskohle, vol. ii (1888), p. 66, pls. and wondcuts. 
Crgarorgrs, L. Agassiz, Poiss. Foss., vol. ii, pt. ii (1843), p. I8o (name only); J. Phillips, Geol. Oxford, I87I, p. I82.

cyclus, L. Agassiz, ibid., p. 180 (name only); J. Phillips, Geol. Oxford, I87 I, p. 182, woodcut xli, f. I-4.

Loc. Stonesfield Slate. [Scales ; Oxford Mus.]

CTENOPETALS, Davis. "Ctenoptychius.

crenatus, Davis. $\%$ Ctenoptychius lobatus.

serratus, Davis. $\%$ Ctenoptychius serratus.

CTrar optyciru Us, L. Agassiz, Poiss. Foss., vol. iii (1838), p. 99.

Ctenopetalus, J. W. Davis (ex Agassiz, MS.), Ann. Mag. Nat. Hist. [5] vol. viii, I 88 I, p. 426.

Harpacodus, J. W. Davis (ex Agassiz, MS.), ibid., p. 426.

Peripristis, O. St. John (ex Agassiz, MS.), Proc. Amer. Phil. Soc., vol. xi, 1871, p. 434 .

Petalodopsis, J. W. Davis (non W. J. Barkas), Trans. Roy. Dublin Soc. [2] vol. i (1883), p. 498.

aciculatus, W. J. Barkas. v. Callopristodus aciculatus.

aptcalls, L. Agassiz, Poiss. Foss., vol. iii (1838), p. 99, pl. xix, f. I ; E. W. Binney, Trans. Manchester Geol. Soc., vol. i (I841), p. I69, pl. v, f. I9 ; R. Garner, Nat. Hist. Stafford., I 844 , p. 446 , pl. E, f. I 2 ; J. Morris, Cat. Brit. Foss., 1854 , p. 324 ; T. P. Barkas, Coal Meas. Palaont., 1873, p. 18, f. 21 ; W. J. Barkas, Monthly Rev. Dental Surgery, vol. ii, 1874 , pp. 443, 482, f. xiv, xv ; J. Ward, [Proc.] N. Staffs. Nat. Field Club, 1875, p. 218, f. 12, and Trans. N. Staffs. Inst. Mining Engin., vol. $x, 1889$, p. I4I, pl. ii, f. 17, I8, 20 ; A. S. Woodward, Cat. Foss. Fishes B. M., pt. i, 1889, p. 50.

Pealodus apicalis, W. J. Barkas, Monthly Rev. Dental Surgery, vol. ii, 1874 , p. 538 .

LOC. Coal-Meas. ; Lanarkshire, Northumberland, Yorkshire, Lancashire, and Staffordshire. [Tooth ; Brit. Mus.] T.

crenctus, L. Agassiz, Poiss. Foss., vol. iii (1843), p. 173 (name only)

LOC. Coal-Meas.; Carluke. [Tooth ; Hunterian Mus., Glasgow.]

cuspidatus, L. Agassiz, ibid., p. 173 (name only).

LOC. Coal-Meas.; Glasgow. [Tooth; Hunterian Mus., Glasgow.]

dentatus (Owen), L. Agassiz, Poiss. Foss., vol. iii (1843), pp. 173, 383 (name only); J. Morris, Cat. Brit. Foss., 1854, p. 324 ; A. S. Woodward, Cat. Foss. Fishes B. M., pt. i, 1889, p. 51 . Ctenoptychius macrodus, L. Agassiz, Poiss. Foss., vol. iii (1843), pp. 173, 383 (name only); J. E. Portlock, Rep. Geol. Londonderry, 1843 , p. 467 , pl. xiv, f. 7 (inaccurate fig.); J. Morris, Cat. Brit. Foss., 1854 , p. 324. [Tooth; olim Capt. Jones Coll.] 
CTFAO OPYCIIUS dentatus (continued) -

Petalodus dentatus, R. Owen, Odontogr. (1840), p. 62 .

Harpacodus dentatus, J. Morris and G. E. Roberts, Quart. Journ. Geol. Soc., vol. xviii, 1862, p. 100 (name only); J. W. Davis, Ann. Mag. Nat. Hist. [5] vol. viii, I881, p. 426, and Trans. Roy. Dublin Soc. [2] vol. i (1883), p. 514 , pl. 1xi, f. Io.

T. (Harpacodus.)

LOC. L. Carb. Limest.; Armagh. [Tooth ; olim Capt. Jones Coll.]

denticulatus, Agassiz. v. Callopristodus pectinatus.

lobatus (Etheridge), A. S. Woodward, Cat. Foss. Fishes B. M., pt. i, 1889, p. 51 .

Petalodus? lobatus, R. Etheridge, jun., Geol. Mag. [2] vol. ii, 1875, p. 244 , pl. viii, f. 5,6 .

Ctenopetalus crenatus, J. W. Davis, Trans. Roy. Dublin Soc. [2] vol. i (1883), p. 513. pl. lxi, f. 9 (broken tooth), and Quart. Journ. Geol. Soc., vol. xl, I884, p. 623, pl. xxvii, f. 18. [Tooth ; York Mus.]

LOC. L. Carb. Limest.; Lanarkshire. U. Carb. Limest.; Yorkshire and Derbyshire. [Tooth; Edinburgh Mus.]

macrodus, Agassiz. $\%$. Ctenoptychius dentatus.

obtusus, Barkas. v. Callopristodus pectinatus.

? ord1, [-ii] J. W. Davis, Quart. Journ. Geol. Soc., vol. xxxvii, I88I, p. 422, pl. xxii, f. 8 ; [-ii] A. S. Woodward, Cat. Foss. Fishes B. M., pt. i, 1889 , p. 54 .

Loc. Rhretic ; Aust Cliff, near Bristol. [Tooth?; J. W. Davis Coll.]

pectinatus, Agassiz. v. Callopristodus pectinatus.

priscus, L. Agassiz, Poiss. Foss. V. G. R. (1844), pp. III, I24 ; J. Morris, Cat. Brit. Foss., 1854, p. 324 . An indeterminable ichthyolite (A. S. Woodward, Cat. Foss. Fishes B. M., pt. i, I 889, p. 54).

Loc. Old Red Sandst.; Scotland. [Type unknown.]

serratus (Owen), L. Agassiz, Poiss. Foss., vol. iii (1843), pp. 173, 383 (name only) ; J. Morris, Cat. Brit. Foss., 1854, p. 324; F. M'Coy, Brit. Palæoz. Foss. (1855), p. 626, pl. iii I, f. 21-23; A. S. Woodward, Cat. Foss. Fishes B. M., pt. i, I 889, p. 52.

Petalodus serratus, R. Owen, Odontogr. (1840), p. 62.

Ctenopetalus serratus, J. Morris and G. E. Roberts, Quart. Journ. Geol. Soc., vol. xviii, I862, p. Ioo (name only); J. W. Davis, Ann. Mag. Nat. Hist. [5] vol. viii, 1881, p. 426, and Trans. Roy. Dublin Soc. [2] vol. i (1883), p. 512, pl. lxi, f. 6-8.

T. (Ctenopetalus.)

LoC. L. Carb. Limest.; Armagh. Carb. Limest.; Shropshire (Davis) and Derbyshire $\left(\mathrm{M}^{\circ} \mathrm{Coy}\right) . \quad$ [Tooth ; olim Capt. Jones Coll.] 


\section{CXINOPTYCIIUS (continued)-}

tripartitus (Davis), A. S. Woodward, Cat. Foss. Fishes B. M., pt. i, r 889 , p. 5 t.

Petalodopsis tripartitus, [-is] J. W. Davis, Trans. Roy. Dublin Soc. [2] vol. i (1883), p. 499, pl. lx, f. 6, and Quart. Journ. Geol.

Soc., vol. xl, 1884, pl. xxvi, f. 16. T. (Petalodopsis, Davis.) Loc. Yoredale Rocks; Wensleydale. [Tooth ; York Mus.]

unilateralis, T. P. Barkas. $v$. Euctenius unilateralis.

sp., T. P. Barkas, 1869. थ. Euctenius unilateralis.

CURTODUS, Sauvage. \% Asteracanthus.

CYATrASPIS, E. R. Lankester, Rep. Brit. Assoc., IS64 (1865), Sect., p. 58, and Fishes O. R. Sandst., pt. i (Pal. Soc., 1868), p. 26.

banksi (Huxley and Salter), [-ii] E. R. Lankester, Fishes O. R. Sandst., pt. i (Pal. Soc., i868), p. 26, pl. ii, f. 9-11, pl. iv, f. 6.

Pteraspis banksii, T. H. Huxley and J. WV. Salter, Quart. Journ. Geol. Soc., vol. xii, I856, p. 100, pl. ii, f. 2 ; T. H. Huxley, Quart. Journ. Geol. Soc., vol. xiv, 1858, p. 274, pl. xv.

LOC. Downton Sandst.; Herefordshire. [Head-shield.] $\boldsymbol{T}$.

symondsi, E. R. Lankester, Rep. Brit. Assoc., 1864 (1865), Sect., p. 58, and Fishes O. R. Sandst., pt. i (Pal. Soc., 1868), p. 27, pl. vi, f. 5 .

LOC. L. Old Red Sandst. (Cornstones); Herefordshire. [Internal cast of head-shicld; Mus. Pract. Geol.]

CצBIU M, Cuvier, Règne Animal, ed. 2, vol. ii, 1829, p. 199.

macropomum, L. Agassiz, Poiss. Foss., vol. v, pt. i (1843), p. 62, pl. xxvi, f. I-3 ; J. Morris, Cat. Brit. Foss., 1854 , p. 324.

Loc. London Clay; Sheppey. [Head; Paris Mus.]

CYCIA Trinus, L. Agassiz, Poiss. Foss., vol. iii (1843), p. 382.

macropterus, L. Agassiz, ibill., p. 382 , pl. xliv, f. 1 ; J. Morris, Cat. Brit. Foss., 1854 , p. 324.

LOC. L. Lias; Lyme Regis. [Pectoral fin; Bristol Mus.] $\boldsymbol{T}$.

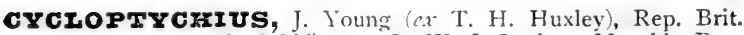
Assoc., I 865 (I866), p. 3 I 8 ; W. J. Barkas, Monthly Rev. Dental Surgery, vol. iii, 1875, p. 500.

carbonarius, J. Young (ex T. H. Huxley), ibid., p. 319 ; R. H. 'Traquair, Geol. Mag. [2] vol. i, 1874, p. 24I, pl. xii ; J. Ward, [Proc.] N. Staffs. Nat. Field Club, 1875 , p. 240 , and Trans. N. Staffs. Inst. Mining Engin., vol. x, I889, pl. v. f. 3-5.

Cycloptychius, A. Hancock and T. Atthey, Ann. Mag. Nat. Hist. [4] vol. i, i 868, p. 362 ; T. P. Barkas, Coal Meas. Palæont. I 873 , p. 36, f. I 40 .

Loc. Coal-Meas. (Deep-mine Ironstone); Longton, Staffordshire. Coal-Nleas.; Newcastle-upon-Tyne. [Fishes; J. Ward Coll.] 
Cxoxormycrivs (continucd)-

concentricus, R. H. Traquair, Trans. Roy. Soc. Edinb., vol. $\mathrm{xxx}, 188 \mathrm{r}$, p. 37 , pl. ii, f. $17-20$.

LOC. Calcif. Sandst. (Cement-stone Group); Glencartholm, Eskdale. [Fishes; Geol. Surv. Scotland.]

Cxaropodus, R. H. Traquair, Geol. Mag. [2] vol. viii, I88 I, p. 35 .

crenulatus, R. H. Traquair, ibid., p. 35 , and ibid., vol. ix, 1882, p. 541. Figure in forthcoming Cat. Foss. Fishes B. M., pt. ii, pl. i, f. I.

Loc. L. Carb.; Scottish Coalfield. [Dermal spines; Edinburgh Mus.]

Cyzronodus, J. W. Davis, Quart. Journ. Geol. Soc., vol. xl, I 884 , p. 630 ; A. S. Woodward, Cat. Foss. Fishes B. M., pt. i, 1889, p. 216.

gibbus, J. W. Davis, Quart. Journ. Geol. Soc., vol. xl, i884, p. 63 I, pl. xxvii, f. I, 6 .

Loc. Yoredale Rocks; Wensleydale, Yorkshire. [Teeth; York Mus.]

T.

hornei, A. S. Woodward, Cat. Foss. Fishes B. M., pt. i, I889, p. 216, pl. vi, f. 26.

LOC. U. Carb. Limest. (incl. Yoredale Rocks) ; Richmond and Wensleydale, Yorkshire. ['Tooth ; Brit. Mus.]

DACTYLODUS, Newberry and Worthen. $\%$ Polyrhizodus.

DApgDius, H. T. De la Beche, Trans. Geol. Soc. [2] vol. i (1822), p. 45 (Dapedium).

Tetragonolepis, L. Agassiz (non Bronn), Poiss. Foss., vol. ii, pt. i $(1835)$, p. 18 I.

Echnodus, P. M. C. Egerton, Quart. Journ. Geol. Soc., vol. x, 1854 , p. 367 , (withdrawn P'alæont. Indica [4] vol. i, pt. 2, I 878, p. 8.)

angulifer: Tetragonolepis angulifer, L. Agassiz, Poiss. Foss., vol. ii, pt. i (1837), p. 213, pl. xxiii.

Echmodus angulifer (P. M. G. Egerton, Quart. Journ. Geol. Soc., vol. $x, 1854$, p. 367 ), J. Morris, Cat. Brit. Foss., 1854, p. 316.

Tetragonolepis traillii, L. Agassiz, Neues Jahrb., 1832, p. 147, and Poiss. Foss., vol. ii, pt. i (1833), p. 7 (name only).

LOC. Lias; Stratford-on-Avon. [Fish; olim Weaver Coll., Birmingham.]

arenatıs, L. Agassiz, Poiss. Foss., vol. ii, pt. i (1843), p. 304 (name only).

LOC. L. Lias ; Lyme Regis.

cole1, L. Agassiz, Poiss. Foss., vol. ii, pt. i (1835), p. 195, pl. xxv b, f. $1-7$, pl. xxv c ; J. Morris, Cat. Brit. Foss., 1854 , p. 324. LOC. L. Lias ; Lyme Regis. Imperfect fish; Brit. Mus.] 
DAPEDIUS (continued) -

dorsalis , Tetragonolepis dorsalis, L. Agassiz, Poiss. Foss., vol. ii, pt. i (1837), p. zis, pl. xxi, f. I, 2, pl. xxi a, f. I.

Echmodus dorsalis (P. M. G. Egerton, Quart. Journ. Geol. Soc., vol. x, 1854, p. 367), J. Morris, Cat. Brit. Foss., 1854, p. 316.

LOC. Lias; Byrford, Gloucestershire. [Fish ; Brit. Mus.]

fimbriatus, Agassiz. $\%$. Eugnathus latus.

granulatus, L. Agassiz, Poiss. Foss., vol. ii, pt. i (1835), p. 190, pl. xxv, f. 2-6; W. C. Williamson, Phil. Trans., 1849, p. 445 , pl. xl, f. 5, 6 (D. gremulosus) ; J. Morris, Cat. Brit. Foss., 1854, p. 324.

Tetragonolepis pustulatus, L. Agassiz, Poiss. Foss., vol. ii, pt. i (1837), p. 20I, pl. xxiii $c$. [Imperfect head and abdominal region; Brit. Mus.]

A.chmodus pustulatus (P. M. G. Egerton; Quart. Journ. Geol. Soc., vol. x, 1854 , p. 367), J. Morris, Cat. Brit. Foss., 1854, p. 317 .

LOC. L. Lias ; Lyme Regis. [Imperfect head, etc. ; Oxford Mus.]

gramulosıs, Williamson. $\%$. Dapedius granulatus.

heteroderma : Tetragonolepis heteroderma, L. Agassiz, Neues Jahrb., 1832, p. 147, and Poiss. Foss., vol. ii, pt. i (1837). p. 206, pl. xxiii e, f. I. [Aichmodus heteroderma (P. M. G. Egerton, Quart. Journ. Geol. Soc., vol. x, 1854, p. 367), J. Morris, Cat. Brit. Foss., I854, p. 316.7 Recorded upon very doubtful evidence from the L. Lias of Lyme Regis.

leachi : Tetragonolepis leachii, L. Agassiz, Poiss. Foss., vol. ii, pt. i (1837), p. 203, pl. xxiii $d$.

Aichmodus leachii (P. M. G. Egerton, Quart. Journ. Geol. Soc., vol. $x, 1854$, p. 367), J. Morris, Cat. Brit. Foss., 1854, p. 316.

Pholidotus leachii, W. C. Williamson, Phil. Trans., I849, p. 444.

Tetragonolepis striatus, L. Agassiz (in coll.), H. G. Bronn, Index Palæont., I848, p. I261.

LOC. L. Lias ; Lyme Regis. [Head and scales ; Brit. Mus.]

lelosomus: Tetragonolepis leiosomus, L. Agassiz, Poiss. Foss., vol. ii, pt. i (1837), p. 202, pl. xxiii a, f. 3 .

Echmodus leiosomus (P. M. G. Egerton, Quart. Journ. Geol. Soc., vol. $x, 1854$, p. 367), J. Morris, Cat. Brit. Foss., 1854, p. 316. [Immature fish.]

Loc. L. Lias; Lyme Regis. [Fish ; Brit. Mus.]

micams, L. Agassiz, Poiss. Foss., vol. ii, pt. i (1843), p. 304 (name only); J. F. Blake, in Tate and Blake, Yorksh. Lias, 1876, p. 257 ('doubtful formation and locality:')

LOC. 'U. Lias; Whitby.' [Specifically indeterminable scales ; Brit. Mus.] 
DAPEDUS (continued)-

montlfer (Agassiz), J. Morris, Cat. Brit. Foss., I854, p. 324.

Tetragnnolepis monilifer, L. Agassiz, Poiss. Foss., vol. ii, pt. i (1837), p. 212, pl. xxi $a$, f. 25.

Tetragonolepis striolatus, L. Agassiz, ibid. (1843), p. 304 (name only). [specimen labelled by Agassiz in Brit. Mus.]

LOC. L. Lias; Barrow-on-Soar. [Fish, wanting head; Bristol' Mus.]

orbicularis : Echmodus orbicularis, J. Morris, Geol. Mag., vol. vi, 1869, p. 337, pl. x.

LOC. L. Lias; Lyme Regis. [Fish; W. H. Hudleston Coll.]

orb1s, L. Agassiz, Poiss. Foss., vol. ii, pt. i (1837), p. 2 I 8, pl. xxv $d$; W. C. Williamson, Phil. Trans., 1849, p. 445 ; J. Morris, Cat. Brit. Foss., 1854, p. 324.

LOC. L. Lias; Barrow-on-Soar. [Fish, wanting tail ; Scarborough Mus.]

ovalis : Tetragonolepis ovalis, L. Agrassiz, Poiss. Foss., vol. ii, pt. ir (1837), p. 209, pl. xxi, f. 3. [Echmodus aralis (P. M. G. Egerton, Quart. Journ. (ieol. Soc., vol. x, 1854, p. 367), J. Morris, Cat. Brit. Foss., 1854 p. 317 i. J. F. Blake, in Tate and Blake, Yorksh. Lias, I876, p. 258.] Recorded, upon uncertain evidence, from the U. Lias of Whitby.

pholidotus: Tetragonolepis pholidotus, L. Agassiz, Neues Jahrb., 1832 , p. 147, and Poiss. Foss., vol. ii, pt. i (1837), p. 207, pl. xxiii e, f. 2. [Achmodus pholidotus (P. M. G. Egerton, Ouart. Journ. Ceol. Soc., vol. x, 1854, p. 367), J. Mlorris, Cat. Brit. Foss.. 1854, p. 317.] Recorded, upon uncertain evidence, from the L. Lias of Lyme Regis.

politus, H. De la Beche, Trans. Geol. Soc. [2] vol. i (1822), p. 45, pl. vi, f. I-4 (Dapedium politum); L. Agassiz, Poiss. Foss., vol. ii, pt. i ( 1835$)$, p. 185 , pl. xxv, f. I ; J. Morris, Cat. Brit. Foss., 1854, p. 324 .

Tetragonolepis confluens, L. Agassiz, Poiss. Foss., vol. ii, pt. i (1835), p. 199, pl. xxiii a, f. 1. [Head, etc. ; Brit. Mus.]

Echmodus confluens (P. M. G. Egerton, Quart. Journ. Geol. Soc., vol. x, 1854, p. 367), J. Mo:ris, Cat. Brit. Foss., 1854, p. 316.

LOC. L. Lias ; Lyme Regis. [Fish ; unknown.]

T.

punctatus, L. Agassiz, Poiss. Fos๔., vol. ii, pt. i (1835), p. 192, pl. xxva, pl. xxv, f. 7-9; J. Morris, Cat. Brit. Foss., 1854 , p. 324 .

? Tetragonolepis speciosus, L. Agassiz, Poiss. Foss, vol, ii, pt. i (1835), p. 199, pl. xxiii b. [Fish; Brit. Mus.]

? Echmodus specinsus (P. M. (. E. Egerton, Quart. Journ. Geol. Soc., vol. x, 1854 , p. 367 ), J. Morris, Cat. Brit. Eoss., I 854 , p. 317.

LOC. L. Lias ; Lyme Regis. [Fish ; Oxford Mus.] 
DAPEDIUS (continued) -

radiatus : Tetragonolepis ratiatus, L. Agassiz, Poiss. Foss., vol. ii, pt. i (1837), p. 20I, pl. xxiii $a$, f. 2.

Echmodus radiatus (P. M. G. Egerton, Quart. Journ. Geol. Soc., vol. x, 1854, p. 367), J. Morris, Cat. Brit. Foss., 1854, p. 317.

LOC. L. Lias ; Lyme Regis. [Imperfect head and trunk; Brit. Mus.]

DAPTINUS, Cope. v. Saurocephalus.

intermedius, Newton. \%. Saurocephalus intermedius.

Deltodopsis sp., St. John and Worthen. v. Sandalodus morrisi.

D\&ITODUS, J. Morris and G. F. Roberts (ex Agassiz, MS.), Quart. Journ. Geol. Soc., vol. xviii, 1862, p. 100 (name only); A. S. Woodward, Cat. Foss. Fishes B. M., pt. i, I889, p. 195.

TæNiodus, O. St. John and A. H. Worthen (ex De Koninck, MS.), Pal. Illinois, vol. vii, 1883, p. 75 (pars).

alfformis (M'Coy), J. W. Davis, Trans. Roy. Dublin Soc. [2] vol. i (1883), p. 43I, pl. liii, f. I 2.

Pecilodus aliformis, F. M'Coy, Ann. Mag. Nat. Hist. [2] vol. ii, 1848 , p. 129, and Brit. Palroz. Foss. (1855), p. 638, pl iii G, f. 10 ; J. Morris, Cat. Brit. Foss., 1854, p. 340 .

LOC. U. Carb. Limest. ; Derbyshire. [Tooth ; Woodwardian Mus.]

expansus, Davis. \%. Deltodus sublævis and Deltoptychius acutus.

glbbus, A. S. Woodward, Cat. Foss. Fishes B. M., pt. i, I889, p. 197, pl. vi, f. 19, 20.

LOC. L. Carb. Limest. ; Bristol. [Teeth ; Brit. Mus.]

rugosus, A. S. Woodward, Cat. Foss. Fishes B. MI., pt. i, 1889, p. 198. pl. vi, f. 2 I, 22.

LOC. Carb. Limest. ; Oreton, Shropshire. [Teeth ; Brit. Mus.]

subløvis ( $\mathrm{I}^{6} \mathrm{C}$ oy), J. Morris and G. E. Roberts, Quart. Journ. Geol. Soc., vol. xviii, 1862, p. 100 (name only); J. W. Davis, Trans. Roy. Dublin Soc. [2] vol. i (1883), p. 428, pl. lii, f. 7-9; A. S. Woodward, Cat. Foss. Fishes B. M., pt. i, 1889. p. 196.

Pacilodus sublavis, L. Agassiz, Poiss. Foss., vol. iii (I843), p. 174 (name only); J. Morris, Cat. Brit. Foss., 1854, p. 340; F. M'Coy, Brit. Palæoz. Foss. (1855), p. 640, pl. iii I, f. 7-9.

Pacilodus parallilus, L. Agassiz, Poiss. Foss., vol. iii (1843), p. 174 (name only); J. Morris, Cat. Brit. Foss., 1854, p. 340 ; F. M'Coy, Brit. Palcoz. Foss. (1855), p. 640, pl. iii I, f. 6. [Tooth; Woodwardian Mus.]

Deltodus expansues, J. W. Davis (pars), Trans. Roy. Dublin Soc. [2] vol. i (1883), p. 431, pl. liii, f. II (nion f. 9. 10). [Tooth; Brit. Mus.] 
DExTODUs subløvis (continued)-

Pecilodus jonesii, J. W. Davis (errore), ibid., p. 442, pl. liii, f. 22. [Tooth ; Brit. Mus.]

LOC. L. Carb. Limest.; Armagh. [Teeth; Woodwardian Mus.] sp., Morris and Roberts. $v$. Sandalodus morrisi.

DExTOPTyCrIUs, J. Morris and G. E. Roberts (ex Agassiz, MS.), Quart. Journ. Geol. Soc., vol. xviii, I862, p. Ioo (name only); J. W. Davis, Trans. Roy. Dublin Soc. [2] vol. i (1883), p. 432.

acutus (M'Coy), J. Morris and G. E. Roberts, Quart. Journ. Geol. Soc., vol. xviii, I862, p. I00 (name only); J. W. Davis, Trans. Roy. Dublin Soc. [2] vol. i (1883), p. 433, pl. liii, f. 13-17 ; A. S. Woodward, Cat. Foss. Fishes B. M., pt. i, 1889, p. 212.

Cochliodus acutus, L. Agassiz, Poiss. Foss., vol. iii (1843), p. 174 (name only); J. Morris, Cat. Brit. Foss., 1854, p. 322 ; F. M'Coy, Brit. Palæoz. Foss. (1855), p. 62 I, pl. iii I, f. 24.

Cochliodus compactus, R. Owen, Geol. Mag., vol. iv, I867, pl. iv, f. I. [Tooth; Woodwardian Mus.]

Deltodus expansus, J. W. Davis (pars), Trans. Roy. Dublin Soc. [2] vol. i (1883), p. 43I, pl. liii, f. 9, Io (non f. II). [Teeth; Brit. Mus.]

? Deltoptychius plicatus, J. IV. Davis, Quart. Journ. Geol. Soc., vol. xl, 1884, p. 628, pl. xxvi, f. 13, I4, and Geol. Mag. [3] vol. iii, 1886, p. 152. [Teeth; York Mus.]

LOC. L. Carb. Limest.; Armagh and Ayrshire. U. Carb Limest.; Yorkshire and Derbyshire. [Tooth; Woodwardian Mus.]

gibberulus, J. Morris and G. E. Roberts (ex Agassiz, MS.), Quart. Journ. Geol. Soc., vol. xviii, I862, p. 100 (name only); J. W. Davis, Trans. Roy. Dublin Soc. [2] vol. i (1883), p. 435, pl. liii, f. 18, 19; A. S. Woodward, Cat. Foss. Fishes B. M., pt. i, 1889, p. 214 , pl. i, f. 21.

Cochliodus contortus, L. Agassiz (errore), Poiss. Foss., vol. iii (1838), p. I I5, pl. xiv, f. 21, 29-33 (Psammodus contortus on plate). [Teeth; Bristol Mus.]

LOC. L. Carb. Limest. ; Wexford, Gloucestershire, Somersetshire, and Shropshire. U. Carb. Limest.; Derbyshire. [Teeth ; Brit. Mus.]

plicatus, Davis. v. Deltoptychius acutus.

Dras DRoDUS, R. Owen, Microscopic Journal, vol. i, 1841, p. 4, and Odontogr. (1845), p. 171 ; C. H. Pander, Saurodipt., Dendrodont., etc., devon. Syst., 1860, p. 27 ; J. V. Rohon, Mém. Acad. Imp. Sci. St. Pétersbourg [7] vol. xxxvi, no. 14 (1889); R. H. Traquair, Geol. Mag. [3] vol. vi, 1889, p. 490.

Lamnodus, L. Agassiz, Poiss. Foss. V. G. R. (1844), p. 83. 
Drad DRODU (continued)-

biporcatus, R. Owen, Microscopic Journal, vol. i, I8+1, p. 5, woodcuts 1, 2, and p. I9, woodcut 5, and Odontogr. (1841), p. I71, pl. lxii A, 1. 1, pl. lxii B ; P. Duff, Geol. Moray, 1842, p. 67 , pl. vi, f. 5 .

Holoptychius or Megalichthys? R. I Murchison, Silur. Syst., 1839, p. 600, pl. il bis, f. $8,9$.

Lamnodus biporcatus, L. Agassiz, Poiss. Foss. V. G. R. (1844), pp. 84,144, pl. c, f. 7-9, 14, 19, pl. xxviii, f. 6, 7 ; J. Morris, Cat. Brit. Foss., 1854 , p. 33 I. [Teeth.] T. (Lamnodus.)

Dendrodus compressus, R. Owen, Microscopic Journal, vol. i, I84I, p. 17, woodcut 3; P. Duff, Geol. Moray, 1842, p. 67, pl. vi, f. 7 .

Dendrodus hastatus, R. Owen, Odontogr. (1841), p. 175.

Lamnodus hastatus, L. Agassiz, Poiss. Foss. V. G. R. (1844), p. 87, pl. C, f. I-6, IJ-13.

Lamnodus panderi, L. Agassiz, Poiss. Foss., vol. ii, pt. ii (1843), p. 162 (name only).

Dendrodus strigatus, L. Agassiz (errore), Poiss. Foss., vol. ii. pt. ii (1843), p. I62, pl. lv $a$, f. I 9, 20.

Dendrodus latus, P. I) uff, Geol. Moray, 1842 , p. 67 , pl. vi, f. 4 ; L. Agassiz, Poiss. Foss. V. G. R. (1844), p. 82, pl. xxviii, f. I, 2. Tooth of Holoptychius, H. Miller, Old Ked Sandst., I $8+1$, pl. ix, f. 4 .

Lamnodus sulcatus, L. Agassiz, Poiss. Foss. V. G. R. (1844), p. I45, pl. xxviii $a$, f. IS ; J. Morris, Cat. Brit. Foss., I854, p. 331 .

LOC. U. Old Red Sandst.; Scat Craig. [Teeth.]

$\mathbf{x}$.

incurvus, Duff. \%. Cricodus incurvus.

strigatus, R. Owen, Microscopic Journal, vol. i, 1841, p. 17, woodcut I ; P. Duff, Geol. Noray, 1842, p. 67, pl. vi, f. 6; L. Agassiz, Poiss. Foss. V. G. R. (1844), p. 80 , pl. c, f. 10, 20-22, pl. xxviii a, f. I, 2 ; J. Morris, Cat. Brit. Foss., 1854 , p. 324 .

Dendrodus sigmoideus, R. Owen, Microscopic Journal, vol. i, I841, p. 17, woodcut 2; P. Duff, Geol. Moray, 1842, p. 68, pl. vi, f. 8-10; L. Agassiz, Poiss. Foss. V. G. R. (I844), p. 82 , pl. xxviii, f. 3 , xxviii a, f. 3-5 ; J. Morris, Cat. Brit. Foss., 1854, p. 324. [Tooth.]

Loc. U. Old Red Sandst.; Scat Craig. [Tooth.]

IENDROPTICHIUS, Young. v. Strepsodus.

sp., Young. \%. Strepsodus sauroides.

DERCETrs, L. Agassiz, Poiss. Foss.. vol. ii, pt. ii (1843), p. 258 ; A. S. Woodward, ?'roc. Geol. Assoc., vol. x (1888), p. 318.

? Pelargorhynchus, IV. von der Marck, Zeitschr. deutsch. geol. Ges., vol. x, 1858 , p. 242. 
Dracerrs (continued) -

elongatus, L. Agassiz, Poiss. Foss., Feuill., p. 55 (1835) (pars, name only), and vol. ii, pt. ii (1843), p. 258, pl. Ixvi a, f. I, 2, 5-8 (non f. 3, 4); F. Dixon, Geol. Sussex, 185o, pl. xxxiv, f. 5 ; J. Morris, Cat. Brit. Foss., 1854, p. 325 ; W. Davies, Geol. Mag. [2] vol. vi, I879, p. 145; A. S. Woodward, Proc. Geol. Assoc., vol. x (1888), p. 318. [See also Murcena lewesiensis.]

LOC. Chalk; S. E. England. [Imperfect fishes; Brit. MIus.] T.

DICentrodus, R. H. Traquair, Geol. Mag. [3] vol. v, I888, p. 86.

bicuspldatus, R. H. Traquair, Geol. Mag. [3] vol. v, 1888, p. 86 ; A. S. Woodward, Cat. Foss. Fishes B. M., pt. i, 1889, p. 26, pl. vi, f. 7-9.

Cladodus bicuspidatus, R. H. Traquair, Geol. Mag. [2] vol. viii, I88 I, p. 35.

LOC. M. Carb. Limest. (Blackband Ironstone); Borough Lee, near Edinburgh. [Teeth; Edinburgh Mus.]

DICIrmodus, J. W. Davis, Trans. Roy. Dublin Soc. [2] vol. i, (1883), p. 410.

scitulus, J. W. Davis, ibid., p. 4 Io, pl. li, f. 29, and Quart. Journ. Geol. Soc., vol. xl, 1884, p. 623, pl. xxvii, f. 12.

Loc. Yoredale Rocks ; Wensieydale, Yorkshire. [Tooth; York Mus.]

T.

DICREN ODUs, H. Romanovsky, Bull. Soc. Imp. Nat. Moscou, I 853 , pt. i, p. 408.

Carcharopsis, L. Agassiz, Poiss. Foss., vol. iii (1843), p. 313 (undefined); J. W. Davis, Trans. Roy. Dublin Soc. [2] vol. i (1883), p. 381 .

Pristicladodus, F. M'Coy, Brit. Palroz. Foss. (1855), p. 642 .

dentatus ( $\mathrm{M}^{\prime} \mathrm{C}$ oy), A. S. Woodward, Cat. Foss. Fishes B. M., pt. i, 1889, p. 28.

Carcharopsis prototypus, L. Agassiz, Poiss. Foss., vol. iii (1843), p. 313 (name only). [Tooth; Brit. Mus.]

x. (Carcharopsis.)

Pristicladodus dentatus, F. M'Coy, Brit. Paleoz. Foss. (1855), p. 642, pl. iii G, f. 2 ; J. IV. Davis, Trans. Roy. Dublin Soc. [2] vol. i (1883), p. 384, pl. xlix, f. 22, and Quart. Journ. Geol. Soc., vol. xl, I884, p. 620, pl. xxvii, f. 4 ; R. H. Traquair, Geol. Mag. [3] vol. v, I888, p. 103. T. (P'risticladoilus.)

Carcharopsis colei, J. W. Davis, Trans. Roy. Dublin Soc. [2] vol. i (1883), p. 383, pl. xlix, f. 26. [Tooth; Brit. Mus.]

LOC. L. Carb. Limest.; Armagh and Ayrshire. U. Carb. Limest.; Yorkshire and Derbyshire. [Tooth; Woodwardian Mus.] 
DICRENODUS (continued) -

goughi (M'Coy), A. S. Woodward, Cat. Foss. Fishes B. M., pt. i, 1889, p. 29.

Pristicladodus goughi, F. M'Coy, Brit. Palæoz. Foss. (1855), p. 643 , pl. iii $\mathrm{K}$, f. 11 .

LOC. Carb. Limest.; Kendal. [Tooth; Woodwardian Mus.]

DICTyodus, R. Owen, Rep. Brit. Assoc., 1838 (1839), Sect., p. I42; L. Dollo and R. Storms, Zool. Anzeiger, I888, p. 265.

SPhyrenodus, L. Agassiz, Poiss. Foss., vol. v, pt. i (1843), p. 98.

? crassidens : Sphyranodus crassidens, L. Agassiz, ibid., p. 99 (name only).

LOC. London Clay ; Sheppey. [Fragments of jaws ; Brit. Mus.] gracilis, Dixon. v. Dictyodus tenuis.

priscus: Sphyrenodus priscus, L. Agassiz, Poiss. Foss., vol. v, pt. i (1843), p. 98, pl. xxvi, f. 4-6; J. Morris, Cat. Brit. Foss., 1854 , p. 34 j.

Dictyodus, R. Owen, Rep. Brit. Assoc., 1838 (1839), Sect., p. 142. Sphyranodus priscus (Dictyodus), R. Owen, Odontogr. (184I), p. 129, pl. liv.

LOC. London Clay; Sheppey. [Head; Brit. Mus.]

T.

tenuis : Sphyranodus temis, F. Dixon, Geol. Sussex, I850, p. I12, pl. xi, f. 24 (misprinted $S$. gracilis, ibid., p. 205, and copied by J. Morris, Cat. Brit. Foss., 1854, p. 344 ; also E. T. Newton, in F. Dixon, Geol. Sussex, ed. 2, 1878, p. 251.)

Loc. Bracklesham Beds. [Portion of jaw ; Brit. Mus.]

DICTy O Pу Gæ, P. M. G. Egerton, Quart. Journ. Geol. Soc., vol. iii, I 847 , p. 276 ; R. H. Traquair, Quart. Journ. Geol. Soc., vol. xxxiii, 1877, p. 565 .

catoptera (Agassiz), R. H. Traquair, ibid., p. 567.

Palconiscus catopterus, L. Agassiz, Poiss. Foss., vol. ii, pt. i (1843), p. 303 (name only); R. I. Murchison, Proc. Geol. Soc., vol. ii (1836), p. 206 ; P. M. G. Egerton, Quart. Journ. Geol. Soc., vol. vi, 1850, p. 4 , and ibid., vol. xiv, 1858 , pl. xi, f. 4 ; W. King, Permian Foss. (Pal. Soc., I850), p. 226 ; J. Morris, Cat. Brit. Foss., I854, p. 336.

LOC. Keuper; Roan Hill, Tyrone. [Fishes; Brit. Mus. and Mus. Pract. Geol.]

superstes (Egerton), K. A. von Zittel, Handb. Palæont., vol. iii (1887), p. 203.

Palconiscus superstes, P. M. G. Egerton, Quart. Journ. Geol. Soc., vol. xiv, 1858, p. 164, pl. xi, f. 1-3.

Loc. Keuper; Rowington, Warwickshire. [Fish ; P. B. Brodie Coll.] 
DIDYMas PIS, E. R. Lankester, Geol. Mag., vol. iv, I867, p. 152 .

grindrodi, E. R. Lankester, ibid., p. 153, pl. viii, f. 4-8, and Fishes O. R. Sandst., pt. i (Pal. Soc., 1870), p. 59, pl. xiii, f. I, 2, and woodcut 33 .

Loc. L. Old Red Sandst. (Passage lieds); Ledbury, Herefordshire. [Head-shield; Oxford Mus.]

T.

DIMILEUS, J. W. Davis (ex Agassiz, MS.), Trans. Roy. Dublin Soc. [2] vol i (1883), p. 4;8. [Genus non det.]

woodii, J. W. Davis (e.r Agassiz, MS.), ibid., p. 478, pl. lviii, f. 24. Indeterminable tooth (A. S. Woodward, Cat. Foss. Fishes B. M., pt. i, 1889, p. 99).

LOC. U. Carb. Limest. ; Richmond, Yorkshire. [Tooth ; Brit. Mus.]

r.

DIODONTOPSODUS, Davis. \% Pristodus.

DIPIACANTrus, L. Agassiz, Poiss. Foss. V. G. R. (1844), pp. $34,40$.

Rhadinacanthus, R. H. Traquair, Geol. Mag. [3] vol. v, i888, p. 512 .

cressispinus, Agassiz. \%. Diplacanthus striatus.

cressissimus, Duff. v. Diplacanthus striatus.

? curtus: Euthacanthus curtus, J. Powrie, Trans. Edinb. Geol. Soc., vol. i (1870), p. 293, pl. xii, f. 7.

LOc. L. Old Red Sandst. ; Forfarshire. [Fish, wanting head; J. Powrie Coll.]

gibbus, M'Coy. $\approx$. Diplacanthus striatus.

gracilis, Egerton. $v$. Ischnacanthus gracilis.

longispinus, L. Agassiz, Poiss. Foss. V. G. R. (1844), pp. 34, 42, pl. xiii, f. 5, pl. xiv, f. 8, 9 ; J. Morris, Cat. Brit. Foss., I854, p. 325 .

'Ichthyolite,' H. Miller, O. R. Sandst., I841, pl. viii, f. I.

Diplacanthus perarmatus, F. M'Coy, Ann. Mag. Nat. Hist. [2] vol. ii, I848, p. 301 , and Brit. Palæoz. Foss. (1855), p. 585 , pl. ii B, f. 3 ; J. Morris, Cat. Brit. Foss., 1854, p. 325. [Fish; Woodwardian Mus.]

Rhadinacanthus longispinus, R. H. Traquair, Geol. Mag. [3] vol. v, I 888 , p. 5 I2.

T. (Rhadinacunthus.)

LOC. L. Old Red Sandst.; Nairnshire, Cromarty, and Orkney Isles. [Fish ; Forres Mus.]

perarmatus, $\mathrm{M}^{\prime} \mathrm{Coy}$. v. Diplacanthus longispinus.

striatulus, Agassiz. $\%$. Diplacanthus striatus.

strlatus, L. Agassiz, Poiss. Foss. V. G. R. (1844), pp. 34, 4I, pl. xir, f. I-5; J. Morris, Cat. Brit. Foss., 1854, p. 325 ; R H Traquair, Geol. Mag. [3] vol. v, 1888, p. 512. 
DXPXACANTIUS striatus (continued)-

'Ichthyolite,' H. Miller, O. R. Sandst., I84I, pl. viii, f. 2.

Diplacanthus striatulus, L. Agassiz, Poiss. Foss. V. G. R. (1844), pp. 34, 42, pl. xiii, f. 3, 4 ; J. Morris, Cat. Brit. Foss., 1854, p. 325. [Imperfect fishes ; Forres and Brit. Mus.]

Diplocanthus crassisimus, P. Duff, Geol. Moray, 1842, p. 7 I, pl. x, f. 2.

Diplacanthus crassispinus, L. Agassiz, Poiss. Foss. V. G. R. (1844), pp. 34, 43, pl. xiii, f. I, 2, pl. xiv, f. 6, 7 ; J. Morris, Cat. Brit. Foss., 1854, p. 325. [Imperfect fishes; Brit. Mus. (pars).]

Diplacanthus gibbus, F. M'Coy, Ann. Mag. Nat. Hist. [2] vol. ii, I848, p. 301 , and Brit. Palæoz. Foss. (1855), p. 584, pl. ii B, f. 4 ; J. Morris, Cat. Brit. Foss., I854, p. 325. [Fish; Woodwardian Mus.]

LOC. L. Old Red Sandst.; Cromarty, Banffshire, Morayshire, Ross-shire, and Orkney Isles. [Imperfect fishes; Edinburgh Mus. (pars).]

DIPIACOTS, J. W. Davis, Quart. Journ. Geol. Soc., vol. xl, I884, p. 632 ; emend. A. S. Woodward, Cat. Foss. Fishes B. M., pt. i, 1889, p. 216.

bulboides, J. W. Davis, Quart. Journ. Geol. Soc., vol. xl, 1884, p. 633, pl. xxvii, f. 20 ; A. S. Woodward, Cat. Foss. Fishes B. M., pt. i, I889, p. 216, pl. vi, f. 27.

LOC. Yoredale Rocks; Wensleydale, Yorkshire. [Portion of tooth ; York Mus.]

DIXI0DUS, L. Agassiz, Poiss. Foss., vol. iii (I843), p. 204. [A provisional generic name applied to the teeth of the Pleuracanthidæ.]

DitTodus, R. Owen, Trans. Odontol. Soc., vol. v, 1867, p. 325 (pars).

Ochlodus, R. Owen, ibid., p. 346.

Aganodus, R. Owen, ibid., p. 359.

PternotuUs,, R. Owen, ibid., p. 363 .

equilateralis, J. Ward, Trans. N. Staffs. Inst. Mining Engin., vol. $\mathrm{x}, \mathrm{I} 889$, p. 139 , pl. ii, f. 2.

LOC. Coal-Meas. ; Longton. [Tooth ; J. Ward Coll.]

gibbosus, E. W. Binney (ex Agassiz), Trans. Manchester Geol. Soc., vol. i, 1841, p. 169, pl. v, f. 17, 18; L. Agassiz, Poiss. Foss., vol. iii (1843), p. 204, pl. xxii $b$, f. I (non f. 2-5); W. C. Williamson, Phil. Trans., 1851, p. 680; J. Morris, Cat. Brit. Foss., 1854, p. 325 ; A. Hancock and T. Atthey, Nat. Hist. Trans. Northumb. and Durham, vol. iii (1870), p. III ; J. Ward, Trans. N. Staffs. Inst. Mining Engin., vol. x, 1889, p. 138, pl. ii, f. 3 ; A. S. Woodward, Cat. Foss. Fishes B. M., pt. i, 1889, p. Io. [See also Diplodus tenuis.] 
DIPX0mצ' gibbosus (continued)-

Diplodus gibbus, R. Garner, Nat. Hist. Staffs., I 844 , pl. E, f. I I (name a misprint).

Dittodus divergens, R. Owen, Trans. Odontol. Soc., vol, v, 1867, p. 334, pl. ii. [Micro. section of tooth.]

Ochlodus crassus, R. Owen, ibit., p. 346, pl. v. [Micro, section of tooth.]

'. (Ochlodus.)

Aganodus apicalis, R. Owen, ibid., p. 359, pl. ix. [Micro. section of tooth.]

T. (Aganodus.)

Aganodus undatus, R. Owen, ivid., p. 362, pl. x. [Micro. section of tooth.]

Pternodus productus, R. Owen, ibid., p. 363, pl. xi. [Micro. section of tooth ; Brit. Mus.]

Pleuracanthus (Diplodus) gibbosus, J. Ward, [Proc.] N. Staffs. Nat. Field Club, 1875 , p. 224.

LOC. Coal-Meas. ; Northumberland, Lancashire, and Staffordshire. [Tooth; Brit. Mus.]

$\boldsymbol{x}$.

minutus, L. Agassiz, Poiss. Foss., vol. iii (1843), p. 205, pl. xxii 6, f. 6-8; J. Morris, Cat. Brit. Foss., I854, p. 325.

Pleuracanthus (Diplodus) minutus, J. Ward, [Proc.] N. Stafis. Nat. Field Club, 1875 , p. 225.

LOC. Calcif. Sandst.; Burdiehouse. [Imperfect teeth; olim Sir J. Robison Coll.]

moorei, A. S. Woodward, Ann. Mag. Nat. Hist. [6] vol. iii, I889, p. 299, pl. xiv, f. 4, 5 .

Diplodus, A. S. Woodward, Cat. Foss. Fishes B. M., pt. i, 1889, p. 15.

LOC. Keuper; Ruishton, near Taunton. [Teeth; Bath Mus.]

parvulus, R. H. Traquair, Geol. Mag. [2] vol. viii, I881, p. 36 ; A. S. Woodward, Cat. Foss. Fishes B. M., pt. i, 1889, p. 12, pl. vi, f. 5,6 .

LOC. M. Carb. Limest. (Blackband Ironstone); Borough Lee, near Edinburgh. [Teeth; Edinburgh Mus.]

tenuis, A. S. Woodward, Cat. Foss. Fishes B. M., pt. i, 1889, p. II, pl. vi, f. 2-4; J. Ward, Trans. N. Staffs. Inst. Mining Engin., vol. x, 1889, p. 140, pl. ii, f. I.

Diplodus gibbosus, L. Agassiz (pars), Poiss. Foss., vol. iii (1843), p. 204, pl. xxii b, f. 2-5 ; ? T. P. Barkas, Coal Meas. Palæont., 1873, p. 16, pl. i, f. 6, 7, 9-13.

Pleuracanthus (Xenacanthus) gibbosus, J. W. Salter, Foss. S. Welsh Coalfield (Mem. Geol. Surv., I86I-Iron Ores Gt Brit., pt. iii), p. 224 , pl. i, f. 10.

Diplodus, W. J. Barkas, Monthly Rev. Dental Surgery, vol. ii, I 874 , p. 346, f. i-v.

Loc. Coal-Meas.; S. Scotland, England, and S. Wales. [Teeth ; Brit. Mus.]

sp., Woodward. v. Diplodus moorei. 
DIPIOMISTUS, E. D. Cope, Bull. U.S. Geol. Surv. Territ., vol. iii, 1877 , p. 808 .

vectensis (Newton), A. S. Woodward, Geol. Mag. [3] vol. vi, I 889 , p. 230.

Cluper vectensis, E. T. Newton, Quart. Journ. Geol. Soc., vol. xlv, I889, p. I12, pl. iv.

LOC. Osborne Beds; Isle of Wight. [Fishes; G. W. Colenutt Coll., Ryde.]

DI PLOPTERAX, M'Coy. \%. Diplopterus.

DIPIOPTgRts, L. Agassiz, Poiss. Foss., vol. ii, pt. i (1835), p. II 3 ; F. M'Coy, Ann. Mag. Nat. Hist. [2] vol. ii, 1848, p. 305, and Brit. Palæoz. Foss. (1855), p. 586 (Diplopterax); P. M. G. Egerton, Ann. Mag. Nat. Hist. [2] vol. iii (I849), p. 53 .

Gyroptychius, F. M'Coy, Ann. Mag. Nat. Hist. [2] vol. ii, I848, p. 308 (pars).

affinis, Agassiz. v. Diplopterus agassizi.

agassizi, T. S. Traill, Trans. Roy. Soc. Edinb., vol. xv (1841), p. 89 (D. agassis); F. M'Coy, Brit. Palæoz. Foss. (I855), p. 586 (Diplopterax) ; J. Morris, Cat. Brit. Foss., 1854, p. 325; R. H. Traquair, Geol. Mag. [3] vol. v, I888, p. 5 I6.

Diplopterus macrocephalus, L. Agassiz, Poiss. Foss. V. G. R. (1844), p. 54, pls. xvi, xvii ; J. Morris, Cat. Brit. Foss., I854, p. 325. [Fish; Brit. Mus. and Forres Mus.]

Diplopterus affinis, L. Agassiz, Poiss. Foss. V. G. R. (1844), p. 55 ; J. Morris, Cat. Brit. Foss., I 854 , p. 325 ; F. M'Coy, Brit. Palæoz. Foss. (1855), p. 586 (Diplopterax). [Fish ; unknown.]

Diplopterus borealis, L. Agassiz, Poiss. Foss. V. G. R. (1844), p. 55, pl. xviii ; C. H. Pander, Saurodipt., Dendrodont., etc., devon. Syst., 186o, p. 23, pl. iii, f. 23-27, pl. iv. [Fish; olim T. S. Traill Coll.]

Diplopterus gracilis, F. M'Coy, Ann. Mag. Nat. Hist. [2] vol. ii, 1848, p. 305, and Brit. Paleoz. Foss. (1855), p. 586, pl. ii c, f. I (Diplopterax) ; J. Morris, Cat. Brit. Foss., I 854, p. 325. [Fish; Woodwardian Mus.]

Gyroptychius diplopteroides, F. M'Coy, Ann. Mag. Nat. Hist.

[2] vol. ii, 1848, p. 309, and Brit. Palæoz. Foss. (1855),

p. 597, pl. ii c, f. 3 ; J. Morris, Cat. Brit. Foss., 1854, p. 328.

[Fish; Woodwardian Mus.]

LOC. L. Old Red Sandst.; Orkney, Nairnshire, and Banffshire. [Imperfect fishes; olim T.S. Traill Coll.]

T.

borealis, Agassiz. $\%$. Diplopterus agassizi.

carbonarius, Agassiz. $v$. Megalichthys pygmæus.

dalgleisiensis, Anderson. v. Glyptolæmus kinnairdi. 
DIPIOPTrRUS (continued)-

gracilis, I'Coy. \%. Diplopterus agassizi.

macrocephalus, Agassiz. \%. Diplopterus agassizi.

robertsoni, Agassiz. '. Megalichthy's laticeps.

DIPRIACantrivs, $\mathrm{F}$. $\mathrm{M}^{\circ} \mathrm{C} \mathrm{Coy}, \mathrm{Ann}$. Mag. Nat. Hist. [2] vol. ii, 1848, p. 12 I, and Proc. Camb. Phil. Soc., vol. i (1848), p. 65.

falcatus, $\mathrm{M}^{\prime} \mathrm{Coy}$. v. Erismacanthus jonesi.

stokesi, [-ii] F. M'Coy, Ann. Mag. Nat. Hist. [2] vol, ii, 1 848 , p. 12I, and Brit. Palæoz. Foss. (1855), p. 627 , pl. iii K, f. I 8; [-ii] J. Morris, Cat. Brit. Foss., 1854, p. 325; [-ii] J. W. I) avis, Trans. Roy. Dublin Soc. [2] vol. i (1883), p. 360 , pl. xlviii, f. Io.

T.

LOC. L. Carb. Limest.; Armagh. [Spine ; Woodwardian Mus.]

DIPTgRon OTUS, P. M. G. Egrerton, Quart. Journ. Geol. Soc, vol. $x, 1854$, p. 369. [Name inappropriate, the dorsal fin being apparently divided by accident in breaking of matrix.]

cyphus, P. M. G. Egerton, ibid., p. 360 , pl. xi ; J. Morris, Cat. Brit. Foss., I 854 , p. 325.

LoC. Keuper; Bromsgrove. [Fish ; Mus. Pract. Geol.] T.

DIPxrR US, A. Sedgwick and R. I. Murchison, Trans. Geol. Soc. [2] vol. iii (1828), p. I43; H. Miller, Footprints of Creator, I 849, p. 59, woodcuts 18-22; R. H. Traquair, Ann. Mag. Nat. Hist. [5] vol. ii, I 878 , p. I.

Catopterus, L. Agassiz, Poiss. Foss., vol. ii, pt. i (1833), p. 3.

Polyphractus, L. Agassiz, Poiss. Foss. V. G. R. (1844), pp. 5, 29.

brachypygopterus, Sedgwick and Murchison. $v$. Dipterus valenciennesi.

macrolepidotus, Sedgwick and Murchison. $\because$ Thursius macrolepidotus.

macropterus, R. H. Traquair, Geol. Mag. [3] vol. v, 1888, p. 508, and ibid. [3] vol. vi, 1889, p. 97, pl. ii.

Loc. L. Old Red Sandst.; John o' Groats, Caithness. [Fish ; Edinburgh Mus.]

macropygopterus, Sedgwick and Murchison. 1\%. Dipterus valenciennesi.

platycephalus, Pander. \%. Dipterus valenciennesi.

valenciennes1, A. Sedgwick and R. 1. Murchison, Trans. Geol. Soc. [2] vol. iii (1828), p. I43, pl. xvi, f. I-3; [-ii] R. H. Traquair, Geol. Mlag. [3] vol. v, 1888, p. 507.

Catopterus analis, L. Agassiz, Poiss. Foss., vol. ii, pt. i (1833), p. 3 .

T. (Catopterus.)

Dipterus macropygopterus, A. Sedgwick and R. I. Murchison, Trans. Geol. Soc. [2] vol. iii (1828), p. 143, pl. xv, f. 1-3; F. M'Coy, Brit. l'aleoz. Foss. (1855), p. 502. [Fish; Geol. Soc. London.] 


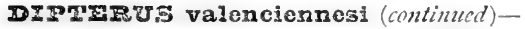

Dipterus brachypygotterus, A. Sedgwick and R. I. Murchison, Trans. Geol. Soc. [2] vol. iii (1828), p. I43, pl. xvii, f. I-3; F. M'Coy, Brit. Palæez. Foss. (I855), p. 592. [Fish ; Geol. Soc. London.]

Polyfhractus platycephalus, L. Agassiz, Poiss. Foss. V. G. R. (1844), pp. 5, 29, pl. xxvii, f. I, pl. xxxi, f. 5. [Cranial roof, and operculum; Brit. Mus.] T. (Polyphractus.)

Megalichthys priscus, L. Agassiz, Poiss. Foss., vol. i (1844), p. xxxiv (name only).

Dipterus platycephalus, C. H. Pander, Ctenodipt. devon. Syst., 1858, p. 7, pl. i, f. 5, pl. ii, f. 2, 9, pl. iii, pl. iv, f. 23, 27, pl. v, f. 15-19, pl. vii, f. 5, I1.

LOC. L. Old Red Sandst.; Caithness, Ross-shire, Cromarty, Nairnshire, and Banifshire. [Fishes; Geol. Soc. London.]

DITA

I.

Impar, R. Owen, ibid., p. 107, pls. iv, v.

LOC. Kim. Clay; Oxfordshire and Wiltshire. [Jaws; Brit. Mius.]

$\mathbf{T .}$

DITTODCS, Owen. ¿. Diplodus and Rhizodopsis.

divergens, Owen. $\because$ Diplodus gibbosus.

parallelus, Owen. $\because$. Rhizodopsis sauroides.

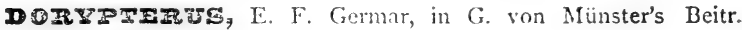
Petrefakt., pt. v, I 842 , p. 35 .

hofimann1, E. F. Germar, iuid., p. 35, pl. xiv, f. 4 ; A. Hancock and R. Howse, Quart. Journ. Geol. Soc., vol. xxvi, 1870, p. 623, pls. xlii, xliii, and Nat. Hist. Trans. Northumb. and Durham, vol. iv (1872), p. $2+3$, pls. ix, $\mathrm{x}$.

LoC. Marl-Slate; Midderidge. [Fishes; Newcastle-upon-Tyne i. Hus.]

DREPANEPHORUS, Egerton. *'Cestracion.

canaliculatus, Egerton. \%. Cestracion canaliculatus.

s1. A. S. Woodward. \%. Cestracion rugosus.

IECIIENUS, L. Agassiz, Rep. Brit. Assoc., 1844 (1845), p. 308 (name only).

politus, L. Agassiz, ibid., p. 308 (name only).

LOC. London Clay; Sheppey.

2.

ECFII ODUG, J. W. Davis, Quart. Journ. Geol. Soc., vol. xl, I884, p. 63 I.

paradorus, J. W. Davis, ibid., p. 63 I, pl. xxvii, f. 7 .

LOC. Yoredale Rocks; Wensleydale, Yorkshire. [Dermal fossil; York Mus.] 
mDAPrODON, W. Buckland, Proc. Geol. Soc, vol. ii (1838), p. 687 ; P. M. G. Egerton, Quart. Journ. Geol. Soc., vol. iii, I 847, p. 35 1, pl. xiii, f. 2, 3 .

Passalodon, W. Buckland, Proc. Geol. Soc., vol. ii (1838), p. 687.

Psittacodon, L. Agassiz, Poiss. Foss., vol. iii (1843), p. 340 (pars).

agassizi (Buckland), [-ii] E. T. Newton, Chimeroid Fishes Brit. Cret. Rocks (Miem. Geol. Surv., 1878), p. 12, pl iii.

Chimara agassizii, W. Buckland, Proc. Geol. Soc., vol. ii (1835), p. 206, and Phil. Miag. [3] vol. viii, I836, p. 5.

Ischyodus agassizi, P. M. G. Egerton, Proc. Geol. Soc., vol. iv $(1843)$, p. 156 ; J. Morris, Cat. Brit. Foss., I 854 , p. 330.

Chimera (/schyodon) agassizit, L. Agassiz, Poiss. Iross., vol. iii (1843), p. 34 I, pl. xl a, f. 3, 4 (? f. 5), pl. xl c, f. 16 (non f. 14, 15). [See also /schyodus thurmanni.]

LOC. Chalk-Marl and L. Chalk; Sussex, Kent, and Surrey. [Mandib. tooth; Brit. Mus.]

buckland1, L. Agassiz, Poiss. Foss., vol. iii (1843), p. 351, pl. $\mathrm{xl} d$, f. $1-4,9-12,19-24$; J. Morris, Cat. Brit. Foss., 1854, p. 325 .

Edaphodon eurygnathus, L. Agassiz, Poiss. Foss., vol. iii (1843), p. 352 ; F. Dixon, Geol. Sussex, 1850 , p. II I, pl. x, f. 18, 19 J. Morris, Cat. Brit. Foss., 185t, p. 325. [Palatine teeth Brit. Mus.]

LOC. London Clay; Sheppey. Bagshot and Bracklesham Beds; Surrey and Sussex. [Theoretically associated teeth; Brit. Mus. (pars).]

crassus, E. T. Newton, Chimaroid Fishes Brit. Cret. Rocks (Mem. Geol. Surv., 1878), p. 21, pl. vii.

LOC. U. Greensand; Warminster. Cambrilge Greensand. ? L. Chalk; Lewes. [Associated teeth; Mus. Pract. Geol.]

euvygnathus, Agassiz. v. Edaphodon bucklandi.

gigas, P. M. G. Egerton, Quart. Journ. Geol. Soc., vol. iii, 1847, p. 352 ; J. Morris, Cat. Brit. Foss., 1854 , p. 325 ; E. T. Newton, Chimaroid Fishes Brit. Cret. Rucks (Mem. Geol. Surv., 1878), p. I7, pl. v, f. I, 2.

Ischyodus gigas, P. M. G. Egerton, Proc. Gcol. Soc., vol. iv (1843), p. 211 ; F. Dixon, Geol. Sussex, 1850, descr. to pl. xxxiv, f. 8.

LOC. Chalk; Sussex. [Vomerine teeth ; unknown.]

luxleyi, Seeley. $\quad$. Edaphodon sedgwicki.

Iaminosus, E. T. Newton, Chimæroid Fishes Brit. Cret. Rocks (Mem. Geol. Surv., 1878), p. 24, pl. viii.

Loc. Gault; Folkestone. Cambridge Greensand. [Mandib. tooth; Brit. Mus.] 
IDAPHODON (continut) -

leptognathus, L. Agassiz, Poiss. Foss., vol. iii (1843), p. 352, pl. xl $d$, f. 5-8, 13-18; J. Morris, Cat. Brit. Foss., 1854, p. 325 .

Edaphodon, P. M. G. Escrton, Quart. Journ. Geol. Soc., vol. iii, I 847, p. 35 I, pl. xiii, f. 2,3 .

Loc. Bagshot and Bracklesham Beds; Surrey and Sussex. Barton Clay; Hampshire. [Mandib. and palatine teeth; Brit. Mus. (pars).]

T.

mantellí (Buckland), P. M. G. Egerton, Quart. Journ. Geol. Soc., vol. iii, 1847, p. 352 ; F. Dixon, Geol. Sussex, 1850, p. 203 , pl. xxxiv, f. 6, 7 ; J. Morris, Cat. Brit. Foss., 1854, p. 325 ; E. T. Newton, Chimæroid Fishes Brit. Cret. Rocks (Mem. Geol. Surv., I878), p. I4, pl. iv, f. I-9.

Chimara mantellii, W. Buckland, Proc. Geol. Soc., vol. ii (1835), p. 206, and Phil. Mag. [3] vol. viii, I 836, p. 5.

Ischyodus mantclli, P. M. G. Egerton, Proc. Geol. Soc., vol. iv (1843), p. 156.

Chimara (Psittacodon) mantellii, L. Agassiz, Poiss. Foss., vol. iii (1843), p. 348 , pl. xl $a$, f. I, 2.

T. (Psittacodon.)

LOC. Chalk and Chalk-Marl; Sussex and Kent. [Mandib. teeth ; Brit. Mus.]

reed1, [-ii] E. T. Newton, Chimeroid Fishes Brit. Cret. Rocks (Mem. Geol. Surv., I878), p. 19, pl. vi.

LOC. Cambridge Greensand. [Dentition; York Mus.]

sedgwicki (Agassiz), P. M. G. Egerton, Quart. Journ. Geol. Soc., vol. iii, 1847 , p. 352 ; F. Dixon, Geol. Sussex, 1850, p. 203 ; J. Norris, Cat. Brit. Foss., I854, p. 326 ; [-ii] E. T. Newton, Chimaroid Fishes Brit. Cret. Rocks (Mem. Geol. Surv., 1878), p. 7, pls. i, ii.

Chimara (Psittacodon) sedgavickii, L. Agassiz, Poiss. Foss., vol. iii (1843), p. 349, pl. xl, f. 17, 18.

Ischyodus sedswicki, P. M. G. Egerton, Proc. Geol. Soc., vol.iv (1843), p. 156.

Edaphodus huxleyi, H. G. Seeley, Ann. Mag. Nat. Hist. [3] vol. xiv, 1864, p. 276 . [Portions of teeth; Woodwardian Mus.]

LOC. L. Greensand; Atherfield. Gault; Folkestone. Red Chalk; Norfolk. Chalk-Marl and L. Chalk; Kent and Sussex. Variety in Cambridge Greensand. [Mandib. tooth; Geol. Soc. London.]

IGבzTOrIA, I. Cocchi, Annali R. Mus. Fis. Stor. Nat. Firenze, n.S., vol. i, 1866, pp. 90, 121.

Isodonta, I. Cocchi, ibial, p. I22, pl. iv, f. I, 2.

LOC. London Clay; Sheppey. [Teeth; Brit. Mus.] 
zxASMODECTES (Newton), A. S. Woodivard, Proc. Geol. Assoc., vol. x (1888), p. 301.

Elasmoginathus, E. T. Newton (non J. E. Gray, 1867), Chimeroid Fishes Brit. Cret. Rocks (Mem. Geol. Surv., I 878$),$ p. 43.

willetti (Newton), [-ii] A. S. Woodward, Proc. Geol. Assoc., vol. $x$ (1888), p. 301.

Elasmognathus willetii, E. T. Newton, Chimaroid Fishes Brit. Cret. Rocks (Mem. (ieol. Surv., 1878), p. 43, pl. xii, f. 13-15.

LOC. L. Chalk; Sussex and Kent. [Mandib. teeth; Brighton MIus.]

T.

mIASmodus, l'. M. G. Egerton, Proc. (ieol. Soc., vol. iv (I843), p. 156.

greenoughi (E. grecnori), P. M. G. Egerton, ibid., p. 156. Of doubtful locality, recorded as British by J. Morris, Cat. Brit. Foss., I 854, p. 326 ; now known to be obtained from the Poudingue de la Malogne (U. Senonian), Ciply, Belgium.

hunteri, P. M. G. Egerton, Proc. Geol. Soc., vol. iv (i 843), p. 156, and Quart. Journ. Geol. Soc., vol. iii, 1847, p. 35 I, and Figs. and Descript. Brit. Organic Remains, dec. vi (Mem. Geol. Surv., I852), no. I, pl. i; F. Dixon, Geol. Sussex, 1850, p. 11 I, pl. x, f. II, I2 ; J. Morris, Cat. Brit. Foss., I854, p. 326.

Extinct Chimcra, R. Owen, Odontogr., vol. i (1840), p. 66.

Loc. London Clay; Sheppey. Bracklesham Beds. [Teeth; Mus. R. Coll. Surgeons and Brit. Mus.]

$\mathbf{T}$.

ELASHOGNATHCS, Newton. v. Elasmodectes.

willettii, Newton. $v$. Elasmodectes willetti.

בrovrCFrrys, C. G. Giebel, Fauna der Vorwelt, vol. i, pt. iii, I 848 , p. 249 ; R. H. Traquair, Proc. Roy. Soc. Edinb., vol. ix, 1877 , p. 275 .

aitkeni, R. H. Traquair, Geol. Mag. [3] vol. iii, 1886, p. 440; ]. Ward, Trans. N. Staffs. Inst. Mining Engin., vol. x, 1889 , pl. vi, f. 9 .

Acrolcpis sp., J. Aitken, Trans. Manchester Geol. Soc., vol. xiii, 1874 , p. 36.

LOC. Coal-Meas.; Lancashire and Derbyshire. Millstone Grit ; Staffordshire and Yorkshire. [Fish; olim J. Aitken Coll.]

binneyi, R. H. Traquair, Geol. Mag. [3] vol. v, I888, p. 25 I.

LOC. L. Coal-Meas. (Dalemoor-Rake Ironstone); Stanton-byDale, Derbyshire. [Fish; Manchester Mus.]

bucklandi (Arassiz), R. H. Traquair, Quart. Journ. Geol. Soc., vol. xxxiii, 1877, pp. 553, 575, and Proc. Roy. Soc. Edinb., vol. ix, 1877, p. 428 .

Pygopterus bucklandi, L. Agassiz, in S. Hibbert, Trans. Roy. Soc. Edinb., vol. xiii, 1835 , p. 217 , pl. vii, f. 2 ; J. Morris, Cat. Brit. Foss., 1854 , p. 343.

Loc. Calcif. Sandst.; Burdiehouse. [Fish; unknown.] 
IIONTCITTZTS (continued)-

caudalis, R. H. Traquair, Ganoid Fishes Brit. Carb. Form. (Pal. Soc., 1877 ), p. 53 , pl. v, f. I-4.

LOC. Coal-Meas. (Knowles Ironstone) ; Fenton, North Staffordshire. [Imperfect fish ; J. Ward Coll.]

dunsi, R. H. Traquair, Proc. Roy. Phys. Soc. Edinb., vol. v, 1880, p. 126.

LOC. Calcif. Sandst.; Broxburn, Linlithgowshire ; and ? Water of Leith, near Edinburgh. [Fish ; Free Church College Mus., Edinburgh.]

egertoni (Agassiz), R. H. Traquair, Quart. Journ. Geol. Soc,, vol. xxxiii, 1877, p. 553 .

Palconiscus egertoni, L. Agassiz, Poiss. Foss., vol. ii, pt. i, (1843), p. 302 (name only); P. M. G. Egerton, Quart. Journ. Geol. Soc., vol. vi, I 8 ;o, p. 5 , and Figs. and Descript. Brit. Organic Remains, dec. vi (Mem. Geol. Surv., 1852), no. 2, pl. ii ; J. Morris, Cat. Brit. Foss., I854, p. 336 ; A. Hancock and T. Atthey, Nat. Hist. Trans. Nurthumb. and Durham, vol. iii (1870), p. 96.

LOC. Coal-Meas.; Staffordshire, Lancashire, Yorkshire, and Northumberland. [Imperfect fish ; Brit. Mus.]

intermedius, R. H. Traquair, Proc. Roy. Soc. Edinb., vol. ix, 1877 , p. 279.

Amblypterus punctatus, L. Agassiz, Poiss. Foss., vol. ii, pt. i (1835), p. 109, pl. iv c, f. 3, 5-8 (non f. 4).

LOC. Calcif. Sandst.; Wardie. [Fish ; Edinburgh Mus.]

microlepidotus, R. H. Traquair, Geol. Mag. [3] vol. iii, I886, p. $44 \mathrm{I}$; J. Ward, Trans. N. Staffs. Inst. Mining Engin., vol. $\mathrm{x}, \mathrm{I} 889$, pl. vi, f. $\mathrm{I}$.

Loc. Coal-Meas. (Knowles Ironstone Shale); Longton, North Staffordshire. [Fish; J. Ward Coll.]

nəmopterus (Agassiz), R. H. Traquair, Quart. Journ. Geol. Soc., vol. xxxiii, 1877, p. 553, and Proc. Roy. Soc. Edinb., vol. ix, 1877 , p. 278.

Amblypterus nemopterus, L. Agassiz, Poiss. Foss., vol. ii, pt. i (1835), p. 107, pl. iv 6, f. 1, 2 ; J. Muris, Cat. Brit. Foss., 1854, p. 317 .

Loc. Calcif. Sandst.; Edinburgh. [Fish ; Brit. Mus.]

oblongus, R. H. Traquair, Ganoid Fishes Brit. Carb. Form. (Pa!. Soc., 1877), p. 55, pl. vi, f. I, 2.

LOC. Coal-Meas. (Knowles Ironstone); Fenton. [Imperfect fish ; J. Ward Coll.]

ortholepis, R. H. Traquair, Geol. Mag. [3] vol. i, I884, p. 10.

Loc. Calcif. Sandst. (Cement-Stone Group); Eskdale, Dumfriesshire. [Fish; Brit. Mus.]

ovatus, R. H. Traquair, Proc. Roy. Soc. Edinb., vol.ix, 1877, p. 427. LOC. Calcif. Sandst. ; Burdiehouse. [Fish; Edinburgh Mus.] 


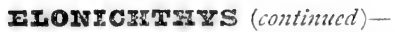

pectinatus, R. H. Traquair, Proc. Roy. Soc. Edinb., vol. ix, I877, p. 430, and Proc. Roy. Phys. Soc. Edinb., vol. v, 1880, p. 12 I, and Geol. Mag. [2] vol. ix, 1882, p. 545, and Trans. Edinb. Geol. Soc., vol. v, I887, p. 315.

LOC. Carb. Limest.; Gilmerton and Borough Lee, near Edinburgh; Carluke, Lanarkshire. Calcif. Sandst.; Abden, Fife. [Fish ; Edinburgh Mus.]

? portlock (Egerton), [-ii] R. H. Traquair, Quart. Journ. Geol. Soc., vol. xxxiii, 1877 , p. 553.

Amblypterus portlocki, P. M. G. Egerton, Quart. Journ. Geol. Soc., vol.vi, 1850, p. 2 ; J. Morris, Cat. Brit. Foss., 1854, p. 317.

Loc. Coal-Meas.; Derry, Ireland. [Scales; Brit. Mus. (pars).]

pulcherrimus, R. H. Traquair, Trans. Roy. Soc. Edinb., vol. $\mathrm{xxx}, \mathrm{I} 88 \mathrm{I}, \mathrm{p} .24$, pl. i, f. 9-12.

LOC. Calcif. Sandst. (Cement-stone Group); Glencartholm, Eskdale, Dunfriesshire. [Fish ; Geol. Surv. Scotland.]

robisoni (Hibbert), R. H. Traquair, Quart. Journ. Geol. Soc., vol. xxxiii, 1877, p. 553.

Palconiscus robisoni, S. Hibbert, Trans. Roy. Soc. Edinb., vol. xiii (1835), p. I9I, pl. vi, f. 6, 7, pl. vii, f. I-3; L. Agassiz. Poiss. Foss., vol. ii, pt. i (1835), p. 88, pl. x a, f. I, 2 ; J. Morris, Cat. Brit. Foss., 1854, p. 337.

LOC. Calcif. Sandst.; Burdichouse. [Fishes; olim S. Hibbert Coll.]

semistriatus, R. H. Traquair, Ganoid Fishes Brit. Carb. Form. (Pal. Soc., 1877), p. 49, pl. iii, f. 9-12, pl. iv, f. I-3, and Quart. Journ. Geol. Soc., vol. xxxiii, I877, p. 577 ; H. Bolton, Trans. Manchester Geol. Soc., vol. xx (1889), p. 2 I 5.

Pygopterus, T. P. Barkas, Coal Meas. Palaont., 1873, p. 37, f. 130 (? and f. 131).

LOC. Coal-Meas. ; Staffordshire, Lancashire, and Northumberland. [Head and scales; Mus. Pract. Geol.]

serratus, R. H. Traquair, Trans. Roy. Soc. Edinb., vol. xxx, I 88 I, p. 22 , pl. i, f. $5^{-8}$.

Loc. Calcif. Sandst. (Cement-stone Group); Glencartholm, Eskdale, Dumfriesshire. [Fish ; Geol. Surv. Scotland.]

striolatus (Agassiz), R. H. Traquair, Quart. Journ. Geol. Soc., vol. xxxiii, 1877, p. 553, and Ganoid Fishes Brit. Carb. Form. (Pal. Soc., 1877), p. 57, pl, vii, f. 4-15.

Palconiscus striolatus, L. Agassiz, Poiss. Foss., vol. ii, pt. i (1835), p. 91, pl. xa, f. 3, 4 ; J. Morris, Cat. Brit. Foss., 1854 , p. 337.

LOC. Calcif. Sandst.; Burdiehouse, near Edinburgh. [Fish ; Edinburgh Mus.]

tonuiserratus, R. H. Traquair, Proc. Roy. Phys. Soc. Edinb., vol. v, I880, p. I 19.

LOC. Calcif. Sandst.; West Calder. [Fish; olim T. Stock Coll.] 
INOFrODUS, L. Agassiz, l'oiss. Foss., vol. v, pt. i (1843), p. 64.

Eurygnathus, J. IV. Davis (non Wollaston), Trans. Roy. Dublin Soc. [2] rol. iii (1887), p. 6oI.

halocyon, Agassiz. a'. Enchodus lewesiensis.

lewesiensis (Mantell), A. S. Woodward, Proc. Geol. Assoc., vol. x (1888), p. 315 , pl. i, f. 5,6 (E. levesiensis).

Esox lewesiensis, G. A. Mantell, Foss. S. Downs, 1822, p. 237, pl. xxxiii, f. 2-4, pl. xli, f. I, 2.

Enchodus halocyon, L. Agassiz, Poiss. Foss., Feuill., p. 55 (1835), and vol. v, pt. i (1843), p. 64, pl. xxy c, f. 1-16; F. Dixon, Geol. Sussex, 1850, p. 373, pl. xxx, f. 27, pl. xxxi, f. II ; J. Morris, Cat. Brit. Foss., I854, p. 326.

LOC. Chalk; S. E. England. [Fragments of jaws, etc. ; Brit. Mus.]

FNDACTIS, P. M. G. Egerton, Figs. and Descript. Brit. Organic Remains, dec. ix (Mem. Geol. Surv., 1858), no. 4.

agassizi, P. M. G. Egerton, ibid., no. 4, pl. iv.

LOC. L. Lias ; Lyme Regis. [Imperfect fish ; Brit. Mus.]

ERISICHTHE, Cope. ' ' 'rotosphyrana.

dixoni (Leidy), Cope. $\because$. I'rotosphyræna ferox.

IRISMA CANTruU, F. M'Coy, Ann. Mag. Nat. Hist. [2] vol. ii, I848, p. I19, and Proc. Camb. Phil. Soc., vol i (1848), p. 65.

Cladacanthus, J. W. Davis ( $e x$ Agassiz, MS.), Trans. Roy. Dublin Soc. [2] vol. i (1883), p. 364.

jonesi, [-ii] F. M'Coy, Ann. Mag. Nat. Hist. [2] vol. ii, 1848, p. I I9, and Brit. Palcoz. Foss. (1855), p. 628, pl. iii $\mathrm{K}$, f. 26, 27 ; [-ii] J. Morris, Cat. Brit. Foss., 1854, p. 326.

Cladacanthus paradoxus, L. Agassiz, Poiss. Foss., vol. iii (1843), p. 176 (name only); J. Morris, Cat. Brit. Foss., 1854, p. 321 ; J. W. Davis, Trans. Roy. Dublin Soc. [2] vol. i (I883), p. 365 , pl. xlvii, f. I-5, and Quart. Journ. Geol. Soc., vol. xl, 1884, p. 617, pl.xxvi, f. 1-5. [Spines; olim Enniskillen Coll.]

T. (Cladacanthus.)

Dipriacanthus falcatus, F. M'Coy, Ann. Mag. Nat. Hist. [2] vol. ii, I848, p. I2 I ; J. Morris, Cat. Brit. Foss., 1854, p. 325. [Spine ; olim Capt. Jones Coll.]

IOC. L. Carb. Limest. ; Armagh. Yoredale Rocks ; Wensleydale, Yorkshire. [SPines; olim Capt. Jones Coll.]

T.

major, J. W. Davis, Trans. Roy. Dublin Soc. [2] vol. i (1883), p. 366 , pl. xlvii, f. 6,7 .

10C. L. Carb. Limest.; Armagh. [Imperfect spines; olim Enniskillen Coll.]

ISOx, Linnæus, Syst. Nat., ed. 12, vol. i, 1766, p. 515.

lewesiensis, Mantell. \%. Enchodus lewesiensis. 
ISOx (continued)-

lucius, Linnæus, Syst. Nat., ed. 12, vol. i, 1766, p. 516; E. T. Newton, Vert. Forest Bed (Mein. Geol. Surv, 1882), p. 123, pl. xviii, f. II-I 4 .

Esox sp., L. Agassiz, Ann. Mag. Nat. Hist., vol. viii, 1842, p.61 ; J. Morris, Cat. Brit. Foss., 1854 , p. 326. [Jaws and teeth ; Brit. Mus.]

Loc. Forest-Bed Series; Norfolk. Pleistocene ; Copford and Ilford, Essex. [Jawes and preopcraulum; Mus. Pract. Geol.]

IUCEPXATAS PIS, Lan'iester. $\%$. Cephalaspis.

agassizi, Lankester. $\%$. Cephalaspis agassizi.

asper, Lankester. $v$. Cephalaspis asper.

lyelli (Agassiz), Lankester. $v$. Cephalaspis lyelli.

pagei (Powrie), Lankester. $v$. Cephalaspis pagei.

powriei, Lankester. $v$. Cephalaspis powriei.

झUCTENIUS. R. H. Traquair, Geol. Mag. [2] vol. viii, I88I, pp. 36,334 .

elegans, R. H. Traquair, ibill, p. 36.

'Kammplatten,' T. Stock, Aun. MIag. Nat. Hist. [5] vol. viii, r88r, p. 90.

LOC. M. Carb. Limest. (Blackband Ironstone); Borough Lee, Edinburgh. [Dermal defences; Edinburgh Mus.] T.

unllateralis (IV. J. Barkas), R. H. Traquair, Geol. Mag. [2] vol. viii, I881, p. 335 ; J. Ward, Trans. N. Staffs. Inst. Min. Engin., vol. x (1889), pl. ii, f. I4.

Ctenoptychizs, T. P. Barkas, Geol. Mag., vol. vi, 1869, p. 43.

Ctenoptychius unilateralis, IV. J. Barkas, Monthly Rev. Dental Surgery, vol. ii, 1874 , p. 484 , f. xvi, xvii ; J. Ward, [Proc.] N. Staffs. Nat. Field Club, 1875, p. 220.

LOC. Coal-Meas. ; Staffordshire, Northumberland, and South Scotland. [Dermal defence; T. P. Barkas Coll.]

FUGINATrus, L. Agassiz, Poiss. Foss., vol. ii, pt. ii (1843), p. 97. ? Conodus, L. Agassiz, ibid., p. 105 (name only).

Heterolepidotus, P. M. G. Egerton, Figs. and Descript. Brit. Organic Remains, dec. xiii (Nem. Geol. Surv., I872), no. 2.

Lissolepis, J. W. Davis, Ann. Mag. Nat. Hist. [5] vol. xiii, 1884 , p. 448.

? chirotes, L. Agassiz, Poiss. Foss., vol. ii, pt. ii (1843), p. 102, pl. lvii $b$; J. Morris, Cat. Brit. Foss., 1854, p. 326. [? Osteorachis.]

Conodus ferox; L. Agassiz, ibial., p. 105 (name only). [Jaws; Brit. Mus.] T. (Conodus.)

'Knochenfisch?', K. A. v. Zittel, Handb. Palæont., vol. iii (I887), p. 213.

LOC. L. Lias ; Lyme Regis. [Imperfect fish ; Brit. Mus.] 


\section{DUGMATZUS (continued)-}

fasciculatus, L. Agassiz, Poiss. Foss., vol. ii, pt. ii (1843), p. 105 (name only).

LOC. U. Lias ; Whitby.

latus: Heterolepidotus latus, P. M. G. Egerton, Figs. and Descript. Brit. Organic Remains, dec. xiii (Mem. Geol. Surv., 1872), no. 2, pl. ii.

T. (Heterolepidotus.)

? Dapedius fimbriatus, L. Agassiz, Poiss. Foss., vol. ii, pt. i (1835), p. 196.

? Lepidotus fimbriatus, L. Agassiz, ibid. (1837), p. 247, pl. xxxiii $b$; W. C. Williamson, Phil. Trans., I849, p. 444; J. Morris, Cat. Brit. Foss., 1854, p. 331. [Scales; Oxford Mus.]

? Semionotus rhombifer, L. Agassiz, Poiss. Foss., vol. ii, pt. i (1837), p. 228, pl. xxvi $a$; W. C. Williamson, Phil. Trans., 1849, p. 444, pl. xli, f. 9 ; J. Morris, Cat. Brit. Foss., 1854, p. 314. [Imperfect fish ; Brit. Mus.]

? Hetcrolepidotus minor, J. W. Davis, Proc. Yorks. Geol. and Polyt. Soc., n.s., vol. viii (1884), p. 403, pl. xxii. [Imperfect fish; J. W. Davis Coll.] (If truly distinct, requires a new specific name).

LOC. L. Lias; Lyme Regis. [Fish ; Brit. Mus.]

leptodus, L. Agassiz, Poiss. Foss., vol. ii, pt. ii (1843), p. 105 (name only).

LOC. L. Lias; Lyme Regis.

mandibularis, L. Agassiz, Poiss. Foss., vol. ii, pt. ii (1843), p. 105 (name only).

LoC. L. Lias ; Lyme Regis.

minor, L. Agassiz, Poiss. Foss., vol. ii, pt. ii (1843), p. 103, pl. Iviii $a$, f. I ; J. Morris, Cat. Brit. Foss., 1854, p. 326.

LOC. L. Lias; Lyme Regis. [Portion of head and trunk; Oxford Mus.]

operculariz, L. Agassiz, Poiss. Foss., vol. ii, pt. ii (1843), p. 104 ; J. Morris, Cat. Brit. Foss., 1854, p. 326.

LOC. L. Lias; Lyme Regis. [Fish ; unknown.]

ornatus, L. Agassiz, Poiss. Foss., vol. ii, pt. ii (1843), p. 105 (name only).

LOC. L. Lias; Lyme Regis.

orthostomus, L. Agassiz, Poiss. Foss., vol. ii, pt. ii (1843), p. 98, pl. lvii $a$; J. Morris, Cat. Brit. Foss., 1854 , p. 326.

Lepidosteus? dentosus, C. Koenig, Icon. Foss. Sect. (1825), pl. xii, f. I 40.

Eugnathus speciosus, L. Agassiz, Poiss. Foss., vol. ii, pt. ii (1843), p. 100, pl. lvii ; J. Morris, Cat. Brit. Foss., 1854, p. 326. [Head and scales; Oxford Mus.]

Eugnathus scabriusculus, L. Agassiz, Poiss. Foss., vol. ii, pt. ii (1843), p. 105 (name only). [Jaws, etc.; Brit. Mus.]

LOC. L. Lias ; Lyme Regis. [Fish; Brit. Mus.] 
IUGMATIUS (continued) -

phllpotæ, [-iæ] L. Agassiz, Poiss. Foss., vol. ii, pt. ii (1843), p. IOI, pl. Iviii ; J. Morris, Cat. Brit. Foss., 1854, p. 326.

Heterolepidotus sauroides, P. M. G. Egerton, Figs. and Descript. Brit. Organic Remains, dec. xiii (Mem. Geol. Sirv., 1872), no. 3, pl. iii. [Fish; Brit. Mus.]

Ophiopsis sauroides, K. A. v. Zittel, Handb. Palæont., vol. iii (1887), p. 217.

LOC. L. Lias ; Lyme Regis. [Fish, wanting jaws ; Oxford Mus.]

polyodon, Agassiz. v. Platysiagum sclerocephalum.

scabriusculus, Agassiz. v. Eugnathus orthostomus.

serratus: Lissolepis serratus, J. W. Davis, Ann. Mag. Nat. Hist. [5] vol. xiii, 1884, p. 449 , pl. xvi.

r. (Lissolepis.)

LOC. L. Lias; Lyme Regis. [Fish ; J. W. Davis Coll.]

sorrulatus : Helerolepidotus serrulatus, P. M. G. Egerton, Figs. and Descript. Brit. Organic Remains, dec. xiii (Mem. Geol. Surv., 1872), no. 2.

Lepidotus sermulatus, L. Agassiz, Poiss. Foss., vol. ii, pt. i (1843), p. 305, pl. xxxi ; J. Morris, Cat. Brit. Foss., 1854, p. 332 .

Loc. L. Lias ; Barrow-on-Soar. [Imperfect fish ; Brit. Mus.]

speciosus, Agassiz. v. Eugnathus orthostomus.

tenuidens, L. Agassiz, Poiss. Foss., vol. ii, pt. ii (1843), p. 105 (name only).

LOC. L. Lias; Street, Somersetshire. [Maxilla; Brit. Mus.]

2UXEIASPIS, E. R. Lankester, Fishes O. R. Sandst., pt. i (Pal. Soc., 1870), p. 56.

Sclerodus, L. Agassiz, in Murchison's Silur. Syst., r839, p. 606 (inappropriate).

Plf.ctrodus, L. Agassiz, ibid., p. 606; P. M. G. Egerton, Quart. Journ. Geol. Soc., vol. xiii, 1857, p. 288 (inappropriate).

pustuliferus (Agassiz), E. R. Lankester, Fishes O. R. Sandst., pt. i (Pal. Soc., 1870), p. 58, pl. xiii, f. 9-14.

Sclerodus pustuliferus, L. Agassiz, in Murchison's Silur. Syst., I839, pp. 606, 704, pl. iv, f. 27-32, 60-62; J. Morris, Cat. Brit. Foss., 1854, p. 344.

T. (Sclerodus.)

Plectrodus (Sclerodus) pustuliferus, R. I. Murchison, Siluria, 1854, pl. xxxv, f. 9-12.

Plectrodus mirabilis, L. Agassiz, in Murchison's Silur. Syst. 1839 , pp. 606, 704, pl. iv, f. 14-16; R. I. Murchison, Siluria, 1854, pl. xxxv, f. 3-8 ; J. Morris, Cat. Brit. Foss., 1854, p. 339.

T. (Plectrodus.)

Plectrodus pleiopristis, L. Agassiz, in Murchison's Silur. Syst., 1839 , p. 606, pl. iv, f. 18, 19, 22-24; J. Morris, Cat. Brit. Foss., 1854, p. 339. 
IUIERRASPIS pustuliferus (continued)-

Plectrodus pustuliferus, E. R. Lankester, Rep. Brit. Assoc., 1867 (1868), Sect., p. 63.

P'terygotus pustulifer, J. W. Salter, Quart. Journ. Geol. Soc., vol. ix, 1853, p. 14 (referred to Crustacea).

I.OC. Downton Sandst.; Ludlow. [Cornua of head-shields; Brit. Mus. (pars).]

sp.: Plectrodus, P. M. G. Egerton, Quart. Journ. Geol. Soc., vol. xiii, I 857 , p. 288 , pl. x, f. 2-4.

LOC. Downton Sandst.; Ludlow. [Portions of cornua ; Ludlow and Brit. Mus.]

IU xycormus, A. Wagner, Abh. k. bay. Akad. Wiss., math.phys. Cl., vol. ix (1563), p. 707.

grandis, A. S. Woodward, Geol. Mag. [3] vol. vi, r889, p. 449, and Rep. Brit. Assoc., 1889.

Loc. Kim. Clay; Ely. [Head; Woodwardian Mus.]

EURYGNATHUS, L. Agassiz (non Wollaston), Rep. Brit. Assoc., 1844 (I845), p. 307 (name only).

cavifrons, L. Agassiz, ibid., p. 307 (name only).

LOC. London Clay; Sheppey. [Imperfect skull; Brit. Mus.] $\mathbf{T}$.

EURTGNATHUS, Davis. v. Enchodus.

EURYNOTUS, L. Agassiz, Poiss. Foss., vol. ii, pt. i (1835), p. I 53 ;

P. M. G. Egerton, Quart. Journ. Geol. Soc., vol. vi, I850,

p. 2 ; J. Young, Quart. Journ. Geol. Soc., vol. xxii, 1866,

p. 314 ; R. H. Traquair, Trans. Roy. Soc. Edinb., vol. xxix, 1879, p. 348 .

Plectrolepis, L. Agassiz, Poiss. Foss., vol. ii, pt. i (1843), p. 306 (name only); P. M. G. Egerton, Quart. Journ. Geol. Soc., vol. vi, 1850, p. 3.

? aprion (?), R. H. Traquair, Trans. Roy. Soc. Edinb., vol. xxx, I \&8 I, p. 54, pl. v, f. 20.

Loc. Calcif. Sandst. (Cement-stone Group); Tweeden Burn, Liddesdale, Dumfriesshire. L. Carb.; Moyheeland, Draperstown, Ireland. [Scales; Geol. Surv. Scotland.]

crenatus, L. Agassiz, Poiss. Foss., vol. ii, pt. i (1835), p. 154, pls. xiv $a, b$; S. Hibbert, Trans. Roy. Soc. Edinb., vol. xiii (18.35), pl. vii, f. 4 ; J. Morris, Cat. Brit. Foss., 1854, p. 326 ; R. H. Traquair, Trans. Roy. Soc. Edinb., vol. xxix, 1879, p. 349, pl. iii, f. 1-16, and ibid., vol. xxx, 1881, p. 54.

Plectrolepis nugosus, L. Agassiz, Poiss. Foss., vol. ii, pt. i (1843), p. 306 (name only); P. M. G. Egerton, Quart. Journ. Geol. Soc., vol. vi, 1850 , p. 4 ; J. Morris, Cat. Brit. Foss., 1854 , p. 339. [Fish; Brit. Mus.]

T. (Plectrolepis.) 
FURYNOTUS crenatus (continued)-

Platysomus declizus (L. Agassiz, MS.), J. Morris, Cat. Irit. Foss., 1854, p. 339. [Distorted fish ; Brit. Mus.]

LOC. L. Carb.; South Scotland. [Imperfect fishes; Edinburgh Mus. (pars).]

T.

fimbriatus, L. Agassiz, Poiss. Foss., vol. ii, pt. i (1835), p. I57, pl. xiv $c$, f. I-3; J. Morris, Cat. Brit. Foss., 1854, p. 326 ; R. H. Traquair, Trans. Roy. Soc. Edinb., vol. xxiv, 1867, p. 7 Io, pl. xlv, f. 8, 9 .

LOC. Calcif. Sandst.; Wardie, near Edinburgh. [Portions of trunk ; Oxford Mus.]

בUזX P OMA, T. H. Huxley, Figs. and Descript. Brit. Organic Remains, dec. xii (Mem. Geol. Surv., I866), p. 32.

egertoni (Egerton), T. H. Huxley, ibid., p. 32.

Macropoma egertoni, L. Agassiz, Poiss. Foss., vol. ii, pt. ii (1843), p. 174 (name only); J. Morris, Cat. Brit. Foss., 1854, p. 332 ; P. M. G. Egerton, Figs. and Descript. Brit. Organic Remains, dec. ix (Mem. Geol. Surv., 1858), no. 10, pl, x.

Loc. Gault; Speeton, Yorkshire. [Anterior half of fish; Brit. Mus.]

T.

EURYSOMUS, Young. $\%$. Globulodus.

macrurus (Agassiz), Young. \%, Globulodus macrurus.

EUTHACANTHUS, Powrie. v. Climatius.

curtus, Powrie. v. Diplacanthus curtus.

elegans, Powrie. $\%$. Climatius gracilis.

gracilis, Powrie. v. Climatius gracilis.

grandis, Powrie. $v$. Climatius grandis.

menicoli, Powrie. $\%$. Climatius macnicoli.

rISSODUS, O. St. John and A. H. Worthen, Pal. Illinois, vol. vi, I875, p. 413.

patton1, R. Etheridge, jun., Geol. Mag. [2] vol. iv, 1877, p. 306, pl. xiii, f. 2,3 .

LOc. L. Carb. Limest.; South Scotland. [Tooth; Geol. Surv. Scotland.]

GADOPSIS, L. Agassiz, Rep. Brit. Assoc., 1844 (1845), p. 3oS (name only).

breviceps, L. Agassiz, ibid., p. 308 (name only).

LOC. London Clay; Sheppey. [Head.]

T.

GADUS, Linnæus, Syst. Nat., ed. 12, vol. i, 1766, p. 435. [See also Platax woodwardi.]

morrhua, Linnæus, ibid., p. 436 : E. T. Newton, Vert. Forest Bed (Mem. Geol. Surv., 1882), p. 127, pl. xix, f. 4, 5.

LOC. Forest Bed; Norfolk. [Dentary and articular; Mus. Pract. Geol.] 
GADUS (continued)-

pollachius, Linnæus, Syst. Nat., ed. 12, vol. i, 1766, p. 439; E. T. Newton, Vert. Forest Bed (Mem. Geol. Surv., 1882), p. I28, pl. xviii, f. 24.

Loc. Weybourn Crag; Norfolk. [Otolith; Mus. Pract. Geol.]

GAImOCrRD O, J. Müller and F. G. Henle, Syst. Beschreib. Plagiostom., 1841, p. 59.

Protogaleus, R. Molin, Sitz. math.-nat. Cl. k. Akad. Wiss. Wien, vol. xl, 1860, p. 583 .

latidens, L. Agassiz, Poiss. Foss., vol. iii (1843), p. 23I, pl. xxvi, f. 22, 23 (? f. 20, 21) ; F. Dixon, Geol. Sussex, 1850, p. 202, pl. xi, f. 22, 23 ; J. Morris, Cat. Brit. Foss., 1854, p. 326 ; A. S. Woodward, Cat. Foss. Fishes B. M., pt. i, 1889, p. 444.

Loc. Bracklesham Beds. [Tooth; Paris Mus.]

? minor, L. Agassiz, Poiss. Foss., vol. iii (1843), p. 232, pl. xxvi, f. 15-19 (? f. 20, 21), pl. xxvi a, f. 64-66; A. S. Woodward, Cat. Foss. Fishes B. M., pt. i, 1889, p. 446.

Protogaleus minor, R. Molin, Sitz. math.-nat. Cl. k. Akad. Wiss. Wien, vol. xl, I860, p. 583 .

LOC. Eocene; London and Hampshire Basins. [Teeth; Brit. Mus.]

? sp., A. S. Woodward, Cat. Foss. Fishes B. M., pt. i (1889), p. 447. Loc. London Clay; Highgate. [Tooth; Brit. Mus., no. 43135a.]

GArzU8, Cuvier, Règne Animal, vol. ii, 1817, p. 127.

can1s, C. L. Bonaparte (ex Rondelet), Fauna Italica, Pesci, I84 I; E. T. Newton, Vert. Forest Bed (Mem. Geol. Surv., 1882), p. $13 \circ$, pl. xix, f. 7 .

Loc. Forest-Bed Series; Norfolk. [Teeth; Mus. Pract. Geol.] pristodontus, Agassiz. $\%$. Corax falcatus and C. pristodontus. sp., A. S. Woodward, Cat. Foss. Fishes B. M., pt. i, 1889, p. 452.

LOC. London Clay ; Highgate. [Teeth ; Brit. Mus., no. 43134a.]

sp., A. S. Woodward, ibid., p. 452.

LOC. Barton Clay; Highcliff. [Teeth; Brit. Mus., nos. $40242 a$, 40245.]

GANACRODUS, R. Owen, Trans. Odontol. Soc., vol. v, I867, p. 349. Undetermined Palæoniscid genus (A. Hancock and T. Atthey, Nat. Hist. Trans. Northumb. and Durham, vol. iii (1870), p. 100).

hastula, R. Owen, ibid., p. 349, pl. vi.

Loc. Coal-Meas.; Newsham. [Micro. section of tooth.]

GANODUS, L. Agassiz (e.* Egerton, MS.), Poiss. Foss., vol. ii (1843), p. 339.

Psittacodon, L. Agassiz, ibid., p. 340 (pars).

bucklandi, Egerton. v. Ischyodus bucklandi. 
GANODUS (continued) -

colei, Agassiz. \%. Ischyodus colei.

curvidens, P. M. G. Egerton, Proc. Geol. Soc., vol. iv (1843), p. I54 (Chimeru), and Quart. Journ. Geol. Soc., vol. iii, 1847 , p. 352 ; J. Morris, Cat. Brit. Foss., I854, p. 327.

Chimara (Ganodus) curitents, L. Agassiz, Poiss. Foss., vol. iii (1843), p. 348. [? Ganodus nugulosus.]

I.OC. Stonesfieid Slate. [Mandib. tooth; Rrit. Mus.]

dentatus, P. M. G. Egerton, Quart. Journ. Geol. Soc, vol. iii, 1847 , p. 352 ; J. Morris, Cat. Brit. Foss., 1854 , p. 327.

I.oc. Stonesfield Slate. [Mandib. tooth ; Brit. Mus.]

emargimatus, Egerton. \%. Ischyodus emarginatus.

falcatus, P. M. G. Egerton, Proc. Geol. Soc., vol. iv (I843). P. 154 (Chimera), and Quart. Journ. Geol. Soc., vol. iii, 1847 , p. 352 ; J. Morris, Cat. Brit. Foss., I854, p. 327.

Chimara (Psittacodon) falcata, L. Agassiz, Poiss. Foss., vol. iii (1843), p. 349, pl. xl c, f. 13. [? Ganodus oweni.]

I.oc. Stonesfield Slate. [Mandib. tooth; Brit. Mus.]

neglectus, P. M. C. Egerton, Proc. Geol. Soc., vol. iv (1813), p. 153 (Chimere), and Quart. Journ. Geol. Soc, vol. iii, I847, p. 352 ; J. Morris, Cat. Erit. Foss., 1854, p. 327.

Chimara (Ganodus) neglecta, L. Agassiz, Poiss. Foss., vol. iii (I843), p. 347 , pl. xl $c$, f. II.

Loc. Stonesfield Slate. [Mandib. tooth; Brit. Mus.]

owent, L. Agassiz (ex Buckland, MS.), Poiss. Foss., vol. iii (1 $8+3)$, p. 347 , pl. xl, f. 6, 7 (Chimara (Ganodus) owenii); J. Morris, Cat. Brit. Foss., 1854 , p. 327.

Loc. Stonesfield Slate. [Mandib. tooth; Brit. Mus.]

psittacinus, P. M. G. Egerton, Proc. Geol. Soc., vol. iv (1843), p. 153 (Chimera), and Quart. Journ. Geol. Soc., vol. iii, I847, p. 352 ; J. Morris, Cat. Brit. Foss., 1854, p. 327.

Chimara (Psittacodon) psittacina, L. Agassiz, Poiss. Foss., vol. iii $(18+3)$, p. 35o, pl. xl c, f. 12. [? Ganodus oweni.]

LOC. Stonesfield Slate. [Mandib. tooth ; Brit. Mus.]

rugulosus, P. M. G. Egerton, Proc. Geol. Soc., vol. iv (1843), p. 154 (Chimara), and Quart. Journ. Geol. Soc, vol. iii, 1847, p. 352 ; J. Morris, Cat. Brit. Foss., 1854, p. 327.

Chimara (Ganodus) rugulosa, L. Agassiz, Poiss. Foss., vol. iii (1843), p. 347 .

Loc. Stonesfield Slate. [Mandib. tooth ; Brit. Mus.]

GANOLODUS, Owen. $\%$ Rhizodopsis and Megalichthys.

cragyesi, Owen. $v$. Rhizodopsis sauroides.

sicula, Owen. $\because$. Megalichthys hibberti.

undatus, R. Etheridge, Foss. Brit. Islands, pt. i, 1888, p. 328 :

misprint for Aganndus undalus. 
GANOPRISTODUS, R. H. Traquair, Geol. Mag. [2] vol. viii, I88 I, p. 37 .

splendens, R. H. Traquair, Geol. Mag. [2] vol. viii, I881, p. 37, and ibid. [2] vol. ix, 1882, p. 543 .

LOC. M. Carb. Limest. (Blackband Ironstone); Borough Lee, near Edinburgh. [Jaws ; Edinburgh Mus.]

T.

GANORIYNC3UE, R. H. Traquair, Geol. Mag., vol. x, 1873, p. 555 .

woodwardi, R. H. Traquair, ibid., p. 555, pl. xiv.

LOC. Unknown. [End of snout; Brit. MIus.]

T.

GEISACANTIIUS, St. John and Vorthen. v. Cosmacanthus.

GIOBUエ0DUs, G. von Münster, Peitr. Petrefakt., pt. v, I842, p. 47.

Eurysonus, J. Young, Quart. Journ. Geol. Soc., vol. xxii, I 866, p. 3I I ; R. H. Traquair, Trans. Roy. Soc. Edinb., vol. xxix, I879, p. 358.

macrurus (Agassiz), R. Howse, Guide Coll. Local Foss. Mus. Nat. Hist. Soc., Newcastle-upon-Tyne, 1889, p. 24.

'Fossil Fish,' A. Sedgwick, Trans. Geol. Soc. [2] vol. iii (1829), p. II 8 , pl. xii, f. I, 2.

Platysomus macrumes, L. Agassiz, Poiss. Foss., vol. ii, pt. i (1835), p. 170, pl. xviii, f. 1, 2; W. King, Permian Foss. (Pal. Soc., 1850), p. 227, pl. xxvi, f. I ; J. Morris, Cat. Brit. Foss., 1854 , p. 339.

Eurysomus macnurus, J. Young, Quart. Journ. Geol. Soc., vol. xxii, 1866, p. 3 II ; R. H. Traquair, Trans. Roy. Soc. Edinb., vol. xxix, I 879, p. 359, pl. iv, f. I0, I I. T. (Eurysomus.) LOC. Marl-Slate; Midderidge and Ferryhill. [Fish; unknown.]

Grossonus, F. M'Coy, Ann. Mag. Nat. Hist. [2] vol. ii, I848, p. 127, and Proc. Camb. Phil. Soc., vol. i (1848), p. 66.

lingua-bovis, F. M'Coy, Ann. Mag. Nat. Hist. [2] vol. ii, 1848, p. 127 ; J. Morris, Cat. Brit. Foss., 1854, p. 327 ; A. S. Voodward, Cat. Foss. Fishes B. M., pt. i, 1889, p. 60.

Glossodus marginatus, F. M'Coy, Ann. Mag. Nat. Hist. [2] vol. ii, 1848, p. 128, and Brit. Palæoz. Foss. (1855), p. 629, pl. iii K, f. i ; J. Morris, Cat. Brit. Foss., 1854, p. 327 ; J. W. Davis, Trans. Roy. Dublin Soc. [2] vol. i (1883), p. 5 Io, pl. lxi, f. 3-5.

LOC. L. Carb. Limest. ; Armagh. [Teeth ; olim Capt. Jones Coll.]

marginatus, $\mathrm{M}^{\prime} \mathrm{Coy}$. $v$. Glossodus lingua-bovis.

GIYPXa NODUS, J. W. Davis, Trans. Roy. Dublin Soc. [2] vol. i (1883), p. 386. 
GIXPHANODUS (continued)-

tenuis, J. W. Davis, ibid., p. 386, pl. xlix, f. 24, 25, and Quart. Journ. Geol. Soc., vol. xl, I884, p. 621, pl. xxvii, f. 8 .

? Abraded teeth of Petralodus (A. S. Woodward, Cat. Foss. Fishes B. M., pt. i, 1889, p. 47).

LOC. Yoredale Rocks; Wensleydale. [Tooth ; York Mus.] T.

GLIPHIS, Agassiz. \%". Carcharias.

hastalis, Agassiz. $\approx$. Carcharias (I'rionodon) hastalis.

GIYPTOCEPIIAIUS, L.Agassiz, Rep. Brit. Assoc., I $S_{44}$ (1845), p. 308 (name only); A. Günther, Introd. Study Fishes, 1880, p. 684 .

realictus, L. Agassiz, ibid., p. 308 (name only).

LOC. London Clay; Sheppey. [Head.]

T.

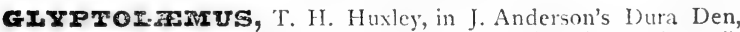
1859, p. 63 (wrongly engraved on plate, 'Glyptola'mus, Agass.'), and Figs. and Descript. Brit. Organic Remains, dec. $x$ (Mem. Geol. Surv., 1861), p. 4 I.

kinnairdi, T. H. Huxley, in Anderson's Dura Den, 1859, p. 63, pls. iii and iv, and Figs. and Descript. Brit. Organic Remains, dec. x (Mem. Geol. Surv., I861), p. 4I, pls. i, ii.

? Diplopterus dalgleisiensis, J. Anderson ('ex Agassiz'), Dura Den, 1859 , p. 71 , pl. i, f. 4. (Assigned to Glyptolemus by J. Powrie, Quart. Journ. Geol. Soc., vol. xviii, 1862, p. 435.) [Head; Mis. l'ract. Geol.]

I.oc. U. Old Red Sandst.; Dura Den, Fife. [Fishes; Mus. Pract. Geol.]

r.

GIX Prox.tris, L. Agassiz, l'oiss, Foss. V. C. R. (1844), p. 62.

Pi.atygnathus, L. Agassiz, ibid., pp. 6I, 76.

elegans, Agassiz. \%. Glyptolepis leptopterus.

leptopterus, L. Agassiz, Poiss. Foss., vol. ii, pt. ii (18.43), p. 179, and Poiss. Foss. V. G. R. (1844), p. 63, pls. xx, xxi; J. Morris, Cat. Brit. Foss., 1854 , p. 327 ; F. M Coy, Brit. Palrez. Foss. (1855), p. 590 ; R. H. Traquair, Geol. Mag. [3] vol. v, I 888, p. 513 .

Glyptolepis, H. Miller, Old Red Sandst., 1841, p. 81, pl. v, f. 2.

Glyptolepis elegans, L. Agassiz, Poiss. Foss., vol. ii, pt. ii (1843), p. 179, and Poiss. Foss. V. G. R. (1844), p. 65, pl. xix, f. 4 , 5 ; J. Morris, Cat. Brit. Foss., 1854, p. 327. [Portions of fishes; unknown.]

Holoptychius sedswickii, F. M'Coy, Ann. Mag. Nat. Hist. [2] vol. ii, 1848 , p. 311 , and Brit. Palicoz. Foss. (1855), p. 595, pl. ii D, f. 6; J. Morris, Cat. Brit. Foss., 1854, p. 320. [Fish; Woodwardian Mus.]

LOC. L. Old Red Sandst. ; Nairnshire, Banffshire, Cromarty, and Orkney. [Portions of fishes; Brit. Mus.] 
GIYPTOITPIS (continued)-

microlepidotus, Agassiz. \%. Gyroptychius microlepidotus.

paucidens (Agassiz), R. H. Traquair, Geol. Mag. [3] vol. v, 1888, p. 513 .

Platygnathus paucidens, L. Agassiz, Poiss. Foss. V. G. R. (1844), pp. 6r, 78, pl. xxviii, f. II ; J. Morris, Cat. Brit. Foss., $185+$, p. 339.

T. (Platygnathus.)

Jaws and scales, etc., of Asterolepis, H. Miller, Footprints of Creator, 1849 , p. 71, woodcuts 25, 26, 30-33, 42.

גoc. L. Old Red Sandst.; Orkney and Caithness. [Manảib. ramus; Brit. Mus.]

I sp., J. Powrie, Quart. Journ. (ienl. Soc., vol. xviii, I862, p. 435. [Vithdrawn in Geologist, I863, p. 96.]

I.OC. U. Old Red Sandst.; Dura Den, Fifeshire.

GIYPTOPOMUS, L. Agassiz, loiss. Foss. V. G. R. (1844), p. 57 ; J. Anderson, Dura Jen, 1859 , p. 55 ; T. H. Huxley, Figs. and Descript. Brit. Organic Remains, dec, xii (Mem. Geol. Surv., I866), no. I.

Platygnathus, L. Agassiz, Poiss. Foss. V. G. R. (1844), p. 77 (pars).

minor, L. Agassiz, Poiss. Foss. V. G. R. (1844), p. 57, pl. xxvi ; J. Anderson, Dura Den, I859, p. 55, pl. ii ; J. Morris, Cat. Brit. Foss., I854, p. 327 ; T. H. Huxley, Figs. and Descript. Brit. Organic Remains, dec. xii (Mem. Geol. Surv., 1866), p. 4 , pl. i, f. I.

Platygnathus minor, L. Agassiz, Poiss. Foss., vol, ii, pt. ii (1843), p. I62 (name only), and Poiss. Foss. V. G. R., lettering of pl. xxvi.

I.Oc. U. Old Red Sandst.; Dura Den. [Portion of fish; Brit. Mus.]

$\boldsymbol{T}$.

sp., Huxley. \%. Holoptychius flemingi.

GLYPTOSTEUS, Agassiz. \%. Bothriolepis.

reticulatus, Agassiz. v. Bothriolepis giganteus.

GNATIACANTrits, J. W. Davis, Trans. Roy. Dublin Soc. [2] vol. i (1883), p. 363 .

striatus, J. W. Davis, ibid., p. 364, pl. xlviii, f. 12.

LOC. L. Carb. Limest.; Armagh. [Spine; Brit. Mus.]

triangularis, J. W. Davis, Trans. Roy. Dublin Soc. [2] vol. i (1883), p. 363 , pl. xlviii, f. I I.

Loc. L. Carb. Limest.; Armagh. [Spine ; Brit. Mus.]

T.

Gompracantrius, J. W. Davis, Quart. Journ. Geol. Soc., vol. $x l, 1884$, p. 618 .

acutus, J. W. Davis, ibial., p. 6ı8, pl. xxvi, f. 9.

LOC. Yoredale Rocks; Wensleydale, Yorkshire. [Spine; York Mus.] 
GOAxPIrODUS, A. E. Reuss, Verstein. böhm. Kreideform., pt. ii, 1846, p. 99 .

sp., A. S. Woodward, Cat. Foss. Fishes B. M., pt. i, 1889, p. 338. LOC. U. Chalk ; Gravesend and Charing, Kent. [Teeth; Brit. Mus., nos. 41702 and P. 334.]

GONATODUS, R. H. Traquair, Quart. Journ. Gcol. Soc., vol. xxxiii, I877, p. 555, and Proc. Roy. Soc. Edinb., vol. ix, 1877, p. 265.

Microconodus, R. H. Traquair, Ganoid Fishes Brit. Carb. Form. (Pal. Soc., 1877), p. 12 (name only).

macrolepis, R. H. Traquair, Quart. Journ. Geol. Soc., vol. xxxiii, 1877 , p. 556, and Proc. Roy. Soc. Edinb., vol. ix, 1877, p. $27 \mathrm{I}$.

Loc. M. Carb. Limest. (Blackband Ironstone); Gilmerton. [Fish; Edinburgh Mus.]

molyneuxi, R. H. Traquair (ex Ward, MS.), Geol. Mag. [3] vol. v, 1888, p. 252 ; J. Ward, Trans. N. Staffs. Inst. Mining Engin., vol. x, I889, pl. vi, f. 2 , Ir.

Microconodus molyneuxi, R. H. Traquair, Ganoid Fishes Brit. Carb. Form. (Pal. Soc., 1877), p. 33 (name only).

\section{T. (Microconodus.)}

Loc. Coal-Meas. (Deep-Mine Ironstone); Longton, Staffordshire. [Fish; J. Ward Coll.]

parvidens, R. H. Traquair, Geol. Mag. [2] vol. ix, 1882, p. 546.

Loc. M. Carb. Limest. (Blackband Ironstone); Borough Lee, near Edinburgh. [Fish; Edinburgh Mus.]

punctatus (Agassiz), R. H. Traquair, Quart. Journ. Geol. Soc., vol. xxxiii, 1877, p. 555, and Proc. Roy. Soc. Edinb., vol. ix, 1877, p. 265.

Amblypterus punctatus, L. Agassiz, Poiss. Foss., vol. ii, pt. i (1835), p. 109, pl. iv c, f. 4 (non f. $3,5-8$ ); J. Morris, Cat. Brit. Foss., 1854, p. 317 . [See also Elonichthys intermedius.]

? Amblypterus anconoachmodus, R. Walker, Trans. Edinb. Geol. Soc., vol. ii, pt. i (1872), p. I18.

LOC. Calcif. Sandst.; Wardie and ? Pitcorthie, Fifeshire. [Imperfect fish ; Edinburgh Mus.]

T.

GONIOBATIS, Le Hon. v. Aetobatis.

GONIOGNATHUS, L. Agassiz, Poiss. Foss., vol. v, pt. i (1843), p. 63 (name only).

coryphanoides, L. Agassiz, ibid., p. 63 (name only).

T.

LOC. London Clay; Sheppey. [Imperfect skull; Brit. Mus.]

maxillaris, L. Agassiz, ibid., p. 63 (name only).

Loc. London Clay; Sheppey. [Imperfect skull; Brit. MIus.] 
GRAPTOLEPS, L. Agassiz, Poiss. Foss., vol. ii, pt. ii (1843), p. 106 (name only).

ormatus, L. Agassiz, ibid., p. I63 (name only).

LOC. Coal-Meas.; Carluke. [Ornamented plate.]

GXIACANTIIUS, L. Agassiz, Poiss. Foss., vol. iii (IS37), p. 17; R. H. Traquair, Ann. Mag. Nat. Hist. [5] vol. xiii (1884), p. 38, and Proc. Roy. Phys. Soc. Edinb., vol. viii (1884), p. 91 ; J. Thomson, Trans. Geol. Soc. Glasgow, vol. iii, pt. i (IS68), p. I30.

Mitrodus, R. Owen, Trans. Odontol. Soc., vol. v, 1867, p. 338 .

almuicensis, L. Agassiz, Poiss. Foss., vol. iii (1837), p. 19, pl. i $a$, f. 8 (undefined); J. Morris, Cat. Brit. Foss., I854, p. 327.

I.oc. Carb. Limest.; Alnwick, Northumberland. [Fragment of spine.]

denticulatus, J. W. Davis, Ann. Mag. Nat. Hist. [5] vol. vi, IS80, p. 372 , woodcuts.

1.OC. M. Coal-Meas.; Tingley, Yorkshire. [Extremity of spine; J. W. Davis Coll.]

formosus, L. Agassiz, Poiss. Foss., vol. iii (1837), p. 17, pl. v, f. $2-6$; E. WV. Binney, Trans. Manchester Geol. Soc., vol. i. iI 41), p. 168 ; J. Morris, Cat. Brit. Foss., I854, p. 327 ; J, Ward, [Proc.] N. Staffs. Nat. Field Club, 1875 , p. 216, f. 3 , and Trans. N. Staffs. Inst. Mining Engin., vol. x (1889), pl. iii, f. I ; R. H. Traquair, Ann. Mag. Nat. Hist. [5] vol. xiii, Is + , p. 46.

'Fish spine?,' J. De C. Sowerby, Zool. Journ., vol. i, IS25, p. 252, pl. viii, f. 9, and ibid., vol. ii, i \&z6, p. 22.

Gyracanthus tubirchlatus, L. Agassiz, Poiss. Foss, vol. ii $(1837)$, p. 19, pl. i a, f. I-7; J. Morris, Cat. Brit. Foss., I $55+$ p. 327 ; A. Hancock and T. Atthey, Nat. Hist. Trans. Northumb. and Durham, vol. iii (1870), p. IoS, and ibid., vol. iv $(1872)$, p. 421 ; A. Hancock and T. Atthey, Ann. Mag. Nat. Hist. [4] vol. i, IS6S, p. 36S, and ibid. [4] vol. ix, IS72, p. 200 ; J. Ward, [Proc.] N. Stafts. Nat. Field Club, IS75, p. $217 ;$ J. IV. I)avis, Quart. Journ. Geol. Soc., rol. xxii, is -6, p. 334. [Spine.]

G. Matuthus, T. P. Barkas, Coal Meas. Palxont., IS73, p. 15, f. $1-5$.

[The spine from the Calcif. Sandst. of Burdiehouse, figured by S. Hibbert, Trans. Roy. Soc. Edinb., vol, xiii (1835), pl. xi, f. I, and assigned to Gyacanthus formosus by Agassiz, is of doubtful species.]

L.oc. Coal-Meas.; Stafiordshire, Lancashire, Yorkshire, Northumberland, and South Scottish Coalfield. [Spine.]

$\mathbf{x .}$

noblls, R. H. Traquair, Geol. Mag. [2] vol. x, $188_{3}, p .542$, and Anm. Mag. Nat. Hist. [5] vol. xiii, ISS4, p. 44 , and Proc. Roy. Phys. Soc. Edinb., vol, viii, IS84, p. 100. 
GXRACANTHUS nobilis (continued) -

Gyracanthus tuberculatus, R. H. Traquair (non Agassiz), Geol. Mag. [2] vol. viii, I88 I, p. 35, and? T. Stock, Trans. Edinb. Nat. Field Club, vol. i ( $188_{3}$ ), p. 50.

Gyracanthus formosus, R. H. Traquair (non Agassiz), Geol. Mag. [2] vol. ix, 1SS2, p. $5+2$.

LOC. M. Carb. Limest.; Edinburgh and Fifeshire. [Spine; R. H. Traquair Coll.]

obliquus, F. M'Coy, Ann. Mag. Nat. Hist. [2] vol. ii, 1848, p. 117, and Brit. Palcez. Foss. (1855), p. 629, pl. iii $\mathrm{K}$, f. 13,$14 ; \mathrm{J}$. Morris, Cat. Brit. Foss., 1854 , p. 327.

Loc. L. Carb. ; Moyheeland. [Spine; Woodwardian Mus.]

ornatus, L. Agassiz, Poiss. Foss., vol. iii (I843), p. I77 (name only).

Loc. Coal-Meas. ; Wales. [Spine.]

tuberculatus, Agassiz. $\%$ Gyracanthus formosus.

youngi, [-ii] R. H. Traquair, Geol. Mag. [2] vol. x, I883, p. 543, and Ann. Mag. Nat. Hist. [5] vol. xiii, 1884, p. 47, and Proc. Roy. Phys. Soc. Edinb., vol. viii, I884, p. 103.

Loc. M. Carb. Limest.; Scottish Coalfield. LSpine; R. H. Traquair Coll.]

sp., R. Howse, Trans. Tyneside Nat. Field Club, vol. i, I $\delta 48$, p. 237 ; assigned to $G$. formosus by II: King, Permian Foss. (Pal. Soc., I 850$)$, p. 221.

LOC. L. Permian; Westoe. [Spine; Newcastle-upon-Tyne Mus.]

(dermal tubercles of). $\%$ Mitrodus quadricornis.

GצroDus, L. Agassiz, Poiss. Foss., vol. ii, pt. ii $(18+3)$, p. 223 ; R. Owen, Rep. Brit. Assoc., 1838 (1839), Sect., p. 143; J. J. Heckel, Denkschr, k. k. Akad. Wiss. Wien, math.naturw. $\mathrm{Cl}$, vol. $\mathrm{xi}, 1856$, p. 200.

Sphærodus, L. Agassiz, Poiss. Foss., vol. ii, pt. i (1833), p. I5, and ibicl., pt. ii (I S43), p. 209 (pars).

angustus, Agassiz. \%. Cœlodus angustus.

coccoderma, P. MI. G. Egerton, Quart. Journ. Geol. Soc., vol. xxy, 1869 , p. $38_{3}$, woodcuts 3,4 .

Loc. Kim. Clay; Kimeridge. [Vomer; Mus. Pract. Geol.]

conicus, Dixon. $\%$ Gyrodus cretaceus.

cretaceus, L. Agassiz, Poiss. Foss., vol. ii, pt. ii (1843), p. 233 , pl. Ixix $a$, f. 13; F. Dixon, Geol. Sussex, 1850, p. 370, pl. xxx, f. 15 ; J. Norris, Cat. Brit. Foss., 1854, p. 328 ; A. S. Woodward, Proc. Geol. Assoc., vol. x (1888), p. 308.

Gyrodus mammillaris, L. Agassiz, Poiss. Foss., vol. ii, pt. ii (1843), p. 236, pl. 1xxiii $a$, f. I, 2.

Pycnodus marginalis, L. Agassiz, ibid., pt. ii (1S43), p. 199. [Tooth ; olim Manchester Mus.] 


\section{GYRODUS cretaceus (continued) -}

Spharodus mammillaris, L. Agassiz, ibid., pt. i (1833), p. 15 (pars). Gyrodus conicus, F. Dixon, Geol. Sussex, r 850 , p. 370, pl. xxxii,

f. 8 ; J. Morris, Cat. Brit. Foss., 1854, p. 328. [Teeth.]

Loc. Chalk ; Sussex. [Iomer ; Brit. Mus.]

cuvieri, L. Agrassiz, Poiss. Foss.,vol. ii, pt. ii (1843), p. 230, pl. Ixixa, f. $21-23$; J. Morris, Cat. Brit. Foss., 1854, p. 328 .

Gyrodus minor, L. Agassiz, Poiss. Foss., vol. ii, pt. ii (1843), p. 234 , pl. lxix $a$, f. 14 ; J. Morris, Cat. Brit. Foss., 1854, p. 328. [Mandib. dentition.]

'Jaw-bone and teeth of a reptile,' J. Phillips, Geol. Yorkshire, I 829, p. 124, pl. ii, f. 55. [Type of $G$. minor, Agassiz.]

I.OC. Kim. Clay; Ely and Weymouth. Speeton Clay; Yorkshire Coast. [Mandib. dentition.]

goweri, P. M. G. Egerton, Quart. Journ. Geol. Soc., vol. xxv, I869, p. 379 , woodcuts 1,2 .

LOC. M. Jurassic ; Culgower, Sutherland. [Trunk; Marcus Gunn Coll. and olim Dunrobin Mus.]

lavior, L. Agassiz, Poiss. Foss., vol. ii, pt. ii (1843), p. 233, pl. lxix a, f. 12 ; J. Morris, Cat. Brit. Foss., 1854, p. 328. [Splenial dentition of Pycnodus toliapicus from the London Clay of Sheppey in Enniskillen Coll., Brit. Mus., erroneously identified with this species.]

I.OC. Unknown. [Splenial dentition; Paris Mus.]

mammillaris, Agassiz. \%. Gyrodus cretaceus.

mantelli, Agassiz. v. Cuelodus mantelli.

millor, Agassiz. \%. Gyrodus cuvieri.

ornatissimus, J. F. Blake, Quart. Journ. Geol. Soc., vol. xxxi, 1875 , p. 223; R. Damon, Geol. Weymouth, ed. 3, 1888, Suppl., pl. xi, f. I-3, 6. [? G. cuvieri.]

I.OC. L. Kim. Clay; Weymouth. [Dentition; Brit. Mus.]

perlatus, Agassiz. v. Mesodon bucklandi.

punctatus, L. Agassiz, Poiss. Foss., vol. ii, pt. ii (1843), p. 231, pl. lxix $a$, f. 24 .

I.OC. Corallian; Malton, Yorkshire. [Vomer; York Mus.]

trigonus, Agassiz. v. Mesodon trigonus.

sp.: Sphcrodus sigas, P. M. G. Egerton (non Agassiz), Quart. Journ. Geol. Soc., vol. xxv, 1869, p. 385, woodcut 5.

I.OC. Kim. Clay; Kimeridge. [Vomer.]

GXROIIPIS, L. Agassiz, Poiss. Foss., vol. ii, pt. i (I833), pp. 6, 172 , and ibid., pt. ii (I843), p. $28 ;$; R. H. Traquair, Quart. Journ. Geol. Soc., vol. xxxiii, 1877, p. 567 ; W. Dames, l'alæont. Abhandl., vol. iv, 1888, p. 135.

albert1i, L. Agassiz, Poiss. Foss., vol. ii, pt. i (1835), p. 173, pl. xix, f. $1-6$, and ibid., pt. ii (1843), p. 285 ; R. Etheridge, Proc. 
GYROIEPIS albertii (continucd)-

Cardiff Naturalists' Soc. vol. iii, I872, pl. ii, f. I 8 ; J. Morris, Cat. Brit. Foss., I 854 , p. 328 ; W. Dames, Palæont. Abhandl., vol. iv, I888, p. 143, pl. xi, f. I, pl. xii, f. I, pl. xv, f. I ; A. S. Woodward, Trans. Leicester Lit. and Phil. Soc., n.s., pt. xi (1889), p. 20.

Gyrolcpis tenuistriatus, L. Agassiz, Poiss. Foss., vol. ii, pt. i (1835), p. I74, pl. xix, f. IO-12, and ibid., pt. ii (1843), p. 285 ; J. Morris, Cat. Brit. Foss., 1854, p. 328.

Gy'rolepis maximus, L. Agassiz, Poiss. Foss., vol. ii, pt. i (1835),

p. 175 , pl. xix, f. 7-9, and ibid., pt. ii (1843), p. 285.

LOC. Rhatic; Gloucestershire, Monmouthshire, Somersetshire, Devonshire, and Leicestershire. [Scales.]

giganter.s, Agassiz. \%. Holoptychius nobilissimus.

maximus, Agassiz. $\tau^{\prime}$. Gyrolepis albertii.

rankinei, Agassiz. v. Acrolepis rankinei.

temuistriatus, Agassiz. \%. Gyrolepis albertii.

GIRONCIILS, Agassiz. \% Mesodon.

oblongus, Agassiz. $v$. Mesodon oblongus.

GYROPRISTIS, L. Agassiz, Poiss. Foss., vol. iii (1843), p. 177 (name only).

obliquus, L. Agassiz, iỏid, p. I77 (name only); W. King, Permian Foss. (Pal. Soc., 1850 ), p. 222 ; J. Morris, Cat. Brit. Foss., 1854 , p. 328 .

LOC. ? Permian; Belfast. ['Ichthyodorulite.']

GYROPTyCIIUs. F. M'Coy, Ann. Mag. Nat. Hist. [2] vol. ii, 1848, p. 307, and Brit. Palæen. Foss. (1855), p. 596 ; emend. R. H. Traquair, Geol. Mag. [3] vol. v, 1888, p. 514. [Sce also Diplopterus.]

angustus, M'Coy. v. Gyroptychius microlepidotus.

diplopteroides, $\mathrm{M}$ 'Coy. v. Diplopterus agassizi.

microleridotus (Agassiz), R. H. Traquair, ibid., p. 514.

Glyptolepis microlepidotus, L. Agassiz, Poiss. Foss. V. G. R. (1844), p. 65, pl. xxi a, f. $3-7$; J. Morris, Cat. Brit. Foss., 1854 , p. 327.

Gy'roptychius angustus, F. M'Coy, Ann. Mag. Nat. Hist. [2] vol. ii, 1848 , p. 308 , and Brit. Palæoz. Foss. (1855), p. 596, pl. ii c, f. 2 ; J. Morris, Cat. Brit. Foss., 1854, p. 328; R. H. Traquair, Trans. Roy. Soc. Edinb., vol. xxvii (1875), p. 395 ; C. H.Pander, Saurodipt., Dendrodont., etc., devon. Syst., 1860, p. 79, pl. vi, f. I-7, pl. vii, f. 1-3; T. H. Huxley, Figs. and Descript. Brit. Organic Remains, dec. x (Mem. Geol. Surv., 1861), p. 3, woodcut 3 (Gyroptychizs). [Imperfect fish ; Woodwardian Mus.]

LOC. L. Old Red Sandst. ; Lethen Bar and Orkney. [Portions of fishes; Brit. Mus. and Forres Mus.] 
GXrostrus, L. Agassiz, Poiss. Foss., vol. ii, pt. ii (1843), p. 179 (name only); A. S. Woodward, Proc. Geol. Assoc., vol. xi (1889), p. 36.

mirabilis, L. Agassiz, Poiss. Foss., vol, ii, pt. ii (1843), p. 179 (name only); J. Morris, Cat. Brit. Foss., 1854 , p. 328 ; P.M.G. Egerton, Phil. Trans., 1858, p. $883 ;$ J. F. Hiake, in Tate and Blake, Yorksh. Lias, 1876 , p. 256 , pl. ii, f. 2,3 ; A. S. Woodward, Proc. Geol. Assoc., vol. xi (1889), p. 32, woodcuts 2-7.

Scpict incomposita, M. Simpson, Foss. Yorkshire Lias, 1855, p. 2 I. [Bone; Whitby Mus.]

LOC. U. Lias; Whitby, [Various bones; Brit. Mus.] $\mathbf{2}$.

HALECOPSIS, L. Agassiz, Poiss. Foss., vol. v, pt. ii (1843), p. 139, and Rep. Brit. Assoc., 1844 (1845), p. 308 (name only).

laevis, L. Agassiz, ibid. (1S43), p. 139, and iürl., 1844, p. 308 (name only).

LOC. Londun Clay; Sheppey. [Imperfect fish; Brit. Mus.] $\boldsymbol{x}$.

HARPACANTrys, R. H. Traquair, Ann. Mag. Nat. Hist. [5] vol. xviii, 1886, p. 493.

fimbriatus (Stock), R. H. Traquair, ibid., p. 493, woodcut.

Tristychius fimbriatus, T. Stock, Ann. Mag. Nat. Hist. [5] vol. xii, 1883, p. 177, pl. vii, f. I.

LOC. M. Carb. Limest.; Gilmerton, near Edinburgh. [Spine ; Edinburgh Mus.]

I ARPACODU, Davis. \% Ctenoptychius.

clavatus, Davis. \% Venustodus clavatus.

dentrtus (Owen), Davis. $\%$. Ctenoptychius dentatus.

HARPACTES. Egerton. \%. Harpactira.

velox, Egerton. $\approx$. Harpactira velox.

IARPACTIRA, P. M. G. Egerton, Geol. Mag. [2] vol. iii, 1876, p. 576 .

HaRpaCtes, P. M. G. Egertom, ibil., p. $4 \frac{11}{1}$ (preoccupied).

velox, P. M. G. Egerton, ibid., p. 4tI (Harpactes).

LOC. L. Lias; Lyme Regis. [Fish ; Brit. Mus.]

s.

I2EエODUS, L. Agassiz, Poiss. Foss, vol. iii (1838), p. 104.

[A provisional generic name principally applied to undetermined anterior teeth of Cochlioronil.te (A. S. Woodward, Cat. Foss. Fishes B. M., pt. i, 1889, p. 218).]

Lophodes, H. Romanovsky. Bull. Soc. Imp. Nat. Moscou, vol. xxxvii, pt. ii, 1864, p. I60.

acutus, J. W. I)avis, Trans. Roy. Dublin Soc. [2] vol. i (1883), p. 455 , pl. lix, f. 7 .

LOC. L. Carb. Limest, ; Armagh. [Tooth ; Brit. Mus.] 
IrIXODS (continued)-

angularis: Lophodus angularis, J. W. Davis, Quart. Journ. Geol. Soc., vol. xl, I884, p. 628, pl. xxvii, f. 25.

LOC. Yoredale Rocks ; Wensleydale. [Tooth; York Mus.]

appendiculatus, F. M'Coy, Ann. Mag. Nat. Hist. [2] vol. ii, I 848 , p. 123 ; J. Morris, Cat. Brit. Foss., 1854 , p. 328.

LOC. L. Carb. Limest. ; Armagh. [Tooth ; olim Capt. Jones Coll.]

bifurcatus: Lophodus bifurcatus, J. W. Davis, Trans. Roy. Dublin Soc. [2] vol. i (1883), p. 408, pl. li, f. 25, and Quart. Journ. Geol. Soc., vol. xl, I884, p. 628, pl. xxvii, f. 5 .

LOC. Yoredale Rocks; Wensleydale. [Tooth ; York Mus.]

cinctus (Agassiz), M'Coy. v. Chomatodus cinctus.

clavatus, J. IV. Davis, Trans. Roy. Dublin Soc. [2] vol. i ( $188_{3}$ ), p. 455 , pl. lix, f. 5,6 .

LOC. L. Carb. Limest.; Armagh. [Teeth ; Brit. Mus.]

conicus: Lophodus conicus, J. W. Davis, Quart. Journ. Geol. Soc., vol. xl, 1884 , p. 627 , pl. xxvi, f. 18 .

LOC. Yoredale Rocks; Wensleydale. [Tooth; York Mus.]

crassus, J. W. Davis, Trans. Roy. Dublin Soc. [2] vol. i ( 1883 ), p. 453, pl. lix, f. 1, 2. [Including Chomatodus cinctus, Agassiz (pars).]

LOC. L. Carb. Limest.; Armagh. [Teeth ; Brit. Mus.]

didymus, Agassiz. v. Psephodus magnus.

expansus, J. W. Davis, Trans. Roy. Dublin Soc. [2] vol. i (1883), p. 457 , pl. lix, f. 10 .

Chomatodus linearis, L. Agassiz (pars), Poiss. Foss., vol. iii (1838), p. 108, pl. xii, f. 7, 8, I2, 13 ; J. W. Davis, Trans. Roy. Dublin Soc. [2] vol. i (1883), p. 508, pl. Ixi, f. 1. [Teeth; Bristol Mus. (purs).]

LOC. L. Carb. Limest.; Armagh. [Tooth ; Brit. Mus.]

gribberulus, L. Agassiz, Poiss. Foss., vol. iii (I838), p. 106, pl. xii, f. I, 2 (Psammodus on plate); J. Morris, Cat. Brit. Foss., 1854, p. 328.

Lophodus gibberulus, J. W. Davis, Trans. Roy. Dublin Soc. [2] vol. i (1883), p. 405, pl. li, f. I9.

LOC. L. Carb. Limest.; Armagh. [Tooth ; Bristol Mus.]

laevissimus, Agassiz (non F. M'Coy, Brit. Palæoz. Foss. (1855), p. 630 , pl. iii I, f. 17 ; non J. W. Davis, Trans. Roy. Dublin Soc. [2] vol. i (1883), p. 404); J. Morris, Cat. Brit. Foss., 1854, p. 328. v. Psephodus lævissimus.

Ievis : Lophodus levis, J. W. Davis, Trans. Roy. Dublin. Soc. [2] vol. $i$ (1883), p. 409, pl. li, f. 26, 27, and Quart. Journ. Geol. Soc., vol. xl, 1884, p. 627 (L. lavis).

Loc. Yoredale Rocks; Wensleydale. [Tooth ; York Mus.] 
IrIODUS (continued) -

mammillaris, L. Agassiz, Poiss. Foss., vol. iii (1843), p. 173 (name only); J. Morris, Cat. Brit. Foss., I 854 , p. 328 ; F. M'Coy, Brit. Palæoz. Foss. (I855), p. 631, pl. iii I, f. 16.

Lophodus mammillaris, J. W. Davis, Trans. Roy. Dublin Soc. [2] vol. i (1883), p. 406, pl. li, f. 20.

LOC. L. Carb. Limest.; Armagh. [Tooth; Woodwardian Mus.]

mitratus, L. Agassiz, Poiss. Foss., vol, iii (1843), p. 173 (name only). [? H. simplex.]

Loc. Coal-Meas.; Carluke. [Tooth.]

obliquus: Chomatodus obliquus, F. M'Coy, Ann. Mag. Nat. Hist. [2] vol. ii, 1848, p. 124, and Brit. Palæoz. Foss. (1855), p. 6i8, pl. iii K, f. 3 (Chomatodus (Helodus) obliquus); J. Morris, Cat. Brit. Foss., 1854, p. 321.

LOC. L. Carb. Limest.; Armagh. [Tooth; Woodwardian Mus.]

planus (Agassiz), M'Coy. v. Psephodus magnus.

pusllus (M'Coy), A. S. Woodward, Cat. Foss. Fishes B. M., pt. i, 1889, p. 227.

Polyrhizodus pusillus, F. M'Coy, Ann. Mag. Nat. Hist. [2] vol. ii, 1848, p. 126, and Brit. Palæoz. Foss. (1855), p. 642, pl. iii K, f. 2 ; J. Morris, Cat. Brit. Foss., 1854, p. 340.

LOC. L. Carb. Limest.; Armagh. [Tooth; Woodwardian Mlus.]

? reticulatus : Lophodus reticulatus, J. W. Davis, Trans. Roy. Dublin Soc. [2] vol. i (1883), p. 407, pl. li, f. 22, and Quart. Journ. Geol. Soc., vol. xl, I884, p. 626, pl. xxvii, f. 2.

Loc. Yoredale Rocks; Wensleydale. [Tooth ; York Mus.]

richmondiensis, J. IV. Davis, Trans. Roy. Dublin Soc. [2] vol. i (1883), p. 456 , pl. lix, f. 8.

Loc. U. Carb. Limest.; Richmond, Yorkshire. [Tooth ; Brit. Mus.]

rudis, $\mathrm{M}^{\prime} \mathrm{Coy}$. v. Psephodus magnus.

simplex, L. Agassiz, Poiss. Foss., vol. iii (1838), p. r04, pl. xix, f. 8-10; J. Morris, Cat. Brit. Foss., I 854 , p. 328 ; J. IV. Salter, Foss. S. Welsh Coalfield (Mem. Geol. Surv., 186I - Iron Ores Gt. Brit., pt. iii), p. 225, pl. i, f. 17; A. Hancock and T. Atthey, Ann. Mag. Nat. Hist. [4] vol. ix, 1872 , p. 26r ; J. Ward, [Proc.] N. Staffs. Nat. Field Club, 1875 , p. 220, and Trans. N. Staffs. Inst. Mining Engin., vol. x, 1889, pl. ii, f. I3; W. J. Barkas, Monthly Kev. Dental Surgery, vol. iii, 1875, p. IOI, f. xlv-xlviii ; R. H. Traquair, Geol. Mag. [3] vol. v, I888, p. 84; A. S. Woodward, Cat. Foss. Fishes B. M., pt. i, 1889, p. 172.

Loc. Coal-Meas.; Staffordshire, Shropshire, Yorkshire, Northumberland, South Wales, and Lanarkshire. [Tooth ; Brit. Mus.]

subteres, Agassiz. v. Orodus ramosus. 
IrIODUS (continued) -

tenuis, J. W. Davis, Trans. Roy. Dublin Soc. [2] vol. i (1883), p. 454 , pl. lix, f. 3, 4. [See also Chomatodus cinctus.]

LOC. L. Carb. Limest.; Armagh. [Teeth ; Brit. Mus.]

trlangular1s, J. W. Davis, ibid., p. 456, pl. lix, f. 9.

Loc. L. Carb. Limest.; Armagh. [Tooth ; Brit. Mus.]

turgldus, L. Agassiz, Poiss. Foss., vol. iii (1838), p. I06, pl. xv, f. 1-12 (Psammodus on plate); J. Morris, Cat. Brit. Foss., 1854 , p. 328 ; F. $\mathrm{I}^{6} \mathrm{Coy}$, Brit. Palæoz. Foss. $(18 ; 5)$, p. 632. [See also Chomatodus cinctus.]

LOC. L. Carb. Limest. ; Bristol. [Teeth ; Bristol Mus.]

HEMICLADODUS, Davis. ¿. Cheirodus.

unicuspidatus, Davis. v. Cheirodus sp.

HEIICYCLASPIS, Lankester. v. Cephalaspis.

murchisoni (Egerton), Lankester. v. Cephalaspis murchisoni.

HETEROLEPIDOTUS, Egerton. v. Eugnathus.

grendis, Davis. $\approx$. Platysiagum grandis.

latus, Egerton. \%. Eugnathus latus.

manseli (Egerton), Zittel. $\%$. Lepidotus manseli.

minor, Davis. $\approx$. Eugnathus latus.

sauroides, Egerton. v. Eugnathus philpota.

serrulatus (Agassiz), Egerton. v. Eugnathus serrulatus.

mISTrowoтus, P. M. G. Egerton, Ann. Mag. Nat. Hist. [2] vol. xiii, 1854 , p. 434 , and Figs. and Descript. Brit. Organic Remains, dec. viii (Mem. Geol. Surv., 1855), no. 5.

angularis, P. M. G. Egerton, ibid., p. 434, and ibid., pl. v ; J. Morris, Cat. Brit. Foss., 1854, p. 329 ; J. C. Mansel-Pleydell, Geol. Mag. [3] vol. vi, 1889, p. 24I, pl. vii. LOC. Purbeck Beds; Swanage. [Imperfect fish; Brit. Mus.]

IOXASPIS, E. R. Lankester (er Salter, MS.), Geol. Mag., vol. x, 1873, p. 242.

sericea, E. R. Lankester, ibid., pp. 24I, 331, pl. x, and woodcut. LOC. L. Old Red Sandst. ; Abergavenny. [Head-shield: Brit. Mus.]

r.

IOxOxMAGS, P. M. G. Egerton, Figs, and Descript. Brit. Organic Remains, dec. x (Mem. Geol. Surv., 1861), p. 19; T. H. Huxley, ibid., dec, xii (1866), p. 26, and ibia., dec, xiii (1872), no. 10. [? Undina.]

gulo, P. M. G. Egerton, Figs. and Descript. Brit. Organic Remains, dec. x (Mem. Geol. Surv., I861), p. 19; T. H. Huxley, ibid., dec.xii(1866), p.26, pl.vi, and ibid., dec.xiii (1872), no. 1o, pl.x. Loc. L. Lias; Lyme Regis. [Fish, wanting head; Mus. Pract. Geol.]

T. 
IrOIOPTYCrIUS, L. Agassiz, in Murchison's Silur. Syst., I 839, p. 599 (Holoptychus); F. M'Coy, Brit. Palæoz. Foss. (1855), p. 593 .

Platygnathus, L. Agassiz, Poiss. Foss. V. G. R. (1844), pp. 6I, 76 (pars).

andersoni, Agassiz. $\quad$. Holoptychius flemingi.

falcatus, L. Agassiz, Poiss. Foss., vol. ii, pt. ii (1843), p. I80 (name only). [? Genus.]

Loc. Coal-Meas. ; Greenside, Glasgow.

flemingi, [-ii] L. Agassiz, Poiss. Foss. V. G. R. (I 844), p. 7 I, pl. xxii, f. I ; J. Morris, Cat. Brit. Foss., 1854, p. 329 ; J. Anderson, Dura Den, 1859 , p. 57 ; [-ii] R. H. Traquair, Geol. Mag. [3] vol. v, I888, p. 513 .

Holoptychius andersoni, L. Agassiz, Poiss. Foss. V. G. R. (I844), p. 72 , pl. xxii, f. 3 ; J. Morris, Cat. Brit. Foss., 1854, p. 329 ; J. Anderson, Dura Den, 1859 , p. 57 , pl. vii, pl. viii, f. 1, 2 ; F. M'Coy, Brit. Palieoz. Foss. (1855), p. 594. [Head and trunk; Brit. Mus.]

Platygnathus jamesoni, L. Agassiz, Poiss. Foss. V. G. R. (1844), p. 77 , pl. Xxv ; J. Morris, Cat. Brit. Foss., 1854, p. 339 ; J. Anderson, Dura Den, 1859 , p. 56, pl. i, f. 2. [Tail ; Brit. Mus.]

Glyptopomus, T. H. Huxley (errore), Figs. and Descript. Brit. Organic Remains, dec. x (Mem. Geol. Surv,, I86I), p. 4, woodcut 4, and ibid., dec. xii (I866), pl. i, f. 2. [Head; Brit. Mus.]

LOC. U. Old Red Sandst. ; Dura Den. [Group of scales ; olim Fleming Coll.]

garneri (Murchison), Agassiz. $\%$. Strepsodus sauroides.

giganteus, L. Agassiz, Poiss. Foss. V. G. R. (1844), pp. 73, 140, pl. xxiv, f. 3-IO ; J. Morris, Cat. Brit. Foss., 1854, p. 329 ; F. M'Coy, Brit. Palæoz. Foss. (1855), p. 594.

Holoptychius, R. I. Murchison, Silur. Syst., I839, p. 600 , pl. ii bis, f. 3. [Scale. $]$

Holoptychius princeps, F. M'Coy, Ann. Mag. Nat. Hist. [2] vol. ii, 1848, p. 310 ; Brit. Palæoz. Foss. (1855), p. 595 ; J. Morris, Cat. Brit. Foss., 1854, p. 329. [Scale; Woodwardian Mus.]

LOC. U. Old Red Sandst.; Scat Craig and Alves, Elgin. [Scales; unknown.]

granulatus, Agassiz. $\%$. Rhizodopsis sauroides.

hibberti, Agassiz. v. Rhizodus hibberti.

hopkinsi, $\mathrm{I}^{\prime} \mathrm{C} \mathrm{C}$. $\%$. Acrolepis hopkinsi.

minor, L. Agassiz, Poiss. Foss., vol. ii, pt. ii (1843), p. 180 (name only). [Rhizodopsis.]

LOC. Coal-Meas.; Leeds.

murchisoni, Agassiz. $v$. Holoptychius nobilissimus. 
HOxOPTYCizIUS (continucd)-

nobllissimus, L. Agassiz, in Murchison's Silur. Syst., 1839, p. 599, pl. ii bis, f. I, 2 , and Poiss. Foss. V. (.. R. (1844), pp. 73, 140, pl. xxiii, pl. xxiv, f. 2 , pl. xxxi $a$, f. 26 ; H. Miller, Old Red Sandst., 1841, p. 162, pl. ix, f. 2 ; J. Morris, Cat. Brit. Foss., I854, p. 329 ; F. M ${ }^{\circ}$ Coy, Brit. Palicoz. Foss. (185j), p. 595.

Gyrolepis giganteus, L. Agassiz, Poiss. Foss., vol. ii, pt. i (1835), p. 175, pl. xix, f. I3.

Holoptychius murchisoni, L. Agassiz, Poiss. Foss. V. G. R. (1844), p. 72, pl. xxii, f. 2 ; J. Morris, Cat. Brit. Foss., 18 54 , p. 329. [Scales; unknown.]

LOC. U. Old Red Sandst.; Elgin and Clashbennie. [Fish, wanting tail ; Brit. Mus.]

portlocki (Agassiz), Portlock. $\%$ Archichthys portlocki.

prince'ps, $\mathrm{M}^{\circ} \mathrm{Coy}$. v. Holoptychius giganteus.

sauroides, Williamson, Binney, Kirkby and Atthey. v. Rhizodopsis sauroides and Strepsodus sauroides.

sedgricki, M'Coy. \%. Glyptolepis leptopterus.

striutus, L. Agassiz, Poiss. Foss., vol. ii. pt. ii (1843), p. 180 (name only).

Loc. Milistone Grit ; Edinburgh.

sp., Kirkby and Atthey. $v$ Rhizodopsis sauroides.

HOLOPTYCHUS, Agassiz. Misprint for Holoptychius.

IrorURUS, R. H. Traquair, Trans. Roy. Soc. Edinb., vol. $x x x$ ( 1881 ), p. 43.

fulcratus, R. H. Traquair, ibid., p. 46, pl. iii, f. 13, 14 .

LOC. Calcif. Sandst. (Cement-stone Group); Glencartholm, Eskdale, Dumfriesshire. [Imperfect fish; Geol. Surv. Scotland.]

ischypterus, R. H. Traquair, Trans. Roy. Soc. Edinb., vol. $x x x$ (188r), p. 66, pl. iii, f. 15, 16.

LOC. Calcif. Sandst. (Cement-stone Group); Valley of Tweed, near Coldstream Bridge. [Fish ; Geol. Surv. Scotland.]

parki, R. H. Traquair, Trans. Roy. Soc. Edinb., vol. $\operatorname{xxx}$ (1881), p. 44 , pl. iii, f. 9-12.

LOC. Calcif. Sandst. (Cement-stone Group); Glencartholm, Eskdale. [Fïsh ; Geol. Surv. Scotland.]

moiracantruts, I. Agassiz, Poiss. Foss. V. G. R. (1844), p. 113 .

macrodus, F. M'Coy, Ann. Mag. Nat. Hist. [2] vol. ii, 1848, p. 115, and Brit. Palæoz. Foss. (1855), p. 632, pl. iii K, f. 20 ; J. Morris, Cat. Brit. Foss., 1854, p. 329; J. W. Davis, Trans. Roy. Dublin Soc. [2] vol. i (1883), p. 362, pl. xlviii, f. 14 .

r.oc. L. Carb. Limest.; Armagh. [Spine; Woodwardian Mus.] 
MOMACANTHUS (continued)-

microdus, F. M'Coy, Ann. Mag. Nat. Hist. [2] vol. ii, 1848, p. I 15, and Brit. Palæoz. Foss. (1855), p. 633, pl. iii K, f. 19; J. Morris, Cat. Brit. Foss., I 854, p. 329; J. W. Davis, Trans. Roy. Dublin Soc. [2] vol. i (1883), p. 361, pl. xlviii, f. 7-9.

LOC. L. Carb. Limest.; Armagh. [Spine; Woodwardian Mus.]

HOMALODUS, Davis. v. Psammodus.

quadratus, Davis. $v$. Psammodus trapeziformis.

trapeziformis, Davis. $\%$. Psammodus trapeziformis.

momonotus, L. Agassiz, in F. Dixon, Geol. Sussex, 1850, p. 372 ; A. S. Woodward, Proc. Geol. Assoc., vol. x (1888), p. 329 .

dorsalis, F. Dixon, Geol. Sussex, I850, p. 372, pl. xxxv, f. 2 ; J. Morris, Cat. Brit. Foss., 1854, p. 329; A. S. Woodward, Proc. Geol. Assoc., vol. x (1888), p. 329.

$\mathbf{T}$. IOC. Chalk; S. E. England. [Imperfect fish ; Brighton Mus.]

IOMOSTEUS, $H$. Asmuss, Das vollkomm. Hautskelet d. bisher bekannt. Thierreihe (Inaug. Dissert. Dorpat, 1856), p. 8 (Homostius).

Asterolepis, L. Agassiz (non Eichwald), Poiss. Foss. V. G. R. (1844), p. 89 (pars); H. Miller (non Eichwald), Footprints of Creator, 1849, p. 70 (pars).

mllleri, R. H. Traquair, Geol. Mag. [3] vol. v, 1888, p. $5 \mathrm{II}$, and ibid. [3] vol. vi, 1889, p. 1, pl. i, f. I.

'Asterolepis of Stromness,' H. Miller, Footprints of the Creator, I849, p. 70, woodcuts $24,27-29,36,37,39-41$ (? $34,35,38,44$ ).

Asterolepis asmusiz, J. Morris, Cat. Brit. Foss., 1854, p. 318.

Asterolepis, J. Miller, Geol. Mag., vol. vi, 1869, p. 384 ("hyoid" $=$ dorsal plate).

roc. L. Old Red Sandst. ; Caithness and Orkney. [Carapace ; Edinburgh Mus.]

HOMотноR AX, Agassiz. v. Bothriolepis.

flemingi, Agassiz. v. Bothriolepis hydrophilus.

aOPIONCxUS, J. W. Davis, Quart. Journ. Geol. Soc., vol. xxii, 1876, p. 336.

elegans, J. W. Davis, ibid., vol. xxxv, 1879, p. 183, pl. x, f. 12-14. LOC. L. Coal-Meas.; Yorkshire. [Spine; J. W. Davis Coll.] T.

EOPגOPTERXX, L. Agassiz, Poiss. Foss., vol. iv (1843), p. I31.

leweslensis (Mantell), A. S. Woodward, Proc. Geol. Assoc., vol. $\times$ (1888), p. 327.

Zeus leavesiensis, G. A. Mantell, Foss. S. Downs, 1822, p. 234, pls. xxxv, xxxvi. 
HOPTOPTERXX lowesiensis (continued)-

Beryx ornatus, L. Agassiz, Poiss. Foss., Feuill., p. 55 (1835), and vol. iv (1843), p. I I 5 , pl. xiv $a$, pl. xiv $b$, f. I, 2, pl. xiv $c$, f. I-6, pl. xiv $d$; F. Dixon, Gcol. Sussex, 18;o, p. 371, pl. xxxvi, f. 3 , pl. xxxiv, f. I, 4, 5 .

Beryx lewesiensis, J. Morris, Cat. Brit. Foss., 1854, p. 318.

Loc. Chalk; S. E. England. [Imperfect fishes; Brit. Mus.]

superbus (Dixon), J. W: Davis, Trans. Roy. Dublin Soc. [2] vol. iii (1887), p. 514 ; A. S. Woodward, Proc. Geol. Assoc., vol. x (1888), p. 328.

Beryx superbus, F. Dixon, Geol. Sussex, 1850, p. 372, pl. xxxvi, f. 5 ; J. Morris, Cat. Brit. Foss., 1854 , p. 318.

LOC. Chalk; S. E. England. [Fish; Brit. Mus.]

HOPLOPYGUS, Agassiz, ש. Cœlacanthus.

binneyi, Agassiz. $\approx$. Cœlacanthus lepturus.

IxzBODOPSIS, W. J. Barkas, Geol. Mag. [2] vol. i, 1874, p. 54\% (name only), and Monthly Rev. Dental Surgery, vol. vii, $187 \&$, p. 191 ; A. S. Woodward, Cat. Foss. Fishes B. M., pt. i, 1889, p. 240.

wardi, W. J. Barkas, Geol. Mag. [2] vol. i, 1874, p. 546 (name only), and Monthly Rev. Dental Surgery, vol. vii, 1878, 1 . I9I, f. i-vi ; A. S. Woodward, Cat. Foss. Fishes B. M., pt. i, I889, p. 240.

Loc. Coal-Meas.; Burnley, Lancashire. [Fragment of jaw with teeth ; J. Ward Coll.]

$\mathbf{T}$.

EצBODus, L. Agassiz, Poiss. Foss., vol. iii (1837), p. 41 ; A. S. Woodward, Cat. Foss. Fishes B. M., pt. i, 1889, p. 250.

Sphenonchus, L. Agassiz, Poiss. Foss., vol. iii (1843), p. 201 (pars).

? Meristodon, L. Agassiz, ibid (1843), p. 286.

acutus, L. Agassiz, ibid. (1837), p. 45, pl. x, f. 4-6 ; J. Morris, Cat. Brit. Foss., 1854, p. 329.

LOC. L. Kim. Clay; Shotover, near Oxford. [Spine; Brit. Mus.]

apicalis, L. Agassiz, Poiss. Foss., vol. iii (1837), p. 43, pl. x, f. 22 (non p. 195, pl. xxiii, f. 16-20) ; J. Morris, Cat. Brit. Foss., 1854, p. 329 ; A. S. Woodward, Cat. Foss. Fishes B. M., pt. i, 1889, p. 301 .

LOC. Stonesfield Slate. [Spine ; Bristol Mus.]

austiensis, Davis. v. Hybodus cloacinus.

basanus, P. M. G. Egerton, Quart. Journ. Geol. Soc., vol. i, 1845, p. 199, pl. iv ; J. Morris, Cat. Brit. Foss., 1854. p. 329 ; A. S Woodward, Cat. Foss. Fishes B. M., pt. i, 1889, p. 273, pl. xii, f. I-5. 
HYBODUS basanus (continted) -

? Hybodus sulcatus, L. Agassiz, Poiss. Foss., vol. iii (1837), p. 44, pl. x b, f. 15, I6; J. Morris, Cat. Brit. Foss., 1854, p. 330.

Hybodus, A. S. Woodward, Geol. Mag. [3] vol. iii, I886, p. 258. LOC. U. Wealden; I. of Wight and Sussex. [Head; olim Capt. Ibbetson Coll.]

cartnatus, L. Agassiz, Poiss. Foss., vol. iii (1837), p. 52, pl. ix, f. 13,14 ; J. Morris, Cat. Brit. Foss., 1854, p. 329. ?=Acrodus anningice (A. S. Woodward, Cat. Foss. Fishes B. M., pt. i, 1889, p. 294).

LOC. L. Lias ; Lyme Regis. [Spine ; Brit. Mus.]

cloacinus, F. A. Quenstedt, Der Jura, I858, p. 34, pl. ii, f. 15 ; A. S. Woodward, Cat. Foss. Fishes B. M., pt. i, I889, p. 256 (? pl. x, f. 8-14), and Trans. Leicester Lit. and Phil. Soc., n.s., pt. xi (I889), p. 19.

Hybodus reticulatus, R. Etheridge (non Agassiz), Proc. Cardiff Naturalists' Soc., vol. iii (1872), pl. ii, f. II.

? Hybodus austiensis, J. W. Davis, Quart. Journ. Geol. Soc., vol. xxxvii, I88I, p. 416, pl. xxii, f. I. [Spine; J. W. Davis Coll.]

LOC. Rhætic; Somersetshire, Gloucestershire, Devonshire, Leicestershire, and S. Wales. ? L. Lias; Lyme Regis, Dorset. [Teeth; Brit. MIus.]

complanatus, R. Oiven, Geol. Mag., vol. vi, 1869, p. 482.

LOC. L. Greensand; Maidstone. [Spine ; Brit. Mus.]

crassispinus, L. Agassiz, Poiss. Foss., vol. iii (1837), p. 48, pl. viii b, f. 7 ; J. Morris, Cat. Brit. Foss., 1854, p. 329.

LOC. L. Lias; Lyme Regis. [Spine ; Brit. Mus.]

crassus, L. Agassiz, P'oiss. Foss., vol. iii (1837), p. 47, pl. x, f. 23 ; J. Morris, Cat. Brit. Foss.y 1854, p. 329 ; A. S. Woodward, Cat. Foss. Fishes B. M., pt. i, I889, p. 30 I.

LOC. Inf. Oolite ; Rutlandshire. [Spine ; Brit. Mus.]

curtus, Agassiz. v. Acrodus anningize and Hybodus reticulatus. davisi, W. J. Barkas. $\%$. Sphenacanthus hybodoides.

delabechei, [-eii] E. Charlesworth, Mag. Nat. Hist., n.s., vol. iii, 1839, p. 242, pl. iv ; J. Morris, Cat. Brit. Foss., I854, p. 329 ; E.C. H. Day, Geol. Mag., vol. ii, I 865, p. 565 ; A. S. Woodward, Cat. Foss. Fishes B. M., pt. i, I889, p. 259, pl. viii, pl. x, f. I-5, and Ann. Rep. Yorksh. Phil. Soc., I 888 (1889), p. 58, pl. i. Hybodus pyramidalis, L. Agassiz, Poiss. Foss., vol. iii (1843), p. I 82 , pl. xxii $\iota$, f. 20,21 ; J. Morris, Cat. Brit. Foss., 1854 , p. 330. [Group of teeth ; Brit. Mus.]

LOC. L. Lias; Lyme Regis. [Teeth and spines; Mus. Pract. Geol.]

dorsalis, L. Agassiz, Poiss. Foss., vol. iii (1837), p. 42, pl. x, f. I ; J. Morris, Cat. Brit. Foss., 1854, p. 330 ; A. S. Woodward, Cat. Foss. Fishes B. M., pt. i, 1889, pp. 302, 303.

Loc. Stonesfield Slate. [Spine; Brit. Mus.] 
ITY BODUS (continued) -

dubius, Agassiz. v. Hybodus lawsoni.

aubrisieusis, Mackie. $\%$. Synechodus dubrisiensis.

ensatus, L. Agassiz, Poiss. Foss., vol. iii (1837), p. 5 1, pl. ix, f. 12 ;

J. Morris, Cat. Brit. Foss., 1854, p. 330.

LOC. L. Lias; Lyme Regis. [Spine ; Oxford Mus.]

formosus, Agassiz. \%. Hybodus reticulatus.

grossiconus, L. Agassiz, Poiss. Foss., vol. iii (1843), p. I\& pl. xxiii, f. 26-4I (? f. 25); J. Morris, Cat. Brit. Foss., 1854, p. 330 ; J. Phillips, Geol. Oxford, I871, p. 177, woodcut xxxvii, f. 4-6; A. S. Woodward, Cat. Foss. Fishes B. X1, pt. i, 1889 , p. 270 , pl. xi, f. 4 .

LOC. Gt. Oolite (incl. Stonesfield Slate); Oxfordshire, Gloucestershire, and Somersetshire. Forest Marble; Wiltshire. [Teeth; Brit. Mus. (pars).]

jugosus, Phillips. \%. Hybodus polyprion.

keuperiuls, Murchison and Strickland. $v$. Acrodus keuperinus.

Iøviusculus, L. Agassiz, Poiss. Foss., vol. iii (1837), p. 46, p?. x, f. $24-26$; J. Morris, Cat. Brit. Foss., 1854, p. 330.

LOC. Rhetic ; Aust Cliff. [Spine ; Bristol Mus.]

Iawsont, P. Duff, Geol. Noray, I8 42 , pp. 6I, 63, pl. iv, f. I, 2, 5, 6 ; J. Morris, Cat. Brit. Foss., 1854, p. 330 ; A. S. Woodward, Cat. Foss. Fishes B. M., pt. i, I 889 , p. 255.

? Tooth of Sphenonchus, 1. Duff, Geol. Moray, 1842, p. 63, pl. iv, f. II.

Hybodus dubius, L. Agassiz, Poiss. Foss., vol. iii (1843), p. I88, pl. xxii $a$, f. 8-Io; J. Morris, Cat. Brit. Foss., 1854, p. 330. [Teeth; olim Robertson Coll.]

? Sphenonchus martini, L. Agassiz, Poiss. Foss., vol. iii (1843), p. 203, pl. xxii $a$, f. I5-17 ; J. Morris, Cat. Brit. Foss., 1854, p. 344 .

toc. Rhretic; Linksfield, near Elgin. [Spines and tecth; J. Powrie Coll.]

Teptodus, L. Agassiz, Poiss. Foss., vol. iii (1837), p. 44, pl. x, f. 2, 3 ; J. Morris, Cat. Brit. Foss., 1854, p. 330.

LOC. Kim. Clay; Shotover. [Spine; Bristol Mus.]

1evis, A.S. Woodward, Cat. Foss. Fishes B. M., pt. i, 1889, p. 269 , pl. xi, f. 5-7.

LOC. Stonesfield Slate. [Teeth ; Brit. Mus.]

marginalis, L. Agassiz, Poiss. Foss., vol. iii (1837), p. 43, pl. x, f. I8-2 I ; J. Morris, Cat. Brit. Foss., I 854, p. 330 ; J. Phillips, Geol. Oxford, I871, p. 178, woodcut xxxviii, f. 6 (H. marginatus, crrore); A. S. Woodward, Cat. Foss. Fishes B. i1., pt. i, i889, p. 302.

Loc. Stonesfield Slate. [Spincs; Brit. Mus.] 


\section{HXBODUS (contiuned) -}

medtus, L. Agassiz, Poiss. Foss., vol. iii (1843), p. I84, pl. xxiv, f. 25 ; J. Morris, Cat. Brit. Foss., 1854, p. 330 ; A. S. Woodward, Cat. Foss. Fishes B. M., pt. i, 1889, p. 264, pl.vii, f.2, pl.ix. LoC. L. Lias ; Lyme Regis. [Teeth ; Oxford Mus.]

minor, L. Agassiz, Poiss. Foss., vol. iii (1837), p. 48, pl. viii b, f. 2, 3 (spine), and ibid. (1843), p. I83, pl. xxiii, f. $21-24$ (teeth); J. Morris, Cat. Brit. Foss., 1854, p. 330 ; R. Etheridge, Proc. Cardiff Naturalists' Soc., vol. iii (1872), pl. ii, f. 12-14; A. S. Woodward, Cat. Foss. Fishes B. M., pt. i, I889, p. 254, and Trans. Leicester Lit. and Phil. Soc., n.s., pt. xi (1889), p. 18.

[? Teeth of Nimacanthus and spine of Acrodus minimus.]

LOC. Rhætic; Somersetshire, Gloucestershire, Devonshire, Leicestershire, and S. Wales. [Spine ; Bristol Mus.-Teeth; Brit. Mus.]

obtusus, L. Agassiz, Poiss. Foss., vol. iii (1843), p. 186, pl. xxiii, f. 43,44 ; J. Morris, Cat. Brit. Foss., I 854 , p. 330 ; H. M. Platnauer, Ann. Rep. Yorksh. Phil. Soc., 1887, p. 35, pl. i, f. I-16; A. S. Woodward, Cat. Foss. Fishes B. Mi., pt. i, 1889, p. 272 , pl. xi, f. $8-13$.

Glossopetra minima palmata, Luidius, Lithophyl. Brit., I760, p. $65, \mathrm{pl} . \mathrm{xv}, \mathrm{f} .1283$.

LOC. Oxford Clay; Huntingdonshire and Wiltshire. Coral Rag; Yorkshire. L. Kim. Clay ; Oxfordshire and Dorsetshire. [Tecth; York Mus. and Brit. Mus.]

plicatilis, Agassiz : recorded in error from Axmouth by Egerton, in J. Morris, Cat. Brit. Foss., I854, p. 330.

polyprion, L. Agassiz, Poiss. Foss., vol. iii (1843), p. 185, pl. xxiii, f. I-I5; J. Morris, Cat. Brit. Foss., I854, p. 330; J. Phillips, Geol. Oxford, I87 I, p. 177, woodcut xxxvii, f. 2, 3 ; A. S. Woodward, Geol. Mag. [3] vol. iii, I886, p. 257, pl. vi, f. I, 2, and Cat. Foss. Fishes B. M., pt. i, 1889, p. 268, pl. xi, f. $1-3$.

Hybodus jusosus, J. Phillips, Geol. Oxford, 1871, p. 177, woodcut xxxvii, f. I. [Tooth; Oxford Mus.]

LOC. Gt. Oolite (incl. Stonesfield Slate); Oxfordshire, ? and Somersetshire. [Teeth; Paris Mus.] [See also Notidanus davicsi.]

punctatus, J. W. Davis, Quart. Journ. Geol. Soc., vol. xxxvii, 1881, p. 417 , pl. xxii, f. 2 .

I.oc. Rhætic ; Aust Cliff, near Bristol. [Spine; J. Wr. Davis Coll.] pyramidalis, Agassiz. i'. Hybodus delabechei.

raricostatus, L. Agassiz, Poiss. Foss., vol. iii (1843), p. 187, pl. xxiv, f. 24 ; J. Morris, Cat. Brit. Foss., 1854 , p. 330 ; A.S. Woodward, Cat. Foss. Fishes B. M., pt. i, I889, p. 257, pl. x, f. 15 (? pl. $x$, f. 6,7 ).

Loc. L. Lias ; Lyme Regis. [Tooth ; Bristol Mus.] 


\section{IFYBODUS (continued) -}

reticulatug, L. Agassiz, Poiss. Foss., vol. iii (1837 and 1843), pp. 50, 180, pl. ix, f. I-9, pl. xxiv, f. 26 , pl. xxii $a$, f. 22, 23 ; IV. C. Williamson, Phil. Trans., I849, p. 465 , pl. xliii, f. 33 ; J. Morris, Cat. Brit. Foss., 1854, p. 330 ; A. S. Woodward, Cat. Foss. Fishes B. M., pt. i, I 889, p. 266, pl. x, f. I6-18.

'Fossil jaw with a triple row of teeth,' H. T. De la Beche, Trans. Geol. Soc. [2] vol. i (1822), p. 44, pl. v, f. 3.

Iyybodus curtus, L. Agassiz, Poiss. Foss., vol. iii (1837), p. 49, pl. viii $b$, f. 4-6 (pars).

? Hybodus formosus, L. Agassiz, Poiss. Foss., vol. iii (1837), p. 5 I, pl. ix, f. IO, II ; J. Morris, Cat. Brit. Foss., I854, p. 330. [Spine; Oxford Mus.]

Loc. L. Lias; Lyme Regis. [Associated teeth, fin-spines, and cartilage ; Oxford Mus.]

striatulus, L. Agassiz, Poiss. Foss., vol. iii (1837), p. 44, pl. viii $b$, f. I ; J. Morris, Cat. Brit. Foss., I854, p. 330 ; A. S. Woodward, Cat. Foss. Fishes B. M., pt. i, 1889, pp. 276, 304 .

? Oxyrhina (Meristodon) paradoxu, L. Agassiz, Poiss. Foss., vol. iii (1843), p. 286, pl. xxxvi, f. 53-56. [Imperfect teeth; Brit. Mus.]

T. (Meristodon.)

? Oxyrhina paradoxa, J. Morris, Cat. Brit. Foss., 1854, p. 336.

? Hybodus, sp. inc. (? striatulus, Agassiz), A. S. Woodward, Cat. Foss. Fishes B. M., pt. i, 1889, p. 276, pl. xi, f. 14, 15.

'Dorsal fin of fish allied to Silurus,' G. A. Mantell, Illustr. Geol. Sussex, I827, p. 58 , pl. x, f. 4 .

Loc. Wealden; Sussex. [Spine; Brit. Mus.]

strictus, L. Agassiz, Poiss. Foss., vol. iii (1837), p. 45, pl. x, f. 7-9; J. Morris, Cat. Brit. Foss., 1854 , p. 330; A. S. Woodward, Cat. Foss. Fishes B. M., pt. i, I889, pp. 275, 303.

? Hybodus (? strictus, Agassiz), A. S. Woodward., ibid., p. 275.

Loc. Purbeck Beds; Swanage. [Spine; olim Cumberland Coll.]

subcarinatus, L. Agassiz, Poiss. Foss., vol. iii (1837), p. 46, pl. x, f. 10-12 ; J. Morris, Cat. Brit. Foss., 1854, p. 330 ; A. S. Woodward, Cat. Foss. Fishes B. M., pt. i, 1889, p. 304.

'Dorsal fin of fish,' T. Webster, Trans. Geol. Soc. [2] vol. ii (1829), p. 35, pl. vi, f. 9.

Loc. Wealden; Sussex. [Spine ; olim Cumberland Coll.]

sulcatus, Agassiz. v. Hybodus basanus.

undulatus, L. Agassiz, Poiss. Foss, vol. iii (1843), p. I 88 , pl. xxii $a$, f. I I ; J. Morris, Cat. Brit. Foss., I 854 , p. 33 o.

LOC. Rhxtic; Linksfield, near Elgin. [Tooth; olim Robertson Coll.]

8p. Inc., A. S. Woodward, Cat. Foss. Fishes B. M., pt. i, I889, p. 276, pl. xi, f. 16 .

Loc. Wealden; Tilgate Forest, Sussex. Neocomian; I. of Wight. [Tooth; Brit. Mus.] 
HX BODUS (continued) -

? sp., A. S. Woodward, Cat. Foss. Fishes B. M., pt. i, 1889, p. 277. LOC. Chalk; S. E. England. [Group of teeth; Brit. Mus., no. 453 II.]

s1)., J. Morris, Cat. Brit. Foss., 1854, p. 330. (?. Synechodus.)

IXPSOCORMUs, A. Wagner, Abh. k. bay. Akad. Wiss., Cl. ii, vol. ix, 1863, p. 677 .

leedsi, A. S. Woodward, Geol. Mag. [3] vol. vi, I $\$ \$ 9$, p. 450, and Rep. Brit. Assoc., I889.

LOC. Oxford Clay; Peterborough. [Jaws; A. N. Leeds Coll.]

tenuirostris, A. S. Woodward, ibid., p. 45r, and ibid.

LOC. Oxford Clay; Peterborough. [Jaws; A. N. Leeds Coll.]

sp., A. S. Woodward, Geol. Mag. [3] vol. vi, r889, p. 45 r.

LOC. Kim. Clay; Weymouth. [Portions of jaws; Brit. Mus., no. 42368.]

IIITSODON, Agassiz. $\tau$ '. Pachyrhizodus and Portheus.

lenesiensis, Agassiz. \%. Cladocyclus lewesiensis, Pachyrhizodus gardneri, Portheus mantelli, and Portheus sp.

minor, Dixon. v. Ichthyodectes minor.

oblongus, L. Agassiz, Poiss. Foss., vol. v, pt. i (1843), p. ror (name only).

Loc. London Clay; Sheppey.

toliapicus, L. Agassiz, Poiss. Foss., vol. v, pt. i (1843), p. 10 I (name only.)

LOC. London Clay; Sheppey. [Imperfect cranium; Brit. Mus.]

ICrTryodrcts, E. D. Cope, Proc. Amer. Phil. Soc.., vol. xi (1871), p. 536.

elogans, E. 'T. Newton, Quart. Journ. Geol. Soc., vol. xxxiii, I 877 , p. 52 I, pl. xxii, f. I5.

LOC. L. Chalk; Dorking, Surrey. [Jaw; Brit. Mus.]

minor, E. T. Newton, ibid., p. 520, pl. xxii, f. 14.

Hypsodon minor, F. Dixon, Geol. Sussex, 1850, descr. to pl. xxxii*, f. 9 (name and fig. only).

Loc. Chalk; Sussex. [Jaw ; Brit. Mus.]

Ichthyolithus clackmammensis, Fleming. v. Megalichthys hibberti.

ICTINOCEPIIALS, Page. \% Ischnacanthus.

granulatus, Page. \% Ischnacanthus gracilis.

ISCINA AANTIrUs, J. Powrie, Quart. Journ. Geol. Soc., vol. xx, 1864, p. 419.

ICTiNocephalus, D. Page, Rep. Brit. Assoc., 1858 (1859), Sect., p. 105. 
ISCINACANTIXUS (continucl) -

gracilis (Egerton), J. Powrie, ilid., p. 419; R. H. Traquair, Geol. Mlag. [3] vol. v, i888, p. 5 I2.

Diplacanthus stacilis, P. M. G. Egerton, Figs. and Descript. Brit. Organic Remains, dec. $x$ (Mem. Geol. Surv., 1861), p. 69, pl. ix ; J. Powrie, Trans. Edinb. Gcol. Soc., vol. i (1870), p. 290 , pl. x, f. 2.

Ictinocephalus granululat, D. Page, Rep. Brit. Assoc., 1858 (1859), Sect., p. 105 (name only).

T. (Ictinocephaluis.)

Loc. L. Old Red Sandst.; Forfarshire. [Fish ; J. Powrie Coll.]

ISCrY 0 DUs, P. M.G. Egerton, Proc. Geol. Soc., vol.iv(1843), p. I 55.

agassizi, Egerton. \%. Edaphodon agassizi.

agassizi, Bcnsted (iton Egerton). $\because$ Ischyodus thurmanni.

beaumont1, P. M. G. Egerton, Proc. Geol. Soc., vol. iv (1843), pp. $155,156$.

Chimara (Ischyodon) beaumontii, L. Agassiz, Poiss. Foss., vol. iii (1843), p. 346.

Ischyodus, P. M. G. Egerton, Quart. Journ. Geol. Soc., vol. iii, 1847, p. 35 I, pl. xiii, f. I.

Loc. Kim. Clay; Dorsetshire. [Tecth; Brit. MIus.]

brevirostris, Agassiz. $\%$ Ischyodus thurmanni.

bucklandi, P. M. G. Egcrton, P'roc. Geol. Soc., vol. iv (1843), pp. $153,156$.

Chimara (Ischyodon) bucklandi, L. Agassiz, Poiss. Foss., vol. iii (1843), p. 343 , pl. xl $c$, f. 19 .

Ganodus bucklindi, P. M. G. Egerton, Quart. Journ. Geol. Soc, vol. iii, I 847 , p. 352 ; J. Morris, Cat. Brit. Foss., I854, p. 326. Loc. Stonesfield Slate. [Mandib. tooth; Brit. Mus.]

cole1: Chimara (Ganodus) colei, L. Agassiz, Poiss. Foss., vol. iii (1843), p. 346, pl. xl, f. 8-10.

Ganodus colei, J. Morris, Cat. Brit. Foss., 1854, p. 327.

Loc. Stonesfield Slate. [Mandib. and palatine teeth; Brit. Mus.]

egertond (Buckland), P. M. G. Egerton, Proc. Geol. Soc., vol. iv (I $8+3$, p. I56; J. Morris, Cat. Brit. Foss., 1854, p. 330 ; J. Phillips, (ieol. Oxford, 1871, p. 306, pl. xii, f. 24.

Chimara cgertonii, W. Buckland, Proc. Geol. Soc., vol. ii ( 1835 ), p. 206, and Phil. Mag. [3] vol. viii, 1836, p. 5 .

Chimara (Ischyodon) egertoni, L. Agassiz, Poiss. Foss., vol. iii $(1843)$, p. 340, pl. xl $c$, f. I-IO.

LOC. Kim. Clay; Oxfordshire and Dorsetshire. [Teeth; Oxford Mus.]

emarginatus, [-a] P. M. G. Egerton, Proc. Geol. Soc., vol, iv (1843), pp. 154, I 56.

Ganodus emarginatus, J. Norris, Cat. Brit. Foss., I854, p. 327. LOC. Stonesfield Slate. [Mandib. tooth ; Brit. Mus.] 
ISCrYYODS (continued)-

gigas, Egerton. v. Edaphodon gigas.

? Incisus, E. T. Newton, Chimæroid Fishes Brit. Cret. Rocks (Mem. Geol. Surv., 1878), p. 38, pl. xii, f. 3-10.

LOC. Gault ; Folkestone. Cambridge Greensand. L. Chalk ; Kent and Sussex. [Mandib. tooth ; Brit. Mus.]

jolensoni, Agassiz. v. Myriacanthus paradoxus.

latus, E. T. Newton, Chimaroid Fishes Brit. Cret. Rocks (Mem. Geol. Surv., 1878), p. 32, pl. x.

LOC. Cambridge Greensand. [Mandib. tooth; Mus. Pract. Geol.]

mantelli, Egerton. $\%$. Edaphodon mantelli.

orthorhinus, Egerton. v. Myriacanthus granulatus.

planus, E. T. Newton, Chimæroid Fishes Brit. Cret. Rocks (Mem. Geol. Surv., 1878), p. 37, pl. xii, f. I, 2.

LOC. Cambridge Greensand. [Mandib. tooth; T. Jesson Coll.]

sedgwicki, Egerton. v. Edaphodon sedgwicki.

thurmanni, F. J. Pictet and P. Campiche, Foss. Terr. Cretacé St. Croix (Pal. Suisse, I858), p. 76, pl. ix, f. 8.

Ischyodus brevirostris, P. M. G. Egerton, Proc. Geol. Soc., vol. iv (I843), p. I 56 (name only); J. Morris, Cat. Brit. Foss., 1854, p. 330; E. T. Newton, Chimæroid Fishes Brit. Cret. Rocks (Mem. Geol. Surv, I878), p. 27, pl. ix. [Teeth ; Brit. Mus.]

Chimara (Ischyodon) brevirostris, L. Aigassiz, Poiss. Foss., vol. iii (1843), p. 344 (name only).

Chimera (Ischyodon) agassizi, L. Agassiz (crrore), ibit., pl. xl $c$, f. 14, 15. [Palatine tooth; Brit. Mus.]

Ischyodus agassizii, W. H. Bensted (c\%ore), Geologist, vol. v, 1862, p. 378 .

LOC. Gault ; Folkestone. Cambridge Greensand. Varieties also in L. Greensand, Maidstone, and L. Chalk, Newmarket. [Tecth; Brit. Wus.]

townsendi (Buckland), I'. M. G. Egerton, Proc. Geol. Soc., vol. iv (1843), p. 156 (I. townshendi); J. Morris, Cat. Brit. Foss., 1854, p. 330 (1. townshendi); [-ii] E. T. Newton, Chimaroid Fishes Brit. Cret. Rocks Mem. Geol. Surv., 1878), p. 33, pl. xi, and l'roc. Geol. Assoc., vol. vii (1881), p. II6, woodcuts.

Chimera tou'nsendii, IV. Buckland, Proc. Geol. Soc., vol. ii (1835), p. 206, and Phil. Mag. [3] vol. viii, 1836, p. 5.

Chimara (Ischyodon) toa'nsendii, L. Agassiz, Poiss. Foss., vol. iii (1843), p. 343, pl. xl, f. 20-22, pl. xl c, f. 17, I8.

LOC. Portlandian; Oxfordshire, Wiltshire, and Dorsetshire. Derived in Potton Bone-bed (Neocomian). [Mandib. tooth ; Brit. Mus.] 
ISOCorUM, P. M. G. Egerton, Figs. and Descript. Brit. Organic Remains, dec. xiii (Mem. Geol. Surv., 1872), no. 4.

granulatum, P. M. G. Egerton, ibil., no. 4, pl. iv. LOC. L. Lias ; Lyme Regis. [Fish ; Brit. Mus.]

T.

ISODIUS, Morris (ex $\left.\mathrm{M}^{\prime} \mathrm{Coy}\right) . \quad \tau^{\prime}$. Isodus.

ISOdUs, F. M'Coy, Ann. Mag. Nat. Hist. [2] vol. ii, I848, p. 3, and Proc. Camb. Phil. Soc., vol. i (1848), p. 65.

leptognathus, F. $M 1^{6} \mathrm{Coy}$, Ann. Mag. Nat. Hist. [2] vol. ii, I848, p. 3 ; J. Morris, Cat. Brit. Foss., I854, p. 331 (Isodius).

LOC. Carboniferous; Draperstown, Moyheeland. [Jaw ; olim R. Griffith Coll.]

T.

JANASSA, G. von Münster, Beitr. Petrefakt., pt. i (1839), p. 67.

Climaxodus, F. M'Coy, Ann. Mag. Nat. Hist. [2] vol. ii, 1848, p. 128, and I'roc. Camb. Phil. Soc., vol. i (1848), p. 66.

bituminosa (Schlotheim), G. von Münster, Beitr. Petrefakt., pt. i (1839), p. 122; H. B. Geinitz, Dyas (1861), p. 24, pl. iv, f. 5 , pl. v, f. I-4; A. Hancock and R. Howse, Ann. Mag. Nat. Hist. [4] vol. v, 1870, p. 47 , pls. ii, iii ; K. A. von Zittel, Handb. Palzont., vol. iii (I887), p. 98, woodcut IIo; A. S. Woodward, Cat. Foss. Fishes B. M., pt. i, I889, p. 35.

Trilobites bituminosus, E. Schlotheim, Petrefaktenkunde (1820), p. 39, and ibid., Nachtr., pt. ii (1823), pp. 39, 87, pl. xxii, f. 9. Loc. Marl-Slate; „Durham. [Dentition; Brit. Mus. and Nerucastle-upon-Tyne Mus.]

$\mathbf{T}$.

clavata ( $\mathrm{M}^{\prime} \mathrm{Coy}$ ), A. S. Woodward, Cat. Foss. Fishes B. M.. pt. i, 1889, p. 37 , pl. i, f. 3.

Chomatodus clavatus, F. M'Coy; Brit. Palæoz. Foss. (185j), p. 617 , pl, iii K, f. 10 .

Chomatodus truncatus, L. Agassiz, Poiss. Foss., vol. iii $(18+3)$, p. I74 (name only); F. MI'Coy, Brit. Palæoz. Foss. (1855), p. 618, pl. iii I, f. I ; J. Morris, Cat. Brit. Foss., 1854, p. 321. [Tooth; Woodwardian Mus.]

Petalorhynchus psittacinus, J. W. Davis (errore), Trans. Roy. Dublin Soc. [2] vol.i (1883), p. 518 (pars). [Teeth; Brit. Mus.]

LOC. Carb. Limest.; England, Scotland, and Ireland. ['Tooth ; Woodwardian Mus.]

Imbricata (M'Coy), A. Hancock and R. Howse, Nat. Hist. Trans. Northumb, and Durham, vol. iii (1870), p. 338 ; A. S. Woodward, Cat. Foss. Fishes B. M., pt. i, 1889, p. 38, pl. i, f. I, 2.

Climaxodus imbricatus, F. M'Coy, Ann. Mag. Nat. Hist. [2] vol. ii, 1848, p. 129, and Brit. Palxoz. Foss. (1855), p. 620, pl. iii G, f. 5 ; J. Morris, Cat. Brit. Foss., I854, p. 322.

T. (Climaxodus.)

LOC. U. Carb. Limest. ; South Derbyshire. [Tooth; Woodwardian Mus.] 
JANASSA (continued)-

linguæformis (Atthey), A. Hancock and R. Howse, Nat. Hist. Trans. Northumb. and Durham, vol. iii (1870), p. 330, pl. ix ; J. Ward, [l'roc.] N. Staffs. Nat. Fie!d Club, I875, p. 222 , f. 8 ; A. S. Wondward, Cat. Foss. Fishes B. M., pt. i, เ 889 , p. 36 .

Climarodus linguaformis, T. Atthey, Ann. Mag. Nat. Hist. [4] vol. ii, IS68, p. 321 ; I. Ward, Trans. N. Stafís. Inst. Mining Engin., vol. x, I889, p. I 40, pl. ii, f. I9.

Climaxodus ow utus, T. P. Barkas, Geol. Mag., vol. v, IS68, p. 496, woodcut, and ibid., vol. vi, 1869, p. 42. [Tooth; T. l. Barkas Coll.]

Climaxodus a'crmiformis, T. P. Barkas, Geol. Mag., vol. vi, I 869, p. 38 I. [Tooth; T. P. Barkas Coll.]

Cimaxodius, T. P. Barkas, Coal Meas. Palreont., 1873, p. 20, f. $35-38$.

Janassa ovatus, J. minutus, and J.processus, W. J. Barkas, Monthly Rev. Dental Surgery, vol. iii, I875, p. I53, f. xlixlviii. ['Teeth; T. P. Barkas Coll.]

LOC. Coal-Meas. ; Staffordshire, Northumberland, Yorkshire, and South Scotland. [Tooth; Newcastle-upon-Tyne Mus.]

minutus, W. J. Barkas. $\because$. Janassa linguxformis.

oratus, W. J. Barkas. $v$. Janassa linguæeformis.

jocessus, W. J. Barkas. \%. Janassa linguæformis.

IAIxOSTRAXON, E. R. Lankester, Fishes O. R. Sandst., pt. i (Pal. Soc., 1870), p. 6 I.

podura, E. R. Lankester, ibil., p. 6r, pl. xiii, f. 20,2 I, pl. xiv, f. 6. LOC. L. Old Red Sandst.; Herefordshire. [Dermal calcifications; Oxford Mus.]

$\mathbf{T .}$

KOKKODERMA, Quenstedt. ¿'. Coccoderma.

$\boldsymbol{L A B O D U S , ~ D a v i s . ~} \%$ Copodus. planus, Davis. \% Copodus planus.

prototypus, Davis. $\%$. Copodus prototypus.

LABROPHAGUS, L. Agassiz, Rep. Brit. Assoc., I844 (1845), p. 308 (name only).

esorinus, L. Agassiz, ihid., p. $30 S$ (name only).

I.OC. London Clay; Sheppey. [Imperfect skull; Brit. MIus.] T.

IABRUS, Linnæus, Syst. Nat., ed. 12, vol. i, 1766, p. 473.

sp. From Red Crag; Suffolk. [Lower pharyngeal ; Brit. Mus., no. P. $5 ; 65$.]

IAmNA, Cuvier, Règne Animal, vol. ii, I817, p. 126.

Otodus, L. Agassiz, Poiss. Foss., vol. iii (1843), p. 266.

acuminata, Agassiz. $\because$. Oxyrhina mantelli. 
IAMNA (continued) -

appendiculata, L. Agassiz, Poiss. Foss., Feuill., p. 54 (1835); A. S. Woodward, Cat. Foss. Fishes B. M., pt. i, 1889, p. 393. Squalus mustelus?, G. A. Mantell, Foss. S. Downs, 1822, p. 226, pl. xxxii, f. 2, 3, 5, 6, 9, I I.

Otodus appendiculatus, L. Agassiz, Poiss. Foss., vol. iii (1843), p. 270 , pl. xxxii, f. I-25; F. Dixon, Geol. Sussex, 1850, descr. to pl. xxx, f. 25 , pl. xxxi, f. 17 ; J. Morris, Cat. Brit. Foss., 1854, p. 335 ; A. S. Woodward, Proc. Geol. Assoc., vol. x (1888), p. 292.

LOC. Gault; Folkestone. Cambridge Greensand. U. Greensand; Dorsetshire and Wiltshire. Chalk; Sussex, Surrey, Kent, Wiltshire, and Norfolk. [Teeth; Brit. Mus.]

compressa, Agassiz. v. Lamna macrota.

contortidens, Agassiz. Recorded (? errore) by Morris, Cat. Brit. Foss., 1854, p. 331, from the Eocene of Kyson (Kingston), Suffolk. (? Odontaspis elegans.)

cuspidata, Agassiz. $\%$. Odontaspis cuspidata.

denticulata, Agassiz. $v$. Odontaspis cuspidata.

dubia, Agassiz. $v$. Odontaspis cuspidata.

elegans, Agassiz. v. Odontaspis elegans and Lamna macrota.

hopei, Agassiz. $\%$. Odontaspis cuspidata.

macrorhiza, E. D. Cope, Vert. Cret. Form. West (Rep. U. S. Geol. Surv. Territ.), vol. ii (1875), p. 297, pl. xlii, f. 9, IO ; A. S. Woodward, Cat. Foss. Fishes B. M., pt. i, 1889, p. 399. LOC. Gault; Folkestone. Cambridge Greensand. [Teeth; Brit. Mus.]

macrota (Agassiz), A. S. Woodward, Cat. Foss. Fishes B. M., pt. i, 1889 , p. 402.

Otodus macrotus, L. Agassiz, Poiss. Foss., vol. iii (1843), p. 273 , pl. xxxii, f. 29-31 ; J. Morris, Cat. Brit. Foss., 18 54 , p. 335.

Lamna elegans, L. Agassiz (errore), Poiss. Foss., vol. iii (1843), pl. xxxv, f. 6, 7, pl. xxxvii $a$, f. 58 .

Lamna compressa, L. Agassiz, ibid., p. 290, pl. xxxvii a, f. 35-42 ; J. Morris, Cat. Brit. Foss., 1854, p. 331.

Otodus lanceolatus, F. Dixon (non Agassiz), Geol. Sussex, 1850, p. 204 , pl. xi, f. 20, 21 ; J. Morris, Cat. Brit. Foss., I 854 , p. 335 .

LOC. Eocene; London and Hampshire Basins. [Teeth ; Paris Mus.]

? obliqua (Agassiz), A. S. Woodward, Cat. Foss. Fishes B. M., pt. i, I 889, p. 404 .

Otodus obliquus, L. Agassiz, Poiss. Foss., vol. iii (1843), p. 267, pl. xxxi, pl. xxxvi, f. 22-27; F. Dixon, Geol. Sussex, 1850, p. 204, pl. x, f. 32-35, pl.xv, f. I i ; J. Morris, Cat. Brit. Foss., 1854, p. 335 .

T. (Otodus.) 
IAMNA ? obliqua (continued)-

Otodus lanceolatus, L. Agassiz, Poiss. Foss., vol. iii (I843), p. 269, pl. xxxvii, f. 19-23.

Carcharodon obliquus, F. Noetling, Abh. Geol. Specialk. Preuss. u. Thüring. Staaten, vol. vi, pt. iii (1885), p. 84, pl. vi, f. 4-6.

Otodus, E. Charlesworth, Mag. Nat. Hist., n.s., vol. iii, 1839, p. 35I, woodcut; R. Owen, Odontography (1840), descr. to pl. v, f. 5 .

'Dens Squali,' G. Brander, Foss. Hanton., 1766, p. 16, pl. ix, f. II 5 .

LOC. Eocene; London and Hampshire Basins. [Teeth ; Paris Mus. and Strassburg Mus.]

v'haphiodon, Agassiz. \%. Scapanorhynchus rhaphiodon and Scapanorhynchus subulatus.

semiplicata (Agassiz), A. S. Woodward, Cat. Foss. Fishes B. M., pt. i, I889, p. 397.

Otodus semiplicatus, L. Agassiz, Poiss. Foss., vol. iii (1843), p. 272, pl. xxxvi, f. 32 (? f. 33); A. S. Woodward, Proc. Geol. Assoc., vol. x (1888), p. 292.

LOC. Chalk; Kent. [Teeth; Brit. Mus.]

subulata, Agassiz. $v$. Scapanorhynchus subulatus.

sulcata (Geinitz), A. S. Woodward, Cat. Foss. Fishes B. M., pt. i, I 889 , p. 398.

Otodus sulcatus, H. B. Geinitz, Char. Schicht. u. Petref. sächs.böhm. Kreidegeb., Nachtr. (1843), p. 5, pl. iv, f. 2.

Otodus crassus, A. S. Woodward (non Agassiz), Proc. Geol. Assoc., vol. x (1888), p. 292.

Loc. Chalk; Sussex, Surrey, and Kent. [Teeth; Brit. Mfus.]

? verticalis (Agassiz), J. Morris, Cat. Brit. Foss., 1854, p. 331 .

Lamna (Odontaspis) verticalis, L. Agassiz, Poiss. Foss., vol. iii, (1843), p. 294, pl. xxxvii $a$, f. 3 I, 32.

LOC. 'London Clay ; Sheppey.' [Very doubtful.]

vincent1 (Winkler), A. S. Woodward, Cat. Foss. Fishes B. M., pt. i, 1889, p. 403 .

Otodus vincenti, T. C. Winkler, Archiv. Mus. Teyler, vol. iv (1876), p. 25, pl. ii, f. 9, 10.

LOC. Eocene; London and IIampshire Basins. [Teeth; Brit. Mus.]

sp., A. S. Woodward, Cat. Foss. Fishes B. M., pt. i, 1889, p. 400.

LOC. U. Chalk; Norwich. [Teeth ; Brit. Mus., no. 48956 b.]

LAMNODUS, Agassiz. v. Dendrodus.

biporcatus (Owen), Agassiz. v. Dendrodus biporcatus.

hastatus (Owen), Agassiz. \% Dendrodus biporcatus.

panderi, Agassiz. $v$. Dendrodus biporcatus.

sulcatus, Agassiz. $v$. Dendrodus biporcatus. 
LAPARUS, L. Agassiz, Rep. Brit. Assoc., 1844, p. 308 (name only). alticeps, L. Agassiz, ibid., p. 308 (name only).

LOC. London Clay; Sheppey. [Imperfect skull; Brit. Mus.] T.

ImgDsזCHTHxs, A. S. Woodward, Geol. Mag. [3] vol. vi, I889, p 451 .

problematicus, A. S. Woodward, ibicl., p. 451, and Rep. Brit. Assoc., I 889 .

T.

Loc. Oxford Clay; Peterborough. [Bones; A. N. Leeds Coll.]

IFGม NoTUS, P. M. G. Egerton, Figs. and Descript. Brit. Organic Remains, dec. viii (Mem. Geol. Surv., 1855), no. 7, p.4.

cothamensis, P. M. G. Egerton, ibid., pl. vii, f.9-12; J. Morris, Cat. Brit. Foss., 1854, p. 331 .

LOC. Rhætic (Cotham Marble); Aust Cliff, near Bristol. [Fishes; Bristol Mus.]

T.

LEIOSPIIEN, Agassiz. v. Sphenonchus.

ImPIDOSTrus, Lacépède, Hist. Nat. Poiss., vol. v, I803, p. 33 I.

NaISIA, G. v. Münster, Beitr. Petrefakt., pt. vii (1846), p. 34.

dentosus, König. \%. Eugnathus orthostomus.

fimbriatus, S. V. Wood, Lond. Geol. Journal (1846), pp. 6, 122, pl. ii, f. 9, and Quart. Journ. Geol. Soc., vol. x, 1854 , p. 156, pl. iii, f. 1 .

Naisia ?apicalis (Münster), J. Morris, Cat. Brit. Foss., 1854, p. 334. LOC. U. Eocene; Hordwell, Hants. [Scales and head-bones ; Brit. Mus.]

sp. Ind. From Bracklesham Beds; Sussex. [Vertebra; Brit. Mus., no. P. 5442.]

ImPIDOTUS, L. Agassiz, Poiss. Foss., vol. ii, pt. i (1837), p. 233 ; R. Owen, Rep. Brit. Assoc., I838 (1839), Sect., p. I43 (teeth); J. E. Lee, Geologist, vol. iii, 1860, p. 458 , pl. xii (structure of scales).

SPherodus, L. Agassiz (pars), Poiss. Foss., vol. ii, pt. i (1833), p. 15, and pt. ii (1843), p. 209.

elvensis (H. D. de Blainville), F.A. Quenstedt, 'Ueber Lepidotus im Lias $\epsilon^{\prime}$ (Tübingen, 1847).

Cyprinus elvensis, H. D. de Blainville, Nouv. Dict. d'Hist. Nat., vol. xxvii, I818, p. 394.

Lepidotus gigas, L. Agassiz, Neues Jahrb., 1832, p. 145, and Poiss. Foss., vol. ii, pt. i (1837), p. 235, pls. xxviii, xxix ; J. Morris, Cat. Brit. Foss., 1854, p. 331 ; B. Thompson, Journ. Northampton. Nat. Hist. Soc., vol. iv (1886), p. 27, with plate.

'Fossil Fish,' G. Baker, Hist. Northampton, vol. i, 1830, p. 440, with plate.

LOC. M. Lias; Rothersthorpe, Northamptonshire. [Fish; Brit. Mus.]

T. 
IXPXDOTUS (continued)-

fimbriatus, Agassiz. v. Eugnathus latus.

fittoni, Agassiz. $v$. Lepidotus mantelli.

gigas, Agassiz. v. Lepidotus elvensis.

latissimus, Agassiz. $v$ Lepidotus semiserratus.

macrocheirus, P. M. G. Egerton, Quart. Journ. Geol. Soc., vol. i, 1845 , p. 230 ; J. Morris, Cat. Brit. Foss., 1854, p. 332 .

Loc. Oxford Clay; Christian Malford. [Portion of fish ; Marquis of Northampton Coll.]

? manseli : Semionotus manselii, P. M. G. Egerton, Figs. and Descript. Brit. Organic Remains, dec. xiii (Mem. Geol. Surv, I872) no. 8, pl, viii.

Heterolepidotus? manseli, K. A. v. Zittel, Handb. Palæont., vol. iii (1887), p. 205.

LOC. Kim. Clay ; Dorsetshire. [Caudal region, etc. ; Brit. Mus.]

mantelli, L. Agassiz, Poiss. Foss., vol, ii, pt. i (1837), p. 262, pl. $\mathrm{xxx}$, f. Io-I 5 , pl. $\operatorname{xxx} a$, f. $4-6$, pl. $\mathrm{xxx} b$, f. $2, \mathrm{pl}$. $\mathrm{xxx} c$, f. I-7; R. Owen, Odontography (1841), p. 69, pl. xxx, f. I, pl. xxxi ; W. C. Williamson, Phil. Trans., I849, p. 444; J. Morris, Cat. Brit. Foss., 1854, p. 332.

Lepidotus subdenticulatus, L. Agassiz, Poiss. Foss., vol. ii, pt. i (1833), p. 9.

Lepidotus fittoni, L. Agassiz, Poiss. Foss., vol. ii, pt. i (1843), p. 265 , pl. xxx, f. 4-6, pl. $\operatorname{xxx} a$ (excl. f. $4,5,6$ ), pl. $\operatorname{xxx} b$ (excl. f. 2) ; J. Morris, Cat. Brit. Foss., 1854, p. 331 ; J. E. Lee, Geologist, vol. iii, I86o, p. 458, pl. xii. [Portion of fish; Brit. Mus.]

Tetragonolepis mastodontcus, L. Agassiz, Poiss. Foss., vol. ii, pt. i (1837), p. 216, pl. xxiii e, f. 3,4 (non f. 5). (Founded upon fragment of jaw figured in Trans. Geol. Soc. [2] vol. ii, pl. vi, f. 5, 6).

Echmodus mastodontens (P. M. G. Egerton, Quart. Journ. Geol. Soc., vol. x, 1854, p. 367); J. Morris, Cat. Brit. Foss., 1854, p. 317.

LOC. Wealden; Tilgate Forest. [Portions of fishes; Brit. Mus.]

maximus, A. Wagner, Abl. k. bay. Akad. Wiss., Cl. ii, vol. ix, I 863, p. 629; R. Etheridge and H. Willett, Quart. Journ. Geol. Soc., vol. xlv, 1889 , p. 356 , pl. xv.

Sphcerodus gigils, L. Agassiz, Poiss. Foss., vol. ii, pt. i (1833), p. 15, and pt. ii (I837), p. 210 , pl. 1xxiii, f. 83-94 ; R. Owen, Odontography (1841), p. 71, pl. xxxiii ; J. Morsis, Cat. Brit. Foss., I 854 , p. 344.

LOC. Kim. Clay; Shotover. Remanié in Neocomian Bone-bed, Potton. [Teeth; Brit. Mus. and Woodwardian Mus.] 
minor, L. Agassiz, Poiss. Foss., vol. ii, pt. i (1837), p. 260, pl. xxxiv ; J. MIorris, Cat. Brit. Foss., I854, p. 332.

LOC. Purbeck Beds; I. of Purbeck and Portland. [Fish ; School of Mines, Paris.]

palliatus, L. Agassiz, Poiss. Foss., vol. ii, pt. i (I837), p. 25j, pl. xxix $c$, f. 2,3 ; H. E. Saurage, Mém. Soc. Géol. France [3] vol. i (1877), p. I8, pl. i, f. I, pl. ii.

LOC. Kim. Clay; Weymouth. [Type-scales ; Brit. Mus. (from Boulogne). Scales; Brit. Mus., no. 42364.]

pectinatus, P. M. G. Egerton, Proc. Geol. Soc., vol. iv (1843), p. 183 , and Figs. and Descript. Brit. Organic Remains, dec. vi (Mem. Geol. Surv., 1852), no. 3, pl. iii ; J. Morris, Cat. Brit. Foss., 1854 , p. 332.

I.oc. U. Lias; Whitby. [Imperfect fish ; Brit. Mus.]

punctutus, L. Agassiz, Poiss. Foss., vol. ii, pt. i (1843) p. 30\% (nume only). Gemis non det., A. S. Woodward, Proc. Geol. As soc., vol. x (1888), p. 304, pl. i, f. 2.

I.oc. Chalk; Kent. [Scales; Brit. Mus., and S. J. Hawkins Coll.]

rugosus, L. Agassiz, Poiss. Foss., vol. ii, pt. i (1837), p. 246, pl. xxxiii $a$, f. I-8; J. Morris, Cat. Brit. Foss., 1854, p. 332.

LOC. L. Lias; Lyme Regis. U. Lias; Whitby. [Scales, etc.; Brit. Mus., Oxford Mus., and Whitby Mus.]

semiserratus, L. Agassiz, Poiss. Foss., vol. ii, pt. i (1837), p. 240 , pls. xxix $a, b$; W. C. Williamson, Phil. Trans., 1849 , p. 44 I, pl. xl, f. 3, 4 ; J. Morris, Cat. Brit. Foss., I854, p. 332 Lepidotus latissimus, L. Agassiz, Poiss. Foss., vol. ii, pt. i (1833), p. 8 (undefined). [Scales ; Paris Mus.]

Lcfidotus umbonatus, L. Agassiz, ibid., p. 8 (undefined). [S'cales. I 'Pikes,' Young and Bird, Geol. Yorksh., 1822, p. 26I, pl. xvi, f. 7,8 .

LOC. U. Lias; Whitby. [Imperfect fishes; Whitby Mus. and Scarborough Mus.]

servulatus, Agassiz. v. Eugnathus serrulatus.

subdenticulatus, Agassiz. $v$. Lepidotus mantelli.

tuberculatus, L. Agassiz, Poiss. Foss., vol. ii, pt. i (1837), p. 256 , pl. $\operatorname{xix} 6$, f. 7 ; J. Morris, Catt. Brit. Fuss., 1854 , p. 332 .

I.OC. Stonesfield Slate. [Scale; Oxford Mus.]

umbonatus, Agassiz. $\%$. Lepidotus semiserratus.

unguiculatus, L. Agassiz, Poiss. Foss., vol. ii, pt. i (1837). p. 25 I, pl. xxix $c$, 1. 1, pl. xxx, f. 7-9; J. Morris, Cat. Bit. Foss., 1854, p. 332 ; J. Phillips, Geol. Oxford, 1871 , p. 182, woodcut xli, f. 5-7.

Loc. Stonesfield Slate. [Sc le ; Brit. Mus.] 
ImpRACANTriUs, R. Owen (cx Egerton, MS.), Geol. Mar., vol. vi, 1869, p. $48 \mathrm{I}$.

cole1, P. M. G. Egerton, in L. Agassiz, Poiss. Foss., vol. iii (1843), p. 177 (name only); J. Morris, Cat. Brit. Foss., I854, p. 332 ; R. Owen, Geol. Mag., vol. vi, I869, p. 48I, woodcut ; J. IV. Davis, Quart. Journ. Geol. Soc., vol. xxxii, 1876, p. 335.

LOC. Coal-Neas.; North Wales and Yorkshire. [Spine; Brit. Mus.]

$\mathbf{T}$.

ImPTACANTrivs, L. Agassiz, Pniss. Foss., vol. iii (I837), p. 27. [Dorsal fin-spines of Chimeroid Fishes.]

jenkinsomi, $\mathrm{M}^{\prime} \mathrm{Coy}$. Acondylacanthus jenkinsoni.

junceus, $\mathrm{M}$ 'Coy. $\because$ Acondylacanthus junceus.

priscus, Agassiz. \%. Cosmacanthus priscus.

semistriatus, L. Agassiz, Poiss. Foss., vol. iii (I837), p. 28, pl. vii, f. 3-8; J. Morris, Cat. Brit. Foss., I 854 , p. 332 ; J. Phillips, Geol. Oxford, IS7I, p. I78, woodcut xxxviii, f. 9. [? Ganodus.]

Loc. Stonesfield Slate. [Spine; Brit. Mus.]

serratus, L. Agassiz, Poiss. Foss., vol. iii (1837), p. 29, pl. vii, f. I, 2; J. Phillips, Geol. Oxford, 1871, p. 178, woodcut xxxviii, f. 8. [? Ganodus.]

LOC. Stonesfield Slate. [Spine; Brit. Mus.]

temuispinus, Agassiz. v. Myriacanthus granulatus.

IEPTOCHELIS, Salter. \%. Onchus murchisoni.

IEPTOZEPIS, L. Agassiz, Neues Jahrb., I832, p. 146, and Poiss. Foss., vol. ii, pt. ii (1843), p. 129.

brodiei, P. M. G. Egerton, in Brodie's Foss. Insects, I845, p. 15, pl. i, f. I-3 ; J. Morris, Cat. Brit. Foss., I854, p. 332.

LOC. Purbeck; Vale of Wardour, Wilts. [Fish; P. B. Brodie Coll.]

bronzi, [-ii] L. Agassiz, Neues Jahrb., IS32, p. 146, and Poiss. Foss., vol. ii, pt. ii (1835), p. I33; J. Morris, Cat. Brit. Foss., I 854, p. 332 .

Loc. L. Lias; Lyme Regis. [Fishes; Brit. Muss.]

caudalis, L. Agassiz, Poiss. Foss., vol. ii, pt. ii (1843), p. 133 ; J. Morris, Cat. Brit. Foss., 1854, p. 332.

LOC. L. Lias ; Lyme Regis. [Fishes ; Brit. Mus.]

concentricus, P. M. G. Egerton, Quart. Journ. Geol. Soc., vol. v, 1849 , p. 35 ; J. Morris, Cat. Brit. Foss., I854, p. 332. LOC. U. Lias ; Dumbleton. [Fish ; Bath Mus.]

constrictus, P. M. G. Egerton, Figs. and Descript. Brit. Organic Remains, dec. vi (Mem. Geol. Surv., 1852), no. 9, pl. ix ; J Morris, Cat. Brit. Foss., 1854, p. 332.

LOC. Lias; Ilminster. [Fishes; Bath Mus.] 
TEPTOLEPS (continued)-

costalis, P. M. G. Egerton, Quart. Journ. Geol. Soc., vol. i, IS+5, p. 231 ; J. Morris, Cat. Brit. Foss., 1854, p. 332.

Loc. Oxford Clay; Christian Malford, Wiltshire. [Imperfect fishes; Brit. Ilus.]

filipemnis, L. Agassiz, Poiss. Foss., vol. ii, pt. ii (1843), p. 134 (name only).

Loc. L. Lias; Street, Somerset. [Fish; Brit. Mus.]

macrophthalmus, P. M. G. Egerton, Quart. Journ. Geol. Soc., vol. i, 1845 , p. $23 \mathrm{I}$, and Figs. and Descript. Brit. Organic Remains, dec. vi (Mem. Gcol. Surv., I 852), no. 8, pl. viii; J. Morris, Cat. Brit. Foss., 1854, p. 332.

Loc. Oxford Clay; Christian Malford, Wiltshire. [Imperfect fishes ; Brit. Mus.]

nanus, P. M. G. Egerton, in Brodie's Foss. Insects, I845, p. I5, pl. i, f. 5 ; J. Norris, Cat. Brit. Foss., 1854, p. 332.

Loc. Purbeck; Vale of Wardour. [Fish; P. B. Brodie Coll.]

saltviciensis, M. Simpson, Foss. Yorksh. Lias, 1855, p. I9; J. IF. Blake, in Tate and Blake, Yorksh. Lias, 1876, p. 260, pl. iii, f. i. LOC. U. Lias; Whitby. [Fish : Whitby Mus.]

İUCIScUS, Cuvier (ex Klein), Règne Animal, vol. ii, 1817, p. 194. ? cephalus (Linnæus), E. 'T. Newton, Vert. Forest Bed (Mem. Geol. Surv., I 882), p. I24.

Cyprimus cephalus, Linnæeus, Syst. Nat., ed. 12, vol. i, 1766, p. 527. LOC. Forest-Bed Series; Norfolk. [Pharyngeal; Mus. Plact. Geol.]

erythrophthalmus (Linnæus), E. T. Newton, Vert. Forest Becl (Mem. Geol. Surv., 1882), p. 126, pl. xviii, f. 18.

Cyprinus erythrophthalmus, Linnæus, Syst. Nat., ed. 12, vol. i, I766, p. 530.

Loc. Forest-Bed Series (U. Freshwater Bed); West Runton. [Pharyngeal tooth; Mus. Pract. Geol.]

rutilus (Linnæus), E. T. Newton, Vert. Forest Bed (Mem. Geol. Surv., I8S2), p. 125, pl. xviii, f. 17.

Cyprimus rutilus, Linnzeus, Syst. Nat., ed. 12, vol. i, I766, p. 520. LOC. Forest-Bed Series (U. Freshwater Bed); West Runton. [Pharyngeal; A. Savin Coll.]

IISPACANTrEUs, J. W. Davis, Trans. Roy. I) ublin Soc. [al vol. i (1883), p. 359 .

? gracilis, J. W. Davis, ibid., p. 359, pl. xlviii, f. 6.

Loc. Carb. Limest. ; Kendal Fells, Westmoreland. [Spine; Geol. Soc. London.]

retrogradus, $J$. W. Davis, ibid., p. 359, pl. xlviii, f. 5 .

LOC. L. Carb. Limest. ; Armagh. [Spine: Brit. Mus.] T. 
LISSOLEPIS, Davis. \%. Eugnathus.

serratus, Davis. v. Eugnathus serratus.

LOPHACANTHUS, Stock. v. Pleuracanthus.

teylori, Stock. $v$. Pleuracanthus cylindricus.

IOPצrosmomUs, P. M. G. Egerton, Figs. and Descript. Brit.

Organic Remains, dec. vi (Mem. Geol. Surv., 1852), no. Io.

dizoni, P. M. G. Egerton, ibid., pls. $x, x^{*}$; A. S. Woodward, Proc.

Geol. Assoc., vol. x (I888), p. 303.

Loc. Chalk ; S. E. England. [Head, etc. ; Brit. Mus.] '

LOPIIODUS, Romanovsky. v. Helodus and Psephodus.

angularis, Davis. v. Helodus angularis.

bifurcatus, Davis. $\%$. Helodus bifurcatus.

conicus, Davis. v. Helodus conicus.

didymus (Agassiz), Davis. v. Psephodus magnus.

gibberulus (Agassiz), Davis. 2\%. Helodus gibberulus.

levissimus, Davis. v. Psephodus magnus and Psephodus levissimus.

levis, Davis. $v$. Helodus levis.

mammillaris, Davis. $\%$. Helodus mammillaris.

reticulatus, Davis. $\%$ Helodus reticulatus.

serratus, Davis. v. Venustodus serratus.

sinuosus, Davis. v. Psephodus magnus.

LOIMODUS, Symonds. \% Acrodus keuperinus.

LOXOSTOMUS, L. Agassiz, Rep. Brit. Assoc., 1844 (1845), p. 303 (name only).

muncus, L. Agassiz, ibid., p. 308 (name only).

LOC. London Clay; Sheppey. [Imperfect head ; Brit. Mus.] $\mathbf{T}$.

IMACROPOMA, L. Agassiz, Poiss. Foss., Feuill., p. 55 (1835), and vol. ii, pt. ii (1843), p. 174 .

egertoni, Agassiz. v. Eurypoma egertoni.

mantell, [-ii] L. Agassiz, Poiss. Foss., Feuill., p. 55 (1835), and vol. ii, pt. ii $(1843)$, p. 174 , pls. Ixv $a-d$; W. C. Williamson, Phil. Trans., 1849 , [0. 462 , pl. xlii, f. 25,26 , pl. xliii, f. 27-30 ; [-ii] 'T. H. Huxley, Figs. and Descript. Brit. Organic Remains, dec. xii (Mem. Geol. Surv., I 866), p. 27, pls. vii, viii ; J. Morris, Cat. Brit. Foss., 1854, p. 332 ; A. S. Woodward, Proc. Geol. Assoc., vol. x (1888), p. 303 .

Amia? leviesiensis, G. A. Mantell, Foss. S. Downs, 1822, p. 239, pls. xxxvii, xxxviii.

I.Oc. Chalk ; S. E. England. [Imperfect fishes; Brit. Mus.] T. substriolutum, Huxley. v. Coccoderma substriolatum. 
IMACROPOIMA (continuci)-

(coprolites of), L. Agassiz, Poiss. Foss., vol. ii (1843), p. 177, pl. lxi $a$, f. 3-I1.

'Cones of unknown vegetables,' J. Parkinson, Organic Remains, vol. i, I820, p. 447 , pl. vi, f. xv, xvii; G. A. Mantell, Foss. S. Downs, 1822, p. IO3, pl. ix, f. 5, i1. [Brit. Mus.]

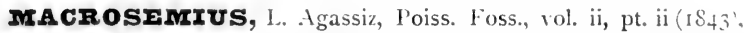
p. 150 .

brevirostris, L. Agassiz, ihid., p. I66 (name only). LOC. Stonesfield Slate. [Mandib. ramus; Brit. Mus.]

MEGAIICYTHYS, L. Agassiz, Poiss. Foss., vol. ii, pt. ii ( 1843 , pp. 89,154 ; T. H. Huxley, Figs. and Descript. Brit. Organic Remains, dec. $x$ (Mem. Geol. Surv., 1861), p. 12; J. Young, Quart. Journ. Geol. Soc., vol. xxii, 1866, p. 606; W. J. Barkas, Monthly Rev. Dental Surgery, vol. iv (1875), pp. 60, 197, $25 \mathrm{I}$, f. Lxi-lxxix. [See also Rhizodus.]

Centrodus, F. M'Coy, Ann. Mag. Nat. Hist. [2] vol. ii, I $\$ 48$, p. 4, and ibid., vol. iii, $18+9$, p. 140.

Parabatrachus, R. Owen, Quart. Journ. Geol. Soc., vol. ix, 1853, p. 67.

Rhomboptychius, J. Young (cx Huxley, MS.), Quart. Journ. Geol. Soc., vol. xxii, I866, p. 604.

Ganolodus, R. Owen, Trans. Odontol. Soc., vol v, IS67, p. 354 (pars).

coccolepis, J. Young, Rep. Brit. Assoc., I869 (1870), Sect., p. 102 ; J. Ward, [Proc.] X. Staffs. Nat. Field Club, 1875, p. 229.

LOC. Coal-Meas. ; Lanarkshire, Northumberland, and N. Staffordshire. [Head-bones; J. Thomson Coll.]

falcatus, S. Hibbert, Trans. Roy. Soc. Edinb., vol. xiii (1835), p. 214 ; J. Morris, Cat. Brit. Foss., 1854 , p. 333.

LOC. Coal-Meas. ; Greenside, Glasgow.

hibbert1, L. Agassiz, Poiss. Foss., vol. ii, pt. ii (I8+3), p. 90, pls. lxiii, lxiii a, lxiv ; R. Garner, Nat. Hist. Staffordsh., 1844, p. 446, pl. E, f. 1o ; W. C. Williamson, Phil. Trans., I849, p. 450 , pl. xli, f. 15, pl. xlii, f. I6-19; J. Morris, Cat. Brit. Foss., 1854 , p. 333 ; F. M'Coy, Brit. Palæoz. Foss. (1855), p. 610 ; J. IV. Salter (ex Egerton, MS.), Foss. S. Welsh Coalfield Mem. Geol. Surv. - Iron Ores Gt. Brit., pt. iii, 186I), p. 224, pl. i, f. 16 ; J. Ward, [Proc.] N. Staffs. Nat. Field Club, I875, p. 227 , f. 10, 14, and Trans. N. Staffs. Inst. Mining Engin., vol.. (I889), pl. ii, f. 4 ; R. Etheridge, jun., Geol. Alag. [2] vol. v, 1878, p. 269 ; L. C. Miall, Quart. Journ. Geol. Soc., vol. xl, 1884, p. 347 , woodcuts $1-4,6$. [See also hhizodus hi'berti.]

Ichthyolithus clackmannensis, J. Fleming, Edinb. New Phil. Mlag., vol. xix, 1835, p. 314 , pl. iv, f. I-3. [Portions of fish; Edinburgh Mus.] 
IIEGIICFTrys hibberti (continued) -

Megalichthys maxillaris, L. Agassiz, Poiss. Foss., vol. ii, pt $\mathrm{ii}_{f}$ (1843), p. 96. [Head; Leeds Mus.]

Parabatrachus colei, R. Owen, Ouart. Journ. Geol. Soc., vol. ix, 1853, p. 67 , pl. ii, f. I. Maxilla of Megalichthy's, J. Young, Proc. Nat. Hist. Soc. Glasgow, vol. i (1868), p. 174, pl. i, f. 1-3. [Maxilla; Brit. Mus.] T. (Parabatrachus.)

Ganolodus sicula, R. Owen, Trans. Odontol. Soc., vol. v, I867, p. 354 , pl. vii. Tooth of 1/cgalichthys, A. Hancock and 'T. Atthey, Nat. Hist. Trans. Northumb. and Durham, vol. iii (1870), p. 9o. [Micro. section of tooth ; Brit. Mus.]

T. (Ganolodus.)

Centrodus striatulus, F. M'Coy, Ann. Mag. Nat. Hist. [2] vol. ii, I848, p. 4, and Brit. Paleoz. Foss. (1855), p. 61 I, pl. iii G, f. I; J. Morris, Cat. Brit. Foss., 1854, p. 319. (Assigned to Megalichthys by J. Young, Quart, Journ. Geol. Soc., vol. xxii, I866, p. 607.) [Tooth; Woodwardian Mus.] T. (Centrodus.)

I.oc. Coal-Meas. ; S. Wales, Staffordshire, Yorkshire, Lancashire, Northumberland, and South Scottish Coalfield. [Head and scales; Leeds Mus.]

$\mathbf{T}$

laticeps, R. H. Traquair, Proc. Roy. Phys. Soc. Edinb., vol. viii, (IS84), p. 67, pl.iv, and Geol. Mag. [3] vol. i, 1884, p. I15, pl. v, f. 1-6.

? Diplopterus robertsoni, L. Agassiz, Poiss. Foss., vol. i (1844), p. xxxvi (name only).

LOC. Calcif, Sandst.; Burdiehouse, near Edinburgh. [Portions of fishes ; Edinburgh Mus.]

maxillaris, Agassiz. $\%$ Megalichthys hibberti.

miscus, Agassiz. \%. Dipterus valenciennesi.

pygmøus, R. H. Traquair, in Mem. Geol. Surv. Scotland, 1879, Expl, to Sheet 3I, p. 76 (name only), and in J. Ward, Trans. N. Staffs. Inst. Mining Engin., vol. x (1889), pl. vi, f. 7, 8.

Diplopterus carbonarius, L. Agassiz, Poiss. Foss., vol. ii, pt. ii (1843),p. 162 (name only). [Fragments labelled by Agassiz; Brit. Mus.]

1Tegalichthys hibberti "young"), E.W. Binney, Trans. Manchester Geol. Soc., vol. i (1841), p. 163, pl. v, f. 1, 2. [Mandib. ramus.]

I.OC. Coal-Meas, : Lanarkshire, Northumberland, and Staffordshire. [Mandib. ramus; Geol. Surv. Scotland.]

rugosus, J. Young, Rep. Brit. Assoc., 1869 (1870), Sect., p. 102; J. Ward, [Proc.] N. Staffs. Nat. Field Club, 1875, p. 229.

? Rhomboptychizs, J. Young (ex Huxley, MS.), Quart Journ Geol. Soc., vol. xxii, I866, pp. 597, 604, woodcuts I, 2 ; J. Ward, [Proc.] N. Staffs. Nat. Field Club, 1875 , p. 230, f. 6, and Trans. N. Staffs. Inst. Mining Engin., vol. x, 1889, pl. ii, f. 6, pl. riii, f. 9. [Head and portion of trunk ; Andersonian Mus., Glasgow.]

LOC. Coal-Meas.; Lanarkshire and N. Staffordshire. [Headbones; J. Thomson Coll.] 
MIEGALODON, Agassiz. \%. Portheus.

lewesiensis, Mantell. $v$. Portheus mantelli.

sauroides, Agassiz. v. Portheus mantelli.

IMIGAIOPS, B. L. G. de Lacépède, Hist. Nat. Poissons, vol. v', 1803, p. 289.

priscus, L. Agassiz, Poiss. Foss., vol, v, pt. ii (1843), p. 114 (name only).

I.OC. London Clay; Sheppey. [Skull ; Brit. Mus.]

MaGAIURUS, L. Agassiz, Poiss. Foss., vol. ii, pt. ii (1843), p. 145.

austeni, P. M. (G. Egerton, Figs. and Descript. Brit. Organic Remains, dec. ix (Mem. Geol. Surv., 18;8), no. 9, pl. ix.

LOC. Purbeck; Swanage. [Imperfect fish ; Brit. Mlus.]

damonl, P. M. G. Egerton, ibid., no. 8, pl. viii.

LOc. Purbeck; Bincombe, near Weymouth. [Fish ; Brit. Mus.]

MEIRISTOLON, Agassiz. \%. Hybodus.

paraloxa, Agassiz. $\approx$. Hybodus striatulus.

MERLINES, L. Agassiz, Rep. Brit. Assoc., 1844 (I 845), p. 308 (name only).

cristutus, L. Agassiz, iuid, p. 308 (name only).

LOC. London Clay; Sheppey. [Imperfect head; Brit. Mus.]

MESACANTHES, Traquair. $\%$ Acanthodes.

mitchelli (Egerton), Traquair. $\%$ Acanthodes mitchelli.

perchi (Egerton), Traquair. v. Acanthodes peachi.

pusillu.s (Agassiz), Traquair. $v$. Acanthodes pusillus.

mrsodon, A. Wagner. Abh. k. bay. Akad. Wiss., Cl. ii $(18 j \mathrm{I})$, p. 56 .

Grronchus, L. Agassiz, Poiss. Foss., vol. ii, pt. ii $\left(1 S_{43}\right)$, p. 202 (incompletely defined).

? biserialis: PJcnodus biseriatis, L. Agassiz, ibid., p. 199 (name only).

LOC. Stonesfield Slate. [Mandib. dentition; Brit. Mus.]

bucklandi (Agassiz), A. S. Woodward, Geol. Mag. [3] vol. vi, r 889 , p. 454 .

Pycnodus bucklandi, L. Agassiz, Poiss. Foss., vol. ii, pt. ii (18+3), p. 192, pl. lxxii $a$, f. I5-22; R. Owen, Odontogr. (18+1), pl. xxxiv, f. 2 ; J. Morris, Cat. Brit. Foss., 1854, p. 342 ; J. Phillips, Geol. Oxford, 1871, p. 179, woodcut xaxix, f. 4, 5. [See also Hesodon sp.]

l'ycnodus didymus, L. Agassiz, Poiss. Foss., vol. ii, pt. ii (1843), p. 193, pl. Ixxii a, f. 24,25 ; R. Owen, Odontugr. (1841) pl. xxxiv, f. 3 ; J. Morris, Cat. Brit. Foss., 1854 , p. 342 ; J. Phillips, Geol. Oxford, 1871, p. 179, woodcut xxxix, f. 6. [Mandib. dentition; Brit. Mus.] 
MESODON bucklandi (continued)-

Pycnodus obtusus, L. Agassiz, Poiss. Foss., vol. ii, pt. ii (1 $\$_{43}$ ), p. 199; J. Murris, Cat. Brit. Foss., 1854, p. 342. [Vomer; Brit. Mus.]

Pycnodus ovalis, L. Agassiz, ibid., p. 195, pl. lxxii a, f. 5 ; J. Morris, Cat. Brit. Foss., 1854, p. 342. [Vomer; Bristol Mus.]

? Gyrodus perlatus, L. Agassiz, ibid., p. 236 ; J. Morris, Cat. Brit. Foss., 1854, p. 328. [Scales; Brit. Mus.]

Ioc. Stonesfield Slate. [Vomer; Oxford Mus.]

discoides: Pycnodus discoides, L. Agassiz, Poiss. Foss., vol. ii, pt. ii (1843), p. 199 (name only).

LOC. Gt. Oolite; Little Gibraltar, Oxford. [Vomer; Brit. Mus.]

Ilasstcus (Egerton), J. J. Heckel, Denkschr. k. k. Akad. Wiss. Wien., math.-naturw. Cl, vol. xi, 18;6, p. 202.

Pyinodus liassicus, P. M. G. Egerton, Ann. Mag. Nat. Hist. [2] vol. xiii, I854, p. 436, and Figs. and Descript. Brit. Organic Remains, dec. viii (Mem. Geol. Surv., 1855), no. Io, pl. x ; J. Morris, Cat. Brit. Foss., 1854, p. 342.

LoC. L. Lias; ? Barrow-on-Soar, and Tewkesbury. [Imperfect fish ; Brit. Mus.]

oblongus (Agassiz), K. A. von Zittel, Handb. Palæont., vol. iii (1887), p. 247 .

Gyronchus oblengus, L. Agassiz, Poiss. Foss., vol. ii, pt. ii (1843), p. 202, pl. Jxix a, f. 10, II ; J. Morris, Cat. Brit. Foss., 1854 , p. 328 .

LOC. Stonesfielà Slate. [Vomer; Bristol Mus.]

rugulosus (Agassiz), A. S. Woodward, Geol. Mag. [3] vol. vi, I 889, p. 454.

Pyonodus rugulosus, L. Agassiz, P'oiss. Foss., vol. ii, pt. ii (1839-43), p. I94, pl. 1xxii a, f. 23; R. Owen, Odontogr. (IS4 I), descr. to pl. xxxiv, f. I ; J. Morris, Cat. Brit. Foss., I854, p. 342 ; ? J. Phillips, Geol. Oxford, 1871, p. 179, woodcut xxxix, f. I.

P'ycnodus parans, L. Agassiz, Poiss. Foss., vol. ii, pt. ii (1843), p. 199 ; J. Morris, Cat. Brit. Foss., 1854, p. 342. [Mandib. dentition ; Brit. Mus.]

Loc. Gt. Oolite; Northamptonshire. Stonesfield Slate. [Vomer ; Oxford Mus.]

trigonus (Agassiz), A. S. Woodward, Geol. Mag. [3] vol. iv, I889, p. 454.

Gyrodus trigonus, L. Agassiz, Poiss. Foss., vol. ii, pt. ii (1843), p. 232, pl. lxix «, f. 15 ; J. Morris, Cat. Brit. Foss., 1854, p. 328 ; J. Phillips, Geol. Oxford, 1871, p. 179, woodcut xxxix, f. 7.

Pycnodus trigonus, W. Buckland, Geol. and Mineral., ed. 2, 1837, vol. ii, p. 45. pl. xxvii $c$, f. 3 ; L. Agassiz, P'oiss. Foss., vol. ii, pt. ii $(1843)$, p. 199 (name only). [Vomer; Oxford Mus.] 
IMESODON trigonus (continued)-

Wicrodon trigonnes, L. Agassiz, Poiss. Foss., vol. i (1844), p. xlii Pycnodus latirostris, L. Agassiz, Poiss. Foss., vol. ii, pt. ii (1843), p. 199 (undefined). [Mandib. dentition; Brit. Mus.]

1.OC. Stonesfield Slate. [Vomer; Brit. Mus.]

umbonatus : l'ycnodus umbonatus, L. Agassiz, Poiss. Foss., vol. ii, pt. ii (1843), p. 194, pl. Ixxii $a$, f. I-4 ; J. Morris, Cat. Brit. Foss., 1854, p. $3+3$.

LOC. Forest Marble; England. [Mandib. dentition.]

sp.: Pycnodus bucklandi, R. Damon (non Agassiz), Geol. Weymouth, ed. 3, I888, Suppl., pl. viii, f. 9.

I.OC. Portland Stone; Portland. [Mandib. dentition; Brit. Mus.]

MESOGOMH'IICS, Davis. ¿. Copodus.

lingu, Davis. $\approx$ '. Copodus lingua.

MrSOLmPIS, J. Young, Quart. Journ. Geol. Soc., vol. xxii, 1\$66, p. 313 ; K. H. Traquair, Trans. Roy. Soc. Edinb., vol. xxix (1879), p. 355.

? Pododus, L. Agassiz, Poiss. Foss., vol. ii, pt. ii (1843), p. 83 (name only).

micropterus, R. H. Traquair, Trans. Roy. Soc. Edinb., vol. xixix (1879), p. 356, pl. iv, f. 8 .

I.Oc. L. Coa!-Nleas. (Dalemoor-Rake Ironstone); Stanton-byDale, Derbyshire. [Fish; Manchester Mus.]

scalaris, J. Young, Quart. Journ. Geol. Soc., vol. xxii, i86, p. 313 ; J. Ward, [Proc.] N. Staffs. Nat. Field Club, 1876 , p. 235 , and Trans. N. Staffs. Inst. Mining Engin., vol. x (1889), pl. vii, f. IO-I 4 ; R. H. Traquair, Trans. Roy. Soc. Edinb., vol. xxix (1879), p. 355 , pl. iv, f. 1-5.

Loc. Coal-Neas.; North Staffordshire. [Fish; J. Ward Coll.]

wardi, J. Young, Quart. Journ. Geol. Soc., vol. xxii, I866, p. 313 , pl. xxi, f. I, 3 ; J. Ward, [Proc.] N. Staffs. Nat. Field Club, I 875 , p. 234 ; R. H. Traquair (?), Trans. Roy. Soc. Edinb., vol. xxix (1879), p. 355 .

T.

LOC. Coal-Nleas.; North Staffordshire. [Fish; J. Ward Coll.]

? sp., R. H. Traquair, Trans. Koy. Soc. Edinb., vol. xxix (1879), p. 355 .

? Podotus capitutus, L. Agassiz, Poiss. Foss., vol. ii, pt. ii (1843), p. 163 (name only).

2. (Pododus.

LOC. Coal-Nleas.; Carluke. [Teeth.]

masorormodus, A. S. Woodward, Cat. Foss. Fishes B. M., pt. i, I889, p. 61 .

protlematicus, A. S. Woodward, ibid., p. 61, pl. i, f. $18,19$.

LOC. L. Carb. Limest.; Armagh. [Tooth; Brit. Mus.] 
METOPACANTHUS, Zittel. v. Myriacanthus.

orthorhimes (Egerton), Zittel. v. Myriacanthus granulatus.

MICROBRACrIUS, R. H. Traquair, Geol. Mag. [3] vol. v, I 888, p. 5 Io.

dicki, R. H. Traquair, ibil., p. 510 , and Ann. Mag. Nat. Hist. [6] vol. ii, 1888, p. 502, pl. xviii, f. 7, S.

Pterichthys dickii, C. W. Peach, Rep. Brit. Assoc., I867 (1868), Sect., p. 72 (name only).

LOC. L. Old Red Sandst.; Jolnn-o'-Groats, Caithness. [Imperfect fish; Edinburgh Mus.]

$\mathbf{T}$.

MICROCONODUS, Traquair. \%. Gonatodus.

molyneuxi, Traquair. \%. Gonatodus molyneuxi.

MICRODON, L. Agassiz, Poiss. Foss., vol, ii, pt. ii i $8+3)$, p. 204.

nuchalis, Dixon. \%. Platax nuchalis.

occipitalis, F. Dixon, Geol. Sussex, I8jo, p. 369, pl. xxxii*, f. 2; J. Morris, Cat. Brit. Foss., I854, p. 333. Pycnodont genus non det., A. S. Woodward, Proc. Geol. Assoc., vol. x (1888), p. 308.

LOC. Chalk ; Lewes. [Fragment of fish ; Brighton Mus.]

pagoda (Blake), A. S. Woodward, Geol. Mag. [3] vol. vi, 1889, p. 454 .

Pycnodus pagodn, J. F. Blake, Quart. Journ. Geol. Soc., vol. xxxvi, I880, p. 228 , pl. x, f. Io.

Loc. Portland Stone; Upway, near Weymouth. [Vomer.]

quincuncialis: Pycnodus quincunciulis, J. F. Blake, Quart.

Journ. Geol. Soc., vol. xxxi, 1875, p. 223; R. Damon, Geol.

Weymouth, ed. 3, 1888, Suppl., pl. xi, f. 10.

LOC. L. Kim. Clay; Weymouth. [Vomer; Brit. Mus.]

radiatus, L. Agassiz, Poiss. Foss., vol. ii, pt. ii (1843), p. 208, pl. lxix $c$, f. I, 2 ; R. Owen, Odontogr. (I841), p. 73, pl. xliii, f. I ; J. Morris, Cat. Brit. Foss., 18 ; t, p. 333.

LOC. Purbeck; Swanage. [Fishes; olim H. E. Strickland and Johnson Colls.]

trigomus, Agassiz. \%esodon trigonus.

MIOG.1NODUS, Owen. \%. Loxomma. [Asphint.]

laniarils, Owen. \%. Loxomma allmanni. [AMPHBBA.]

MITRODUS, Owen. \%. Gyracanthus.

qualricormis, R. Owen, Trans. Odontol. Soc, vol. v, i 867, p. 338, pl. iii. 'Tubercles of Gyracinthus, A. Hancock and T. Atthey, Nat. Hist. Trans. Northumb, and Durham, vol. iii (1870), p. 1 IO.

Loc. Coal-Meas.; Newsham. [Micro. section of dermall tubercle ; Brit. Mus.] 
'Murcena ? lewesiensis,' G. A. Mantell, Foss. South Downs, I822, p. 232, pl. xxxiv, f. I I, pl. xl, f. 2. (Same specimens figured as Dercetis elongatus by L. Agassiz, Poiss. Foss., vol. ii, pt. ii (1843), pl. Ixvi $a$, f. 3,4 .) $=$ Worm-burrows from the Chalk, lined with various scales of fishes (W. 1)avies, Geol. Mag. [2] vol. vi, 1879, p. 145.) [Brit. Mus.]

MILACODUS, Davis. $\because$ Copodus.

quadretus, Davis. $\%$. Copodus spatulatus.

sestmini, Davis. $\%$. Copodus spatulatus.

variabilis, Davis. $\approx$. Copodus variabilis.

MILAT, Davis. $\%$ Copodus.

batoides, Davis. थ. Copodus oblongus.

IMYIroBatrs, Cuvier 'ex Duméril), Règne Animal, vol. ii, i I I , p. 137 . [Myliobates, auct.]

acuta, [-us] L. Agassiz, Poiss. Foss., vol. iii (I843), p. 33I, pl. xlv, f. 14-17; [-us] J. Morris, Cat. Brit. Foss., 1854, p. 333.

LOC. London Clay; Sheppey. [Spine ; olim J.S. Bowerbank Coll.]

canaliculata, [-us] L.Agassiz, Poiss. Foss., vol. iii (I843), p. 33I, pl. xlv, f. I $8-20$; [-us] J. Morris, Cat. Brit. Foss., 1854, p. 333. LOC. London Clay; Sheppey. [Spine; olim J. S. Bowerbank Coll.]

colei, L. Agassiz, Poiss. Foss., vol. iii (18+3), p. 325 (name only). LOC. London Clay; Sheppey. [Teeth.]

contractus, Dixon. vyliobatis dixoni.

dixoni, L. Agassiz, Poiss. Foss., vol. iii (1843), p. 3 I9; F. Dixon, Geol. Sussex, 1850, p. 198, pl. x, f. I, 2, pl. xi, f. I4, pl. xii. f. 3 ; J. Morris, Cat. Brit. Foss., 1854 , p. 333 ; A. S. Woodward, Ann. Mag. Nat. Hist. [6] vol. i, I888, p. 4I, pl. i, f. I-4, and Cat. Foss. Fishes B. M., pt. i, 1889, p. IC9, woodcut +.

Myliobatis heteropleums, L. Agassiz, Poiss. Foss., vol. iii (1843), p. 323 , pl. xlvii, f. 6-8; J. Morris, Cat. Brit. Foss., 18 ; p. 333. [Lower teeth ; Brit. Mus.]

Myliobatis contractus, F. Dixon, Geol. Sussex, I850, p. 200 , pl. xi, f. I7 ; J. Morris, Cat. Brit. Foss., 1854, p. 333. [Lower teeth ; Brit. Mus.]

Myliobatis striatus, F. Dixon (non Agassiz), Geol. Sussex, I 8;0, descr. to pl. xii, f. 2. [Lower teeth ; Brit. Mus.]

LOC. Bracklesham Beds; Sussex. Barton Clay; Hampshire. [Upper teeth ; Brit. Mus.]

edwardsi, Dixon. $v$. Myliobatis striata.

goniopleurus, L. Agassiz, Poiss. Foss., vol. iii (1 $\delta_{43}$ ), p. $31 \%$, pl. xlvii, f. 9, 10 ; J. Morris, Cat. Brit. Foss., 1854, p. 333 ; A. S. Woodward, Ann. Mag. Nat. Hist. [6] vol. i, I888, p. 44 , and Cat. Foss. Fishes B. M., pt. i, 1889, p. 115, pl. iii, f. 5 .

LOC. London Clay; Sheppey. Bracklesham Beds; Sussex [Teeth ; Brit. Mus.] 
MYIIOBATIS (continued) -

gyrata, [-us] L. Agassiz, Poiss. Foss.. vol. iii (1843), p. 323, pl. xlvi, f. I-3; [-us] J. Morris, Cat. Brit. Foss., 1854 , p. 333 . [Unsatisfactorily defined.]

LOC. London Clay ; Sheppey. [Teeth; Paris Mus.]

heteropleurus, Agassiz. \% Myliobatis dixoni.

imegularis, Dixon. $\%$. Myliobatis striata.

jugrulis, L. Agassiz, Poiss. Foss., vol. iii (1843), p. 324, pl. xlvii f. 13,14 ; J. Morris, Cat. Brit. Foss., 1854, p. 333. "Unsatis. factorily defined.]

LOC. London Clay; Sheppey. [Teeth ; olim S. Hibbert Coll.]

lateralis, L. Agassiz, Poiss. Foss., vol. iii (1843), p. 331, pl. xlv, f. 24-27; J. Morris, Cat. Brit. Foss., 1854, p. 333.

Loc. London Clay; Sheppey. [Spine; olim G. Cumberland Coll.]

latidens, A. S. Woodward, Ann. Mag. Nat. Hist. [6] vol. i, I 888 , p. 45 , pl. i, f. II, I2, and Cat. Foss. Fishes B. M., pt. i, 1889, p. I I 8 .

LOC. Bracklesham Beds ; Sussex. [Teeth ; Brit. Mus.]

marginalis, L. Agassiz, Poiss. Foss., vol. iii (1843), p. 33I (name only),

I.OC. Barton Clay; Hampshire. [Spine ; Brit. Mus.]

nitidus, Agassiz. \%. Myliobatis toliapica.

owent, L. Agassiz, Poiss. Foss., vol. iii (1843), p. 33r, pl. xlv, f. 11-13; J. Morris, Cat. Brit. Foss., 1854, p. 333 .

1yyliobatis, F. Dixon, Geol. Sussex, 1850, descr. to pl. x, f. 10.

LOC. Bracklesham Beds; Sussex. [Spine; R. Coll. Surgeons Mus.]

puectatus, Agassiz. $\%$. Myliobatis striata.

striata, [-us] W. Buckland, Geol. and Mineral., ed. 2, 1837, vol. ii, p. 46 , pl. xxvii $d$, f. 14 (name and fig. only); [-us] L. Agassiz, Poiss. Foss., vol. iii $(1843)$, p. 320 ; [-us] J. Mlorris, Cat. Brit. Foss., 1854 , p. 333 ; [-us] A. S. Woodward, Ann. Mag. Nat. Hist. [6] vol. i, 1888, p. 42 , pl. i, f. 5-9, and Cat. Foss. Fishes B. M., pt. i, 1889, p. I12. [See also M. diteni and M.toliapica.] IYvliobatis punctatus, L. Agassiz, Poiss. Foss., vol. iii (1843), p. 322, pl. xlvii, f. 11, 12 ; J. Morris, Cat. Brit. Foss., I 84 , p. 333. [Abraded teeth; Brit. Mus.]

Ifyliobatis irregularis, F. Dixon, Geol. Sussex, I850, p. 199, pl. xi, f. 15; J. Morris, Cat. Brit. Foss., 1854, p. 333. [Teeth; Brit. Mus.]

Iyliobatis edwardsi, F. Dixon, ibid., p. 199, pl. xi, f. 16; [-ii] J. Morris, Cat. Brit. Foss., 1854, p. 333. [Teeth ; Brit. Mus.] LoC. Bracklesham Beds; Sussex. Barton Clay; Hampshire. [Teeth; Oxford Mus.] 
IMXTOBATIS (continued)-

suturalis, Agassiz. \%. Myliobatis toliapica.

tollapica, [-us] L. Agassiz, Poiss. Foss., vol. iii (1843), p. 32 I, pl. xlvii, f. I 5-20; [-us] ? F. Dixon, Geol. Sussex, 1850, descr. to pl. x, f. $3-5$; [-us] J. Morris, Cat. Brit. Foss., I 854, p. 333 ; [-us] A. S. Woodward, Ann. Mag. Nat. Hist. [6] vol. i, I 888 , p. 45, and Cat. Foss. Fishes B. M., pt. i, 1889, p. 116. [Spines figured by L. Agassiz, Poiss. Foss., vol. iii (1843), p. 33I, pl.xlv, 1. $21-23$, and F. Dixon, Geol. Sussex, 1850 , pl. x, f. 36 .]

Myliobatis suturalis, L. Agassiz, l'oiss. Foss., vol. iii (1843), p. 322 , pl. xlvi, f. 12-16.

Myliobutis nitidus, L. Agassiz, P'oiss. Foss., vol. iii (1843), p. 325;

J. Morris, Cat. Brit. Foss., 1854, p. 333. [Teeth ; Brit. Mus.]

Myliobatis striatus, R. Owen (non Agassiz), Ann. Mag. Nat.

Hist. [I] vol. xix, 1847, p. 25, woodcut.

LOC. London Clay; Sheppey. Bracklesham Beds ; Sussex. Barton Clay; Hampshire. [Lower teeth ; Brit. Mus.]

? tumidens, A. S. Woodward, Cat. Foss. Fishes B. M., pt. i, I889, p. 119 .

LOC. Red Crag; Suffolk. [Tooth; Brit. Mus.]

MYRIACANTHUs, L. Agassiz, l'oiss. Foss, vol. iii (1837), p. 37.

Prognathodus, P. M. G. Egerton, Quart. Journ. Geol. Soc., vol. xxviii, 1872, p. 236.

Metopacanthus, K. A. von Zittel, Handb. Palacont., rol. iii (I887), p. IIO.

granulatus, L. Agassiz, Poiss. Foss., vol. iii (1837), p. 40, pl. viii $a$, f. 16 ; J. Morris, Cat. Brit. Foss., I 854 , p. 333 ; A. S. Woodward, Ann. Mag. Nat. Hist. [6] vol. iv, 1889, p. 279.

Leplacanthus tenuispinus, L. Agassiz, Poiss. Foss., vol. iii (1837), p. 27, pl. i, f. 12, 13; J. Morris, Cat. Brit. Foss., I 85 , p. 332 . [Fragment of spine ; Brit. Mus.]

T. (Leptacanthus.)

Ischyodus orthorhinus, P. M. G. Egerton, Quart. Journ. Geol. Soc., vol. xxvii, 187 I, p. 275 , pl. xiii. [Head, etc.; Brit. Mus.]

Metopacanthus orthorhinus, K. A. von Zittel, Handb. Paleont., vol. iii (1887), p. $110 . \quad$ T. (Metopacanthus.)

LOC. L. Lias ; Lyme Regis. [Iorsal fin-spine ; Brit. Mus.]

paradoxus, L. Agassiz, Poiss. Foss, vol. iii (1837), p. 38 , pl. vi ; J. Morris, Cat. Brit. Foss., 1854, p. 333; A. S. Woodward, Ann. Mag. Nat. Hist. [6] vol. iv, 1889, p. 279.

'External defensive organ,' H. T. De la Beche, Trans. Geol. Soc. [2] vol. i (1822), p. 44, pl. v, f. I, 2.

Myriacanthus retrorsus, L. Agassiz, Poiss. Foss., vol. iii (I 837), p. 39 , pl. viii $a$, f. 14, 15; J. Morris, Cat. Brit. Foss., 1854, p. 333. [Base of spine; Oxford Mus.] 
MYRIACANTIUUS paradoxus (continued)-

Chimcera (Ischyodon) johnsonit, L. Agassiz, Poiss. Foss., vol. iii (I 843 ), p. 344, pl. xl $\epsilon$, f. 22.

1schyodus johnsoni, J. Morris, Cat. Brit. Foss., 1854, p. 330. [Teeth ; Brit. Mus.]

Prognathodus guentheri, P. M. G. Egerton, Quart. Journ. Geol. Soc., vol. xxviii, 1872 , p. 233 , pl. viii. [Head, with teeth ; Brit. Mus.]

T. (Prognathodus.)

I.OC. L. Lias; Lyme Regis. [Dorsal fin-spines ; Brit. Mus. and Bristol Mus.]

retroisus, Agassiz. \%. Myriacanthus paradoxus.

WI R IOLEIIS, Egerton, MS. (non Quart. Journ. Geol. Soc., vol. $\mathrm{xx}, 1864$, p. 3), quoted by Etheridge, Foss. Brit. Islands, pt. i, I888, p. 333. (Carboniferous?)

MIIIPRISTIS, Cuvier, Mém. Acad. Sci. Inst. France, vol. viii, 1825 (1829), p. cxxix.

toliapicus, L. Agassiz, Rep. Brit. Assoc., I 844 (1845), p. 307 (name only).

Loc. London Clay; Sheppey. [Imperfect head; Brit. Mus.]

NAISIA, Münster. $\%$ Lepidosteus.

apicalis (Münster), Morris. \%. Lepidosteus fimbriatus.

NamACANTzUS, L. Agassiz, Poiss. Foss., vol. iii (i 837), p. 25 ; A. S. Woodward, Trans. Leicester Lit. and Phil. Soc., n.S., pt. xi, I889, p. I 8 .

brevis, J. Phillips. Geol. Oxford, I871, p. 178, woodcut xxxviii, f. 3-5 (figs. only).

Nemacanthus breaispinus, L. Agassiz, Poiss. Foss., vol. iii (1843), p. 177 (name only).

LOC. Stonesfield Slate. [Spine; Oxford Mus.]

brevispimus, Agassiz. \%. Nemacanthus brevis.

filifer, Agassiz. \% Nemacanthus monilifer.

minor, J. W. Davis, Quart. Journ. Geol. Soc., vol. xxxvii, ISSI, p. 419, pl. xxii, f. 5 .

LOC. Rhatic ; Wainlude Cliff. [Spine; Geol. Soc. London.]

monilifer, L. Agassiz, Poiss. Foss., vol. iii (1837), p. 26, pl. vii, f. 10-15; J. Norris, Cat. Brit. Foss., 1854, p. 334 ; J. W. Davis, Quart. Journ. Geol. Soc., vol. xxxvii, I881, p. 418.

Nemacanthus fliffor, L. Agassiz, P'oiss. Foss., vol. iii (1837), p. 26, pl. vii, f. 9 ; J. W. Davis, Quart. Journ. Geol. Soc., vol. xxxvii, I88I, p. 418. [Spine; Bristol Mus.]

Loc. Rhrtic; Aust Cliff. [Spines; Bristol Mus.]

miscus, $\mathrm{I}^{\circ} \mathrm{Coy}$ \% Cosmacanthus priscus. 


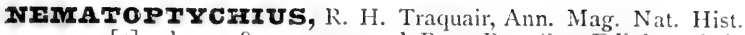
[4] vol. xv, r 875, p. 259, and Proc. Roy. Soc. Edinb., rol. ix (1877), p. 262.

gracilis, R. H. Traquair, Proc. Roy. Soc. Edinb., vol. ix (IS77), p. 262.

LOC. Carb. Limest. (Blackband Ironstone); Gilmerton. [Fish ; Edinburgh Mus.]

greenocki, [-ii] R. H. Traquair, Ann. Mag. Nat. Hist. [4] vol. xv, I 875, p. 258 , pl. xvi, f. 9-II, and Quart. Journ. Geol. Soc., vol. xxxiii, 1877, p. 577, and Proc. Roy. Phys. Soc. Edinb., vol. v, 1879, pp. $118,128$.

Pygopterus greenockii, L. Agassiz, Poiss. Foss., vol. ii, pt. ii (1843), p. 78 (name only); J. Morris, Cat. Brit. Foss., I 854, p. 343 ; R. H. Traquair, Proc. Roy. Soc. Edinb., vol. v (i 866 ), p. 597, and Trans. Roy. Soc. Edinb., vol. xxiv, 1867, 1). 701 , pl. xlv.

LOC. Calcif. Sandst. ; Wardie and Water of Leith, near Edinburgh; Burntisland, and near Anstruther, Fifeshire. Carb. Limest. ; Loanhead, Gilmerton, and Borough Lee, near Edinburgh, and Possil, Lanarkshire. [Imperfect fish ; Edinburgh Mus.]

T.

Nemopteryx mandibularis, Agassiz. v. Pygopterus mandibularis.

Ngorifombormsis, A. S. Woodward, Proc. Geol. Assoc, vol. $\mathrm{x}$ (1888), p. 304 .

excelsus, A. S. Woodward, ibid., p. 304, pl. i, f. I.

LOC. L. Chalk ; Kent. [Imperfect fish; Brit. Mus.]

T.

NOTrosomrs, L. Agassiz, Poiss. Foss., vol. ii, pt. i (1843), p. 292 (name only); P. M. G. Egerton, Figs. and Descript. Brit. Organic Remains, dec. ix (Mem. Geol. Surv., 1858), no. 6, pl. vi.

octostychius, L. Agassiz, Poiss. Foss., vol. ii, pt. i (18+3), p. 292 (name only); J. Morris, Cat. Brit. Foss., I 854 , p. 334 ; P. M. G. Egerton, Figs. and Descript. Brit. Organic Remains, dec. ix (Mem. Geol. Surv, 1858), no. 6, pl. vi.

LOC. L. Lias; Street, Somersetshire. [Fish, wanting head; Brit. Mus.]

$\mathbf{T}$

MOTIDANU $\mathbf{3}$, Cuvier, Règne Animal, vol. ii, is I7, p. I2S.

Xiphodolimia, J. Leidy, Journ. Acad. Nat. Sci. Philad. [2] vol. viii (1877), p. 252.

amalthei, A. Oppel, Württ. Jahresh., vol. x, 1854, p. 62, pl. i, f. 1. Recorded from the U. Lias of Whitby by Tate and Blake, Yorkshire Lias, 1876, p. 256. (Doubtful tooth, A. S. Woodward, Geol. Mag. [3] vol. iii, I886, p. 525, and Cat. Foss. Fishes 13. M., pt. i, 1889, p. I68.) [Tooth; Whitby Mus.] 


\section{NOTIDANUS (continued)-}

daviesi, A. S. Woodward, Geol. Mag. [3] vol. iii, I886, p. 212, pl. vi, f. 8.

Hybodus polyprion, J. Phillips (non Agassiz), Geol. Oxford, 1871, p. 305 , pl. xii, f. I 8.

Loc. Oxford Clay; Oxford. ['Tooth ; Oxford Mus.]

glgas, E. Sismonda, Mem. R. Accad. Sci. Torino [2] vol. xix (1857), p. 460, f. I3; A. S. Woodward, Geol. Mag. [3] vol. iii, I886, p. 255, woodcut I ; H. M. Platnauer, Ann. Rep. Yorksh. Phil. Soc., I886, pl. i, f. 4 ; A. S. Woodward, Cat. Foss. Fishes B. M., pt. i, 1889, p. 165, woodcut 7.

Notidanus meneghinit, R. Lawley, Atti Soc. Tosc., vol. iii (1877), p. 72 , pl. ii, f. 4 ; A. S. Woodward, Geol. Mag. [3] vol. iii, 1886 , p. 255 , woodcut 2 ; H. M. Platnauer, Ann. Rep. Yorksh. Phil. Soc., 1886, pl. i, f. 5. [Tooth; York Mus.]

LOC. Red Crag ; Suffolk. [Teeth; York Mus.]

lanceolatus, A. S. Woodward, Geol. Mag. [3] vol. iii, I $8 S 6$. p. 214 , pl. vi, f. 16, and Cat. Foss. Fishes B. M., pt. i, 1889, p. 160.

LOC. Gault ; ? Folkestone, Kent. [Tooth ; Brit. Mus.]

meneghinii (Lawley), A. S. Woodward. $v$. Notidanus gigas.

microdon, L. Agassiz, Poiss. Foss., Feuill, p. 54 (1835), and vol. iii (I843), p. 22 I, pl. xxvii, f. I, pl. xxxvi, f. I, 2 ; F. Dixon, Geol. Sussex, I850, descr. to pl. xxx, f. 30 ; J. Morris, Cat. Brit. Foss., 1854, p. 334 ; A. S. Woodward, Geol. Mag. [3] vol. iii, I886, p. 213, pl. vi, f. 10-15, and Proc. Geol. Assoc., vol. x (1888), p. 287, and Cat. Foss. Fishes B. M., pt. i, I889, p. 160.

'Tooth of Squalus?,' G. A. Mantell, Foss. S. Downs, 1822, p. 227 , pl. xxxii, f. 22.

Notidanus pectinatus, L. Agassiz, Poiss. Foss., vol. iii (1843), p. 22 I, pl. xxxvi, f. 3 ; J. Morris, Cat. Brit. Foss., 1854, p. 334 .

Loc. Cambridge Greensand. U. and L. Chalk ; Sussex, Kent, Surrey, and Norfolk. [Teeth; Brit. Mus.]

orpiensis, Winkler. $\%$ Acanthias orpiensis.

pectinatus, Agassiz. $v$. Notidanus microdon.

primigenius, L. Agassiz, Poiss. Foss., vol. iii (1843), p. 218, pl. xxvii, f. 6-8, I3-I7 (? f. 4, 5); A. S. Woodward, Genl. Mag. [3] vol. iii, 1886, p. 216, pl. vi, f. 19, 20, 22 ( $n$ กn f. 21 ), and Cat. Foss. Fishes B. M., pt. i, 1889, p. 163.

Notidanus recurvus, L. Agassiz, Poiss. Foss., vol. iii (1843) r. p. 220, pl. xxvii, f. 9-12. [Teeth.]

LOC. Barton Clay; Hampshire. [Teeth; Brit. .llus.]

recurvus, Agassiz. v. Notidanus primigenius. 
NOTIDANUS (continucd)-

serratissimus, L. Agassiz, Poiss. Foss., vol. iii ( 1843 ), p. $2=2$, pl. xxxvi, f. 4, 5 ; J. Morris, Cat. Brit. Foss., 1854, p. 334 A. S. Woodward, Geol. Mag. [3] vol. iii, I886, p. 214, pl. vi, f. $23^{-26}$, and Cat. Foss. Fishes B. M., pt. i, I? p. 162.

Dens Squali, G. Brander, Foss. Hanton., 1776, p. I6, f. III.

LOC. London Clay; Sheppey and Middlesex. [Teeth, clim J. S. Bowerbank Coll.]

serratus, O. Fraas, Württ. Jahresh., vol. xi, 1855, p. 98; F.

Quenstedt, Der Jura, I858, p. 784 , pl. xcvi, f. $44 ;$ A. Woodward, Geol. Mag. [3] vol. iii, 1886, p. 2I2, pl. vi, f. ?and Cat. Foss. Fishes B. M., pt. i, 1889, p. 159.

LOC. Oxford Clay; Scarborough. [Tooth; Brit. Mus.]

sp. (anterior teeth, Xiphodolamia), A. S. Woodward, Cat. Focs.

Fishes B. M., pt. i, 1889, p. 163.

LOC. London Clay; Sheppey. [Teeth; Brit. Mus., no. 431 \& I

OCHLODUS, Owen. ¿. Diplodus.

crassus, Owen. $\%$ Diplodus gibbosus.

ODONTAsprs, L. Agassiz, Poiss. Foss., vol. iii ( $\left.1 \delta_{3} \delta\right)$, p. 87.

cuspidata (Agassiz), A. S. Woodward, Cat. Foss. Fishes B. 1?, pt. i, 1889, p. 368.

Lamna cuspidata, L. Agassiz, Poiss. Foss., vol. iii (1843), p. $20 n$. pl. xxxvii $a$, f. $43-50$.

Lamna denticulata, L. Agassiz, ibid., p. 291, p. xxxvij :, f. $51-53$.

Lamna (Odontaspis) hopei, L. Agassiz, ibid., p. 293, pl. xxxvii .", f. $27-30$.

Lamna (Odontaspis) dubia, L. Agassiz, ibid., p. 295, pl. xxxvii is, f. 24-26.

Lamna hopei, J. Morris, Cat. Brit. Foss., 1854, p. 331.

LOC. Eocene; London and Hampshire Basins. [Teeth; Bri.. Mus.]

dubia, Agassiz. \% Odontaspis cuspidata.

elegans (Agassiz), A. S. Woodward, Cat. Foss. Fishes B. X, pt. i, I889, p. 361 .

Ornithoglossum sessile vulgaris, Luidius, Lithophyl. Brit., $17 \mathrm{C}=$ p. 64, pl. xv, f. 1266.

Dentes Squali, G. Brander, Foss. Hanton., 1776, p. 16, pl. ix, f. 113,114 .

Lamna, E. Charlesworth, Mag. Nat. Hist., n.s., vol, iii, I $\varepsilon_{3}$ ? p. $45 \mathrm{I}$, woodcut 6I $a$.

Lamna elegans, L. Agassiz, Poiss. Foss., vol. iii (1843), p. 2i , pl. xxxv, f. I-5 (non f. 6, 7), pl. xxxvii $a$, f. 59 (non f. 58 );

Owen (ex Agassiz), Odontography (I840), p. 32, pl. v, f. 3,4 , 
ODONTASPIS elegans (continued) -

pls. vi, vii; F. Dixon, Geol. Sussex, 18jo, p. 203, pl. x, f. 28-3I ; J. Morris, Cat. Brit. Foss., 1854, p. 331. [See also Lamna macrota.]

LOC. Eocene; London and Hampshire Basins. [Teeth ; Brit. Mus.]

hopei, Agassiz. v. Odontaspis cuspidata.

2.Teuphiodon, Agassiz. ¿'. Scapanorhynchus rhaphiodon.

rutoti (Winkler), A. S. Woodward, Cat. Foss. Fishes B. M., pt. i, I 889, p. 361 .

Otodus nutoti, T. C. Winkler, Archiv. Mus. Teyler, vol iv, fasc. i, 1876 , p. 4 , pl. i, f. 3,4 .

Lamna, E. Charlesworth, Mag. Nat. Hist., n.s., vol. iii, I839, p. $45 \mathrm{I}$, woodcut $6 \mathrm{I} \mathrm{c}$.

LOC. L. Eocene ; London Basin. [Teeth; Brit. Mus.]

subulatu, Agassiz. z'. Scapanorhynchus subulatus.

OACFUs, L. Agassiz, Poiss. Foss., vol. iii (1837), p. 6.

arcuatus, Agassiz. v. Byssacanthus arcuatus.

fulcatus, L. Agassiz, Poiss. Foss., vol. iii (1843), p. I77 (name only). LOC. L. Carb. Limest.; Armagh. [Spine.]

namatus, Agassiz. \%'. Physonemus hamatus.

major, R. Etheridge, Rep. Brit. Assoc., I871 (1872), Sect., p. IIo.

LOC. L. Old Red Sandst.; Llidiart-y-Warn Quarry, near Hay, Brecon. [Spine; Brit. Mus.]

murchisoni, L. Agassiz, Poiss. Foss., vol. iii (1837), p. 6, pl. i, f. I, 2 ; R. I. Murchison, Silur. Syst. (1839), p. 607, pl. iv, f. 9, II ; J. Morris, Cat. Brit. Foss., 1854, p. 334.

Leptocheles munchisoni, J. W. Salter, Quart. Journ. Geol. Soc., vol. ix, 1853, p. If (regarded as Crustacean).

LOC. U. Ludlow; Herefordshire and Radnorshire. L. Old Red Sandst. (Passage Beds); Ledbury. [Spines; olim Murchison Coll.]

T.

plicatus, Agassiz. \%. Ctenacanthus plicatus.

rectus, Agassiz. $\%$. Ctenacanthus rectus.

semistriatus, L. Agassiz, Poiss. Foss., vol. iii (I 837), p. 8, pl. i, f. 9, and Poiss. Foss. V. G. R. (I844), p. I 18, pl. xxxiii, f. 37; R. I. Murchison, Silur. Syst. (1839), p. 596, pl. ii, f. I2, I3; J. Morris, Cat. Brit. Foss., 1854, p. 334.

LOC. Old Red Sandst.; Southstone Rock. [Spine; olim Murchison Coll.]

suivulutus, L. Agassiz, Poiss. Foss., vol. iii (1843), p. 177 (name only). [Genus non det.]

Loc. Coal-Meas.; Ruabon. [Spine; Brit. Mus.]

wleatus, Agassiz. \%. Ctenacanthus sulcatus. 
ONCHU士 (continued) -

tenuistrfatus, L. Agassiz, Poiss. Foss., vol. iii (1837), p. 7, pl. i, f. Io ; R. I. Murchison, Silur. Syst. (1839), p. 607, pl. iv, f. 12, 13, 57-59; J. Morris, Cat. Brit. Foss., 1854, p. 334 ; F. M'Coy, Brit. Palæoz. Foss. (1855), p. 577.

LOC. Ludlow Bone-bed and Downton Sandst.; Herefordshire. L. Old Red Sandst. (Passage Beds); Ledbury. [Spine; olim Murchison Coll.]

Omyczodus, J. S. Newberry, Geol. Surv. Obio, vol. i, pt. ii, 1873, p. 296.

anglicus, A. S. Woodward, Geol. Mag. [3] vol. v, 1888, p. 500, woodcut.

LOC. L. Old Red Sandst. (Passage Beds); Herefordshire. [Presymphysial teeth; Oxford Mus.]

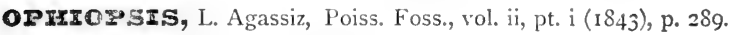

brevicops, P. M. G. Egerton, Figs. and Descript. Brit. Organic Remains, dec. vi (Mem. Geol. Surv., 1852), no. 6, pl. vi; J. Morris, Cat. Brit. Foss., I854, p. 334.

LOC. L. Purbeck.; Oakleigh Quarry, near Tisbury, Wiltshire. [Fish; Mus. Pract. Geol. and Brit. Mus.]

dorsalis, L. Agassiz, Poiss. Foss., vol. ii, pt. i (1843), p. 291, pl. xxxvi, f. 5 ; J. Morris, Cat. Brit. Foss., I 354 , p. 334.

Loc. Purbeck; Swanage. [Fish ; Brit. Mus.]

flesheri (Agassiz), K. A. von Zittel, Handb. Palæont., vol. iii (r887), p. 217.

Pholidophomes flesheri, L. Agassiz, P'oiss. Foss., vol. ii, pt. i (I843), p. 28I, pl. xxxvii, f. 8 ; J. Morris, Cat. Brit. Foss., $1854, \mathrm{p} .338$.

LOC. Inf. Oolite; Blisworth. [Imperfect fish; counterpart in Brit. Mus.]

penicillatus, L. Agassiz, Poiss. Foss., vol. ii, pt. i (1843), p. 290, pl. xxxvi, f. 2-4 ; J. Morris, Cat. Brit. Foss., 1854, p. 334.

LOC. Purbeck; ? Swanage. [Fish; Brit. Mus.]

sauroides (Egerton), Zittel. v. Eugnathus philpotæ.

Oracantrius, L. Agassiz, Poiss. Foss., vol, iii (1837), p. 13.

Pnigeacanthus, O.St. John and A. H. Worthen, Pal. Illinois, vol. vi, I875, p. 480 .

Phoderacanthus, J. W. Davis, Trans. Roy. Dublin Soc. [2] vol. i (1883), p. 533.

armigerus, R. H. Traquair, Trans. Edinb. Geol. Soc., vol. v (1887), p. 313 (name only), and Geol. Mag. [3] vol. v, I888, p. 86.

LOC. Calcif. Sandst.; Abden, Fife, and Eskdale, Dumfries. Carb. Limest.; Broadstone, Ayrshire. [Spine; R. Craig Coll. Also portion of fish; Edinburgh Mius.] 


\section{ORACANTrUS (continued) -}

confuens, Agassiz. \%. Oracanthus milleri.

milleri, L. Agassiz, Poiss. Foss., vol. iii (1837), p. I3, pl. iii, f. 1-4 ; J. Morris, Cat. Brit. Foss., 1854, p. 334 ; F. M'Coy, Brit. Palæoz. Foss. (1855), p. 634 ; R. Etheridge, jun., Geol. Mag. [2] vol. iv, 1877 , p. 307 , pl. xiii, f. 4-6; J. W. Davis, Trans. Roy. Dublin Soc. [2] vol. i (1883), p. 525, pl. lxiii, f. I-4, pl. lxiv, pl. lxv, f. 3, 4 (? also pl. lxii).

Oracanthus confluens, L. Agassiz, Poiss. Foss., vol. iii (1843), p. 177 (name only). [Spine.]

Oracanthus minor, L. Agassiz, ibid. (1843), p. 16, pl. iii, f. 5, 6 ; J. Morris, Cat. Brit. Foss., I854, p. 335. [Fragment of spine; Bristol Mus.]

LOC. L. Carb. Limest.; Gloucestershire and Armagh. [Spines; Bristol Mus.]

minor, Agassiz. in Oracanthus milleri.

pustulosus, L. Agassiz, Poiss. Foss., vol. iii (1837), p. 15, pl. ii, f. 3,4 ; J. Morris, Cat. Brit. Foss., I 854 , p. 335 .

Phoderacanthus grandis, J. W. Davis, Trans. Roy. Dublin Soc. [2] vol. i (1883), p. 534, pl. lxv, f. I. [Spine; Brit. Mus.]

T. (Phoderacanthus.)

LOC. L. Carb. Limest.; Bristol. [Spine; Bristol Mus.]

sp., Davis. v.Asterolepis verrucosus and Coccosteus carbonarius.

ORODUs, L. Agassiz, Poiss. Foss., vol. iii (1838), p. 96.

angustus, Davis. \% Orodus elongatus.

catenatus, J. E. Portlock (ex Agassiz, MS.), Rep. Geol. Londonderry, I 843 , p. 46 I (name only); J. Morris, Cat. Brit. Foss.. I854, p. 335 ; J. W. Davis, Trans. Roy. Dublin Soc. [2] vol. (I883), p. 395, pl. 1i, f. 5 ; A. S. Woodward, Cat. Foss. Fishes B. M., pt. i, I 889 , p. 234.

LOC. L. Carb. Limest. ; Armagh. [Tooth ; Brit. Mus.]

cinctus, L. Agassiz, Poiss. Foss., vol. iii (1838), p. 96, pl. xi, f. I-4 : J. Morris, Cat. Brit. Foss., I 854 , p. 335 ; J. W. Davis, Trans. Roy. Dublin Soc. [2] vol. i (1883), p. 392, pl. 1, f. 8, 9; A. S. Woodward, Cat. Foss. Fishes B. M., pt. i, 1889, p. 230.

LOC. L. Carb. (Bone-bed in L. Limest. Shales); Avon Gorge, near Bristol. [Teeth; Bristol Mus.]

compressus, F. M'Coy, Ann. Mag. Nat. Hist. [2] vol. ii, 1848, p. I3I ; J. Morris, Cat. Brit. Foss., I 854 , p. 335 ; J. W. Davis, Trans. Roy. Dublin Soc. [2] vol. i (1883), p. 394 , pl. I, f. II.

LOC. L. Carb. Limest. ; Armagh. [Tooth ; Geol. Soc. London.]

elongatus, J. W. Davis (ex Agassiz, MS.), Trans. Roy. Dublin Soc. [2] vol. i (1883), p. 394, pl. li, f. I-3; A. S. Woodward, Cat. Foss. Fishes B. M., pt. i, 1889, p. 233. 
ORODUs elongatug (continued)-

Orodus angustus, J. E. Portlock (ex Agassiz, MS.), Rep. Geol. Londonderry, I843, p. 461 (name only); J. Morris, Cat. Brit. Foss., 1854, p. 335 ; J. W. Davis (ex Agassiz, MS.), Trans. Roy. Dublin Soc. [2] vol. i (1883), p. 395, pl. li, f. 4. [Tooth ; Geol. Soc. London.]

I.OC. L. Carb. Limest. ; Armagh. [Teeth ; Brit. Mus.]

? glbbus, J. E. Portlock (ex Agassiz, MS.), Rep. Geol. Londonderry, I 843 , p. 461 (name only); J. Morris, Cat. Brit. Foss., I854, p. 335 ; J. W. Davis, Trans. Roy. Dublin Soc. [2] vol. i (1883), p. 396 , pl. li, f. 6,7 ; A. S. Woodward, Cat. Foss. Fishes B. M., pt. i, I889, p. 236. [? P'sephodus magnus (pars).] LoC. L. Carb. Limest. ; Armagh. [Teeth ; Brit. Mus.]

moniliformis, J. W. Davis, Trans. Roy. Dublin Soc. [2] vol. i (1883), p. 398, pl. li, f. Io-12 ; A. S. Woodward, Cat. Foss. Fishes B. M., pt. i, 1889, p. 234.

Orotus ormatus, J. IV. Davis (non Newberry and Worthen), Trans. Roy. Dublin Soc. [2] vol. i (1883), p. 397, pl. li, f. 9. [Tooth ; Brit. Mus.]

I.OC. L. Carb. Limest.; Armagh. U. Carb. Limest. (incl. Yoredale Rocks); Richmond and Wensleydale, Yorkshire. [Teeth; Brit. Mus.]

ormatus, Davis. v. Orodus moniliformis.

porosus, F. M'Coy, Ann. Mag. Nat. Hist. [2] vol. ii, I848, p. 131 ; J. Morris, Cat. Brit. Foss., 1854 , p. $33 ;$; J. W. Davis, Trans. Roy. Dublin Soc. [2] vol. i (1\$83), p. 393, pl. 1, f. Io.

LOC. L. Carb. Limest. ; Armagh. [Tooth ; Geol. Soc. London.]

ramosus, L. Agassiz, Poiss. Foss, vol. iii (1838), p. 97, pl. xi, f. 5-8; J. Morris, Cat. Brit. Foss., 1854, p. 335; W. H. Baily, Figs. Charact. Brit. Foss. (1875), p. 120, pl. xli, f. Io ; J. W. Davis, Trans. Roy. Dublin Soc. [2] vol. i (1883), p. 390, pl. 1, f. I-7 ; A. S. Woodward, Cat. Foss. Fishes B. M., pt. i, 1889, p. 23I. [Very doubtful tooth figured by J. E. Portlock, Rep. Geol. Londonderry, 1843, p. 467 , pl. xiv A, f. 8.]

Helodus subteres, L. Agassiz, Poiss. Foss., vol. iii (1838), p. 105 , pl. xii, f. 3, 4 (I'scmmodus on plate); J. Morris, Cat. Brit. Foss., 1854, p. 328. [Abraded tooth ; Bristol Mus.]

Oredus subteres, J. W. Davis, Trans. Roy. Dublin Soc. [2] vol. i (1883), p. 399, pl. li, f. I5.

LOC. L. Carb. Limest. ; Somersetshire and Gloucestershire. Carb. Limest. ; Shropshire and Nonmouthshire. [Teeth ; Brit. Mus. and Bristol Mus.]

reedi, J. W. Davis, Trans. Roy. Dublin Soc. [2] vol. i (IS83), p. 398 , pl. li, f. 13.

Loc. Carb. Limest. ; Settle, Yorkshire. [Tooth ; York Mus.]

sculptus, J. W. Davis, ibid. (1883), p. 396, pl. 1i, f. 8.

LOC. L. Carb. Limest. ; Bristol. [Tooth; Bristol Mus.] 
ORODU (continued) -

subteres (Agassiz), Davis. \%. Orodus ramosus.

? tenuis, J. W. Davis, ibid. (I883), p. 399, pl. li, f. I4; A. S. Woodward, Cat. Foss. Fishes B. M., pt. i, 1889, p. 236.

LOC. L. Carb. Limest. ; Armagh. [Abraded tooth ; Brit. Mus.]

OROGNATHUS, L. Agassiz, Poiss. Foss., vol. ii, pt. ii (1843), pp. 83,105 (name only).

conideus, L. Agassiz, ibid., p. I62 (name only).

LoC. Coal-Meas.; Carluke.

I.

ORTIIACANTIUS, Agassiz. $\%$ Pleuracanthus.

cylimdricus, Agassiz. \%. Pleuracanthus cylindricus.

OATzaconds, A. S. Woodward, Cat. Foss. Fishes B. M., pt. i, I 889, p. 349.

Sphexouus, L. Agassiz (non Sphenodon, Gray, 1831, non Sphenodun, Lund, I 839), Poiss. Foss., vol. iii (1843), p. 288.

sp., A. S. WVoodward, Geol. MIag. [3] vol. vi, r889, p. 362.

Loc. Coral Rag; Malton, Yorkshire. [Teeth; Malton Mus.]

ORTHAGORISCL'S', J. G. Schneider, Blochii Syst. Ichthy., 1801, p. 509 (Orthragoriscus).

sp., F. Dixon, Geol. Sussex, I850, descr. to pl. xxxii, f. 3, 4; J. Morris, Cat. Brit. Foss., I854, p. 335. Chelonian dentary bone, A. S. Woodward, Iroc. Geol. Assoc., vol. x (1888), p. 276.

Loc. Chalk ; Sussex. [Brit. Mus.]

ORTHOGNATHES, T. P. Barkas. ¿. Rhizodopsis. reticulosus, T. I'. Barkas. \% Rhizodopsis sauroides.

OSmrzRorDES, L. Agassiz, P'oiss. Foss., vol.v, pt. i (1834), p. 14, and pt. ii (I 443$)$, p. IO3.

? crassus, F. Dixon, Geol. Sussex, 1850, p. 376 ; J. Morris, Cat. Brit. Foss., 1854 , p. 335 ; A. S. Woodward, Proc. Geol. Assoc., vol. $x$ (I888), p. 322 .

LOC. Chalk ; Sussex. [Anterior portion of fish ; Brighton Mus.]

granulatus. L. Agassiz, Poiss. Foss., vol. v, pt. i (1834), p. 14 (name only); J. Morris, Cat. Brit. Foss., 1854, p. 335 .

Loc. Chalk; Lewes. [? Pomognathus eupterygius.]

lewesiensis, L. Agassiz, Poiss. Foss., vol.v, pt. i (1834), p. 14, and pt. ii $(1843)$, p. 105, pl. lx b, f. I, 2, 5-7, pl. lx $c$ (non pl. lx b, f. 3,4 : see Pomognathus eupterygius); J. Morris, Cat. Brit. Foss., 1854 , p. 335 ; A. S. Woodward, Proc. Geol. Assoc., vol. x (1888), p. 322.

Salmo lewesiensis, G. A. Mantell, Foss. South Downs, 1822, p. 235, pl. xl, f. I, pl. xxxiii, f. 12. 
OSMrRoIDES lewesiensis (ionlinued)-

'Scales of unknown fish,' G. A. Mantell, ibid., pl. xxxiv, f. I-

Osmeroides mantelli, L. Agassiz, Neues Jahrb., 1839, p. II I (name only); G. A. Mantell, Wonders of Geol., ed. 3, vol. ;, I839, p. 427 ; J. Morris, Cat. Brit. Foss., I854, p. 335.

LOC. Chalk; S. E. England. [Fishes; Brit. Mus.] $\Xi$ mantelli, Agassiz. \%. Osmeroides lewesiensis.

OSTzOIEPIS, A. Valenciennes, in Sedgwick and Murchisrn, Trans. Geol. Soc. [2] vol. iii (1828), p. I44; L. Agassiz, Yoiss. Foss., vol. ii, pt. i $(1835)$, p. I17; H. Miller, Footprints of the Creator, 1849 , p. 5I, woodcuts $12-15$.

Pleiopterus, L. Agassiz, Poiss. Foss., vol. ii, pt. i (1835), p. Ir 3. Tripterus, F. M'Coy, Ann. Mag. Nat. Hist. [2] vol. ii, I 848 , p. 306.

Triplopterus, F. M'Coy, Brit. I'alreoz. Foss. (i 855 ), p. 589.

arenatus, Agassiz. ¿'. Osteolepis macrolepidotus.

brevis, $\mathrm{M}^{\prime} \mathrm{C}$ oy. \%. Osteolepis macrolepidotus.

macrolepidotus, L. Agassiz, P'oiss. Foss., vol. ii, pt. i ( 1833 ), p. 119 , pl. ii $b$, f. $1-4$, pl. ii $c$, f. 5-6; J. Morris, Cat. Init. Foss., 1854 , p. 335 ; F. MI'Coy, Brit. Palæoz. Foss. (I 855 ), p. 588 ; C. H. Pander, Saurodipt., Dendrodont., etc., devon. Syst., I860, p. II, pl. ii, f. 2, 6-9, pl. iii, f. I-20; R. H. Traquair, Geol. Mag. [3] vol. v, I888, p. 5 I 5 ; ? A. Valenciennes, Trans. Geol. Soc. [2] vol. iii (I828), p. I44. [See also Thursius macrolepidotus.]

Ostcolepis microlepidotus, L. Agrassiz, Poiss. Foss., vol. ii, pt. i (1835), p. I2I, pl. ii $c$, f. I-4: J. Morris, Cat. Brit. Foss, I854, p. 335 ; F. M'Coy, Brit. I'alæoz. Foss. (1855), p. 588.

Osteolepis arenatus, L. Agassiz, Poiss. Foss., vol. ii, pt. i (1835), p. I22, pl. ii $d$, f. I-4; F. M Cov, Brit. Palroz. Foss. (1855), p. 587 ; J. Morris, Cat. Brit. Foss., 1854, p. 335. [Fish; Brit. Mus.]

Osteolepis major, L. Agassiz, Poiss. Foss. V. G. R. (I844), p. 51, pl. xix, f. I-3; F. M'Coy, Brit. Palieoz. Foss. (1855), p. 588 ; J. Morris, Cat. Brit. Foss., 1854, p. 335. [Fish; Brit. Mit.]

Osteolepis, H. Miller, Old Red Sandst., I841, p. 72, pl. iv.

Osteolepis brevis, F. 11 'Coy, Ann. Mag. Nat. Hist. [2] vol. :", I848, p. 305, and Brit. Palæoz. Foss. (1855), p. 587, pl. ii D, f. 4 ; J. Morris, Cat. Brit. Foss., I854, p. 335. [Crushed fish; Woodwardian Mus.]

Tripterus pollexfeni, F. M'Coy, Ann. Mag. Nat. Hist. [2] vol. i, 1848, p. 306 ; J. Morris, Cat. Brit. Foss., I 854 , p. 346 . [Portion of fish ; Woodwardian Mus.]

Triplopterus pollexfeni, F. M'Coy, Brit. Palwoz. Foss. (1 $85 \vdots$ ', p. 589 , pl. ii D, f. 5 .

LOC. L. Old Red Sandst.; Banffshire, Nairnshire, Cromar:y, Ross-shire, Caithness, and Orkney. [Imperfect fishes; $01 . \mathrm{m}$ T. S. Traill Coll.] 


\section{OSTMOIEPIS (continued) -}

major, Agassiz. \%. Osteolepis macrolepidotus.

microlepirlotus, Agassiz. \%. Osteolepis macrolepidotus.

microlepidotus, C. H. Pander, Saurodipt., Dendrodont., etc., devon. Syst., I860, p. Io and passim, pl. i, f. I-6, 8-1o, pl. ii, f. 1, 3-5, 10-14.

? Osteolepis microlepidotus, A. Valenciennes, Trans. Geol. Soc. [2] vol. iii (1828), p. 144 .

LOC. L. Old Red Sandst.; Caithness. [Fishes; Imp. Acad. Sci. St. Petersburg.]

OSTFOPIAX, F. M['Coy, Ann. Mag. Nat. Hist. [2] vol, ii, I848, p. 6, and Proc. Camb. Phil. Soc., vol. i (1848), p. 65.

orosa, [-us] F. M'Coy, Ann. Mag. Nat. Hist. [2] vol. ii, 1848, p. 6, and Brit. Palæoz. Foss. $(1855)$, p. 613, pl. iii k, f. I2; [-us] J. Morris, Cat. Brit. Foss., 1854, p. 335.

LOC. Carb.; Cultra, co. Down. [Dermal Plate; Woodwardian Mus.?

T.

OSTEORIACkIS, P. M. G. E.gerton, Figs. and Descript. Brit. Organic Remains, dec. xiii (Mem. Geol. Surv., 1872), no. 5 (Osteorachis).

macrocephala, [-us] P. M. G. Egerton, ibid., no. 5, pl. v. I.OC. L. Lias ; Lyme Regis. [Fish ; Hrit. Mus.]

() STINASI'IS, Trautschold. \%'. P'etrodus.

OSTRACACANTruU, J. W. I)avis, Rep. Brit. Assoc., 1879 (r880), p. 343 (Ostracocanthus), and Quart. Journ. Geol. Soc., vol, xxxvi, 1880, p. 64.

dllatatus, J. W. Davis, ibid., p. 343, and ibid., p. 64, woodcut 3 . LOC. Cannel Coal ; Tingley, Yorkshire. [Dermal Spine; J. W. Davis Coll.]

TODUS, Agassiz. \%. Lamna.

appendiculatus, Agassiz. v. Lamna appendiculata.

crassus, Agassiz. Recorded (? errore) by J. Morris, Cat. Brit. Fos5., I854, p. 335 ; see also Lermm sulcatir.

lanceoletus, Agassiz. \%'. Lamna obliqua.

lerrecolutu,s, Dixon. $\%$. Lamna macrota.

macrotus, Agassiz. v. Lamna macrota.

obliqums, Agassiz. \%. Lamna obliqua.

rutoti, Winkler. $\%$ Odontaspis rutoti.

semiplicutus, Agassiz. v. Lamna semiplicata.

sulcutus, Geinitz. \% Lamna sulcata.

vincenti, Winkler. $v$. Lamna vincenti.

voodwardi, C. Hasse, Palæontographica, vol. xxxi (1884), p. 8, pl. ii, f. $13^{-15}$ (vertebra). [? Lamma appendiculata.]

LOC. Cambridge Greensand. [Vertebra ; Anat. Inst., Univ. of Breslau.] 
OXYGNATrUS, P. M. G. Egerton, Ann. Mag. Nat. Hist. [2] vol. xiii, 1854 , p. 435 , and Figs. and Descript. Brit. Organic Remains, dec. viii (Mem. Geol. Surv., 1855), no. 9.

ornatus, P. M. G. Egerton, ibid., pls. ix, ix*; J. Morris, Cat. Brit. Foss., 1854, p. 336.

Loc. L. Lias; Lyme Regis. [Fishes; Brit Mus.]

T.

OXXGONIUs, L. Agassiz, in P. B. Brodie's Foss. Insects, I845, p. 16.

tenuis, L. Agassiz, ibid., p. 16, pl. i, f. 4 ; J. Morris, Cat. Brit. Foss., 1854, p. 335.

LOC. Purbeck; Vale of Wardour. [Fish; P. B. Brodic Coll.]

OxyarinA, L. Agassiz, P’oiss. Foss., vol. iii (1838), p. 86.

T.

angustidens, A. E. Keuss, Verstein. böhm. Kreideform., pt. i (1845), p. 6, pl. iii, f. 7-13; A. S. Woodward, Cat. Foss. Fishes B. M., pt. i, 1589 , p. 380 .

LOC. U. Greensand; Wiltshire. Chalk; Sussex, Surrey, Kent, and Cambridgeshire. [Teeth; Brit. Muss.]

crassidens, F. Dixon, Geol. Sussex, 18 80 , p. 367, pl. xxxi, f. 13; J. Morris, Cat. Brit. Foss., 1854, p. 336 ; A. S. Woodward, Cat. Foss. Fishes B. M., pt. i, 1889, p. 382.

Oxyrhina mantelli, L. Agassiz (errore), Poiss. Foss., vol. iii (I843), pl. xxxiii, f. 6. [Tooth ; Brit. Mus.]

LOC. Chalk ; Sussex. [Tooth ; Brit. Mus.]

hastalis, L. Agassiz, Poiss. Foss., vol. iii (1843), p. 277, pl. xxxiv (excl. f. 1, 2, ? I4); A. S. Woodward, Cat. Foss. Fishes B. M., pt. i, 1889, p. 385 .

Including $O$.xiphodon, $O$. trigonodon, $O$. plicatilis, $O$. retroflext, and O. quadrans, L. Agassiz, Poiss. Foss., vol. iii.

Loc. Red and White Crags ; Suffolk. [Teeth; Brit. Mus.]

macrorhiza, F. J. Pictet and P. Campiche, Foss. Terr. Crét. St. Croix (Pal. Suisse, I8;8), p. 83 , pl. x, f. 6-I 8 ; A. S. Woodward, Cat. Foss. Fishes B. M., pt. i, I889, p. 38 I.

LOC. Gault; Folkestone. Cambridge Greensand. [Teeth; Brit. Mus.]

mantelli, [-ii] L. Agassiz, Poiss. Foss., Feuill., p. $54\left(1 \Omega_{35}\right)$, and vol. iii (1843), p. 280 , pl. xxxiii, f. $1-5,7-9$ (non f. 6); F. Dixon, Geol. Sussex, 1850, descr. to pl. xxx, f. 2t; J. Morris, Cat. Brit. Foss., I854, p. 336 ; A. S. Woodward, Proc. Geol. Assoc., vol. x (I888), p. 291 , and Cat. Foss. Fishes B. M., pt. i, IS89, p. 376, pl. xvii, f. 9-21. [See also Oxyrhina crassitens.]

Squalus zyganu?, G. A. Mantell, Foss. S. Downs, I8z2, p. 227. pl. xxxii, f. $4,7,8,10,11,26,28$.

Lamna acuminata, L. Agassiz, Poiss. Foss., vol. iii (1843), p. 292, pl. xxxviia, f. 54 (? non f. 55-57, non ibid., Feuill., p. 54, 
OXIRIINA mantell (continued)-

1835); F. Dixon, Geol. Sussex, 1850 , descr. to pl. $x x x$, f. 19;

J. Norris, Cat. Brit. Foss., I 854, p. 331. [Teeth; Brit. Mus.]

LOC. U. Greensand; 1)orsetshire and Somersetshire. Chloritic

Marl ; Ventnor, I. of Wight. Cambridge Greensand. Chalk ;

Sussex, Surrey, Kent, and Essex. [Teeth; Brit. Mus.]

parectoxa, Agassiz. \%. Hybodus striatulus.

plicetilis, Agassiz. \%. Oxyrhina hastalis.

qurelterus, Agassiz. \%. Oxyrhina hastalis.

retioflexe, Agassiz. \% Oxyrhina hastalis.

trigonodom, Agassiz. ¿. Oxyrhina hastalis.

xiphodon, Agassiz. i'. Oxyrhina hastalis.

PACH CEIIfALCS. L. Agassiz, Poiss. Foss., vol. v, pt. ii (1843), p. I 39 (name only), and Rep. Brit. Assoc., 1844 (1845), p. 308 (name only).

cristatus, L. Agassiz, ibid., p. 139 (name only), and ibid., p. 308 (name only).

LOC. London Clay; Sheppey. [Imperfect head; Brit. Mus.] T.

PACrix CORMUS, L. Agassiz, Poiss. Foss., vol. ii, pt. ii (1843),

p. IIO.

(reutiostris, L. Agassiz, ibid., p. II (undefined).

LOC. U. Lias; Whitby.

brenchialis (Agassiz, MS.), J. Morris, Cat. Brit. Foss., I854, p. 336 (name only).

LOC. L. Lias ; Lyme Regis. [Fish; Brit. Mus.]

curtus, L. Agassiz, Poiss. Foss., vol.ii, pt. ii (1843), p. 112, pl. lix ; J. Morris, Cat. Brit. Foss., 1854, p. 336.

Loc. U. Lias; Whitby. [Fishes; Brit. Mus. and Scarborough Mus.]

gracilis, L. Agassiz, Poiss. Foss., vol. ii, pt. ii (1843), p. II4 (unclefined).

Uraus gracilis, L. Agassiz, Neues Jahrb., 1832, p. 142 (name only).

LOC. U. Lias; Whitby.

beterurus, L. Agassiz, Pniss. Foss., vol. ii, pt. ii (1843), p. II3, pl. lviii $a$, f. 4,5 ; J. Morris, Cat. Brit. Foss., 1854, p. 336.

Loc. L. Lias; Lyme Regis. [Tail ; Oxford Mus.]

latipennis, L. Agassiz, l'oiss. Foss., vol. ii, pt. ii (1843), p. 114 (undefined); J. Morris, Cat. Brit. Foss., 1854, p. 336 ; P. M. G. Egerton, Figs. and Descript. Brit. Organic Remains, dec. ix (Mem. Geol. Surv, 1858), no. 3, pl. iii.

LOC. L. Lias; Lyme Regis. [Head, pectoral fin, scales; Brit. Mus.] 
PACFrCokntus (continued)-

latirostris, L. Agassiz, l'oiss. Foss, vol. ii, pt. ii (1843), p. 114 (undefined).

LOC. U. Lias; Whitby.

latus, L. Agassiz, ibicl., p. 114 (undefined).

LOC. U. Lias; Whitby. [Imperfect fish; Brit. Mus.]

leptosteus, L. Agassiz, iuil., p. II (name only).

LoC. L. Lias ; Lyme Regis.

macropterus, L. Agassiz, ibid., p. III, pl. lix $a$; recorded by J. F. Blake, in Tate and Blake, Yorksh. Lias, I876, p. 260.

Loc. U. Lias; Whitby. [Fish; York Mus.]

macrurus, L. Agassiz, I'oiss. Foss., vol. ii, pt. ii (1843), p. II 3 , pl. Iviii a, f. 3 ; J. Morris, Cat. Brit. Foss., 1854, p. 336.

LOc. L. Lias; Lyme Regis. [Tail ; Oxford Mus.]

sp. From Oxford Clay; Peterborough. [Portions of fishes; Brit. Mus., Woodwardian Mus., and A. N. Leeds Coll.]

PACFrRfizod US, F. Dixon ( $c x$ Agassiz, MS.), Geol. Sussex, 1850 , p. 374 ; E. D. Cope, Vert. Cret. Form. West (Rep. U.S. Geol. Surv. Territ., vol. ii, 1875), p. 220.

Megalodon, L. Agassiz (non Sowerby), Poiss. Foss., Feuill., p. 55 (1835) (pars).

Hypsodon, L. Agassiz, Neues Jahrb., 1838, p. 108, and Poiss. Foss., Feuill., p. 104 (1837), and vol. v, pt. i (1843), pp. 8, 99 (pars).

Rhaphiosaurus, R. Owen, Trans. Geol. Soc. [2] vol. vi (1840), p. 413 (Raphiosaurus).

Acrodontosaukus, J. W. Mason, Quart. Journ. Geol. Soc., vol. xxv, 1869, p. 444.

basalis, F. Dixon, Geol. Sussex, I850, p. 374, pl. xxxir, f. 9, 10 ; I. Morris, Cat. Brit. Foss., 1854, p. 336.

LOC. Chalk; Steyning, Sussex. [Fragment of mandib. ramus; Brit. Mus.]

T.

gardner1 (Mlason), A. S. IVoodward, Proc. Geol. Assoc., vol. x (1888), p. 3I4.

Hypsodon lcatcsiensis, L. Agassiz (pars), Neues Jahrb., I 839, p. 120 , and P'oiss. Foss., Feuill., p. 104 (1837), and vol. v, pt. i (1843), p. 99, pl. $\operatorname{xva} a$, f. I, 2,4 , pl. $\operatorname{xxvb}$, f. 4,5 ; E. T. Newton, Quart. Journ. Geol. Soc., vol. xxxiii, 1877, p. 508.

'Reptile or Fish,' Toulmin Smith, Lond. Geol. Journ. (1846), p. 2I, woodcut. [Group of bones; Brit. Mus.]

Acrodontosaunus gardneri, J. WV. Mason, Quart. Journ. Geol. Soc., vol. xxv, i 869 , p. 444 , pl. xix. T. (Acrodontosaurus.) LOC. Chalk; S.E. England. [Portion of mandib. ramus; Brit. Mus.] 
PACHYRTIZODUS (continued) -

glyphodus, C. C. Blake and S. J. Nackie, Geologist, vol. vi, I863, p. 134.

LOC. Gault ; Folkestone. [Fragment of jaw ; Brit. Mus.]

gracilis (Owen), A. S. Woodward (ex W. Davies and O. C. Marsh, MS.), Proc. Geol. Assoc., vol. x (1888), p. 314.

Mosusaurus gracilis, R. Owen, in F. Dixon, Geol. Sussex, 1850, p. 380, pl. xxxvii, f. I, and Rept. Cret. Form. (Pal. Soc., I851), p. 3I, pl. ix, f. I. [For other figures see Reptilia.]

Loc. Chalk; Sussex. [Jaws; Brighton Mus.]

subulidens (Owen), A. S. Woodward, Ann. Mag. Nat. Hist. [6] vol. iv, 1889, p. 350.

Rhaphiosaurus subulidens, R. Owen, Rep. Brit. Assoc., I841, p. 190, and Rept. Cret. Form. (Pal. Soc., 1851), p. I9, pl. x, f. 5,6 .

T. (Rhaphiosumus.)

Rhaphiosaums lucius, R. Owen, in F. Dixon, Geol. Sussex, I 850 , p. 385 , pl. xxxix, f. $1-3$.

LoC. L. Chalk; Cambridge. Chalk; Sussex. [Mandib. ramus; Woodwardian Mus.]

PAImonrscus, H. D. de Blainville, Dict. Hist. Nat., vol. xxvii, I 818, p. 320 (Palaoniscum); L. Agassiz, Poiss. Foss., vol. ii, pt. i (1834), p. 4 I.

PALEOThrissum, H. D. de Blainville, Dict. Hist. Nat., vol. xxvii, I818, p. 320.

abbsi, Kirkby. $v$. Acentrophorus abbsi.

altus, Kirkby. \%. Acentrophorus altus.

? angustus, L. Agassiz, Poiss. Foss., vol. ii, pt. i (1834), p. 57, pl. ix, f. 1-5. Doubtfully recorded from Permian of Fulwell by J. W. Kirkby, Quart. Journ. Geol. Soc., vol. xx, I864, p. 356. [? not Palconiscus, according to Traquair, Quart. Journ. Geol. Soc., vol. xxxiii, 1877, p. 558.$]$

carinatus, Agassiz. $\%$. Rhadinichthys carinatus.

crtopterus, Agassiz. $\%$ Dictyopyge catoptera.

comptus, L. Agassiz, Poiss. Foss, vol. ii, pt. i $(1 \$ 35)$, p. 97, pl. $\mathrm{x} b$, f. I-3 (P. comtus); W. King, lermian Foss. (Pal. Soc., I850), p. 223, pl. xxi, f. 1; W. C. Williamson, Phil. Trans., 1849 , p. 445 , pl. xl, f. 7 ; J. Morris, Cat. Brit. Foss., 1854, p. 336.

Palcoothrissum magnum and I'. macrocephalum, A. Sedgwick, Trans. Geol. Soc. [2] vol. iii (1829), p. I17, pl. viii, f. 1, 2, pl. ix, f. 2.

Palconiscus freieslebeni, F. M'Coy (? nnn Blainville), Brit. Palroz. Foss. (1855), p. 606; R. Howse, Guide Coll. Local Foss. Mus. Nat. Hist. Soc. Newcastle-upon-Tyne, I889, pp. 21,24 .

LOC. Marl-Slate; Midderidge and Cullercoats. L. Magnesian Limest.; Down Hill, near Boldon. [Fishes.] 
PAIfEONISCUS (continued)-

egertoni (Agassiz), Egerton. \%. Elonichthys egertoni.

elegans (Sedgwick), L. Agassiz, Poiss. Foss., vol. ii, pt. i (1835), p. 95 , pl. $x b$, f. 4,5 ; W. King, Permian Foss. (Pal. Soc., 1850), p. 223, pl. xxii, f. I $a$; J. Morris, Cat. Brit. Foss., 1854 , p. 336 ; F. M'Coy, Brit. Palzeoz. Foss. (1855), p. 605.

Palcothrissum elegans, A. Sedgwick, Trans. Geol. Soc. [2] vol. iii (1829), p. 117, pl. ix, f. I.

LOC. Permian; Durham. [Fish.]

freieslebeni, Blainville, recorded in error by J. Phillips in Murchison's Silur. Syst., 1839, p. 89, and J. Morris, Cat. Brit. Foss., I 854 , p. 336, from Spirorbis Limestone in U. Coal-Meas., Ardwick : see also Palconiscus comptus.

glaphyrus, Agassiz. $v$. Acentrophorus glaphyrus.

hancocki, Atthey. $v$. Rhadinichthys hancocki.

longissimus, L. Agassiz, Poiss. Foss., vol. ii, pt. i (1835), p. 100, pl. $x c$, f. 4 ; W. King, Permian Foss. (Pal. Soc., 1850), p. 225 , pl. xxi, f. 2 ; J. Morris, Cat. Brit. Foss., I 854, p. 337.

Loc. Pernian; Durham. [Fish ; counterpart in Brit. Mus.]

macrophthalmus, L. Agassiz, Poiss. Foss., vol. ii, pt. i (1835), p. 99, pl. x $c$, f. 3 ; W. King, Permian Foss. (Pal. Soc., 1850), p. 225 , pl. xxii, f. 2 ; J. Morris, Cat. Brit. Foss., I854, p. 337 ; F. M'Coy, Brit. Palæoz. Foss. (1855), p. 607.

Loc. Permian; Durham. [Fish; Geol. Soc. London.]

monensis, Egerton. $\%$. Rhadinichthys monensis.

ornatissimus, Agassiz. $\%$. Rhadinichthys ornatissimus.

robisoni, Agassiz. $\%$. Elonichthys robisoni.

striolatus, Agassiz. $v$. Elonichthys striolatus.

wardi (Young), Ward. $\%$. Rhadinichthys wardi.

latus, Kirkby. $\%$. Acentrophorus altus.

superstes, Egerton. v. Dictyopyge superstes.

varians, Kirkby. v. Acentrophorus varians.

PAImOSPINAX, P. M. G. Egerton, Figs. and Descript. Brit. Organic Remains, dec. xiil (Mem. Geol. Surv., I872), no. 7.

priscus (Agassiz), P. M. G. Egerton, ibid., pl. vii, and Quart. Journ. Geol. Soc., vol. xxix, 1873, p. 420 ; J. W. Davis, Ann. Mag. Nat. Hist. [5] vol. vii, 1881 , p. 429 , pl. xx ; C. Hasse, Neues Jahrb., 1883, vol. ii, p. 66 ; A. S. Woodward, Geol. Mag. [3] vol. v, 1888, p. 499, and Cat. Foss. Fishes B. M., pt. i, 1889, p. 322 , pl. vii, f. I.

Thyellina prisca, L. Agassiz, Poiss. Foss., vol. iii (1843), p. 378 , pl. xxxix, f. I, 2 ; J. Morris, Cat. Brit. Foss., 1854, p. 345.

Cestracion (Acrodus), C. Hasse, Palæontographica, vol. xxxi (I884), p. 6, pl. ii, f. 8, 9.

LOC. L. Lias; Lyme Regis, Dorsetshire. [Vertebral column; Oxford Mus.]

$\mathbf{T}$. 
PALFOSPINAX (continued) -

sp., A. S. Woodward, Cat. Foss. Fishes B. M., pt. i, 1889, p. 325. Loc. Rhætic; Holwell, near Frome, Somersetshire. [Teeth and spines; Bath Mus.]

PALAOTHRISSUM, De Blainville. v. Palæoniscus. elegans, Sedgwick. v. Palæoniscus elegans.

macrocephalum, Sedgwick. \%. Palæoniscus comptus. magmum, Sedgwick. $\%$. Palæoniscus comptus.

PAMPHRACTUS, Agassiz. v. Bothriolepis.

andersoni, Agassiz. $v$. Bothriolepis hydrophilus.

hydrophilus, Agassiz. v. Bothriolepis hydrophilus.

PARבXUS, L. Agassiz, Poiss. Foss. V. G. R. (I845), p. 120; J. Powrie, Quart. Journ. Geol. Soc., vol. xx, I864, p. 423.

falcatus, J. Powrie, Trans. Edinb. Geol. Soc., vol. i, 1870, p. 294, pl. xiii, f. 9 .

LOC. L. Old Red Sandst.; Forfar. [Imperfect fish ; J. Powrie Coll.]

Incurvus, L. Agassiz, Poiss. Foss. V. G. R. (1845), p. 120, pl. xxxiii, f. 26, 27 ; J. Morris, Cat. Brit. Foss., 1854, p. 337 ; J. Powrie, Quart. Journ. Geol. Soc., vol. xx, 1864, p. 424, pl. xx, f. I, and Trans. Edinb. Geol. Soc., vol. i (1870), p. 293 , pl. xii, f. 8.

LOC. L. Old Red Sandst.; Forfarshire. [Dorsal fin-spine ; Rossie Priory.]

PASSALODON, Buckland. v. Edaphodon.

PELARGORHYNCHUS, W. v. d. Marck. v. Dercetis.

PELECOPTERUS, Cope. v. Protosphyræna (fins of).

PERCA, Linnæus, Syst. Nat., ed. I2, vol. i, I766, p. 48 r.

fiviatilis, Linnæus, ibid., p. $48 \mathrm{I}$; E. T. Newton, Vert. Forest Bed (Mem. Geol. Surv., I882), p. I20, pl. xviii, f. I-8.

LOC. Forest-Bed Series; Norfolk. [Preoperculum, supraclavicle, post-temporal, and scales; $A$. Savin Coll. and Mus. Pract. Geol.]

PEİCOSTOMA, L. Agassiz, Rep. Brit. Assoc., I 844 (1845), p. 307 (name only).

angustum, L. Agassiz, ibid., p. 307 (name only).

LOC. London Clay; Sheppey. [Imperfect head; Brit. Mus.]

PERIODUS, Agassiz. v. Pycnodus.

koenigi, Agassiz. v. Pycnodus toliapicus.

marginalis, L. Agassiz, Poiss. Foss., vol. i (1844), p. xlii (name only). Probably teeth of Mesodon from the English Oolite. 
PETALODOPSIS, W. J. Barkas. ". Ctenodus. mirabilis, W. J. Barkas. v. Ctenodus obliquus.

PETALODOPSIS, Davis, $v$. Ctenoptychius.

tripartitus, Davis. $v$. Ctenoptychius tripartitus.

PrTazodus, R. Owen, Odontography (I84I), p. 6I.

Chomatodus, L. Agassiz, Poiss. Foss., vol. iii (1838), p. 107 (pars).

Antliodus, J. S. Newberry and A H. Worthen, Pal. Illinois, vol. ii, I866, p. 33.

acuminatus, L. Agassiz, l'oiss. Foss., vol. iii (1843), pp. 174, 384 ; J. Morris, Cat. Brit. Foss., 1854, p. 337 ; F. M'Coy, Brit. Palæoz. Foss. (1855), p. 635, pl. iii G, f. 4 ; J. W. Davis, Trans. Roy. Dublin Soc. [2] vol. i (1883), p. 494, pl. lix, f. 22-24, and Quart. Journ. Greol. Soc., vol. xl, 1884, p. 624, pl. xxvi, f. Io; A. S. Woodward, Cat. Foss. Fishes B. M., pt. i, r 889 , p. 42 , pl. i, f. $4-7$.

Chomatodus acuminatus, L. Agassiz, Poiss. Foss., vol. iii (1838), p. 108, pl. xix, f. II-13.

Petalodus hastingsii, R. Owen, Odontogr. (I84I), p. 6i, pl. xxii, f. 3-5; ? J. E. Portlock, Rep. Geol. Londonderry, I843, p. 468, pl. xiv, f. 1o; [-ix] J. Morris, Cat. Brit. Foss., 1854, p. 337. [See also P. hastingsia.] [Portion of tooth; Brit. Mus.]

Petalodus rhombus, F. M[Coy, Ann. Mag. Nat. Hist. [2] vol. ii, I 848 , p. 125 ; J. Morris, Cat. Brit. Foss., 1854, p. 337. [Tooth ; olim Capt. Jones Coll.]

Petalodus inequilateralis, J. W. Davis, Trans. Roy. Dublin Soc. [2] vol. i (1883), p. 497, pl. 1x, f. 3, 4. [Teeth; Brit. Mus.]

Loc. U. Carb. Limest.; Durham, Yorkshire, Derbyshire, Fifeshire, and Ayrshire. [Tooth ; olim Murchison Coll.] T.

? acutus: Chomatodus acutus, J. W. Davis, Trans. Roy. Dublin Soc. [2] vol. i (1883), p. 509, pl. lxi, f. 2. ? Petalodus (A. S. Woodward, Cat. Foss. Fishes B. M., pt. i, I889, p. 46.) LOC. L. Carb. Limest.; Armagh. [Tooth; Brit. Mus.]

apicalis (Agassiz), IV. J. Barkas. $\%$. Ctenoptychius apicalis.

davis1, [-ii] A. S. Woodward, Cat. Foss. Fishes B. MI., pt. i, 1889, p. 46.

Loc. L. Carb. Limest.; Armagh. [Teeth : Brit. Mus.]

dentatus, Owen. $v$. Ctenoptychius dentatus.

Gabellula, A. S. Woodward, Cat. Foss. Fishes B. MI., pt. i, 1889, p. 45 , pl. i, f. 8 .

Loc. Carb. Limest.; Shropshire. [Tooth; Brit. Mus.]

grandis, J. W. Davis, Trans. Roy. Dublin Soc. [2] vol. i (1883), p. 496, pl. 1x, f. I.

LOC. L. Carb. Limest.; Armagh. [Imperfect tooth ; Brit. Mus.] 


\section{PETAIODUS (continued)-}

hastingsiæ, L. Agassiz, Poiss. Foss., vol. iii (1 843 ), pp. 174, 384 name only : [-ii] F. M.Coy, Brit. Palæoz. Foss. (I855), p. 635 ; W. H. Baily, Figs. Char. Brit. Foss. (1875), p. 120, pl. xli, f. I3; J. W. Davis, Trans. Roy. Dublin Soc. [2] vol. i ( $\mathrm{ISS}_{3}$ ), p. 493. pl. lix, f. I6, 17, I9 (non f. 18, 20, 21), and Geol. Mas. [3] vol. iii, 1886, p. 149; A. S. Woodward, Cat. Foss. Fishes B. MI., pt. i, ISSg, p. 44. [Non P. hastingsii, Owen; see Petalodus acuminatus and Petalorhynchus psittacinus.]

Petalodus lavissimus, L. Agassiz, Poiss. Foss., vol. iii ( $18_{43}$ ), pp. $174,3_{4}$ (name only).

LOC. L. Carb. Limest.; Armagh and Tyrone.? U. Carb. Limest.; Derbyshire, Yorkshire, and Northumberland. [Teeth.]

inequilateralis, Davis. $\because$. Petalodus acuminatus.

lavissimus, Agassiz. \%. Petalodus hastingsiæ.

? lamelliformis : Cismatodus haneiliformis, J. IV. Davis, Quart. Journ. Geol. Soc., vol. xl, is $S_{4}$, p. 625 , pl. xxvii, f. 23 .

Loc. Yoredale Rocks; IVensleydale, Yorkshire. [Tooth; York Mus.]

linearis (Agassiz), A. S. Woodward, Cat. Foss. Fishes B. M., pt. i, 1889, p. 45

Chomatodus linearis, L. Agassiz, Poiss. Foss., vol. iii (1838), p. 108, pl. xii, f. 6, 9, 10 (?f. 5, 11, non f. 7, 8, 12, 13). (Psammodus on plate.)

LOC. L. Limest. Shales (Bone-bed); Bristol. [Teeth; Bristol Mus.]

? lobatus, Etheridge, jun. थ. Ctenoptychius lobatus.

marginalis, Davis. ข. Polyrhizodus magnus.

psittacinus, M'Coy. ¿'Petalorhynchus psittacinus.

radicans, Agassiz. $\because$ Polyrhizodus magnus.

rectus, Agassiz. \%. Polyrhizodus magnus.

recurvus, J. W. Davis, Trans. Roy. Dublin Soc. [2] vol. i (1883), p. 497 , pl. lx, f. 2.

LOC. L. Carb. Limest.; Bristol. [Tooth; olim Enniskillen Coll.] vhombus, IICOy. \%. Petalodus acuminatus.

sagittatus, $\mathrm{M}^{\circ} \mathrm{Coy} . \approx$ Petalorhynchus psittacinus.

serratus ( $\left.\mathrm{I}^{\prime} \mathrm{Coy}\right)$, Owen. $v$. Ctenoptychius serratus.

(new variety), W. J. Barkas, Monthly Rev. Dentai Surgery, vol. ii, is 7 t, p. 537 , f. xxvii, xxviii.

LOC. Coal-Neas. ; Northumberland.

PETAIORHY NCHUS, T. S. Newbern and A. H. Worthen (ex Agassiz, MS.), Pal. Illinois, vol. ii, I866, p. 32.

? benniei, Etheridge, jun. v. Pristodus benniei. 
PrTAIORHYRCFus (continued)-

psittacinus ( $M 1^{\prime} \mathrm{Coy}$ ), J. Morris and G. E. Roberts, Quart. Journ. Geol. Soc., vol. xviii, I862, p. Ior (name only); J. W. Davis, Rep. Brit. Assoc., 1881 (1882), p. 646, and Trans. Roy. Dublin Soc. [2] vol. i (1883), p. 5 I6, pl. lxi, f. 12-16, and Geol. Mag. [3] vol. iii, I886, p. I49; A. S. Woodward, Cat. Foss. Fishes B. M., pt. i, I889, p. 40.

Petalodus psittacinus, L. Agassiz, Poiss. Foss., vol. iii (1843), pp. 174, 384 (name only); J. Morris, Cat. Brit. Foss., I 54 , p. 337 ; F. M'Coy, Brit. Palzeoz. Foss. (185j), p. 636, pl. iii I, f. 4 . Petalodus sagittatus, L. Agassiz, Poiss. Foss., vol. iii (I843), pp. 174,384 (name only); F. M'Coy, Brit. Palæoz. Foss. (1855), p. 635, pl. iii I, f. 2, 3. [Tooth; Woodwardian Mus.] I'tulodus hastingsice, J. W. Davis (errore), Trans. Roy. Dublin Sic. [2] vol. i (1883), p. 49t, pl. lix, f. I8, 20, 21. [Teeth; Bit. Mus.]

LOC. L. Carb. Limest. ; Armagh. [Tooth; Woodwardian Mus.]

psittacinus, var. minor, A. S. Woodward, Cat. Foss. Fishes B. M., pt. i, is89, p. 41, pl. i, f. 9.

LOC. U. Carb. Limest.; S. Derbyshire. [Tooth; Brit. Mus.]

PzTRODUS, F. M'Coy, Ann. Mag. Nat. Hist. [2] vol. ii, 1848, p. I32, and Proc. Camb. Phil. Soc., vol. i (I848), p. 66.

Ostinaspis, H. Trautschold, Nouv. Mém. Soc. Imp. Nat. Moscou, vol. xiii (1874), p. 298.

patelliformis, F. MI'Coy, Ann. Mag. Nat. Hist. [2] vol. ii, r84S, p. I 32 , and Brit. Palæoz. Foss. (1855), p. 637, pl. iii G, f. 6-8; J. Morris, Cat. Brit. Foss., 1854, p. 337 ; J. W. Davis, Trans. Roy. Dublin Soc. [2] vol. i (1883), p. 400, pl. li, f. I6; A. S. Woodward, Cat. Foss. Fishes B. MI., pt. i, I889, p. 246.

LOC. U. Carb. Limest.; Lancashire and Derbyshire. [Dermal tubercles; Woodwardian Mus.]

$\mathbf{x .}$

sp., A. S. Woodward, Cat. Foss. Fishes B. M., pt. i, I889, p. 246.

LOC. Yoredale Rocks; Todmorden, Lancashire. [Dermal tubercle; Brit. Mus., no. P. 5855.]

I'HACODUS, F. Dixon, Geol. Sussex, I 850, p. 37 I (undefined).

pumetretes, F. Dixon, ibid., p. 37 I, pl, xxx, f. I6 ; J. Morris, Cat. Brit. Foss., I 854 , p. 337 . 'Indeterminable,' A. S. Woodivard, Proc. Geol. Assoc., vol. x (1888), p. 308.

LOC. Chalk; Sussex, [Teeth; Brit. Mus.] T.

s\%, recorded (? errore) by J. Morris, Cat. Brit. Foss., I 854 , p. 337 , from L. Chalk, Charing (Harris Coll.).

PHALACRUS, L. Agassiz, Rep. Brit. Assoc., I844 (1845), p. 30 S (name only).

cybioides, L. Agrassiz, ibit., p. 308 (name only).

LOC. London Clay; Sheppey. [Imperfect head; Brit. Mus.] 


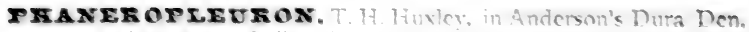

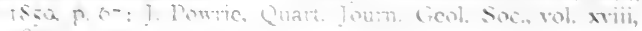
$\operatorname{lis} 2, p, 4=4$

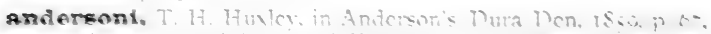

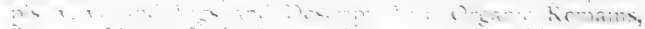

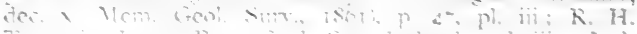

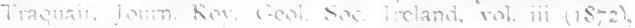

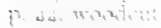

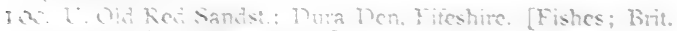

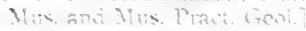

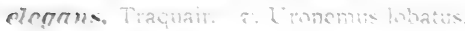

FEA

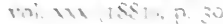

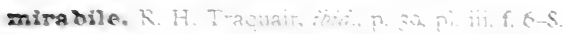

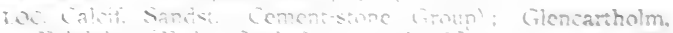

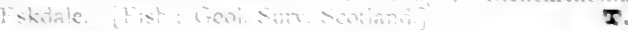

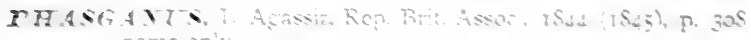
name on!

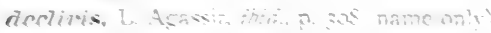

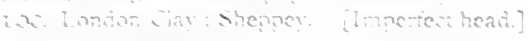

I.

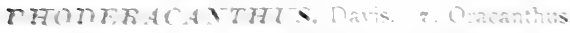

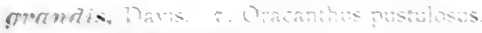

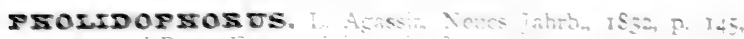

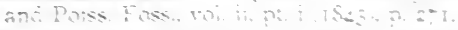

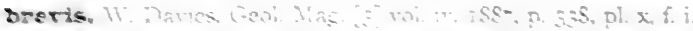

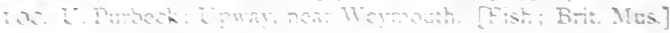

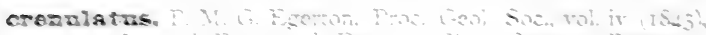

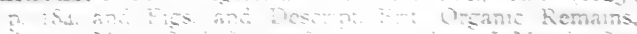

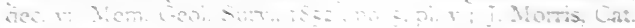

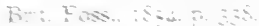

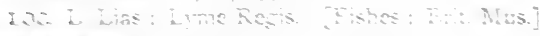

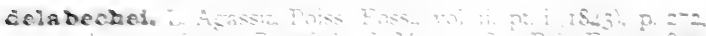

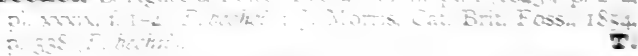

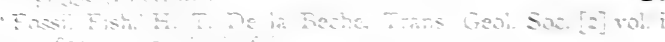

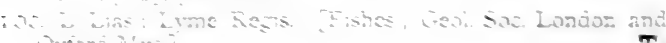

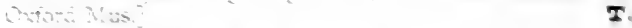

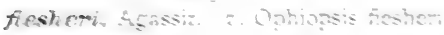

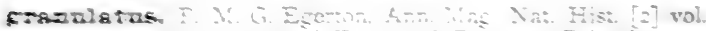

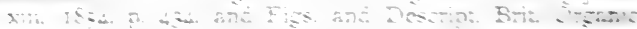

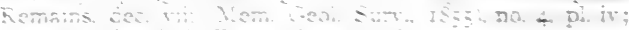

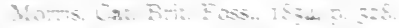

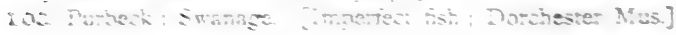


PROLTDOPHOZTS "untinul

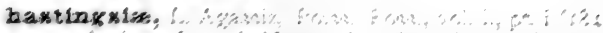
G.t. $x$ int it ?

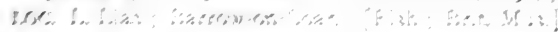

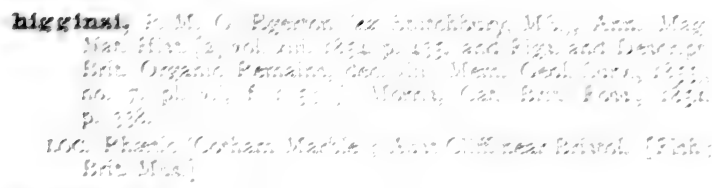

Iatiascaloss.

3.0.

lis:-

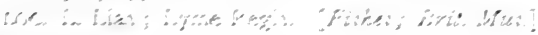

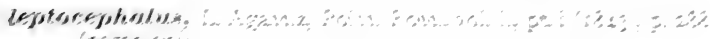

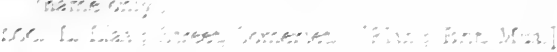

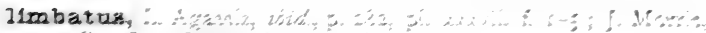

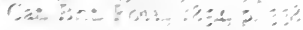

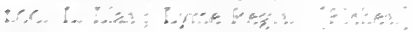

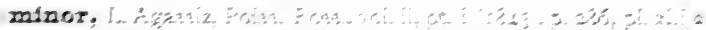

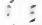

'숭

Anpadirioncres: :5.

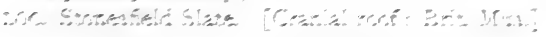

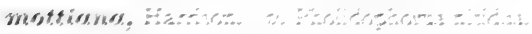

aftidus,

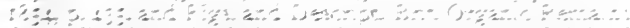

tas

bermis

ir. or.

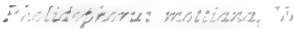

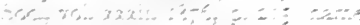

Lerses: in at

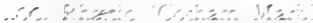

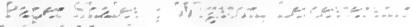

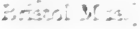

ozgchins.

IL xrax 5

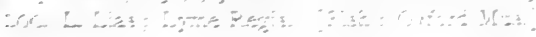




\section{PFOIIDOPXORUS (continued)-}

ornatus, L. Agassiz, Poiss. Foss., vol. ii, pt. i (I843), p. 2So, pl. xxxvii, f. 6, 7 ; J. Morris, Cat. Brit. Foss., I854, p. 338.

LOC. Purbeck; Swanage. [Fish; Brit. Mus.]

pachysomus, P. M. G. Egerton, Figs. and Descript. Brit. Organic Remains, dec. vi (Mem. (reol. Surv., 1852), no. 4, pl. iv; J. Morris, Cat. Brit. Foss., 1854, p. 338.

LOC. L. Lias; Lyme Regis. [Fish ; Brit. Mus.]

purbeckensis, IV. Davies, Geol. Mag. [3] vol. iv, I887, p. 337, pl. x, f. $2-4$, and in R. Damon, Geol. Weymouth, ed. 3, I888, Suppl., pl. xix, f. I.

LOC. L. Purbeck ; I. of Portland. [Fish ; Brit. Mus.]

stricklandi, L. Agassiz, Poiss. Foss., vol. ii, pt. i (1843), p. 284 , pl. xlii $a$, f. 3,4 ; J. Morris, Cat. Brit. Foss., I854, p. 338. LOC. L. Lias ; Barrow-on-Soar. [Fish; Brit. Mus.]

Pholidotus leuchi (Agassiz), Williamson. v. Dapedius leachi.

Pz:OLxDURUs, A. S. Woodward, Proc. Geol. Assoc., vol. xi (1889), p. 3 I.

disjectus, A. S. Woodward, ibid., p. 3I, pl. i, f. 4, 5 .

LOC. U. Chalk; Gravesend. [Portion of tail ; Brit. Mus.]

PHRICACANTIIUS, Davis. \%. Pleuracanthus.

biserialis, Davis. \%. Pleuracanthus cylindricus.

PIEYIIODUS, L. Agassiz, I'oiss. Foss., vol. ii, pt. ii (1843), p. 233 ; I. Cocchi, Annali R. Mus. Fis. Stor. Nat. Firenze, n.s., vol. i (I $866 ;$, p. 89 . [The following species, or probably varieties, are founded upon examples of the pharyngeal dentition obtained from the London Clay of Sheppey, or rarely, in a derived state, from the Red Crag of Suffolk.]

bowerbanki, I. Cocchi, Annali R. Mus. Fis. Stor. Nat. Firenze, n.s., vol. i (1866), p. Ioo, pl. ii, f. 2, 3. [Brit. Mus.]

coiei, I. Cocchi, ibid., p. 9r, pl. i, f. i. [Brit. Mus.]

hexagonalis, I. Cocchi, ibid., p. 92, pl. i, f. 2, 3. [Brit. Mus.]

irregularis, L. Agassiz, Poiss. Foss., vol. ii, pt. ii (1843), p. $24 \mathrm{I}$ (name only); I. Cocchi, Ann. R. Mus. Fis. Stor. Nat. Firenze, n.S., vol. i (IS66), p. I12, pl. iii, f. 7. [Brit. Mus.]

marginalis, L. Agassiz, Puiss. Foss., vol. ii, pt. ii (1843), p. 240, pl. 1xix $\pi$, f. 8,9 ; J. Morris, Cat. Brit. Foss., I854, p. 338 ; I. Cocchi, Ann. R. Mus. Fis. Stor. Nat. Firenze, n.s., vol. i (1866), p. 98, pl. ii, f. i. [Oxford Mus.]

medius, L. Agassiz, Poiss. Foss., vol, ii, pt. ii (I $8+3$ ), p. 24 I (name oniy ; I. Cocchi, Ann. R. Mus. Fis. Stor. Nat. Firenze, n.s., vol. i (1866́), p. I I4, pl. ii, f. 10-12. [Brit. Mus.] 
PYYIIODUS (continued)-

petiolatus, R. Owen, Cat. Mus. R. Coll. Surg., 1850, no. 675; I. Cocchi, Ann. R. Mus. Fis. Stor. Nat. Firenze, n.s., vol, i (1866), p. Io9, pl. iii, f. I.

Phyllodus, R. Owen, Odontogr. (I845), p. I39, pl. xlvii, f. I, 2. [Mus. R. Coll. Surgeons.]

planus, L. Agassiz, Poiss. Foss., vol. ii, pt. ii (I843), p. 239, pl. Ixix $a$, f. 4,5 ; J. Morris, Cat. Brit. Foss., 1854, p. 338 ; I. Cocchi, Ann. R. Mus. Fis. Stor. Nat. Firenze, n.s., vol. i (1866), p. 94, pl. i, f. 4-6. [Oxford Mus.]

polyodus, L. Agassiz, Poiss. Foss., vol. ii, pt. ii (I843), p. 240 , pl. Lxix a, f. 6, 7 ; J. Morris, Cat. Brit. Foss., 1854, p. 338 ; I. Cocchi, Ann. R. Mus. Fis. Stor. Nat. Firenze, n.s., vol. i (1866), p. I07, pl. iii, f. S. [Oxford Mus.]

secundarius, I. Cocchi, ibid. (1866), p. I02, pl. ii, f. 7, pl. vi, f. 2. [Brit. Mus.]

speciosus, I. Cocchi, ibid. (1866), p. 96, pl. i, f. 7, 8, pl. ii, f. $5,6$. [Brit. Mus.]

submedius, I. Cocchi, ibid. (IS66), p. II 8 , pl. ii, f. I4. [13rit. Mus.]

toliapicus, L. Agassiz, I'oiss. Foss., vol. ii, pt. ii (1843), p. 239, pl. lxix $a$, f. I-3; R. Owen, Odontogr. (18.45), p. I38, pl. xliv, f. 2 ; J. Morris, Cat. Brit. Foss., 1854, p. 338 ; I. Cocchi, Ann. R. Mus. Fis. Stor. Nat. Firenze, n.s., vol. i (1866), p. I04, pl. ii, f. S, 9. [Oxford Mius.]

PrỹI0IzPIs, L. Agassiz, l'oiss. Foss. V. G. R. (1344), p. 67. concentricus, L. Agassiz, ibid., p. 67, pl. xxiv, f. I ; J. Morris, Cat. Brit. Foss., I 854 , p. 338 ; W. Davies, Geologist, vol. v, I862, p. 458 ; A. Fritsch, Fauna der Gaskohle, vol. ii, I\&SS, p. 89 , woodcut I69.

LOC. U. Old Red Sandst. ; Clashbennie. Devonian; Meadfoot, Torquay. [Scale; olim Murchison Coll.]

temuissimus, Agassiz. \% Rhizodus hibberti.

Prysonzmus, F. M'Coy (ex Agassiz, MS.), Brit. Paliec l. Mi-s. (1855), p. 638 .

arcuatus, F. $\mathrm{I}^{\circ} \mathrm{Coy}$, Ann. Mag. Nat. Hist. [2] vol. ii, IS 4 , p. 117 , and Brit. Palæoz. Foss. (1855), p. 638 , pl. iii $1, f .29$; J. Morris, Cat. Brit. Foss., 1854, p. 338 ; J. W. Davis, Trans. Roy. Dublin Soc. [2] vol. i (I883), p. 367 , pl. slvii, f. S.

LOC. L. Carb. Limest.; Armagh and Gloucestershire. [Spine; Woodwardian Mus.]

I.

attenuatus, J. W. Davis, ibid., p. 369, pl, xlvii, f. Io.

LOC. L. Carb. Limest.; Armagh. [Spine; olim Enniskillen Coll.]

hamatus (Agassiz), J. W. Davis, ibid., p. 370, pl. xlvii, f. 9, I T, and Quart. Journ. Geol. Soc., vol. xl, 1884, p.617, pl. xxvi, f. 6. 
PExSONgrus hamatus (continued) -

Onchus hamatus, L. Agassiz, Poiss. Foss., vol. iii (1837), p. 9, pl. i, f. 7,8 ; J. Morris, Cat. Brit. Foss., 1854, p. 334.

LOC. L. Carb. Limest.; Gloucestershire. U. Carb. Limest. incl. Yoredale Rocks); Yorkshire. [Spine; Bristol Mus.]

? subteres, L. Agassiz, Poiss. Foss., vol. iii (1843), p. I76 (name only); J. Morris, Cat. Brit. Foss., I854, p. 338 ; F. M'Coy, Brit. Palreoz. Foss. (1855), p. 638, pl. iii I, f. 30 ; J. W. Davis, Trans. Roy. Dublin Soc. [2] vol. i (1883), p. 368, pl. xlvii, f. 12. [Genus doubtful.]

LOC. L. Carb. Limest.; Armagh. [Spine; Geol. Soc. London.]

I'INACODUS, Daris. \%. Copodus.

gelasimus, Davis. v. Copodus angulatus.

gonoplax, Davis. $\because$. Copodus cornutus.

RESODUs, R. Owen, Odontography (1845), p. I 38.

oweni, [-ii] L. Agassiz, l'oiss. Foss., vol. ii, pt. ii (1843), p. 247 (name only); R. Owen, Odontography (1845), p. I38, pl. xlvii, f. 3, and Cat. Foss. Rept. and Pisces Mus. R. Coll. Surgeons, 1854, p. 167.

LOC. London Clay; Sheppey. [Vomerine or parasphenoid teeth; R. Coll. Surgeons.]

T.LACOTHORAX. Agassiz. \%. Bothriolepis.

paradoxus, Agassiz. $\because$. Bothriolepis major.

PZATAX, Cuvier, Règne Animal, vol. ii, 1817, p. 334.

? nuchalis, A. S. Woodward, Ann. Mag. Nat. Hist. [5] vol. xx, I887, p. 342.

Microdon muchalis, F. Dixon, Geol. Sussex, I850, p. 369, pl. xxiii, f. 7 ; J. Morris, Cat. Brit. Foss., I854, p. 333.

LOC. Chalk; Sussex. [Portion of fish ; Brit. Mus.]

woodwardi, [-ii] L. Agassiz, Poiss. Foss., vol. iv (1843), p. 250, pl. xix, f. 3 ; J. Morris, Cat. Brit. Foss., 1854, p. 339 ; E. T. Newton, Vert. Forest Bed (Mem. Geol. Surv., I882), p. I22, pl. xix, f. I-3. Figs. given by S. Woodward, Geol. Norfolk, i $\$ 33$, pl. iii, f. $31-33$. [Some of these fossils may be referable to Gadus.]

LOC. Red and White Crags; Suffolk. Norwich Crag, Weybourn Crag, and Forest Bed; Norfolk. [Various bones.]

PEATESSA, Cuvier, Rìgne Animal, vol. ii, I817, p. 220.

sp., E. T. Newton, Vert. Forest Bed (Mem. Geol. Surv., I882), p. 128.

LOC. Forest Bed; Overstrand, Norfolk. [Maxilla; A. Savin Coll.] 
PLATYACANTIIUS, F. M'Coy, Ann. Mag. Nat. Hist. [2] vol, ii, 1848, p. 120, and Proc. Camb. Phil. Soc., vol. i (1848), p. 65 (Platycanthus).

isosceles, F. M'Coy, Ann. Mag. Nat. Hist. [2] vol. ii, I848, p. I20; J. Aorris, Cat. Brit. Foss., 1854, p. 339. ? Oracanthus (J. W. Davis, Trans. Roy. Dublin Soc. [2] vol. i (1883), p. 525). LOC. L. Carb. Limest.; Armagh. [Dermal plate; Geol. Soc. London.]

PLATYCANTHUS, M'Coy. v. Platyacanthus.

PLATYGNATHUS, Agassiz. v. Glyptolepis, Glyptopomus, and Holoptychius.

jamesoni, Agassiz. v. Holoptychius flemingi.

minor, Agassiz. v. Glyptopomus minor.

pancialens, Agassiz. v. Glyptolepis paucidens.

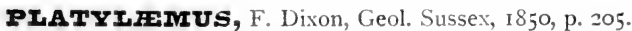

Pseudospherodon, F. Noetling, Abh. Geol. Specialk. Preussen u. Thüring. Staaten, vol. vi, pt. iii, 1885, p. I02.

colei, F. Dixon, Geol. Sussex, I850, p. 205, pl. xii, f. II-I3, pl. x, f. 23 ; J. Morris, Cat. Brit. Foss., I 854, p. 339.

LOC. Bracklesham Beds. [Pharyngeals; Brit. Mus.] T.

PIATYSIAGUMx, P. M. G. Egerton, Figs. and Descript. Brit. Organic Remains, dec, xiii (Mem. Geul. Surv., 1872), no. 6.

grandis : Heterolcpidotus grandis, J. W. Davis, Journ. Linn. Soc., Zoology, vol. xviii, 1885 , p. 293 , pl. vii.

LOC. L. Lias; Lyme Regis. [Fish ; J. W. Davis Coll.]

sclerocephalum, P. M. G. Eyerton, ibid., no. 6, pl. vi, and Quart. Journ. Geol. Soc., vol. xxix, 1873, p. 419.

? Eugnathus polyodon, L. Agassiz, Poiss. Foss., vol. ii, pt. ii (1835), p. ro4, pl. Iviii $a$, f. 2 ; J. Morris, Cat. Brit. Foss., I 854 , p. 326. [Jaw ; Oxford Mus.]

LOC. L. Lias; Lyme Regis. [Fish ; Brit. Mus.]

$\mathbf{T}$.

PIATySomUs, L. Agassiz, Poiss. Foss., vol. ii, pt. i ( 1835 ), pp. 6, I6I ; J. Young, Quart. Journ. Geol. Soc., vol. xxii, I866, p. 302 ; R. H. Traquair, Trans. Roy. Soc. Edinb., vol. $\operatorname{xxix}($ I 879$)$, p. 368.

Teclivis, Agassiz. $\%$. Eurynotus crenatus.

forsteri, A. Hancock and T. Atthey, Ann. Mag. Nat. Hist. [4] vol. ix, I872, p. 254 , pl. xvii, f. 3 , and Nat. Hist. Trans. Northumb. and Durham, vol. iv (I872), p. 413, pl. xv, f. 3 ; R. H. Traquair, Trans. Roy. Soc. Edinb., vol. xxix (1879), p. 369 , pl. vi, f. Iо.

LOC. Coal-Meas. ; Newsham, Northumberland. [Imperfect fish ; Newcastle-upon-Tyne Mus.] 


\section{PIATYSOMUS (continue. 7 ) -}

macrures, Agassiz. \% Globulodus macrurus.

rarvulus, L. Agassiz, Poiss. Foss., vol. ii, pt. i (1843), p. 303 (name only); J. Morris, Cat. Brit. Foss., 1854, p. 339 ; J. Young, Quart. Journ. Geol. Soc., vol. xxii, I866, p. 303 , pl. xxi, f. 2 ; W. C. Williamson, Thil. Trans., I 849 , p. 449 , pl. xli, f. I2-I4; J. Ward, [Proc.] N. Staffs. Nat. Field Club, 1875 , p. 235 , and Trans. N. Staffs. Inst. Mining Engin., vol. x (1889), pl. viii, f. I-4; R. H. 'Traquair, Trans. Roy. Soc. Edinb., vol. xxix (i 879), p. 369, pl. vi, f. 3-9, I I.

LoC. Coal-Neas.; Northumberland, Staffordshire, and Yorkshire. [Portions of fishes; Leeds Mus.]

premers, Agassiz. v. Platysomus striatus.

rotundus, A. Hancock and T. Atthey, Ann. Mag. Nat. Hist. [4] vol. ix, 1872 , p. 252 , pl. xvii, f. 2 , and Nat. Hist. Trans. Northumb. and Durbam, vol. iv (I872), p. 4Ir, pl. $x v$, f. 2.

Loc. Coal-Meas.; Newsham. [Fish; Newcastle-upon-Tyne Mins.]

striatus, L. Agassiz, Poiss. Foss., vol. ii, pt. i (I 835 ), p. 168, pl. xvii, f. $1-4$; W. King, Permian Foss. (Pal. Soc., I 850), p. 23I, pl. xxvii, f. I, pl. xxviii, f. I ; J. Morris, Cat. Brit. Foss., I 854 , p. 339 ; F. M 'Coy, Brit. Palæoz. Foss. (I855), p. 6I4; R. H. Traquair, Trans. Koy. Soc. Edinb., vol. xxix (I879), p. 369, pl. vi, f. I, 2. [See also $P$. tenuistriatus.]

'Flat Fish,' W. R. Clanny, Annals Philosophy, vol. vi, ISI5, p. II 5 (with plate). [Fish; Sunderland Mus.]

'Fish (? Chatodont)', N. J. Winch, Trans. Geol. Soc., vol. iv, (i8I7), p. 9, pl. ii.

'Fossil Fish,' A. Sedgwick, Trans. Geol. Soc. [2] vol. iii (IS29), pl. xii, f. 3,4 .

Platysomus paruus, L. Agassiz, Poiss. Foss., vol. ii, pt. i (IS35), p. I 7o, pl. xviii, f. 3 .

Loc. L. Magnesian Limest. ; Pallion Quarry, Sunderland. MarlSlate; Cullercoats and Midderidge. [Fish; Newcastleupon-Tyne Mus.]

superbus, R. H. 'Traquair, Trans. Roy. Soc. Edinb., vol. xxx, I881, p. 58 , pl. vi.

LOC. Calcif. Sandst. (Cement-stone Group); Glencartholm, Eskdale, Dumfriesshire. [Fish ; (Geol. Surv. Scotland.]

tenuistriatus, R. H. Traquair, Trans. Roy. Soc. Edinb., vol. xxix (1879), p. 369.

? Platysomus striatus, J. Young (non Agassiz), Quart. Journ. Geol. Soc., vol. xxil, I 866 , p. 305.

LOC. L. Coal-Neas. (1)alemoor-Rake Ironstone); Stanton-byDale, Derbyshire. [Fish; Mus. Pract. Geol.] 
PLECTRODUS, Agassiz. v. Eukeraspis.

mirabilis, Agassiz. v. Eukeraspis pustulifera.

pleiop'istis, Agassiz. \%. Eukeraspis pustulifera.

pustulifeu,s (Agassiz), Lankester. \%. Eukeraspis pustulifera.

PLECTROLEPIS, Agassiz. \%. Eurynotus.

mugosus, Agassiz. \%. Eurynotus crenatus.

PLEIOPTERU', Agassiz. \% Osteolepis.

PIETHODUs, F. Dixon, Geol. Sussex, 1850, p. 366; A. S. Woodward, Proc. Geol. Assoc., vol. x (1888), p. 33 I.

expansus, F. Dixon, Geol. Sussex. I 850 , p. 366, pl. xxxiii, f. 2 ; J. Morris, Cat. Brit. Foss., I 854, p. 339 ; A. S. Woodward, Proc. Geol. Assoc., vol. x (1888), p. 331.

LOC. Chalk and Gault ; S. F. England. Cambridge Greensand. [Tooth; Brighton Mus.]

oblongus, F. Dixon, Geol. Sussex, I\$50, p. 366, pl. xxxii", f. 4 ; J. Morris, Cat. Brit. Foss., I 854 , p. 339 ; A. S. Woodward, Proc. Geol. Assoc., vol. x (1888), p. 33 I.

LOC. Chalk; S. E. England. [Tooth; Brighton Mus.]

$\mathbf{T}$

PIEURACANTrits, L. Agassiz, l'oiss. Foss., vol. iii ( I 837), p. 66.

Orthacanthus, L. Agassiz, Poiss. Foss., vol. iii (I843), pl. xlv.

Compsacanthus, J. S. Newberry, Proc. Acad. Nat. Sci. Philad., 1856, p. 100.

Lophacanthus, T. Stock, Ann. Mag. Nat. Hist. [5] vol. v, 1880, p. 217.

Anodontacanthus, J. W. Davis, Quart. Journ. Geol. Soc., vol. xxxvii, I88I, p. 427.

? Phricacanthus, J. W. Davis, ibia., vol. xxxv, I879, p. I86.

alatus, J. W. Davis, Quart. Journ. Geol. Soc., vol. xxxvi, I880, p. 329 , pl. xii, f. 4 ; J. Ward, Trans. N. Staffs. Inst. Mining Engin., vol. x (I889), p. I36.

LOC. M. Coal-Meas.; Tingley, Yorkshire. Coal-Meas.; North Staffordshire. [Spine; J. W. Davis Coll.]

alternidentatus, J. W. Davis, ibid., p. 328, pl. xii, f. 3.

LOC. Coal-Meas.; Middleton, near Leeds. [Spine ; Leeds MIus.]

altus, R. Etheridge, Foss. Brit. Islands, pt. i, r888, p. 337: misprint for alatus.

attenuidentatus, R. Etheridge, iöid., p. 357: misprint for altcmidentatus.

cylindricus (Agassiz), J. W. Daris, Quart. Journ. Geol. Soc., vol. xxxvi, I880, p. 33r, woodcut; $A$. S. Woodward, Cat. Foss. Fishes B. M., pt. i, I\$89, p. S.

Orthacanthus cylindriuz, L. Agassiz, Loiss. Foss., vol. iii $\left(\mathrm{I} \varepsilon_{+3}\right)$, p. 177 , pl. xlv, f. 7-9 (no description); J. Morris, Cat. Brit. Foss., I 854 , p. 335 ; ]. Ward, [1'roc. N. Staffs. Nat. Field 
PIEURACANTrus cylindricus (continued) -

Club, 1875 , p. 217 , and Trans. N. Staffs. Inst. Mining Engin., vol. x (1889), p. 137.

T. (Orthacanthus.)

Orthocanthus, T. P. Barkas, Coal Meas. P'alaont., I873, p. 20, f. $39-42$.

? Phricacanthus biserialis, J. II. Davis, Quart. Journ. Geol. Soc., vol. xxxv, 1879, p. I86, pl. x, f. 16, 17. J. W. Davis Coll.] [Abraded spine;

Lophacanthus taylori, T. Stock, Ann. Mag. Nat. Hist. [5] vol. v, I880, p. 217, woodcut. [Abraded spine; J. Taylor Coll.]

T. (Lophacanthus.)

? Anodontacunthus acutus, J. W. Davis, Quart. Journ. Geol. Soc., vol. xxxvii, I88I, p. 428, pl. xxii, f. Io. [Abraded spine; J. W. Davis Coll.] T. (Anodontacanthus.)

Pleuracanthus (Orthaianthus) cylindricus, R. H. Traquair, Geol. Mag. [3] vol. v, I888, p. IOI.

LOC. Coal-Meas.; England and S. Scotland. [Spine.]

denticulatus, J. WV. Davis, Quart. Journ. Geol. Soc., vol. xxxvi, I 880, p. 334 , pl. xii, f. 7 .

Loc. L. Coal-Meas. (Better-bed Coal); Clifton, near Halifax. [Spine ; J. W. Davis Coll.]

elegans, R. H. Traquair, Geol. Mag. [2] vol. viii, ISSI, p. 36.

Loc. M. Carb. Limest. (Blackband Ironstone); Borough Lee, Edinburgh. [Spine ; Edinburgh Mus.]

erectus, Davis. \%. Pleuracanthus levissimus.

gibbosus, Ward. ¿. Diplodus gibbosus.

gracillimus, R. H. Traquair, Geol. Mag. [2] vol. ix, I882, p. 540. LoC. M. Carb. Limest. (Blackband Ironstone); Borough Lee, Edinburgh. [Spine; Edinburgh .Mus.]

horridulus, R. H. Traquair, Geol. Mag. [2] vol. ix, I882, p. 54 I. LOC. M. Carb. Limest. (Blackband Ironstone); Borough Lee, Edinburgh. [Spine; Edinburgh Mus.]

1ævissimus, L. Agassiz, Poiss. Foss., vol. iii $\left(\mathrm{I}_{37} \mathrm{j}\right.$, p. 66, pl. xlv, f. 4, 5; J. Morris, Cat. Brit. Foss., I854, p. 339 ; J. Ward, [Proc.] N. Staffs. Nat. Field Club, 1875 , p. 225, and Trans. N. Staffs. Inst. Mining Engin., vol. x (1889), p. 134, pl. iii, f. 8 ; J. W. Davis, Quart. Journ. Geol. Soc., Vol. xxxvi, 1880, p. 325 ; R. H. Traquair, Geol. Mag. [3] vol. v, 1888, p. IOI ; A. S. Woodward, Cat. Foss. Fishes I3. M., pt. i, 1889, p. 5.

Pleuracanthus planus, L. Agassiz, Poiss. Foss, vol. iii (1843), p. 177 (name only); J. IV. I) avis, (guart. Journ. Geol. Soc., vol. xxxvi, I8So, p. 329. [Spine; Hrit. Mus.]

Compsacanthus major, J. W. Davis, itidt. p.62, woodcut. [Spine; J. W. Davis Coll.]

Pleuracanthus erectus, J. W. Daris, ibil., p. 326, woodcut. [Spine; J. W. Davis Coll.] 
PIEURACANTHUS lævisgimus (continued) -

Pleuracanthus pulchellus, J. WV. Davis, ibid., p. 327 , pl. xii, f. 2. [Spine ; J. WV. Davis Coll.]

Anodontacanthus fastigiatus, J. WV. Davis, ibid., vol. xxxvii, I88 I, p. 428, pl. xxii, f. 12. [Spine.]

? Anodontacanthus obtusus, J. W. Davis, ibid., p. 428, pl. xxii, f. I I. [Spine ; J. W. Davis Coll.]

Pleuracanthus, T. P. Barkas, Coal Meas. Palæont., 1873, p. I7, f. $14-16$.

LOC. Coal-Meas. ; England and S. Scotland. [Spine.] T.

plamus, Agassiz. थ. Pleuracanthus levissimus.

pulchellus, Davis. \%. Pleuracanthus lævissimus.

robustus, J. IV. Davis, Quart. Journ. Geol. Soc., vol. xxxvi, I880, p. 330 , pl. xii, f. 5 ; A. S. Woodward, Cat. Foss. Fishes B. M., pt. i, I889, p. 7 ; J. Ward, Trans. N. Staffs. Inst. Mining Engin., vol. x (1889), p. 136.

? Compsacanthus triungularis, J. WV. Davis, Quart. Journ. Geol. Soc., vol. xxxvi, ISSO, p. 62, woodcut. [Spine; J. W. Davis Coll.]

LOC. M. Coal-Meas.; Yorkshire. Coal-Meas.; North Staffordshire. [Spine; J. W. Davis Coll.]

tenuis, J. IV. Davis, Quart. Journ. Geol. Soc., vol. xxxvi, I88o, p. 327, pl. xii, f. I.

Loc. L. Coal-Meas. (Better-bed Coal); Clifton, near Halifax. [Spine; J, W. Davis Coll.]

wardi, J. W. Davis, ibid., p. 334, pl. xii, f. 6 ; J. Ward, Trans.

N. Staffs. Inst. Mining Engin., vol. x (1889), p. 136.

LOC. Coal-Meas. (Ragmine); Fenton. [Spine; J. Ward Coll.]

PLEUTODUS (Agassiz), Hancock and Atthey. v. Pleuroplax.

affinis, Agassiz. \%. Pleuroplax rankinei.

fulcutus, Traquair. \%. Pleuroplax falcatus.

rankinei (Agassiz), Hancock and Atthey. $v$. Pleuroplax rankinei. woorli, Davis. v. Pleuroplax woodi.

PLEUROGOMPHUS, Davis. \%. Copodus.

auriculatus, Davis. $\approx$. Copodus auriculatus.

PIEUROPHOxIS, I. M. ( . Egerton, Figs. and Descript. Brit. Organic Remains, dec. ix (Mem. Geol. Surv., 1858), no. 7.

attenuata, [-us] P. MI.G. Egerton, ihid., no. 7, pl. vii, f. I ; J. Morris, Cat. Brit. Foss., 1854, p. 329 (Egerton, MS.)

Loc. M. Purbeck; Apsel Lane, near Sutton Mandeville. [Fish.]

crassicauda, [-us] P. MI. G. Egerton, Figs. and Descript. Lrit. Organic Remains, dec. ix (Mem. Geol. Surv., I8;8), no. 7, pl. vii, f. 2.

Loc. M. Purbeck; Durdlestone Bay, Swanage. [Fish; P. B. Brodie Coll.] 
PIIUROProzis (continued)-

longicauda, [-us] P. M. G. Egerton, ibil., no. 7, pl. vii, f. 4 .

LOC. M. Purbeck; Durdlestone Bay. [Fish; P. B. Brodie Coll.]

serrata, [-us] P. M. G. Escrton, ibil., no. 7, pl. vii, f. 5-9.

LOC. Purbeck; Hartwell, near Aylesbury. [Imperfect fishes; olim John Lee Coll., Hartwell.]

PIפUIOPIAX, A. S. Woodward, Cat. Foss. Fishes B. M., pt. i, I889, p. 173 .

Pievrodes, A. Hancock and T. Atthey (cx Agassiz, MS.) (non Pleurodon, Wood, 1840, nec Harlan, I842), Ann. Mag. Nat. Hist. [4] vol. ix, 1872 , p. 249.

attheyi (Barkas', A. S. Woodward, Cat. Foss. Fishes B. M., pt. i, I889, p. I75, pl. vi, f. IO, II ; J. Ward, Trans. N. Staffs. Inst. Mining Engin., vol. x (1889), pl. ii, f. 9.

Pacilodus angustus, L. Agassiz, Poiss. Foss., vol. iii (IS43), p. 174 (name only).

Pacilodus attheyi, WV. J. Barkas, Monthly Rev. Dental Surgery, vol. iii, 1874 , p. 57 , f. xl-xliv.

Loc. Coal-Meas. ; Northumberland and Lanarkshire. [Tooth; IV. T. Barkas Coll.]

fulcuta: Pleurodus fulcutus, R. H. Traquair, Trans. Edinb. Geol. Soc., vol. v (IS87), p. 3i 3 (name only).

Loc. Calcif. Sandst.; Abden, Fife. [Spine.]

rankinei (Hancock and Atthey), A. S. Woodward, Cat. Foss. Fishes B. M., pt. i, I 889 , p. I73; J. Ward, Trans. N. Staffs. Inst. Mining Engin., vol. x (I889), pl, ii, f. 8, Io.

Pleurodus runkinei, L. Agassiz, Poiss. Foss., vol. iii (IS43), p. I 74 (name only); [-ii] J. Morris, Cat. Brit. Foss., I 854, p. 339 ; A. Hancock and T. Atthey, Ann. Mag. Nat. Hist. [4] vol. ix, 1872 , p. 249 , pl. xvii, f. 1 ; J. Ward, [Proc.] N. Staffs. Nat. Field Club, 1875 , p. 222 , f. 13 .

Pleurodus affinis, L. Agassiz, Poiss. Foss., vol. iii (1843), p. 174 (name only); J. Niorris, Cat. Brit. Foss., I854, p. 339 ; J. W. Salter (ex Egerton, MS.) Foss. S. Weish Coalfield (Mem. Geol. Surv. - Iron Ores Gt. Brit., pt. iii, I86I), p. 225, pl. i, f. I8, ig (figs. only); J. Ward, [Proc.] N. Staffs. Nat. Field Club, I875, p. 223 ; J. W. Davis, Quart. Journ. Geol. Soc., vol. xxxii, 1876 , p. 337 , and ibit., vol. xxxv, I879, p. I 81 , pl. $x$, f. I-II. [Teeth.]

Pleurodus, T. P. Barkas, Coal Meas. Palkont., I 873 , p. I 8 , f. 24-27; W. I. Barkas, Monthly Rev. Dental Surgery, vol. iii, 1874, p. 6, f, xxxri-xxxix.

LOr. Coal-Meas.; N. and S. Wales, Staffordshire, Yorkshire, Northumberland, and S. Scotland. [Anterior portion of fish; Newcastle-upon-Tyne .11us.] 
PIEUROPIAX (continued)-

woodi (Davis), A. S. Woodward, Cat. Foss. Fishes B. M., pt. i, 1889, p. 175 .

Pleurodus reoodi, J. W. Davis, Trans. Roy. Dublin Soc. [2] vol. i (1883), p. 458 , pl. lix, f. I2-15, and Quart. Journ. Geol. Soc., vol. xl, is84, p. 6z4, pl. xxvii, f. I4-I7, and Geol. Mag. [3] vol. iii, 1886, p. 156; [-ii] R. H. Traquair, Trans. Edinb. Geol. Soc., vol. v (I887), p. 313 .

Pleurodus sp., Wr. J. Barkas, MLonthly Rev. Dental Surgery, vol. iii, I874, p. 9.

LOC. Calcif. Sandst.; Abden, Fife. U. Carb. Limest.; Yorkshire and Derbyshire. [Teeth; York MIus.]

PIINTy OPIORUS, A. C. L. C. Gïnther, Geol. Mag., vol. i, I 864 , p. II 5 .

robustus, A. C. L. G. Günther, ibid., p. 115, pl. vi ; A. S. Woodward, Proc. Geol. Assoc., vol. x (1888), p. 319.

LOC. L. Chalk; Kent. [Fish; Brit. Mus.]

$\boldsymbol{T}$.

PNIGEACAN'THUS, St. John and Worthen. ¿. Oracanthus.

PODOCEPHALUS, L. Agassiz, Poiss. Foss., vol. v, pt. ii (I843), p. 139 (name only), and Rep. Brit. Assoc., I844 (1845), p. 307 (name only).

mitidus, L. Agassiz, ibid. (1843), p. I39 (name only), and ibid., I 844 , p. 307 (name only).

LOC. London Clay; Sheppey. [Imperfect head; Brit. Mus.] T.

PODODUS, Agassiz. $\%$ Mesolepis.

cupitatus, Agassiz. \%. Mesolepis sp.

PCrimodus, F. M'Coy (ex Agassiz), Brit. Palreoz. Foss. (I855), p. 638 ; emend., A. S. Woodward, Cat. Foss. Fishes B. M., pt. i, 1889, p. 201 .

aliformis, M'Coy. $\tau^{\circ}$. Deltodus aliformis.

angustus, Agassiz. v. Pleuroplax attheyi and $\mathrm{X} y$ strodus striatus. attheyi, Barkas. $\tau$. Pleuroplax attheyi.

corrugetus, Davis. ¿'. Pcecilodus jonesi.

clongretus, R. H. Traquair, Trans. Edinb. Geol. Soc., vol. v (1887), p. 3it name only.

LOC. Calcif. Sandst.; Abulen, Fife. [Tooth.]

fureolutus, R. Etheridge, Foss. Brit. Islands, pt. i, 1S8S, p. 338 : misprint for forecolitus.

foveolatus, F. M'Coy, Ann. Mag. Nat. Hist. [2] vol. ii, 1848 , p. 129, and 13rit. Palæoz. Foss. (185j), p. 639, pl. iii G, f. I i ; J. Morris, Cat. Brit. Foss., 1854, p. $340 ;$ J. W. Davis, Trans. Roy. Dublin Soc. [2] vol. i (1883), p. 445, pl. liii, f. 26.

Loc. Carb. Limest.; Derbyshire. [Tooth; Woodwardian Mus.] 
PacrIodus (continued) -

gibbosus, J. W. Davis, Trans. Roy. Dublin Soc. [2] vol. i (I883), p. 445 , pl. liii, f. 27 ; A. S. Woodward, Cat. Foss. Fishes B. Mi., pt. i, I889, p. 204, pl. vi, f. 23.

LOC. L. Carb. Limest.; Armagh and Sligo. [Tooth; Brit. Mus.]

Jonesi, [-ii] L. Agassiz, Poiss. Foss., vol. iii (I843), p. I74 (name only); [-ii] J. E. Portlock, Rep. Geol. Londonderry, I 843, p. 468, pl. xiv a, f. 6 (fig. only); J. Morris, Cat. Brit. Foss., I 854, p. 340 ; [-ii] F. M'Coy, Brit. Palæoz. Foss. (I 855), p. 639 ; [-ii] J. W. Davis, Trans. Roy. Dublin Soc. [2] vol. i (1883), p. 442, pl. liii, f. 20, 2 I, 23 (non f. 22), and Geol. Mag. [3] vol. iii, I886, p. I 52 ; [-ii] A. S. Woodward, Cat. Foss. Fishes B. M., pt. i, I889, p. 202. [See also Deltodus sublavis.] Pecilodus obliquus, L. Agassiz, Poiss. Foss., vol. iii (1843), p. 174 (name only); J. Morris, Cat. Brit. Foss., 1854, p. 340; F. M'Coy, Brit. Palcoz. Foss. (I 855), p. 640, pl. iii I, f. 5 ; J. W. Davis, Trans. Roy. Dublin Soc. [2] vol. i (1883), p. 443 , pl. liii, f. 24. [Tooth; Woodwardian Mus.]

Pacilodus transwersus, L. Agassiz, Poiss. Foss., vol. iii (1843), p. 174 (name only); J. E. Portlock, Rep. Geol. Londonderry, 1843 , p. 468 , pl. xiv $a$, f. 7 (fig. only); J. Morris, Cat. Brit. Foss., 1854, p. 340. [Imperfect tooth; Brit. Mus.]

Pacilodus corrugatus, J. W. Davis, Trans. Roy. Dublin Soc. [2] vol. i (1883), p. 444, pl. liii, f. 25, and Quart. Journ. Geol. Soc., vol. xl, I884, p. 625 , pl. xxvii, f. 21 . [Imperfect teeth; York Mus.]

LOC. L. Carb. Limest.; Armagh and Ayrshire. U. Carb. Limest.; Northumberland, Yorkshire, and Derbyshire. [Teeth; Woodwardian Mus.]

T.

obliquus (Agassiz), M'Coy. $\%$ Pocilodus jonesi.

parallelus (Agassiz), $\mathrm{M}^{\prime} \mathrm{C}$ oy. \%. Deltodus sublævis.

sublavis (Agassiz), M'Coy. थ. Deltodus sublævis.

trunsversus, Agassiz. v. Pœcilodus jonesi.

POLYPHRACTUS, Agassiz. \%. Dipterus.

platycephalus, Agassiz. $\%$ Dipterus valenciennesi.

POLYPLOCODUS, Pander. \%. Cricodus.

POIy RHIzODUs, F. M'Coy, Ann. Mag. Nat. Hist. [2] vol. ii, 1848 , p. 125, and Proc. Camb. Phil. Soc., vol. i (1848), p. 65.

Dactylodus, J. S. Newberry and A. H. Worthen, Pal. Illinois, vol. ii, 1866, p. 33 .

attenuatus, J. W. Davis, Trans. Roy. Dublin Soc. [2] vol. i (1883), p. 505, pl. lx, f. I4 ; A. S. Woodward, Cat. Foss. Fishes B. Mi., pt. i, 1889, p. 58 .

IOC. L. Carb. Limest.; Armagh. [Tooth; Brit. Mus.] 
POIYRHIZODUS (continued) -

cole1, J. W. Davis, Trans. Roy. Dublin Soc. [2] vol. i (I883), p. 502, pl. lx, f. 9, I0, and Quart. Journ. Geol. Soc., vol. xl, 1884, p. 622, pl. xxvii, f. 13 ; A. S. Woodward, Cat. Foss. Fishes B. M., pt. i, I889, p. 57.

LOC. L. Carb. Limest.; Armagh. Yoredale Rocks; Wensleydale, Yorkshire. [Tooth; Brit. Mus.]

constrictus, Davis. \%. Polyrhizodus magnus.

elongatus, J. W. Davis, Trans. Roy. Dublin Soc. [2] vol. i (1883), p. 503, pl. 1x, f. 16 ; A. S. Woodward, Cat. Foss. Fishes B. M., pt. i, I 889 , p. 58.

LOC. L. Carb. Limest.; Armagh. [Tooth ; Brit. Mus.]

magnus, F. M'Coy, Ann. Mag. Nat. Hist. [2] vol. ii, I848, p. 126, and Brit. Palæoz. Foss. (1855), p. 64I, pl. iii k, f. 6-8; A. S. Woodward, Cat. Foss. Fishes B. M., pt. i, 1889, p. 57.

Petalodus radicans, L. Agassiz, Poiss. Foss., vol. iii (I 843 ), pp. 174, 384 (name only).

?Petalodus marginalis, L. Agassiz, ibid., pp. 174, 384 (name only).

Polyrhizodus radicans, J. Morris, Cat. Brit. Foss., I854, p. 340; J. W. Davis, Trans. Roy. Dublin Soc. [2] vol. i ( $\left(8 S_{3}\right)$, p. 500, pl. lx, f. 7,8 .

Polyrhizodus constrictus, J. W. Davis, ibid., p. 506, pl. 1x, f. 15. [Tooth ; Brit. Mus.]

? Petalodus rectus, L. Agassiz, Poiss. Foss., vol. iii (1843), pp. I74, 384 (name only); J. E. Portlock, Rep. Geol. Londonderry, 1843, p. 468, pl. xiv, f. 9 ; J. Morris, Cat. Brit. Foss., I 854 , p. 337 .

LOC. L. Carb. Limest.; Armagh. [Tooth.]

$\mathbf{T}$.

pusillus, $\mathrm{M}^{\prime} \mathrm{Coy}$. $v$. Helodus pusillus.

ralicans, Davis. ข. Polyrhizodus magnus.

sinuosus, J. W. Davis, Trans. Roy. Dublin Soc. [2] vol. i ( $188_{3}$ ), p. 504 , pl. lx, f. II-I3 ; A. S. Woodward, Cat. Foss. Fishes B. M., pt. i, 1889, p. 58 .

Loc. L. Carb. Limest.; Armagh. [Tooth ; Brit. Mus.]

sp., A. S. Woodward, Cat. Foss. Fishes B. M., pt. i, 1889, p. 59. LOC. Carb. Limest.; Oreton, Shropshire. [Imperfect tooth; Brit. Mus., no. P. 227 a.]

POMOGNATIIUS, F. Dixon, Geol. Sussex, I 850, p. 367 ; A. S. Woodward, Proc. Geol. Assoc., vol. x (1888), p. 317.

Phylactocephalus, J. W. Davis, Trans. Roy. Dublin Soc. [2] vol. iii (1887), p. 605.

eupterygius, F. Dixon, Geol. Sussex, 1850, p. 367, pl. xxxv, f. 6,7 ; J. Morris, Cat. Brit. Foss., 1854, p. 340 ; A. S. Woodward, Proc. Geol. Assoc., vol. x (1888), p. 318. 
POMOGNATIXS eupterygius (continued)-

Osmeroides lewesiensis, L. Agassiz (errore), Poiss. Foss., vol. v (I843), pl. lx b, f. 3, 4. [Head; Brit. Mus.]

LOC. Chalk ; S. E. England. [Fish; Brighton Mus.]

POMOPHR ICTUS, L. Agassiz, Rep. Brit. Assoc., 1844, p. 307 (name only).

egertoni, L. Agassiz, ibid., p. 307 (name only).

LOC. London Clay; Sheppey. [Imperfect head; Brit. Mus.]

POzTrevs, E. D. Cope, Proc. Amer. Phil. Soc., i871, p. 173, and ibid., 1872 , p. 333 .

Megalodon, L. Agassiz (non Sowerby), Poiss. Foss., Feuill., p. 55 (1835) (pars).

Hypsodon, L. Agassiz, Poiss. Foss., Feuill., p. I04 (1837), and vol. v, pt. i (1843), pp. 8, 99 (pars).

daviesi, [-ii] E. T. Newton, Quart. Journ. Geol. Soc., vol. xxxiii, 1877, p. 5 I1, pl. xxii, f. 13.

LOC. L. Chalk; Kent. [Maxilla ; Brit. Mus.]

gaultinus, E. T. Newton, ibid., p. 512, pl. xxii, f. I-I2, and woodcut.

LOC. Gault ; Folkestone. [Head, etc.; Mus. Pract. Geol.]

mantelli, E. T. Newton, ibid., p. 510.

'Unknown Fish,' G. A. Mantell, Foss. S. Downs, I822, p. 24I, pl. xlii.

Megalodon sauroides, L. Agassiz, Poiss. Foss., Feuill., p. 55 (I 835).

Megalodon? lewesiensis, G. A. Mantell (pars), Descript. Cat. Geol., etc., Mus. Sussex Sci. and Lit. Inst., Brighton, ed. 5 (1836), p. 30.

Hypsodon lervesiensis, L. Agassiz, Poiss. Foss., vol. v (I843), pp. 8, 99, pl. xxv b, t. I, 2 .

LOC. Chalk; S. E. England. [Portions of jaws; Brit. Mus.]

sp. ind., E. T. Newton, Quart. Journ. Geol. Soc., vol. xxxiii, 1877 , p. 510.

Hypsodon lewesiensis, L. Agassiz, Poiss. Foss., vol. v (1843), p. 99, pl. $x x v 6$, f. 3 .

LOC. Chalk ; Lewes. [Portion of jaw ; Brit. Mus.]

PRIONOIEPIS, P. M. G. Egerton, in F. Dixon, Geol. Sussex, 1850, p. 368.

Aspidopleurus, F. J. Pictet and A. Humbert, Nouv. Rech. Poiss. Foss. Mt. Liban, 1866, p. I09.

angustus, P. M. G. Egerton, in F. Dixon, Geol. Sussex, 1850, pl. xxxii*, f. 3 ; J. Morris, Cat. Brit. Foss., 1854, p. 340 ;

A. S. Woodward, Proc. Geol. Assoc., vol. x (1888), p. 306.

LOC. U. and L. Chalk; S. E. England. [Scales; Brit. Mus.] T. 
PRISTACANTrt $\mathbf{3}$, L. Agassiz, Poiss. Foss., vol. iii (I 837), p. 35. securis, L. Agassiz, ibid., p. 35, pl. viii $a$, f. II-I3; J. Morris, Cat. Brit. Foss., 1854, p. 340 ; J. l'hillips, Geol. Oxford, 1871, p. 178, woodcut xxxviii, f. Io.

LOC. Stonesfield Slate. [Portion of spine; R. Coll. Surgeons Mus.]

T.

PRISTICLADODUS, M'Coy. $\%$. Dicrenodus.

concimmus, Davis. \%. Pristodus concinnus.

lentutus, $\mathrm{M}^{\prime} \mathrm{Coy}$. $v$. Dicrenodus dentatus.

goughi, $\mathrm{M}^{6} \mathrm{Coy}$. v. Dicrenodus goughi.

PRISTIS, J. Latham, Trans. Linn. Soc., vol. ii (I794), p. 276.

reutidens, L. Agassiz, Poiss. Foss., vol. iii (1843), p. 382** (name only).

LOC. Bagshot Sands. [Rostral tooth.]

bisulcata, [-us] L. Agassiz, ibid., p. $382^{*}$, pl. xli ; [-us] J. Morris, Cat. Brit. Foss., I 854 , p. 340 ; [-us] A. S. Woodward, Cat. Foss. Fishes B. M., pt. i, 1889, p. 73 .

Pristis hastingsia, L. Agassiz, Poiss. Foss., vol. iii (1843), p. $382 *$ (name only). [Rostral tooth ; Brit. Mus.]

Pristis, F. Dixon, Geol. Sussex, I850, pl. xii, f. 6, 7.

Pristis? bisulcatus, W. Dames, Sitz. math-phys. Cl. k. preuss. Akad. Wiss., I883, pt. i, p. I39.

Loc. Bracklesham Beds; Sussex. Barton Clay; Hampshire. [Portion of rostrum ; Brit. Mus.]

contorta, [-us] F. Dixon, Geol. Sussex, r850, p. 202, pl. xii, f. 9, 10 (misprinted $P$. distortus on p. III); [-us] J. Morris, Cat. Brit. Foss., I 854 , p. 340 ; [-us] A. S. Woodward, Cat. Foss. Fishes B. M., pt. i, I889, p. 74.

LoC. Bracklesham Beds; Sussex. [Rostral tooth ; Brit. Mus.]

listortus, Dixon. v. Pristis contorta.

hastingsioe, Agassiz. $\%$. Pristis bisulcata.

sibthorpi, W. Buckland. Recorded by J. Morris, Cat. Brit. Foss., 1854 , p. 340, but the name does not occur at the reference given.

PRISTODUS, J. W. Davis (ex Agassiz, MS.), Trans. Roy. Dublin Soc. [2] vol. i (1883), p. 519 ; A. S. Woodward, Cat. Foss. Fishes B. M., pt. i, 1889, p. 62.

Diodontopsodus, J. W. Davis, Rep. Brit. Assoc., I881, p. 646.

benniei (Etheridge), R. H. Traquair, Geol. Mag. [3] vol. v, I888, p. Ior ; A. S. Woodward, Cat. Foss. Fishes B. M., pt. i, I 889, p. 64, pl. i, f. I 3 .

Pctalorhynchus ? benniei, R. Etheridge, jun., Geol. Mag. [2] vol. ii, 1875, p. 243 , pl. viii, f. 3,4 .

LOC. U. Carb. Limest.; S. Scotland and Derbyshire. [Tooth ; Edinburgh Mus.] 
PRISTODUS (continued)-

concinnus (Davis), R. H. Traquair, Geol. Mag. [3] vol. v, ISSS, p. I02 ; A. S. Woodward, Cat. Foss. Fishes B. M., pt. i, 1889, p. 64.

Pristicladodus concinnus, J. W. Davis, Trans. Roy. Dublin Soc. [2] vol. i (1883), p. 385 , pl. xlix, f. 23, and Quart. Journ. Geol. Soc., vol xl, I\$84, p. 62 I, pl, xxvi, f. 15.

LOC. U. Carb. Limest.; Yorkshire. [Imperfect tooth; York Mus.]

falcatus, J. Morris and G. E. Roberts (ex Agassiz, MS.), Quart. Journ. Geol. Soc., vol. xviii, 1862, p. IoI (name only); J. IV. Davis, Trans. Roy. Dublin Soc. [2] vol. i, i 883 , p. 5 I9, pl. 1xi, f. 17-22, and Quart. Journ. Geol. Soc., vol. xl, I 884, p. 623, pl. xxvi, f. 19, 20; R. H. Traquair, Geol. Mag. [3] vol. v, I 888, p. Ior ; A. S. Woodward, Cat. Foss. Fishes B. M., pt. i, 1889, p. 63.

LOC. U. Carb. Limest.; Yorkshire. [Teeth; Brit. Mus.]

x.

PROGNATHODUS, Egerton. \%. Myriacanthus.

guentheri, Egerton. \%. Myriacanthus paradoxus.

PROTOGALEUS, Molin. \%. Galeocerdo.

minor (Agassiz), Molin. \%. Galeocerdo minor.

Protosprizina, J. Leidy, Trans. Amer. Phil. Soc., vol, xi, (I857), p. 95 .

SaUrocephalus. L. Agassiz (non Harlan), Poiss. Foss., vol. v, pt. i (1843), p. IO2.

Erisichthe, E. D. Cope, Vert. Cret. Form. West (Rep. U. S. Geol. Surv. Territ, vol. ii, I875), p. 217 ; Bull. U.S. Geol. Surv. Territ., vol. iii, I877, p. 82 I.

ferox, J. Leidy, Trans. Amer. Phil. Soc., vol. xi (1857), p. 95; E. T. Newton, (2uart. Journ. Geol. Soc., vol. xxxiv, I 878, p. 788; A. S. Woodward, Proc. Geol. Assoc., vol. x (I888), p. 32 I.

'Undetermined,' G. A. Mantell, Foss. South Downs, I822, p. 227 , pl. xxxiii, f. 7-9.

Saurocephalus lanciformis, L. Agassiz (non Harlan), Poiss. Foss., Feuill., p. 55 (I835), and vol. v, pt. i (I843), p. I02, pl. $x x v c$, f. 2I-29; F. Dixon, Geol. Sussex, I850, p. 374, pl. xxx, f. 2 I, pl. xxxi, f. 12, pl. xxxii*, f. I, pl. xxxiv, f. 1 I ; J. Morris, Cat. Brit. Foss., I854, p. 343.

Saurocephalus lanceolatus, L. Agassiz, Poiss. Foss., vol. v, pt. i (I883), p. 8 (misprint).

Xiphias dixoni, J. Leidy, Trans. Amer. Phil. Soc., vol. xi (1857), p. 95. [Rostrum; Brit. Mus.]

Erisichthe dixoni, E. D. Cope, Bull. U. S. Geol. Surv. Territ., vol. iii, 1877, p. 821 ; W. Davies, Geol. Mag. [2] vol. v, I878, p. 260 , pl. viii, f. 3 .

Loc. Chalk; S. E. England. [Teeth ; Brit. Mus.] 
PROTOSPHYR HRA (continued)-

minor (Agassiz), A. S. Woodward, Proc. Gcol. Assoc., vol. x (1888), p. $32 \mathrm{I}$.

Tetraptenus minor, L. Agassiz, Poiss. Foss., vol. v, pt. i (1843), p. 9I, pl. lx $a$, f. 9, IO ; J. Morris, Cat. Brit. Foss., I 854 , p. $3+5$.

Loc. Chalk; S. E. England. [Rostrum; Brit. Mus.]

(fins of). Probably described under the names of Ptychodus arcuatus, Ag., $P$. articulatus, Ag., $P$. gibberulus, Ag., and $P$. spectabilis, Ag. (L. Agassiz, Poiss. Foss., vol. iii (1837), pp. 56-59, pls. $\times a, \times b$, f. I 8), and also assigned to Pelecopterus, E. D. Cope, Vert. Cret. Form. West (Rep. U. S. Geol. Surv. Territ., vol. ii, 1875), pp. $244 a-f$. See A. S. Woodward, Proc. Geol. Assoc., vol. x (IS88), p. 32 I.

PSAIIODUS, P. M. G. Egerton, in L. Agassiz, Poiss. Foss., vol. iii (1843), p. 35 I, and Proc. Geol. Soc., vol. iv (1843), p. 157 .

compressus, P. M. G. Egerton, ibid., p. I56 ; J. Morris, Cat. Brit. Foss., I 854 , p. 340.

LOC. London Clay; Sheppey. [Nandib. tooth; R. Coll. Surgeons Mus.]

r.

PSAmIMODUS, L. Agassiz, Poiss. Foss., vol. iii (183S), p. I10; R. Owen, Rep. Brit. Assoc, I 838 (1839), p. 140.

Homalodus, J. IV. Davis, Trans. Roy. Dublin Soc. [2] vol. i (I883), p. 48 I.

Astrabodus, J. W. Davis, Quart. Journ. Geol. Soc., vol. xl, I884, p. 629 .

canaliculatus, M'Coy. v. Psammodus rugosus.

cinctus, Agassiz. $\%$. Chomatodus cinctus.

contortus, Agassiz. $v$. Deltoptychius gibberulus.

cornutus, Agassiz. \%. Copodus cornutus.

expansus (Davis), A. S. Woodward, Cat. Foss. Fishes B. M., pt. i, I889, p. 105.

Astrabodus expansus, J. IV. Davis, Quart. Journ. Geol. Soc., vol. xl, 1884, p. 630, pl. xxvi, f. I I, 12. T. (Astrabodus.)

LOC. Yoredale Rocks; Wensleydale, Yorkshire. [Teeth; York Mus.]

gibberulus, Agassiz. $\approx$. Helodus gibberulus.

lavissimus, Agassiz. \%. Psephodus lævissimus.

linearis, Agassiz. v. Petalodus linearis.

porosus, Agassiz. \% Psammodus rugosus.

rugosus, L. Agassiz, Poiss. Foss., vol. iii (1838); p. III, pl. xii, f. 14-18, pl. xix, f. 15; J. E. Portlock, Rep. Geol. Londonderry, 1843, p. 466, pl. xiv $a$, f. I ; J. Morris, Cat. Brit. Foss., 1854, p. 341 ; F. M'Coy; Brit. Palizoz. Foss. (1855), p. 644 ; 
PSAMrMODUS rugosus (continued)-

J. Morris and G. E. Roberts, Quart. Journ. Geol. Soc., vol. xviii, I862, p. I02 ; R. Etheridge, jun., Geol. Mag. [2] vol. iv, I 877 , p. 308 , pl. xiii, f. 7-9; J. W. Davis, Trans. Roy. Dublin Soc. [2] vol. i (1883), p. 459, pls. lvi, lvii, and Quart. Journ. Geol. Soc., vol. xl, 1884, p. 629, and Geol. Mag. [3] vol. iii, I886, p. I56; A. S. Woodward, Cat. Foss. Fishes B. M., pt. i, I889, p. IоO.

Psammodus porosus, L. Agassiz, Foiss. Foss., vol. iii (1838), p. II 2, pl. xiii, f. I-I 8 ; J. E. Portlock, Rep. Geol. Londonderry, I 843 , p. 465 , pl. xiv $a$, f. 2 ; J. Morris, Cat. Brit. Foss., I 854 , p. 34 I ; W. H. Baily, Figs. Char. Brit. Foss., I 875 , p. I20, pl. xli, f. 9. [Abraded teeth ; Bristol Mus.]

Psammodus nugosus, var. porosus, F. M'Coy, Brit. Palæoz. Foss. (1855), p. 644.

Psammodus canaliculatus, F. M'Coy, Ann. Mag. Nat. Hist. [2] vol. ii, 1848, p. 122, and Brit. Palæoz. Foss. (1855), p. 643, pl. iii G, f. I2 ; J. Morris, Cat. Brit. Foss., I854, p. 340. [Tooth; Woodwardian Mus.]

LOC. L. Carb. Limest.; Gloucestershire, Armagh, Sligo, and Wexford. U. Carb. Limest. ; Richmond, Yorkshire. Carb. Limest. ; Ayrshire, Westmoreland, Derbyshire, and North Wales. [Teeth; Bristol Mus. and Brit. Mus.]

T.

? salopiensis, A. S. Woodward, Cat. Foss. Fishes B. M., pt. i, I 889, pp. 105, 187 .

'A palate, or part of a palate,' J. Morris and G. E. Roberts, Quart. Journ. Geol. Soc., vol. xviii, I862, p. 105, pl. iii, f. 5.

LOC. Carb. Limest. ; Farlow, Shropshire. [Teeth ; Brit. Mus.] subteres, Agassiz. \%. Orodus ramosus.

trapeziformis (Davis), A. S. Woodward, Cat. Foss. Fishes B. M., pt. i, I889, p. I05.

Homalodus trapeziformis, J. W. Davis, Trans. Roy. Dublin Soc. [2] vol. i (1883), p. 482, pl. lviii, f. 30. T. (Homalodus.) Homalodus quadratus, J. W. Davis, ibid., p. 482, pl. Iviii, f. 3 I. [Tooth ; Brit. Mus.]

LOC. L. Carb. Limest. ; Armagh. [Tooth ; Brit. Mus.] turgidus, Agassiz. v. Helodus turgidus.

PSAmmostreds, L. Agassiz, Poiss. Foss. V. (.. R. (1845), p. 103.

granulatus, F. M'Coy, Ann. Mag. Nat. Hist. [2] vol. ii, 1848, p. 7 ; J. Morris, Cat. Brit. Foss., 1854, p. 34 I.

LOC. L. Carb. ; Kesh, Co. Fermanagh. [Dermal plate; olim R. Griffith Coll.]

vermicularis, F. M'Coy, Ann. Mag. Nat. Hist. [2] vol. ii, I848, p. 7 ; J. Morris, Cat. Brit. Foss., I854, p. 34I.

LOC. Carb. ; Fallaghloon, Maghera. [Dermal plate; olim R. Griffith Coll.] 
PSEPYODUs, J. Morris and G. E. Roberts (ex Agassiz, MS.), Quart. Journ. Geol. Soc., vol. xviii, 1862, p. ror ; R. H. Traquair, Trans. Geol. Soc. Glasgow, vol. vii (1884), p. 396, pl. xvi (reprinted, Geol. Mag. [3] vol. ii, 1885, p. 337, pl. viii).

Helodus, L. Agassiz, I'oiss. Foss, vol. iii (I838), p. IO4 (pars).

Lophodus, H. Romanovsky, Bull. Soc. Imp. Nat. Moscou, vol. xxxvii, I864, pt. ii, p. I60 (pars).

Aspidodus, J. S. Newberry and A. H. Worthen, Pal. Illinois,
vol. ii, I 866, p. 92 .

TENIODUS, O. St. John and A. H. Worthen, Nal. Illinois, vol. vii,
I 883 , p. 75 (pars).

dubius, A. S. Woodward, Cat. Foss. Fishes B. M., pt. i, 1880, p. $1_{3}$, pl. vi, f. I 3 (? f. I2).

LOC. Carb. Limest.; Oreton, Shropshire. [Tooth; Brit. Mus.]

læovissimus (Agassiz), A. S. Woodward, ibid., I889, p. I8I, pl. vi,
f. I6.

Helodus lavissimus, L. Agassiz, Poiss. Foss., vol. iii (1838), p. $104, \mathrm{pl}$ xiv, f. I-I 4 (non f. I 5 ) (lettered Psammodus on plate).

LOC. L. Carb. (Bone-bed in L. Limest. Shales); Avon Gorge, near Bristol. [Teeth; Bristol Mus.]

magnus ( $\left.\mathrm{I}^{\prime} \mathrm{C} \mathrm{Coy}\right), \mathrm{J}$. Morris and G. E. Roberts, Quart. Journ. Geol. Soc., vol. xviii, I862, p. 101 ; J. W. Davis, Trans. Roy. Dublin Soc. [2] vol. i ( 1883$),$ p. 439 , pl. Iv, f. I-1 4 , and Geol. Mag. [3] vol. iii, I886, p. I 51 ; R. H. Traquair, Trans. Geol. Soc. Glasgow, vol. vii (I 884), p. 392, pl. xvi (reprinted, Geol. Mag. [3] vol. ii, I 885 , p. 337 , pl. viii); A. S. Woodward, Cat. Foss. Fishes B. M., pt. i, 1889 , p. I77.

Cochliodus magnus, L. Agassiz, Poiss. Foss., vol. iii (1843), p. I74 (name only); J. E. Portlock, Rep. Geol. Londonderry, I 8.43 , p. 466, pl. xiva, f. 4 (fig. only); J. Morris, Cat. Brit. Foss., 1854 , p. 322 ; F. M $I^{\circ}$ Coy, Brit. Palizoz. Foss. (1855), p. 622.

Helodus planus, L. Agassiz, Poiss. Foss., vol. iii (1843), p. I73 (name only); J. Morris, Cat. Brit. Foss., I854, p. 328 ; F. I'Coy, Brit. Palæoz. Foss. (1855), p. 63I, pl. iil I, f. I2-I5. Helodus planus $=$ Cochliodus magnus, J. E. Portlock, Rep.
Geol. Londonderry, 1843 , p. 462 .

Helodus didymus, L. Agassiz, Poiss. Foss., vol. iii (1843), p. 173
(name only); J. Morris, Cat Brit Foss., M'Coy, Brit. Palæoz. Forre Cat. Brit. Foss., 1854 , p. 328 ; F. (pars). Helodus lavissimus, F. M'Coy, Brit. Pal: $\mathrm{I}^{\circ} \mathrm{Oz}$. Foss. (IS55), p.
$\quad 630$ (pars).

Helodus rudis, F. M'Coy, Ann. Mag. Nat. Hist. [2] vol. ii, 1848, p. 123, and Brit. Palæoz. Foss. (1855), p. 631, pl. iii k, f. 4; J. Morris, Cat. Brit. Foss., 1854, p. 328 ; J.W. Davis, Trans. Roy. Dublin Soc. [2] vol. i (1883), p. 45 ;, pl. lix, f. II.

Lophodus larissimus, J. W. Davis, ibid. (1883), p. 404, pl. li,
f. I 8 (pars). 


\section{PSDPXODUS magnus (continued)-}

Lophodus didymus, J. WV. Davis, ibid. (1883), p. 407, pl. li, f. 2 ? (pars).

'Selachian Fish,' J. Coutts, Trans. Geol. Soc. Glasgow, vol. vii (1883), p. 164. [Associated teeth; Edinburgh Mus.]

? Loploodus simuosus, J. W. Davis, Trans. Roy. Dublin Soc. [2] vol. i (1883), p. 409, pl. li, f. 28. [Tooth ; York Mus.]

LOC. L. Carb. Limest. ; Armagh, Lanarkshire, Northumberland, and Gloucestershire. Carb. Limest.; Ayrshire, Westmoreland, Derbyshire, Denbighshire, and Flintshire. [Teeth ; Woodwardian Mus.]

T.

salopiensis, A. S. Woodward, Cat. Foss. Fishes B. M., pt. i, I889, p. 182 , pl. vi, f. 14,15 .

LOC. Carb. Limest. ; Oreton, Shropshire. [Teeth ; Brit. Mus.]

simplex, J. W. Davis, Geol. Mag. [3] vol. iii, r886, p. I5 I, f. r, 2.

LOC. Carb. Limest. ; Derbyshire. [Teeth ; T. Parker Coll.]

sp., A. S. Woodward, Cat. Foss. Fishes B. M., pt. i, I889, p. I84, pl. vi, f. 17 .

L.OC. L. Carb. Limest. ; Hook Point, WVaxford. ['Tooth; Brit. Mus., no. 23712.]

sp., A. S. Woodward, ibid., p. I8 4 , pl. vi, f. I 8 .

Loc. Carb. Limest. ; Oreton, Shropshire. [Tooth; Brit. Mus. no. P. 227 C.]

PSEUDOSPHARODON, Noetling. v. Platylemus.

PSITTACODON, Agassiz. $\tau$. Edaphodon and Ganodus.

fulcatus, Agassiz. $\%$ Ganodus falcatus.

mantellii, Agassiz. v. Edaphodon mantelli.

psittceinus, Agassiz. \%. Ganodus psittacinus.

sellguickii, Agassiz. $\%$ Edaphodon sedgwicki.

PTgRAsPI i, T. H. Huxley, ()uart. Journ. Geol. Soc., vol. xii, I856, p. Ion, and Rep. Brit. Assoc., I 858 (1859), Sect., p. 82 (emend. $e x$ R. Kner, Haidinger's Naturw. Abhandl., vol. i, 1847, p. 165); E. R. Lankester, Rep. Brit. Assoc., I864 (1865), Sect., p. 58, and Quart. Journ. Geol. Soc., vol. xx, I864, p. 194 (scales), and Fishes O. R. Sandst., pt. i (Pal. Soc., 1868), p. 28; ? J. W. Salter, Quart. Journ. Geol. Soc., vol. xxiii, I867, p. 333 (tracks).

banlisii, Huxley and Salter. $\tau$ ' Cyathaspis banksi.

crouchi, E. R. Lankester (ex Salter, MS.), Rep. Brit. Assoc., 1864 (1865), Sect., p. 58, and Fishes O. R. Sandst., pt. i (Pal. Soc., 1868), p. 3o, pl. iii, pl. iv, f. 5 , pl. vi, f. $4,7,8$, pl. vii, f. $4,8,11$; A. S. Woodward, Proc. Zool. Soc., I887, p. 478, woodcut.

LOC. L. Old Red Sandst. (Cornstones); Herefordshire. [Headshields; Ludlow Mus., Brit. Mus., and Oxford Mus.] 
PTERASPIS (contimued) -

dunensis (Roemer): erroneously recorded as British by $R$. Etheridge, Foss. Brit. Islands, pt. i, I 888 , p. 175.

lloydii (Agassiz), Kner. $\%$. Scaphaspis lewisi.

ludensis, Salter. \%. Scaphaspis ludensis.

mitchelli, J. Powrie, Geolngist, vol. vii, 1864, p. 170; E. R. Lankester, Rep. Brit. Assoc., 1864 (1865), Sect., p. 58, and Fishes O. R. Sandst., pt. i (Pal. Soc., I 868), p. 33, pl. v, f. I, 2,6, IO, II.

LOC. L. Old Red Sandst.; Forfarshire. [Head-shield; Dundee Mus.]

rostrata (Agassiz), [-us] T. H. Huxley, Quart. Journ. Geol. Soc., vol. xvii, I86I, p. I66, woodcuts; [-us] E. R. Lankester, Rep. Brit. Assoc., I 864 (1865), Sect., p. 58 , and Fishes O. R. Sandst., pt. i (Pal. Soc., 1868), p. 32, pl. iv, f. I, 2, 3, 7, 8, pl. v, f. 4 , pl. vi, f. I, 2, 3, 6, 10, pl. vii, f. $3,5,9,12,13,16,17,19$.

Cephalaspis rostratus, L. Agassiz, Poiss. Foss., vol. ii, pt. i (1835), p. I48, pl. i b, f. 6, 7 ; J. Morris, Cat. Brit. Foss., 1854, p. 320 .

Loc. L. Old Red Sandst. (Cornstones); Herefordshire. [Headshield; olim Murchison Coll.]

T.

trumcatus, Huxley and Salter. $\tau$. Scaphaspis truncata.

sp., J. Powrie, Geologist, vol. vi, I863, p. 67, woodcut.

Loc. L. Old Red Sandst.; South Scotland. [Head-shield: J. Powrie Coll.]

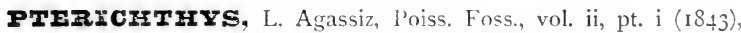
p. 302, and Poiss. Foss. V. G. R. (1844), p. 6.

cancriformis, Agassiz. \%. Pterichthys productus.

cormutus, Agassiz. \%. Pterichthys testudinarius.

dickii, Peach. \%. Microbrachius dicki.

hydrophilus (Agassiz), Miller. z'. Bothriolepis hydrophilus.

hydrophyllıs, R. Etheridge, Foss. Brit. Islands, pt. i, 1888, p. 175 : misprint for Pterichthys hydrophilus.

latus, Agassiz. \%. Pterichthys milleri.

macrocephalus, Egerton. $\%$ Bothriolepis macrocephalus.

major, Agassiz. v. Bothriolepis major.

milleri, L. Agassiz, Poiss. Foss., vol. ii, pt. i ( 1843 ), p. 302, and Poiss. Foss. V. G. R. (1844), p. I 5 , pl. i, f. I-3 ; J. Morris, Cat. Brit. Foss., I854, p. 341 ; R. H. 'Traquair, Geol. Mag. [3] vol. v, I888, p. 509 .

Pterichthys latus, L. Agassiz, Poiss. Foss., vol. ii, pt. i ( $8+3$ ), p. 302 , and Poise. Foss. V. G. R. (I 844$)$, p. I2, pl. iii, f. 3,4 ; P. M. G. Egerton, Quart. Journ. Geol. Soc., vol. iv, 1848, p. 312 ; J. Morris, Cat. Brit. Foss., IS54, p. 341. [Fishes; Brit. Mus. and Forres Mus.] 
Prgricrirys milleri (continued)-

Pterichthys quadratus, P. M. G. Egerton, Quart. Journ. Geol. Soc., vol. iv, 1848 , p. 313 , pl. x ; J. Morris, Cat. Brit. Foss., 1854, p. 34I ; R. H. Traquair, Geol. Mag. [3] vol. v, 1888, p. 509. [Fish; Geol. Soc. London.]

LOC. L. Old Red Sandst. ; Cromarty, Nairnshire, and Banffshire. [Fishes; Edinburgh Mus.]

oblongus, L. Agassiz, P’oiss Foss., vol. ii, pt. i (1843), p. 302, and Poiss. Foss. V. G. R. (18+t), p. 18, pl. iii, f. 1, 2 ; P. M. G. Egerton, Quart. Journ. Geol. Soc., vol. iv, I848, p. 313; J. Morris, Cat. Brit. Foss., 1854, p. 341 ; F. M'Coy, Brit. Palæoz. Foss. (1855), p. 600 ; K. H. Traquair, Geol. Mag. [3] vol. v, 1888, p. 509.

LOC. L. Old Red Sandst.: Banffshire, Nairnshire, and Cromarty. [Fishes; Elgin Mus.]

paucideus ('Agassiz'), R. Etheridge, Foss. Brit. Islands, pt. i, I888, p. 175 : mis-quotation, the name not existing.

productus, L. Agassiz, Poiss. Foss., vol. ii, pt. i (1843), p. 302, and P'oiss. Foss. V. G. R. (1844), p. 16, pl. v, f. I-4; P. M. G. Egerton, Quart. Journ. Geol. Soc., vol. vi, 1848, p. 312 ; J. Morris, Cat. Brit. Foss., 1854, p. 34I ; F. M'Coy, Brit. Palæoz. Foss. (1855), p. 600; R. H. Traquair, Geol. Mag. [3] vol. v, I 888, p. 509 .

Pterichthys cancriformis, L. Agassiz, Poiss. Foss., vol. ii, pt. i (1843), p. 302, and Poiss. Foss. V. G. R. (1844), p. 17, pl. i, f. 4,5 ; J. Morris, Cat. Brit. Foss., I 854 , p. 341 ; F. M'Coy, Brit. Palieoz. Foss. (1855), p. 599. [Fishes ; Brit. Mus.]

LOC. L. Old Red Sandst. ; Nairnshire and Orkney. [Imperfect fishes; Forres Mus. and Brit. Mus.]

quadratus, Egerton. \%. Pterichthys milleri.

testudinarius, L. Agassiz, Poiss. Foss., vol, ii, pt. i (1843), p. 302, and Poiss. Foss. V. G. R. (I844), p. I4, pl. iv, f. I-3 ; P. M. G. Egerton, Quart. Journ. Geol. Soc., vol. iv, I848, p. 312 ; J. Morris, Cat. Brit. Foss., I854, p. 341 ; F. M'Coy, Brit. Palreoz. Foss. (1855), p. 600.

Pterichthys cormutus, L. Agassiz, Poiss. Foss., vol. ii, pt. i (1843), p. 302, and Poiss. Foss. V. G. R. (1844), p. 17, pl. ii, f. I-5; P. M. G. Egerton, Quart. Journ. Geol. Soc., vol. iv, 1848, p. 313 ; J. Morris, Cat. Brit. Foss., 1854 , p. 34 I ; R. H. Traquair, Geol. Mag. [3] vol. v, i 888, p. 509, and Ann. Mag. Nat. Hist. [6] vol. ii, I888, p. 485 , pl. xvii, t. I-3. [Fishes; Brit. Mus.]

LOC. L. Old Red Sandst.; Lethen Bar, Nairnshire. [Head and trunk; Edinburgh Mus.]

PTERNODUS, Owen. $\because$ Diplodus.

productus, Owen. $\because$ Diplodus gibbosus. 
PTYCHACANTHUS, L. Agassiz, Poiss. Foss., vol. iii (1837), p. 22. = Tristychius and undetermined genera.

dubils, L. Agassiz, Poiss. Foss., vol. iii (1843), p. 176, and Poiss. Foss. V. G. R. (I844), p. II 8 , pl. xxxiii, f. 22, 23 ; J. Morris, Cat. Brit. Foss., I854, p. 341. [Undetermined ichthyolite.] LOC. L. Old Red Sandst. ; Abergavenny. [Spine ; olim Murchison Coll.]

sublcevis, Agassiz. \%. Tristychius arcuatus.

? s1., L. Agassiz, in Murchison's Silur. Syst. (1839), p. 597, pl. i, f. 9, 10 ; J. Morris, Cat. Brit. Foss., 1854, p. 341 .

LOC. L. Old Red Sandst.; Abergavenny and Herefordshire. [Spine; olim Murchison Coll.]

PTYCHOCEPHALUS, L. Agassiz, Poiss. Foss., vol. v, pt. ii (I843), p. I39 (name only), and Rep. Brit. Assoc., I 844 $(18+5)$, p. 307 (name only).

radiatus, L. Agassiz, ibid. (1843), p. 139 (name only), and ibid., $18+4$, p. 307 (name only).

LOC. London Clay; Sheppey. [Imperfect head; Brit. Mus.] T.

PTYCzrodus, L. Agassiz, Poiss. Foss., Feuill., p. 54 (1835), and vol. iii (1839), p. 150 ; A. S. Woodward, Cat. Foss. Fishes B. M., pt. i, I889, p. 132 .

Aulodus, F. Dixon, Geol. Sussex, 1850, p. 366.

acutus, L. Agassiz, Poiss. Foss., vol. iii (1 843), p. 177 (name only): recorded from Gault of Folkestone by J. Morris, Cat. Brit. Foss., 1854, p. 34r.

altior, Agassiz. $\quad$ '. Ptychodus mammillaris and P. rugosus.

arcuatus, Agassiz. $\%$ Protosphyrena (fins of).

articulatus, Agassiz. $\%$. Protosphyræna (fins of).

concentricus, named variety of $P$. polygyrus (L. Agassiz, Poiss. Foss., vol. iii (1839), p. 156).

decurrens, L. Agassiz, Poiss. Foss., Feuill., p. 54 (1835), and vol. iii (1839), p. I 54, pl. xxv b, f. 1, 2, 4, 6-8 (non f. 3, 5); R. Owen, Odontogr. (1840), descr. to pls. xviii, xix ; F. Dixon, Geol. Sussex, 1850, p. 362, pl. xxx, f. 7, 8, pl. xxxi, f. I, pl. xxxii, f. 5 ; J. Morris, Cat. Brit. Foss., 1854, p. 341 ; A. S. Woodward, ()uart. Journ. Geol. Soc,, vol. xliii, 1887, p. 123, pl. x, f. I-10, 13, and Cat. Foss. Fishes B. M., pt. i, 1889, p. 138. [See also P. mammillaris.]

'Palate of Unknown Fish,' J. Parkinson, Organic Remains, vol. iii, I81 I, p. 262, pl. xviii, f. I2.

Ptychodus depressus, F. Dixon, Geol. Sussex, 1850, p. 363 , pl. xxxi, f. 9 ; J. Morris, Cat. Brit. Foss., 1854, p. 34I. [Tooth ; Brit. Mus.]

I.OC. U. and L. Chalk; Sussex, Kent, Surrey, Cambridgeshire, and Norfolk. [Teeth ; Brit. Mus. (pars).]

depressus, Dixon. $\because$ Ptychodus decurrens. 


\section{PTYCrodus (continued) -}

gibbemulus, Agassiz. v. Protosphyrona (fins of).

latissimus, L. Agassiz, Poiss. Foss., Feuill., p. 54 (I 835 ), and vol. iii (1843), p. I57, pl. xxv a, f. I-6 (? f. 7, non f. 8), pl. xxv b, f. $24-26$; R. Owen, Rep. Brit. Assoc., I838 (I839), p. 140 , and Odontogr. (IS40), descr. to pl. xvii, f. I, 2 ; J. Morris, Cat. Brit. Foss., I 854 , p. 342 ; O. Rees, Proc. Geol. Assoc., vol. vii (1873), p. I17; A. S. Woodward, Cat. Foss. Fishes B. M., pt. i, I889, p. 147. [See also P. mammillaris and $P$. polygyrus.]

'Palate of Unknown Fish,' J. Parkinson, Organic Remains, vol. iii, 18I I, p. 262, pl. xix, f. I8.

'Tooth allied to Diodon,' G. A. Mantell, Foss. S. Downs, I822, p. 23I, pl. xxxii, f. I9.

Ptychodus paucisulcatus, F. Dixon, Geol. Sussex, 1850, p. 363, pl. xxx, f. 3 ; J. Morris, Cat. Brit. Foss., I854, p. 342 ; A. S. Woodward, Quart. Journ. Geol. Soc., vol. xliii, I887, p. 127, pl. x, f. 12. [Tooth; Brit. Mus.]

LOC. U. and L. Chalk ; Sussex, Kent, Surrey, Suffolk, Hertfordshire, and Wiltshire. [Teeth ; ? Brit. Mus. (pars).]

mammillaris, L. Agassiz, Poiss. Foss., Feuill., p. 54 (1835), and vol. iii (I \&39), p. I5 l, pl. xxy b, f. I2-20 (? f. II) ; F. Dixon, Geol. Sussex, 1850, p. 36I, pl. xxx, f. 6, pl. xxxi, f. 4 ; J. Morris, Cat. Brit. Foss., I 854, p. 342 ; A. S. Woodward, Cat. Foss. Fishes B. M., pt. i, I889, p. I33.

Ptychodus decurrens, L. Agassiz (crore), Poiss. Foss., vol, iii (I839), p. I54, pl. $\mathrm{xxv} b$, f. 3,5 .

Ptychodus altior, L. Agassiz, Poiss. Foss., Feuill., p. 54 (1835), and vol. iii (1839), p. 155, pl. xxy b, f. 9, Io.

Ptychodus latissimus, F. Dixon (errore), Geol. Sussex, I850, pl. xxxi, f. 3 .

'Tooth allied to Diodon,' G. A. Mantell, Foss. S. Downs, I822, p. 231, pl. xxxii, f. 17, 18, 20, 2I, 25, 27, 29.

LOC. U. and L. Chalk; Sussex, Kent, Surrey, and Wiltshire. [Teeth; Brit. Mus. $(p(r) s)$.

marginalis, named variety of $P$. polyerws (L. Agassiz, Poiss. Foss., vol. iii (1839), p. 157).

mortoni, G. A. Mantell, Descript. Cat. Genl., etc., Mus. Sussex Sci. and Lit. Inst. Brighton, ed. 5 (1836), p. 27 ; S. G. Morton, Journ. Acad. Nat. Sci. Philad., vol. viii (1839), p. 215 , pl. xi, f. 7 ; J. Morris, Cat. Brit. Foss., I854, p. 342. Recorded from Chalk of sussex by F. Dixon, Geol. Sussex, 1850, p. 364 , pl. xxxi, f. 6,7 , but doubtful; similar tooth in Oxford Museum from Chalk of Winchester.

multistriatus, A. S. Woodward, Cat. Foss. Fishes B. M., pt. i, r889, p. I46, pl. v, f. 4-6.

Loc. L. Chalk; Kent. [Teeth; Brit. Mus.] 
PTYCHODUS (continued) -

oweni, F. Dixon, Geol. Sussex, 1850, p. 364, pl. xxxi, f. 2 ; J. Morris, Cat. Brit. Foss., 1854 , p. 342 ; A. S. Woodward, Cat.

Foss. Fishes B. M., pt. i, I889, p. I38, pl. v, f. 8.

LOC. U. Chalk; Kent and Sussex. [Tooth ; Brit. Mus.]

prencisulcutes, Dixon. 7 . Ptychodus latissimus.

polygyrus, L. Agassiz, Poiss. Foss., Feuill., p. 55 (1835), and vol. iii (1839\%, p. 156, pl. xxv, f. 4-II, pl. xxv 6, f. 21-23; IV. Buckland, Geol. and Mineral., ed. 2, 1837, vol. ii, p. 48, pl. xxvii $f$; F. Dixon, Geol. Sussex, 1850, p. 363 , pl. xxx, f. 9, pl. xxxi, f. IO; J. Morris, Cat. Brit. Foss., I854, p. 342; S. J. Mackie, Geologist, vol. vi, I863, p. I6I, pl. ix; A. S. Woodward, Quart. Journ. Geol. Soc., vol. xliii, I887, p. 127, pl. x, f. I I, and Cat. Foss. Fishes B. M., pt. i, I889, p. I43, pl. v, f. 7 . [Tarieties are named $P$. concentricus, $P$. marginalis, and $P$. sulcatus, q.v.]

'Teeth allied to Diodon,' G. A. Mantell, Foss. S. Downs, I822, p. 23I, pl. xxxii, f. 23, 24.

Ptychodus latissimus, L. Agassiz (errore), Poiss. Foss., vol. iii (I843), p. I 57, pl. xxv a, f. 8 ; F. Dixon, Geol. Sussex, I850, descr. to pl. xxx, f. 1, 2. [Tooth; Oxford Mus.]

Loc. U. and L. Chalk ; Sussex, Kent, Essex, and Hampshire. [Teeth ; Brit. Mus. (pars).]

rugosus, F. Dixon, Geol. Sussex, 1850, p. 362, pl. xxxi, f. 5 ; J. Morris, Cat. Brit. Foss., 1854, p. 342 ; A. S. Woodward, Cat. Foss. Fishes B. M., pt. i, I889, p. 136, pl. v, f. I-3.

Ptychodus altior, F. Dixon (non Agassiz), Geol. Sussex, I850, p. $362, \mathrm{pl} . \mathrm{xxx}, \mathrm{f}$. IO.

LOC. U. and L. Chalk; Kent, Sussex, Surrey, and Yorkshire. [Tooth.]

spectabilis, Agassiz. $\%$. Protosphyræna (fins of).

sulcatus, named variety of P.polygyrus (L. Agassiz, Poiss. Foss., vol. iii (1839), p. 156).

PTYCrorgPIS, L. Agassiz, Poiss. Foss., vol. ii, pt. ii (1 $8+3)$, p. 107.

bollensis, L. Agassiz, Neues Jahrb., 1832, p. I42, and Poiss. Foss., vol. ii, pt. ii $(1843)$, p. 108, pl. lviii b; W. C. Williamson, Phil. Trans., 1849 , p. 444 ; J. Morris, Cat. Brit. Foss., I 854, p. 342 .

Loc. L. Lias; Lyme Regis. U. Lias; Whitby. [Fish; Brit. Mus.]

T.

ourtus, P. M. G. Egerton, Ann. Mag. Nat. Hist. [2] vol. xiii, 1854 , p. 435 , and Figs. and Descript. Brit. Organic Remains, dec. viii (Mem. Geol. Surv., 1855), no. 8, pl. viii ; J. Morris, Cat. Brit. Foss., 1854, p. 342.

Loc. L. Lias ; Lyme Regis. [Fish ; S. H. Beckles Coll.] 
PTYCHOIXRIS (continued)-

gracllis, J. W. Davis, Ann. Mag. Nat. Hist. [5] vol. xiii, I884, p. 335, pl. $x$.

LOC. L. Lias ; Lyme Regis. [Fish ; J. W. Davis Coll.]

minor, P. M. G. Egerton, Figs. and Descript. Brit. Organic Remains, dec. vi (Mem. Geol. Surv., 1852), no. 7, pl. vii ; J. Morris, Cat. Brit. Foss., 1854 , p. 342.

LOC. L. Lias; Barrow-on-Soar, Leicestershire. [Fishes; Brit. Mus.]

PצCaOdUs, L. Agassiz, I'oiss. Foss., vol. i (1833), pl. G, f. I (name and fig. only), and ibia., vol. ii, pt. ii (1843), p. I83; J. J. Heckel, Denkschr. k. k. Akad. Wiss. Wien, math.naturw. Cl., vol. xi, I856, p. 204.

Periodus, L. Agassiz, Poiss. Foss, vol. ii, pt. ii (1843), p. 201. angustus, Agassiz. v. Coelodus angustus. biserialis, Agassiz. \%. Mesodon biserialis.

bowerbanki, P. M. G. Egerton, Geol. Mag. [2] vol. iv, 1877, p. 52, pl. iii, f. 2.

LOC. London Clay; Sheppey. [R. mandib. dentition; Brit. Mus.]

bucklandi, Agassiz. \%. Mesodon bucklandi.

cretuceus, Agassiz. \%. Cœlodus cretaceus.

didymus, Agassiz. \%. Mesodon bucklandi.

discoides, Agassiz. \%. Mesodon discoides.

elongatus, Agassiz. v. Colodus angustus.

hugii, L. Agassiz, Poiss. Foss., vol. 1i, pt. ii (1843), p. 195, pl. lxxii a, f. $49-5+$. Erroneously recorded from Stonesfield Slate by J. Morris, Cat. Brit. Foss., I854, p. 342.

lativostris, Agassiz. \%. Mesodon trigonus.

liassicus, Egerton. \%. Mesodon liassicus.

mantelli, Agassiz. \%. Colodus mantelli.

marginalis, Agassiz. \%. Gyrodus cretaceus.

microdon, Agassiz. \%. Colodus mantelli.

minor, L. Agassiz, Poiss. Foss., vol, ii, pt. ii (1843), p. 200 (name only). ? = Gyrodus minor, Agassiz.

obtusus, Agassiz. \%. Mesodon bucklandi.

oualis, Agassiz. $\because$ Mesodon bucklandi.

pachyrhinus, P. M. G. Egerton, Geol. Mag. [2] vol. iv, 1877, p. 54, pl. iv, f. 1, 2 .

Loc. London Clay; Sheppey. [Vomer; Brit. Mus.]

pagola, Blake. $\%$ Microdon pagroda.

parallelus, Dixon. v. Coelodus parallelus.

parvus, Agassiz. \%. Mesodon rugrulosus.

quincuncialis, Blake. \%. Microdon quincuncialis. 
PYCNODUS (continued) -

mudis, J. Phillips, Geol. Oxford, 1871, p. 180, woodcut xl, f. I (name and fig. only). [? Genus.]

LOC. Stonesfield Slate. [Mandib. ramus ; Oxford Mus.]

rugulosus, Agassiz. v. Mesodon rugulosus.

toliapicus, L. Agassiz, Poiss. Foss., vol. ii, pt. ii (1843), p. 196, pl. Ixxii $a$, f. 55 ; J. Morris, Cat. Brit. Foss., 1854, p. 343 ; P. M. G. Egerton, Geol. Mag. [2] vol. iv, 1877, p. 53 .

Periodus koenigii, L. Agassiz, Poiss. Foss., vol. ii, pt. ii (1843), p. 20I, pl. Ixxii $a$, f. 61, 62 ; F. Dixon, Geol. Sussex, 1850, p. 205 , pl. x, f. 13 ; J. Morris, Cat. Brit. Foss., I854, p. 337. [Abraded mandib. dentition ; Brit. Mus.] T. (Periodus.)

LOC. London Clay; Sheppey. [Mandib. dentition; Oxford Mus.] trigonus, Agassiz. \%. Mesodon trigonus.

tristychius, L. Agassiz, Poiss. Foss., vol. ii, pt. ii (1843), p. 199 (name only). [?.Mesodon.]

LOC. ? Stonesfield Slate. [Teeth ; olim Bristol Mus.]

umbonatus, Agassiz. 2'. Mesodon umbonatus.

PYGOPTrR שS, L. Agassiz, Poiss. Foss., vol. ii, pt. ii (1834), p. 74 ; R. H. Traquair, Quart. Journ. Geol. Soc., vol. xxxiii, 1877, p. 574 .

bucklandi, Hibbert. $z$. Elonichthys bucklandi.

greenocki, Agassiz. $\tau^{\prime}$. Nematoptychius greenocki.

jamesoni, L. Agassiz, Poiss. Foss., vol. ii, pt. ii (1843), p. 78 (name only).

LOC. Calcif. Sandst. ; Burdiehouse.

latus, P. M. G. Egerton, in King's Permian Foss. (Pal. Soc., 1850), p. 233, pl. xxiv, f. I ; J. Morris, Cat. Brit. Foss., I854, p. 343 ;

R. H. Traquair, Quart. Journ. Geol. Soc., vol. xxxiii, I877, p. 575 .

Loc. Magnesian Limest.; Ferry Hill, Durham. [Fish; Brit. Mus.]

mandibularis, L. Agassiz, Poiss. Foss., vol. ii, pt. i (1 834 ), p. 76, pls. liii, liii $a$; W. King, Permian Foss. (Pal. Soc., 1850), p. 232, pl. xxiii, f. I ; J. Morris, Cat. Brit. Foss., r 854, p. 343 ; F. M'Coy, Brit. Palæeoz. Foss. (1855), p. 608 ; R. H. Traquair, Quart. Journ. Geol. Soc., vol. xxxiii, 1877, p. 575.

'Unknown fish,' A. Sedgwick, Trans. Geol. Soc. [2] vol. iii (1829), p. II $8, \mathrm{pl}$. $\mathrm{x}$.

Nemoptery $x$ mandibularis, L Agassiz, Neues Jahrb., I833, p. 476.

Pygopterus sculptus, L. Agassiz, Poiss. Foss., vol. ii, pt. ii (1834), p. 77.

Pygopterus scoticus, L. Agassiz, ibid., pt. i, p. ro; H. G. Bronn, Leth. Geogn., ed. 2, vol. i, 1837, p. 128.

Loc. Magnesian Limest.; Durham. [Fish; olim Miss Surtees Coll.] 
PIGOPTERU (continued) -

scoticus, Bronn. v. Pygopterus mandibularis.

sculptus, Agassiz. v. Pygopterus mandibularis.

sp., T. P. Barkas. v. Elonichthys semistriatus.

RAJA, Linnaus, Syst. Nat., ed. I2, I766, vol. i, p. 39j. [Raia, auct.] antique, Agassiz. v. Raja clavata.

batis, Linnæus, ibid., p. 395 ; E. T. Newton, Vert. Forest Bed (Mem. Geol. Surv., I882), p. I31, pl. xix, f. 9.

Loc. Weybourn Crag; East Runton, Norfolk. [Teeth; Mus. Pract. Geol.]

clavata, Linnæus, Syst. Nat., ed. 12, I766, vol. i, p. 397 ; E. T. Newton, Vert. Forest Bed (Mem. Geol. Surv., I 882), p. I32, pl. xix, f. Iо, I I ; A. S. Woodward, Cat. Foss. Fishes B. M., pt. i, 1889, p. 87 .

Raja antiqua, L. Agassiz, Poiss. Foss., vol. iii (I843), p. 37 r, pl. xxxvii, f. 33 ; J. Morris, Cat. Brit. Foss., I854, p. 343. [Dermal tubercles ; olim Buxton Coll.]

LOC. Red and White Crags; Suffolk. Norwich and Weybourn Crags; Norfolk. [Dermal tubercles; Mus. Pract. Geol.]

similis, A. S. Woodward. \%. Arius sp.

RHALDODERMA, Reis. \% Coelacanthus.

huxleyi (Traquair), Reis. $\%$. Coelacanthus huxleyi.

tingleyense (Davis), Reis. \%. Colacanthus tingleyensis.

RHADIN ACANTHUS, Traquair. \%. Diplacanthus.

longispinus (Agassiz), Traquair. v. Diplacanthus longispinus.

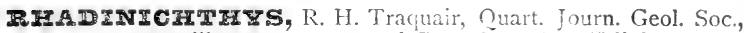
vol. xxxiii, I 877 , p. 559, and Proc. Koy. Soc. Edinb., vol. ix, 1877 , p. $43 \mathrm{I}$.

? angustulus, R. H. Traquair, Trans. Roy. Soc. Edinb., vol. xxx (I881), p. 33, pl. ii, f. IO, II.

LOC. Calcif. Sandst. (Cement-stone Group); Glencartholm, Eskdale. [Fish; Geol. Surv. Scotland.]

brevis, R. H. Traquair, Proc. Roy. Soc. Edinb., vol. ix, 1877, p. 440.

Loc. Calcif. Sandst.; Wardie. [Fish ; Edinburgh Mus.]

carinatus (Agassiz), R. H. Traquair, Quart. Journ. Geol. Soc., vol. xxxiii, 1877 , p. 559, and Proc. Roy. Soc. Edinb., vol. ix, I 877 , p. $44 \mathrm{I}$.

Palconiscus carinatus, L. Agassiz, Poiss. Foss., vol. ii, pt. i (1835), p. 104, pl. iv b, f. I, 2 ; J. Morris, Cat. Brit. Foss., I 854 , p. 336.

LOC. Calcif. Sandst.; Wardie. [Imperfect fish; Edinburgh Mus.] 
RIXADINICHTIXY (continued)-

delicatulus, R. H. Traquair, Trans. Roy. Soc. Edinb., vol. xxx (I88I), p. 29, pl. ii, f. 6-9.

Loc. Calcif. Sandst. (Cement-stone Group); Glencartholm, Eskdale. [Fish; Geol. Surv. Scotland.]

ferox, R. H. Traquair, Proc. Roy. Soc. Edinb., vol. ix, 1877, p. 435. LOc. Calcif. Sandst. ; Wardie. [Fish ; Edinburgh Mus.]

? fusiformis, R. H. Traquair, Trans. Roy. Soc. Edinb., vol. xxx (1881), p. 34, pl. iii, f. 1-5.

LOC. Calcif. Sandst. (Cement-stone Group); Glencartholm, Eskdale. [Fish; Geol. Surv. Scotland.]

geikiei, R. H. Traquair, Proc. Roy. Soc. Edinb., vol. ix, I 877 , p. 438 , and Trans. Roy. Soc. Edinb., vol. $x x x$ (188I), p. 25, pl. i, f. $13-18$.

LOC. Calcif. Sandst.; Redhall and Colinton, Mid-Lothian ${ }^{-}$; Glencartholm, Eskdale, Dumfriesshire. [Fish; Geol. Surv. Scotland.]

var. elegantulus, R. H. Traquair, Trans. Roy. Soc. Edinb., vol. xxx (1881), p. 27, pl. ii, f. I-5.

LOC. Calcif. Sandst. (Cement-stone Group); Glencartholm. [Fish; Geol. Surv. Scotland.]

grossarti, R. H. Traquair, Proc. Roy. Phys. Soc. Edinb., vol. iv, I 878, p. 244.

Loc. Coal-Meas.; Lanarkshire. [Imperfect fishes; Grossart Coll., and Geol. Surv. Scotland.]

bancocki: Palconiscus hancocki, T. Atthey, Ann. Mag. Nat. Hist. [4] vol. xv, 1875, p. 311 , and Nat. Hist. Trans. Northumb. and Durham, vol. v (1877), p. 228.

Loc. Coal-Meas.; Newsham. [Fish; Newcastle-upon-Tyne Mus.]

lepturus, R. H. Traquair, Proc. Roy. Soc. Edinb., vol. ix, IS77, p. 437.

LOC. Calcif. Sandst.; Burntisland. [Imperfect fish ; Edinburgh Mus.]

macconochii, R. H. Traquair, Trans. Roy. Soc. Edinb., vol. xxx (1881), p. 30, pl. ii, f. 12-I6.

Loc. Calcif. Sandst. (Cement-stone Group); Glencartholm, Eskdale. [Fish; Geol. Surv. Scotland.]

macrodon, R. H. Traquair, Geol. Mag. [3] vol. iii, I886, p. 44I.

LOC. Coal-Meas. (Knowles-Ironstone Shale); Fenton. [Fish; J. Ward Coll.]

monensis (Egerton), R. H. Traquair, Proc. Roy. Phys. Soc. Edinb., vol. iv, I878, p. 24 I.

Palceniscus monensis, P. M. G. Egerton, Quart. Journ. Geol. Soc., vol. vi, I85o, p. 5 ; J. Morris, Cat. Brit. Foss., I 854, p. 337.

LOC. Coal-Meas.; North Wales, North Staftordshire, and Lanarkshire. [Scales; Brit. Mus.] 


\section{RIADIAICHTrYs (continued)-}

ornatissimus (Agassiz), R. H. Traquair, Quart. Journ. Geol. Soc., vol. xxxiii, 1877, p. 559, and Proc. Roy. Soc. Edinb., vol. ix, 1877, p. 432.

Palaoniscus ornatissimus, L. Agassiz, Poiss. Foss., vol. ii, pt. i (1843), p. 92, pl. x $a$, f. 4, 6, 8 (non f. 5, 7) ; J. Morris, Cat. Brit. Foss., I854, p. 337 .

LOC. Calcif. Sandst.; Burdiehouse. [Imperfect fish; Edinburgh Mus.]

\section{T.}

planti, R. H. Traquair, Geol. Mag. [3] vol. v, I888, p. 253; J. Ward, Trans. N. Staffs. Inst. Mining Engin., vol. x (I889), pl. iv, f. 6.

LOC. Coal-Meas.; Lancashire and N. Staffordshire. [Fish ; Salford Mus., Manchester.]

tenuicauda, R. H. Traquair, Proc. Roy. Soc. Edinb., vol. ix, 1877, p. 443 .

LOC. M. Carb. Limest. (Edge Coal); Wallyford, Edinburgh, and Possil, Glasgow. [Fish; Edinburgh Mus.]

tuberculatus, R. H. Traquair, Trans. Roy. Soc. Edinb., vol. $\mathrm{xxx}(188 \mathrm{I})$, p. 3I, pl. iv, f. I-3.

LOC. Calcif. Sandstones (Cement-stone Group) ; Glencartholm, Eskdale. [Fish; Geol. Surv. Scotland.]

wardi (Ward), R. H. Traquair, Quart. Journ. Geol. Soc., vol. xxxiii, 1877 , p. 559, and Proc. Roy. Phys. Soc. Edinb., vol. iv, I878, p. 239 : J. Ward, Trans. N. Staffs. Inst. Mining Engin., vol. x (1889), pl. vi, f. Io.

Palconiscus wardi, J. Young, Proc. Nat. Hist. Soc. Glasgow, vol. ii, pt. i (1875), p. 66 (name only); J. Ward, [Proc.] N. Staffs. Nat. Field Club, 1875, p. 239.

LOC. Coal-Meas.; North Staffordshire, Lancashire, and Lanarkshire. [Fish; J. Ward Coll.]

RmAmpHODUs, J. W. Davis, Trans. Roy. Dublin Soc. [2] vol. i (1883), p. 402.

dispar, J. W. Davis, ibid., p. 402, pl. li, f. I7.

LOC. L. Carb. Limest.; Armagh. [Tooth; Brit. Mus.] $\boldsymbol{T}$.

RyINOBATUS, J. G. Schneider, Blochii Syst. Ichthyol., I\&oI, p. 353.

sp., A. S. Woodward, Cat. Foss. Fishes B. M., pt. i, I889, p. 83, and Geol. Mag. [3] vol. vi, I889, p. 396.

Loc. Kim. Clay; Oxfordshire. [Vertebre; Bıit. Mus., no. 46332 a.]

RHINOCEPHALUS, L. Agassiz, Poiss. Foss., vol. v (1 843), p. 142 (name only), and Rep. Brit. Assoc., 1844 (1845), p. 308 (name only). 
RHINOCEIIIALS (continued)-

planiceps, L. Agassiz, ibid. (18+3), p. I 42 (name only), and ibid., 1844 (I845), p. 308 (name only).

T. LOC. London Clay; Sheppey. [Imperfect head; Brit. Mus.]

R IrIx OPTrRA, J. Müller and J. Henle (cx Kuhl), Syst. Beschre.l). Plagiostom., I84I, p. I $8 \mathrm{I}$.

Zygobates, L. Agassiz, Poiss. Foss., vol. iii (1843), p. 328.

daviesi, A. S. Woodward, Cat. Foss. Fishes B. M., pt. i, 1889, p. 126, pl. iii, f. 6 .

LoC. London Clay; Sheppey. [Dentition; Brit. Mus.]

woodwardi (Agassiz), A. C. L. G. Günther, Introd. Study of Fishes, 1880, p. 346, woodcut 132 ; A. S. Woodward, Cat. Foss. Fishes B. MI., pt. i, I889, p. 127.

Zygobates woodwardi, L. Agassiz, Poiss. Foss., vol. iii (1843), pp. 329, 333, pl. R, f. 6, 7 ; J. Morris, Cat. Brit. Foss., 1854 , p. 346.

LOC. Red Crag; Suffolk. Norwich Crag; Norfolk. [Teeth; olim Buxton Coll.]

RHIPIDOLEPIS, L. Agassiz, Rep. Brit. Assoc., I844 (1845), p. 308 (name only).

elegans, L. Agassiz, ibid., p. 308 (name only).

LOC. London Clay; Sheppey.

RIIzoD orsis, T. H. Huxley, Figs. and Descript. Brit. Organic Remains, dec. xii (Mem. Geol. Surv., I 866), p. 3 I (footnote); J. Young, Quart. Journ. Geol. Soc., vol. xxii, I866, p. 596 ; T. Atthey, Ann. Mag. Nat. Hist. [4] vol. $x x, 1877$, p. 129 ; R. H. Traquair, Ann. Mag. Nat. Hist. [4] vol. xx, I877, p. 24t, and Proc. Roy. Soc. Edinb., vol. ix, 1877, p. 444, and Proc. Roy. Phys. Soc. Edinb., vol. iv, 1878, p. 135, and Trans. Roy. Soc. Edinb., vol. xxx (188I), p. 167, and Geol. Mag. [3] vol. v, I888, p. 515 .

DitTonus, R. Owen, Trans. Odont. Soc.,vol.v, i 867, p. 325 (pars).

Ganol.odus, R. Owen, ibid., p. 354 (pars).

Characodus, R. Owen, ibid., p. 366.

Gastrodus, R. Owen, ibid., p. 370 .

Orthognathus, T. P. Barkas, Coal Meas. Palxont., 1873, p. 38 ; W. J. Barkas, Monthly Rev. Dental Surgery, vol. iv $(1876)$, p. 53o, f. lxxxix, xc.

sauroides (Williamson), J. Young, Quart. Journ. Geol. Soc., vol, xxii, 1866, p. 596, woodcut 8 ; A. Hancock and T. Atthey, Nat. Hist. Trans. Northumb. and Durham, vol. iii (1870), pp. 85, 9i, and Ann. Mag. Nat. Hist. [4] vol. i, 1868, p. 34); J. Ward, [Proc.] N. Staffs. Nat. Field Club, 1875, p. 23I, and Trans. N. Staffs. Inst. Mining Engin., vol. x (1889), pl. viii, f. 5-7; W. J. Barkas, Monthly Rev. Dental Surgery, vol. iv (1876), p. 433, f. lxxxii-lxxxv; R. H. Traquair, Trans. Koy. Soc. Edinb., vol. $x \times x$ (1881), p. 169, woodcuts $1-3$. 
RIIZODOPSIS sauroides (continued)-

'Fossil Salmon,' W. C. Williamson, Phil. Mag. [3] vol. xi, I837, p. 300, pl. ii, f. 4-6. [Scales.]

Holoptyclius, sp. now., E. W. Binney, Trans. Manchester Geol. Soc., vol. i (184I), p. 165, pl. v, f. 6. [Dentary; Manchester Mus.]

Holoptychius sauroides, II. C. Williamson, Phil. Trans., 1849, p. 457 , pl. xlii, f. 21-23.

Holoptychius granulutus, L. Agassiz, Poiss. Foss., vol. ii, pt. ii (Is+3), p. iso (name only); E. W. Binney, Trans. Manchester Geol. Soc., vol. i (18+1), p. 165, pl. v, f. 8, 10. [Scales.]

Rhizodus sramulatus, J. W. Salter, Foss. S. Welsh Coalfield (Mem. Geol. Surv. - Iron Ores Gt. Brit., pt. iii, I861), p. 223, pl. i, f. 4-6 (also teeth, pl. i, f. I-3, 7-9).

Holoptychius sp. indet., J. W. Kirkby and T. Atthey, Trans. Tyneside Nat. Field Club, vol. vi (I864), p. 235, pl. vi, f. 4.

Dittodus parallelus, R. Owen, Trans. Odontol. Soc., vol v, I867, p. 325 ,pl.i. [Micro. section of jaw; Brit. Mus.] $\mathbf{T}$. (Dittodus.)

Ganolodus craggesii, R. Owen, ibid., p. 356, woodcut. [Mandib. ramus; Brit. Mus.]

Characodus confertus, R. Owen, ibid., p. 366, pl. xiii. [Micro. section of tooth.]

m. (Characodus.)

Gastrodus prapositus, R. Owen, ibid., p. 37o, pls. xiv, xv. [Micro. section of jaw; Brit. MIus.] $\boldsymbol{x}$. (Gastrodus.)

Orthognathus reticulosus, T. P. Barkas, Coal Meas. Palæont., I 873 , p. 38 , f. 143 , I 4 . [Portions of jaws; T. P. Barkas Coll.]

r. (Orthognathus).

Rhizodopsis, T. P. Wrrkas, Coal Meas. Palæont., I873, p. 23, f. 59-66.

LOC. Coal-Meas.; al! English, Welsh, and Scottish Coalfields. [Scales.]

RFIzODU5, R. Owen, Odontogr. (I840), p. 75 ; F. M'Coy, Proc. Camb. Thil. Soc., vol. i (1848), p. 66, and Brit. Palrez. Foss. (1855), p. 611 ; I. Young, Quart. Journ. Geol. Soc., vol. xxit, IS66, 1p. 317, 599 ; L. C. Miall, Quart. Journ. Geol. Soc., vol. Xxxi, I 875 , p. 624, woodcut; W. J. Barkas, Monthly Rev. I)entii Surgery, vol. is 1875 , p. 394, f. lxxx, lxxi; R. H. Tratur, Ann. Wag. Nat. Hist. [t] rol. xx, 1877 , p. 244, and Proc. Roy. Soc. Edinb., vol. ix, 1877, p. 444, and Proc. Roy. I'hys. Soc. Edinb., vol. iv, I873, p. I35.

Megalichthys, L. Agassiz and S. Hibbert, Trans. Roy. Soc. Edinb., vol. xiii ( $1 \$ 36$ ), p. 202 (non Megalichthys, L. Agassi7, I'oiss. Foss., rol. ii, pt. ii (1 $8+3)$, p. 89 ).

ferox: given by J. Morris (Cat. Brit. Foss., 1854, p. 343), as described in Owen's Odontography, but name does not occur.

(IIoloptychius) ganneri, Ward. ¿'. Strepsodus sauroides. 
RIIZODUฐ (continued) -

gracilis, F. M'Coy. \%. Rhizodus hibberti.

granulutus (Agassiz), Salter. \%. Rhizodopsis sauroides.

hibberti (Agassiz and Hibbert), [-ii] R. Owen, Odontogr. (I 841 ), p. 75, pl. xxx1, f. 2, pls. xxxvi, xxxvii; F. M'Coy, Brit. I'alicoz. Foss. (1855), p. 6I2 ; J. Young, Trans. Geol. Soc. Glasgow, vol. ii (I865), p. $3^{8}$ (exc. scales, which are probably $R$. ornatus, R. H. Traquair, Proc. Roy. Soc. Edinb., vol. ix, I877, p. 659); R. H. Traquair, Ann. Mag. Nat. Hist. [4] vol. xv, I875, p. 266, and ibid. [4] vol. xix, 1877, p. 303, and Proc. Roy. Soc. Edinb., vol. ix, 1877, p. 658. [See also Archichthy's pirtlocki and A. sulcidens.]

Afgralichtlyys hibherli, I. Agassiz and S. Hibbert, Trans. Roy. Soc. Edinb., vol. xiii (1836), p. 202, pl. viii, f. I, 2, pl. ix, f. 2, 3. 9, 10 ; W. Buckland, Geol. and Mineral., ed. 2 , 1837, vol. ii, p. 43 , pl. xxvii, f. 12 .

Ifolnf,jchius hibberti (Agassiz), W. Buckland, ibid., I 837 , vol. ii, 1). 43 , pl. xxvii, f. 9-1I, I3, I4 (Holyptycluzs); L. Agassiz, Poiss. Foss., vol. ii, pt. ii (18+3), p. I80 (name only); J. Morris, Cat. Brit. Foss., 1854 , p. 329.

I'hyllolepis tomissimus, L. Agassiz, Poiss. Foss., vol. ii, pt. ii (I843), p. I 80 (name only). [Scales.]

Rhizodus gracilis, F. M'Coy, Brit. Paleoz. Foss. (1855), p. 6II, pl. iii G, f. 17. [Dentary; Woodwardian Mus.]

Rhizodus, J. Young, Quart. Journ. Geol. Soc., vol. xxii, i\$66, p. 597, woodcuts 4, 5 ; T. Stock, Geol. Mag. [2] vol. viii, I881, p. 77, and Trans. Edinb. Geol. Soc., vol. iv, I88 I, p. 38.

LOC. Calcif. Sandst. and Carb. Limest.; Edinburgh. Possil Ironstone (Carb. Limest.); Airdrie and Glasgrow. Redesdale Ironstone; Scremerston, Northumberland. [Teeth; olim S. Hibbert Coll.]

I.

Inneeiformis ('Newberry'), J. W. Kirkby and T. Atthey, Trans. Tyneside Nat. Field Club, vol vi (I864), p. 234, pl. vi, f. I-3. =Loxomma allmanni. [AMPHiBA.]

ornatus, R. H. Traquair, Proc. Roy. Soc. Edinb., vol. ix, IS77, p. 659.

Rhizodus, L. C. Miall, Quart. Journ. Geol. Soc., vol. xxxi, 1875 , p. 624, woodcut. [See also lihizodus hibberti.]

I.OC. Calcif. Sandst.; Burdiehouse and Pittenweem. M. Carlo. Limest. (Blackband Ironstonc); Gilmerton. [Various remains; Edinburgh Mus.]

porkoekii (Agassiz), Etheridge, \%. Archichthys portlocki.

striarus, R. Etheridse, Foss. Brit. Islands, pt. i, ISSS, p. 3+1. $=$ Holoptschizs striatus, Ag.

I HOMLOMTICHIUS, loung. \% Megalichthys.

sp., Youn. r'. Megalichthys rugosus. 
RHONCHUS, L. Agassiz, Rep. Brit. Assoc., I 844 (1845), p. 308 (name only) (R/loncus).

carangoides, L. Agassiz, ibid., p. 308 (name only).

Loc. London Clay; Sheppey. [Imperfect head; Brit. Mus.] T.

RHYMODUS, Davis. \%. Copodus.

convexus, Davis. \% Copodus convexus.

oblongus, Davis. \%. Copodus oblongus.

transversus, Davis. \%. Copodus prototypus.

RHYNCHORHINTS, L. Agassiz, Poiss. Foss., vol. v, pt. ii (I843), p. 139 (name only), and Rep. Brit. Assoc., I844 (1845), p. 308 (name only).

branchialis, L. Agassiz, Poiss. Foss., vol. v', pt. ii (1843), p. I39 (name only).

LOC. London Clay; Sheppey. [Imperfect head; Brit. Mus.] T.

SAGENODUS, Owen. ¿'. Ctenodus.

incequalis, Owen. \%. Ctenodus obliquus.

SALMO, Linnæus, Syst. Nat., ed. 12, vol. i, 1766, p. 509.

lewesiensis, Mantell. \%. Osmeroides lewesiensis.

SANDAIODUS, J. S. Newberry and A. H. Worthen, Pal. Illinois, vol. ii, I866, p. I02.

Vaticinodus, O. St. John and A. H. Worthen, Pal. Illinois, vol. vii, 1883 , p. 8 .

minor, J. W. Davis, Quart. Journ. Geol. Soc., vol. xl, r884, p. 626, pl. xxvi, f. I7 ; A. S. Woodward, Cat. Foss. Fishes B. M., pt. i, 1889, p. 187.

Loc. Yoredale Rocks; Wensleydale, Yorkshire. [Tooth; York Mus.]

morris1, [-ii] J. W. Davis, Trans. Roy. Dublin Soc. [2] vol. i (1883), p. 437 , pl. liv, f. I-I6; [-ii] A. S. Woodward, Cat. Foss. Fishes B. M., pt. i, I889, p. I85.

Deltodus sp., J. Morris and G. E. Roberts, Quart. Journ. Geol. Soc., vol. xviii, 1862, p. 105, pl. iii, f. I-3 (? f. 4).

Vaticinodus sp., O. St. John and A. H. Worthen, Pal. Illinois, vol. vii, 1883, p. 81 .

Deltodopsis sp., O. St. John and A. H. Worthen, ibid., p. I60. LOC. L. Carb. Limest.; Bristol and Oreton. [Teeth; Brit. Mus. and Bristol Mus.]

SARGODON, T. Plieninger, Württemb. Jahresh., I847, p. I65.

tomicus, T. Plieninger, ibid., p. 165, pl. i, f. 5-10 ; ? A. S. Woodward, Trans. Leicester Lit. and Phil. Soc., n.s., pt. xi (I889), p. 20.

T.

LOC. Rhretic; Gloucestershire, Somersetshire, and ? Leicestershire. [Teeth; Brit. Mus., Bath Mus., and Leicester Mus.] 
SAURICrryxs, L. Agassiz, Poiss. Foss., vol. ii, pt. ii (1843), p. 84 ; R. Owen, Odontogr. (184j), p. 170.

Stylorhynchus, K. Martin, Zeitschr. deutsch. Geol. Ges., vol. xxv, 1873, p. 725 .

acuminatus, L. Agassiz, Poiss. Foss., vol ii, pt. ii (1843), p. 86, pl. lv $a$, f. $1-5 ;$ R. Etheridge, Proc. Cardiff Naturalists' Soc. vol. iii, r872, pl. ii, f. 7-IO; A. S. Woodward, Ann. Mag. Nat. Hist. [6] vol. iii, 1889, p. 301, pl. xiv, f. 7, 8, and Trans. Leicester Lit. and Phil. Soc., n.s., pt. xi (1889), p. 20.

Saurichthys longidens, L. Agassiz, Poiss. Foss., vol. ii, pt. ii (1843), p. 87, pl. 1v a, f. 17, I8. [Tooth from Aust Cliff.]

Saurichthys apicalis, J. Morris (nom Münster), Cat. Brit. Foss., 1854 , p. 343 ; R. Etheridge, Proc. Cardiff Naturalists' Soc. vol. iii, 1872 , pl. ii, f. 5, 6. [Teeth from Aust Cliff.]

LOC. Rhetic; Gloucestershire, Somersetshire, Devonshire, and Leicestershire. [Teeth ; Bristol Mus.]

SAUR OCEPIfAIUs, R. Harlan, Journ. Acad. Nat. Sci. Ihilad., vol. iii (1824), p. 337 .

Daptinus, E. D. Cope, Proc. Acad. Nat. Sci. Philad., I873, p. 339 ; E. T. Newton, Quart. Journ. Geol. Soc., vol. xxxiv, I878, p. 439.

intermedius: Daptinus intermedius, E. T. Newton, ibial., p. 440, pl. xix.

LOC. L. Chalk; Dover. [Head and portion of trunk; Brit. Mus.]

lanceolatus, L. Agassiz, Poiss. Foss., vol. v, pt. i (1844), p. 8. Misprint: $v$. Protosphyrzena ferox.

lanciformis, Agassiz. $\%$. Protosphyrena ferox.

striatus, L. Agassiz, Poiss. Foss., vol. v, pt. i (18.43), pp. 8, 102, pl. xxv $c$, t. 17-20; F. Dixon, Geol. Sussex, 1850, p. 375, pl. xxxy, f. 5 ; J. Morris, Cat. Brit. Foss., 1854, p. 343.

Cimolichthys?, E. T. Newton, Quart. Journ. Geol. Soc., vol. xxxiv, I 878, p. 793. [ [ jenus non det.]

Loc. Chalk; Sussex. [Fragment of jaw ; Brit. Mus.]

SAURODON, Agassiz (non Hays). $\approx$ '. Cimolichthys.

leamus, Agassiz (non Hays). \%. Cimolichthys lewesiensis.

SAUROPSIS, L. Agassiz, Netles Jahrb., I $\$_{32}$, p. I42, and Poiss. Foss., vol. ii, pt. ii (1843), p. 120.

mordax, L. Agassiz, ibid., p. 122 (name only).

LOC. Stonesfield Slate. [Jaw.]

SCAPANORIY NCIIUS, A. S. Woodward, Cat. Foss. Fishes B. M., pt. i, 1889 , p. 351 .

Rhinognathus, J. W. Davis (non Fairmaire, 1873), Trans. Roy. Dublin Soc. [2] vol. iii (1887), p. 480.

?gigas, A. S. Woodward, Cat. Foss. Fishes B. M., pt. i, 1889, p. 358. LOC. Cambridge Greensand. [Teeth; Brit. Mus.] 


\section{SCAPATOPEYTCHUS (continued)-}

rhaphiodon (Agassiz), A. S. Woodward, ibid, p. 353.

Lamna (Odontaspis) rhaphioa'on, L. Agassiz, Poiss. Foss., Feuill, p. 55 (1835), and vol. iii (1843), p. 296, pl. xxxvii $a$, f. I2-I6 (non f. II).

Oiontaspis rhaphiodon, F. Dixon, Geol. Sussex, 1850, descr. to pl. xxx, f. 32 ; J. Morris, Cat. Brit. Foss., 1854 , p. 332.

Lamna raphiodon, J. Morris, ibid., p. 33I.

LOC. Cambridge Greensand. Chalk; Sussex, Surrey, Kent, Wiltshire, and Norfolk. [Teeth ; Brit. Mus.]

? subulatum (Agassiz), [-us] A. S. Woodward, Cat. Foss. Fishes B. M., pt. i, I889, p. 356.

Squalus comubicus, G. A. Mantell (non Gmelin), Foss. S. Downs, 1822, p. 226, pl. xxxii, f. I.

Lamna (Odontaspis) subulata, L. Agassiz, Poiss. Foss., vol. jii (1843), p. 296, pl. xxxvii $a$, f. 5 (? f. 6, 7).

? Lamm (Odontaspis) rhaphiodon, L. Agassiz (errore), ibid., pl. xxxvii $a$, f. II. [Tooth; Brit. Mus.]

Icamnu subulata, J. Morris, Cat. Brit. Foss., I854, p. 33 I.

I.OC. Gault ; Folkestone. Cambridge Greensand. U. Greensand; Wiltshire. Chalk; Sussex, Surrey, Kent, Essex, Wiltshire, and Norfolk. [Teeth; Brit. Mus.]

sp. (cf. Odontaspis studeri, Pictet), A. S. Woodward, Cat. Foss. Fishes B. M., pt. i, I889, p. 359.

LOC. Neocomian ; Folkestone. [Teeth ; Brit. Mus., no. P. 5759 .]

SCAPIASPIJ, E. R. Lankester, Rep. Brit. Assoc., I864 (I865), Sect., pp. 5-8, and Fishes O. R. Sandst., pt. i (Pal. Soc., 1868), p. 19. [A provisional genus probably founded upon ventral shields.]

P'TERAspis, R. Kner (non Huxley), Haidinger's Naturw. Abhandl., vol. i, 1847, p. 159 (pars).

cormubicus (M'Coy), Lee. $\%$ Steganodictyum cornubicum.

lewisi (Agassiz), J. E. Lee, Geol. Mag. [2] vol. ix, I882, pl. iii, f. I.

Cephalaspis lewisii, L. Agassiz, Poiss. Foss., vol. ii, pt. i (I835), p. I 49, pl. ib, f. 8 ; J. Morris, Cat. Brit. Foss., I854, p. 319.

Cephalaspis lloydii, L. Agassiz, Poiss. Foss., vol. ii, pt. i (I835), p. 150 , pl. ib, f. 9-1I ; J. Morris, Cat. Brit. Foss., I 854 , p. 320. [Shield; olim Murchison Coll.]

Pteraspis lloydii, R. Kner, Haidinger's Naturw. Abhandl., vol. i, 1847, p. I 59, pl. v; 'T. H. Huxley, Quart. Journ. Geol. Soc., vol. xiv, I858, p. 267, and ibid., vol. Xvii, I861, p. I64.

T. (Ptcraspis, Kner, non Huxley.)

Scaphaspis lloydii, E. R. Lankester, Rep. Brit. Assoc., I 864 (1865), Sect., p. 58, and Fishes O. R. Sandst., pt. i (Pal. Soc., 1868), p. 20, pl. i, pl. vii, f. I, 6, 17, I 8 .

IOC. L. Old Red Sandst. (Cornstones) ; Herefordshire and Monmouthshire. [Shield; olim R. I. Murchison Coll.] 
SCAPHASPIS (continued)-

lloydi (Agassiz), Lankester. ¿'. Scaphaspis lewisi.

Iudensis (Salter), E. R. Lankester, Fishes O. R. Sandst., pt. i (Pal. Soc., I868), p. 25, pl. ii, f. 4 .

Pteraspis ludensis, J. W. Salter, Ann. Mag. Nat. Hist. [3] vol. iv, 1859 , p. 46 , woodcut.

LOC. L. and U. Ludlow; Herefordshire. [Shield.]

recta, [-us] E. R. Lankester, Fishes O. R. Sandst., pt. i (Pal. Soc., 1868), p. 23, pl. ii, f. 5-8, 12, I3, pl. vii, f. 2.

LOC. Old Red Sandst. (Cornstones); Herefordshire. [Shields; Brit. Mus. (pars).]

truncata (Huxley and Salter), -us] E. R. Lankester, Fishes O. R. Sandst., pt. i (Pal. Soc., I S68), p. 24, pl. ii, f. I-3.

Pteraspis truncatus, T. H. Huxley and J. W. Salter, Quart. Journ. Geol. Soc., vol. xii, is 856 , p. Ioo, pl. ii, f. I.

LOC. Downton Sandstone and Ludlow Bone-bed; Herefordshire. [Shield; olim R. IV. Banks Coll.]

SCAPHODUS. L. Agassiz, MIS. : J. Morris, Cat. Brit. Foss., I 854 , p. 344 (? Gyronchus).

heteromorphus, L. Agassiz MIS. in Bristol Museum; J. Morris, Cat. Brit. Foss., 1854 , p. $34+$.

LOC. Stonesfield Slate.

SCImWURUS, L. Agassiz, Rep. Irit. Assoc., I $8_{44}$ (I 845 ), p. 295.

bowerbanki, L. Agassiz, ibia., p. 286, and ibid., p. 295, pl., and Edinb. New Phil. Journ., vol. xl, 1846, p. I21, pl. vi; J. Morris, Cat. Brit. Foss., I 854, p. 344.

T.

Loc. London Clay; Sheppey. [Portions of fishes; Brit. Mus.] crassior, L. Agassiz, Poiss. Foss., vol. iv (1843), p. 307 (name only).

LOC. London Clay; Sheppey.

SCLERODUS, Agassiz. ¿. Eukeraspis.

pustuliferus, Agassiz. \%. Eukeraspis pustulifera.

SCOMBRINUS, L. Agassiz, Rep. Brit. Assoc., I844 (1845), p. 30 S' $^{\circ}$ (name only).

uuchalis, L. Agassiz, ibid., p. 308 (name only).

LOC. London Clay; Sheppey. [Imperfect head; Brit. Mus.] T

SCYLLIODUS, Agassiz. \% Cantioscyllium and Scyllium.

antiquus, Agassiz. \% Scyllium antiquum and Cantioscyllium decipiens.

SCyエrIUIM, Cuvier, Règne Animal, vol, ii, I S I7, p. 124.

Thyellina, L. Agassiz, Poiss. Foss., vol. iii (1843), p. 37 .

Scylliodus, L. Agassiz, ibia., p. 377 (pars). 
SCYIIIUIM (continued)-

antiquum (Agassiz), A. S. Woodward, Cat. Foss. Fishes B. M., pt. i, 1889 , p. 340.

Scylliodus antiquus, L. Agassiz, Poiss. Foss., vol. iii (1843), p. 378 , pl. xxxviii, f. 1, 3 (non f. 2).

Loc. L. Chalk; Kent. [Anterior portion of fish ; Brit. Mus.]

dubium, A. S. Woodward, Cat. Foss. Fishes B. M., pt. i, 1889, p. 34I, pl. xvi, f. 7,8 .

LOC. L. Chalk ; Dover. [Teeth ; Brit. Mus.]

sp. : vertebræ described by C. Hasse, Palæontographica, vol. xxxi (I884), p. 7, pl. ii, f. IO, II, and compared with the recent S. edruardsi.

LOC. U. Chalk; Dorking, Surrey. [Vertebræ; Anat. Inst. Univ. of Breslau.]

SELACHE, Cuvier. $\%$ Cetorhinus.

maxima (Linnæus). $\quad$ '. Cetorhinus maximus.

? sp. : F. Dixon, Geol. Sussex, I850, p. I Io, pl. xi, f. 25. [Probably a malformed tooth of Odontaspis.]

Loc. Bracklesham Beds. [Tooth ; Brit. Mus.]

SמMIONOTUS, L. Agassiz, Neues Jahrb., 1832, p. 144, and Poiss. Foss., vol. ii, pt. i (1837), p. 222.

brodie1, E. T. Newton, Quart. Journ. Geol. Soc., vol. xliii, I887, p. $539, \mathrm{pl}$. xxii, f. $1-8$.

Loc. U. Keuper; Shrewley, Warwick, and Colwick Wood, Nottingham. [Imperfect fishes ; P. B. Brodie Coll.]

joassi, A. S. Woodward, Ann. Mag. Nat. Hist. [5] vol. xx, 1887, p. 179, pl. viii. [Genus doubted by W. Deecke, Palæontographica, vol. xxxv (1889), p. I03.]

LOC. L. Oolite; Brora, Sutherlandshire. [Fish; Dunrobin Mus.]

manseli, Egerton. $\tau$. Lepidotus manseli.

rhombifer, Agassiz. r'. Eugnathus latus.

SIxURUs, Linnæus, Syst. Nat., ed. 12, vol. i, 1766, p. 501.

egertoni, Dixon. $\tau$. Arius egertoni.

SI'HAROIUUS, Agassiz. \%. Lepidotus.

gigas, Agassiz. $v$. Lepidotus maximus.

mammillaris, Agassiz. \%. Gyrodus cretaceus.

GPHAGODUS, L. Agassiz, in Murchison's Silur. Syst., 1839, p. 606.

pristodontus, L. Agassiz, ibid., p. 606, pl. iv, f. I-3.

'Shagreen,' J. Morris, Cat. Brit. Foss., 1854, p. 344.

LOC. U. Ludlow Bone-bed. [Dermal tubercles.] 
SPFrgacAntrus, L. Agassiz, l'oiss. Foss., vol. iii (1837), p. 23.

aquistriatus (Davis), A. S. Woodward, Cat. Foss. Fishes B.M., pt. i, 1889, p. 244.

Ctenacanthus aquistriatus, J. W. Davis, Quart. Journ. Geol. Soc., vol. $x x x v, 1879$, p. 185, pl. x, f. 15.

LOC. L. Coal-Meas. ; Yorkshire. [Spine; J. W. Davis Coll.]

costellatus (Traquair), A. S. Woodward, Cat. Foss. Fishes B. M., pt. i, 1889 , p. $2+2$.

Ctenacanthus costellatus, R. H. Traquair, Geol. Mag. [3] vol. i, I 884 , p. 3, pl. ii, and ibid., vol. v, r888, p. 8 I.

LOC. Calcif. Sandst. ; Eskdale, Dumfriesshire. [Fish; Brit. Mus.]

hybodoldes (Egerton), A. S. Woodward, Cat. Foss. Fishes B. M., pt. i, 1889, p. 242; J. Ward, Trans. N. Staffs. Inst. Mining Engin., vol. x (1889), pl. iii, f. 2.

Ctenacanthus hybodoides, P. M. G. Egerton, Quart. Journ. Geol. Soc., vol. ix, 1853, p. 280, pl. xii ; J. Morris, Cat. Brit. Foss., I 854, p. 323 ; J. Thomson, Trans. Geol. Soc. Glasgow, vol. iv, pt. i (I87 I), p. 59, pls. ii, iii ; J. Ward, [Proc.] N. Staffs. Nat. Field Club, 1875 , p. 215 ; J. IV. Davis, Quart. Journ. Geol. Soc., vol. xxxii, 1876 , p. 335.

Ctenacanthus nodosus, P. M. G. Egerton, Quart. Journ. Geol. Soc., vol. ix, 1853, p. 281 ; J. Morris, Cat. Brit. Foss., I854, p. 323. [Spine; Brit. Mus.]

Ctenacanthus major; J. Thomson (non Agassiz), Rep. Brit. Assoc., 1869 (1870), Sect., p. 102.

Cladodus elegans, T. P. Barkas, English Mechanic, vol. xii (1871), p. 403, woodcut. [Tooth.]

Ctenacanthus (? and Cladodus), T. P. Barkas, Coal Meas. Palæont., 1873, p. 2I, f. 43-58 (? p. I9, f. 3I-34).

Hybodus, W. J. Barkas, Geol. Mag. [2] vol. i, 1874, p. 163, and Proc. Roy. Soc. N. S. Wales, vol. xi (1878), p. 145.

? Hybodus davisi, W. J. Barkas, Monthly Rev. Dental Surgery, vol. vii, 1878, p. 193. [Tooth; J. W. Davis Coll.]

LOC. Coal-Meas. ; South Scotland, Northumberland, Yorkshire, Derbyshire, Staffordshire, and North Wales. [Spine; Brit. Mus.]

minor (Davis), A. S. Woodward, Cat. Foss. Fishes B. M., pt. i, 1889 , p. 244.

Ctenacanthus minor, J. W. Davis, Geol. Mag. [2] vol. vi, I879, p. 531, woodcut.

LOC. L. Coal-Meas. (Black-bed Coal); Dudley Hill, near Bradford, Yorkshire. [Spine; Bradford Mus.]

gerrulatus, L. Agassiz, Poiss. Foss., vol. iii (1837), p. 24, pl. i, f. II-I3 ; J. Morris, Cat. Brit. Foss., I 854, p. 344 ; A. S. Woodward, Cat. Foss. Fishes B. M., pt. i, I889, p. 242. 
SPHENACAN TIIUS serrulatus (intinued)-

Ctenacanthus? scrrulatus, R. H. Traquair, Geol. Mag. [3] vol. i, I 884 , p. 6.

Loc. Calcif. Sandst.; Hurdiehouse, near Edinburgh. [Spine ; Edinburgh Mus.]

sp., A. S. Woodward, Cat. Foss. Fishes B. M., pt. i, I889, p. 244.

LOC. U. Coal-Meas. (Ardwick Limest.); Ardwick, near Manchester. [Spine; Brit. Mus., no. 4 I25I $a$.]

SIIIENONCHUS, L. Agassiz, Poiss. Foss., vol. iii (1843), p. 20 r. A provisional genus founded upon head-spines of Hybodus (E. Charlesworth, Mag. Nat. Hist., n.s., vol. iii, 1839, p. 242), Acrodus (A. S. Woodward, Cat. Foss. Fishes B. M., pt. i, 1889, p. 290), and Asteracanthus (A. S. Woodward, Ann. Mag. Nat. Hist. [6] vol. ii, I888, p. 340). Said to have been originally named Leiosphen in collections (L. Agassiz, Poiss. Foss., vol. iii (1843), p. 20I).

clongatus, L. Agassiz, Poiss. Foss., vol. iii (1843), p. 202, pl. xxii (t, f. I8; J. Morris, Cat. Brit. Foss., I854, p. 344.

LOC. Wealden; Sussex. [Spine ; Brit. Mus.]

Ilamatus, L. Agassiz, Poiss. Foss., vol. iii (1843), p. 202, pl. xxii cr, f. 12-14; J. Morris, Cat. Brit. Foss., I854, p. 344. 'Tooth,' H. T. De La Beche, Trans. Geol. Soc. [2] vol. i (1822), p. 44, pl. iv, f. 4, 5 ; W. Buckland, Geol. and Mineral., ed. 2 (I837), pl. xxvii $d$, f. 6,7 .

LOC. L. Lias; Lyme Regis. [Spine.]

martini, Agassiz. \%. Hybodus lawsoni.

(Iyborlus) obtusus, J. W. Davis, Quart. Journ. Geol. Soc., vol. xxxvii, I88I, p. 420 , pl. xxii, f. 7.

Loc. Rhrtic; Aust Cliff, near Bristol. [Spine; J. W. Daris Coll.]

sp : 'Tooth of Fish,' 'T. Webster, Trans. Geol. Soc. [2] vol. ii (1826), p. $35, \mathrm{pl}$. vi, f. 8 .

LOC. Wealden; Sussex. [Spine.]

s1. Recorded from Lias of Weston, near Bath, and Wealden of Isle of Wight ( $\mathrm{E}$. Charlesworth and $\mathrm{H}$. Woods, Mag. Nat. Hist., n.s., vol. in, I839, pp. 279, 282): also from Kimeridge Clay, Weymouth; I'urbeck Beds, Swanage; Wealden, Sussex; and Grey Chalk, Dover (A. S. Woodward, Cat. Foss. Fishes 13. M1., pt. i, I889, pp. 303, 305).

SPIIYRANODUS, Agassiz. ¿ Dictyodus.

corssidens, Agassiz. \%. Dictyodus crassidens.

gracilis, Dixon. \% Dictyodus tenuis.

priscus, Agassiz. \%. Dictyodus priscus.

tenuis, Dixon. \%. Dictyodus tenuis. 
SPIMAx, Cuvier, Rigne Animal, vol. ii, 1817, p. I29.

major, L. Agassiz, Poiss. Foss., vol. iii (1837), p. 62, pl. x 6, f. 8 , 1o-rt (? pl. xl a, f. 6, vertebre). Dorsal fin-spines of undetermined species of Cestraciont Sharks, genera Cestracion and Synechodus (A. S. Woodward, Cat. Foss. Fishes B. M., pt. i, i 889, p. 337).

LOC. Gault ; Folkestone. U. Greensand ; Maidstone. Cambridge Greensand. Chalk; S. and S.E. England. [Brit. Mus.]

SQUAmoraJA, H. Riley, Proc. (ieol. Soc., vol. i (I833), p. 48t, and Trans. Geol. Soc. [2] vol.v (1837), p. 83 (Squalo-raia).

Spinacorhinus, L. Agassiz, Poiss. Foss., Feuill., p. 94 (i $\$ 37$ ).

polyspondyla, L. Agassiz, Poiss. Foss., vol. iii (I843), p. 3 SI ; J. Morris, Cat. Brit. Foss., I 854 , p. 345 ; W. Davies, Geol. Mag., vol. ix, 1872 , p. 145 , pl. iv ; C. Hasse, Palæontographica, vol. xxxi (1885), p. 4, pl. i, f. 2, 3 ; A. S. Woodward, Proc. Zool. Soc., I 886, p. 527, pl. lv, f. I-5, 7, 8.

Squaloraia dolichognathus, H. Riley, Proc. Geol. Soc., vol. i (1833), p. 483, and Trans. Geol. Soc. [2] vol. v (1837), p. $8_{3}$, pl. iv.

Spinacorhinus polyspondylus, L. Agassiz, Poiss. Foss., Feuill., p. 94 (1837), and vol. iii (1836), pls. xlii, xliii.

LOC. L. Lias; Lyme Regis. [Imperfect skeleton; Bristol Mus.]

T.

tenuispina, A. S. Woodward, Proc. Zool. Soc., I886, p. 530, pl. lv, f. 6.

LOC. L. Lias ; Lyme Regis. [Head-spine ; Brit. Mus.]

SQUATrNA, A. Duméril (cx Aldrovandi), Zool. Analyt., 1806, p. 102.

cranei, A. S. Woodward, Quart. Journ. Geol. Soc., vol. xliv, IS88, p. 144, pl. vii, f. I-6, and Proc. Geol. Assoc., vol. x (IS88), p. 294, and Cat. Foss. Fishes B. M., pt. i, 1889, p. 70.

? 'Tooth of Squaloid Fish,' F. Dixon, Geol. Sussex, 1850, descr. to pl. $\mathrm{xxx}, \mathrm{f} .35$.

LOC. L. Chalk; Sussex. [Head, etc.; Brighton Mus.]

sp. ind. Teeth of undetermined species recorded from the Gault of Folkestone; U. Chalk of Brighton, Lewes, and Norwich; Lower Eocene of Chislehurst, Kent ; London Clay of Highgate ; and the Red Crag of Suffolk (A. S. Woodward, Cat. Foss. Fishes B. M., pt. i, I889, p. 7I). Vertebre also recorded from the Chalk of Dorking, Surrey (ibid.). [Brit. Mus.]

STAGONOLEI'IS, Agassiz. Transferred to Reptilia. robertsoni, Agassiz. Transferred to Reptilia.

STrGaNoDICTyUm, F. M'Coy, Ann. Mag. Nat. Hist. [2] vol. viii, I85 I, p. $48 \mathrm{r}$, and Brit. Palaeoz. Foss. (1855), descr. to pl. ii A ; J. W. Salter, Geol. Mag., vol. v, 1868, p. 247 ("=Pteraspis'); C. W. Peach, Geol. Mag., vol. v, I 868, p. 568. 
STMGANODICTYUM (continucl)-

cornubicum, F. M'Coy, Ann. Mag. Nat. Hist. [2] vol. viii, I85 I, p. 482, and Brit. Palzeoz. Foss. (1855), descr. to pl. ii A, f. I-3. Scaphaspis cormubicus, J. E. Lee, Geol. Mag. [2] vol. ix, 1882, p. I05, pl. iii, f. 2, 3 .

LOC. Devonian; Polperro, Cornwall. [Fragments of shields ; Wvodwardian Mus.]

STEMIMATODUs, O. St. John and A. H. Worthen (non Heckel), Pal. Illinois, vol. vi, 1875 , p. 328. [A provisional name applied to certain Selachian dermal tubercles.]

sp., A. S. Woodward, Cat. Foss. Fishes B. M., pt. i, 1889, p. 247 ; J. Ward, Trans. N. Staffs. Inst. Mining Engin., vol. x (1889), pl. ii, f. 22.

Loc. Coal-Meas.; Fenton, North Staffordshire. [Tubercles; Brit. Mus., nos. 35000-1.]

STznostoma, F. Dixon (ex Agassiz, MS.), Geol. Sussex, I880, p. 373 ; A. S. Woodward, Proc. Geol.Assoc., vol.x(1888), p. 328.

pulchellum, [-a] F. Dixon, Geol. Sussex, 1850, p. 373, pl. xxxvi, f. 2; J. Morris, Cat. Brit. Foss., 1854, p. 345 ; [-a] A. S. Woodward, Proc. Geol. Assoc., vol. x (1888), p. 329.

LOC. Chalk ; S. E. England. [Imperfect fish ; Brit. Mus.]

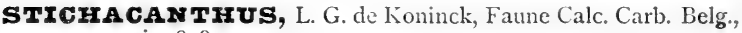
pt. i, 1878, p. 70 .

coemansi, L. G. de Koninck, ibid., p. 7 I, pl. vi, f. 4, 5 .

LOC. L. Carb. Limest.; Shropshire and Gloucestershire. [Spine; brit. Mus.]

tortworthensis, J. W. Davis, Trans. Roy. Dublin Soc. [2] vol. i (1883), p. 532, pl. lxv, f. 2 .

LOC. L. Carb. Limest.; Gloucestershire and Shropshire. [Spine; Earl of Ducie Coll.]

STrATODUs, E. D. Cope, Vert. Cret. Form. West (Rep. U. S. Geol. Surv. Territ., vol. ii, 1875), p. 219.

anglicus, A. S. Woodward, P'roc. Geol. Assoc., vol. x (1888), p. 314 , pl. i, f. 3 .

Loc. Chalk ; Sussex. [Jaws; Brit. Mus.]

STREBIODUS, J. Norris and (i. E. Roberts $x$ Agassiz, MS.), Quart. Journ. Geol. Soc., vol. xviii, 1862, p. IOI (name only); R. Owen, Geol. Mag., vol. iv, 1867, p. 62.

colei, J. Morris and (.. E. Roberts (ex Agassiz, MS.), Quart. Journ. Geol. Soc., vol. xviii, I862, p. 101 (name only); J. W. Davis, Trans. Roy. Dublin Soc. [2] vol. i (1883), p. 425, pl. liii, f. 5, 6 ; A. S. Woodward, Cat. Foss. Fishes B. M., pt. i, 1889, p. 210 , pl. vi, f. 25.

LOC. L. Carb. Limest. ; Armagh. U. Carb. Limest. ; Yorkshire and Derbyshire. [Teeth; Brit. Mus.] 
STRבBTODUS (continucd)-

egertoni, J. Morris and G. E. Roberts (e.r Agassiz, MS.), Quart. Journ. Geol. Soc, vol. xviii, I 862, p. 101 (name only); J. WV. Davis, Trans. Roy. I ublin Soc. [2] vol. i (1883), p. 426 , pl. liii, f. 7,8; A.S. Woodward, Cat. Foss. Fishes B.M., pt. i, 1889, p. 211 . Loc. L. Carb. Limest.; Bristol. [Teeth ; Brit. Mus.]

oblongus (Portlock), J. Morris and G. E. Roberts, Quart. Journ. Geol. Soc., vol. xviii, I862, p. Ior (name only); R. Owen, Geol. Mag., vol. iv, 1867, p. 62, pl. iii, f. 3 ; J. WV. Davis, Trans. Roy. Dublin Soc. [2] vol. i (I883), p. 424, pl. liii, f. I-4; A. S. Woodward, Cat. Foss. Fishes B. M., pt. i, 1 889, p. 209.

Cochliodus oblongus, L. Agassiz, Poiss. Foss., vol. iii (1843). p. 174 (name only); J. E. Portlock, Rep. Geol. Londonderry, I 843 , p. 466 , pl. xiv $a$, f. 5 , IO ; J. Morris, Cat. Brit. Foss., 1854 , p. 322 ; F. M'Coy, Brit. Palæoz. Foss. (1855), p. 623, pl. iii H, f. 19, pl. iii I, f. 28 .

Loc. L. Carb. Limest.; Armagh. [Teeth; Mus. Pract. Geol.] T.

STzzPsodus, J. Young ( $c x$ Huxley), Quart. Journ. Geol. Soc., vol. xxii, 1866, p. 602 ; T. P. Barkas, Coal Meas. Palæont., I 873 , p. 32 ; W. J. Barkas, Monthly Rev. Dental Surgery, vol. iv (1876), pp. 481, 529, f. lxxxvi-Ixxxviii.

DendroptychiUs, J. Young (ex Huxley, MS.), Quart. Journ. Geol. Soc., vol. xxii, I866, p. 601 .

LABYRINThODONTOSAURUS, T. P. Barkas, Coal Meas. Palæont., 1873, p. 75.

gauroides (Binney), J. Young, Quart. Journ. Geol. Soc., vol. xxii, I 866, p. 602, woodcut 3 ; G. Lyon, Trans. Edinb. (ieol. Soc., vol. ii $(1872)$, p. I25; J. Ward, [Proc.] N. Staffs. Nat. Field Club, 1875, p. 232, and Trans. N. Staffs. Inst. Mining Engin., vol. x (1889), pl. ii, f. 5 ; R. H. Traquair, Trans. Roy. Soc. Edinb., vol. xxx (ISSI), p. I8.

Holoptychius sauroides, E. W. Binney, Trans. Manchester Geol. Soc., vol. i (1841), p. 165, pl. v, f. 7 ; L. Agassiz, Poiss. Foss., vol. ii, pt. ii (1843), p. I80 (name only); J. W. Kirkby and T. Atthey, Trans. Tyneside Nat. Field Club, vol. vi (1S64), p. 234 , pl. vi, f. 5,6 .

Holoptychius garneri, L. Agassiz, Poiss. Foss., vol. ii, pt. ii (18+3), p. I80 (name only).

Rhizodus (Holoptychius) gameri, J. Ward, [Proc.] N. Staffis. Nat. Field Club, 1875, p. 233, f. 4. [Scale; J. Ward Coll.]

Dendroptychius, J. Young, Quart. Journ. Geol. Soc., vol. xxii, 1866, p. 601, woodcut $7 ; \mathrm{J}$. Ward, [Proc.] N. Staffs. Nat. Field Club, 1875, p. 233. [Scale; Mus. Pract. Geol.]

T. (Dendroptychizus.)

Labyrinthodontosaurus simmi, T. P. Barkas, Coal Meats. Palicont., 1873 , pp. 75,94 , f. 194, 223, 224. [Fragment of mandible; T. P. Barkas Coll.] T. (Labyrinthodontosaurus.) LOC. Calcif. Sandst. ; Eskdale and Liddesdale, Dumfries. CoalMeas.; Yorkshire, Lancashire, Staffordshire, and Northumberland. [Tooth; Manchester Mus.] 
STE:SSODUS (continued) -

striatulus, R. H. Traquair, Geol. Mag. [2] vol. ix, I882, p. 544.

LOC. Calcif. Sandst.; Abden, Fife. M. Carb. Limest. (Blackband Ironstone); Borough Lee, near Edinburgh. [Teeth ; Edinburgh Mus.]

STrOBIIODUS, A. Wagner, Abh. k. bay. Akad. Wiss., Cl. ii, vol. vi, 1851 , p. 75 .

Thlattodus, R. Owen, Geol. Mag., vol. iii, I866, p. 55.

suchoides (Owen), K. A. v. Zittel, Handb. Palæont., vol. iii (1887), p. 230 ; A. S. WVoodward, Geol. Mag. [3] vol. vi, I889, p. 450. Thlattodus suchoides, R. Owen, Geol. Mag., vol, iii, 1866, p. 55, pl. iii.

T. (Thlattodus.)

LoC. Kim. Clay ; Downham, Norfolk. [Jaws ; Brit. Mus.]

sp., A. S. Woodward, Geol. Mag. [3] vol. vi, I889, p. 450.

Loc. P'urbeck; Swanage. [Head; Brit. Mus., no. 469I1.]

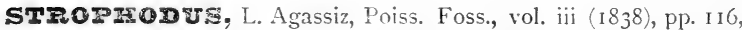
163 , pl. K, f. 3-5. [A 'generic' name to be provisionally retained for those teeth of Asteracanthus not yet discovered in association with their respective fin-spines. ]

asper, L. Agassiz, Poiss. Foss., vol. iii (1838), p. 128 b, pl. x b, f. I-3 ; J. Morris, Cat. Brit. Foss., 1854 , p. 345 . Crustacean fragment (A. S. Whodward, Proc. Geol. Assoc., vol. x (I888), p. 293, and Cat. Foss. Fishes B. M., pt. i, I889, p. 319), from the Chalk of Lewes. [Brit. Mus.]

farosus, Agassiz. v. Strophodus magnus.

lingualis, J. Phillips, Geol. Oxford, I87 I, p. I77, woodcut xxxvii, f. 8 (fig. only); A. S. Woodward, Cat. Foss. Fishes B. M., pt. i, 1889, p. 319, pl. xr, f. 9-12.

LOC. Stonesfield Siate; Oxfordshire. Forest Marble; Wilt. shire. [Teeth ; Brit. Mus.]

magnus, L. Agassiz, Poiss. Foss., vol. iii (1838), p. I26, nl. xviii, f. I I-I 5 ; J. Morris, Cat. Erit. Foss., 1854, p. 345 ; J. Phillips, Geol. Oxford, IS7I, p. I77, woodcut xxxvii, f. 7 ; A. S. Woodward, Cat. Foss. Fishes B. M., pt. i, I889, p. 314, pl, xv, f. 4-8. Siliquastrum, Luidius, Lithophyl. Brit., 1760, p. 73, pl. xvi. f. I 442, I 445, I 448, I 449 .

Siliquastrum minustriansulum, Luidius, ibid., p. 75, pl.xvi, f. I48S Strophodus far'osus, L. Agassiz, Poiss. Foss., vol. iii (1843) p. 175 (name only); A. S. Woodward, Science Gossip, 1885, p. 107 , woodcut 78 . [Tooth; Brit. Mus.]

? Strophodus ricaurz, H. M. Platnauer, Ann. Rep. Yorksh. Phil. Soc., I 886 (1887), p. 36, pl. i, f. I, 2. [Thoth; York Mus.]

I.OC. Inferior Oolite; Lincolnshire. Gt. Oolite (incl. Stonesfield Slate); Oxfordshire, Northamptonshire, Gloucestershire, and Wiltshire. Forest Marble; Wiltshire. Cornbrash : Northamptonshire. [Tceth.] 
STROPFODUS (continucl)-

radiato-punctatus, L. Agassiz, Poiss. Foss., vol. iii ( $183 S$ ), p. 128, pl. xviii, f. 27 ; J. Morris, Cat. Brit. Foss., 1854 , p. 345 .

LOC. Kelloways Rock ; Yorkshire. [Tooth ; Scarborough Mus.] reticulatus, Agassiz. $\approx$ '. Asteracanthus ornatissimus.

rigauxi, Platnauer (? Sauvage). $\%$ Strophodus magnus.

subreticulatus. Agassiz. \%. Asteracanthus ornatissimus. sulcutus, Agassiz. \%. Cestracion sulcatus.

tenuis, L. Agassiz, Poiss. Foss., vol. iii (1838), p. 127, pl. xviii, f. 16-25; J. Morris, Cat. Brit. Foss., 1854, p. 345 ; J. Phillips, Geol. Oxford, 1871, p. I77, wooduut xxxvii, f. 9 ; A. S. Woodward, Cat. Foss. Fishes B. M., pt. i, 1889, p. 317, pl. xv, f. 2,3 .

LOC. Gt. Oolite (incl. Stonesfield Slate); Oxfordshire, Lincolnshire, Northamptonshire, and W'iltshire. Cornbrash; Northamptonshire. Forest Marble; Oxfordshire and Wiltshire. [Teeth.]

sp., A. S. Woodward, Cat. Foss. Fishes B. M., pt. i, I889, p. 319 , pl. $x v$, f. 13 .

LOC. Forest Marble; Miltshire. [Tooth; Brit. Mus., no. P. 5886.]

ST2 p. 288, and Geol. Mag. [3] vol. v, I888, p. 496.

dubrisiengis (Mackie), A. S. Woodward, Proc. Geol. Assoc., vol. x (1888), p. 288, and Geol. Mag. [3] vol. v, I888, p. 496, woodcut, and Cat. Foss. Fishes B. M., pt. i, I889, p. 327, woodcut 12 and pl. xi, f. $17-20$, pl. xii, f. 6 .

Hybodus dubrisiensis, S. J. Mackie, Geologist, vol. vi, I863, p. 24I, pl. xiii; A. S. Woodward, I'roc. Zool. Soc.,'1886, p. 213 , pl. $x x$.

Loc. Chalk; Kent. [Jaws; Bit. Mus.]

z.

recurvus (Trautschold), A. S. Woodward, Cat. Foss. Fishes B. MI., pt. i (1889, p. 329, pl. xi, f. 22, 23.

Sphenodus recurvins, H. Trautschold, Bull. Soc. Imp. Nat. Moscou, 1877, pt. ii, p. 335, pl. v, f. 4 .

LOC. Gault; Folliestone. [Teeth; Brit. MIus.]

tenuis, A. S. Woodward, Cat. Foss. Fishes B. M., pt. i, I889, p. 329, pl. i, f. 2 I.

Loc. Neocomian; Maidstone. [Tooth; Lrit. Mus.]

sp., A. S. Woodward, iurd., p. 330, pl. xii, f. 7.

Loc. U. Chalk; Norwich. [Teeth; Brit. Mus., nos. 48953-4, P. 5846-7.]

TAYIODES, St. John and Worthen. \% Deltodus.

TARRASIUI, R. H. Traquair, Trans. Roy, Soc. Edinb., vol. xx. (1881), p. 6I. 
TARRASIUS (continued)-

problematicus, R. H. Traquair, ibid., p. 62, pl. iv, f. 4-6.

LOC. Calcif. Sandst. (Cement-stone Group); Tarras Foot and Glencartholm, Eskdale, Dumfriesshire. [Fish; Geol. Surv. Scotland.]

TשRATrCITIYS, C. Koenig, Icones Foss. Sect., 1825, p. 4 .

antiquitatis, C. Koenig, ibit., p. 4, pls. vi and vii, f. 79 and $79^{*}$; J. Morris, Cat. Brit. Foss., I 854 , p. 345.

LOC. London Clay; Sheppey. [Remains of skeleton; Brit. Mus.]

$\mathbf{r}$.

TrTRA GON OIEPTS, H. G. Bronn (non Agassiz), Neues Jahrb., I830, p. 30 ; P. M. G. Egerton, Quart. Journ. Geol. Soc., vol. ix, 1853, p. 274 .

discus, P. MI. G. Egerton, ibid., p. 278, pl. xi, f. 5 ; J. Morris, Cat. Brit. Foss., I 854 , p. 345.

LOC. U. Lias; Dumbleton. [Head and trunk; P. B. Brodie Coll.]

TETR AGONOLEPIS, Agassiz (non Bronn). v. Dapedius.

angulifer, Agassiz. $v$. Dapedius angulifer.

confluens, Agassiz. \%. Dapedius politus.

dor'sulis, Agassiz. $\%$. Dapedius dorsalis.

heteroderma, Agassiz. v. Dapedius heteroderma.

leachi, Agassiz. $v$. Dapedius leachi.

leiosomus, Agassiz. \%. Dapedius leiosomus.

mastodonteus, Agassiz. $\%$ Lepidotus mantelli.

monilifer, Agassiz. $\because$ Dapedius monilifer.

ovalis, Agassiz. \% Dapedius ovalis.

pholidotus, Agassiz. $\%$ Dapedius pholidotus.

pustulatus, Agassiz. $\%$. Dapedius granulatus.

radiatus, Agassiz. \% Dapedius radiatus.

speciosus, Agassiz. \%. Dapedius punctatus.

striolatus, Agassiz. \%. Dapedius monilifer.

trailli, Agassiz. \%. Dapedius angulifer.

TETrAPTURUS, C. S. Rafinesque-Schmalz, Caratt. Nuov. Gen. Spec. Anim. Sicilia, 18 so, p. 54. (Tetrapterus, L. Agassiz (emend.), Neues Jahrb., I839, p. I20).

minor, Agassiz. \%. Protosphyræna minor.

priscus, L. Agassiz, Poiss. Foss., vol. v, pt. i (1843), p. 9I, pl. xxxi ; J. Morris, Cat. Brit. Foss., 1854, p. 345.

Tetraptumes, L. Agassiz, Neues Jahrb., I834, p. 303.

Loc. London Clay; Sheppey. [Imperfect head; Paris Mus.] 
TrmexODUS, L. Agassiz, in Murchison's Silur. Syst. (1839), p. 606. parvidens, L. Agassiz, ibid., pp. 606 and 704, pl. iv, f. 34-36; F. M'Coy, Brit. Palæoz. Foss. (1855), p. 576 ; J. Morris, Cat. Brit. Foss., I854, p. 345.

LOC. U. Ludlow Bone-bed. [Shagreen?]

T.

THLATTODUS, Owen. $v$ Strobilodus.

suchoides, Owen. $v$. Strobilodus suchoides.

Tririssonotus, L. Agassiz, Poiss. Foss., vol. ii, pt. ii (r843), p. I28 (name only); P. M. G. Egerton, Figs. and Descript. Brit. Organic Remains, dec. ix (Mem. Geol. Surv., 1858), no. 2.

colei, L. Agassiz, Poiss. Foss., vol. ii, pt. ii (1843), p. 128 (name nnly) ; P. M. G. Egerton, Figs. and Descript. Brit. Organic Remains, dec. ix (M̂em. Geol. Surv., 1858), no. 2, pl. ii.

LOC. L. Lias; Lyme Regis. [Fish, wanting tail; Brit. Mus.] $\mathbf{T}$.

Trrarsso PATrR, A. C. L. G. Günther, Figs. and Descript. Brit. Organic Remains, dec, xiii (Mem. Geol. Surv., 1872), no. I.

salmoneus, A. C. L. G. Günther, ibid., no. I, pl. i.

LOC. Gault; Folkestone. [Fish ; Woodwardian Mus.]

Trrerssops, I. Agassiz, Poiss. Foss., vol. ii, pt. i (1833), p. I2, and pt. ii (1843), p. 123.

sp. (cf. T. heckeli, Thiollière), A. S. Woodward, Geol. Mag. [3] vol. vi, 1889, p. 455, and Rep. Brit. Assoc., I889.

LOC. Kim. Clay; Dorset. [Portions of fishes; Brit. Mus., no. P. 922.]

sp., A. S. Woodward, ibic., p. 455, and ibid., 1889.

LOC. Portland Stone; I. of Portland. [Fish ; Brit. Mus., no. P. 5538.]

TriuRsIUS, R. H. Traquair, Geol. Mag. [3] vol, v, I888, p. 516.

macrolepidotus (Sedgwick and Murchison), R. H. Traquair, ibid., p. 516 .

Diptcrus macrolepidotus, A. Sedgwick and R. I. Murchison, Trans. Geol. Soc. [2] vol. iii (1829), p. I43, pl. xvi, f. 2 (? f. 4, 5) ; L. Agassiz, Poiss. Foss., vol. ii, pt. i (1837), p. 115, pl. ii, f. 4 ; J. Morris, Cat. Brit. Foss., I854, p. 325.

? Osteolepis macrolepidotus, C. H. Pander, Saurodipt., Dendrodont., etc., devon. Syst., I860, p. 9, pls. i-iii (pars).

Diplopterax macrolepilotus, F. M'Coy (non Agassiz), Brit. Palæoz. Foss. (1855), p. 587.

Loc. L. Old Red Sandst.; Caithness. [Fish; Geol. Soc. London.]

T.

pholidotus, R. H. Traquair, Geol. Mag. [3] vol. v 1888, p. 516. LOC. L. Old Red Sandst.; Caithness. [Fish; Edinburgh Mus. 
TrYצrIINA, Agassiz. \%. Scyllium.

prisca, Agassiz. \%. Palæospinax priscus.

TrNCA, Cuvier, Règne Animal, vol. ii, I817, p. 193.

vulgaris, Cuvier, ibid., p. 193 ; E. T. Newton, Vert. Forest Bed (Mem. Geol. Surv., 1882), p. 127, pl. xviii, f. 21-23.

LOC. Forest-Bed Series (U. Freshwater Bed); West Runton, Norfolk. [Pharyngeal and tooth; A. Savin Coll., and Mus. Pract. Geol.]

TOzrodus, J. W. Davis (ex Agassiz, MS.), Trans. Roy. Dublin Soc. [2] vol. i (1883), p. 446.

convexus, J. Morris and G. E. Roberts (ex Agassiz, MS.), Quart. Journ. Geol. Soc., vol. xviii, I862, p. IOI (name only); J. W. Davis, Trans. Roy. Dublin Soc. [2] vol. i (I883), p. 446, pl. lv, f. I5-18; O. St. John and A. H. Worthen, Pal. Illinois, vol. vii, I883, p. 172 ; A. S. Woodward, Cat. Foss. Fishes B. M., pt. i, I889, p. I9I.

Cochliodus contortus, L. Agassiz (crrore), Poiss. Foss., vol. iii (1838), p. I1 5, pl. xiv, f. 23-25.

LOC. L. Carb. Limest. ; Bristol. [Teeth ; Bristol Mus.]

T.

TOMO GNATHUS, F. Dixon, Geol. Sussex, 1850, p. 376.

leiodus, Dixon. v. Tomognathus mordax.

mordax, F. Dixon, Geol. Sussex, I850, p. 376, pl. xxxv, f. I ; J. Morris, Cat. Brit. Foss., 1854, p. 346 ; A. S. Woodward, Proc. Geol. Assoc., vol. x (1888), p. 3 I 3.

Tomognathus leiodus, F. Dixon, Geol. Sussex, 1850, p. 377, pl. xxx, f. 31 ; J. Morris, Cat. Brit. Foss., 1854, p. 346. [Mandib. rami ; Brit. Mus.]

Loc. Chalk; Sussex. [Jaws.]

TRIPLOPTERUS, M'Coy. ข. Osteolepis. pollexfeni, M'Coy. $\%$ Osteolepis macrolepidotus.

TRIPTERUS, M'Coy. v. Osteolepis. pollexfeni, $\mathrm{M}^{\prime} \mathrm{Coy}$. v. Osteolepis macrolepidotus.

Tristrchoptraus, P. M. G. Egerton, Rep. Brit. Assoc., I860 (1861), Sect., p. 78, and Figs, and Descript. Brit. Organic Remains, dec. x (Mem. Geol. Surv., I86I), p. 5 I.

alatus, P. M. G. Egerton, Rep. Brit. Assoc., 1860 (1861), Sect., p. 78, and Figs. and Descript. Brit. Organic Remains, dec. x (Mem. Geol. Surv., 186I), p. 5 I, pls. iv, v; R. H. Traquair, Proc. Roy. Soc. Edinb., vol. viii, 1875, p. 513, and Trans. Roy. Soc. Edinb., vol. xxvii (1876), p. 383, pl. xxxii.

LOC. L. Old Red Sandst.; Caithness. [Trunk; Mus. Pract. Geol. and Brit. Mus.] 
TRISTyCrIU: L. Agassiz, I'oiss. Foss, vol. iii (1837), p. 21 : R. H. Traquair, Geol. Mag. [3] vol. v, 1888, p. 83.

Prychacantius, L. Agassiz, Poiss. Foss., vol. iii (1837), p. 22.

arcuatus, L. Agassiz, ibid. (1837), p. 22, pl. i $a$, f. 9-I I ; J. Morris, Cat. Brit. Foss., I 854 , p. 346 ; T. Stock, Ann. Mag. Nat. Hist. [5] vol. xii, I883, p. I77, pl. vii ; R. H. Traquair, Geol. Mag. [3] vol. v, I888, p. 83, and ibid., vol. vi, 1889, p. 27 ; A. S. Woodward, Cat. Foss. Fishes B. M., pt. i, 1889, p. 245 .

Ptychacanthus sublavis, L. Agassiz, Poiss. Foss., vol. iii (I 837), p. 23 , pl. v, f. I-3 ; J. Morris, Cat. Brit. Foss., I854, p. $34 x$. [Spine; olim Jameson Coll.]

LOC. Calcif. Sandst. ; S. Scotland. [Spine ; Anderson's College, Glasgow.]

T.

fimbriatus, Stock. v. Harpacanthus fimbriatus.

minor, J. E. Portlock, Rep. Geol. Londonderry, I843, p. 464, pl. xiv, f. 6; J. Morris, Cat. Brit. Foss., 1854, p. 346. [ = ? Sphenacanthus or Hoplonchus.]

LOC. Carboniferous; Fermanagh. [Spine ; Mus. Pract. Geol.]

URAUS, L. Agassiz, Neues Jahrb., 1832, p. 142. = Caturus and Pachycormus.

gracilis, Agassiz. v. Pachycormus gracilis.

URonmrus, L. Agassiz, Poiss. Foss., vol. ii, pt. ii (1843), p. 17 \& ; R. H. Traquair, Journ. Roy. Geol. Soc. Ireland, n.s., vol. iii (1873), p. 4I.

lobatus, L. Agassiz, Poiss. Foss., vol. ii, pt. ii (1843), p. 178 ; R. H. Traquair, Journ. Roy. Geol. Soc. Ireland, n.s., vol. iii (1873), p. 4I, pl. v; J. Morris, Cat. Brit. Foss., I85t, p. 346.

Phaneropleuron elegans, R. H. Traquair, Geol. Mag., vol. viii, I871, p. 534. [Name withdrawn, Journ. Roy. Geol. Soc. Ireland, n.s., vol. iii (1873), p. 47.]

LOC. Calcif. Sandst.; Burdiehouse, near Edinburgh. [Imperfect fish ; Brit. Mus.]

$\mathbf{T}$.

? magnus, R. H. Traquair, Geol. Mag. [2] vol. i, 1874, p. 554.

Loc. Coal-Meas. (Blackband Ironstone); Airdrie, Lanarkshire. [Tail; Brit. Mus.]

VมN UsmoDUs, O. St. John and A. H. Worthen, Pal. Illinois, inl. vi, 1875, p. 344 .

? clavatus (Davis), A. S. Woodward, Cat. Foss. Fishes B. M., pt. i, 1889, p. 225 .

Harpacodus clavatus, J. W. Davis, Trans. Roy. Dublin Soc. [2] vol. i (1883), p. 5 I5, pl. lxi, f. 20.

Loc. L. Carb. Limest. ; Armagh. [Tooth ; Brit. Mus.] 
VมNบSTODUS (continued) -

denticulatus $\left(\mathrm{M}^{\circ} \mathrm{C}\right.$ oy), A. S. Woodward, Cat. Foss. Fishes B. M., pt. i, 1889, p. 225 .

Chomatodus (Hclodus) denticulatus, F. M'Coy, Ann. Mag. Nat. Hist. [2] vol. ii, 1848, p. 124, and Brit. Palæoz. Foss. (1855), p. 618 , pl. iii $\mathrm{K}$, f. 9 .

LOC. L. Carb. Limest. ; Armagh. [Tooth ; Woodwardian Mus.]

serratus (Davis), A. S. Woodward, Cat. Foss. Fishes B. M., pt. i, I889, p. 225 , pl. i, f. I6, I7.

Lophodus serratus, J. W. Davis, Trans. Roy. Dublin Soc. [2] vol. i (1883), p. 408, pl. li, f. 23, 24, and Quart. Journ. Geol. Soc., vol. xl, 1884, p. 627, pl. xxvii, f. 19.

Loc. Yoredale Rocks ; Wensleydale. U. Carb. Limest.; South Derbyshire. [Teeth; York Mus.]

WARDICITzrys, R. H. Traquair, Ann. Mag. Nat. Hist. [4] vol. xv, I875, p. 266.

cyclosoma, R. H. Traquair, ibid., p. 262 , pl. xvi, f. I-5, and Trans. Roy. Soc. Edinb., vol. xxix (1879), p. 36r, pl. iv, f. 12-I ; ; T. Stock, Ann. Mag. Nat. Hist. [5] vol. vii, I88I, p. 490 .

LOC. Calcif. Sandst.; Wardie, near Edinburgh. [Fish; Edinburgh Mus.]

\section{$\mathbf{T}$.}

WODNIrA, G. von Münster, Beitr. Petrefakt., pt. vi, 1843, p. 48 .

striatula, G. von Münster, ibid., p. 48, pl. i, f. I ; R. Howse, Guide Coll. Local Foss. Mus. Nat. Hist. Soc., Newcastleupon-Tyne, 1889, p. 23.

LOc. Marl-Slate; Thickley. [Spines and shagreen; Newcastleupon-Tyne Mus. and W. Dinning Coll.]

XENACANTHUS, Beyrich. v. Pleuracanthus.

gibbosus (Agassiz), Salter. $\%$. Diplodus gibbosus.

XFNoDoramiA, J. Leidy, Journ. Acad. Nat. Sci. Philad. [2] vol. viii (1877), p. $25 \mathrm{I}$.

sp., A. S. Woodward, Cat. Foss. Fishes B. M., pt. i, 1889, p. 429.

LOC. L. Chalk; Guildford. [Tooth; Brit. Mus., no. 49954.]

Xiphias dixoni, Leidy. \%. Protosphyrena ferox.

XIPIODOLAMIA, Leidy. v. Notidanus sp.

XYSTroDus, J. Morris and G. E. Roberts (ex Agassiz, MS.), Quart. Journ. Geol. Soc., vol. xviii, I862, p. IoI (name only); J. W. Davis, Trans. Roy. Dublin Soc. [2] vol. iii (1883), p. 447 ; A. S. Woodward, Cat. Foss. Fishes B. M., pt. i, 1889 , p. 192.

angustus, Davis. $\%$ Xystrodus striatus. 
XYSTRODUS (continued)-

? egertoni, J. W. Davis, Trans. Roy. Dublin Soc. [2] vol. i (1883), p. 450 , pl. lv, f. 22,$23 ;$ A. S. Woodward, Cat. Foss. Fishes B. MI., pt. i, I889, p. 194.

LOC. L. Carb. Limest.; Bristol. [Teeth; Brit. MIus.]

parkeri, J. W. Davis, Geol. Mag. [3] vol. iii, I886, p. 153, woodcuts 3,4 .

LoC. Carb. Limest.; Chapel-en-le-Frith, Derbyshire. ['Teeth; T. Parker Coll.]

pulchellus, J. W. Davis, Trans. Roy. Dublin Soc. [2] vol. i (1883), p. 450 , pl. lv, f. 24.

Loc. Yoredale Rocks ; Wensleydale. [Tooth ; York Mus.]

striatus ( $\left.\mathrm{I}^{\circ} \mathrm{Coy}\right), \mathrm{J}$. Morris and G. E. Roberts, Quart. Journ. Geol. Soc., vol. xviii, I862, p. IOI (name only); J. W. Davis, Trans. Roy. Dublin Soc. [2] vol. i (1883), p. 448, pl. liv, f. 7Io ; O. St. John and A. H. Worthen, Pal. Illinois, vol. vii, 1883, p. I83 ; A. S. Woodward, Cat. Foss. Fishes B. M., pt. i, 1889, p. 193 .

Cochliodus striatus, L. Agassiz, Poiss. Foss., vol. iii (1S43), p. 174 (name only); J. Morris, Cat. Brit. Foss., I854, p. 322 ; F. M'Coy, Brit. Palæoz. Foss. (I855), p. 624, pl. iii I, f. 27.

Xy'strodus angustus, J. Morris and G. E. Roberts (ex Agassiz, MS.), Quart. Journ. Geol. Soc., vol. xviii, 1862, p. IOI (name only); J. W. Davis, Trans. Roy. Dublin Soc. [2] vol. i (1883), p. 449, pl. lv, f. 19-2I.

LOc. L. Carb. Limest.; Armagh, Northumberland, and Ayrshire. U. Carb. Limest.; Derbyshire. [Tooth; Woodwardian AIus.]

ZENASPIS, Lankester. \% Cephalaspis.

T. salweyi (Egcrton), Lankester. \% Cephalaspis salweyi.

ZYGOBATES, Agassiz. $\because$ Rhinoptera.

woodwardi, Agassiz. $\%$. Rhinoptera woodwardi.

\section{MISCELLANEOUS PISCES.}

'Dentes Squali,' Brander, 1766. ‘r. Lamna obliqua, Notidanus serratissimus, and Odontaspis elegans.

'Dorsal fin of fish allied to Silurus,' Mantell, 1827. i' Hybodus striatulus.

'External defensive organ,' De la Beche, I8z2. \% Myriacanthus paradoxus.

'Fish (? Chatodon),' Winch, 1817. ¿'. Platysomus striatus.

'Fish Spine,' Sowerby, I825. ¿'. Gyracanthus formosus. 
'Tlat fish,' Clanny, I8I 5. v. Platysomus striatus.

'Fossil Fish,' Sedgwick, 1829. \%. Acrolepis sedgwicki and Globulodus macrurus. [The original of pl. xi of Sedgwick's memoir is referable to Colacanthus granulosus.]

'Fossil Fish,' De la Beche, I822. v. Pholidophorus delabechei.

'Fossil jaw with triple row of teeth,' De la Beche, 1822. $\%$. Hybodus reticulatus.

'Ornithoglossum sessile vulgaris,' Luidius, 1760 . $v$. Odontaspis elegans.

Otoliths, E. T. Newton, Vert. Forest Bed (Mem. Geol. Surv., 1882), p. I28, pl. xviii, f. 25,25 .

LOC. Weybourn Crag; East Runton, Norfolk. [Mus. Pract. Geol.]

'Otolithus (incerte sedis) crassus,' Koken, 1884. $v$. Arius crassus.

'Palate,' Sowerby, I826. v. Ctenodus cristatus.

'Palate, or part of a palate,' Morris and Roberts, 1862. v. Psammodus salopiensis.

'Palate of unknown fish,' Parkinson, 1811. v. Ptychodus decurrens and P. latissimus.

'Reptile or Fish,' Toulmin Smith, 1846. $\approx$. Pachyrhizodus gardneri.

'Selachian Fish,' Coutts, 1883. \%. Psephodus magnus.

'Siliquastrum,' Luidius, 1760. $\approx$. Strophodus magnus.

'Siliquastrum minus triangulum,' Luidius, 1760. v. Strophodus magnus.

'Tooth of fish,' Webster, I829. \%. Sphenonchus sp.

'Teeth allied to Diodon,' Mantell, 1822. \%. Ptychodus latissimus, $P$. mammillaris, and $P$. polygyrus.

'Tooth of Squalus,' Mantell, IS22. \%. Notidanus microdon.

'Undetermined,' Mantell, I822. $\%$ '. Protosphyræna ferox.

'Unknown fish,' Mantell, I8z2. \% Portheus mantelli.

The problematical tooth-like bodies, named Conodonts, and sometimes ascribed to Fishes, have been discovered by $\mathrm{C}$. Moore in the Carboniferous Limestone of England, and by C. J. Smith in the Lower Carboniferous of Scotland: see G. J. Hinde, Quart. Journ. Geol. Soc., vol. xxxr, 1879, p. 35 r. 
$199 \quad)$

\section{A M PHIBIA.}

AMPHICWLOSAURUS, T. P. Barkas. v. Loxomma.

taylori, T. P. Barkas. v. Loxomma allmanni.

AMTPISAURUS, T. P. Barkas. $v$. Anthracosaurus.

amblyodus, T. P. Barkas. v. Anthracosaurus russelli.

ANISOPUS, Owen. $\%$. Rhombopholis.

scutulatus, Owen. $v$. Rhombopholis scutulata.

AxTrRACOsAurus, T. H. Huxley, Quart. Journ. Geol. Soc, vol. xix, I 863 , p. 56 ; A. Hancock and T. Atthey, Nat. Hist. Trans. Northumb. and Durham, vol. iii, 1870, p. 319 ; L. C. Miall, Rep. Brit. Assoc., 1874 (1875), p. I59.

Amphisaurus, T. P. Barkas, Coal Meas. Palæont., i873, pp. 72,91 .

? Leptognathosiurus, T. P. Barkas, ibid., p. Io6.

? Streptodontosiurus, T. P. Barkas, ibid., p. I07.

edgei, W. H. Baily, Rep. Brit. Assoc., 1878 (1879), p. 530, and ibid., 1883 (1S84), p. 496.

Loc. Coal-Meas.; Jarrow, Kilkenny, Ireland. [Skull, vertebræ, etc.; Geol. Surv. Ireland.]

russelli, T. H. Huxley, Quart. Journ. Geol. Soc., vol. xix, 1863, p. 56, woodcuts I, 2; A. Hancock and T. Atthey, Nat. Hist. Trans. Northumb. and Durham, vol. iii (1870), p. 313, and ibid., vol. iv (1872), p. 385 , pl. xii, and Ann. Mag. Nat. Hist. [4] rol. vii, I871, p. 73, pl. vi; T. P. Barkas, Coal Meas. Palcont., 1873 , p. 65 , f. I 86 ; T. Atthey, Nat. Hist. Trans. Northumb. and Durham, vol. v, 1876 (1877), p. 307, pls. ix-xii, and Ann. Mag. Nat. Hist. [4] vol. xviii, 1876, p. I 46, pls. viii-xi; J. Ward, [Proc.] N. Staffs. Nat. Field Club, I 875, p. 248 .

Anthracosaums, A. Hancock and T. Atthey, Ann. Mag. Nat. Hist. [4] vol. iv, I869, p. 270; J. Thomson, Rep. Brit. Assoc., i 868 (1 869), Sect., p. 79.

? Pteroplax comuta?, T. P. Barkas, Ann. Mag. Nat. Hist. [4] vol. iii, 1869 , p. 419. 
ANTIRACOSAURUS russelli (iontinucd) -

Amphisaurus amblyodus, T. P. Barkas, Coal Meas. Palæont., I873, pp. 72, 91, f. 192, 221, 222.

? Leptognathosaurus elongatus, T. P. Barkas, ibid., p. 106, f. 236. Gen. et sp. non det. (J.. C. Miall, Rep. Brit. Assoc., 1874 (1875), p. 187.) [Mandib. ramus; 'T. P. Barkas Coll.]

T. (Leptognithosaumus.)

? Streffodontosaumus carinatus, T. P'. Barkas, Coal Meas. Palkont., 1873, p. 107, f. 237. ? Labyrinthodont (L. C. Miall, Rep. Brit. Assoc., 1874 (1875), p. 191). [Mandib. ramus; T. P. Barkas Coll.] T. (Streptodontosaurus.

Loc. Coal-Meas.; Lanarkshire, Northumberland, and North Staffordshire. [Skull; Mus. Pract. Geol.]

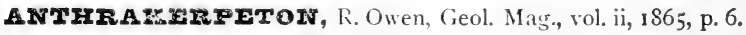

crassosteum, R. Owen. Geol. Mag., vol. ii, 1865, p. 6, pls. i, ii, woodcuts 1, 2, and Trans. Cardiff Naturalists' Soc., 1868-69 (IS70), p. I07, pls. i, ii, and woodcuts I, 2.

Loc. Coal-Meas.; Llantrissent, Glamorganshire. [Ribs, etc.; Brit. Mus.]

T.

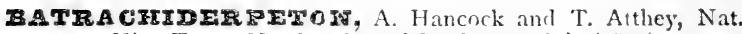
Hist. Trans. Northumb, and Durham, vol. iv (187 I), p. 209; L. C. Miall, Rep. Brit. Assoc., 1874 (1875), p. 165, pl. vi, f. 3,4 .

lineatum, A. Hancock and T. Atthey, Nat. Hist. Trans. Northumb. and Durham, vol. iv (I871), p. 208, pl. iv; T. P. Barkas, Coal Neas. Palæont., 1873 , p. 77, f. 196 ; L. C. Miall, Rep. Brit. Assoc., 1874 (1875), p. 165.

Loc. Coal-Nleas. (Low Main Seam); Newsham, near Newcastle-upon-Tyne. [Skull; Newcastle-upon-Tyne Mus.] $\mathbf{I}$.

BRACHYSCELIS, T. H. Huxley, Trans. Roy. Irish Acad., vol. xxiv (Sci.), 1867, p. 369 (name only).

sp., T. H. Huxley, ibid., p. 369 (undescribed).

LOC. Coal-Mleas.; Jarrow, Kilkenny, Ireland.

BUTO, J. N. Laurenti, Syn. Rept., 1768, p. 25.

6p., E. T. Newton, Geol. Mag. [2] vol. ix, r882, p. 9, and Vert. Forest Bed (Mem. Geol. Surv., I882), p. I18, pl, xvii, fig. 8.

IOC. Forest Bed; West Runton. [Iliac bones; A. Savin Coll., and Mus. Pract. Geol.]

CERATERPETON. \% Keraterpeton.

Cheirotherium anglomm (named 'from embodying the ideas of English geologists') applied to a theoretical tail-less restoration of the supposed maker of the Storeton footprints by S. Haughton, Man. Geology, 1865, p. 275, woodcut $4 \mathbf{I}$ [restoration]. [See also 'Ichnites' at end of REPTILIA.] 
DAsycres, T. H. Huxley, Quart. Journ. Gcol. Soc., vol. xv, 1859 , p. 647 , note, and appendix to Howell, Mem. WVarwick. Coalfield (Mem. Geol. Surv., 1859), p. 52 ; L. C. Miall, Rep. Brit. Assoc., 1874 (I875), p. I58, pl. v, f. 3 .

bucklandi (Lloyd), T. H. Huxley, appendix to Howell, Mem. Warivick. Coalfield (Mem. Geol. Surv., 1859), p. 52, woodcuts I, 2, and Quart. Journ. Geol. Soc., vol. xv, I859, p. 647, note; L. C. Miall, Rep. Brit. Assoc., 1874 (1875), p. 159; J. Phillips, Geol. Oxford, 1871, p. 96, woodcut xxv, f. I, 2.

Labyrinthodon bucklandi, Lloyd, Rep. Brit. Assoc., I 849 (1850), Sect., p. 56 ; J. Morris, Cat. Brit. Foss., 1854, p. 350. I.OC. Permian; Kenilworth. [Skull ; Warwick Mus.]

DASצGMATrus, T. H. Huxley, Mem. Geol. Surv. United Kingdom, Mlonogr. iii, 1877, p. 45.

Iongidens, T. H. Huxley, ibid., p. 45, pl. iv, f. I.

'Lower jaw of Stagonolepis,'? T. H. Huxley, Quart. Journ. Geol. Soc., vol. xv, i859, p. 454 .

Loc. U. Trias; Elgin. [Mandib. ramus; Elgin Mus.]

T.

DIADETOGIA AFES, L. C. Miall, Rep. Brit. Assoc., I 873 (1874), p. 248 , pl. ii, f. 3 (name only), and Quart. Journ. Geol. Soc., vol. xxx, 1874, p. 425.

varvicensis, L. C. Miall, Quart. Journ. Geol. Soc., vol. xxx, I874, p. 425 , pl. xxvi, f. 3 , pl. xxviii, and Rep. Brit. Assoc., I 874 (I875), p. I 58 .

LOC. Keuper; Warwick. [Mandib. ramus; Warwick Mus.] $\mathbf{x}$.

DISCOSPONDYLCS, T. H. Huxley, Trans. Roy. Irish Acad., vol. xxiv (Sci.), 1867, p. 369 (name only).

sp., T. H. Huxley, ibid., p. 369 (undescribed).

LOC. Coal-Meas. ; Jarrow, Kilkenny, Ireland.

Dorrcriosoma, T. H. Huxley, Trans. Roy. Irish Acad., vol. xxiv (Sci.), I867, p. 366.

emersoni, T. H. Huxley, ibid., p. 366 , pl. xxi, f. 3 ; L. C. Miall, Rep. Brit. Assoc., 1874 (1875), p. 171 .

IOC. Coal-Meas.; Jarrow, Kilkenny, Ireland. [Head and vertebral column; Dublin Univ. Mus.]

ERP vol. xxiv (Sci.), is67, p. 368. [? Ichthyerpeton.]

rugosus, T. H. Huxley, ibid., p. 368 , pl. xxiii, f. 2 ; L. C. Miall, Rep. Brit. Assoc., 1874 (1875), p. 186.

LOC. Coal-Meas.; Jarrow, Kilkenny, Ireland. [Head; Dublin Univ. Mus.]

T.

GASTRODUG, Owen. $\because$ Rhizodopsis. [PISCES.] prapositus, Owen. $\tau^{\prime}$. Rhizodopsis sauroides. [PISCES.] 
ICHTry ERRgTON, T. H. Huxley, Trans. Roy. Irish Acad., vol. xxiv (Sci.), 1867, p. 367.

bradleyæ, T. H. Huxley, ibid., p. 367 , pl. xxiii, f. I ; L. C. Miall, Rep. Brit. Assoc., 1874 (1875), p. I66.

LOC. Coal-Meas. ; Jarrow, Ireland. [Portion of trunk; Dublin Univ. Mus.]

EgratgrpgroN, T. H. Huxley, Trans. Roy. Irish Acad, vol. xxiv (Sci.), 1867, p. 354 ; L. C. Miall, Rep. Brit. Assoc., 1874 (1875), p. 170, pl. vii, f. 2.

galvani, T. H. Huxley, ibid., p. 354, pl. xix, f. $1-4$; L. C. Miall, Rep. Brit. Assoc., 1874 (1875), p. I7 I ; W. H. Baily, Charact. Brit. Foss. (1875), p. 121, pl. xli, f. 16; J. Ward, Trans. N. Staffs. Inst. Mining Engin., vol. x, I889, pl. ix, f. 2.

LOC. Coal - Meas. ; Jarrow, Ireland. [Imperfect skeletons ; Dublin Univ. Mus. (pars).]

IABYRINTHODON, R. Owen, Trans. Geol. Soc. [2] vol. vi, I84I (I842), p. 504 ; ? W. S. Symonds, Rep. Brit. Assoc., I 862 (1863), Sect., p. 96 (Scutes from Keuper bone-breccia, Pendock).

bucklanali, Lloyd. v. Dasyceps bucklandi.

giganteus (Jaeger), Morris. v. Mastodonsaurus giganteus.

iaegeri, v. Meyer. v. Mastodonsaurus giganteus.

? laniarius, R. Owen, Trans. Geol. Soc. [2] vol. vi, 184I (1842), pp. 512, 516. [? Mastodonsaurus pachygnathus.]

LOC. Keuper; Warwick. [Tooth; Warwick Mus.]

lavisi, H. G. Seeley, Quart. Journ. Geol. Soc., vol. xxxii, 1876, p. 278 , pl. xix.

LOC. Keuper ; Sidmouth. [Imperfect mandib. ramus.]

leptognathus, R. Owen, Trans. Geol. Soc. [2] vol. vi, I84I (1842), p. 516, pl. xliii, f. I-3, pl. xliv, f. $7-9$ (non pl. xlv, f. 5-10), and Odontography (I84I), p. 207, pl. lxiii A, f. I-3, pl. lxiii B, and Palæont., ed. 2, 1861, p. 211 ; J. Morris, Cat. Brit. Foss., 1854, p. 350 ; L. C. Miall, Quart. Journ. Geol. Soc., vol. xxx, I874, pp. 425, 430, and Rep. Brit. Assoc., I874 (1875), p. 158.

Loc. Keuper ; Coton End, Cubbington, and Warwick. [Portion of snout ; Warwick Mus.]

pachygnathus, Owen. v. Mastodonsaurus pachygnathus.

salamandroides, Owen. $v$. Mastodonsaurus giganteus.

scutulatus, Owen. $v$. Rhombopholis scutulata.

ventricosus, R. Owen, Trans. Geol. Soc. [2] vol. vi, I84I (I842), pp. 512,515 ; J. Morris, Cat. Brit. Foss., 1854, p. 350.

LOC. Trias; Coton End. [Tooth; Warwick Mus.]

LABITINTHODONTOSAURUS, T. P. Barkas. v. Strepsodus. [PISCES.]

simmi, T. P. Barkas, v. Strepsodus samroides. [P1SCES.] 
I Geol. Soc., vol. xxvi, 1870 , p. 557 ; L. C. Miall, Rep. Brit. Assoc., 1873 (1874), p. 245. [? Labyrinthodont.]

duffi, [-ii] A. Hancock and R. Howse, ibid., p. 557, pl. xxxviii, and Nat. Hist. Trans. Northumb. and Durham, vol. iv (1871), p. 22 I, pl. vi [lettered viii]; L. C. Miall, Rep. Brit. Assoc., 1874 (1875), p. 187.

Loc. Magnesian Limest.; Midderidge, Durham. [Fragments of head and trunk; Newcastle-upon-Tyne Mus. and Brit. Mus.]

IEPTER $\operatorname{xxiv}($ Sci.), 1867, p. 362 .

dobbsi, [-ii] T. H. Huxley, ibid., p. 362, pl. xxi, f. 1, 2 ; [-ii] L. C. Miall, Rep. Brit. Assoc., 1874 (1875), p. 168.

Loc. Coal-Meas. ; Jarrow, Ireland. [Skeleton; Dublin Univ. Mus.]

r.

LEPTOGNATHOSACRCS, T. P. Barkas. v. Anthracosaurus. elongatus, T. P. Barkas. $\%$. Anthracosaurus russelli.

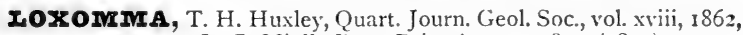
p. 293 ; L. C. Miall, Rep. Brit. Assoc., 1873 (1874), p. 225, pls. i, ii, and ibid., 1874 (1875), p. 162.

Mioganodus, R. Owen, Trans. Odontol. Soc., vol. v, I867, p. 357.

Orthosaurus, T. P. Barkas, Coal Meas. Palæont., I873, pp. $61,102$.

Megalocephalus, T. P. Barkas, ibid., p. 69.

? Macrosaurus, T. P. Barkas, ibid., p. 58.

?AMPhicclosaurus, T. P. Barkas, ibid., p. I04.

allmanni, T. H. Huxley, Quart. Journ. Geol. Soc., vol. xviii, r862, p. 293, pl. xi, f. I, 2 ; A. Hancock and T. Atthey, Ann. Mag. Nat. Hist. [4] vol. v, I 870 , p. 374, and ibid. [4] vol. vii, I871, p. 77, also Nat. Hist. Trans. Northumb. and Durham, vol. vi (187I-72), pp. 20I, 390; D. Embleton and T. Atthey, Nat. Hist. Trans. Northumb. and Durham, vol. v (1875), p. 196, pls. i-iv; T.Atthey, ibid., vol. viii (1874), p. 46, also Ann. Mag. Nat. Hist. [4] vol. xir, 1874, p. 38, pls. iv-vii ; L. C. Miall, Rep. Brit. Assoc., I873 (I874), p. 225 , pls. i, ii, and ibid., I874 (1875), p. 163, pl. vi, f. I ; J. Ward, [Proc.] N. Staffs. Nat. Field Club, I 875, p. 249 , and Trans. N. Staffs. Inst. Mining Engin., vol. x (1889), pl. ix, f. 1 ; D. Embleton, Rep. Brit. Assoc., 1889, and Nat. Hist. Trans. Northumb. and Durham, vol. viii (1889), pl. vi.

Mioganodus laniarius, R. Owen, Trans. Odontol. Soc, vol. v, I867, p. 357, pl. viii. [Micro. section of tooth; Brit. Mus.]

T. (Mioganodus:)

Loxomma, T. P. Barkas, Coal Meas. Palæont., 1873, p. $7 \mathrm{I}$, f. 190 . 
Ioxoaran allmanni (continued)-

Orthosaurus pachycephalus, T. P. Barkas, Coal Meas. Palæont., 1873 , pp. 61, 102, f. $183-185,232$. [Skull; T. P. Barkas Coll.]

T. (Orthosaurus.)

Megalocephalus macromma, T. P. Barkas, ibid., p. 69, f. 189. [Hinder portion of skull; T. P. Barkas Coll.]

T. (Megalocephalus.)

?.Macrosaurus polyspondylus, T. P. Barkas, ibid., p. 58, pl. vii. [Vertebral column; Newcastle-upon-Tyne Mus.]

T. (Macrosaurus.)

?Amphicalosaurus taylori, T. P. Barkas, ibid., p. 104, f. 234. [Vertebræ; T. P. Barkas Coll.] T. (Amphicalosaurus.)

? 'Tooth from Shelton.' R. Garner, Nat. Hist. Staffordshire, I 844 , p. 225 , pl. E, f. I3.

? Rhizodus lanceiformis, A. Hancock and T. Atthey, Ann. Mag. Nat. Hist. [4] vol. i, 1868, p. 271 (assigned to Pteroplax brevicornis by J. Young, Rep. Brit. Assoc., I869 (1870), Sect., p. IOI). [Teeth; Newcastle-upon-Tyne Mus.]

Loc. M. Carb. Limest. (Edge-Coal Series); Gilmerton, near Edinburgh. Coal-Meas.; Lanarkshire, Northumberland, N. Staffordshire, and Shropshire. [Hinder portion of skull and sternal plates; olim Edinburgh Mus.]

T.

MACROSAURUS, T. P. Barkas. \%. Loxomma.

poiyspoudylus, T. P. Barkas. ¿. Loxomma allmanni.

MASTODONSAURUS, C. F. Jaeger, Foss. Rept. Württembergs, I 828 , p. 35 ; L. C. Miall, Kep. Brit. Assoc., 1873 (1874), p. 226, pl. iii, f. I, 2 , and ibid., 1874 (1875), p. I 5 I, pl. iv, f. I, 2.

SAlainandoides, C. F. Jaeger, Foss. Rept. Württembergs, I 828 , p. 38 (wille Jaeger, Bull. Soc. Géol. France, vol. iii, 1833, p. 86$)$.

? giganteus (Jaeger), L. C. Miall, Quart. Journ. Geol. Soc., vol. xxx, I874, p. +30 , woodcut 2 , and Rep. Brit. Assoc., 1874 (1875), p. 152 .

Salamandroides giganteus, C. F. Jaeger, Foss. Rept. Württemhergs, I828, p. 38 , pl. iv, f. $4^{-6}$, pl. v, and Bull. Soc. Géol. France, vol. iii, 1833, p. 86.

Labyrinthodon giganteus, J. Morris, Cat. Brit. Foss., I 854, p. 350. Mastodonsumrus juegeri, F. Holl, Handb. Petref, vol. i, I 829, p. 88 ; H. von Meyer, Palæologica, I832, p. 107.

Salumandroides jägeri, F. von Alberti, Beitr. Monogr. Bunter Sandstein, 1834, p. 120.

Labyrinthodon jageri, R. Owen, Trans. Geol. Soc. [2] vol. v I $8+1$ (18+2), p. 506, woodcut 1, p. 537, pl. xliv, f. 4-6, pl. xlvii, f. I-3, and Odontography (1841), p. I98, pl. lxiii, f. I, pl. lxiv, pl. Ixiv $a$, and Palæont., ed. 2, I86I, p. 2 Io, woodcuts 86, 87.

Labyrinthodon salamandroides, R. Owen, Rep. Brit. Assoc., 1841 (18+2), p. 188.

LOC. Keuper; Guy's Cliff, Warwick. [Portions of skull and jaws; Warwick Mus.] 
MASTODONSAURTS (continued) -

pachygnathus (Owen), L. C. Miall, Quart. Journ. Geol. Soc., rol. xxx, 1874 , p. 418 , pl. xxvi, pl. xxvii, f. I, 2 (?4), and Rep. Brit. Assoc., I 874 (1875), p. I 52.

Labyrinthodon pachygnathus, R. Owen, Trans. Geol. Soc. [2] vol. vi, 1841 (1842), p. 526, pl. xliii, f. 4-I1, pl. xliv, f. 1-3 (? pl. xlvi, f. 6, 7), and Odontography, p. 205 (1841), pl. lxiii $\mathrm{A}$, f. 4, 5, pl. lxiv B, f. I, 2, and Palæont., ed. 2, 186I, p. 2 I 2 ; J. Morris, Cat. Brit. Foss., I 854 , p. 350.

LOC. Keuper; Coton End, Cubbington, and Warwick. [Portions of upper jaw ; Warwick Mus.]

IMFGAIERPETON, J. Young, Rep. Brit. Assoc., I869 (I8;0), Sect., p. IOI.

plicidens, J. Young, ibid., p. IOI ; L. C. Miall, Rep. Brit. Assoc., 1874 (1875), p. 187.

LOC. Coal-Meas. ; Lanarkshire. [Skull with teeth; J. Thomson Coll.]

simplex, J. Young, Rep. Brit. Assoc., I 869 (1870), Sect., p. Ior ; L. C. Miall, Rep. Brit. Assoc., I874 (I875), p. I88.

LOC. Coal-Meas.; Lanarkshire. [Skull with teeth; J. Thomson Coll. ]

MEGALOCEPHALCS, T. P. Barkas. थ. Loxomma.

macromma, T. P. Barkas. $\%$. Loxomma allmanni.

MESOSAURUS, T. P. Barkas, Coal Meas. Palæont., 1873, p. 1 if (name only).

taylori, T. P. Barkas, ibid. (name only); L. C. Miall, Rep. Brit. Assoc., 1874 (1875), p. 188 (no information).

LOC. Coal-Meas.; Northumberland.

MRפTOPIAS, H. von Meyer (non Eichwald, 1842), Neues Jahrb., 1842 , p. 302 ; L. C. Miall, Rep. Brit. Assoc., I 874 (1875), p. 157, pl. v, f. 2.

? diagnosticus, $H$. von Meyer, Neues Jahrb., 1842, p. 302, and Saurier des Muschelkalkes, 1847-52, pp. 146, 167,pls. 1x, 1xi, f. 3, lxiv, f. 10 ; L. C. Miall, Rep. Brit. Assoc., 1874 (1875), p. 157.

LOC. Rhæetic ; Aust Cliff, near Bristol. [Fragments of skull and jaws; Bristol Mus.]

T.

MOIGE, B. Merrem, Tent. Syst. Amphib., I 820 , p. 185.

Triton, J. N. Laurenti, Syn. Rept., 1768, p. 147 (preoccupied).

cristata (Laurenti), G. A. Boulenger, Cat. Batrachia Caudata, B. M., ed. 2, 1882, p. 8.

Triton cristatus, J. N. Laurenti, Syn. Rept., 1768, p. 147 ; E. T. Newton, Geol. Mag. [2] vol. ix, 1882, p. 9, and Vert.

Forest Bed (Mem. Geol. Surv., 1882), p. 119, pl. xvii, f. 9.

LOC. Forest Bed; West Runton. [Femur; Muts. Pract. Geol.] 
OPFID RPETON, T. H. Huxley, Trans. Roy. Irish Acad, vol. $\operatorname{xxiv}($ Sci.), 1867, p. 364 .

brownriggi, [-ii] T. H. Huxley, ibid., p. 364 , pl. xxii, f. I-4 ; [-ii] L. C. Miall, Rep. Brit. Assoc., 1874 (1875), p. 17 I.

Ophiderpeton nanum, A. Hancock and T. Atthey, Ann. Mag. Nat. Hist. [4] vol. i, I 868 , p. 276, and Nat. Hist. Trans. Northumb. and Durham, vol. iii (1870), p. 79. [Skeleton; Newcastle-upon-Tyne Mus.]

LOC. Coal-Meas. ; Jarrow, Kilkenny, Ireland, and Northumberland. [Imperfect skeleton; Dublin Univ. Mus.]

nanum, Hancock and Atthey. $\%$. Ophiderpeton brownriggi.

ORTHOSAURUS, T. P. Barkas. v. Loxomma.

pachycephalus, T. P. Barkas. v. Loxomma allmanni.

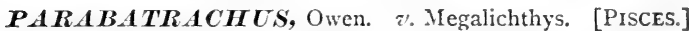
colei, Owen. $v$. Megalichthys hibberti. [PISCEs.]

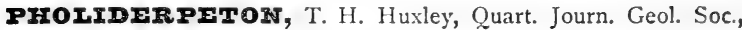
vol. xxv, I869, p. 310.

scutigerum, T. H. Huxley, ibid., p. 310 , pl. xi ; L. C. Miall, Rep. Brit. Assoc., 1874 (I875), p. 166.

Pholiderpeton, J. Ward, [Proc.] N. Staffs. Nat. Field Club, I 875 , p. 249.

'New Carboniferous Labyrinthodont,' L. C. Miall, Proc. Geol. Polyt. Soc. Yorkshire, vol. v (1870), p. 15.

LOC. M. Coal-Meas.; Toftshaw, near Bradford. Coal-Mleas.; Fenton, N. Staffordshire. [Portions of skeleton; Philosophical Society's Mus., Bradford.]

PHOIIDOGASTrR. T. H. Huxley, Quart. Journ. Geol. Soc., vol. xviii, 1862, p. 294.

pisciformis, T. H. Huxley, ibid., p. 294, pl. xi, f. 3, 4; L. C. Miall, Rep. Brit. Assoc., I 874 (1875), p. 166.

LOC. L. Carb.; Edinburgh. [Imperfect skeleton; Brit. Mus.]

PrgRormAx, A. Hancock and T. Atthey, Ann. Mag. Nat. Hist. [4] vol. i, 1868, p. 266, and Nat. Hist. Trans. Northumb. and Durham, vol. iii (1870), p. 67, and ibid., vol. iv (1872), p. 206; L. C. Miall, Rep. Brit. Assoc., 1873 (1874), p. 226, and ibid., 1874 (1875), p. 165.

brevicornis, J. Young, Rep. Brit. Assoc., I869 (1870), Sect., p. IOI.

LOC. Coal-Meas.; Lanarkshire. [Skull with teeth; J. Thomson Coll.]

ornuta, A. Hancock and T. Atthey, Ann. Mag. Nat. Hist. [4] vol. i, 1868, p. 266, pl. xiv, pl. xv, f. I, 2, and Nat. Hist. Trans. Northumb. and Durham, vol. iii (1870), p. 67, pl. i, pl. ii, f. I, 2, and ibid., vol. iv (1871), p. 207; T. Atthey, 
PTERopraz cornuta (continued)-

Ann. Mlag. Nat. Hist. [4] vol. xx, 1877 , p. 369, pls. xii, xiii, and Nat. Hist. Trans. Northumb. and Durham, vol. vii $(1 \$ 78)$, p. 176, pls. xv, xvi ; L. C. Miall, Rep. Brit. Assoc., 1873 (1S74), p. 225 , pl. iii, f. 3 , and ibid., 1874 (1875), p. 165, pl. vii, f. I; J. Ward, [Proc.] N. Staffs. Nat. Field Club, 1875, p. 249. [See also Anthracosaurus russelli.]

LOC. Coal-Meas.; Newsham, near Newcastle-upon-Tyne, and Longton, N. Staffordshire. [Middle portion of skull ; Newcastle-upon-Tyne Mus.]

T.

RANA, Linnaus, Syst. Nat., ed. 12, vol. i, 1766, p. 354.

? esculenta, Linnrus, ibid., p. 357 ; E. T. Newton, Geol. Mag. [2] vol. ix, I882, p. 9, and Vert. Forest Bed (Mem. Geol. Surv., I882), p. II 8 .

Loc. Forest Bed; West Runton. [Humeri; A. Savin Coll. and Mus. Pract. Geol.]

temporaria, Linnæus, Syst. Nat., ed. 12, vol. i, 1766, p. 357 ; E. T. Newton, Vert. Forest Bed (Mem. Geol. Surv., 1882), p. 117, pl. xvii, f. 7 .

Rana temporaria ?, E. T. Newton, Geol. Mag. [2] vol. ix, 1882, p. 8.

LOC. Forest Bed; West Runton and Sidestrand. [Humeri; A. Savin Coll. and Mus. Pract. Geol.]

RFOMBOPIIOIIS, R. Owen, Palieontology, ed. 2, 186I, p. 215 (as sub-genus). [A substitution for Anisopus, which had been previously used by Templeton for a proposed genus of Amphipodous Crustacea.] ? Labyrinthodont (L. C. Miall, Quart. Journ. Geol. Soc., vol. xxx, 1874, p. 433).

scutulata : Labyrinthodon (Anisupus) scutulatus, R. Owen, Trans. Geol. Soc. [2] vol. vi, 1841 (1842), p. 538, pl. xlvi, f. $I-5$.

Labyrinthodon (Rhombopholis) scutulatus, R. Owen, Palæont., ed. 2, I86I, p. 214, woodcut 88.

Labyrinthodon scutulatus, J. Morris, Cat. Brit. Foss., I854, p. 350.

? 'Tooth, found at Leamington,' Murchison and Strickland, Trans. Geol. Soc. [2] vol. v (1840), descr. to pl. xxviii, f. 9.

LOC. Keuper; Leamington. [Portion of skeleton; Warwick Mus.]

$\mathbf{T}$

SALAMANDROIDES, Jaeger. v. Mastodonsaurus.

giganteus, Jaeger. v. Mastodonsaurus giganteus.

jaegeri, Alberti. v. Mastodonsaurus giganteus.

STREPTODONTOSAURUS, T. P. Barkas. v. Anthracosaurus. cavinatus, T. P. Barkas. v. Anthracosaurus russelli.

TRITON, Laurenti. $v$. Molge. cristatus, Laurenti. $v$. Molge cristata. 
UROCORDYxUS, T. H. Huxley, Trans. Roy. Irish Acad., vol. xxiv (Sci.), I867, p. 359.

reticulatus, A. Hancock and T. Atthey, Ann. Mag. Nat. Hist. [4] vol. iv, I869, p. I82, and Nat. Hist. Trans. Northumb. and Durham, vol. iii (1870), p. 310; L. C. Miall, Rep. Brit. Assoc., 1874 (1875), p. 169.

LOC. Coal-Meas.; Northumberland. [Portion of skeleton ; Newcastle-upon-Tyne Mus.]

wandesfordi, [-ii] T. H. Huxley, Trans. Roy. Irish Acad. (Sci.), vol. xxiv, I 867 , p. 359 , pl. xx, f. I, 2 ; [-ii] L. C. Miall, Rep. Brit. Assoc., I874 (I875), p. I69; ? J. Ward, [Proc.] N. Staffs. Nat. Field Club, I875, p. 249.

Loc. Coal-Meas.; Jarrow, Ireland, ? and Longton, N. Staffordshire. [Imperfect skeleton; Dublin Univ. Mus.]

T.

\section{MISCELLANEOUS AMPHIBIA.}

'Ova of Batrachia,' G. A. Mantell, Quart. Journ. Geol. Soc., vol. viii, I 852 , p. I07, woodcuts $\mathrm{I}-3$; J. Morris, Cat. Brit. Foss., 1854, p. 347. [Doubtful fossil.]

LOC. Old Red Sandst.; Forfarshire.

Undetermined Labyrinthodonts recorded from the Coal-Measures of Newsham by A. Hancock and T. Atthey, Ann. Mag. Nat. Hist. [4] vol. i, I 868, p. 277, and Nat. Hist. Trans. Northumb. and Durham, vol. iii (187o), p. 8o (sternal plate); and from the Yoredale Beds of Wensleydale, by L. C. Miall, Quart. Journ. Geol. Soc., vol. xxx, 1874, p. 775, and J. W. Davis, Rep. Brit. Assoc., 1883 (1884), p. 492.

'New Carboniferous Labyrinthodont,' Miall, 1870. v. Pholiderpeton scutigerum.

'Tooth from Shelton,' Garner, 1844. $v$. Loxomma allmanni.

'Tooth, found at Leamington,' Murchison and Strickland, 1840. \%. Rhombopholis scutulata. 


\section{$209 \quad)$ \\ REPTILIA.}

ACANTroprrours, T. H. Huxley, Geol. Mag., vol. iv, 1867, p. 65.

eucercus, H. G. Seeley, Quart. Journ. Geol. Soc., vol. xxxv, 1879, p. 632.

LOC. Cambridge Greensand. [Vertebræ; Woodwardian Mus.]

horr1da, [-us] T. H. Huxley, Geol. Mag., vol. iv, 1867, p. 65, pl.v; [-us] H. G. Seeley, Quart. Journ. Geol. Soc., vol. xxxv, 1879, p. 596, woodcuts 2, 3 ; [-us] R. Lydekker, Cat. Foss. Rept. B. M., pt. i, 1888, p. I83, woodcut 34 .

? Acanthopholis?, H. G. Seeley, Quart. Journ. Geol. Soc., vol. xxxv, I879, p. 594, woodcut I. [Axis vertebra; Woodwardian Mus.]

Loc. Chalk-Marl; Folkestone. ?Cambridge Greensand. [Scutes, vertebræ, etc.; Mus. Pract. Geol.]

T.

macrocercus, H. G. Seeley, Index to Aves, etc., Woodwardian Mus., r869, p. xvii (name only).

LOC. Cambridge Greensand. [Vertebra; Woodwardian Mus.]

? platypus, H. G. Seeley, ibid., p. xvii (name only), and Ann. Mag.

Nat. Hist. [4] vol. viii, I871, p. 305, pl. vii. [?Macruroscuurus.]

LOC. Cambridge Greensand. [Metapodium; Woodwardian Mus.]

stereocercus, H. G. Seeley, Index to Aves, etc., Woodwardian Mus., I869, p. xvii (name only), and Quart. Journ. Geol. Soc., vol. xxxv, 1879, p. 628 .

LOC. Cambridge Greensand. [Vertebræ; Woodwardian Mus.]

ACRODONTOSAURUS, Mason. v. Pachyrhizodus. [PISCES.] gardneri, Mason. v. Pachyrhizodus gardneri. [PISCES.]

ALLIGATOR, Wood (non Cuvier). $\%$. Diplocynodon.

hantoniensis, Wood. v. Diplocynodon hantoniensis.

ALLOPLEURON, Baur. v. Chelone.

hoffmanni (Gray), Baur. $v$. Chelone hoffmanni. 
ATOPIOSA שR US, H. G. Seeley, Quart. Journ. Geol. Soc., vol. xxxv, I879, p. 600.

curtonotu.s, H. G. Seeley, ibid., p. 600, pls. xxxiv, xxxv.

LOC. Cambridge Greensand. [Remains of skeleton; Woodwardian Mus.]

major, H. G. Seeley, ibid., p. 63I.

LOC. Cambridge Greensand. [Cervical vertebra; Woodwardian Mus.]

ANOSTymaA, J. Leidy, Proc. Acad. Nat. Sci. Philad., 1871, p. 102 .

anglica, R. Lydekker, Ann. Mag. Nat. Hist. [6] vol. iii, 1889, p. 54 (Anostira), and Cat. Foss. Rept. B. M., pt. iii, 1889, p. I43. [? Identical with $A$. radulina, E. D. Cope, Proc. Amer. Phil. Soc., vol. xii (I872), p. 555, according to G. Baur, Ann. Mag. Nat. Hist. [6] vol. iii, I889, p. 273.]

Loc. U. Eocene ; Hordwell. [Left xiphiplastron ; Brit. Mus.]

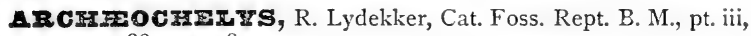
I889, p. 218.

valdensis, R. Lydekker, ibid., p. 219 , woodcut 50.

Emys, G. A. Mantell, Illustr. Geol. Sussex, r827, p. 6I, pl. vii, f. 3 (fig. only).

'Plastron from the Wealden,' R. Lydekker, Quart. Journ. Geol. Soc., vol. xlv, I889, p. 5 I I, woodcuts I, 2 .

LOC. Wealden; Cuckfield. [Hyo-and xiphiplastrals; Brit. Mus.]

ARGIII0C5rzIz:, R. Lydekker, Quart. Journ. Geol. Soc., vol. xlv, I889, p. 236.

antiqua (Koenig), R. Lydekker, Cat. Foss. Rept. B. M., pt. iii, I889, p. 4I, woodcut Io.

Chelone antiqua, C. Koenig, Icones Foss. Sect., I825, pl. xvii f. 232 .

Chelone breviceps, R. Owen (pars), Proc. Geol. Soc., vol. iii (I841), p. 570, and Rep. Brit. Assoc., I841 (I842), p. I78, and Rept. London Clay, pt. i (Pal. Soc., 1849), p. Io, pl. i, and Brit. Foss. Rept., vol. ii, I $\$ 84$, pl. xvii A (Chelonia).

Puppigenus breviceps, E. D. Cope (pars), Trans. Amer. Phil. Soc., vol. xiv (1870), p. 235.

LOC. London Clay; Sheppey. Red Crag; Suffolk (derived). [Skull ; Brit. Mus.]

convexa (Owen), R. Lydekker, Cat. Foss. Rept. B. M., pt. iii, I 889 , p. 48.

Chelone convera, R. Owen (pars), Rep. Brit. Assoc., I84I (1842), p. I78, and Proc. Geol. Soc., vol. iii (I841), p. 575, and Rept. London Clay, pt. i (Pal. Soc., 1849), p. 2 I, pl. vii ; J. Mcrris, Cat. Brit. Foss., 1854, p. 347. 
ARGIIIOCHETYS convexa (continued)-

Chelone declivis, R. Owen (pars), Rept. London Clay, pt. i (Pal. Soc., I849), p. 30, pl. xiv, and in F. Dixon, Geol. Sussex, I850, p. 229, woodcut ; J. Morris, Cat. Brit. Foss., I854, p. 347. [Carapace; Brit. Mus.]

Puppigerus conviexus, E. D. Cope, Trans. Amer. Phil. Soc., vol. xiv (1870), p. 235.

LOC. London Clay; Sheppey and Bognor. [Shell.]

cuneiceps (Owen), R. Lydekker, Cat. Foss. Rept. B. M., pt. iii, I889, p. 44.

Chelone cunciceps, R. Owen, Rept. London Clay, pt. i (Pal. Soc., 1849), p. 33, pl. xv ; J. Morris, Cat. Brit. Foss., I854, p. 347.

Chclone convexa, R. Owen (pars), in F. Dixon, Geol. Sussex, 1850 , p. 120, pl. xiii, f. I-3, and Brit. Foss. Rept., vol. ii, I884, pl. xxv (Chelonia), f. I-3. [Immature cranium; Brit. Mius.]

LOC. London Clay; Sheppey. [Cranium; Brit. Mus.] F.

subcristata (Owen), R. Lydekker, Cat. Foss. Rept. B. M., pt. iii, I 889, p. 47.

Chelone subcristata, R. Owen, Rep. Brit. Assoc., I84I (I842), p. 179, and Proc. Geol. Soc., vol. iii (1841), p. 576, and Rept. London Clay, pt. i (Pal. Soc., 1849), p. 24, pl. viii, and Brit. Foss. Rept., vol. i (1884), p. 24, pl. xv (Chelonia); J. Morris, Cat. Brit. Foss., 1854, p. 348.

? Chelone subcarinata, T. Bell, in R. Owen, Rept. London Clay, pt. i (Pal. Soc., 1849), p. 37, pl. viii A ; J. Morris, Cat. Brit. Foss., 1854, p. 348. [Shell ; Brit. Mus.]

Puppigerus subcristatus, E. D. Cope, Trans. Amer. Phil. Soc., vol. $\operatorname{xiv}(1870)$, p. 235.

? Puppigerus subcarinatus, E. D. Cope, ibid., p. 235.

LOC. London Clay; Sheppey. [Shell ; Brit. Mus.]

sp. $\boldsymbol{a}$ (cf. A. antiqua), R. Lydekker, Cat. Foss. Rept. B. M., pt. iii, I 889 , p. 4 I.

LoC. Bracklesham Beds. [Mandible; Brit. Mus., no. R. 1447.]

sp. $\boldsymbol{b}$, R. Lydekker, ibid., p. 44.

Chelone sp., R. Owen, Rept. London Clay, pt. i (Pal. Soc., I849), p. 43 , pl. xix D, f. I, 2 ; J. Morris, Cat. Brit. Foss., I 854, p. 348. Loc. 'U. Eocene; Hordwell.' [? Pracklesham Beds.] [Mandible; Brit. Mus., no. 38099.]

Azrstosucrius, H. G. Seeley, Quart. Journ. Geol. Soc., vol. xliii, 1887 , p. 22 r.

Calamospondylus, W. Fox, Geol. Mag., vol. iii, r866, p. ${ }_{3} \delta_{3}$ (name only).

pusillus (Owen), H. G. Seeley, Quart. Journ. Geol. Soc., vol. xliii, 1887, p. 221, pl. xii, f. 13, 14, and woodcut, and ibid., vol. xlv, I 889 , p. 396 ; R. Lydekker, Cat. Foss. Rept. B. M., pt. i, 1888 , p. 158 . 
ARISTOSUCHus pusillus (continued)-

Poikilopleuron pusillus, R. Owen, Rept. Weald. and Purb. Form., Suppl. vii (Pal. Soc., 1876), p. 2, pi. i.

Coclurus pusillus, O. C. Marsh, Amer. Journ. Sci. [3] vol. xxvii, 1884, p. 335.

Calamospondylus ozveni, W. Fox, Geol. Mag., vol. iii, 1866, p. 383 (name only). T. (Calamospondylus.)

I.oc. Wealden; Brook, I. of Wight. [Sacrum, etc.; Brit. Mus.]

AULACOCHELYS, Lydekker. \%. Trionyx.

circumsulcata (Owen), Lydekker. $v$. Trionyx circumsulcatus.

BAPTANODON, O. C. Marsh, Amer. Journ. Sci. [3] vol. xix, I880, p. 49I. [R. Lydekker states that a right humerus (Brit. Mus., no. 43989), named by him (Cat. Foss. Rept. B. M., pt. ii, 1889, p. 9) Ophthalmosaurus (?) cantabrigiensis, may belong to this genus.]

BOTIRTOSPONDYIUS, R. Owen, Rept. Mesoz. Form., pt. ii (Pal. Soc., 1875), p. 15.

elongatus, Owen. v. Morosaurus brevis.

magnus, Owen. v. Ornithopsis hulkei.

robustus, R. Owen, Rept. Mesoz. Form., pt. ii (Pal. Soc., 1875), p. 2 I, pl. vi ; R. Lydekker, Cat. Foss. Rept. B. M., pt. i, 1888, p. 17 I.

LOC. Forest Marble; Bradford. [Vertebra; Brit. Mus.]

suffossus, R. Owen, Rept. Mesoz. Form., pt. ii (Pal. Soc., I 875), p. 15, pls. iii, iv, v ; R. Lydekker, Cat. Foss. Rept. B. M., pt. i, I888, p. I7o, and Geol. Mag. [3] vol. v, I888, p. 452 (B. suffosus).

LOC. Kim. Clay; Swindon. [Vertebræ; Brit. Mus.]

I.

BRACHYDECTES, Owen. ข. Oweniasuchus.

major, Owen. v. Oweniasuchus major.

minor, Owen. $v$. Oweniasuchus minor.

CAIAMOSPONDYIUS, R. Lydekker (non Fox), Geol. Mag. [3] vol. vi, 1889, p. I2I.

foxi, R. Lydekker, ibid., p. I20, woodcut.

LOC. Wealden; I. of Wight. [Cervical vertebra ; Brit. Mus.]

CALAMOSPONDYLUS, Fox. v. Aristosuchus.

oweni, Fox. $v$. Aristosuchus pusillus.

CAMPTONOTUS, Marsh. v. Camptosaurus. 
CAmptosaur us, O. C. Marsh, Amer. Journ. Sci. [3] vol. xxix, 1885, p. 169.

Camptonotus, O. C. Marsh (non Uhler, 1864), ibid. [3] vol. xviii, I879, p. 501 .

Cumnoria, H. G. Seeley, Rep. Brit. Assoc., I887 (I888), p. 698.

leeds1, R. Lydekker, Quart. Journ. Geol. Soc, vol. xlv, I889, p. 47 , woodcut 3 .

LOC. Oxford Clay ; Peterborough. [Femur; A. N. Leeds Coll.]

prestwichi (Hulke), R. Lydekker, Quart. Journ. Geol. Soc., vol. xlv, I 889 , p. 48 .

Iguanodon prestivichi, [-ii] J. W. Hulke, Quart. Journ. Geol. Soc., vol. xxxvi, I880, p. 433, pls. xviii-xx; R. Lydekker, Quart. Journ. Geol. Soc., vol. xliv, I888, p. 47 , and Cat. Foss. Rept. B. M., pt. i, I888, p. I96.

Cumnoria prestwichi, H. G. Secley, Rep. Brit. Assoc., 1887 (1888), p. 698.

LOC. Kim. Clay; Cumnor Hurst. [Remains of skeleton ; Oxford Mus.]

valdensis, R. Lydekker, Quart. Journ. Geol. Soc., vol. xlv, I8\&9, p. 48.

'Camptonotus,' R. Lydekker, Geol. Mag. [3] vol. v, I88s, p. 453.

?'Young Iguanodon,' R. Owen, Rept. Weald. and Purb. Form., Suppl. iii (Pal. Soc., I862), p. 19, pl. x.

Hypsilophodon sp., R. Lydekker, Cat. Foss. Rept. B. M., pt. i, I 888, p. 195 .

Loc. Wealden; I. of Wight. [Femur; Brit. Mus.]

CARDIODON, R. Owen, Odontography (I84I), p. 29 I.

rugulosus, R. Owen, ibid., p. $29 \mathrm{I}$, pl. $\mathrm{Xxxy}$ A, f. 7 , and Brit. Foss. Rept., vol. i, 1884, p. 625, pl. lxxxv (Dinosauria), f. 2-5 ; J. Morris, Cat. Brit. Foss., 18 54 , p. 347.

[Probably tooth of Cetiosaurus o.roniensis, Phillips, according to Lydekker, Quart. Journ. Geol. Soc., vol. xlv, I889, p. 245.]

Loc. Forest Marble; Bradford, Wiltshire. [Teeth; Chaning Pearce Coll.]

$\mathbf{T}$.

CrTARTIR OSA URUS, H. (;. Seeley, ( )uart. Journ. Geol. Soc., vol. xxix, 1873 , p. 505 ; J. W. Hulkc, Proc. Geol. Soc., I 883 , p. 50.

walkeri, H. G. Secley, ibid., p. 505, woodcut I.

Ichthyosaurus walkeri, H. G. Seeley, Index to Aves, etc., Woodwardian Mus., r869, pp. xvii, 65.

LOC. Cambridge Greensand. [Femur; Woodwardian Mus.] $\mathbf{T}$. 
CETIOSA

Ceteosaurus, J. Phillips, Geol. Oxford, i 87 I, p. 245, woodcuts Ixxiv (plan of occurrence at Enslow)-cxii. [Phillips' localities for Coteosemrns are:-Blisworth, Buckingham, bletchingdon, Chapelhouse, Chipping Norton, Cuddesdon, Enslow Bridge, Essendine, Enstone, Garsington, Glympton, Kirtlington, Sarsden, Stony Stratford, Staple Hill, Thame.]

? brachgures, R. Owen, Rep. Brit. Assoc., 184I (1842), p. Ioo; J. Morris, Cat. Brit. Fuss., 1854 , p. 347.

Loc. Wealden; Tetham. [Dorsal and caudal vertebrx; Brit. Mus.]

brevis, Owen. a..Morosaurus brevis and Iguanodon bernissartensis. gidymptonensis, J. Phillips, Geol. Oxford, I87I, p. 29I, woodcuts xcv, xcvi ; R. Lyackker, Cat. Foss. Rept. B. M., pt. i, I 888, p. I 38.

Loc. Forest Marble; Glympton, Oxfordshire, and Cogenhoe, Northamptonshire. [Caudal vertebre; Oxford Mus.]

lemerocristutus, Huke. $\%$ Ornithopsis humerocristatus.

Iongua, R. Owen, Rip. Brit. Assoc., IS+1 (IS42), p. IOI, and Rept. Mesoz. Form., pt. ii (Pa1. Soc., 1875), p. 27, pl. x, and Brit. Foss. Rept., vol. i (1884), p. 413, pl. xxiv (Crocodilia); J. Miorris, Cat. 13rit. Foss., 185t, p. 347; J Phillips, Geol. Oxford, I87I, p. 390 . [See also C.oxoniensis.]

Loc. [? L. Oolite; White Nab, Yorkshire. (Vertebra; Scarborough Mus.)]. Fortland Stone; Garsington, Oxfordshire. [Caudal vertebre; Oxford Mus. (pars).]

mealius, Owen. v. Cetiosaurus oxoniensis.

oxozlezsis, J. Phillips, Geol. ()xford, 187I, p. 29I, woodcuts xcviii-cxi (? purs); R. Lydekker, Cat. Foss. Rept. B. M., pt. i, IS88, p. 137 . [Sec also Caralioilon.]

? Cetiosaums midius, R. Owen, Rep. Brit. Assoc., I84I (1842), p. I00; J. Morris, Cat. Lrit. loss., 1854 , p. 347. [Vertebra.]

Cetiosumus lonus, R. Owen (purs), Rept. Nesoz. Form., pt. ii (Pal. Soc., IS75), p. 27, woodcuts $1-10$.

(Ornithopsis) oxmichsis, H. (;. Seeley, Quart. Journ. Geol. Soc., vol. xlv, r889, p. 392, woodcut 2.

'Thigh bone,' J. Platt, Phil. Trans., 1758, p. 524, pl. xix.

LOC. Gt. Oolite and Forest Marble; Bibury, Blisworth, and Enslow.* [Portions of skeleton; Oxford Mis.]

'Cetiostumoid twoth,' G. S. Boulger, Proc. W. Lond. Sci. Assoc., vol. i $(1875)$, p. 99 .

LoC. Git. Oolite; Sapperton. [Tooth; Brit. Mus.]

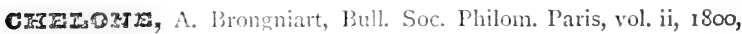
p. 89 (Chelinili).

Allopleuron, (i. Baur, Science, vol. xi, 1888, p. 144.

* Prestwich (Geology, vol. ii, r8s8, p. 2II) states that the original bones of this species, now in the? Uxford Mus., were ublained from the forest Marble, not the Gt. Oolite, as noted by thillips. 
Cris IONI (continued) -

acuticeps, Owen. $\%$. Lytoloma trigoniceps. [R. Lydekker, Cat. Foss. Rept. B. M., pt. iii, 1889 , p. 55, remarks :-'This name would appear to be due to an error, since in the text all the specimens (figs. 8, 10, and 18 ) are referred to Chelone longiccps.]

altimentum, R. Owen, in S. J. Mackie, Geologist, vol. v, I862, p. 74 (name only).*

antiqu, Koenig. $\%$. Argillochelys antiqua.

belli, Mantell. \%. Hylæochelys belli.

benstedi (Mantell), Owen. $v$. Cimoliochelys benstedi.

breviceps, Owen. $\%$ Argillochelys antiqua and Lytoloma planimentum.

comperi, Owen. $\because$. Chelone hoffmanni.

convexa, Owen. \%. Argillochelys convexa, A. cuneiceps, and Lytoloma planimentum.

costata, Owen. $\approx$ Lytoloma crassicostatum, Hylæochelys belli, and Plesiochelys sp.

crussicostuta, Owen. \% Lytoloma crassicostatum.

cuneiceps, Owen. $v$. Argillochelys cuneiceps.

declicis, Owen. \% Argillochelys convexa.

depressimentum, R. Owen, in S. J. Mackie, Geologist, vol.v, 1862 , p. 74 (name only).*

gigas, Owen. $\%$ Eosphargis gigas.

harriciensis, Samuel Woodward. v. Lytoloma planimentum.

? hoffmanni (Gray), R. Lydekker, Cat. Foss. Rept. B. M., pt. iii, I 889, p. 30.

Chelonia hoffmanni, J. E. Gray, Syn. Rept., I831, p. 54.

Allopleuron hoffmanni, G. Baur, Science, vol. xi, 1888, p. I44.

'Large Turtle,' P. Camper, Phil. Trans., vol. lxxvi, I786, p. 45 I.

Chelone camperi?, R. Owen, Rept. Cret. Form., pt. i (Pal. Soc., I 851), p. 9, pl.v ; J. Morris, Cat. Brit. Foss., I 854, p. 347.

Loc. Chalk; Kent. [Portions of carapace; Brit. Mus.]

jessoni, R. Lydekker, Quart. Journ. Geol. Soc., vol. xlv, I889, p. 23I, woodcut I, and Cat. Foss. Rept. B. M., pt. iii, I889, p. 36.

Loc. Cambridge Greensand. [Mandib. symphysis; Brit. Mus.]

latiscutata, Owen. v. Lytoloma crassicostatum.

7ongiceps, Owen. v. Lytoloma longiceps and Chelone acuticeps.

obovata, R. Owen, Rep. Brit. Assoc., IS+I (I 842 ), p. I7o, and Brit. Foss. Rept, vol. ii (I884), descr, to pl. ix (Chelonia);

J. Morris, Cat. Brit. Foss., I 854 , p. 348.

LOC. Purbeck; Swanage. [Shell and limb-bones; Chaning Pearce Coll. 1

* MS. names temporarily given to specimens from the Cambridge Greensand in the British Museum. 


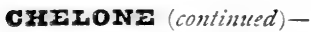

plamicejs, Owen. $v$. Stegochelys planiceps.

planimentum, Owen. v. Lytoloma crassicostatum and L. planimentum.

pulchriceps, Owen. $v$. Rhinochelys pulchriceps.

subcurinrta, Bell. $\%$ Argillochelys subcristata.

subcristatu, Owen. $v$. Argillochelys subcristata.

sulcimentum, R. Owen, in S. J. Mackie, Geologist, vol. v, I862, p. 74 (name only).*

trigomiceps, Owen. v. Lytoloma trigoniceps.

uncimentum, R. Owen, in S. J. Mackie, Geologist, vol. v, I862, p. 74 (name only).*

? sp., R. Lydekker, Cat. Foss. Rept. B. M., pt. iii, ı889, p. 3 o.

LOC. U. Eocene ; Hordwell. [Left scapulo-precoracoid; Brit. Mius, no. 304Iod.]

sp., R. Lydekker, Cat. Foss. Rept. B. M., pt. iii, 1889, p. 35.

LOC. L. Chalk; Dover [Pectoral girdle; Brit. Mus., no. 25960], Rochester [Various bones; Brit. Mus., no. 36813], and Lewes [Costal ; Brit. Mus., no. 49918].

s)., Owen, I $842 . \quad \%$. Cimoliochelys benstedi.

s)., Owen, 1849. $\%$. Argillochelys sp.

sp., Ow»n, i $850 . \%$. Eosphargis gigas and Lytoloma trigoniceps.

sp., Owen, 1851. \%. Lytoloma sp.

Chelys? blakii, Mackie and Blake. z'. Protochelys stricklandi.

CIIONIROSTEOSAURUS, Owen. v. Ornithopsis.

gigas, Owen. $\tau$. Morosaurus brevis and Ornithopsis hulkei.

agmus, Owen. $\%$ Ornithopsis hulkei.

GIRYSEMYS, J. E. Gray; Cat. Tortoises B. M., I844, p. 27.

blcarinata (Bell), R. Lydekker, Cat. Foss. Rept. B. M., pt. iii, I 889 , p. I I9.

Emy's bicarinata, T. Bell, in R. Owen, Rept. London Clay, pt. i, (Pal. Soc., 1849), p. 73, pls. xxv, xxvi ; J. Morris, Cat. Brit. Foss., I 854 , p. 348.

Clemmys bicarinata, R. Lydekker and G. A. Boulenger, Geol. Mag. [3] vol. iv, 1887, p. 275.

LOC. London Clay; Sheppey. [Imperfect shell ; Brit. Mus.]

testudiniformis (Owen), R. Lyclekker, Cat. Foss. Rept. B. M., pt. iii, I889, p. II 8 .

Emy's testudiniformis, R. Owen, Rep. Brit. Assoc., I84I (1842), p. I6r, and Rept. London Clay, pt. i (Pal. Soc., I849), p. 67, pl. xxiv, f. 6 (non f. I-5: $\%$. Ocadia owenz).

* MS. names temporarily given to specimens from the Cambridge Greensand in the British Museum. 
CrIRYSIMYS testudiniformis (continued)-

Emys toliapicus, R. Owen, Rep. Brit. Assoc., I84I (1842), p. I63.

'Enys de Sheppey,' Cuvier, Ossem. Fossiles, ed. 2, vol. v, I824, p. 235, pl. xv, f. I 4, I5. [Carapace; Deluc Coll., Geneva.]

LOC. London Clay; Sheppey. [Imperfect shell ; Brit. Mus.]

CIMIOCHELIS, Owen. v. Cimoliochelys.

CImorrocrralys, R. Owen, Rep. Brit. Assoc., I 84 I (I842), p. I76 (Cimochelys) ; A. S. Woodward, Proc. Geol. Assoc., vol. $x$ (1888), p. 275 .

benstedi (Mantell), A. S. Woodward, ibid., p. 276.

Emys benstedi, G. A. Mantell, Phil. Trans., I 841, p. 153, pls. $\mathrm{xi}$, xii.

Chelone (or Cimochelys) benstedi, R. Owen, Rep. Brit. Assoc. 184 I (I842), p. 173.

Chelone, R. Owen, Trans. Geol. Soc. [2] vol. vi (1842), p. 4 I2, pl. xxxix, f. 5, and Rept. Cret. Form. (Pal. Soc., 1851), p. 4, pls, i-iii.

Chelonia benstedi, G. A. Mantell, Medals, vol. ii, 1844, p. 772, f. 144.

Chelone benstedi, J. Morris, Cat. Brit. Foss., 1854, p. 347 ; L. Rütimeyer, Verh. Naturh. Ges. Basel, vol. vi, 1873, p. I I 8 ; R. Lydekker, Quart. Journ. Geol. Soc., vol. xlv, I889, p. 233, and Cat. Foss. Rept. B. M., pt. iii, 1889, p. 34.

LOC. L. Chalk; Burham, Kent. [Shell; Brit. Mus.]

T.

crmoziosaurus, J. Leidy, Proc. Acad. Nat. Sci. Philad., I85I (I852), p. 325 (Cimoliasaurus).

Elasmosaurus, E. D. Cope, ibid., r868 (1868), p. 92.

Polycotylus, E. D. Cope, Trans. Amer. Phil. Soc., vol. xiv (1870), p. 34.

Mauisaurus, J. Hector, Trans. New Zealand Inst., vol. vi, I 874 , p. 346.

Murenosaurus, H. G. Seeley, Quart. Journ. Geol. Soc., vol. $\mathrm{xxx}, 1874$, pp. 197, 437, 448 .

Colymbosaurus, H. G. Seeley, ibid., p. 447.

bernardi (Owen), R. Lydekker, Cat. Foss. Rept. B. M., pt. ii, I889, p. 185 .

Plesiosaumus bernardi, R. Owen, in F. Dixon, Geol. Sussex, 1850, pp. 396, 399, pl. xxxvii, f. 8, 9, pl. xl, and ed. 2, 1878, p. 423, same pls. and figs., and Rept. Cret. Form. (Pal. Soc., 1851), p. 6o, pl. xviii, and ibid., Suppl. iv (Pal. Soc., 1864), p. 7, pls. iv, v ; J. Morris, Cat. Brit. Foss., 1854 , p. 352.

?Plesiosaurus pachyomus, R. Owen (pars), Rep. Brit. Assoc., I 839 (1840), p. 74, and Rept. Cret. Form. (Pal. Soc., I851), p. 64 , pl. xx; J. Morris, Cat. Brit. Foss., 1854, p. 352. [Vertebrx; Woodwardian Mus.] 


\section{CIMOLIOSAURUS bernardi (continued)-}

Plesiosaums ichthyospondylus, H. G. Seeley, Index to Aves, etc., Woodwardian Mus., I869, p. xvii (name given to P. bernardi, Owen, I 864).

Loc. Cambridge Greensand. Clalk ; Houghton, Lewes, Scaddlescombe Pit, and Southeran Pit, Sussex. [Cervical vertebra.]

brachistospondylus (Hulke), R. Lydekker, Geol. Mag. [3] vol.v, I888, p. 354, and Cat. Foss. Rept. B. M., pt. ii, I889, p. 200. "

Plesiosaurus brachistospondylus, J. W. Hulke, Quart. Journ. Geol. Soc., vol. xxvi, I870, p. 6I I, pl. xli, f. 7-9.

LOC. Kim. Clay; Kimeridge and Shotover. Potton Bone-bed. [Dorsal vertebræ, etc. ; Brit. Mus.]

brevior, R. Lydekker, Cat. Foss. Rept. B. M., pt. ii, I889, p. 243, woodcut 75 .

LOC. Cornbrash; Stilton. Kim. Clay; Weymouth. [Cervical vertebre; Brit. Mus.]

cantabrigiensis, R. Lydekker, Cat. Foss. Rept. B. M., pt. ii, 1889 , p. 183 , woodcut 60.

Plesiosaumus neocomicnsis, R. Owen (non Pictet and Campiche), Rept. Cret. Form., Suppl. iv (Pal. Soc., I864), p. II, pl. vi.

Loc. Cambridge Greensand. Gault; Folkestone. [Cervical vertebrie; Brit. Mus.]

constrictus (Owen), R. Lydekker, Cat. Foss. Rept. B. M., pt. ii, I 889, p. 2 I 2.

Plesiosaumis constriclus, R. Owen, in F. Dixon, Geol. Sussex, I850, p. 398, pl. xxxvii, f. 6, 7, and ed. 2, 1878, p. 425, same pl. and figs., and Rept. Cret. Form. (Pal. Soc., 1851), p. 6I, pl. ix, f. 6, 7 ; J. Morris, Cat. Brit. Foss., 1854, p. 352.

Mauisaums garineri, H. G. Seeley, Quart. Journ. Geol. Soc., vol. xxxii, 1877, p. $54 \mathrm{I}$, pl. xxiii. [Portion of skeleton; Brit. Mus.]

Plesiosaumes gindneri, G. F. Whidborne, Quart. Journ. Geol. Soc., vol. xxxvii, I88I, table facing p. 480.

LOC. Gault; Folkestone. Chalk; Dorsetshire, Southeram Pit and Steyning, Sussex. [Cervical vertebræ; Brit. Mus.]

durobrivensis, R. Lydekker, Cat. Foss. Rept. R. M., pt. ii, I889, p. viii.

I.OC. Oxford Clay; Peterborough. [Portions of slieleton; A. N. Leeds Coll.]

erraticus (Phillips), R. Lydekker, Cat. Foss. Rept. B. M., pt. ii, I889, p. 245 (refers to some Stonesfield remains in the British Museum as possibly indicating a distinct species, for which Phillips' name may be adopted.)

Plesiosaurus erraticus, J. Phillips, Geol. Oxford, I871, p. I83 (name only).

LOC. Stonesfield Slate. [Vertebræ and humerus; Brit Mus.] 


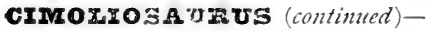

eurymerus (Phillips), R. Lydekker, Cat. Foss. Rept. B.M., pt. ii, 1889 , p. 205, woodcuts 66-68.

Plesiosaurus eurynerus, J. Phillips, Geol. Oxford, 1871, p. 315. woodcut cxx.

LOC. Oxford Clay ; Bedford, Chippenham, Devizes, Melksham, Peterborough, Weymouth, and Wootton-Bassett. [Pectoral limb ; Woodwardian Nus.]

latispinus (Owen), R. Lydekker, Cat. Foss. Rept. B. M., pt. ii, I889, p. 222.

Plesiosaurus latispinus, R. Owen, Cat. Foss. Rept. Mus. Roy. Coll. Surgeons, $18_{54}$, p. 63, and Rept. Cret. Form., Suppl. iv (Pal. Soc., I864), p. r4, pls. vii, viii, ix.

LOC. L. Greensand; Farringdon and Maidstone. [Imperfect skeleton, wanting head ; Brit. Mus.]

limrophilus (Koken), R. Lydekker, Cat. Foss. Rept. B. MI., pt. ii, 1899 , p. 224, woodcut 69.

Plesiosumrus limnophilus, E. Koken, Palæont. Abhandl., vol. iii (1887), p. 109.

LOC. Wealden; Cuckfield. [Cervical vertebre; Brit. Mus.]

oxonieusis (Phillips), R. Lydekker, Cat. Foss. Rept. B. M., pt. ii, I889, p. 209.

Plesiosumrus oroniznsis, J. Phillips, Geol. Oxford, I871, p. 307, woodcuts cxiii-cxvii ; H. G. Seeley, Quart. Journ. Geol. Soc., vol. xxx, 1874, p. 442 (determines woodcut cxvi to be pectoral arch).

Loc. Oxford Clay ; Devizes, Long Marston, Oxford, Shotover, and Summertown. [Imperfect skeleton, wanting head; Oxford Mus.]

planus (Owen), R. Lydekker, Cat. Foss. Rept. H. M., pt. ii, I889, p. 217.

Plesiosaurus planus, R. Owen, Rept. Cret. Form., Suppl. iv (Pal. Soc., I 864), p. 2, pls. i, ii, iii.

Plesiosumus pachyomus, R. Owen (pars), Rep. Brit. Assoc., 1839 (1840), p. 74, and Rept. Cret. Form. (Pal. Soc., I851), p. 64, pl. xxi, f. I. [Dorsal vertebra ; Woodwardian Mus.] Loc. Cambridge Greensand. [Vertebre ; Brit. Mus.]

plicatus (Phillips), R. Lyclekker, Geol. Mag. [3] vol. v, I 888 p. 351, and Cat. Foss. Rept. B. M., pt. ii, I889, p. 234, woodcuts 70-72. [Sce also Cimoliosaurus richardsoni.]

Plesiosaurus plicatus, J. Phillips (pars), Geol. Oxford, I87r, p. 31 3 , woodcuts cxviii, cxix.

Plesiosaurus infraplanus, J. Phillips, ibid., p. 374. [Cervical vertebra; Oxford Mus.]

? Plesiosaurus hexagonalis, J. Phillips, ibid., p. 375, woodcut clxxvi. [Caudal vertebra; Oxford Mus.] 
CIMOIIOSAURUS plicatus (continued) -

Muranosaurus leedsii, H. G. Seeley, Quart. Journ. Geol. Soc., vol. $\mathrm{xxx}, 1874$, p. 197, pl. xxi. [Skeleton; A. N. Leeds Coll.]

T. (Muranosaurus.)

Plesiosaurus leedsii, G. F. Whidborne, Quart. Journ. Geol. Soc., vol. xxxvii, I881, table facing p. 480 .

Loc. Oxford Clay; Chippenham, Cowley, Northampton, Oxford, Peterborough, Weymouth, and Whittlesea. Portland Oolite; Brill, Shotover, and Stanford. [Cervical vertebræ; Oxford Mus.]

portlandicus (Owen), R. Lydekker, Cat. Foss. Rept. B. M., pt. ii, I889, p. 227, woodcut 70 .

Pliosaurus portlandicus, R. Owen, Rept. Kim. Clay, pt. iii (Pal. Soc., I 869), p. 8, pl. iv, f. I-3 ; J. W. Hulke, Proc. Geol. Soc., vol. xxxix, 1883, p. 54, woodcut $\delta$.

Plesiosaurus winspitensis, H. G. Seeley, Ann. Mag. Nat. Hist. [4] vol. viii, I87I, p. I8I, woodcuts. [Vertebræ; Woodwardian Mus.]

Plesiosaurus carinatus, J. Phillips, Geol. Oxford, 1871, p. 374, woodcut clxxv (name preoccupied by Cuvier: $v$. Thaumatosaurus). [Lumbar vertebra ; Oxford Mus.]

Plesiosaurus phillipsi, H. E. Sauvage, Ann. Sci. Nat. (Zool.) [6] vol. viii, art. 6, 1879, p. 2 I ; G. F. Whidborne, Quart. Journ. Geol. Soc., vol. xxxvii, 1881, table facing p. 480.

Loc. Portland Oolite; Portland, Quainton, and Tisbury. [Pelvic limb; Brit. Mus.]

richardsoni, R. Lydekker, Cat. Foss. Rept. B. M., pt. ii, I889, p. 240, woodcuts 73, 74 ; J. C. Mansel-Pleydell, Proc. Dorset. Nat. Hist. Field Club, vol. x, 1889, p. 171, plate and woodcut.

Plesiosaurus plicatus, J. C. Mansel-Pleydell (non Phillips), Proc. Dorset. Nat. Hist. Field Club, vol. ix, I888, table facing p. 40.

LOC. Oxford Clay; Peterborough, Weymouth, and Whittlesea. [Skeleton ; Nelson Richardson Coll., Weymouth.]

smithi (Owen), R. Lydekker, Cat. Foss. Rept. B. M., pt. ii, i889, p. 215.

Plesiosaurus smithii, R. Owen, Brit. Foss. Rept., vol. iv, I884, Index, p. iv, pls. xxvii, $\operatorname{xxx}$ (Enaliosauria).

Plesiosaurus, R. Owen, Rept. Cret. Form. (Pal. Soc., 185I), pp. 63, 66, pls. xvii, xix.

LOC. Gault; Folkestone. L. Chalk; Burham, Kent. [Paddle bones and dorsal vertebra ; Brit. Mus. (pars).]

trochanterius (Owen), R. Lydekker, Geol. Mag. [3] vol. v, I 888 , p. 353, and Cat. Foss. Rept. B. M., pt. ii, I889, p. I90, woodcuts $62-65$.

Plesiosaurus trochanterius, R. Owen, Rep. Brit. Assoc., 1839 (1840), p. 85 ; J. Phillips, Geol. Oxford, 1871, pp. 316, 364, woodcuts clxii, clxiii. 
CIMOIIOSAURUS trochanterius (continued) -

Pliosuurus trochanterius, R. Owen, Rep. Brit. Assoc., 1841 (1842), p. 64, and Rept. Kim. Clay, pt. iii (Pal. Soc., 1869), p. 7, pl. iii ; J. Morris, Cat. Brit. Foss., 1854, p. 352.

Plesiosaurus affinis, R. Owen, Rep. Brit. Assoc., I839 (1840), p. 86; J. Mlorris, Cat. Brit. Foss., 1854 , p. 352 ; J. Phillips, Geol. Oxford, 1871, p. 366. [Femur ; Brit. Mus.]

Plesiosaurus manselii, J. W. Hulke, Quart. Journ. Geol. Soc., vol. xxvi, 1870, p. 612, pl. xli, f. 1-6, and ibid., vol. xxxix, I 883, p. 52, woodcut 7. [Imperfect skeleton, wanting head; Brit. Mus.]

Muranosaurus manselii, H. E. Sauvage, Ann. Sci. Nat. (Zool.) [6] vol. viii, art. 6, 1879, p. 32 .

Plesiosaunus megadeinus, H. G. Seeley, Index to Aves, etc., Woodwardian Mus., I 869, pp. xx, 97, 121 (insufficiently described). [Coracoids; Woodwardian Mus.]

Plesiosuurus macrodeinus, H. G. Seeley, ibid., p. 143 (errore).

Colymbosaurus megadeirus, H. G. Seeley, Quart. Journ. Geol. Soc., vol. xxx, 1874, pp. $445,447$.

Plesiosaurus brachyspondylus, J. Phillips (non Owen), Geol. Oxford, 1871, p. 368, woodcuts clxviii-clxx. [Vertebre ; Oxford Mus.]

Muranosaurus brachyspondylus, H. E. Sauvage, Ann. Sci. Nat. (Zool.) [6] vol. viii, art. 6, 1879, p. 33.

Plesiosaurus validus, J. Phillips, Geol. Oxford, I871, p. 370, woodcuts clxxi, clxxii. [Vertebræ; Oxford Mus.]

Loc. Oxford Clay ; Christian Malford. Kim. Clay; Baldon, Cumnor-Hurst, Devizes, Ely, Foxhangers, Foxcombe, Heddington, Haddenham, Kimeridge, Newport-Pagnall, Pottern, Shotover, Swindon, Weymouth, and Wheatley. Derived in Potton Bone-bed. [Right femur; Brit. Mus.]

truncatus (Owen), R. Lydekker, Cat. Foss. Rept. B. M., pt. ii, 1889, p. 230.

Plesiosaurus truncatus, R. Owen, Cat. ${ }^{-F}$ Foss. Rept. Mus. Roy. Coll. Surgeons, 1854 , p. 59.

? Plesiosaurus subdepressus, R. Owen, ibid., p. 63. [Vertebra; Mus. R. Coll. Surgeons.]

? Plesiosaurus dadicomus, R. Owen, Rep. Brit. Assoc., 1839 (I840), p. 81 ; J. Morris, Cat. Brit. Foss., I854, p. 352. [Propodial bone ; Brit. Mus.]

Plesiosaurus plicatus, J. Phillips (pars), Geol. Oxford, 1871, p. 373, woodcuts clxxiii, clxxiv. [Vertebræ; Oxford MIus.]

Plesiosaurus plicatus, H. E. Sauvage, Ann. Sci. Nat. (Zool.) [6] vol. viii, art. 6,1879, p. 25.

LOC. Kim. Clay ; Ely, Kimeridge, Oxford, Portland, Shotover, Swindon, Weymouth, and Wootton-Bassett. Portland Oolite; Shotover. Derived in Potton Bone-bed. [Cervical vertebra; Mus. R. Coll. Surgeons.] 
CIMOIZOSAUZUS (continued)-

valdensis, R. Lydekker, Cat. Foss. Rept. B. M., pt. ii, 1889, p. I88, woodcut 6r.

Loc. Wadhurst Clay; Hastings. Wealden; Cuckfield. [Vertebrie ; Brit. Mus.]

spp., R. Lydekker, Cat. Foss. Rept. B. M., pt. ii, r889, p. 2 Io 'Centrum; Cornbrash of Stilton, Brit. MIus., no. $47169 i$ : right humerus; Cornbrash of Rushden, Brit. Mus., no. 28625), p. 227 (Propodial bone; Purbeck of Swanage, Brit. Mus. no. 21974), and p. 244 (Vertebra and portion of mandible; Gt. Oolite of Oxfordshire and Essendine, Brit. Mus., nos. R. 256, 44904).

CLADEIODON, Owen. v. Cladyodon.

CIADyOdON, R. Owen, Rep. Brit. Assoc., I84 I (I842), p. I55. [? Belodon.]

lloydi, [-ii] R. Owen, ibit., p. 155, and Odontography (I84I), p. 268, pl. lxii A, f. 4 (Cladeiodon), and Palæontology, ed. 2, I86I, p. 278 ; J. Morris, Cat. Brit. Foss., I854, p. 348.

Megalosaurus, R. I. Murchison and H. E. Strickland, Trans. Geol. Soc. [2] vol. v (1840), p. 344, pl. xxviii, f. 6, 7 .

LOC. Keuper ; Coton End, Leamington, and Warwick. [Tooth; Geol. Soc. London.]

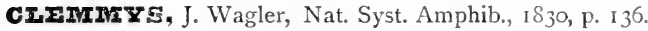

bicarinata (Owen), Lydekker and Boulenger. v. Chrysemys bicarinata.

? mantellii, Fitzinger. v. Hylæochelys belli.

?parkinsonii, Fitzinger. \%. Lytoloma longiceps.

Caruzus, O. C. Marsh, Amer. Journ. Sci. [3] vol. xviii, I879, p. 504 .

dariesi (Seeley), R. Lydelker, Cat. Foss. Rept. B. M., pt. i, I888, p. 156.

Thecospondylus daviesi, H. G. Seeley, Quart. Journ. Geol. Soc., vol. xliv, 1888, p. 79, woodcuts $1-5$.

Calurus, J. W. Hulke, Quart. Journ. Geol. Soc., vol. xliv, $1888_{\text {, }}$ p. 87.

Loc. Wealden; I. of Wight. [Vertebra ; Brit. Mus.] pusillıs, Marsh. v. Aristosuchus pusillus.

COLOBORIY YCHUS, Owen. v. Ornithocheirus.

clavirostris, Owen. $v$. Ornithocheirus clavirostris.

cuvieri (Bowerbank), Owen. $v$. Ornithocheirus cuvieri.

sellgwicki, Owen. v. Ornithocheirus sedgwicki.

COLYMBOSAURUS, Seeley. v. Cimoliosaurus.

megadeims, Seeley. $v$. Cimoliosaurus trochanterius. 
Contasaurus, R. Owen, in F. Dixon, Geol. Sussex, i 850 , p. 386.

orassidens, R. Owen, ibid., pp. 386 , 388, pl. xxxvii, f. 18-20, and ed. 2, I878, p. 413, same pl. and figs., and Rept. Cret. Form. (Pal. Soc., 1851), p. 21, pl. ix, f. 13-15 (Coniosaumes); J. Morris, Cat. Brit. Foss., 1854, p. 348 (Coniosaurus); R. Lydekker, Cat. Foss. Rept. B. M., pt. i, I888, p. 289.

LOC. Chalk; Clayton Pit, Falmer, Hart Hill, near Charing, and Washington, near Worthing. [Associated jaws, teeth, and vertebre; Brighton Mus.]

T.

Cramposauruä, H. G. Seeley, Quart. Journ. Geol. Soc., vol. $\mathrm{xxx}, 1874$, p. 692 .

pottonensis, H. G. Seeley, ibid., p. 69o, pl. xliv.

LOc. Potton Bone-bed. [Portion of cranium; Woodwardian Mus.]

I.

CRIORHY NCHUS, Owen. $\quad \approx$. Ornithocheirus.

simus, Owen. $v$. Ornithocheirus simus.

GrocodruUs, Linnæus, Syst. Nat., ed. 12, vol. i, I766, p. 359.

bollensis, Jaeger. \%. Steneosaurus bollensis.

? cantabrigiensis, H. G. Seeley, Index to Aves, etc., Woodwardian Mus., I 869, p. xvii (name only), and Quart. Journ. Geol. Soc., vol. xxx, 1874, p. 693, woodcuts I-3. Genus non det., R. Lydekker, Cat. Foss. Rept. B. M., pt. i, 1888, p. 75.

LOC. Cambridge Greensand. [Vertebra ; Woodwardian Mus.] champsoides, Owen. $v$. Crocodilus spenceri. cultridens, Owen. $v$. Suchosaurus cultridens.

hastingsice, Owen. $v$. Diplocynodon hantoniensis.

? Icenicus, H. G. Seeley, Quart. Journ. Geol. Soc., vol. xxxii, 1876, p. 437. [Genus non det.]

LOC. Cambridge Greensand. [Vertebra ; Woodwardian Mus.] mantelli, Gray. v. Goniopholis crassidens.

oxoniensis, Conybeare, MS. $v$. Steneosaurus boutillieri.

sauli, Owen. $v$. Miscellaneous Reptilia.

spencer1, W. Buckland, Geol. and Mineral., ed. 2, 1837, vol. i, p. $25 \mathrm{I}$, and vol. ii, p. 36 , pl. xxv, f. I; R. Owen, Rep. Brit. Assoc., 1841 (i842), p. 65, and in F. Dixon, Geol. Sussex, 1850, p. 207, pl. xv, f. 1, 2, and ibid., ed. 2, 1878, p. 253 , pl. xv [16], f. I, 2; R. Lydekker, Geol. Mag. [3] vol. iv, 1887 , p. 3 IO, and Cat. Foss. Rept. B. M., pt. i, 1888, p. 6o, woodcut 9.

Crocedilus toliapicus, R. Owen, Rept. London Clay, pt. ii (Pal. Soc., 1850), pp. 29,34 , pl. ii, f. I, pl. ii A, pl. iv, pl. v, f. I-3, 5, 6 ; J. Morris, Cat. Brit. Foss., 1854, p. 348. [Skull, mandible, and vertebræ; Brit. Mus.] 


\section{CROCODIIUS spenceri (continued)-}

Crocodilus champsoides, R. Owen, Rept. London Clay, pt. ii (Pal. Soc., 1850), p. 31, pl. iii, pl. v, f. 4, 7-9 (? and pl. ii, f. 2) ; J. Morris, Cat. Brit. Foss., 1854, p. 348. [Skull ; Brit. Mus.]

'Crocodile de Sheppey?,' Cuvier, Ossem. Fossiles, ed. 2, vol. v, pt. ii, 1824, p. 165. [Cervical vertebra; Deluc Coll., Geneva.]

LOC. London Clay; Bognor and Sheppey. [Cranium; Brit. Mus.]

superciliosus, De Blainville. v. Metriorhynchus superciliosum. toliapicus, Owen. $\%$. Crocodilus spenceri.

Crocodilian remains, R. Owen, Rept. Weald. and Purb. Form., Suppl. ix (Pal. Soc., 1879), pp. 14, 15, pl. i, f. 4-I2, pl. ii, f. $4,5,11,12$.

LOC. M. Purbeck; Swanage. [Vertebræ and scutes ; Brit. Mus.]

CRYPTODRACO, R. Lydekker, Quart. Journ. Geol. Soc., vol. xlv, I 889, p. 46.

Cryptosaurus, H. G. Seeley (non Geoffroy St. Hilaire), Index to Aves, etc., Woodwardian Mus., I869, p. xxi, and Quart. Journ. Geol. Soc., vol. xxxi, I875, p. 149.

eumerus (Seeley), R. Lydekker, Quart. Journ. Geol. Soc., vol. xlv, I 889, p. 46.

Cryptosaurus eumerus, H. G. Seeley, Index to Aves, etc., Woodwardian Mus., I869, pp. xxi, 93, and Quart. Journ. Geol. Soc., vol. $x x x i, 1875$, p. 149, pl. vi.

LOC. Oxford Clay; Gt. Gransden. [Femur; Woodwardian Mus.]

$\mathbf{T}$.

CRYPTOSAURUS, Seeley. v. Cryptodraco.

eumerus, Seeley. $v$. Cryptodraco eumerus.

CUMNORIA, Seeley. v. Camptosaurus.

prestwichi (Hulke), Seeley. v. Camptosaurus prestwichi.

DACOCrx $\mathbf{x} \mathbf{S}, \mathrm{R}$. Lydekker, Quart. Journ. Geol. Soc., vol. xlv, 1889 , p. $24 \mathrm{r}$.

delabeohei, R. Lydekker, ibid., p. $24 \mathrm{r}$, woodcut $6 . \quad[?=$ Podocnemis? delabechei.]

Loc. London Clay; Sheppey. [Mandib. symphysis; Brit. Mus.]

T.

DACOSAURUS, F. A. Quenstedt, Sonst und Jetzt, 1856, p. 131. [Indistinguishable from Geosaurus, Plieninger, according to Lydekker, Quart. Journ. Geol. Soc., vol. xlv, 1889, p. 57.]

Plesiosuchus, R. Owen, Quart. Journ. Geol. Soc., vol. xl, 1884, p. 153.

lissocephalus, Seeley. \%. Dacosaurus maximus.

manselii (Hulke), A. S. Woodward. v. Dacosaurus maximus. 
Dacosaurus (continucd) -

maximus (Plieninger), F. A. Quenstedt, Der Jura, 1858, p. 785 , woodcut ; R. Lydekker, Cat. Foss. Rept. B. M., pt. i, I 888 , p. 92, woodcut 13 .

Geosaurus maximus, T. Plieninger, Württ. Jahresh., vol, ii, 1846, p. 150.

Dakosaurus lissocebhalus, H. G. Seeley, Index to Aves, etc., Woodwardian Mus., I869, p. 92. [Skull; Woodwardian Mus.]

Steneosaurus rostro-minor=Dakosaurus, J. IV. Hulke, Quart. Journ. Geol. Soc., vol. xxv, I869, p. 390, pls. xvii, xviii.

Liodon primavus, H. E. Sauvage, Comptes Rendus, vol. lxxiii, I87I, p. I 41 .

Dacosaurus primavus, H. E. Sauvage, Bull. Soc. Géol. France [3] vol. i, I 873, p. 380 ,

Steneosaurus mansellii, J. IV. Hulke, Quart. Journ. Geol. Soc., vol. xxvi, 1870, p. 167, pl. ix. [Skull and other parts of skeleton; Brit. Mus.]

Plesiosuchus manselii, R. Owen, Quart. Journ. Geol. Soc., vol. xl, 1884, p. 157, woodcut 3, and Brit. Foss. Rept., vol. iii, 1884, p. 146, pl. xx (Crocodilia). [Idem.]

Dakosaurus manselii, A. S. Woodward, Geol. Mag. [3] vol. ii, 1885, table facing p. 508.

Dakosaurus, J. F. Walker, Ann. Mag. Nat. Hist. [3] vol. xvii, I866, p. 385 ; H. G. Seeley, Geol. Mag., vol. vi, I869, p. I88; J. W. Mason, Quart. Journ. Geol. Soc., vol. xxv, 1869, p. 218 , woodcuts I-4; J. Phillips, Geol. Oxford, 1871, p. $3^{89}$, woodcuts cxci, cxcii.

Loc. Kim. Clay; Culham, Ely, Kimeridge, Shotover, and Weymouth. [Teeth, skull and mandible, vertebra, etc.; Brit. Muts.] Teeth derived, Potton Bone-bed. [Woodvardian Mus.] T.

primcevus, Sauvage. v. Dacosaurus maximus.

DICya DON, R. Owen, Trans. Geol. Soc. [2] vol. vii (IS45), p. 5).

? sp., R. H. Traquair, Rep. Brit. Assoc., I885 (I 886), p. 1024.

LOC. U. Trias; Elgin. [Skull ; Geol. Surv. Scotland.]

DIGERRHUM, Cope. $\%$. Pleurosternum.

bullocki (Owen), Cope. v. Pleurosternum bullocki.

DIMORPIIODON, R. Owen, Rep. Brit. Assoc., 1858 (I850), Sect., p. 97 ; H. G. Seeley, Ann. Mag. Nat. Hist. [3] vol. xv, I865, p. 148, and ibid. [4] vol. iii, 1869, p. 465, and ibid. [4] vol. vi, 1870, p. 129.

macronyx (Buckland), R. Owen, Palxontology, ed. 2, I86 I, p. 273, and Rept. Liassic Form., pt. ii (Pal. Soc., 1870), p. 4l, pls. xvii, xviii, pl. xix, f. $1-4$, pl. xx, and Rept. Mesoz. Form., pt. i (Pal. Soc., 1874), p. 13, pl. i, f. 13, 14, and Brit. Foss. Rept., vol. i (1884), p. 463, pls. xv-xvii (Pterosauria); [H. Woodward] Geol. Mag., vol. v, 1868, p. 536 ; E. T. Newton, Proc. Geol. Assoc., vol. x (1888), p. 414, woodcut II; R. Lyclekker, Cat. Foss. Rept.B. M., pt. i, 1889, p. 37, woodcut 6. 


\section{DIIYORPFODON macronyx (continued)-}

Pterodactylus macronyx, IV. Buckland, Proc. Geol. Soc., vol. i (1829), p. 127, and Trans. Geol. Soc. [2] vol. iii (1835), p. 218 , pl. xxvii ; R. Owen, Odontography (184I), p. 275, pl. lxiii A, f. 7 ; J. Morris, Cat. Brit. Foss., I854, p. 353.

Pterodactylus (Dimnrphodon) macronyx, R. Owen, Rep. Brit. Assoc., I858 (1859), Sect., p. 08, and Rept. Cret. Form., Suppl. i (Pal. Soc., 1859), p. 13, pl. iii, f. 6.

? Pterodactylus marderi, R. Owen, Rept. Mesoz. Form., pt. i (Pal. Soc., 1874), p. 12, pl. i, f. 7-9. [Humerus; Brit. Mus.]

Pterodactylus (Rhamphorhynchus) macronyx, H. vor Meyer, Palæontographica, vol. i (1846), p. 20.

LOC. L. Lias; Lyme Regis. [Portions of skeleton, wanting head; Brit. Mus.]

DIFODOC U5, R. Owen, Brit. Foss. Rept., index to vol. ii, 188, p. ix.

mackesoni, R. Owen, ibid., p. ix ; R. Lydekker, Cat. Foss. Rept. B. M., pt. i, I888, p. 136.

Polyptychodon mackesoni, R. Owen, Page-index to Brit. Foss. Rept., vol. i, 1884, p. vii.

'Gigantic fossil saurian, Rep. Brit. Assoc., 1841 (1842), p. I57, and (' $=$ Polyptychodon') R. Owen, Proc. Geol. Soc., vol. iii (I841-1842), pp. 325, 45I, and Rept. Cret. Form. (Pal. Soc., I851), p. 47, pls. xii, xiii, woodcuts.

LOC. L. Greensand; Hythe. [Limb-bones; Brit. Mus.]

I.

DIPIOCY2 $0 \mathbb{D}$ ㄱ, A. Pomel, Bull. Soc. Géol. France [2] vol. iv, 1847 , p. 383 .

hantoniensis (Wood), R. Lydekker, Geol. Mag. [3] vol. iv, 1887, p. 309, and Cat. Foss. Rept B. M., pt. i, I 888, p. 45.

Alligator hantoniensis, S. V. Wood, Ann. Mag. Nat. Hist., vol. xiv, 1844, p. $35 \mathrm{I}$, and London Geol. Journ., pt. i, 1846, pp. 6, 122, pls. i, vi, vii ; R. Owen, Rept. London Clay, pt. ii (Pal. Soc., I850), p. 42, pl. viii, f. 2 ; J. Morris, Cat. Brit. Foss., I 854 , p. 347 .

Crocodilus hastingsia, R. Owen, Rep. Brit. Assoc., I847 (1848), Sect., p. 66, and Rept. London Clay, pt. ii (Pal. Soc., 1850), p. 37, pls. vi-ix, pl. xii, f. 2, 5 ; J. Morris, Cat. Brit. Foss., 1854, p. 348 ; T. H. Huxley, Quart. Journ. Geol. Soc., vol. xv, 1859 , p. 678 , pl. xxv ; A. S. Woodward, Geol. Mag. [3] vol. ii, I885, p. 509. [Skull, etc.; Brit. Mus.]

LOC. U. Eocene ; Hordwell. [Imperfect skull ; Brit. Mus.]

sp., R. Lydekker, Cat. Foss. Rept. B. M., pt. i, I 888, p. 50.

LOC. L. Miocene: Hempstead. [Portions of jaws ; Brit. Mus., nos. 40215 , R. 980.] U. Eocene ; Lymington. [Frontals and quadrate; Brit. Mus., no. R. 1037.] 
Dozrcyosauxus, R. Owen, in F. Dixon, Geol. Sussex, 1850, p. 388 .

longlcollis, R. Owen, iöid., p. 388 , pl. xxxviii, f. I, 2, pl. xxxix, f. 4 , and ed. 2, 1878, p. 415 , same pls. and figs., and Rept. Cret. Form. (Pal. Soc., I85I), p. 22, pl. x, f. I-4; J. Morris, Cat. Brit. Foss., 1854 , p. 348 ; S. J. Mackie, Geologist, 1863 , p. 267 , pl. xiv ; R. Lydekker, Cat. Foss. Rept. B. M., pt. i, I888, p. 275 .

Raphiosaums, R. Owen (errore), Trans. Geol. Soc. [2] vol. vi (1842), p. 412 , pl. xxxix, f. 3, 4, and Rep. Brit. Assoc., 184 I (;842), p. 145. [Vertebre ; Brit. Mus.]

LOC. L. Chalk; Burham and Lidden Spout, Kent, and Southeram, near Lewes. [Skeleton; Brit. Mus.]

2.

DORATORFy A CFIUS, H. G. Sceley, Quart. Journ. Geol. Soc, vol. xxxi, 1875 , p. 465.

validum (Owen), [-us] H. G. Seeley, Quart. Journ. Geol. Soc., vol. xxxi, I 875 , p. 465 , woodcut ; [-us] J. C. Mansel. Pleydell, Proc. Dorset. Nat. Hist. Field Club, vol. ix, I888, p. 3I.

Pterodactylus validus, R. Owen, Rept. Liassic Form., pt. ii (Pal. Soc., 1870 ), descr. to pl. xix, f. 7 .

Pterodactylus macrums, H. G. Seeley, Index to Aves, etc., Woodwardian Mus., I869, p. 89, and Proc. Camb. Phil. Soc., vol. ii (I869), p. J30. [Mandible and caudal vertebra; Woodwardian Mus.]

Ornithochinus validus, R. Lydekker, Cat. Foss. Rept. B. M., pt. i, I888, p. 26.

Loc. M. Purbeck; Swanage. [Wing-phalanx; Brit. Mus.] $\mathbf{I}$.

FCHINODON, R. Owen, Rept. Weald. and Purb. Form., pt. v (Pal. Soc., I 86I), p. 35, and Palxontology, ed. 2, i 86I, p. 30y.

becklesi, [-ii] R. Owen, Rept. Weald, and Purb. Form., pt. v (Pal. Soc., I86r), p. 35, pl. viii, f. I-9, and ibid., Suppl.v (Pal. Soc., I 874), pl. ii, f. 22 ; R. Lydekker, Cat. Foss. Rept. B. M., pt. i, 1888, p. 247.

Loc. M. Purbeck; Durdlestone Bay. [Jaws; Brit. Mus.] T.

ELASMOSAURUS, Cope. \%. Cimoliosaurus.

בIrys, A. Duméril, Zool. Analytique, I806, p. 76.

benstedi, Mantell. v. Cimoliochelys benstedi.

bicarinatus, Bell. $\%$. Chrysemys bicarinatus.

? comptoni, T. Bell, in R. Owen, Rept. London Clay, pt. i (Pul. Soc., I 849), p. 7 I, pl. xx ; J. Morris, Cat. Brit. Foss., 1854 , p. 348 .

Homopus comptoni, R. Lydekker, Cat. Foss. Rept. B. M.,pt. iii, 1889, p. 93.

LOC. London Clay; Sheppey. [Shell; Brit. Mus.] 
IMYs (continued)-

conybearii, Owen. \% Podocnemis delabechei.

crrassus, Owen. v. Ocadia crassa.

delabechei, Bell. v. Podocnemis delabechei.

hordwellensis, Seeley. v. Ocadia crassa.

lavis, Bell. $v$. Podocnemis bowerbanki.

lutaria, Schneider. v. Emys orbicularis.

mantelli, Owen. v. Hylæochelys belli.

orbicularis (Linnæus), W. T. Blanford, Zool. E. Persia, 1876, p. 308 ; R. Lydekker, Cat. Foss. Rept. B. M., pt. iii, I889, p. 103.

Testudo orbicularis, Linnæus, Syst. Nat., ed. 12, vol. i, I766, p. $35 \mathrm{I}$.

Emys lutaria, J. G. Schneider, Schildkröten, 1783, p. 323; A. Newton, Ann. Mag. Nat. Hist. [3] vol. x, 1862, p. 22.4, pls. vi, vii ; E. T. Newton, Geol. Mag. [2] vol. vi, 1879, p. 304 , pl. viii ; H. B. Woodward, Trans. Norfolk and Norwich Nat. Soc., vol. iii (1880), p. 36.

LOC. Forest Bed; Mundesley. [Portion of carapace; Norwich Mus.] Below Peat ; East Wretham, Norfolk. [Remains of two individuals; Birch Coll.]

parkinsoni, Gray. v. Lytoloma longiceps and L. planimentum. sphenognathus, H. G. Seeley, Index to Aves, etc., Woodwardian Mus., I869, pp. xix, 36 (name only).

LOC. Cambridge Greensand. [? Frontal bone; Woodwardian Mus.]

testudiniformis, Owen. $v$. Chrysemys testudiniformis and Ocadia oweni.

toliapicus, Owen. v. Chrysemys testudiniformis.

PNAIIOCFrIYS, Seeley. $v$. Thalassemys.

chelonia, Seeley. $v$. Thalassemys hugii.

ENGTOMIIASURUS, Kaup. v. Pelagosaurus.

brongniarti, Kaup. v. Pelagosaurus brongniarti.

ENTHEKIODON, Hulke. v. Ichthyosaurus entheciodon.

FOSPIユA RIS, R. Lydekker, Quart. Journ. Geol. Soc., vol. xlv, I889, p. 241 .

glgas (Owen), R. Lydekker, ibid., p. 239, and Cat. Foss. Rept. B. M., pt. iii, 1889, p. 225 .

Chelone gigas, R. Owen, Palæontology, ed. 2, I86r, p. 317, and Rept. London Clay, vol. ii, pt. i (Pal. Soc., I880), p. J, pls. i, ii. 
MOSPHATGIS gigas (continued)-

Chelone sp., R. Owen, ibid., vol. i, pt. ii (Pal. Soc., 1850), descr. to pl. xxix, f. 5 .

LOC. London Clay; Sheppey. [Cranium and femur; Brit. Mus.]

T.

gRgTMOSAURUS, H. G. Seeley, Quart. Journ. Geol. Soc., vol. $\mathrm{xxx}, \mathrm{i} 874, \mathrm{p} .445$.

dubius (Blake), Lydekker. v. Eretmosaurus macropterus.

macropterus: Plesiosaurus macropterus, H. G. Seeley, Ann. Mag. Nat. Hist. [3] vol. xv, 1865 , pp. 49, 232 ; J. F. Blake, in Tate and Blake, Yorksh. Lias, 1876, p. 246.

? Plesiosaurus dubius, J. F. Blake, ibid., p. 246, pl. i, f. 7, 8.

Plesiosaurus rugosus, J. F. Blake (non Owen), ibid., p. 252.

Eretmosaurus? dubius, R. Lydekker, Cat. Foss. Rept. B. M., pt. ii, 1889 , p. 249.

LOC. U. Lias; Lofthouse and Whitby. [Skeleton; Woodwardian Mus.]

rugosus (Owen), R. Lydekker, Cat. Foss. Rept. B. M., pt. ii, 1889, p. 249.

Plesiosaurus mgosus, R. Owen, Rep. Brit. Assoc., 1839 (1840), p. 82, and Rept. Liassic Form., pt. iii (Pal. Soc., 1865), p. 34, pls. xiv, xv, and Rept. Kim. Clay, pt. iii (Pal. Soc., 1869), descr. to pl. iv, f. 4 ; J. Morris, Cat. Brit. Foss., 1854, p. 352.

Eretmosaurus, H. G. Seeley, Quart. Journ. Geol. Soc., vol. xxx, 1874, p. 445 , woodcut 9 .

LOC. L. Lias; Cheltenham, Granby, and Lyme Regis. [Vertebre; ; Bristol, York, and Brit. Mus.]

$\mathbf{T}$.

? sp., R. Lydekker, Cat. Foss. Rept. B. M., pt. ii, 1889, p. 25 I.

LOC. L. Lias; Charmouth. [Cervical vertebre ; Brit. Mus., no. R. 1360.$]$

ERQUELINNESIA, Dollo. v. Lytoloma.

EUCAMEROTUS, Hulke. v. Ornithopsis.

guCrrcosaurus, H. G. Seeley, Quart. Journ. Geol. Soc., vol. $x x x v, 1879$, p. 613 .

tanyspondylus, H. G. Seeley, ibid., p. $61_{3}$, woodcuts 4,5 .

LOC. Cambridge Greensand. [Vertebre; Woodwardian Mus.]

EUCLASTES, Cope. $\%$ Lytoloma.

T.

sp., Dollo. $v$. Lytoloma sp.

EURYSTERNUM (Wagler), Lydekker and Boulenger. \%. Thalassemys ruetimeyeri.

GAvIAIrS, Cuvier, Ann. du Muséum, vol. x, 1807, p. 27 (Gavial); A. Oppel, Ord. Fam. Gatt. Rept., I811, p. 19. 
GAVIALES (continued)-

? dizoni, R. Owen, Rept. London Clay, pt. ii (Pal. Soc., 1850), P. $46, \mathrm{pl} . \mathrm{x}$, and in F. Dixon, Geol. Sussex, I850, p. 208, pl. xil, f. 23-24, woodcuts $1-8$, and ibid., ed. 2, 1878, p. 253, same pl. and figs., and pl. xi [12], f. 18 ; J. Morris, Cat. Brit. Foss., 1854, p. 349 ; R. Lydekker, Cat. Foss. Rept. B. M., pt. i, I888, p. 69 (Giarialis).

LOC. Bracklesham Beds. [Portions of mandible, femur, and a cervical vertebra; Brit. Mus.]

GEOSAUIUS, Plieninger. $\%$ Dacosaurus.

maximas, Plieninger. ¿'. Dacosaurus maximus.

GIGAพTOSAURU:S, II. C. Seeley, Index to Aves, etc., Woodwardian Mus., 1869 , p. 94.

megalonyx, H. G. Seeley, ibid., p. 94. [Doubtfully assigned to Omithopsis humerocristatus by R. Lydekker, Cat. Foss. Rept. B. M., pt. i, 1888, p. 151.]

Loc. Kim. Clay; Ely. [Vertebræ and detached limb-bones; Woodwardian Mus.]

GLOSSOCHELIS, Seeley. \%. Lytoloma.

haviciensis (S. Woodward), Sceley. v. Lytoloma planimentum.

Gorzorzaris, R. Owen, Rep. Brit. Assoc., 18+1 (18+2), p. 69.

carinata, [-us] R. Owen, Brit. Foss. Rept., I884, index to vol. ii, p. $v$, and pl. xiv (Crocodilia) (figures of vertebre only).

LOC. Wealden; Cuckfield. [Vertebre.]

crassidens, R. Owen, Rep. Brit. Assoc, 1841 (1842), p. 69, and Odontography (I8+1), p. 289, pl. lxxii $\mathrm{A}$, f. 9, and Rept. Weald. and Purb. Form., Suppl. viii (Pal. Soc., I878), p. r, pls. i-iii, pl. iv, f. 1-4, and Brit. Foss. Rept., vol. i (I884), p. 427, pls. vii-xiii (Crocodilia); J. IV. Hulke, Quart. Journ. Geol. Soc., vol. xxxiv, 1878, p. 377, pl. xv, f. 1, 2 ; R. Lydekker, Cat. Foss. Rept. B. M., pt. i, iss8, p. 79.

Crocodilus mantelli, J. E. Gray, Syn. Rept., 1831, p. 61.

'Swanage crocodile,' G. A. Mantell, Wonders of Geology, ed. 3, $1839, \mathrm{p}, 387, \mathrm{pl}$. i. [Slab from Purbeck of Swanage, with portions of skeleton ; Brit. Mus.]

'Crocodile,' G. A. Mantell, Illustr. Geol. Sussex, I827, descr. to pl. v, f. 10 [and $\mu l$. vi, f. 8].

Loc. Wealden; Sussex and I. of Wight. Purbeck; Swanage. [Teeth, and imperfect skeleton; Brit. Mus.]

sima, [-us] R. Owen, Rept. Weald. and Purb. Form., Suppl. viii (Pal. Soc., 1878), p. 7, pl. v ; [-us] R. Lydekker, Cat. Foss. Rept. B. M., pt. i, I888, p. $\delta_{3}$.

'Crocodilian skull,' J. W. Hulke, Quart. Journ. Geol. Soc., vol. xxxiv, 1878, p. 379 , pl. xv, f. 3,4 .

Loc. M. Purbeck; Swanage. [Skull; Brit. Mus.] 
GONIOPIZOIIS (continued)-

tenuidens, R. Owen, Rept. Weald. and Purb. Form., Suppl. ix (Pal. Soc., 1879), p. 2, pl. i, f. I ; R. Lydekker, Cat. Foss. Rept. B. MI., pt. i, 1888 , p. 83.

Loc. M. Purbeck; Durdlestone Bay. [Mandible; Brit. Mus.] sp., Phillips. \%. Machimosaurus.

HADROSAURUS, Leidy. v. Trachodon.

IngTrRosuckus, H. G. Seeley, Quart. Journ. Geol. Soc., vol. xliii, 1887, p. 212 . [? Hylaochampsa.]

valdensis, H. G. Seeley, ibid., p. 212, pl. xii, f. 7, 8.

LOC. Wealden; Cuckfield and Hastings, Sussex, and Brook Point, I. of Wight. [Vertebræ; Brit. Mus.] T.

? sp., H. G. Seeley, ibid., p. 215. Genus non dct., R. Lydekker, Cat. Foss. Rept. B. M., pt. i, I 888 , p. 75.

'Lacertian vertebra,' G. A. Mantell, Phil. Trans., I 849, p. 303 , pl. xxix, f. A, A'.

LOC. Wealden; I. of Wight. [Vertebre and limb-bones; Brit. Mus., nos. 36524, R. 188.] M. Purbeck; Durdlestone Bay. [Cervical vertebra; Brit. Mus., no 48244.]

HEXATARSOSTINUS, T. Hawkins, Great Sea Dragons, I 440 , pl. xxviii. = Plesiosaurus ( $\%$. P. hawkinsi).

Homopus comptoni (Bell), Lydekker. \%. Emys comptoni.

IIOPLOSAURUS, P. Gervais, Zool. et Pal. Franç., ed. I, 1848-52, p. 263 (Oplnsaunus); R. Lydekker, Quart. Journ. Geol. Soc., vol. xlv, 1889, p. 396. [Non Hoplosunnus, Seeley, 1881.]

armatus, P. Gervais, Zool. et Pal. Franç., ed. I, 1848-52, p. 263 (Oplosaurus); R. Lydekker, Quart. Journ. Geol. Soc., vol. xlv, i 889, p. 396. [Doubtfully assigned to Ornithopsis hulkei by R. Lydekker, ibid., vol. xliv, 1888, p. 54, pl. iii.]

Cetiosaurus or Pelorosauros?, R. Owen, Brit. Foss. Rept., rol. i, I 884 , p. 422.

'Tooth of unknown saurian,' T. Wright, Ann. Mag. Nat. Hist. [2] vol. x, 1852, p. 9o, woodcuts, and Proc. Cotteswold Nat. Field Club, vol. i, 1853, p. 231, woodcuts.

'Tooth of Carnivorous Reptile,' R. Owen, Rept. Weald. and Purb. Form., Suppl. ii (Pal. Soc., 1859), p. 42.

Loc. Wealden; Brixton Bay, I. of Wight. [Tooth; Brit. Mus.] T.

Hydraspis planiceps, Fitzinger. v. Stegochelys planiceps.

IY工FOCHAMPSA, R. Owen, Rept. Weald. and Purb. Form., Suppl. vi (Pal. Soc., I874), p. I ; R. Lydekker, Geol. Mag. [3] vol. iv, 1887, pp. 310, 5 I 2 ; L. Dollo, Geol. Mlag. [3] vol. iv, I 887 , p. 394 .

vectianus, R. Owen, Rept. Weald. and Purb. Form., Suppl. vi (Pal. Soc., 1874), p. I, and ibid., Suppl. v, pl. ii, f. 23-25; [-a] R. Lydekker, Cat. Foss. Rept. B. M1., pt. i, 1888, p. 77. Loc. Wealden; Brook, I. of Wight. [Cranium; Brit. Mus.] $\mathbf{x}$. 
IXYIFOCHEIXS, R. Lydekker, Quart. Journ. Geol. Soc., vol. xlv, 1889, p. $5^{1} 3$, and Cat. Foss. Rept. B. M., pt. iii, I 889 , p. 185 .

? Plastremys, R. Owen, in C. Parkinson, Quart. Journ. Geol. Soc., vol. xxxvii, 1881, p. 370.

bell (Mantell), R. Lydekker, Cat. Foss. Rept. B. M., pt. iii, 1889, p. 190.

Chelone bellii, G. A. Mantell, Medals of Creation, I844, p. 776, woodcut 146 , and ibid., ed. 2,1854, p. 735 , woodcut 240 , and Petrifactions, 1851 , p. 156, woodcut 33 .

Chelone costata, R. Owen (pars), Rept. Weald. and Purb. Form., pt. i (Pal. Soc., 1853), p. 10, pl. viii. [Portion of carapace; Brit. Mus.]

Emys mantclli, J. E. Gray, Syn. Rept., 1831, p. 33.

Clemmys? mantellit, L. Fitzinger, Ann. Wien. Mus., vol. i, I835, p. 126.

Chelone mantelli, J. Morris, Cat. Brit. Foss., 1854, p. 348.

Platemys mantelli, R. Owen, Rep. Brit. Assoc., 1841 (1842), p. I67, and Rept. Weald. and Purb. Form., pt. i (Pal. Soc., 1853), p. II, pl. ix, f. I ; J. Morris, Cat. Brit. Foss., 1854, p. 351. [Right hyoplastron; Brit. Mus.]

Platemy's dixoni, R. Owen, Rept. Weald. and Purb. Form., pt. i (Pal. Soc., 1853), p. 12, pl. ix, f. 3 ; J. Morris, Cat. Brit. Foss., 1854, p. 351. [Left hyoplastron ; Brit. Mus.]

'Emys de Sussex,' Cuvier, Ossem. Fossiles, ed. 2, vol. v, pt. ii, 1824, p. 232

LOC. Wealden ; Tilgate Forest. [Portion of costal ; Brit. Mus.]

emarginata (Owen), R. Lydekker, Cat. Foss. Rept. B. M., pt. iii, 1889, p. 189.

Pleurosternon emarginatum, R. Owen (pars), Rept. Weald. and Purb. Form., pt. i (Pal. Soc., 1853), p. 6, pl. iv ; J. Morris (pars), Cat. 13rit. Foss., 1854, p. 352.

Platemy's emarginata, G. A. Maack, Palæontographica, vol. xviii (1869), p. 293.

LOC. Purbeck ; Swanage. [Carapace.]

? lata (Owen), R. Lydekker, Cat. Foss. Rept. B. M., pt. iii, 1889, p. 195.

Plastremys lata, R. Owen, Quart. Journ. Geol. Soc., vol. xxxvii, I 881 , p. 370 .

T. (Plastremys.)

LOC. U. Greensand; St. Lawrence Cliff, I. of Wight. [Imperfect shell ; Brit. Mus.]

Iatiscutata (Owen), R. Lydekker, Quart. Journ. Geol. Soc., vol. xlv, I888, p. 5I3, and Cat. Foss. Rept. B. MI., pt. iii, 1889 , p. I86, woodcut 43.

Pleurosternon latiscutatum, R. Owen, Rept. Weald. and Purb. Form., pt, i (Pal. Soc., I853), p. 9, pl. i ; J. Morris, Cat. Brit. Foss., 1854, p. 352. 


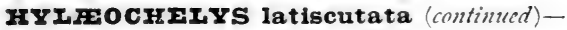

Platemys latiscutata, G. A. Maack, Palæontographica, vol. xviii (1869), p. 295.

Plesiochelys latiscutata, R. Lydekker and G. A. Boulenger, Geol. Mag. [3] vol. iv, p. 272.

Loc. Wealden; Burwash, Sussex. Purbeck; Swanage. [Carapace.]

IXYIFOSAURUS, G. A. Mantell, Proc. Geol. Soc., vol. i (1832), p. 411. [See also Regnosaurus and Megalosaurus ou'eni.]

armatus, G. A. Mantell, Geol. S. E. England, 1833, p. 328, pl. v; J. Morris, Cat. Brit. Foss., 1843, p. 208. [See älso Pleurocaius valdensis.]

Hylcosaurus oweni, [-ii] G. A. Mantell, Medals of Creation, 1844 , p. 734 , and Petrifactions, 1851, p. 314, woodcut 66; [-ii] J. Morris, Cat. Brit. Foss., 1854, p. 349 ; R. Lydekker, Cat. Foss. Rept. B. M., pt. i, 1888, p. 185, woodcut 35.

Hylcosaurus, G. A. Mantell, Phil. Trans., I 841, p. I4 I, pl. x (non pl. vi, f. 9-1 1), and ibid., I 849, pp. 276, 299, pl. xxvii, pl. xxxii, f. 22,23 , and ibid., 1850, p. 391, pl. xxvii, and Wonders of Geology, ed. 6, vol. i, 1848, p. 435, pl. iv; R. Owen, Rept. Weald. and Purb. Form., pt. iv (Pal. Soc., 1857), p. 8, pls. iv-vii, ix-xi (? pl. viii, f. IO, II), and Palreontology, ed. 2, I86I, p. 292, woodcut 99; J. IV. Hulke, Quart. Journ. Geol. Soc., vol. xxxv, 1879, p. 237 , pl. xii, f. 7 (tibia), and Phil. Trans., 1881, p. 660 ; R. Lydekker, Quart. Journ. Geol. Soc., vol, xliv, I888, p. 52, woodcut 3 .

? Hylaosaurus?, J. W. Hulke, Quart. Journ. Geol. Soc., vol. xxx, 1874, p. 516, pl. xxxi. [Tibia and humerus; olim Wilkins Coll., Newport.]

? Suchosaurus, R. Owen, Rep. Brit. Assoc., I841 (1842), p. 69. [Dorsal vertebra; Brit. Mus., no. 2 123.]

'Bones, nature unknown,' G. A. Mantell, Illustr. Geol. Sussex, 1827, p. 8o, pl. ix, f. I I (vertebra), pl. xx, f. 7 (dermal spine?). [Brit. Mus.]

Loc. Wealden; Battle, Bolney, and Tilgate, Sussex. [Portions of skeleton, wanting head; Brit. Mus.]

T.

oveni, auct. $\%$. Hylæosaurus armatus. [In 1844, G. A. Mantell (Medals of Creation, ed. i, vol. ii, p. 734, note \$), proposed to substitute this specific name for that given in 1833 (armatus); there appears to be no justification for the change, and as armatus had already been quoted by J. Morris, Cat. Brit. Foss., 1843, p. 208, the earlier name must be adopted.]

IYPgrODAPEDON, T. H. Huxley, Quart. Journ. Geol. Soc., vol. xv, i 859 , p. 435 , and ibid., vol. $x x v, 1869$, p. 138, and ibit., vol. xliii, 1887, p. 675 . 
IXPERODAPEDON (continued)-

gordoni, T. H. Huxley, Quart. Journ. Gcol. Soc., vol. xv, I859, p. 435 , and ibid., vol. xxv, 1869, p. 138, woodcuts A-D, and ibid., vol. xliii, 1887 , p. 675 , pl. xxvi, woodcuts 1, 4, 7, 8; R. Lydekker, Cat. Foss. Rept. B. M., pt. i, 1888, p. 298, woodcuts 67,68 .

Hyperodapedon, W. Whitaker, Quart. Journ. Geol. Soc., vol. xxv, I869, p. I56 ; A. S. WVoodward, Quart. Journ. Geol. Soc., vol. xliv, 1888, p. 163 .

'Curved spine,' A. T. Metcalfe, Quart. Journ. Geol. Soc., vol. $\mathrm{xl}, \mathbf{1 8 8 4}$, p. 260, woodcut 2. [Premaxilla ; Brit. Mus.]

'Undetermined bone,' H. J. Carter, Quart. Journ. Geol. Soc., vol. xliv, I888, p. 3 I8. [Idem.]

LOC. Keuper ; Lossiemouth, near Elgin, and High Peake, near Sidmouth. [Jaws and parts of skeleton; Elgin Mus.] $\mathbf{T}$.

IIYPSIXOPIXODON, T. H. Huxley, Quart. Journ. Geol. Soc., vol. xxvi, 1870 , p. 3 ; J. W. Hulke, Phil. Trans., I882, p. 1035 ; L. Dollo, Comptes Rendus, vol. cvi, I888, p. 775.

foxi, T. H. Huxley, Quart. Journ. Geol. Soc., vol. xxvi, r87o, p. 3, pls. i, ii ; J. WV. Hulke, Quart. Journ. Geol. Soc., vol. xxix, 1873, p. 522, pl. xviii, and ibid., vol. xxx, 1874, p. 18, pl. iii, and Phil. Trans., I882, p. 1035, pls. Ixxi-lxxxii ; R. Lydekker, Cat. Foss. Rept. B. M., pt. i, I888, p. 193.

Iguanodon foxii, R. Owen, Quart. Journ. Geol. Soc., vol. xxix, 1873, p. 53I, and Rept. Weald. and Purb. Form., Suppl. v (Pal. Soc., 1874), pp. 4, 17, pl. i, f. 9, 10, pl. ii, f. 1, 5, 8-18, woodcuts I, 2.

'Young Iguanodon,' R. Owen, Rept. Weald. and Purb. Form., pt. ii (Pal. Soc., 1855), p. 5, pl. i, and Brit. Foss. Rept., 1884, vol. i, p. 276, pl. vi. [Imperfect skeleton, wanting head; Brit. Mus.]

'Very young Iguanodon,' G. A. Mantell, Phil. Trans., 1849, p. 302, pl. xxix, f. 9. [Cervical vertebræ; Brit. Mus.]

LOC. Wealden ; Cowleaze Chine, Isle of Wight ; and Cuckfield, Sussex. [Skull and mandible; Brit. Mus.]

T.

sp., Lydekker. v. Camptosaurus valdensis.

ICHTrז OSAU RUS [C. Koenig], Syn. Contents Brit. Mus., ed. I3, I 818 , p. 54, and itial., ed. 17, 1820, p. 62; W. D. Conybeare, Trans. Geol. Soc., vol. v (1821), p. 563 ; P. M. G. Egerton, Trans. Geol. Soc. [2] vol. v (1836), p. 187, pl. xiv (vertebræ); R. Owen, Rep. Brit. Assoc., 1839 (1840), p. 86, and Trans. Geol. Soc. [2] vol. vi (1841), p. 199, pl. xx (paddle showing integument), and Rept. Liassic Form., pt. iii (Pal. Soc., I88I), p. 125, pl. xxviii, f. 3 ; G. A. Mantell, Petrifactions, I85I, p. 374 , f. 76 ; J. C. Pearce, Ann. Mag. Nat. Hist., vol. xvii, 1846, p. 44 'embryology); H. Coles, Quart. Journ. Geol. Soc., vol. ix, 1853, p. 79, pl. v (skin); H. G. Scelcy, Rep. Brit. 
ICFTEYTOSAURUS (continued)-

Assoc., r880 (1881), p. 68, pl. i (embryology); V. Kiprijanov, Mém. Acad. Imp. Sci. St. Pétersbourg, vol. xxviii, art. 8 (I881), p. 98, pl. ix, f. 12 (paddle showing integument); J. IV. Hulke, Proc. Geol. Soc., 1883, pp. 45, 47, 50, 55 ; A. S. Woodward, Proc. Zool. Soc., 1886, p. 405, woodcuts I-3 (columella); R. Lydekker, Cat. Foss. Rept. B. M., pt. ii, 1889, p. 12, and Geol. Mag. [3] vol. vi, 1889, p. 388 (paddle showing integument).

Proteosaurus, E. Home, Phil. Trans., 1819, p. 212. [Erroneously quoted as prior to Ichthyosaurus by R. Lydekker, Cat. Foss. Rept. B. M., pt. ii, 1889, p. vii.]

acutirostris, R. Owen, Rep. Brit. Assoc., I839 (I840), p. I2I ; J. Morris, Cat. Brit. Foss., 1854, p. 349; R. Owen, Rept. Liassic Form., pt. iii (Pal. Soc., I $88 \mathrm{I}$ ), p. I2I, pl. xxviii, f. 2 ; R. Lydekter, Cat. Foss. Rept. B. M., pt. ii, 1889, p. 73, woodcut 27.

Ichthyosaurus longipennis, G. A. Mantell (pars), Petrifactions, 1851 , p. 378 .

Ichthyosaurus longirostris, R. Owen (pars), Rept. Liassic Form., pt. iii (Pal. Soc., 1881), p. 121, pl. xxxii, f. 8.

- T. Hawkins, Mem. Ichthyosauri, I834, pl. xiv, and Sea Dragons, $18+0$, pl. xiv.

'Saurian Animal,' J. I'hillips, Geol. Yorkshire, I 835, pt. i, p. I33, pl. xii, f. 2 .

LOC. U. Lias; Whitby. [Skull, etc.; Brit. Mus.]

advena, J. Phillips, Geol. Oxford, 1871, p. 183 (name only).

Loc. Stonesfield Slate. [Vertebrie ; Oxford Mus.]

æqualis, J. Phillips, Geol. Oxford, IS7 I, p. 339.

LOC. Kim. Clay; Shotover. [Caudal vertebra ; Oxford Mus.]

angustidens, H. G. Seeley, Index to Aves, etc., Woodwardian Mus., 1869 , pp. xv, 3 (name only).

LOC. Chalk ; Hunstanton. [Tooth ; Woodwardian Mus.]

bonneyi, H. G. Seeley, Index to Aves, etc., Woodwardian Mus., is69, p. xvii (name only).

LOC. Cambridge Greensand. [Woodwardian Mus.]

brachyspondylus, Owen. \%. Ichthyosaurus thyreospondylus.

breviceps, R. Owen, Rept. Liassic Form., pt. iii (Pal. Soc., I 88I), p. Io9, pl. xxix ; R. Lydekker, Cat. Foss. Rept. B. M., pt. ii, 1889 , p. 52.

? Ichthyosaturus longimanus, R. Owen, Brit. Foss. Rept., vol. iii, 1884, p. I76, pl. $\operatorname{xxx}$ (Ichthyopterygia), f. 2. [Pectoral limb; Brit. Mus.]

LOC. L. Lias; Lyme Regis. [Small skcleton ; Brit. Mus.] 


\section{ICHTHYOSAURUS (continued)-}

campylodon, J. Carter, Rep. Brit. Assoc., I845 (1846), Sect., p. 6o, and London Geol. Journ., vol. i, 1846, p. 8, woodcut ; R. Owen, Rept. Cret. Form. (Pal. Soc, 1851), p. 69, pls. xxii-xxvi, and in F. Dixon, Geol. Sussex, ed. 2, 1878, p. 446, pl. xxxix [44], f. Io, and Brit. Foss. Rept., vol. i (1884), p. 223, pl. i, f. I-10, 13-16, pls. ii-iv, vii (Enaliosauria); J. Morris, Cat. Brit. Foss., I 854, p. 349; R. Lydekker, Cat. Foss. Rept. B. M., pt. ii, I889, p. I5.

Ichthyosaurus communis?, R. Owen, in F. Dixon, Geol. Sussex, I 850 , p. 400, pl. xxxix, f. Io.

Ichthyosaurus compylodon, V. Kiprijanov, Mém. Acad. Imp. Sci. St. Pétersbourg, vol. xxviii, art. 8 (I881), p. 33, pls. i-xvi.

LOC. Cambridge Greensand. Gault ; Folkestone. U. Greensand; Folkestone, Kilmerton, Waddon, and Warminster. Chalk-Marl; Trumpington, near Cambridge. L. Chalk; Cambridge, Dover, and Folkestone. Chalk; Isleham, near Cambridge. [Jaws and teeth; Woodwardian Mus.]

chalarodeirus, H. G. Seeley, Index to Aves, etc., Woodwardian Mus., I869, pp. xx, 106 (name only).

LOC. Kim. Clay; Ely. [Axis vertebra ; Woodwardian Mus.]

chiroligostimus, Hawkins. $v$. Ichthyosaurus platyodon.

chivoparameliostimus, Hawkins. \%. Ichthyosaurus communis and I. intermedius.

chiropolyostimus, Hawkins. $\%$. Ichthyosaurus communis and 1. intermedius.

chirostrongulostinus, Hawkins. 2'. Ichthyosaurus tenuirostris. communis, IV. D. Conybeare, Trans. Geol. Soc. [2] vol. i (1822), p. 108, pl. xv, f. 8, 13, pl. xvi, f. 9-14; W. Buckland, Geol. and Mineral., ed. 2 , 1837 , p. 170, pl. viii, f. I ; R. Owen, Rep. Brit. Assoc., I839 (1840), p. I08, and Trans. Geol. Soc. [2] vol. vi (184I), p. I99, pl. $\mathrm{xx}$, and Odontography; (I84I), p. 276, pl. lxiv B, f. 3, pl. lxxii, f. 4, 7-9, and Rept. Liassic Form., pt. iii (Pal. Soc., 1881), p. 111, pl. xxiv, f. 2, 5 , pl. xxviii, f. I, 4, pl. xxx, f. 3-5; J. C. Pearce, Ann. Mag. Nat. Hist., vol. xvii, 1846, p. 44 (1. communis?) ; J. Morris, Cat. Brit. Foss., 1854 , p. 349 ; S. Haughton, Man. Geology, 1865, p. 272, woodcut $35 ;$ V. Kiprijanov, Mém. Acad. Imp. Sci. St. Pétersbourg, vol. xxviii, art. 8 (1881), p. 98, pl. ix, f. I2 ; R. Lydekker, Cat. Foss. Rept. B. M., pt. ii, 1889, p. 4r, woodcuts I, 19-21. [See also I. campylodon.]

Ichthyosaurus chiropolyostinus, T. Hawkins, Mem. Ichthyosauri, 1834, pls. vii, ix-xi, and Sea Dragons, I840, pls. vii, ix-xi. [Skeleton; Brit. Mus.]

Ichthyosaurus chiroparanckostinus, T. Hawkins, ibid., 1834. pl. xviii, and ibid., 1840, pl. xviii.

LOC. L. Lias; near Bath, Bristol, and Street, Somersetshire; Barrow-on-Soar, Leicestershire; Charmouth and Lyme Regis, Dorsetshire; and Yorkshire. [Tooth; Geol. Soc. London.] T. 
ICHTIYOSAUTUS (continued) -

contformis, R. Harlan, Journ. Acad. Nat. Sci. Philad, vol. iii (I824), p. 339.

Loc. L. Lias ; Bath or Bristol. [Portion of jaw ; Philadelphia Mus., U.S.A.]

conybeare1, [-ri] R. Lydekker, Geol. Mag. [3] vol. v, r888, p. 3Ir, and Cat. Foss. Rept. B. M., pt. ii, 1889, p. 53, woodcut 22.

Ichthyosaums latimanus, R. Owen, Rep. Brit. Assoc, I 839 (I840), p. 123 (imperfect definition); J. Morris, Cat. Brit. Foss., 1854, p. 349. [Skeleton ; olim Bristol Mus.]

LOC. L. Lias; Lyme Regis and Saltford, near Bath. [Skeleton; Brit. Mus.]

crassimanus, Owen. $\%$. Ichthyosaurus trigonodon.

? dilatatus, J. Phillips, Geol. Oxford, I871, p. 307; R. Lydekker, Cat. Foss. Rept. B. M., pt. ii, 1889, p. 3o. [? Ophthalmosaurus, according to Lydekker.]

LOC. Oxford Clay ; Cowley Field and St. Clement's. [Vertebre ; Oxford Mus.] Kim. Clay; Kimeridge and Swindon. [Vertebræ; Brit. Mus.]

doughtyi, H. G. Seeley, Index to Aves, etc., Woodwardian Mus., 1869 , p. xvii (name only).

LOC. Cambridge Greensand. [Woodwardian Mus.]

enthectodon, J. W. Hulke, Quart. Journ. Geol. Soc., vol. xxvii, I871, pp. 3o, 440, pl. xvii ; R. Lydekker, Cat. Foss. Rept. B. M., pt. ii, 1889, p. 32, woodcut 16.

Enthekiodon, J. W. Hulke, Quart. Journ. Geol. Soc., vol. xxvi, 1870, p. 174. [Teeth.]

Ichthyosaurus, R. Owen, Rept. Liassic Form., pt. iii (Pal. Soc., I881), descr. to pl. xxii, f. I-6, 9-1 I.

Ichthyosaurus latimanus, R. Owen (errore), ibid., pl. xxii, f. 7, 8. [Dorsal vertebra; Brit. Mus.]

Loc. ? Oxford Clay; Peterborough. Kim. Clay; Foxhangers, Wiltshire, and Kimeridge. [Skeleton; Brit. Mus.]

fortimanus, Owen. $v$. Ichthyosaurus intermedius.

giganteus, Leach. v. Ichthyosaurus platyodon.

grandipes, Sharpe. $v$. Ichthyosaurus tenuirostris.

hygrodeirus, H. G. Seeley, Index to Aves, etc., Woodwardian Mus., I 869, pp. xx, I06 (name only).

LOC. Kim. Clay; Ely. [Vertebra; Wondwardian Mus.]

immanis, Koenig. $v$. Ichthyosaurus platyodon.

integer, H. G. Bronn, Neues Jahrb., 1844, pp. 386, 679; A. Wagner, Abh. k. bay. Akad. Wiss., vol. vi (1851), p. 532, pl. i, f. 1, Doubtfully recorded from the U. Lias of Kingsthorpe, Yorkshire, by R. Lydekker, Cat. Foss. Rept. B. M., pt. ii, I889, p. 71. [Imperfect skeleton; Brit. Mus., no. 47409.] 


\section{ICITTIYOSAUR US (continued)-}

intermedius, W. D. Conybeare, Trans. Geol. Soc. [2] vol. i (1822), p. 108, pl. xv, f. 9, pl. xvii ; W. Buckland, Geol. and Mineral., ed. 2 , I837, p. I70, pl. viii, f. 2 ; R. Owen, Rep. Brit. Assoc., I839 (1840), pp. I IO, II 7, and Odontogr. (1841), p. 277, pl. lxxiii, f. I, and Rept. Liassic Form., pt. iii (Pal. Soc., I88I), p. I I 3, pl. xxiv, f. 7, pl. xxx, f. I, 2 ; J. Morris, Cat. Brit. Foss., I 854 , p. 349 ; J. F. Blake, in Tate and Blake, Yorkshire Lias, I 876 , p. 254 ; J. W. Hulke, Proc. Geol. Soc., I883, p. 56, woodcut Io ; A. S. Woodward, Proc. Zool. Soc., 1886, p. 406; R. Lydekker, Cat. Foss. Rept. B. M., pt. ii, 1889, p. 55, woodcuts 2, 8, 23, 24, and Geol. Mag. [3] vol. vi, I889, p. 388, woodcut (paddle showing integument).

Ichthyosaurus fortimanus, R. Owen, Brit. Foss. Rept., vol. iii, 1884, p. 176, pl.xxx (Ichthyopterygia), f. I. [Pectoral paddle ; Brit. Mus.]

Ichthyosaurus paramekostinus, T. Hawkins, Sea Dragons, I840, pls. xvii, xx-xxiii. [Skeletons; Brit. Mus.]

Ichthyosaums chiropolyostinus, T. Hawkins (pars), ibid., pl. viii.

Ichthyosaums chiroparamckostinus, T. Hawkins, Mem. Ichthyosauri, I 834 , pls. xvii, xix-xxii.

'Ichthyosaurus tail,' R. Owen, Trans. Geol. Soc. [2] vol.v (1838), p. 5 II, pl. xlii.

Proteosaurus, E. Home, Phil. Trans., 1819, p. 212, pl. xv. [Skeleton; Mus. R. Coll. Surgeons.] '2. (Proteosaurus.)

LoC. L. Lias ; Barrow - on-Soar, Lyme Regis, Robin Hood Bay, Street, Stratford-on-Avon, Scarborough, and Whitby. [Tooth; Geol. Soc. London.]

laticeps, Owen. $v$. Ichthyosaurus latifrons.

latifrons, C. Koenig, Icon. Foss. Sect., I825, pl. xix, f. 250 ; G. A. Mantell, Petrifactions, I85I, p. 385 ; J. Morris, Cat. Brit. Foss., 1854, p. 349; R. Owen, Rept. Liassic Form., pt. iii (Pal. Soc., 188I), p. II9, pl. xxiii, f. 6, pl. xxvii, f. I ; R. Lydekker, Cat. Foss. Rept. B. M., pt. ii, i 889 , p. 89, woodcuts $3 \mathrm{I}-33$.

? Ichthyosaurus longirostris, C. F. Jaeger, Nova Acta Acad. Cæs. Leop.-Carol., vol. xxv, pt. ii, 1856, p. 948, pl. xxx.

Ichthyosaurus longirostris, J. F. Blake, in Tate and Blake, Yorkshire Lias, 1876, p. 254; R. Owen (pars), Rept. Liassic Form., pt. iii (Pal. Soc., 1881), p. 124, pl. xxv, f. 2, pl. xxviii, f. 3, pl. xxxii, f. 7, 9. [See also I. acutirostris.]

Ichthyosaurus laticeps, R. Owen, ibid., p. 120 (errore).

Ichthyosaurus tenuirostris, R. Owen (errore), ibid., pl. xxxii, f. 4 . LOC. L. Lias; Barrow-on-Soar and Lyme Regis. U. Lias; Whitby. [Cranium and vertebral column ; Brit. Mus.]

latimanus, Owen. v. Ichthyosaurus conybearei and I. entheciodon. 
ICITIYOSAŨUS (iontinued)-

lonchiodon, R. Owen, Rep. Brit. Assoc., I839 (I840), p. II6, and Rept. Liassic Form., pt. iii (Pal. Soc., I88I), p. I17, pl. xxiv, f. 6, pl. xxxi, f. 4-7, and Odontography (184I) p. 277, pl. lxxiii, f. 2 ; J. Morris, Cat. Brit. Foss., I854, p. 349 ; R. Lydekker, Cat. Foss. Rept. B. M., pt. ii, 1889, p. 92.

Oligostinus, T. Hawkins, Sea Dragons, I840, pl. ii.

LOC. L. Lias ; Lyme Regis. [Skeleton; Brit. Mus.]

longifroms, Owen. v. Ichthyosaurus zetlandicus.

longimanus, Owen. $\%$. Ichthyosaurus breviceps.

longipennis, Mantell. \%. Ichthyosaurus acutirostris.

longirostris, Owen. $v$. Ichthyosaurus acutirostris and Ichthyosaurus latifrons.

megalodeirus, H. G. Seelcy, Index to Aves, etc., Woodwardian Mus., pp. xxi, I 1о. [? Ophthalmosaums.]

LOC. Oxford Clay; Woodstone Lodge, near Peterborough. [Parts of skeleton; Woodwardian Mus.]

ovalis, J. Phillips, Geol. Oxford, I87 1, p. 339; R. Lydekker, Cat. Foss. Rept. B. M., pt. ii, I889, p. 29, woodcut I5.

LOC. Kim. Clay ; Kimeridge, Portland, Weymouth, Swindon, and Shotover. [Vertebre ; Oxford Mus.]

paramekostimus, Hawkins. v. Ichthyosaurus intermedius.

platymerus, H. G. Seeley, Index to Aves, etc., Woodwardian Mus., 1869, p. xvii (name only).

LOC. Cambridge Greensand. [Woodwardian Mus.]

platyodon, W. D. Conybeare, Trans. Geol. Soc. [2] vol. i, pt. i (1822), p. I08, pl. xv, f. 7, pl. xvi, f. I-7; W. Buckland, Geol. and Mineral., ed. 2, 1837, p. 20, pl. vii (? and pl. x); R. Owen. Odontography (184I), p. 276, pl. Ixxiii, f. 3, 6, and Rept, Liassic Form., pt. iii (Pal. Soc., 1881), p. I15, pl. xxiv, f. 4, pl. xxxi, f. I-3; G. A. Mantell, Petrifactions, I85 I, p. 380 ; J. Morris, Cat. Brit. Foss., I854, p. 349 ; S. Haughton, Manual of Geology, I865, p. 272, woodcut 36 ; V. Kiprijanov, Mém. Acad. Imp. Sci. St. Pétersbourg, vol. xxviii, art. \& (188I), p. I02, pl. xviii (pars); R. Lydekker, Cat. Foss. Rept. B. M., pt. ii, 1889, p. 94, woodcut 34 .

[Regarded as the type of a distinct genus, Temnodontosaurus, by R. Lydekker, in Nicholson and Lydekker, Manual Palieont., ed. 3 (188g), vol. ii, p. xi.]

Ichthyosaums immanis [C. Koenig], Syn. Contents Brit. Mus., ed. 17,1820 , p. 62 (the earliest specific name given to Ichthyosaurian remains, but undefined). [Limb-bones; Brit. Mus., no. R. 809.]

Ichthyosaurus chiroligostinus, T. Hawkins, Mem. Ichthyosauri, I 834 , pls. iii, v, and Sea Dragons, 1840 , pls. iii, v. [Skeleton and portion of skull ; Brit. Mus.]

Oligostinus, T. Hawkins, ibid., I840, pl. vi, f. I-3. [Jaws.] 
ICFTHYOSAURUS platyodon (continued) -

Ichthyosaurus giganteus, Leach, quoted by R. Owen, Rep. Brit. Assoc., I839 (1840), p. I12. [Apparently MS. name.]

'Fossil remains,' E. Home, Phil. Trans., 1814, p. 57 I, pls. xvii-xx. [Skull, etc. ; Brit. Mus.]

LOC. L. Lias; Gloucester, Lyme Regis, Somersetshire, IVarwickshire, and Yorkshire. [Tooth ; Geol. Soc. London.]

polyostimus, Hawkins. = I. chiropolyostinus.

strongylostinus, Hawkins. v. Ichthyosaurus tenuirostris.

tenulrostris, W. D. Conybeare, Trans. Geol. Soc. [2] vol. i, pt. i (1822), p. 108, pl. xv, f. Io; W. Buckland, Geol. and Mineral., ed. 2, 1837, p. 170, pl. ix ; R. Owen, Rep. Brit. Assoc., I 839 (1840), p. II pl. Ixxiii, f. 5, and Rept. Liassic Form., pt. iii (Pal. Soc., I 88 I ), p. I 22 , pl. xxiv, f. 8 , pl. xxv, f. 3 , pl. xxxii, f. I-3, 5, 6; J. Morris, Cat. Brit. Foss., 1854, p. 349 ; J. F. Blake, in Tate and Blake, Yorkshire Lias, 1876, p. 254 ; R. Lydekker, Cat. Foss. Rept. B. M., pt. ii, I 889, p. 83, woodcuts 25, 26.

Ichthyosaurus grandipes, D. Sharpe, Proc. Geol. Soc., vol. i (1830), p. 222. [Imperfect skeleton.]

Ichthyosaurus chirostrongulostinus, T. Hawkins, Mem. Ichthyosauri, 1834, pl. xvi, and Sea Dragons, 1840, pls. xiii, xvi. [Skull and portions of skeleton; Brit. Mus.]

Ichthyosaurus strongylostinus, T. Hawkins, Sea Dragons, 1840 , pls. xii, xv, and Miem. Ichthyosauri, I83t, pl. xv. [Imperfect skeletons ; Brit. Mus.]

Loc. L. Lias; Barrow-on-Soar, Bristol, Glastonbury, Keinton, Keynsham, Lyme Regis, Stratford-on-Avon, Street, and Tewkesbury. U. Lias; Whitby. [Tooth; Geol. Soc. London.]

thyreospondylus, R. Owen, Rep. Brit. Assoc., I 839(1840), p. 124 ; J. Morris, Cat. Brit. Foss., I854, p. 350; ? J. Phillips, Geol. Oxford, I 87 I, pp. 307, 337, woodcuts cxxix-cxxxi ; ? R. Lydekker, Cat. Foss. Kept. B. M., pt. ii, 1889, p. 34.

? Ichthyosaurus brachyspondylus, R. Owen, Rept. Liassic Form. pt. iii (Pal. Soc., 1881), p. 127, pl. xxxiii, f. 3-6. [Vertebræ.]

Loc. Unknown. [Vertebræ; Bristol Mus.] [? Oxford Clay ; Buckingham and Whittlesea. Kim. Clay; Devizes, Nuneham, Portland, Shotover, Weymouth, Wheatley, and Winslow. (Vertebræ; Oxford Mus. and Brit. Mus.)]

trigonodon, C. Theodori, Gelehrte Anz. k. bay. Akad. Wiss., vol. xvi, 1843 , p. 906 ; R. Lydekker, Cat. Foss. Rept. B. M., pt. ii, 1889 , p. 105 .

? Ichthyosaurus crassimanus, R. Owen, Rep. Yorksh. Phil. Soc., I858, p. 9, footnote (name only); J. Phillips, Geol. Yorkshire, ed. 3,1875 , p. 272 (name only); J. F. Blake, in Tate and Blake, Yorkshire Lias, 1876, p. 253, pl. i, f. 9. [Skeleton; York Mus.]

LOC. U. Lias; Kingsthorpe and Lofthouse. [Portions of head; Brit. Mus.] 
ICITTYYOSAURUS (continued)-

trigonus, R. Owen, Rep. Brit. Assoc., I839 (1840), p. 124 ; J. Morris, Cat. Brit. Foss., 1854, p. 350 ; J. Phillips, Gersl. Oxford, 1871, p. 335, woodcuts cxxvi-cxxviii ; V. Kiprijanov, Mém. Acad. Imp. Sci. St. Pétersbourg, vol. xxviii, art. 8 (I88 I), p. 90 ; R. Lydekker, Cat. Foss. Rept. B. M., pt. ii, 1889, p. 22, woodcuts 12-14.

LOC. Kim. Clay; Bury St. Edmunds and Ely ; Foxhangers, Stanton, Swindon, and Wootton-Bassett, Wiltshire ; Shotover, near Oxford; Kimeridge and Weymouth. [Vertebra ; unknown. (Lydekker regards the originals of Phillips' figures as types.)]

uniformis, H. G. Bronn, Index Palæont., 1848, p. 606 ; misquotation from Fleming, Brit. Anim., 1828, p. I54.

walkeri, Seeley. $v$. Cetarthrosaurus walkeri.

zetlandicus, H. G. Seeley, Quart. Journ. Geol. Soc., vol. xxxvi, I 880, p. 635, pl. xxv ; R. Lydekker, Cat. Foss. Rept. B. M., pt. ii, 1889 , p. 76 , woodcuts $28-30$.

Ichthyosaurus longifrons, R. Owen, Rept. Liassic Form., pt. iii (Pal. Soc., I881), p. 118, pl. xxiii, f. I-5, pl. xxiv, f. 1. pl. xxv, f. I, pl. xxvi, f. I, pl. xxvii, f. 2-5. [Cranium ; Brit. Mus.]

LOC. U. Lias; Whitby, and ? Vale of Belvoir, Leicestershire. [Cranium: Woodwardian Mus.]

sp., H. E. Sauvage, Bull. Soc. Géol. France [3] vol. xv, 1887, p. 726, pl. xxvi.

LOC. L. Lias; Watchet, Somersetshire. [Pectoral arch ; Boulogne Mus.]

? sp., R. Lydekker, Cat. Foss. Rept. B. M., pt. ii, I889, p. 94.

Loc. Rhætic ; Aust Cliff. [Tooth ; Brit. Mus., no. R. 1342.]

IGUANA, J. N. Laurenti, Syn. Rept., 1768, p. 47.

ouropæea, H. Filhol, Ann. Sci. Géol., vol. viii, I877, p. 267 (europáana); R. Lydekker, Geol. Mag. [3] vol. v, I888, p. I10, and Cat. Foss. Rept. B. M., pt. i, I888, p. 277, woodcut 6 I.

Proiguana europaana, H. Filhol, Ann. Sci. Géol., vol. viii, I877, p. 338 .

LOC. U. Eocene ; Hordwell. [Vertebre; Brit. Mus.]

IGUANODON,* G. A. Mantell, Phil. Trans., I 825, p. I84; R. Owen, Rep. Brit. Assoc., 1841 (1842), p. 120, and Palæontology, ed. 2, 186I, p. 293; L. Dollo, Bull. Mus. R. Hist. Nat. Belg.,

* The genus Iguanodon was originally described from teeth, and subsequently from detached bones of various individuals. These detached teeth and bones we re considered by the earlier observers to belong to one species, for which the names 1. anglicum, F. Holl, 1829, and I. mantelli, H. von Meyer, 1832, were proposed but it was not until $185 \mathrm{I}$, when $\mathrm{R}$. Owen described a large portion of one individual (the Iguanodon of 'G. F.,' i.e.. Bensted's specimen), that the species manteliz became satisfactorily defined. There is, therefore, doubt as to the specific identity of the various bones described before $185 \mathrm{I}$, and we follow Dollo and Hulke in considering Bensted's specimen, now in the British Museum, as the type of the typical species of the genus. 
IGUANODON (continted) -

vols. i-iii (1882-84), passim; J. W. Hulke, Quart. Journ. Geol. Soc., vol. xxxii, 1876, p. 364 , woodcut I ; H. G. Seeley, Rep. Brit. Assoc., I887 (I888), p. 698.

Streptosponinlus, H. von Meyer, Palæologica, 1832, p. 106 (pars).

[Various portions of skeleton, originally assigned to Iguanodon, are now regarded as referable to the following species of other genera:-Camptosaumus vuldensis, Hypsilophodon foxi, Megalosaurus oweni, Morosaums brevis, Regnosaurus northamptoni.]

anglicum, Holl. $\%$ Iguanodon bernissartensis and I. mantelli.

bernissartensis, G. A. Boulenger, Bull. Acad. Roy. Belg. [3] vol. i, I88I, p. 606 ; L. Dollo, Bull. Mus. Roy. Hist. Nat. Belge, vol. i (1882), p. I76, pl. ix, f. 3, 4 ; R. Lydekker, Quart. Journ. Geol. Soc., vol. xliv, I888, p. 46 (? and p. 52), and Cat. Foss. Rept. B. M., pt. i, 1888, p. 201, woodcuts 40-46. [See also $I$. hollingtoniensis and I. mantelli.]

? Iguanodon anglicum, F. Holl (pars), Handb. Petref., vol. i, 1829 , p. 84 ; H. G. Bronn, Index Palæont., 1848, p. 607. [Teeth; Brit. Mus.]

? Iguanodon mantelli?, J. W. Hulke, Quart. Journ. Geol. Soc., vol. xxxiv, 1878, p. 744, woodcuts I-4. [O's articulare; Brit. Mus.]

Iguanodon mantolli, H. G. Seeley, Quart. Journ. Geol. Soc., vol. xxxix, 1883, p. 6I, woodcuts 4, 5. [Dorsal vertebra; Brit. Mus.]

Iguanodon seclyi, J. W. Hulke, Quart. Journ. Geol. Soc., vol. xxxviii, 1882, p. 135, pl. iv. [Limb-bones and caudal vertebre; J. W. Hulke Coll.]

Iguanodon, W. Buckland, Trans. Geol. Soc. [2] vol. iii (1829), p. 425, pl. xli, f. I, 2; G. A. Mantell, Phil. Trans., 1848, p. 185, pl. xvi, and ibid., 1849, p. 281, pl. xxxi, f. 19, and Petrifactions, 185I, p. 243 , woodcut 53 , and p. 294 , no. 4 ; A. G. Melville, in Mantell, Phil. Trans., 1849, p. 287, pl. xxviii, f. 4, and p. 29I, pl. xxix, f. 9; T. F. Gibson, Quart. Journ. Geol. Soc., vol. xiv, 1858, p. 175 (femur); R. Owen, Rept. Weald. and Purb. Form., pt. ii (Pal. Soc., 1855), p. 20, pl. ii, f. 9, pls. vi, xiii, xvi, xvi A, and ibid., Suppl. ii (Pal. Soc., 1859), p. 26, pl. vii, f. 4-6.

'Horn of Saurian, ? Iguanodon,' G. A. Mantell, Phil. Trans., I 84 I, p. I 49, pl. ix, f. 2.

Cetiosaurus brevis, R. Owen (errore), Rep. Brit. Assoc., 184I (1842), p. 97, and Rept. Weald. and Purb. Form., Suppl. ii (Pal. Soc., 1859), p. 27, pls. viii, ix. [Dorsal vertebræ ; Brit. Mus.]

? Streptospondylus major, R. Owen, Rep. Brit. Assoc., I84I (I842), p. 9I, and Rept. Weald. and Purb. Form., Suppl. ii (Pal. Soc., 1859), p. 22, pl. v, f. I, 2, pl. vi, f. I-3, 5, pl. vii, f. I-3, and Brit. Foss. Rept., vol. i (I884), p. 399, pl. xxi (Crocodilia). [Cervical and dorsal vertebræ ; Brit. Mus.] 


\section{IGUANODON bernissartensis (continued) -}

?Ornithopsis?, H. G. Seeley, Quart. Journ. Geol. Soc., vol. xxxviii, 1882 , p. 367 , woodcuts I-4. [Coracoid ; Woodwardian Mus.]

'Dinosaurian Ilium,' J. W. Hulke, Quart. Journ. Geol. Soc., vol. $\mathrm{xxx}, 1874$, p. $52 \mathrm{I}$, pl. xxxii, figs. $3,4 .{ }^{6}=$ pulbis of Iguanodon,' J. W. Hulke, ibid., vol. xxxii, I876, p. 364.] [Portion of pubis ; olim Wilkins Coll., Newport.]

?'Foot of a young Iguanodon,' R. Owen, ibid., Suppl. i (Pal. Soc., I 858), p. I, pls. i-iii. [S. H. Beckles Coll.]

? 'Large Reptilian Skull from Brook,' J.W. Hulke, ibid., vol. xxvii, 1871, p. 199, pl.xi. [Portion of cranium; J. W. Hulke Coll.]

'Humerus of unknown aquatic animal,' G. A. Mantell, Illustr. Geol. Sussex, I827, p. 80 , pl. xv, f. 8.

LOC. Wealden; Brixton, Brook, and Sandown, I. of Wight ; Cuckfield, Hastings, and Knellstone, Sussex. Neocomian Bone-bed ; Potton. Also dredged off East Coast. [Various portions of skeleton; Brit. Mus., etc.]

dawsoni, R. Lydekker, Quart. Journ. Geol. Soc., vol. xliv, i888, p. 51, woodcuts 1, 2, and Cat. Foss. Rept. B. M., pt. i, I888, p. I96, woodcuts 38,39 .

LOC. Wadhurst Clay; Hastings. ? Wealden; I. of Wight. [Portions of skeleton, wanting head; Brit. Mus.]

fittoni, R. Lydekker, Geol. Mag. [3] vol. vi, 1889, p. 354.

Loc. Wadhurst Clay; Hastings. [Ilim, etc. ; Brit. Mus.]

foxi $i$ (Huxley), Owen. \%. Hypsilophodon foxi.

hoggii, Owen. $\%$. Iguanodon mantelli.

hollingtoniensis, R. Lydekker, Geol. Mag. [3] vol. vi, 1SS9, p. 355. Iguanodon bernissartensis?, R. Lydekker, Cat. Foss. Rept. B. M., pt. i, I888, p. 217 .

LOC. Wadhurst Clay ; Hollington, near Hastings. [Portions of skeleton, wanting head; Brit. Mus., no. R. 1148.$]$

mantelli, H. von Meyer, Palæologica, I832, p. IIo (pars); R. Owen, Rep. Brit. Assoc., 1841 (1842), p. 120, and Rept. Cret. Form., pt. i (Pal. Soc., 1851), p. 105, pls, xxxiii-xxxvii, and ibill., Suppl, ii (Pal. Soc., I858 (1861), p. 27, pl. vii [text altered and re-issued in 1859 (1861)], and ibid., Suppl. iv (Pal. Soc., I872), p. I, pls. i-iii, and ibid., Suppl. v (Pal. Soc., I 874), p. I, pl. i, f. I-7, pl. ii, f. I9-20 ; J. Morris, Cat. Brit. Foss., I 854 , p. 350 ; J. W. Hulke, Quart. Journ. Geol. Soc., vol. xxx, 1874, p. 24, woodcuts I-4 (astragalus), and ibid., vol. xxxvi, 1880, p. 447 (sacrum, 1. Mantelli?); L. Dollo, Bull. Mus. Roy. Hist. Nat. Belge, vol. i (1882), p. 176, pl. ix, f. 1, 2 ; R. Lydekker, Cat. Foss. Rept. B. M1., pt. i, 1888, p. 218 . [See also Iguanodon bernissartensis and Trachodon cantabrigiensis.]

? Iguanodon anglicum, F. Holl (pars), Handb. Petref., vol. i, 1829, p. 81 ; H. G. Bronn, Index Palcont., I848, p. 607. [Teeth; Brit. Mus.] 
IGUANODON mantell (continued)-

? Iguanodon hoggii, R. Owen, Rept. Weald. and Purb. Form., Suppl. v (Pal. Soc, I874), p. 4, pl. i, f. 8. [Fragment of mandible from Purbeck.]

Iguanodon, G. A. Mantell, Phil. Trans., I825, p. 184, pl. xiv (pars), and Illustr. Geol. Sussex, 1827, p. 77, pl. xii, f. 4, and Geol. S. E. England, I 833 , pp. 307,3 Io, pl. iii, f. I-4, pl. ii, f. 7 , and Phil. Trans., I 84 I, p. I 3 I, pls. viii, ix (? pars), and ibid., I 848 , p. I90, pl. xix, and ibid., 1849, p. 28I, pl.xxxi, f. I8, 20, 2 I, and Wonders of Geology, ed. I, I 844 , p. 427, pl. iii, and Medals of Creation, ed. 2, 1854, p. 699, woodcut 224 ; 'G. F.', Phil. Mag., vol. v, 1834, p. 77 ; A. G. Melville, in Mantell, Phil. Trans., 1849 , p. 285 , pl. xxvi, pl. xxviii, f. 7 , pl. xxx ; R. Owen, Rep. Brit. Assoc., I84I (1842), pp. 128, 129, I31, and Odontography (184I), p. 246, and Rept. Weald. and Purb. Form., pt. ii (Pal. Soc., 1854), p. 7, pls. ii (pars), iii-v, vii-ix, xi, xii, xiv, xv, f. I-7, xvii ; J. W. Hulke, Quart. Journ. Geol. Soc., vol. xlii, I886, p. 435, pl. xir (maxilla).

'Hind foot of Iguanodon,' S. H. Beckles, Quart. Journ. Geol. Soc., vol. xiv, I 858 , p. 174 ; R. Owen, Rept. Weald. and Purb. Form., Suppl. iv (Pal. Soc., I872), p. 8, pl. iii (I. mantelli) [S. H. Beckles Coll.]

'Saurien encore plus extraordinaire,' Cuvier, Ossem. Fossiles, ed. 2, 1824, vol. v, p. 35 1, pl. xxi, f. 28-32.

?'Saurian bones from Loxwood,' R. I. Murchison, Trans. Geol. Soc. [2] vol. ii (1825), p. 104, pl. xv. [Brit. Mus.]

'Humerus of unknown animal,' G. A. Mantell, Illustr. Geol. Sussex, 1827, p. 80, pl. xiv, f. 3-6. [Left metatarsal III ; Brit. Mus. 7

'Humerus of Crocodile,' G. A. Mantell, ibid., p. 66, pl. xv, f. I. [Left metatarsal Iv; Brit. Mus.]

LOC. [? M. Purbeck; Swanage.] Wealden; Sussex, Dorsetshire, and I. of Wight. L. Greensand; Maidstone, and Blackgang Chine, I. of Wight. Neocomian Bone-bed; Potton. [Associated portions of skeleton, wanting head; Brit. Mus.]

phulipsi, H. G. Seeley, Index to Aves, etc., Woodwardian Mus., I869, pp. xix, 82.

Loc. Wealden ; Tilgate Forest. [Maxilla ; Woodwardian Mus.]

prestwichi, Hulke. $v$. Camptosaurus prestwichi.

seelyi, Hulke. $v$. Iguanodon bernissartensis.

(FOOTPRints OF), S. H. Beckles, Quart. Journ. Geol. Soc., vol. x, 1854, p. 456, pl. xix, and ibid., vol. xviii, I862, p. 443, woodcuts $2-4$; A. Tylor, Quart. Journ. Geol. Soc., vol. xviii, 1862, p. 247 , woodcut ; Tagart, Quart. Journ. Geol. Soc., vol. ii, I 846, p. 267 ; G. A. Mantell, Geol. I. of Wight, 1854, pp. 237, 240 ; L. Dollo, Bull. Mus. Roy. Hist. Nat. Belge, vol. ii, (I883), p. II 5 , pl. iii, f. 7,8 .

Loc. Wealden; Bexhill, Hastings, Swanage, and I. of Wight. 
ISCHYROSAURUS, Hulke. \% Ornithopsis. manseli, Hulke, MS. v. Ornithopsis manseli.

IACrRTA, Linnxus, Sy'st. Nat., ed. 12, vol. i, 1766, p. 359.

? eocena, R. Owen, Page-index to Brit. Foss. Rept., vol.i(1884), p.vi.

'Pleurodont Eocene lizard,' R. Owen, Rep. Brit. Assoc., 184 I (1842), p. I45, and Rept. London Clay, pt. ii (Pal. Soc., I 850$)$, p. 5o, pl. xiv, f. 43,44 .

'Lacerta, remains of,' J. Morris, Cat. Brit. Foss., 1854, p. 350. LOC. L. Eocene; Kyson (Kingston), Suffolk. [Fragment of jaw.]

ImIODON, R. Owen, Odontography (1841), p. 26I, and Rep. Brit. Assoc., I84I (1842), p. I44.

anceps, R. Owen, ibid., p. 261, pl. lxxii, f. $\mathrm{I}, 2$, and in F. Dixon, Geol. Sussex, I 850 , p. $385, \mathrm{pl}$. xxxvii, f. IO-I2 (and I4), pl. xxxviii, f. 8, 9, and ibid., ed. 2, 1 878 , p. 4 I I, pl. xxxvii, f. Io, etc., as before, and Rept. Cret. Form. (Pal. Soc., $185 \mathrm{I}$ ), p. 42, pl. ix $\iota$, f. I-6, and Ann. Mag. Nat. Hist. [5] vol. iv, I879, p. 53, pl. viii, and ibid. [5] vol. v, 1880, p. 177, pl. viii; J. Morris, Cat. Brit. Foss, 1854 , p. 350 ; T. G. Bayfield, Geol. Mag., vol. i, I864, p. 296 ; R. Lydekker, Cat. Foss. Rept. B. M., pt. i, 1888, p. 265 (Liodon).

Mosasaurus anceps, A. S. Woodward, Proc. Geol. Assoc., vol. $\mathbf{x}$ (I888), p. 280.

Mosasaurus stenodon, E. Charlesworth, London Geol. Journ., pt. i, 1846, p. 23, pls. iv, v.

LOC. U. Chalk; Norfolk, Essex, and Sussex. [Fragment of jaw ; part in Brit. Mus., part destroyed (see Charlesworth, London Geol. Journ., pt. i, 1846, p. 30).]

T.

LEPTOPLEURON, Owen. $\%$ Telerpeton.

lacertimum, Owen. $v$. Telerpeton elginense.

LIODON. $\%$ Leiodon.

HIOPLERODON, Sauvage. \% Pliosaurus. ferox, Sauvage. $v$. Pliosaurus.

grossourrei, Sauvage. ¿. Pliosaurus grossouvrei.

IyтодотA, E. I). Cope, Trans. Amer. Phil. Soc., vol. xiv, i870, p. 144.

Euclastes, E. D. Cope (non Lederer, i863), Proc. Acad. Nat. Sci. Philad., 1867, p. 39.

Glossochelys, H. G. Seeley, Ann. Mag. Nat. Hist. [4] vol. viii, 1871 , p. 227.

Pachyrhynchus, L. Dollo (non Germar, IS24, etc.), Bull. Mus. Roy. Hist. Nat. Belge, vol. iv (1886), p. 130.

Puppigerus, E. D. Cope (pars), Trans. Amer. Phil. Soc., vol. xiv (1870), p. 235.

ERQUelinnesia, L. Dollo, Geol. Mag. [3] vol. iv, 1887, p. 393 (to replace Pachyrhynchus). 
IYTOIOMA (continued)-

cantabrigiense, R. Lydekker, Quart. Journ. Geol. Soc., vol. xlv, I 889. p. 233, woodcuts 2, 3, and Cat. Foss. Rept. B. M., pt. iii, 1889 , p. 68.

Loc. Cambridge Greensand. [Nandible; Brit. Mus.]

crassicostatum (Owen), R. Lydekker, Proc. Zool. Soc., 1889, p. 6o, pls. vi, vii, and Cat. Foss. Rept. B. M., pt. iii, I889, p.60.

Chelone crassicostata, R. Owen, Rept. London Clay, pt. i (Pal. Soc., 1849), pp. 27, 42, pls. xi-xiii B ; J. Morris, Cat. Brit. Foss., 1854 , p. 347.

Thalassochelys crassicostatus, E. D. Cope, Trans. Amer. Phil. Soc., vol. xiv (1870), p. I46.

Puppigerus crassicostatus, E. D. Cope, ibid., p. 235.

? Chelone latiscutata, R. Owen, Rep. Brit. Assoc., 1841 (1842), p. 179, and Proc. Geol. Soc., vol. iii (184I), p. 574, and Rept. London Clay, pt. i (Pal. Soc., I849), p. 20, pl. vi ; J. Norris, Cat. Brit. Foss., I 854, p. 348.

? Puppiserus latiscutatus, E. D. Cope, Trans. Amer. Phil. Soc., vol. xiv (1870), p. 235.

Chelone planimentum, R. Owen, Rep. Brit. Assoc., I84I (1842), p. 178 (pars). [Skull ; Brit. Mus.]

Testudo plana, C. Koenig, Icon. Foss. Sect., 1825, pl. xvii, f. 192. [Portion of carapace; Brit. Mus.]

? Chelone costata, R. Owen (errore), Rept. Weald. and Purb. Form. (Pal. Soc., 1853), p. Io, pl. viii, f. 2. [Femur; Brit. Mus.]

Loc. London Clay; Harwich and Sheppey. [Carapace; Brit. Mus.]

longiceps (Owen), R. Lydekker, Cat. Foss. Rept. B. M., pt. iii, I889, p. 57.

Chelone longiceps, R. Owen, Rep. Brit. Assoc., I84I (1842), pp. 162, 177, and Proc. Geol. Soc., vol. iii (1841), p. 572, and Rept. London Clay, pt. i (Pal. Soc., 1849), p. 16, pls iii-v, and in F. Dixon, Geol. Sussex, ed. 2, 1878, p. 263, pl. xiii, f. 8-10, and Brit. Foss. Rept, vol. i (1884), p. I6, pls. xii, xiii ; J. Morris, Cat. Brit. Foss., I 854, p. 348.

'Fossil Tortoise,' J. Parkinson, Organic Remains, vol. iii, I8II, p. 268, pl. xviii, f. 2. [Carapace and plastron; Brit. Mus.]

'Emyde de l'isle Sheppey,' Cuvier, Ossem. Fossiles, ed. 2, vol.v, pt. ii, I 824, p. 235, pl. xv, f. I3. [Immature shell; Brit. Mus.]

Emy's parkinsonii, J. E. Gray (pars), Syn. Rept., I831, p. 33. [Idem.]

Clemmy's? parkinsonii, L. Fitzinger, Ann. Wien. Mus., vol. i, 1835 , p. 126.

Puppigerus longicops, E. D. Cope, Trans. Amer. Phil. Soc., vol. xir (1870), p. 235.

T. (Puppigerus.)

Pachyrhynchus longiceps, L. Dollo, Bull. Mus. Roy. Hist. Nat. Belge, vol. iv (1886), p. 138.

LOC. London Clay; Sheppey. [Skull.] 
IYTOzOMA (continued)-

planimentum (Owen), R. Lydekker, Cat. Foss. Rept. B. MI., pt. iii, I 889, p. 64. [See also L. crassicostatum.]

Chelone planimentum, R. Owen (pars), Rep. Brit. Assoc., I84 I (1842), p. 178, and Proc. Geol. Soc., vol. iii (1841), p. 576, and Rept. London Clay, pt. i (Pal. Soc., I849), pp. 25, 40, pls. ix, $x, x A$ and Brit. Foss. Rept., vol. $i(1884$ ), p. 25 , pls. xriii, xix (Chelonia); J. Morris, Cat. Brit. Foss., 1854, p. 348.

Chelone broviceps, R. Owen (pars), Rept. Iondon Clay, pt. i (Pal. Soc., 1849), p. Io, pl. ii, and Brit. Foss. Rept., vol. ii (1884), pls. i, xvi, xvii, f. 6 (Chelonia). [Skeleton; Brit. Mus.]

Chelone contexa, R. Owen (pars), Rept. London Clay, pt. i (Pal. Soc., 1849), p. 22, pl. vi, f. 4, pl. vii, f. 3. [Mandible.]

Thalassochelys planimentum, E. D. Cope, Trans. Amer. Phil. Soc., vol. xiv (1870), p. 146.

Packrhynchus flanimentum, L. Dollo, Bull. Mus. Roy. Hist. Nat. Belge, vol. iv, 1886, p. 138.

Puppigenus breviceps, E. I). Cope (pars), Trans. Amer. Phil. Soc., vol. xiv (J870), p. 235.

Chelori, harvicensis, S. Woodward, Syn. Tab., I830, p. 38, pl.

Glossochely's harvicusis, H. G. Seeley, Ann. Mag. Nat. Hist. [4] vol. viii, 1871 , p. 232, woodcut.

T. (Glossochely's.)

Emys parkinsonii, J. E. Gray (pars), Syn. Rept., 1831, p. 33. [Skull; olim F. Dixon Coll.]

'Fossil head of tortoise,' J. Parkinson, Organic Remains, vol. iii, Is'il, p. 27c, pl. xviii, f. 3 .

LOC. London Clay; Harwich and Sheppey. [Skull; Woodwardian Mus. $]$

trigoniceps (Owen), R. Lydekkei, Cat. Foss. Rept. B. M., pt. iii, 1889, p. 53.

Chelone trisoniceps, R. Owen, Rept. London Clay, pt. i (Pal. Soc., 18.9), p. 31, and in F. Dixon, (ieol. Sussex, 1850, p. 218 , pl. xiii, f. 4-7, iा (non 8-10), and ed. $2,1 \& 78$, p. 263 , pl. xiii [14], f. 4-7 and II (8, 9, and 10, referred to C. lonsiceps); J. Morris, Cat. Brit. Foss., 1854, p. 348.

Chelone acuticeps, R. Owen, Brit. Foss. Rept., vol. ii (1884), descr. to pl. xxv, f. 8, 10, is (Chelonia).

Pachyrhynchus trigoniceps, L. Dollo, Bull. Mus. Roy. Hist. Nat. Belige, vol. iv (is86), p. 138 .

Chclone sp., R. Owen, Rept. London Clay, pt. ii (I'al. Soc., I850), descr. to pl. xxix, f. 3, +. [Quadrate bone, etc. ; Brit. Mlus.' LOC. Bracklesham Beds. [Cranium.]

sp., R. Lydekker, Cat. Foss. Rept. B. M., pt. iii, 1889, p. 68.

Chelone sp., R. Owen, Rept. Cret. Form. (Pal. Soc., i8j1), p. II, pl. viiA, f. 4, 5 .

Euclastessp., L. I)ollo, Ann. Soc. Céol. Nord, vol. xv, I888, p. 122.

I.OC. Chalk; Halling, Kent. [Mandible; Brit. Mus., no. 391 I3.] 
IIACEIIODUS, R. Owen, Quart. Journ. Geol. Soc., vol. x, 1854, p. 422.

SAURIllus, R. Owen, ibid., vol. xi, 1855 , p. 12.3.

brodiei, $R$. Owen, ibid., vol. $x, 1854$, p. 422, woodcuts 6-8, and Rept. Weald. and Purb. Form., pt. v (Pal. Soc., I861), p. 33, pl. viii, f. IO, and Palæont., ed. 2, I861, p. 308, woodcut 106; J. Morris, Cat. Brit. Foss., 1854, p. 350 ; K. Lydekker, Cat. Foss. Rept. B. M., pt. i, 1888, p. 289.

? Saurillus obtusus, R. Owen, Quart. Journ. Geol. Soc., vol. xi, 1855 , p. 123, woodcut, and Rept. WVeald. and Purb. Form., pt. v (Pal. Soc., 186I), p. 33. [Jaws; olim W. R. Brodie Coll.

T. (Saurillus.)

LOC. M. Purbeck; Durdlestone Bay. [Jaws; olim W. R. Brodie Coll.]

MACIIMOSAURUS, H. von Neyer, Neues Jahrbuch, 1837, p. 560 (errore Madrimusaurus).

mosæ, F. Liénard, in Sauvage and Liénard, Mém. Soc. Géol. France [3] vol. i, no. 4, 1879, p. II ; R. Lydekker, Cat. Foss. Rept. B. M., pt. i, I888, p. IO4.

Metriorhynchus, E. Deslongchamps, Notes Paléont. (1869), p. 329.

Pliosaurus trochun'erius, R. Owen (errore), Rept. Kim. Clay (Pal. Soc., 1868), p. 7, pl. iii, f. 3-5.

LOC. Kim. Clay; Dorsetshire. [Mandible; Brit. Mus.]

вp. Phillips), H. E. Sauvage, Mém. Soc. Géol. France [2] vol. x, no. 2, 1874, p. 50; A. S. Woodward, Geol. Mag. [3] vol. ii, 1885 , p. 503 .

Goniopholis, J. Phillips, Geol. Oxford, 1871, p. 332.

Loc. Kim. Clay; Hardwick, near Oxford. [Tooth; Oxford Mus.]

MACORHINCHUS, Dunker. ¿'. Pholidosaurus.

meyeri, Dunker. «. Pholidosaurus meyeri.

sp.?, Koken. \%. Steneosaurus geoffroyi and S. laticeps.

MICROSPONDILLS, Meyer. ¿'. Steneosaurus.

bollensis, Meyer. $\approx$. Steneosaurus bollensis.

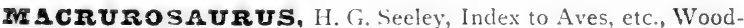
wardian Mus., I869, p. xvii (name only), and Quart. Journ. Geol. Suc, vol. xxxii, I876, p. 440.

gemnus, H. C. Seeley, Index to Aves, etc., Woodwardian Mus., I869, p. xvii (name only), and Quart. Journ. Geol. Soc., vol. xxxii, 1876 , p. $4+0$, woolcuts 1, 2; K. Lydekker, Cat. Foss. Rept. 13. NI., pt. i, I888, p. 153.

LวC. Cambridge Greensand. [Caudal vertebre; Woodwardian inus.] 
MARIMAROSAURUS, R. Owen, Odontography (18+1), p. 29 I. [? Genus.]

obtusus, R. Owen, ibid., p. 291, pl. lxxv A, f. 5 ; A. S. Woodward, Geul. Nag. [3] vol. ii, I885, p. 508 (table).

LOC. Forest Marble; Wiltshire. [Tooth; C. Pearce Coll.] $\boldsymbol{T}$.

MIUISACTUS, Hector. \% Cimoliosaurus.

gardneri, Seeley. $\%$ Cimoliosaurus constrictus.

IMEGAIOSAURUS, W. Buckland, Trans. Geol. Soc. [2] vol. i (1824), p. 390; R. Owen, Rep. Brit. Assoc., I84I (I842), p. 103.

PoEkilopleuron, E. E. Deslongchamps, Mém. Soc. Linn. Normandie, vol. vi, 1834 , p. 37.

bucklandi, $H$. von Meyer, Palæologica, 1832, p. I10; R. Owen, Odontography (1841), p. 269, pl. lxx, f. 8-11, pl. 1xx E, and Rept. Weald and Purb. Form., pt. iii (Pal. Soc., 1856), p. I, pl. xi, f. I-3, 5, 6, 8, 10-12, pl. xii, and Palæont., ed. 2, 186I, p. 288, woodcut 98, and Quart. Journ. Geol. Soc., vol. xxxix, 1883 , p. 334, pl. xi, and Brit. Foss. Rept., vol. iii (I884), p. 166, pl. lxxxvii (Dinosauria); J. Morris, Cat. Brit. Foss., I854, p. 351 ; J. Phillips, Geol. Oxford, 1871, p. I96, woodcuts lvi-lxix, p. 3 19, woodcut cxxiii, p. 390 ; R. Lydekker, Cat. Foss. Rept. B. M., pt. i, 1888 , p. 159. [See also M. oweni.]

Megalosaurus, W. Buckland, Trans. Geol. Soc. [2] vol. i, 1824, p. 390, pls. xi-xliv, and Geol. and Mineral., ed. 2, 1837 , p. 234, pl. xxiii ; G. Cuvier, Ossem. Foss., ed. 2, I 824, vol. v, pt. ii, p. 343 , pl. xxi ; K. Owen, Rept. Weald. and Purb. Form., pt. iii (Pal. Soc., 1856), p. I, pls. i, iv, vi-ix ; T. H. Huxley, Quart. Journ. Geol. Soc., vol. xxv, I869, p. 3II, pl. xii.

Pakilopleuron bucklandi, E. E. Deslongchamps (pars), Mém. Soc. Linn. Normandie, vol. vi, 1838 , p. 37 ; R. Owen, Rep. Brit. Assoc., 1841 (1842), p. 84 (P'oikilopleuron bucklandi).

Poikilopleuron bucklandi $=$ Megalosaurus buiklandi, J. W. Hulke, Quart. Journ. Geol. Soc., vol. xxxy, 1879, p. 233, pl. xii, f. $1,2$.

LOC. Inferior Oolite; Dorsetshire. Gt. Oolite (incl. Stonesfield Slate); Oxfordshire. Forest Marble; Stanton, Wiltshire. Oxford Clay; Dorsetshire and Oxfordshire. Coral Rag; Oxfordshire and Yorkshire. Kim. Clay; Swindon, Wiltshire. Neocomian Bone-bed; Potton. [Portions of jaw; Oxford Mus.]

T.

dunkevi, Lyclekker. v. Megalosaurus oweni.

iusign's, E. E. Deslongchamps, in Lennier, Etudes Géol. et P'aléont. Embouchure Seine, 1870, p. 35; H. E. Sauvage, Mém. Soc. Géol. France [2] vol. x (1\$74), p. 10, pl.v, f. 1-3; R. Lydekker, Cat. Foss. Kept. B. M., pt. i, is88, p. 163.

LOC. Kim. Clay; Foxhangers, Wiltshire. [Tooih; Lrit. .Mus.] 
IRBGAIOSAURUS (continued)-

oweni, R. Lydekker, Geol. Mag. [3] vol. vi, 1889, p. 325.

Megalosaumus, G. A. Mantell, Geol. S. E. England, 1833, p. 26rs woodcut I, and Illustr. (ieol. Sussex, I 827, p. 68 , pl. ix, f. 2, 3, 6, pl. xiii, f. 3 ; R. Owen, Rept. Weald. and l'urb. Form., pt. iii (Pal. Soc., 1856), p. I, pls. ii, iii, v, x; R. Lydekker, Quart. Journ. Geol. Soc., vol. xliv, I888, p. 53.

Poikilopleuron bucklandi, R. Owen, Rep. Brit. Assoc., 1841 (1842), p. 84 , and Brit. Foss. Rept., 1884, vol. i, p. 423, pls. vi, xix (Crocodilia).

Megalosaurus bucklandi, R. Owen (pars), Rept. Weald. and Purb. Form., pt. jii (Pal. Soc., 1856), p. I, pl. xi, f. 4, 7, 9.

'Iguanodon,' G. A. Mantell, Illustr. Geol. Sussex, 1827, pl. xii, f. I (lumbar vertebra), pl. xvii, f. 28, 29 (metatarsal).

'Hylaosaums,' R. Owen, Rept. Weald, and Purb. Form., pt. iv (Pal. Soc., 1857), p. 18, pl. xi ; J. W. Hulke (?), Phil. Trans., I881, p. 660.

Megalosaurus dunkeri, R. Lydekker (non Koken), Quart. Journ. Geol. Soc., vol. xliv, 1888, p. 59, and Cat. Foss. Rept. B. M., pt. i, I888, p. I63.

Loc. Wealden; Tunbridge Wells; Battle, Cuckfield, and Hastings ; Isle of Wight. [Metatarsals; Brit. Mus.]

sp., Murchison and Strickland. v. Cladyodon lloydi.

ILegasternon koenigi. ข. Pleurosternum bullocki.

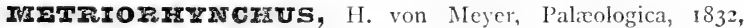
p. I06; E. E. 1)eslongchamps, Notes Paléontologiques (1869), p. 292 ; J. W. Hulke, Proc. Zool. Soc., I888, p. 4 I8, woodcuts I, 2, pl. xviii, f. I-5, pl. xix, f. I, 2.

gracile (Phillips), [-is] A. S. Woodward, Geol. Mag. [3] vol. ii, I 885, p. 502 .

Stcneosaurus gracilis, J. Phillips, Geol. Oxford, I871, p. 384, woodcuts clxxxviii, clxxxix.

LOC. Portland Stone; near Oxford. [Skull and vertebræ; Oxford Mus.]

? hastifer, E. E. Deslongchamps, Notes I'aléontologiques, I869, p. 353 (record only).

LOC. Kim. Clay; England.

moreli, E. E. Deslongchamps, Bull. Soc. Linn. Normandie [2] vol. iii, 1869, p. I87; R. Lydekker; Cat. Foss. Rept. B. M., pt. i, 1888, p. 97.

Mctriorhynitus sp., A. S. Woodward, Geol. Mag. [3] vol. ii, $1885, \mathrm{p} .503$.

LOC. Oxford Clay; Chippenham and Meiksham, Wiltshire. [Portions of skull and mandible; Brit. Mus.]

palpebrosum (Phillips), [-us] A. S. Woodward, Geol. Mag. [3] vol. ii, 1885 , p. 502 , and table, p) 508 .

Steneosaums palpebrosus, J. Phillips, (ieol. Oxford, 1871, p. 380, woodcuts clxxxii-clxxxvii. 
METRIORIIXNCFUS palpebrosum (intinued) -

Sieneosaurus rostro-minor; R. Owen (non Geoffroy), Rep. Brit. Assoc., I84I (1842), p. 82 ; J. Morris, Cat. Brit. Foss., I854, p. 353 .

Loc. Kim. Clay; Garsington and Shotover. [Skull and mandible ; Oxford Mus.]

superciliosum (De Blainville), [-us] E. E. Deslongchamps, Notes Paléontologiques (1869), p. 3 I9 ; [-us] A. S. Woodward, Geol. Mag. [3] vol. ii, I885, table, p. 508.

Crocodilus superciliosus, H. D. I) Blainville (ex Conybeare, MS.), Mém. Soc. Linn. Normandie, vol. ix, 1853, p. II4.

? Stcneosaurus dasycephalus, H. C. Seeley, Index to Aves, etc., Woodwardian Mlus., I869, p. xxi (name only). [Skull; Woodwardian Mus.]

I.oc. Oxford Clay ; Oxford. [Skull.]

I.

sp., Deslongchamps. v. Machimosaurus mosie.

sp., A. S. Woodward. v, Metriorhynchus moreli.

MOROSA UR US, O. C. Marsh, Amer. Journ. Sci. [3] vol. xv, I878, p. 242 .

becklesii, Marsh. \%. Morosaurus brevis.

brevis (Owen), R. Lydekker, in Nicholson and Lydekker, Manual Paliennt., ed. 3, I889, vol. ii, p. 1 i 79.

Cetiosaurus brevis, R. Owen, Rep. Brit. Assoc., I841 (I842), p. 97, and Rept. Weald. and Purb. Form., Suppl. ii (Pal. Suc., I 859), p. 28, pl. x, and Brit. Foss. Rept., vol. i (I884), p. 405 , pls. xxiii, xxxvi (Crocodilia); (.. A. Mantell, Phil. Trans., I 849, p. 296 ; J. Morris, Cat. Brit. Foss., I854, p. 347 ; R. Lydekker, Cat. Foss. Rept. B. M., pt. i, I888, p. I 39.

Pelorosaurus conybearci, G. A. Mantell (pars), I'hil. Trans., I850, p. 388, pls. xxii xxvi, and Petrifactions, I85I, p. 33I, woodcut 72 ; ? R. Owen, Rept. Weald. and Purb. Form., Suppl. ii (Pal. Soc., 1859), p. 38 , pl. xi.

Pelorosanrus becklesii, G. A. Mantell, Proc. Roy. Inst., vol. i (1 852 ), p. 143 (name only); J. Morris, Cat. Brit. Foss., 1854 , p. 351 . [Portions of skeleton, wanting head; S. H. Beckles Coll.]

Bothriospondylus elongatus, R. Owen, Rept. Mesoz. Form., pt. ii (Pal. Soc., 1875), p. 23, pl. vii. [Vertebral centrum; Brit. Mus.]

? Chondrosterosamus gigas, R. Owen (pars), Rept. Weald. and Purb. Form., Suppl. vii (Pal. Soc., 1876, p. 5, pls. iv, v. [Cervical vertebra; Brit. Mus.]

Ornithopsis lulkei, H. G. Seelcy (pars), Amn, Mag. Nat. Hist. [4] rol. v, 1870, p. $283 ;$ J. WV. Hulke, Guart. Journ. Geol. Soc., vol, xxxviii (1882), p. 375.

Morosaunus becklesii, O. C. Marsh, Geol. Mag. [3] vol. vi, I8S9, p. 205.

'Crocodile,' G. A. Mantell, Illustr. (icol. Susscx, 1827, pl. ix, f. 8 , pl. x, f. 3 . 
MOROSAURUS brevis (continued)-

'Vertebra of unknown Saurian,' G. A. Mantell, Phil. Trans., I84I, p. I49, pl. ix, f. 7.

-Tympanic bone of Iguanodon,' G. A. Mantell, Geol. S. E. England, I833, p. 305, pl. ii, f. 5 ; R. Owen, Rep. Brit. Assoc., I84I (1842), p. I24, and Rept. Weald. and Purb. Form., pt. ii (Pal. Soc., 1855), p. I8, pl. x.

LOC. Wealden; Brook, 1. of Wight ; Cuckfield and Hastings, Sussex. [Four associated caudal vertebræ ; Brit. Mus.]

mOSASAURUS, W. D. Conybeare, in Cuvier, Ossem. Foss., ed. 2 , 1824, vol. v, pt. ii, p. 338 .

anceps (Owen), A. S. Woodward. $\%$. Leiodon anceps.

gracilis, Owen. $v$. Mosasaurus sp.; and Pachyrhizodus gracilis. [PISCES.]

hoffimanni, Mantell. v. Mosasaurus sp.

stenodon, Charlesworth. $\%$ Leiodon anceps and Mosasaurus? sp.

? sp.: Mosascurus hoffmannii, G. A. Mantell, Trans. Geol. Soc. [2] vol. iii (1829), p. 207, and Medals of Creation, vol. ii, 1854, p. 708, woodcut 229 ( 11 . hoffinanni, or $1 /$. gracilis).

'Fossil Monitor of Mrestricht,' G. A. Mantell, Foss. S. Downs, I 822, p. 242, pl. xxxiii, f. I3, pl. xli, f. 3. [Named Mosasaurus stenodon by G. A. Mantell, Petrifactions, 1851, pp. 197, I98, woodcut 44.]

Mosasaurus gracilis, R. Owen (pars), in F. Dixon, Geol. Sussex, 1850 , p. 383 , pl.xxxvii, f. 2-5, pl. xxxix, f. 7-9, and Rept. Cret. Form. (Pal. Soc., 1851), p. 40, pl. viii, f. I-3, pl. ix, f. 2-5.

Genus non det., R. Lydekker, Cat. Foss. Rept. B. M., pt. i, 1888, p. 273 .

LOC. Chalk ; Essex, Kent, and Sussex. [Teeth and vertebræ ; Brit. Mus.]

MURAENOSAURUS, Seeley. ¿ Cimoliosaurus.

borchyspondylus (Phillips), Sauvage. $\%$. Cimoliosaurus trochanterius.

lecalsi, Seeley. ข. Cimoliosaurus plicatus.

mareli (Hulke), Sauvage. \% Cimoliosaurus trochanterius.

MISTRIOSAURUS, Kaup. \%. Steneosaurus.

bollensis, D'Alton and Burmeister. $\%$ Steneosaurus bollensis. brevior (Owen), A. S. Woodward. \%. Steneosaurus brevior.

brongniarti, Kaup. \%. Pelagosaurus brongniarti.

chrpmani (Koenig), A.S. Woodward. v. Steneosaurus chapmani. stukelyi, Winkler. $\because$ Steneosaurus bollensis and S. chapmani.

NANNOSUCHUS, R. Owen, Rept. Weald. and Purb. Form., Suppl. ix (Pal. Soc., 1879), p. 6.

gracilidens, R. Owen, ibid., p. 6, pl. ii, f. I-Io, pl. iii, f. 1, 2 ; R. Lydekker, Cat. Foss. Rept. B. M., pt. i, 1889, p. 84.

LOC. M. Purbeck; Durdlestone Bay. [Skull and other parts of skeleton; Brit. Mus.] 
MUTHFTgS, R. Owen, (2uart. Journ. Geol. Soc., vol. x, 1854, p. 420 ; R. Lydekker, Cat. Foss. Rept. B. M., pt. i, 1888, p. 247.

destructor, R. Owen, Quart. Journ. Geol. Soc., vol. x, 1854, p. 420, woodcuts I-4, and Rept. Weald. and Purb. Form., pt. v (Pal. Soc., 1861), p. 31, and ibid., Suppl. ix (Pal. Soc., 1879), p. 16, pl. ii, f. 13-23, and Journ. R. Micros. Soc., 1878, p. 233 , pl. xii, f. 8,9 ; J. Morris, Cat. Brit. Foss., I854, p. 35 I (Nothete's).

T.

Loc. M. Purbeck; Durdlestone Bay. [Jaw and teeth; Brit. Mus.]

OCADIA, J. E. Gray, Suppl. Cat. Shield Rept., pt. i, I870, p. 35.

crassa (Owen), R. Lydekker, Cat. Foss. Rept. B. M., pt. iii, 1889, p. IIO, woodcut 24.

Emys crassus, R. Owen, Rept. London Clay, pt. i (Pal. Soc., 1849), p. 76, pl.xxvii ; J. Morris, Cat. Brit. Foss., 1854, p. 348.

Emys hordwellensis, H. G. Seeley, Quart. Journ. Geol. Soc., vol. xxxii, I876, p. 445, woodcuts I, 2. [Imperfect shell; Woodwardian Mus.]

LOC. U. Eocene ; Hordwell. [Right hyoplastron ; Brit. Mus.]

oweni, R. Lydekker, Cat. Foss. Rept. B. M., pt. iii, 1889, p. 1 I5, woodcut 25.

? Emys testudiniformis, R. Owen (errore, fide Lydekker), Rept. London Clay, pt. i (Pal. Soc., I849), p. 67, pl. xxiv, f. I-3 (? f. 4, 5). [Nuchal and neural plates; Brit. Mus.]

Loc. U. Eocene ; Hordwell. [Portions of shell ; Brit. Mus.]

OLIGOSTINUS, Hawkins, Great Sea Dragons, 1840. = Ichthyosaurus ( $\boldsymbol{v}$. I. lonchiodon).

Omosaurus, R. Owen (non J. Leidy, 1856), Rept. Mesoz. Form., pt. ii (Pal. Soc., 1875), p. 45. [? Stegosaurus, according to O. C. Marsh, Geol. Mag. [3] vol. vi, 1889, p. 207.]

armatus, R. Owen, Rept. Mesoz. Form., pt. ii (Pal. Soc., I875), p. 45 , pls. xi-xxii, and Brit. Foss. Rept., vol. i (1884), p. 556 , pls. Ixiv-lxxv (Dinosauria); W. Davies, Geol. Mag. [2] vol. iii, I876, p. 193, pls. vii, viii ; J. W. Hulke, Quart. Journ. Geol. Soc., vol. xliii, 1887, p. 702 ; R. Lydekker, Cat. Foss. Rept. B. M., pt. i, I888, p. 177.

Stegosaurus armatus, O. C. Marsh, Geol. Mag. [3] vol.vi, 1889, p. 207.

LOC. Kim. Clay ; Swindon. [Portions of skeleton, wanting head; Brit. Mus.]

$\mathbf{T}$.

durobrivensis, J. IV. Hulke, Quart. Journ. Geol. Soc., vol. xliii, I887, p. 699, woodcut 2.

Loc. Oxford Clay (non Kim. Clay); Peterborough. [Pelvis; A. N. Leeds Coll.]

hastiger, R. Owen, Rept. Mesoz. Form., pt. iii (Pal. Soc., 1877), p. 95, pls. xxiii, xxiv ; R. Lydekker, Cat. Foss. Rept. B. M., pt. i, 1888 , p. 179.

LOC. Kim. Clay; Wootton-Bassett. [Dermal spine; Brit. Mus.] 


\section{OMOSaurus (continued)-}

? sp., (?) R. Lydekker, Cat. Foss. Rept. B. M., pt. i, 1888, p. ISo. Loc. Oxford Clay; Bedford. [Dermal spine; Brit. Mus., no. R. $5^{8} 4$ ]. Also Weymouth. [Portion of fibula ; Brit. Mus., no. 40517 ].

Oolithes bathonich, Buckman. $\%$. MiscellanEOUS REPTILIA.

OPITMAIm OSA UR Us, H. G. Seeley, Quart. Journ. Geol.Soc., vol. xxx, 1874, p. 696.

? cantabrigiensis, (?) R. Lydekker, Geol. Mag. [3] vol. v, I 888 , p. 3 IO, and Cat. Foss. Rept. B. M., pt. ii, I889, p. 9, woodcut 6. [See also Baptanodon.]

LOC. Cambridge Greensand. [Right humerus ; Brit. Mus.]

icentcus, H. G. Seeley, Quart. Journ. Geol. Soc., vol. xxx, I874, p. 707, pls. xlv, xlvi ; R. Lydekker, Cat. Foss. Rept. B. M., pt. ii, 1889, p. 9, woodcut 7 .

LOC. Oxford Clay; Oxford and Peterborough. Kim. Clay ; Foxhangers, Wiltshire, and Ilminster. [Fore-limb; A. N. Leeds Coll.]

? sp., (?) R. Lydekker, Cat. Foss. Rept. B. M., pt. ii, I889, pp. 12, 20. LOC. U. Greensand; Warminster. [Right dentary; Brit. Mus., no. R. I192.]

OPLOSAURUS, Gervais. v. Hoplosaurus.

ORNITHO Crisrruts, H. G. Seeley, Index to Aves, etc., Woodwardian Mus., I869, p. xvi, and Ornithosauria, 1870, p. 112 and passim, with 12 plates, and Ann. Mag. Nat. Hist. [4] vol. vii, IS7I, p. 20. [Ornithochirus, Lydekker and others.]

? PAL ÆORnis, G. A. Mantell, Medals of Creation, vol. ii, I844, p. 8o6. [Preoccupied and inappropriate.]

Cimoliornis, R. Owen, Brit. Foss. Mamm. and Birds, 1846, p. 545. [Inappropriate.]

Coloborhynchus, R. Owen, Rept. Mesoz. Form., pt. i (Pal. Soc., 1874$)$, p. 6.

Criorhynchus, R. Owen, ibid., p. 7.

brachyrhinus, H. G. Seeley, Ornithosauria, I870, p. I23.

LOC. Cambridge Greensand. [Fragment of premaxilla; Woodwardian Mus.]

capito, H. G. Seeley, Ornithosauria, I870, p. 126.

LOC. Cambridge Greensand. [Fragment of premaxilla; Woodwardian Mus.]

carteri, H. G. Seeley, Index to Aves, etc., Woodwardian Mus., 1869 , p. xvi (name only), and ibid., p. 6 (Pterodactylus carteri).

Loc. Cambridge Greensand. [Premaxilla; Woodwardian Mus.] 
ORNITIOCIRIRUS (continucd)-

clavirostris: Coloborhynchus clarirostris, R. Owen, Rept. Mesoz. Form., pt. i (Pal. Soc., 1874), p. 6, pl. i, f. I-4.

Loc. Hastings Sands; St. Leonards. [Premaxilla; S. H. Beckles Coll.]

T. (Coloborliynchus.)

? cllfti (Mantell), R. Lydekker, Cat. Foss. Rept. B. M., pt. i, I888, p. 25 .

Palaornis clifti, G. A. Mantell, Medals of Creation, vol. ii, I\$44, p. 806, woodcut I 49.

T: (Palcornis.)

'terodactylus cliftii, J. Morris, Cat. Brit. Foss., 1854, p. 353.

Pterodactylus sp., H. G. Bronn, Index Palæont., 1848, p. 1054.

'Pterodactyle,' R. Owen, Quart. Journ. Geol. Soc., vol. ii, 1846, p. 99, woodcuts 5-7. [Named Pterodactylus syliestris, R. Owen, Brit. Foss. Rept, vol. i (I884), p. 543.]

'Bird-bone,' G. A. Mantell, Illustr. Geol. Sussex, I827, descr. to pl. viii, f. I I, and Trans. Geol. Soc. [2] vol. v (184I), p. 177, pl. xiii, f. 3 (? non f. 5, 6).

Loc. Wealden; Battle, Cuckfield, Hastings, and Tilgate Forest. [Portions of humerus; Brit. Mus.]

colorhinus, H. G. Seeley, Ornithosauria, 1870, p. I24.

LOC. Cambridge Greensand. [Fragments of premaxillæ; WVoodwardian Mus.]

compressirostris (Owen), H. G. Seeley, Ornithosauria, I S7o, p. 92 ; R. Lydekker, Cat. Foss. Rept. B. M., pt. i, 1888, p. I1.

Pterodactylus compressirostris, R. Owen, Proc. Zool. Soc., I 5 I, p. 32, and Rept. Cret. Form. (Pal. Soc., 1851), p. 95, pl. xxiv, pl. xxvii, f. 5, pl. xxviii, f. 8, 9, Io, pl. xxx, f. 4, 5, pl. xixii, f. 2, and in F. Dixon, Geol. Sussex, ed. 2, 1878, p. 428, pl. xxxix [44], f. I I, I2 ; J. Morris, Cat. Brit. Foss., 1854 , p. 353 .

LOC. L. Chalk ; Burham. [Portions of snout ; Brit. Mus.]

crassidens, H. G. Seeley, Ornithosauria, 1870, p. 122.

LOC. Cambridge Greensand. [Fragment of ? premaxilla ; Woodwardian Mus.]

? curtus (Owen), R. Lydekker, Cat. Foss. Rept. B. M., pt. i, i \$88, p. 26. Pterodactylus curtus, R. Owen, Rept. Liassic Form., pt. ii (Pal. Soc., 1870), p. 52, pl. xix, f. 8, 9.

LOC. Wealden; Sussex. [Distal end of tibia ; Brit. Mus.]

cuvieri (Bowerbank), H. G. Seeley, Ornithosauria, I870, p. I I 3 (? pl. xii, f. 5) ; R. Lydekker, Cat. Foss. Rept. B. MI., pt. i, 1888 , p. 12.

Pterodactylus cuvieri, J. S. Bowerbank, Proc. Zool. Soc., I $5_{5} \mathrm{I}$, p. 15 , pl. iv (lettered longirostris); R. Owen, Rept. Cret. Form. (Pal. Soc., I85 I), p. 88, pl. xxviii, f. I-7, pl. xxx, f. I-3, and in F. Dixon, Geol. Sussex, ed. 2, 1878, p. 428; J. Morris, Cat. Brit. Foss., 1854 , p. 353. 
ORNITHOCHמIRUS cuvieri (continued)-

Coloborhynchus cuvieri, R. Owen, Rept. Mesoz. Form., pt. i (Pal. Soc., 1874), p. 6.

LoC. L. Chalk; Burham and Halling, Kent. [Cranial rostrum ; Brit. Mus.]

daviesi (Owen), R. Lydekker, Cat. Foss. Rept. B. M., pt. i, I888, p. 23. [? O. denticulatus.]

Pterodactylus daviesii, R. Owen, Rept. Mesoz. Form., pt. i (Pal. Soc., 1874), p. 2, pl. i, f. $5,6$.

LOC. Gault ; Folkestone. [Mandib. symphysis ; Brit. Mus.]

dentatus, H. G. Seeley, Ornithosauria, 1870, p. II9.

LOC. Cambridge Greensand. [Fragment of premaxilla ; Woodwardian Mus.]

denticulatus, H. G. Seeley, Ornithosauria, I870, p. 122, pl. xii, f. 8,9 .

LOC. Cambridge Greensand. [Fragment of premaxilla ; Woodwardian Mus.]

diomedius (Owen), R. Lydekker, Cat. Foss. Rept. B. M., pt. i, I888, p. I3.

Cimoliornis diomedius, R. Owen (ex Gervais), Brit. Foss. Mamm. and Birds, 1846, p. 545 , woodcut 230.

T. (Cimoliornis.)

'Ornitholite,' R. Owen, Trans. Geol. Soc. [2] vol. vi, I84I (1842), p. 411, pl. xxxix, f, 2.

'Pterodactyle,' R. Owen, Rept. Cret. Form. (Pal. Soc., I851), p. 102, pl. xxxii, f. 4 .

LOC. Chalk; Kent. [Distal end of ulnar metacarpal; Brit. Mus.]

enchorhynchus, H. G. Seeley, Ornithosauria, I870, p. 123.

LOC. Cambridge Greensand. [Premaxilla; Woodwardian Mus.]

eurygnathus, H. G. Seeley, Ornithosauria, 1870, p. 123.

LOC. Cambridge Greensand. [Fragment of ? dentary bone; Woodwardian Mus.]

fitton1 (Owen), H. G. Seeley, Ornithosauria, 1870, p. 118; R. Lydekker, Cat. Foss. Rept. B. M., pt. i, 1888, p. 15.

Pterodactylus fittoni, R. Owen, Rep. Brit. Assoc., I858 (I859), Sect., p. 98, and Rept. Cret. Form., Suppl. i (Pal. Soc., 1859), p. 4 , pl. i, f. $3-5$, pls. ii-iv (pars), and Phil. Trans., 1859 (1860), p. 162, pl. x, f. 7-12, 32-34.

LOC. Cambridge Greensand. [Premaxilla; Woodwardian Mus.]

? giganteus (Bowerbank), A. S. Woodward, Proc. Geol. Assoc., vol. x (1888), p. 284 ; R. Lydekker, Cat. Foss. Rept. B. M., pt. i, 1888, p. 12. 
ORNIFIFOCrIRUs giganteus (continued) -

Pterodactylus giganteas, J. S. Bowerbank, Quart. Journ. Geol. Soc., vol. ii, I846, p. 8, pl. i, and Proc. Zool. Soc., I85I, p. I4 ; R. Owen, Proc. Zool. Soc., 1851, p. 25, and Rept. Cret. Form. (Pal.Soc., 1851), p. 91, pl. xxxi, and in F. Dixon, Geol. Sussex, ed. 2, 1878, p. 427, pl. xxxviii [42], f. 4-7; J. Morris, Cat. Brit. Foss., 1854, p. 353.

Pterodactylus conirostris, R. Owen, in F. Dixon, Geol. Sussex, I 850 , p. 401 , pl. xxxviii, f. 4-7. [Portions of jaws; Brit. Mus.]

LOC. L. Chalk ; Burham. [Portions of jaws, etc. ; Brit. Mus.]

huxleyi, H. G. Seeley, Ornithosauria, 1870, p. I16.

LuC. Cambridge Greensand. [Dentary bone; Mus. Pract. Geol.]

macharorhynchus, H. G. Seeley, Ornithosauria, 1870, p. I13, pl. xii, f. I, 2 .

LOC. Cambridge Greensand. [Dentary bone; Woodwardian Mus.]

microdon, H. G. Seeley, Ornithosauria, i 870, p. I16, pl. xii, f. 6, 7. LOC. Cambridge Greensand. [Premaxilla; Woodwardian Mus.]

nasutus, H. G. Seeley, Ornithosauria, I870, p. I20.

LOC. Cambridge Greensand. [Fragment of premaxilla; Woodwardian Mus.]

nobilis (Owen), R. Lydekker, Cat. Foss. Rept. B. M., pt. i, I888, p. 24.

Pterodactylus nobilis, R. Owen, Rept. Liassic Form., pt. ii (Pal. Soc., 1870), descr. to pl. xix, f. IO.

LOC. Wealden; Brook, I. of Wight. [Portion of wing-phalanx; Brit. Mus.]

oweni, H. G. Seeley, Ornithosauria, 1870, p. Ir 5.

LOC. Cambridge Greensand. [Premaxilla; Woodwardian Mus.]

oxyrhinus, H. G. Seeley, ibid., p. I 7 .

LOC. Cambridge Greensand. [Premaxilla ; Woodwardian Mus.]

platyrhimus, H. G. Seeley, Index to Aves, etc., Woodwardian Mus., 1869, p. xvii (name only).

LOC. Cambridge Greensand.

platystomus, H. G. Seeley, Ornithosauria, I870, p. 120.

LOC. Cambridge Greensand. [Fragment of snout; Woodwardian Mus.]

polyodon, H. G. Seeley, ibid., p. 12 I.

LOC. Cambridge Greensand. [Premaxilla; Woodwardian Mus.]

reedi, H. G. Seeley, ibid., p. 126, and [-ii] Geol. Mag. [2] vol. viii, i 88 i, p. 13, pl. i, f. 3 .

LOC. Cambridge Greensand. [Premaxilla; York Mus.] 
ORNITHOCTrIRUS (continued) -

scaphorhynchus, 11. G. Seeley, Ornithosauria, 1870, p. I19, (O. scaphorynchus).

LOC. Cambridge Greensand. [Fragment of premaxilla; Woodwardian Mus.]

sedgwicki (Owen), H. G. Seeley, ibid., p. II2; R. Lydekker, Cat. Foss. Rept. B. M., pt. i, I888, p. 15.

Pterodactylus sedgwickii, R. Owen, Rep. Brit. Assoc., 1858 (1859), Sect., p. 98, and Phil. Trans., I859 (I860), p. 164, pl. x, f. I-5, I3, I4, 24-26, 28-3I, 35, 36, and Rept. Cret. Form., Suppl. i (Pal. Soc., 1859), p. 2, pl. i, f. I, 2, 6-14, pls. ii-iv (pars), and ibid., Suppl. iii (1861), pl. iv, f. 5-12.

Coloborhynchus sedgrwickii, R. Owen, Rept. Mesoz. Form., pt. i (Pal. Soc., I874), p. 6.

LOC. Cambridge Greensand. [Portions of jaws; Woodwardian Mus.]

simus (Owen), H. G. Seeley, Index to Aves, etc., Woodwardian Mus., I869, p. xvi (Pterodactylus, p. 6), and Ornithosauria, I870, p. 127, and Geol. Mag. [2] vol. viii, I88I, p. I5; ? R. Lydekker, Cat. Foss. Rept. B. M., pt. i, I888, p. I6.

Pterodactylus simus, R. Owen, Rept. Cret. Form., Suppl. iii (Pal. Soc., 1861), p. 2, pls. i, ii, pl. iv, f. 4.

Criorhynchus simus, R. Owen, Rept. Mesoz. Form., pt. i (Pal. Soc., 1874), p. 6.

T. (Criorhynchus.)

?Pterodactylus woodwardi, R. Owen, Rept. Cret. Form., Suppl. iii (Pal. Soc., I86I), p. 4, pl. ii, f. 3. [Portion of jaw ; Woodwardian Mus.]

? Omithocheirus woodwardi, H. G. Seeley, Ornithosauria, 1870, p. 125.

LOC. Cambridge Greensand. [Mandib. symphysis; Woodwardian Mus.]

tenuirostris, H. G. Seeley, Ornithosauria, I870, p. II4.

Loc. Cambridge Greensand. [Premaxilla; Woodwardian Mus.] validus (Owen), Lydekker. v. Doratorhynchus validum. wooduardi (Owen), Seeley. $\%$ Ornithocheirus simus.

xyphorhynchus, H. G. Seeley, ibid., p. I17, and Geol. Mag. [2] vol. viii, 188I, p. I8, pl. i, f. 2.

LOC. Cambridge Greensand. [Portion of mandible; York Mus.]

? sp. (?), R. Lydekker, Cat. Foss. Rept. B. M., pt. i, 1888, p. 25.

LOC. Wealden; Brook, I. of Wight. [Prox. end of humerus ; Brit. Mus., no. R. 558.]

OaNITyo PsIS, H. G. Seeley, Ann. Mag. Nat. Hist. [4] vol. v, I870, p. 279, and Ornithosauria, 1870, p. 103 (note). [See also Iguanodon bernissurtensis.]

Eucamerotus, J. W. Hulke, Quart. Journ. Geol. Soc., vol. xxviii, 1872, p. 36.

Ischyrosaurus, J. W. Hulke (non E. D. Cope, 1870), ibid., vol. $\mathrm{xxx}, \mathrm{I} 874$, p. I6. 
ORNITIXopsis (continued)-

Chondrosteosaurus, R. Owen, Rept. Weald. and Purb. Form., Suppl. vii (Pal. Soc., 1876), p. 5, and Ann. Mag. Nat. Hist. [5] vol. ii, I878, p. 201.

[? Also may include Pelorosaurus, Mantell, and Gigantosaurus, Seeley.]

eucamerotus, Hulke. $v$. Ornithopsis hulkei.

hulkei, H. G. Seeley, Ann. Mag. Nat. Hist. [4] vol. v, I870, p. 283, and Quart. Journ. Geol. Soc., vol. xlv, I889, p. 392, woodcut I ; R. Lydekker, Quart. Journ. Geol. Soc., vol. xliv, 1888, p. 54, pl. iii, f. I-4, and Cat. Foss. Rept. B. M., pt. i, 1888, p. 146, woodcut 24. [See also Morosaurus brevis.]

'Undescribed Wealden Vertebra,' J. W. Hulke, Quart. Journ. Geol. Soc., vol. xxvi, 1870, p. 318, pl. xxii. [Portion of vertebra; J. W. Hulke Coll.]

Bothriospondylus magnus, R. Owen, Rept. Mesoz. Form., pt. ii (Pal. Soc., 1875), p. 24, pls. viii, ix. [The type-vertebra of Ornithopsis hulkei, this name being regarded as inappropriate.] Also Brit. Foss. Rept., vol. iii (1884), p. 172, pl. Ixxxviii (Dinosauria).

? Chondrosteosaurus gigas, R. Owen (pars), Rept. Weald. and Purb. Form., Suppl. vii (Pal. Soc., 1876), p. 5, pls. ii, iii. [Vertebral centrum; Brit. Mus.]

? Chondrosteosaurus magnus, R. Owen, ibid., p. 7, pl. vi, and Brit. Foss. Rept., vol. i (1884), p. 624, pls. Ixxxiii-lxxxv (Dinosauria). [Dorsal vertebra ; Brit. Mus.]

Ornithopsis eucamerotus, J. W. Hulke, Quart. Journ. Geol. Soc., vol. xxxviii, 1882, p. 372, pl. xiv. [Pelvis; Brit. Mus.]

Ornithopsis, J. W. Hulke, ibid., vol. xxxv, 1879, p. 752, woodcuts 1-4. [Vertebra; J. W. Hulke Coll.]

Ornithopsis = Eucamerotus, J. W. Hulke, ibid., vol. xxxvi, 1880, p. 31 , pls. iii, iv. [Vertebre ; Brit. Mus.]

Pelorosaurus armatus, R. Lydekker, Geol. Mag. [3] vol. vi, 1889, p. 325. [Oplosaurus armatus, Gervais, considered to be a tooth of Ornithopsis hulkei: $\%$. Hoplosaurus.]

Loc. Wealden; Brixton Bay and Sandown, I. of Wight; Hastings. [Vertebral centrum; Brit. Mus., no. 28632.] T.

humerocristatus (Hulke), R. Lydekker, Cat. Foss. Rept. B. M., pt. i, 1888, p. I 5 I. [See also O. leedsi.]

Cetiosaurus humerocristatus, J. W. Hulke, Quart. Journ. Geol. Soc., vol. $\mathrm{xxx}, 1874$, p. I6, pl. ii.

Loc. Kim. Clay; Weymouth. [Left humerus ; Brit. Mus.]

Ieedsi, [-ii] J. W. Hulke, Quart. Journ. Geol. Soc., vol. xliii, I887, p. 695 ; H. G. Seeley, (Quart. Journ. Geol. Soc., vol. xlv, 1889, p. 39I.

Ornithopsis humerocristatus?, R. Lydekker, Quart. Journ. Geol. Soc., vol. xliv, I888, p. 58, and Cat. Foss. Rept. B. MI., pt. i, 1888, p. 15 I.

Ioc. Oxford Clay; Peterborough. [Pelvis; A. N. Leeds Coll.] 


\section{ORNITHOPSIS (continued)-}

manseli, R. Lydekker (ex J. W. Hulke, MS.), Cat. Foss. Rept. B. M., pt. i, r888, p. I j2. [? Pelorosaurus.]

Ischyrosaurus manseli, J. W. Hulke, MS. T. (Ischyrosaumus.) 'Saurian humerus,' J. WV. Hulke, Quart. Journ. Geol. Soc., vol. xxv, I869, p. 386, pl. xvi.

Ischyrosaurus, J. W. Hulke, ibid., vol. xxx, 1874, p. 16 .

LoC. Kim. Clay; Kimeridge Bay. [Humerus ; Brit. Mus.]

oxoniensis (Phillips), Seeley. $\%$. Cetiosaurus oxoniensis.

ORNIтHostromA, H. G. Seeley, Ann. Mag. Nat. Hist. [4] vol. vii, I 87 I, p. 35 (footnote), and Quart. Journ. Geol. Soc., vol. xrxii, 1876, p. 499.

sp., H. G. Seeley, ibid., I876, p. 499.

'Proximal end of metacarpal of wing-finger of Pterodactylus,' R. Owen, Rept. Cret. Form., Suppl. i (Pal. Soc., 1859), p. 18, pl. iv, f. $4, \mathbf{j}$.

LOC. Cambridge Greensand. [? Premaxilla; Woodwardian Mius.]

T.

OWgirasucrus, A. S. Woodward, Geol. Mag. [3] vol. ii, 188;, p. 506.

Brachydectes, R. Owen (non E. D. Cope, I868), Rept. Weald. and Purb. Form., Suppl. ix (Pal. Soc., I879), p. 3.

major (Owen), A. S. Woodward, Geol. Mag. [3] vol. ii, 1885, p. 506 ; R. Lydekker, Cat. Foss. Rept. B. M., pt. i, I888, p. 85.

Brachydectes major, R. Owen, Rept. Weald. and Purb. Form., Suppl. ix (Pal. Soc., 1879), p. 3, pl. i, f. 2.

LOC. MI. Purbeck; Durdlestone Bay. [Mandib. ramus; Brit. Mus.]

T.

minor (Owen), A. S. Woodward, Geol. Mag. [3] vol. ii, I885, p. 506 ; R. Lydekker, Cat. Foss. Rept. B. M., pt. i, 1888, p. 85. Brachydectes minor, R. Owen, Rept. Weald. and Purb. Form., Suppl. ix (Pal. Soc., I879), p. 5, pl. i, f. 3 (? and f. 13).

LOC. M.Purbeck; Durdlestone Bay. [Mandib.ramus; Brit. Mus.]

PACHYRHAMPHUS, Fitzinger. \%. Scaphognathus.

PACHIRHYNCHUS, Dollo. v'. Lytoloma.

longiceps (Owen), Dollo. v. Lytoloma longiceps.

planimentum (Owen), Dollo. v. Lytoloma planimentum.

trigoniceps (Owen), Dollo. v. Lytoloma trigoniceps.

PAIFOPFIS, R. Owen, Trans. Geol. Soc. [2] vol. vi (18+1), p. 209 ; R. Lydekker, Geol. Mag. [3] vol. v, I888, p. I12.

? longus, R. Owen, Rept. London Clay, pt. iii (Pal. Soc., I850), p. 66, pl. xiv, f. $35-37,45,46$; J. Morris, Cat. Brit. Foss., 1854, p. 35 I. LOC. L. Eocene; Kyson (Kingston), Suffolk. [Vertebræ.] 
PAxryOPIS (continued)-

porcatus, Owen. $\%$. Palæophis typhæus.

tollapicus, R. Owen, Trans. Geol. Soc. [2] vol. vi (I 84I), p. 209, pl. xxii, and Rept. London Clay, pt. iii (Pal. Soc., 1850), p. 63, pls. xv, xvi, and in F. Dixon, Geol. Sussex, 1850, p. 212 , woodcuts I4, 15, and ibid., ed. 2, 1878, p. 258, same woodcuts, and Palæontology, ed. 2, 186r, pp. 299, 31 3, woodcut I03 (5) ; J. Morris, Cat. Brit. Foss., 1854, p. 351 ; R. Lydekker, Cat. Foss. Rept. B. M., pt. i, 1888, p. 257.

LOC. London Clay; Sheppey. Bracklesham Beds. [Vertebrx; Brit. Mus.]

$\mathbf{T}$.

typhæus, R. Owen, Rept. London Clay, pt. iii (Pal. Soc., I850), p. 56, pl. xiii, f. 5-8, pl. xiv, f. $1-3,7-9,16,17,26-28$, and in F. Dixon, Geol. Sussex, 1850, p. 212 , pl. xii, f. 14, woodcuts 1-3, 7, 13, and ibid., ed. 2, 1878, p. 258, same pl. and figs. (P.typhoeus) ; J. Morris, Cat. Brit. Foss., I854, p. $35 \mathrm{I}$ (P. typhoeus); R. Lydekker, Cat. Foss. Rept. B. M., pt. i, I888, p. 258, woodcut 56.

Palcophis porcatus, R. Owen, Rept. London Clay, pt. iii (Pal. Soc., 1850 ), p. 61, pl. xiv, f. $13-15,18,20,21$, and in F. Dixon, Geol. Sussex, 1850, p. 217, woodcuts 8-11, 16, and ibid., ed. 2, 1878, p. 258, same figs.; J. Morris, Cat. Brit. Foss., 1854, p. 351. [Vertebræ; Brit. Mus.]

LOC. [? London Clay; Sheppey.] Bracklesham Beds. [Vertebræ ; Brit. Mus.]

sp., R. Owen, Rept. London Clay, pt. iii (Pal. Soc., 1850), p. 59, pl. xiv, f. 5, 6, 10-12, 32 .

LOC. Bracklesham Beds. [Vertebra ; Brit. Mus.]

PALAEPTTHON, Rochebrune. v. Paleryx.

PAIFEOSAURUS, H. Riley and S. Stutchbury (non Geoffroy, 1833), Trans. Geol. Soc. [2] vol. v, 1836 (1840), p. 352 ; R. Owen, Rep. Brit. Assoc., I84I (1842), p. I54, and Palæontology, ed. 2, 1861, p. 276.

cylindrodon, H. Riley and S. Stutchbury, Trans. Geol. Soc. [2] vol. v, 1836 (1840), p. 352 , pl. xxix, f. 4 ; J. Morris, Cat. Brit. Foss., 1854, p. 351 ; T. H. Huxley, Quart. Journ. Geol. Soc., vol. xxvi, I870, p. 43 , pl. iii, f. 3 .

LOC. U. Trias (Dolomitic Conglomerate); Redland, Bristol. [Tooth; Bristol Mus.]

platyodon, Riley and Stutchbury. $\%$. Thecodontosaurus platyodon.

? stricklandi, J. W. Davis, Quart. Journ. Gcol. Soc., vol. xxxvii, I 881 , p. 420 , pl. xxii, f. 6.

'Tooth allied to Palcosaurus,' H. E. Strickland, Proc. Geol. Soc., vol. iii (1842), p. 587.

LOC. Rhætic; Combe Hill, near Cheltenham. [Tooth; Geol. Soc. London. 
PAIERYX, R. Owen, Rept. London Clay, pt. iii (Pal. Soc., I850), p. 67 ; R. Lydekker, Geol. Mag. [3] vol. v, I888, p. II2.

Palæopython, A. T. Rochebrune, Nouv. Arch. Muséum [2] vol. iii, I88o, p. 276.

depressus, R. Owen, Rept. London Clay, pt. iii (Pal. Soc., I850), p. 67 , pl. xiii, f. 37,38 ; J. Morris, Cat. Brit. Foss., 1854, p. 35 I; R. Lydekker, Geol. Mag. [3] vol. v, I888, p. 112, and Cat. Foss. Rept. B. M., pt. i, 1888, p. 255.

LOC. U. Eocene ; Hordwell. [Vertebræ; Brit. Mus.]

rhombifer, R. Owen, Rept. London Clay, pt. iii (Pal. Soc., 1850), p. 67 , pl. xiii, f. 29-32 ; J. Morris, Cat. Brit. Foss., 1854, p. 35 I ; R. Lydekker, Geol. Mag. [3] vol. v, I888, p. 112, and Cat. Foss. Rept. B. M., pt. i, 1888, p. 254, woodcut 55.

LOC. U. Eocene ; Hordwell. [Vertebra; Brit. Mus.]

T.

PARAMECOSTINUS, T. Hawkins, Great Sea Dragons, I 840. $=$ Ichthyosaurus ( $v$. I. intermedius).

PATricosaurus, H. G. Seeley, Quart. Journ. Geol. Soc., vol. xliii, 1887 , p. 2 I6.

merocratus, H. G. Seeley, ibid., p. 2 I6, pl. xii, f. 9-12.

LOC. Cambridge Greensand. [Head of femur; Woodwardian Mus.]

PzuAgosaurus, H. G. Bronn, in Bronn and Kaup, Gavialartige Reptilien, I841, p. 28.

Engyommasaurus, J. J. Kaup, Neues Jahrb., 1835, p. 623 (imperfectly defined).

brongniarti (Kaup), E. E. Deslongchamps, Le Jura Normand, pt. iv, 1877, pp. 7, II, pl. i ; A. S. Woodivard, Geol. Mag. [3] vol. ii, 1885, p. 499 ; R. Lydekker, Cat. Foss. Rept. B. M., pt. i, 1888, p. 107.

Engyommasaurus brongniarti, J. J. Kaup, Neues Jahrb., 1835, p. 623 .

T. (Engyommasaurus.)

Mystriosaurus brongniarti, H. G. Bronn, in Bronn and Kaup, Gavialartige Reptilien, I84I, p. 35.

Tclcosaurus chapmanni, R. Owen (errore), Brit. Foss. Rept., I884, pl. xv (Crocodilia).

? Teleosaurus eucephalus, H. G. Seeley, Quart. Journ. Geol. Soc., vol. xxxvi, I880, p. 627, pl. xxiv. [Imperfect cranium; Woodwardian Mus.]

LOC. U. Lias; Whitby. [Skeleton; Brit. Mus.]

? typus, H. G. Bronn, Gavialartige Reptilien, 184I, p. 28. Doubtfully recorded by $\mathrm{R}$. Lydekker, Cat. Foss. Rept. B. M., pt. i, I888, p. 106.

LOC. U. Lias; Whitby. [Imperfect cranium; Brit. Mus.]

$\boldsymbol{P E L I A S , ~ M e r r e m . ~ v . ~ V i p e r a . ~}$

berus, Linnæus. $v$. Vipera berus. 
PexoBAtochexys, H. G. Seeley, Quart. Journ. Geol. Soc, vol. xxxi, 1875, p. 234.

blakei, H. G. Seeley, ibid., p. 234, pl. xiii (P. blakii); J. C. Mansel-Pleydell, Proc. Dorset. Nat. Hist. Field Club, vol. ix, I 888 , p. 6 (P. blakii) ; R. Lydekker, Cat. Foss. Rept. B. M., pt. iii, I889, p. 153, woodcut 37.

LOC. Kim. Clay; Weymouth. [Portions of carapace ; Brit. Mus.]

PשIONEUSTES, R. Lydekker, Quart. Journ. Geol. Soc., vol. xlv, 1889, p. 49, and Cat. Foss. Rept. B. M., pt. ii, 1889, p. 151, and Abstracts Proc. Geol. Soc., Nov. 6, 1889.

øqualis (Phillips), R. Lydekker, Cat. Foss. Rept. B. M., pt. ii, 1889 , p. 152 , woodcut 50.

Plesiosaurus aqualis, J. Phillips, Geol. Oxford, 1871, p. 365, woodcut clxiv.

Plesiosaurus sterrodeirus, H. G. Seeley, Index to Ares, etc., Woodwardian Mus., I869, pp. xx, 98 (name only). [Basioccipital and vertebræ; Woodwardian Mus.]

LOC. Kim. Clay; Broughton-Gifford, Ely, and Swindon. [Femur ; Oxford Mus.]

evansi, (Seeley), Lydekker. v. Pliosaurus evansi.

philarchus (Seeley), R. Lydekker, Quart. Journ. Geol. Soc., vol. xlv, 1889, p. 48 , woodcuts $4-9$, pl. ii, and Cat. Foss. Rept. B. M., pt. ii, 1889, p. 154, woodcuts $47 \mathrm{~A}-49,51-55$.

Plesiosaurus philarchus, H. G. Seeley, Index to Aves, etc., Woodwardian Mus., I 869, pp. xxi, I 39 ; R. Lydekker, Proc. Geol. Soc., I888, p. 89.

Thaumatosaums philarchus, R. Lydekker, Geol. Mag. [3] vol. v, 1888 , p. 352 .

LOC. Oxford Clay ; Christian Malford, Kempston, Peterborough, and Whittlesea. [Imperfect skeleton; Woodwardian Mus.]

$\mathbf{T .}$

PyLorosa URUS, C. A. Mantell, Phil. Trans., 1850, p. 379. $[?=$ Ornithopsis. $]$

armatus (Gervais), Lydekker. $\%$ Ornithopsis hulkei.

becklesii, Mantell. $\because$. Morosaurus brevis.

congbearei, G. A. Mantell, ibid., p. 386, pl. xxi (non pls. xxiixxvi) ; R. Owen, Rept. Weald. and Purb. Form., Suppl. ii (Pal. Soc., 1859), p. 38, pl. xii (non pl. xi); [-ri] J. Morris, Cat. Brit. Foss., I854, p. 351 ; R. Lydekker, Cat. Foss. Rept. B. M., pt. i, I888, p. I45. [See also Morosuturus brezis.]

'Humerus of Ornithopsis,' H.G. Seeley, Quart. Journ. Geol. Soc., vol.xxxviii, 1882, p. 371 , and Geol. Mag. [3] vol. iv, 1887, p. 479.

'Humerus of Cetiosaurus,' H. G. Seeley, Geol. Mag. [3] vol. iv, I 887, p. 84 .

LOC. Wealden; Tilgate Forest. [Humerus; Brit. Mus.] T.

Peltocephalus lavis (Bell), Rütimeyer. \% Podocnemis bowerbanki. 
PrTrosucrus, R. Owen, Rept. Weald. and Purb. Form., Suppl. viii (Pal. Soc., I878), p. 10.

Iæovidens, R. Owen, ibid., p. Io, pl. vi ; R. Lydekker, Cat. Foss. Rept. B. M., pt. i, I888, p. 89.

LOC. M. Purbeck; Swanage. [Skull, wanting extremity of snout : Brit. Mus.]

$\mathbf{T .}$

FIOTIDOSA שRUS, H. von Meyer, Neues Jahrb., I84I, p. 443.

Macrorhynchus, W. Dunker, Program. höch-Gewerbschule in Cassel, I844, p. 44 .

meyeri (Dunker), R. Lydekker, Geol. Mag. [3] vol. iv, I887, p. 3 II, and Cat. Foss. Rept. B. M., pt. i, I888, p. 88.

Macrorhynchus meyeri, W. Dunker, Program. höch-Gewerbschule in Cassel, 1844 , p. $44 ; \mathrm{H}$. von Meyer, in W. Dunker, Monogr. norddeutsch. Wealdenbildungen, I846, p. 74, pl. xx (non f. C).

LOC. Wealden; Brook, I. of Wight. [Occiput, vertebra, and scutes; Brit. Mus.]

PIACOSAURUS, P. Gervais, Zool. et Pal. Franç., ed. i, I848-52, p. 260 .

? margariticeps (Gervais), R. Lydekker, Geol. Mag. [3] vol. v, I888, p. III, and Cat. Foss. Rept. B. M., pt. i, 1888, p. 279, woodcut 62.

l'uranus? margariticeps, P. Gervais, Zool. et Pal. Générales, sér. 2, 1876, p. 6o, woodcut.

LOC. U. Eocene; Hordwell. [Vertebra; Brit. Mus.]

PLASTREMIS, Owen. $\%$ Hylenchelys.

latı, Owen. \%. Hyleochelys lata.

PIATECAREบฐ, E. D. Cope, Proc. Boston Soc. Nat. Hist., vol. xii, 1869, p. 264.

? sp., R. Lydekker, Cat. Foss. Rept. B. M., pt. i, I888, p. 27 I.

Loc. Chalk; Sussex. [Dental crown; Brit. Mus., no. 39424.]

PIATEMES, A. Wagler, Syst. Amphib., I830, p. 135.

bowerbanki, Owen. v. Podocnemis bowerbanki and Pseudotrionyx delheidi.

bullocki, Owen. \%. Pleurosternum bullocki.

concinna (Owen), Maack. \%. Pleurosternum bullocki.

dixoni, Owen. v. Hylxochelys belli.

emargiuctu (Owen), Maack. $\%$. Hylæochelys emarginata.

latiscutata (Owen), Maack. \%. Hylæochelys latiscutata.

mantelli, Owen. $v$. Hylæochelys belli.

ovat (Owen), Maack. \%. Pleurosternum bullocki. 
PIATrmys (continued)-

sp., R. Owen, Rept. Weald. and Purb. Form., pt. i (Pal. Soc., 1853), p. II, pl. ix, f. 2 ; J. Morris, Cat. Brit. Foss., 1854, p. 352. Genus non det. (R. Lydekker, Cat. Foss. Rept. B. M., pt. iii, 1889, p. 202).

Loc. Wealden; Tilgate Forest. [Right hypoplastron; Brit. Mus., no. 2336.]

PIATyCrrezys, A. Wagner, Abh. k. bay. Akad. Wiss., vol. vii, pt. i, 1853 , p. 242.

? anglica, R. Lydekker, Cat. Foss. Rept. B. M., pt. iii, I889, p. 217 , woodcut 49.

LOC. M. Purbeck; Durdlestone Bay. [Portion of carapace; Brit. Mus.]

PLEIOSAURUS, Owen. \%. Pliosaurus.

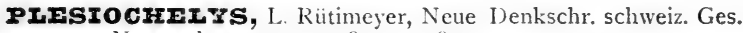
Nat., vol. xxv, art. 2, 1873, p. 28.

brodiei, R. Lydekker, Quart. Journ. Geol. Soc., vol. xlv, I889, p. 238 , woodcuts 4,5 , and Cat. Foss. Rept. B. M., pt. iii, I 889 , p. 20 I.

Plesiochelys vuldensis, R. Lydekker and G. A. Boulenger (pars, errore), Geol. Mag. [3] vol. iv, 1887, p. 272.

LOC. Wealden ; Atherfield, I. of Wight, and Hastings. [Shell ; P. B. Brodie Coll.]

Iatiscutata (Owen), Lydekker and Boulenger. \%. Hylæochelys latiscutata.

valdensis, R. Lydekker, Quart. Journ. Geol. Soc., vol. xlv, 1889, p. 239, and Cat. Foss. Rept. B. M., pt. iii, 1889, p. 199.

I'lesiochelys sancta-verena (L. Rütimeyer), G. A. Boulenger and K. Lydekker, Geol. Mag. [3] vol. iv, I887, p. 272 (errore).

LOC. Wealden ; I. of Wight. [Shell ; Brit. Mus.]

? sp., R. Lydekker, Cat. Foss. Rept. B. MI., pt. iii, I889, p. 198.

LOC. Kim. Clay; Weymouth. [Neurals and head of humerus;

Brit. Mus., nos. 43570, $44178 x-y-z$.]

sp., R. Lydekker, ibid., p. 199.

Loc. Purbeck; Swanage. [Hyo-, hypo-, and xiphi-plastra;

Brit. Mus., nos. R. 1615, $21351 x$, and $23407 x$.]

sp., R. Lydekker, ibid., p. 202.

LOC. Wealden; Cuckfield. [Costal ; Brit. Mus., no. 37715.]

? sp., R. Lydekker, ibid., p. 202.

Chelone? costata, R. Owen (pars), Rept. Weald. and Purb.

Form., pt. i (Pal. Soc., 1853), p. II, pl. ix, f. 4.

LoC. Wealden; Cuckfield. [Hypoplastron; Brit. Mus., no. 2298.] 
PIFSIOSAURUS, W. D. Conybeare, Trans. Geol. Soc., vol. v (I82I), p. 560; T. H. Huxley, Quart. Journ. Geol. Soc, vol. xiv, 1858, p. 281 ; L. Barrett, Rep. Brit. Assoc., I858 (1859), Sect., p. 78, and Ann. Mag. Nat. Hist. [3] vol. ii, 1858, p. 36I, pl. xiii (atlas and axis); R. Owen, Kep. Brit. Assoc., I 839 (I 840 ), p. 49, and Ann. Mag. Nat. Hist., vol. xx, I847, p. 217 (atlas and axis), and Palæontology, ed. 2, 186r, p. 243, and Quart. Journ. Geol. Soc., vol. xxxix, 1883, p. I34; H. G. Seeley, Rep. Brit. Assoc., I887 (1888), p. 697, and Geol. Mag. [3] vol. iv, 1887, p. 562 (development of young.)

aequalis, Phillips. $v$. Peloneustes æqualis.

affinis, Owen. $v$. Cimoliosaurus trochanterius.

arcuatus, Owen. $v$. Thaumatosaurus arcuatus.

bernardi, Owen. $v$. Cimoliosaurus bernardi.

? bitractensis, H. E. Sauvage, Ann. Sci. Géol., vol. xiv, art. 3, I 883 , p. 20, pl. viii, f. 5, pl. ix, f. I, 2 ; R. Lydekker, Cat. Foss. Rept. B. MI., pt. ii, I889, p. 283.

Loc. Rhætic ; Aust Cliff. [Vertebra; Brit. Mus.]

brachistospondylus, Hulke. ${ }^{\prime}$. Cimoliosaurus brachistospondylus.

brachycephalus, Owen. v. Plesiosaurus macrocephalus.

brachyspondylus, Owen. $v$. Pliosaurus brachyspondylus.

brachyspondylus, Phillips. v. Cimoliosaurus trochanterius.

carinatus, Cuvier. $v$. Thaumatosaurus carinatus.

carinatus, Phillips. $v$. Cimoliosaurus portlandicus.

cliduchus, Seeley. $v$. Plesiosaurus dolichodeirus.

coelospondylus, R. Owen, Rept. Liassic Form., pt. i (Pal. Soc., 1865), p. 12 (incompletely defined); J. F. Blake, in Tate and Blake, Yorkshire Lias, 1876, p. 252. [? P. longirostris.]

LoC. U. Lias ; Whitby. [Vertebra ; Whitby Mus.]

compressus, R. Owen, Cat. Foss. Rept. Mus. Roy. Coll. Surgeons, I854, p. 6I.

LOC. Lias; Somerset. [Dorsalvertebra; Mus. R. Coll. Surgeons.] constrictus, Owen. v. Cimoliosaurus constrictus.

conybeare1, [-ri] W. J. Sollas, Quart, Journ. Geol. Soc., vol. xxxvii, 1881, p. 440, pls. xxiii, xxiv ; [-ri] R. Lydekker, Cat. Foss. Rept. B. M., pt. ii, 1889, p. 269.

Plesiosaurus laticeps (R. Owen, MS.), IV. Davies, Geol. Mag., vol. iv, 1867, p. 144 (undefined). [Skeleton; Brit. Mus.]

Plesiosaurus rostratus, R. Owen (pars), Rept. Liassic Form., pt. i (Pal. Soc., 1865), p. 28, pl. xiii. [Skull ; Brit. Mus.]

LOC. L. Lias ; Lyme Regis. [Imperfect skeleton; Bristol Mus.]

costatus, R. Owen, Rep. Brit. Assoc., I839 (1840), p. 80; J. Morris, Cat. Brit. Foss., 1854, p. 352 ; R. Lydekker, Cat. Foss. Rept. B. M., pt. ii, 1889, p. 282.

? Plesiosaurus dibothrius, R. Owen, Cat. Foss. Rept. Mus. Roy.

Coll. Surgeons, 1854, p. 63. [Vertebra; Mus. R. Coll. Surgeons.]

LOC. Rhætic ; Aust Cliff. [Cervical vertebra; Bristol Mus.] 
PIISTOSAURUS (continued)-

cramptoni, Carte and Baily. v. Thaumatosaurus cramptoni.

cycnodeirus, H. G. Seeley, Index to Aves, etc., Woodwardian Mus., I869, pp. xvii, 4I (name only).

LOC. Cambridge Greensand. [Vertebre ; Woodwardian Mus.] dredicomus, Owen. $v$. Cimoliosaurus truncatus.

depressicostatus, R. Owen, Cat. Foss. Rept. Mus. Roy. Coll. Surgeons, 1854 , p. 60.

LOC. Unknown. [Vertebre; Mus. R. Coll. Surgeons.]

dibothrius, Owen. $\%$. Plesiosaurus costatus.

dolichodeirus, W. D. Conybeare, Trans. Geol. Soc. [2] vol. i (1824), p. 389, and Phil. Mag., vol. Ixvii, 1826, p. 272, pl. iii ; W. Buckland, Geol. and Mineral., ed. 2, 1837, vol. ii, p. 29, pl. xvi, f. 2, pl. xviii, f. 2 ; J. Morris, Cat. Brit. Foss., 1854, p. 352 ; R. Owen, Rept. Liassic Form., pt. i (Pal. Soc., 1865), p. I, pls. i-iv; H. G. Seeley, Ann. Mag. Nat. Hist. [3] vol. xv, 1865, p. 50 ; J. W. Hulke (pars), Proc. Geol. Soc., vol. xxxix, 1883, pp. 46, 59, woodcuts 2, 15; R. Lydekker, Cat. Foss. Rept. B. M., pt. ii, I889, p. 255. [See also Plesiosaurus hawkinsi.]

Plesiosaurus cliduchus, H. G. Seeley, Ann. Mag. Nat. Hist. [3] vol. xvi, I865, p. 356, pl. xv ; J. W. Hulke, Proc. Geol. Soc., vol. xxxix, 1883, p. 58, woodcut 14. [Imperfect skeleton; Woodwardian Mus.]

Plesiosuurus homei, J. E. Gray, Syn. Rept., I831, p. 66 (homii).

? 'Large animal,' W. Stukely, Phil. Trans., I719, p. 963, pl. i. [Hinder portion of skeleton ; Brit. Mus.]

LOC. L. Lias ; Charmouth and Lyme Regis, Dorset ; Bath and Watchet, Somerset ; and Elston, near Newark. [Skeleton ; Brit. Mus.]

T.

dubius, Blake. v. Eretmosaurus macropterus.

eleutheraxon, H. G. Seeley, Ann. Mag. Nat. Hist. [3] vol. xvi, 1865, p. 353, pl. xiv, and [-ion] Index to Aves, etc., Woodwardian Mus., 1869, pp. xxi, 137; R. Lydekker, Cat. Foss. Rept. B. M., pt. ii, 1889, p. 259.

Plesiosaurus, L. Barrett, Ann. Mag. Nat. Hist. [3] vol. ii, 18;8, p. 36 I, pl. xiii.

LOC. L. Lias; Street. [Imperfect skeleton, wanting head; Woodwardian Mus.]

? ellpsospondylus, $J$. Phillips (ex Owen, MSS.), Geol. Oxford, 1871 , p. 372 ; R. Lydekker, Cat. Foss. Rept. B. MI., pt. ii, 1889 , p. 172.

Loc. Kim. Clay; Shotover. [Anterior vertebre; Oxford Mus.] erraticus, Phillips. $v$. Cimoliosaurus erraticus.

etheridgei, Huxley. $\%$. Plesiosaurus hawkinsi.

eurymerus, Phillips. v. Cimoliosaurus eurymerus.

euryspondylus, H. G. Seeley, Index to Aves, etc., Woodwardian Mus., 1869, pp. xviii, 4I (name only).

Loc. Cambridge Greensand. [Vertebre ; Woodwardian Mus.] 
PIESIOSAURUS (continued)-

gavdnevi (Seeley), Whidborne. v. Cimoliosaurus constrictus.

giganteus, Conybeare. $v$. Pliosaurus brachydeirus.

grandis, R. Owen, Rep. Brit. Assoc., I839 (1840), p. 83. A name applied to a propodial bone from the Kimeridge Clay, subsequently (ibid., $184 \mathrm{I}$ (1842), p. 64), assigned to Pliosaurus. $v$. Pliosaurus macromerus.

hawkinsi, [-ii] R. Owen, Rep. Brit. Assoc., 1839(1840), p. 57, and Trans. Geol. Soc. [2] vol. v (1838), p. 515, pl. xliii, pl. xliv, f. $4, \mathrm{pl}$. xlv, and Odontography (I84I), p. $28 \mathrm{I}, \mathrm{pl}$. lxxiv, and Rept. Liassic Form., pt. i (Pal. Soc., I865), p. II, pl. xiv, f. 6, pl. xvi ; G. A. Mantell, Petrifactions, 1851, pp. 340, 347, woodcut 73 ; J. Morris, Cat. Brit. Foss., 1854, p. 352 ; H. G. Seeley, Ann. Mag. Nat. Hist. [3] vol. xv, 1865, pp. 50, 233 ; R. Lydekker, Cat. Foss. Rept. B. M., pt. ii, 1889, p. 260, woodcuts $77,79,80$.

Plesiosaurus etheridgei, [-ii] T. H. Huxley, Quart. Journ. Geol. Soc., vol. xiv, I858, p. $28 \mathrm{r}$; [-ii] H. G. Seeley, Ann. Mag. Nat. Hist. [3] vol. xv, 1865, pp. 50, 233. [Skeleton; Mus. Pract. Geol.]

Plesiosaurus dolichodeirus, W. Buckland (crrore), Geol. and Mineral., 1837, pl. xvii, pl. xviii, f. 3 ; J. W. Hulke, Proc. Geol. Soc., vol. xxxix, I883, p. 59, woodcut 15.

? Plesiosaurus hexatarsostinus, T. Hawkins, Great Sea Dragons, 1840, pl. xxriii. [Skeleton; Brit. Mus.]

? Plesiosaumus pentatarsostinus, T. Hawkins, ibid., pl. xxvii. [Skeleton; Brit. Mus.]

Plesiosaurus triatarsostinus, T. Hawkins, ibid., pls. xxiv, $\mathbf{x x v}$ (P.tritarsostinus), and Mem. Ichthyosauri, $1834, \mathrm{pl}$. xxiv, xxv. [Skeleton; Brit. Mus.]

LOC. L. Lias; Bennington, Leicestershire ; Street and Walton. [Skeleton; Brit. Mus.]

hexagonalis, Phillips. \%. Cimoliosaurus plicatus.

hexatarsostiuus, Hawkins. $v$. Plesiosaurus hawkinsi.

homalospondylus, R. Owen, Rept. Liassic Form., pt. i (Pal. Soc., 1865), p. I2, pls. v-viii ; H. G. Seeley, Ann. Mag. Nat. Hist. [3] vol. xv, 1865, pp. 52, 232 ; J. F. Blake, in Tate and Blake, Yorkshire Lias, 1876 , p. 246 ; R. Lydekker, Cat. Foss. Rept. B. M., pt. ii, 1889, p. 253 , woodcut 78.

LOC. U. Lias ; Whitby. [Skeleton; Brit. Mus.]

homei, Gray. v. Plesiosaurus dolichodeirus.

ichthyospondylus, Seeley. v. Cimoliosaurus bernardi.

infiraplanus, Phillips. $\%$. Cimoliosaurus plicatus.

laticeps, Owen. $v$. Plesiosaurus conybearei.

latispinus, Owen. v. Cimoliosaurus latispinus.

leedsi (Seeley), Whidborne. $v$. Cimoliosaurus plicatus.

leptopleuron, R. Owen, Cat. Foss. Rept. Mus. Roy. Coll. Surgeons, 1854, p. 60 .

LOC. Unknown. [Cervical vertebræ; Mus. R. Coll. Surgeons.] 


\section{PIESIOSAURUS ('ontinued) -}

levis, R. Owen, Cat. Foss. Rept. Mus. Roy. Coll. Surgeons, 1854, p. 62. Immature Pliosaurus, according to R. Lydekker, Cat. Foss. Rept. B. M., pt. ii, 1889, p. 139.

LOC. Kim. Clay. [Vertebra; Mus. R. Coll. Surgeons.]

limnophilus, Koken. v. Cimoliosaurus limnophilus.

longirostris, J. F. Blake, in Tate and Blake, Yorkshire Lias, I876, p. 250 , pl. i, f. 6, pl. iii, f. 2 ; R. Lydekker, Cat. Foss. Rept. B. M., pt. ii, I889, p. 27 I. '[? P. colospondylus.]

LOC. Lias; Whitby. [Head.]

macrocephalus, W. Buckland (ex Conybeare, MS.), Geol. and Mineral., ed. 2, 1837, vol. ii, p. 30, pl. xix, f. I ; R. Owen, Rep. Brit. Assoc., 1839 (1840), p. 62, and Trans. Geol. Soc. [2] vol. v (1838), p. 515 , pls. xliii, xlv ; J. Morris, Cat. Brit. Foss., 1854, p. 352 ; S. Haughton, Man. Geology, 1865, p. 274, woodcut 29; H. G. Seeley, Ann. Mag. Nat. Hist. [3] vol. xv, I 865 , p. 50; R. Lydekker, Cat. Foss. Rept. B. M., pt. ii, I889, p. 266 ; M. Browne, Vert. Animals Leicestershire, I889, p. 181. [See also Thaumatosaurus propinquus.]

Plesiosaumus brachycephalus, R. Owen, Rep. Brit. Assoc., I 839 (IS40), p. 69, and Brit. Foss. Rept., vol. iii (1884), p. 178, pl. xv (Enaliosauria); J. Morris, Cat. Brit. Foss., I 854 , p. 352 ; H. G. Seeley, Ann. Mag. Nat. Hist. [3] vol. xv, I 865 , p. 50 ; W. J. Sollas, Quart. Journ. Geol. Soc., vol. xxxvii, 1881, p. 479, pl. xxiv, f. 2. [Skeleton; Bristol Mus.]

LOC. L. Lias; Bitton, Gloucester, Lyme Regis, Street, Weston (near Bath), and Whitby. [Imperfect skeleton; Brit. Mus.]

macrodeirus, Seeley. $\%$ Cimoliosaurus trochanterius.

macromus, R. Owen, Rep. Brit. Asscc., 1839 (I840), p. 72 ; J. Morris, Cat. Brit. Foss., 1854, p. $35^{2}$.

LOC. Lias ; Lyme Regis. [Imperfect skeleton; Oxford Mus.] macroptemis, Seeley. v. Eretmosaurus macropterus.

manseli, Hulke. v. Cimoliosaurus trochanterius.

megacephalus, Stutchbury. v. Thaumatosaurus megacephalus. megadeirus, Seeley. $\%$. Cimoliosaurus trochanterius.

megapleuron, R. Owen, Cat. Foss. Rept. Mus. Roy. Coll. Surgeons, 1854 , p. 60.

LOC. Unknown. [Cervical vertebræ; Mus. R. Coll. Surgeons.] microdeirus, H. G. Seeley, Index to Aves, etc., Woodwardian Mus., 1869, pp. xviii, 40 (name only).

LOC. Cambridge Greensand. [Portions of skeleton; Woodwardian Mus.]

neocomiensis, Owen. v. Cimoliosaurus cantabrigiensis.

ophiodeirus, H. G. Seeley, Index to Aves, etc., Woodwardian Mus., I869, pp. xviii, 44 (name only).

LOC. Cambridge Greensand. [Portion of skeleton; Woodwardian Mus.]

oxoniensis, Phillips. $\%$. Cimoliosaurus oxoniensis.

pachyomus, Owen. $\approx$. Cimoliosaurus bernardi and C. planus. 
PIESIOSAURUS (continued)-

pectopleuron, R. Owen, Cat. Foss. Rept. Mus. Roy. Coll. Surgeons, I 854 , p. 62.

LOC. 'Oolitic Clay; Foxcombe Hill.' [Vertebræ; Mus. R. Coll. Surgeons.]

pentatarsostinus, Hawkins. $ข$. Plesiosaurus hawkinsi.

philarchus, Seeley. $\%$. Peloneustes philarchus.

phillipsi, Sauvage. $v$. Cimoliosaurus portlandicus.

plamus, Owen. $\%$ Cimoliosaurus planus.

platydeirus, H. G. Seeley, Index to Aves, etc., Woodwardian

Mus., 1869, pp. xviii, 4I (name only). [Non Owen, ข. Addenda.]

LOC. Cambridge Greensand. [Vertebre; Woodwardian Mus.] plicatus, Mansel-Pleydell. v. Cimoliosaurus richardsoni.

plicatus, Phillips. v. Cimoliosaurus plicatus and C. truncatus.

plicatus, Sauvage. $v$. Cimoliosaurus truncatus.

pacilospondylus, H. G. Seeley, Index to Aves, etc., Woodwardian Mus., 1869, pp. xviii, 48 (name only).

LOC. Cambridge Greensand. [Vertebre ; Woodwardian Mus.] priscus, J. Parkinson, Introduction, 1822, p. 294. [A general undefined term applied to all the Plesiosaurian remains described in Conybeare's original memoir on the genus.]

propinquus, Blake. v. Thaumatosaurus propinquus.

recentior, J. Parkinson, Introduction, 1822, p. 295. [An undefined specific name applied to miscellaneous remains from the Kimeridge Clay of Weymouth and Headington, near Oxford : ? = Pliosaurus brachy'spondylus.]

rostratus, R. Owen, Rept. Liassic Form., pt. i (Pal. Soc., I865), p. 20, pls. ix-xii (non pl. xiii); emend. R. Lydekker, Cat. Foss. Rept. B. M., pt. ii, 1889, p. 271, woodcut 81. [See also Plesiosaurus conybearei.]

LOC. L. Lias ; Lyme Regis. [Skeleton; Brit. Mus.]

rugosus, Owen. v. Eretmosaurus rugosus.

vugosus, Blake (non Owen). $v$. Eretmosaurus macropterus.

simpsoni, Blake. $v$. Thaumatosaurus propinquus.

smithi, Owen. $v$. Cimoliosaurus smithi.

sterrodeirus, Seeley. v. Peloneustes æqualis.

subconcarus, R. Owen, Cat. Foss. Rept. Mus. Roy. Coll. Surgeons, 1854, p. 61 .

LOC. Lias; Somerset. [Vertebræ; Mus. Roy. Coll. Surgeons.] subdepressus, Owen. $\%$. Cimoliosaurus truncatus.

subplanus, R. Owen, ibid., p. 6I.

LOC. 'Oolitic Clay; Oxfordshire.' [Vertebræ; Mus. R. Coll.Surg.]

subtrigonus, R. Owen, Rep. Brit. Assoc., 1839 (1840), p. 77 ;

J. Morris, Cat. Brit. Foss., I854, p. 352.

LOC. L. Lias ; Weston. [Vertebræ ; Bristol Mus.]

tessarestarsostinus, T. Hawkins, Mem. Ichthyosauri, 1834, p. 40 (undefined). 
PIESIOSAURUS (continued) -

? trigonus, Cuvier, Ossem. Fossiles, ed. 2, vol. v, pt. ii, 1824, p. 486 ; R. Owen, Rep. Brit. Assoc., 1839 (1840), p. 78 ; J. Morris, Cat. Brit. Foss., 1854, p. 352.

LOC. ?Rhretic; Aust Cliff. [Vertebra.]

tritarsostimus, Hawkins. $\%$. Plesiosaurus hawkinsi.

trochanterius, Owen. v. Cimoliosaurus trochanterius.

truncatus, Owen. v. Cimoliosaurus truncatus.

validus, Phillips. v. Cimoliosaurus trochanterius.

winspitensis, Seeley. $v$. Cimoliosaurus portlandicus.

zetlandi, Blake. v. Thaumatosaurus zetlandicus.

zetlandicus, Phillips. v. Thaumatosaurus zetlandicus.

? sp., R. Owen, Rept. Cret. Form. (Pal. Soc., 1851), p. 66, pl. xx, f. 7,8 . [? Cimoliosaurus.]

LOC. L. Greensand ; Shanklin. [Vertebra.]

? sp., R. Owen, in F. Dixon, Geol. Sussex, 1850, p. 399, pl. xxxvii,

f. I 5 , and ed. 2,1878, p. 426 , same pl. and fig. [? Cimoliosaurus.]

LOC. U. Chalk; Houghton, Sussex. [Tooth.]

spp., J. F. Blake, in Tate and Blake, Yorkshire Lias, 1876, p. 252.

[Skeleton, ironstone of Eaton ; two coracoids, ironstone of Skinnington; elongated vertebra, Capricormus beds, Saltburn; dorsal vertebra, Jamesoni beds, Huntcliff.]

PLESTOSUCHUS, Owen. \%. Dacosaurus.

manseli (Hulke), Owen. $\%$. Dacosaurus maximus.

PIEURoccruUs, O. C. Marsh, Amer. Journ. Sci. [3] vol. xxxv, I888, p. 90.

valdensis, R. Lydekker, Geol. Mag. [3] vol. vi, I889, p. 325.

'Teeth of the Hylreosaur ?,' G. A. Mantell, Phil. Trans., 1 841 , p. I 44 , pl. vi, f. 9-1 I, and Petrifactions, 1851, p. 326, woodcut 70 , and Wonders of Geology, ed. 6, 1848, p. 438, woodcut I09; R. Owen, Rept. Weald. and Purb. Form., pt. iv (Pal. Soc., I858), p. 21, pl. viii, f. 6-9 (teeth), and Palæontology, ed. 2, I861, p. 293.

'Teeth of a peculiar form,' G. A. Mantell, Wonders of Geology, ed. 3, vol. i, 1839, p. 403, pl. iii, f. 4 .

LOC. Wealden; Sussex. [Teeth; Brit. Mus.]

PImUROSTrRivim, R. Owen, Rept. Weald. and Purb. Form., pt. i (Pal. Soc., I853), p. 2 (Pleurosternon); R. Lydekker, Quart. Journ. Geol. Soc., vol. xlv, 1889, p. 513.

Megasternon, [J. E. Gray] Syn. Contents Brit. Mus., ed. 44, 1842 , p. 108 (name only, preoccupied).

Digerrhum, E. D. Cope, Trans. Amer. Phil. Soc., vol. xiv (1870), p. 156.

bullock1 (Owen), L. Rütimeyer, Neue Denkschr. schweiz. Nat. Ges., vol. xxv, art. 2, 1873, p. 159; R. Lydekker and G. A. Boulenger, Geol. Mag. [3] vol. iv, 1887, p. 271 ; IV. Davies, Geol. Mag. [3] vol. iv, 1887, p. 380; R. Lydekker, Quart. Journ. Geol. Soc., vol. xlv, 1889, p. 514 , woodcuts 3, 4, and Cat. Foss. Rept. B. MI , pt. iii, I889, p. 206, woodcuts $45,46$. 
PIEUROSTERNUMr bullocki (continued)-

Megastemon kenigii, [J. E. Gray] Syn. Contents Brit. Mus., ed. 44, I 842, p. I08 (name only), and Suppl. Cat. Shield Rept., pt. i, I 870 , p. 77.

Platemys bullockii, R. Owen, Rep. Brit. Assoc., I841 (1842), p. I64, and Rept. London Clay, pt. i (Pal. Soc., 1849), p. 6o, pl. xxi ; J. Morris, Cat. Brit. Foss., I854, p. 351.

Digerrhum bullocki, E. D. Cope, Trans. Amer. Phil. Soc., vol. xiv (1870), p. 156

Pleurosternon concinnum, R. Owen, Rept. Weald. and Purb. Form., pt. i (Pal. Soc., I853), p. 3, pls. ii, iii ; J. Morris, Cat. Brit. Foss., I854, p. 352.

Platemys concinna, G. A. Maack, Palæontographica, vol. xviii (1869), p. 288.

Pleurostemon ovatum, R. Owen, Rept. Weald. and Purb. Form., pt. i (Pal. Soc., I853), p. 8, pl. vii ; J. Morris, Cat. Brit. Foss., I854, p. 352 ; J. C. Mansel-Pleydell, Proc. Dorset. Nat. Hist. Field Club, vol. vi, 1885 , p. 66, pl. iii. [Carapace ; Brit. Mus.]

Platemys ovata, G. A. Maack, Palæontographica, vol. xviii (1869), p. 294.

Pleurosternon emarginatum, R. Owen (pars), Rept. Weald. and Purb. Form., pt. i (Pal. Soc., 1853), p. 6, pls. v, vi, and Palæontology, ed. 2, 186I, p. 3I8, woodcut I09; J. Morris (pars), Cat. Brit. Foss., 1854, p. 352. [Imperfect shell ; Brit. Mus.]

Loc. Purbeck; Durdlestone Bay and Swanage. [Carapace and plastron ; Brit. Mus.]

concinmum, Owen. $v$. Pleurosternum bullocki.

emarginatum, Owen. $v$. Hylæochelys emarginata and Pleurosternum bullocki.

latiscutatum, Owen. $v$. Hylæochelys latiscutata.

ovatum, Owen. v. Pleurosternum bullocki.

oweni, H. G. Seeley, Index to Aves, etc., Woodwardian Mus., I869, pp. xix, 87.

Loc. Purbeck ; Swanage. [Carapace; Woodwardian Mus.]

portlandicum, R. Lydekker, Cat. Foss. Rept. B. M., pt. iii, I 889, p. 2 I 5, woodcut 47.

LOC. Portland Stone; Portland. [Plastron and marginals; Brit. Mus.]

sedgwicki, H. G. Seeley, Index to Aves, etc., Woodwardian Mus., I 869, pp. xix, 86.

Loc. Purbeck; Swanage. [Carapace ; Woodwardian Mus.]

typocardium, H. G. Seeley, ibid., pp. xix, 87.

LOC. Purbeck; Swanage. [Carapace; Woodwardian Mus.]

vansittarti, H. G. Seeley, ibid., pp. xix, 86.

LOC. Purbeck; Swanage. [Carapace; Woodwardian Mus.] 
PIIOSAURUS, R. Owen, Odontography (IS+1), p. 282, and Rep. Brit. Assoc., I 84 I (1842), p. 60, and Palieontology, ed. 2, I86I, p. 252, woodcut 94, and Quart. Journ. Geol. Soc., vol. xxxix, I 883, p. 135 ; J. W. Hulke, Proc. Geol. Soc., vol. xxxix, 1883 , p. 5 I ; R. Lydekker, Cat. Foss. Rept. B. M., pt. ii, I889, p. 120. Liopleurodon, H. E. Sauvage, Bull. Soc. Géol. France [3] vol. i, 1873, p. 377.

braohydeirus, R. Owen, Odontography (I $84 \mathrm{I}$ ), p. 283, and Rep. Brit. Assoc., I84 I (I842), p. 64, and Rept. Kim. Clay, pt. iii (Pal. Soc., 1869), p. 6; J. Morris, Cat. Brit. Foss., I854, p. 352 ; H. G. Seeley, Index to Aves, etc., IVoodwardian Mus., 1869, p. 104 ; J. Phillips, Geol. Oxford, I87I, p. 341, woodcuts cxxxiv-cxli, cxlvi, cxlvii, clx ; R. Lydekker, Cat. Foss. Rept. B. M., pt. ii, I889, p. I23, woodcuts 37, 38. [Referred to $P$. grandis by R. Owen, Rept. Kim. Clay, pt. iii (Pal. Soc., 1869), p. 3.]

Plesiosaurus giganteus, W. D. Conybeare, Trans. Geol. Soc. [2] vol. i (1824), p. 389 (incompletely defined).

LOC. Kim. Clay; Oxfordshire, Lincolnshire, Cambridgeshire, Wiltshire, and Dorsetshire. [Imperfect skeleton; Oxford Mus.]

\section{$\mathbf{T}$.}

brachyspondylus (Owen), H. G. Seeley, Index to Aves, ctc., Woodwardian Mus., I869, p. IO2.

Plesiosaurus brachyspondylus, R. Owen, Rep. Brit. Assoc., I 839 (1840), p. 78.

[Immature Pliosaurus, according to R. Lydekker, Cat. Foss. Rept. B. M., pt. ii, 1889, pp. 139, 140.$]$

Loc. Kim. Clay; Ely. [Vertebra.]

evansi, H. G. Seeley, Index to Aves, etc., Woodwardian Mus., I869, pp. xxi, I I6, and Quart. Journ. Geol. Soc., vol. xxxiii, 1877 , p. 716, woodcuts I-9, and Geol. Mag. [3] vol. iv, 1887, p. $47 \delta$; R. Lydekker, Cat. Foss. Rept. B. M., pt. ii, 1889, p. 128, woodcuts 36,39 . [Referred to Peloneustes by R. Lydekker, Abstr. Proc. Geol. Soc., Nov. 6th, 1889.]

Loc. Oxford Clay; St. Neots and Eynsbury, Huntingdonshire. [Atlas and axis; Woodwardian Mus.]

ferox (Sauvage), R. Lydekker, Cat. Foss. Rept. B. M., pt. ii, I889, p. 145.

Liopleurodon ferox, H. E. Sauvage, Bull. Soc. Géol. France [3] vol. i, 1873, p. 378.

T. (Liopleurodon.)

Polyptychodon ferox, H. E. Sauvage, ibid. [3] vol. viii, 1880, p. 544.

? Pliosaumus pachydeirus, H. G. Seeley, Index to Aves, etc., Woodwardian Mus., 1869, pp. xxi, 118 (name only). [Cervical vertebre; Woodwardian Mus.]

LOC. Oxford Clay; Christian Malford, Wiltshire; ?Great Gransden, Cambridge; Whittlesea and Fletton, near Peterborough; and Weymouth. [Teeth and vertebra; Brit. Mus. and A. N. Leed's [oll.] 
PITOSAURUS (continued) -

gamma, J. Phillips (ex Owen), Geol. Oxford, I87r, p. 358, woodcuts clii-clv. Immature Pliosaurus (R. Lydekker, Cat. Foss. Rept. B. M., pt. ii, I889, p. I40).

LOC. Oxford Clay; Weymouth. Kim. Clay; Shotover. [Vertebra ; Oxford Mus.]

giganteus, Wagner. v. Pliosaurus macromerus.

grandis, Owen. $\%$. Pliosaurus macromerus.

grossouvrei (Sauvage), R. Lydekker, Cat. Foss. Rept. B. M., pt. ii, 1889 , p. 130.

Liopleurodon grossouvrei, H. E. Sauvage, Bull. Soc. Géol. France [3] vol. i, 1873 , p. 379 , pl. vii, f. 2.

Pliosaurus teretidens (Owen, MS.), A. S. Woodward, Geol. Mag. [3] vol. ii, 1885, p. 504, footnote 6 (name only). [Teeth; Brit. Mus.]

LOC. Coral Rag; Heddington, Wiltshire; Wheatley, near Oxford; and Malton, Yorkshire. [Teeth; Brit. Mus.]

macromerus, J. Phillips, Geol. Oxford, I87 I, p. 354, woodcuts cxlviii-cli, clix, clxvi ; R. Lydekker, Cat. Foss. Rept. B. M., pt. ii, I889, p. I 31 , woodcuts $41-43$.

Pliosaurus grandis, R. Owen, Rept. Kim. Clay, pt. i (Pal. Soc., I86 I ), p. I 5, pl. vii, and ibid., pt. ii (Pal. Soc., 1863), p. 27, pl. xii (identified with Plesiosaurus grandis, of Rep. Brit. Assoc., I 839 (1840), p. 83), and ibid., pt. iii (Pal. Soc., 1869), p. 3, pls. i, ii, and Brit. Foss. Rept., vol. iii (1884), p. 154, pl. xviii (Sauropterygia); J. Phillips, Geol. Oxford, 1871, p. 317, woodcuts cxxi, cxxii. [Tooth ; Brit. Mus.]

Pliosaurus giganteus, A. Wagner, Abh. k. bay. Akad. Wiss., vol. vi (1852), p. 696, pl. iv, f. 1-3.

Loc. Kim. Clay; Devizes, Ely, Foxhangers, Gillingham, Hartwell, Kimeridge, Motcombe, Oxford, Peterborough, Shotover, Swindon, Stanton (Drift), and Wootton Bassett. [Femur; Oxford Mus.]

microdeirus, H. G. Seeley, Index to Aves, etc., Woodwardian Mus., 1869, pp. xx, 76 (name only).

LOC. Neocomian Bone-bed; Wicken, Bedfordshire. [Cervical vertebra; Woodwardian Mus.]

nitidus, J. Phillips, Geol. Oxford, 1871, p. 360, woodcuts clviclviii. Immature Pliosaurus (R. Lydekker, Cat. Foss. Rept. B. M., pt. ii, 1889, p. 140). [? P. brachyspondylus.]

LOC. Kim. Clay; Shotover. [Vertebræ; Oxford Mus.]

pachydeivıs, Seeley. $v$. Pliosaurus ferox.

portlandicus, Owen. $v$. Cimoliosaurus portlandicus.

simplex, J. Phillips, Geol. Oxford, 1871, p. 366, woodcut clxv. Immature Pliosaurus (R. Lydekker, Cat. Foss. Rept. B. M., pt. ii, I889, p. 140).

LOC. Kin. Clay ; Shotover. [Femur; Oxford Mus.]

teretidens, Owen, MS. v. Pliosaurus grossouvrei. 
PITOSAURUS (continued) -

trochanterius, Owen. $v$. Cimoliosaurus trochanterius and Machimosaurus mosæ.

Bp., R. Owen, Rep. Brit. Assoc., I841 (1842), p. 64 ; J. Morris, Cat. Brit. Foss., 1854 , p. 352.

LOC. Speeton Clay; Speeton. [Humerus; York Mus.]

PODOCNIMIS, J. Wagler, Syst. Amphib., I 830 , p. I35.

bowerbank1 (Owen), E. D. Cope, Trans. Amer. Phil. Soc., vol. xiv (1870), p. 156 ; R. Lydekker, Cat. Foss. Rept. B. M., pt. iii, I889, p. 17 I.

Platemy's bowerbanki, [-ii] R. Owen, Rep. Brit. Assoc., I84I (1842), p. I63, and Rept. London Clay, pt. i (Pal. Soc., 1849), P. 66, pl. xxiii (non pl. xxix, f. I, 2); J. Morris, Cat. Brit. Foss., 1854, p. 351 .

Emys lavis, T. Bell, in R. Owen, Rept. London Clay, pt. i (Pal. Soc., I849), p. 7o, pl. xxii ; J. Morris, Cat. Brit. Foss., 1854, p. 349. [Imperfect shell ; Brit. Mus.]

Podocnemis lavis, E. D. Cope, Trans. Amer. Phil. Soc., vol. xiv (1870), p. 156.

Peltocephalus lavis, L. Rütimeyer, Neue Denkschr. schweiz. Ges. Nat., vol. xxv, 1873, p. 159.

LOC. London Clay; Sheppey. [Imperfect shell; olim J. S. Bowerbank Coll.]

? delabechei (Bell), R. Lydekker and G. A. Boulenger, Geol. Mag. [3] vol. iv, 1887, p. 274. [? Dacochelys.]

Emys delabechii, T. Bell, in R. Owen, Rept. London Clay, pt. i (Pal. Soc., 1849), p. 74, pl. xxviii ; J. Morris, Cat. Brit. Foss., 1854 , p. 349.

Emys conybearii, R. Owen, Rept. London Clay, pt. i, Suppl. i (Pal. Soc., 1858), p. 77, pls. xiii, xiv (numbered and quoted as xxviii A, xxviii B). [Imperfect shell ; Brit. Mus.]

Dacochelys delabechei (? Bell), R. Lydekker, Cat. Foss. Rept. B. M., pt. iii, 1889, p. 173 .

Loc. London Clay; Sheppey. [Imperfect shell ; Mus. Pract. Geol.] loevis, Cope. v. Podocnemis bowerbanki.

PERILOPLEURON, Deslongchamps. v. Aristosuchus and Megalosaurus.

buclsandi, Deslongchamps. v. Megalosaurus bucklandi and M. oweni.

pusillus, Owen. $v$. Aristosuchus pusillus.

POIACANTIYUS, R. Owen, in W. Fox, Rep. Brit. Assoc., 1865 (1866), Sect., p. 56 ; J. W. Hulke, Phil. Trans., 1881, p. 653.

foxi, J. W. Hulke, ibid., p. 653, pls. Ixx-lxxvi, and ibid., 1887, pt. B, p. I69, pls. viii, ix ; R. Lydekker, Cat. Foss. Rept. B. M., pt. i, 1888 , p. 189.

LOC. Wealden; Barnes Chine, I. of Wight. [Dermal armour and associated vertebræ; Brit. Mus.] 
POLYCOTYLUS, Cope. \%. Cimoliosaurus.

POLYOSTINUS, T. Hawkins, Great Sea Dragons, 1840. Ichthyosaurus ( $v$. I. chiropolyostinus).

POIYPTYCIODON, R. Owen, Rep. Brit. Assoc., I841 (I842), p. 156, and Palæontology, ed. 2, 1861, p. 255 ; H. G. Seeley, Quart. Journ. Geol. Soc., vol. xxxii, I 876, p. 433 (vertebra); W. Davies, Proc. Geol. Assoc., vol. x (1888), p. 277, footnote I. [See also Dinodocus. $\rceil$

continuus, R. Owen, Odontography (184I), descr. to pl. 1xxii, f. 3, and Rept. Cret. Form. (Pal. Soc., 1851), p. 47, pl. xiv, f. 4-6; J. Morris, Cat. Brit. Foss., 1854, p. 352 ; H. E. Sauvage, Mém. Soc. Géol. France [3] vol. ii, art. 4, I882, p. 28 ; R. Lydekker, Cat. Foss. Rept. B. M., pt. ii, I889, p. 179. [See also Dinodocus mackesoni.]

20C. L Greensand; Hythe and Maidstone. Cambridge Greensand. L. Chalk; Frome, Somersetshire, and Vanchurch. U. Chalk ; Kent. [Tooth.]

ferox, Sauvage. v. Pliosaurus ferox.

interruptus, R. Owen, Odontography (I $\delta_{4}$ I), descr. to pl. Ixxii, f. 4 , and in F. Dixon, Geol. Sussex, 1850, p. 378, pl. xxxvii, f. 16, 17, pl. xxxviii, f. 3 , and ibid., ed. 2, 1878, p. 421, pl. xxx [33], f. 22, 23, etc., and Rept. Cret. Form. (Pal. Soc., 1851), p. 55 , pl. x, f. 7-9, pl. xi, pl. xiv, f. I, 2, and ibid., Suppl. iii (Pal. Soc., I86I), p. 20, pl. iv, f. I-3, pls. v, vi, and Quart. Journ. Geol. Soc., vol. xvi, I86o, p. 262 ; J. Morris, Cat. Brit. Foss., 1854, p. 353 ; H. G. Seeley, Quart. Journ. Geol. Soc., vol. xxxii, 1876, p. 436; A. S. Woodward, Proc. Geol. Assoc., vol. x (1889), p. 277 ; R. Lydekker, Cat. Foss. Rept. B. M., pt. ii, I889, p. I73, woodcut 58.

Plectronites maximus corticeus, Luidius, Lithophyl. Brit., I760, p. 66, pl. xvi, f. 1318 .

Loc. U. Chalk; Burham, Charing, Cuxton, Dover, Halling, Maidstone, and Rochester, Kent ; Falmer, Glynde, Lewes, and Steyning, Sussex. L. Chalk; Burham, Charing, Dover and Folkestone, Kent; Houghton and Lewes, Sussex; Dorking, Surrey. U. Greensand; Warminster. Gault ; Folkestone. Cambridge Greensand. [Teeth.]

mackesoni, Owen. $v$. Dinodocus mackesoni.

PRIDDONTOGNATIUS, H. G. Seeley, Quart. Journ. Geol. Soc., vol. xxxi, 1875, p. 439.

phlllipsi, [-ii] H. G. Seeley, ibid., p. 439, pl. xx.

LOC. Cambridge Greensand. [Maxilla; Woodwardian Mus.]

PROIGUANA, Filhol. $v$ Iguana.

europacana, Filhol. v. Iguana europæa. 
PROTrmys, R. Owen, Rept. Cret. Form. (Pal. Soc, 1851), p. 15 , serrata, R. Owen, ibid., p. 15, pl. vii; J. Morris, Cat. Brit. Foss., 1854, p. 353.

LOC. L. Greensand; Maidstone. [Portions of shell; olim Capt. Guise Coll.]

PROTEOSAURUS, Home. $v$ Ichthyosaurus and I. intermedius.

PROTEROSAURUS, Meyer. v. Protorosaurus.

PROTOCrrex $\mathbf{Y S}$, R. Lydekker, Cat. Foss. Rept. B. M., pt. iii, 1889, p. 220.

stricklandi (Phillips), R. Lydekker, ibid., p. 220.

Testudo stricklandi, J. Phillips, Geol. Oxford, 1871, p. 182, woodcut xli, f. ro, II.

'Testudo-remains,' R. Owen, Rep. Brit. Assoc., 1841 (1842), p. 160 ; J. Morris, Cat. Brit. Foss., I 854 , p. 354.

? Chelys (?) blakii, S. J. Mackie and C. C. Blake, Geologist, 1863, p. 4I, woodcut. [Crushed right coracoid ; Brit. Mus.]

'Chelonian scutes,' C. C. Blake, ibid., p. 183.

LOC. Stonesfield Slate. [Epidermal scutes; Oxford Mus.] $\mathbf{x}$.

PROTOROSA URU:G, H. von Meyer, Isis (Oken), I830, p. 518 ; H. G. Seeley, Phil. Trans., 1887, pt. B, p. 204.

huxleyi, A. Hancock and R. Howse, Quart. Journ. Geol. Soc., vol. xxvi, I 870, p. 568, pl. xl (Proterosaurtis), and Nat. Hist. Trans. Northumb. and Durham, vol. iv, 1872, p. 237, pl. vii (Proterosaurus).

LOC. Marl-Slate; Midderidge. [Imperfect skeleton; Newcastle-upon-'Tyne Mus.]

speneri, H. von Meyer, Palreologica, 1832, p. 109; A. Hancock and R. Howse, Quart. Journ. Geol. Soc., vol. xxvi, 1870, p. 566, pl. xxxix (Proterosaurus), and Nat. Hist. Trans. Northumb. and Durham, vol. iv (1872), p. 233, pl. vii (Proterosaurus) ; H. G. Seeley, Phil. Trans., 1887, pt. B, p. 187, pls, xiv-xvi.

Loc. Marl-Slate; Midderidge, Durham. [Portions of skeleton; Mus. R. Coll. Surgeons. Vertebral column; Newcastleupon-Tyne Mus.]

T.

Protostega, E. D. Cope, Proc. Amer. Phil. Soc., vol. xii (1871), p. 175.

anglica, R. Lydekker, Cat. Foss. Rept. B. M., pt. iii, 1889, p. 229, woodcut 53 .

Protostega sp., R. Lydekker, Quart. Journ. Geol. Soc., vol. xlv, I 889, p. 236.

ı.oc. Cambiidge Greensand. Chalk; Lewes and Dorking. [Humerus ; Brit. Mus.] 
PSgPfodrana, $H$. von Meyer, Palæontographica, vol. vi (1858), p. 246.

anglicum, $H$. von Meyer, ibid., vol. xv (I867), p. 26I, pl. xl, f. I-6; R. Lydekker, Cat. Foss. Rept. B. M., pt. iii, I889, p. 23 I.

LOC. Rhætic ; Bristol. [Peripheral scute; Bath Mus.]

PSzPFO IrORUS, H. von Meyer, Neues Jahrb., I 847 , p. 579. rupeliensis, Dollo. v. Psephophorus sp.

вp., R. Lydekker, Cat. Foss. Rept. B. M., pt. iii, 1889, p. 224.

Psephophorus rupeliensis?, L. Dollo, Bull. Mus. R. Hist. Nat. Belge, vol.v (1888), p. 83.

'Leathery turtle,' A. S. Woodward, Proc. Geol. Assoc., vol. x (I887), p. 12.

LOC. Bracklesham Beds. [Fragment of carapace ; Brit. Mus.]

PszUDOTrionyX, L. Dollo, Bull. Mus. R. Hist. Nat. Belge, vol. iv (I886), p. 96.

delhe1di, L. Dollo, ibid., p. 96, pl. i ; R. Lydekker, Cat. Foss. Rept. B. M., pt. iii, I889, p. 145.

Pseudotrionyx delheidi (?), R. Lydekter and G. A. Boulenger, Geol. Mag. [3] vol. iv, 1887, p. 274.

? Platemys bowerbankii, R. Owen (errore), Rept. London Clay, pt. ii, Suppl. i (Pal. Soc., I850), p. I, pl. xxix, f. I, 2.

Pseudotriony.x (?), R. Lydekker, Quart. Journ. Geol. Soc., vol. xlv, I889, p. 242.

LOC. London Clay ; Sheppey. [Cranium, etc.; Brit. Mus.]

PTENODACTYLUS, H. G. Seeley, Index to Aves, etc., Woodwardian Mus., I869, p. xvi (name only). [Non Ptenodactylus, J. E. Gray, I 845.$]$

curieri (Buwerbank), Seeley, ibid. = Ornithocheirus cuvier.

fittoni (Owen), Seeley, ibid. = Ornithocheirus fittoni.

sedgwicki (Owen), Seeley, ibid. = Ornithocheirus sedgwicki.

woodwardi (Owen), Seeley, ibid. ?= Ornithocheirus ? simus.

sp). [Fragments of Pterosaurians from the Cambridge Greensand are also assigned to this genus by Seeley, ibid., under the following undefined specific names :-brachyrhinus, capito, colorhimus, crassidens, dentatus, en horliynchus, eurygnathus, macharorhynchus, macrorhinus, microdon, nasutus, oweni, oxyrhinus, platystomus, polyodon, scaphorhynchus, tenuirostris. This is a "list for the use of students of species" (Seeley, Geol. Mag. [2] vol. viii, 1881, p. 15).]

PTgrodacry ưs, Cuvier, Ann. Muséum, vol. xiii (1809), p. 424 (Pterodactyle).

aclaudi, Owen. \%. Rhamphocephalus depressirostris.

bucklandi, Meyer. $v$. Rhamphocephalus bucklandi. 
PTERODACTYIUS (continued) -

carteri, Seeley. $v$. Ornithocheirus carteri.

clifti, Morris. $\%$. Ornithocheirus clifti.

compressivostris, Owen. $v$. Ornithocheirus compressirostris.

conirostris, Owen. v. Ornithocheirus giganteus.

curtus, Owen. $\%$. Ornithocheirus curtus.

cuvieri, Bowerbank. v. Ornithocheirus cuvieri.

daviesi, Owen. $\%$. Ornithocheirus daviesi.

duncani, Owen. $v$. Rhamphocephalus bucklandi.

fittoni, Owen. $v$. Ornithocheirus fittoni.

giganteus, Bowerbank. v. Ornithocheirus giganteus.

hopkinsi, H. G. Seeley, Proc. Camb. Phil. Soc., vol. i (1864), p. 228 (name only).

LOC. Cambridge Greensand.

kiddii, Owen. \%. Rhamphocephalus bucklandi.

longivostris, Bowerbank. v. Ornithocheirus cuvieri.

macharoihynchus, H. G. Seeley, Proc. Camb. Phil. Soc, vol. i (1864), p. 228 (name only).

LOC. Cambridge Greensand.

macronyx, Buckland. $v$. Dimorphodon macronyx.

macrurus, Seeley. $v$. Doratorhynchus validum.

3 mansel1, [-ii] R. Owen, Rept. Mesoz. Form., pt. i (Pal. Soc., I 874), p. 8, pl. i, f. IO-12, 20, 2 I.

Genus non det., R. Lydekker, Cat. Foss. Rept. B. M., pt. i, 1888, p. 40.

Loc. Kim. Clay ; Weymouth. [Distal end of humerus; Brit. Mus.]

mardevi, Owen. $v$. Dimorphodon macronyx.

nobilis, Owen. $v$. Ornithocheirus nobilis.

oweni, H. G. Seeley, Proc. Camb. Phil. Soc., vol i (1864), p. 228 (name only).

LOC. Cambridge Greensand.

? pleydelli, [-ii] R. Owen, Rept. Mesoz. Form., pt. i (Pal. Soc., 1874), p. 9, pl. i, f. 1 5, 16, 22, 23.

Genus non det., R. Lydekker, Cat. Foss. Rept. B. M., pt. i, I 888 , p. $4 \mathrm{I}$.

LOC. Kim. Clay ; Weymouth. [Distal end of humerus; Brit. Mus.]

? sagittrostris, R. Owen, Rept. Mesoz. Form., pt. i (Pal. Soc., 1874), p. 3, pl. ii. [? Ornithocheirus.]

Loc. Hastings Sands; St. Leonards. [Mandib. rami ; S. H. Beckles Coll.]

sedguickii, Owen. $v$. Ornithocheirus sedgwicki.

simus, Owen. $v$. Ornithocheirus simus.

sylvestris, Owen. $\%$. Ornithocheirus clifti. 
PTIRODACTYIUS (continued)-

validus, Owen. $v$. Doratorhynchus validum.

ขvoodwardi, Owen. \%. Ornithocheirus simus.

sp., R. Owen, Rept. Cret. Form. (Pal. Soc., 1851), p. 102, pl. xxxii,

f. 4,5 ; J. Morris, Cat. Brit. Foss., 1854, p. 353. [? Genus.]

LOC. L. Chalk; Maidstone. [Wing-bones; Brit. Mus., nos. $41638,49005.1$

sp., R. Owen, Rept. Mesoz. Form., pt. i (Pal. Soc., 1874), p. 10, pl. i, f. 24-27.

Loc. Kim. Clay ; Weymouth. [Carpal bones.]

$\boldsymbol{P U P P I G E R U S , ~ C o p e . ~ v . ~ A r g i l l o c h e l y s ~ a n d ~ L y t o l o m a . ~}$

breviceps, Cope. $\%$ Argillochelys antiqua and Lytoloma planimentum.

convexus, Cope. v. Argillochelys convexa.

crassicostatus, Cope. v. Lytoloma crassicostatum.

latiscutatus, Cope. $v$. Lytoloma crassicostatum.

longiceps (Owen), Cope. v. Lytoloma longiceps.

subcarinatus, Cope. $v$. Argillochelys subcristata.

subcristatus, Cope. \%. Argillochelys subcristata.

RAPHIOSAURUS, Owen. v. Rhaphiosaurus.

2rGNOSAURUS, G. A. Mantell, Phil. Trans., 1848, p. 198 ; R. Lydekker, Quart. Journ. Geol. Soc., vol. xliv, 1888, p. 53, and Cat. Foss. Rept. B. M., pt. i, I888, p. 184.

northamptonl, G. A. Mantell, Phil. Trans., 1848, p. 183 ; J. Morris, Cat. Brit. Foss., 1854, p. 353 ; R. Lydekker, Cat. Foss. Rept. B. M., pt. i, I888, p. 184.

Iguanndon, G. A. Mantell, Phil. Trans., 184I, p. 145, pl. v, f. $1,2,5,6,8,9$.

Hylcosaurus?, R. Owen, Rept. Weald. and Purb. Form., pt. iv (Pal. Soc., 1857), p. 19, pl. viii, f. 1-5.

LOC. Wealden; Cuckfield and Tilgate. [Right dentary; Brit. Mus.]

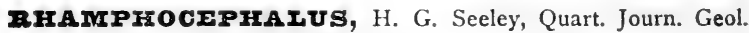
Soc., vol. xxxvi, I88o, p. 27.

bucklandi (Meyer), R. Lydekker, Cat. Foss. Rept. B. M., pt. i, I 888, p. 34 .

Pterodactylus bucklandi, $\mathrm{H}$. von Meyer, Palæologica, 1832, p. I17 ; J. Morris, Cat. Brit. Foss., 1854, p. 353 ; R. Owen, Phil. Trans., 1859 , p. 169 , pl. x, f. $23,37,38$.

Rhamphorhynchus bucklandi, T. H. Huxley, Quart. Journ. Geol. Soc., vol. xv, 1859, p. 658, pl. xxiv, f. 2, 3, 5, 7, 8; J. Phillips, Geol. Oxford, 1871, p. 219, woodcuts lxx-lxxvi. 
RHAMPMOCEPIraxUs bucklandi (continued) -

Pterodactylus duncani, R. Owen, Rept. Mesoz. Form., pt. i (Pal. Soc., I874), p. I I, pl. i, f. I8. [Wing-phalanx; Brit. Mus.]

Pterodactylus kiddii, R. Owen, ibid., p. 11, pl. i, f. 17. [Wingphalanx ; Brit. Mus.]

LOC. Stonesfield Slate. [Mandible.]

depressirostris (Huxley), R. Lydekker, Cat. Foss. Rept. B. M., pt. i, I888, p. 36.

Rhamphorhynchus depressirostris, T. H. Huxley, Quart. Journ. Geol. Soc., vol. xv, I859, p. 663, pl. xxiv, f. I.

? Pterodactylus aclandi, R. Owen, Rept. Mesoz. Form., pt. i (Pal. Soc., I874), p. I I, pl. i, f. I9. [Wing-phalanx; Brit. Mus.]

Loc. Stonesfield Slate. [Portion of mandible; Earl Ducie Coll.]

prestwichi, H. G. Seeley, Quart. Journ. Geol. Soc., vol. xxxvi, I880, p. 27, woodcut.

Loc. Stonesfield Slate; Kyneton, near Stow-on-the-Wold. [Impression of cranial shield; Oxford Mus.]

T.

RIAAmPHORIYNCHUS, H. von Meyer, Neues Jahrb., I 846, p. 463. Not known from British Formations.

bucklandi (Meyer), Huxley. \%. Rhamphocephalus bucklandi.

depressirostris, Huxley. $v$. Rhamphocephalus depressirostris. macronyx (Buckland), Meyer. $v$. Dimorphodon macronyx.

RHAPHIOSACRCS, Owen. ¿'. Dolichosaurus longicollis; also Pachyrhizodus. [PIsCES.]

lucius, Owen. $\%$ Pachyrhizodus subulidens. [PISCES.]

subulidens, Owen. $z^{\prime}$. Pachyrhizodus subulidens. [PISCES.]

ZIrIx OCHEIYs, H. G. Seeley, Index to Aves, etc., Woodwardian Mus., 1869, pp. xviii, 25 ; R. Lydekker, Quart. Journ. Geol. Soc., vol. xlr, i889, p. 227 .* $^{*}$

brachyxhina, R. Lydekker, Quart. Journ. Geol. Soc., vol. xlv, I 889, p. 231, pl. viii, f. 3, and Cat. Foss. Rept. B. M., pt. iii, 1889 , p. 179.

LOC. Cambridge Greensand. [Skull ; T. Jesson Coll.]

oantabrigiensis, R. Lydekker, Ouart. Journ. Geol. Soc., vol. xlv, I889, p. 230, pl. viii, f. 2, and Cat. Foss. Rept. B. M., pt. iii, 1889 , p. 176 , woodcut 42 .

LOC. Cambridge Greensand. [Skull ; Brit. Mus.]

* The following undefined names were applied by H. G. Seeley (Index, etc., p. xviii) to the various forms of crania subsequently described by Lydekker:cardiocephalus, colognathus, dacognathus, dayi, dimerognathus, eurrephalus graptocephalus, grypus, leptognathus, mastocephalus, platjcefhalus, platyrhinus, rheporhinus, sphenicephalus, stenicephalus. 


\section{RHINOCHEIYS (continued) -}

elegans, R. Lydekker, Quart. Journ. Geol. Soc., vol. xlv, 1889, p. 230 , pl. viii, f. 4, 5, and Cat. Foss. Rept. B. M., pt. iii, 1889, p. I7 8 .

LOC. Gault ; Folkestone. Cambridge Greensand. [Skull ; Brit. Mus.]

Jessoni, R. Lydekker, Quart. Journ. Geol. Soc., vol. xlv, 1889, p. 23I, pl. viii, f. 6, and Cat. Foss. Rept. B. M., pt. iii, 1889, p. 180 ,

LOC. Cambridge Greensand. [Skull ; T. Jesson Coll.]

macrorhina, R. Lydekker, Quart. Journ. Geol. Soc, vol. xlv, I889, p. 230, pl. viii, f. 7, and Cat. Foss. Rept. B. M., pt. iii, 1889, p. 177.

LOC. Cambridge Greensand. [Skull ; Brit. Mus.]

pulchriceps (Owen), H. G. Seeley, Index to Aves, etc., Woodwardian Mus., 1869, pp. xviii, 25 ; R. Lydekker, Quart. Journ. Geol. Soc., vol. xlv, I 889 , p. 230 , pl. viii, f. I, and Cat. Foss. Rept. B. M., pt. iii, 1889 , p. 176.

Chelone pulchriceps, R. Owen, Rep. Brit. Assoc, 1841 (1842), p. 172, and Rept. Cret. Form. (Pal. Soc., 185 I), p. 8, pl. vii ^, f. I-3; J. Morris, Cat. Brit. Foss., I 854 , p. 348.

LOC. Cambridge Greensand. [Skull.]

8p., R. Lydekker, Cat. Foss. Rept. B. M., pt. iii, r889, p. 180.

LOC. Cambridge Greensand. [Anterior portion of skull; T. Jesson Coll. (Plaster cast, Brit. Mus., no. R. 1509.)]

RIOMALEOSAURUS, Seeley. v. Thaumatosaurus.

cramptomi (Carte and Baily), Seeley. $v$. Thaumatosaurus cramptoni.

RIXNCHOSA URUS, R. Owen, Rep. Brit. Assoc., I 84 I (1842), p. I45, and Trans. Camb. Phil. Soc., vol, vii, 1842, p. 355, and Ann. Mag. Nat. Hist. [3] vol. iv, 1859, p. 237.

articeps, R. Owen, Rep. Brit. Assoc., 1841 (1842), p. 190, and Trans. Camb. Phil. Soc., vol. vii, 1842, p. 355 , pls. v, vi, and Palicont., ed. 2, 1861, pp. 237, 263, woodcut 91 A ; J. Morris, Cat. Brit. Foss., 1854 , p. 353 ; 'T. H. Huxley, Quart. Journ. Geol. Soc., vol. xliii, I887, p. 689, pl. xxvii, woodcuts 2, 5 ; R. Lydekker, Cat. Foss. Rept. B. M., pt. i, 1888, p. 296.

Loc. Keuper; Grinshill, Shropshire. [Head, etc. ; Shrewsbury Mus. (pars).]

Rysosteus, R. Owen, Rep. Brit. Assoc., 184I (1842), p. 159.

owent : Rysosteus, R. Owen, ibid.; J. Morris, Cat. Brit. Foss., 1854 , p. 353 .

I.OC. Rhretic Bone-bed; Aust Cliff and Westbury. [Anterior dorsal vertebra; olim Johnson Coll. (Bristol.)] 
SAURILLUS, Owen. v. Macellodus.

obtusus, Owen. $v$. Macellodus brodiei.

SAUROSPONDYIUS, H. G. Seeley, Ann. Mag. Nat. Hist. [3] vol. xvi, 1865, p. 145. [? Dolichosaurus.]

dissimilis, H. G. Seeley, ibid., and Index to Aves, etc., Woodwardian Mus., 1869, pp. xv, 3.

Loc. L. Chalk ; Cherry Hinton. [Vertebra; Woodwardian Mus.]

T.

SCAPHOGNATrus, A. Wagner, Sitzungsb. k. bay. Akad. Wiss., vol. i, 1861, p. 519.

PAChYRHAMPHUS, L. J. Fitzinger (non Gray, 1840), Syst. Rept., $1843, \mathrm{p} .35$.

? purdon1, E. T. Newton, Phil. Trans., 1887 (1888), pt. B, p. 503, pls. Ixxvii, Ixxviii.

Scaphognathus, G. Baur, Geol. Mag. [3] vol. vi, 1889, p. I71.

LOC. U. Lias ; Lofthouse, Yorkshire. [Skull; IV. Purdon Coll.]

SOrxIDOsAurus, R. Owen, Encyclopad. Britan., ed. 8, vol. xvii, 1859, p. 150, and Palreontology, ed. 2, 186I, p. 286; H. G. Seeley, Phil. Trans., I889, pt. B, p. 287.

harrison1, R. Owen, Rep. Brit. Assoc., I86I (1862), Sect., p. I21, and Rept. Liassic Form., pt. i (Pal. Soc., I86I), p. I, pls. i-vi, and ibid., pt. ii (Pal. Soc., 1863), p. I, pls. i-ix, and Rept. Weald. and Purb. Form., Suppl. v (Pal. Soc., 1874), p. Io, pl. ii, f. 2 I ; R. Lydekker, Cat. Foss. Rept. B. M., pt. i, 1888, p. I81, woodcut 33 .

Loc. L. Lias; Charmouth and Lyme Regis. [Right femur, tibia, and fibula ; Brit. Mus.]

T.

SPIJNOSPONDYIUs, H. G. Seeley, Quart. Journ. Geol. Soc, vol. xxxix, I $88_{3}$, p. 55 .

gracilis, R. Lydekker, Quart. Journ. Geol. Soc., vol. xliv, I888, p. 47, and Cat. Foss. Rept. B. M., pt. i, 1888, p. 238.

Sphenospondylus, H. G. Seeley, Quart. Journ. Geol. Soc., vol. xxxix, 1883 , p. 55 , woodcuts $1-3$.

Loc. Wealden; Brook, I. of Wight. [Vertebre; Brit. Mus.] $\mathbf{x}$

BTAGONoxmpis, L. Agassiz, Poiss. Foss. V. G. R. (1844), p. 139 ; T. H. Huxley, Quart. Journ. Geol. Soc., vol. xv, 1859 , p. 440 .

robertson1, L. Agassiz, Poiss. Foss. V. G. R. (1844), p. 139, pl. xxxi, f. 13, I4 ; J. Morris, Cat. Brit. Foss., 1854, p. 345; T. H. Huxley, Quart. Journ. Geol. Soc., vol. xv, I859, p. 440, pl. xiv, and ibid., vol. xxxi, 1875, p. 423 , pl. xix, and Mem. Geol. Surv., Monogr. iii, 1877, p. 9, pls. $\mathrm{i}-\mathrm{x}$, xiv-xvi; R. Lydekker, Cat. Foss. Rept. B. M., pt. i, 1888, p. 129. T. LOC. Keuper; Lossiemouth, near Elgin. [Scutes; Elgin Mus.] 
STzGOCמrIxS, R. Lydekker, Quart. Journ. Geol. Soc., vol. xlv, 1889, p. 229.

planiceps (Owen), R. Lydekker, ibid., p. 229, and Cat. Foss. Rept. B. M., pt. iii, I889, p. 233.

Chelone planiceps, R. Owen, Rep. Brit. Assoc., 1841 (1842), p. I68, and Brit. Foss. Rept., vol. ii (1884), pl. viii (Chelonia), f. I-3 ; J. Morris, Cat. Brit. Foss., 1854, p. 348.

Hydraspis planiceps, L. J. Fitzinger, Ann. Wien. Mus., vol. i, I 835 , p. 126.

LOC. Portland Stone ; Portland. [Skull and mandible.]

I.

STEGOSAURUS, Marsh. v. Omosaurus.

STrigosa U vol. xii, I825, p. 146; J. W. Hulke, Proc. Zool. Soc., 1888, p. 43.3, woodcuts 3-6, pl. xviii, f. 6, pl. xix, f. 3-6.

Mystriosaurus, J. J. Kaup, in H. G. Bronn, Lethæa Geognost., vol. i, 1835, p. 525 .

Macrospondylus, H. von Meyer, Nova Acta Acad. Cæs. Leop.-Carol., vol. xv, pt. ii (I83I), p. 196. [See also Dacosaurus.]

bollensis (Jaeger), R. Lydekker, Cat. Foss. Rept. B. M., pt. i, I888, p. 109.

Crocodilus bollensis, G. F. Jaeger, Foss. Rept. Württembergs, I 828 , p. 6.

Teleosaurus bollensis, F. Holl, Handb. Petrefact., vol. i, 1829, p. 87 .

Macrospondylus bollensis, $\mathrm{H}$, von Meyer, Nova Acta Acad. Cæs. Leop.-Carol., vol. xv, pt. ii (183i), p. I97.

Mystriosaurus bollensis, d'Alton and Burmeister, Der Fossil Gavial von Boll, 1854 , p. 73.

Mystriosaurus stukelyi var. bollensis, T. C. Winkler, Archiv. Mus. Teyler, vol. iv, art. 1, 1876, p. 132.

LOC. U. Lias; Whitby. [Imperfect skull and mandible; Brit. Mus.]

boutllieri, E. E. Deslongchamps, Notes Paléontologiques, 1869, p. 230, pl. xvi, f. 2 ; A. S. Woodward, Geol. Mag. [3] vol. ii, I 885, p. 508 (table).

Crocodilus oxoniensis, W. D. Conybeare, MS. (teste Deslongchamps).

Steneosaurus oxoniensis, H. T. De la Beche, MS. (teste Deslongchamps).

LoC. Gt. Oolite; Oxford. [Skull and mandible.]

brevidens (Phillips), R. Lydekker, Cat. Foss. Rept. B. M., pt. i, I888, p. 115 .

Teleosaurus brevidens, J. Phillips, Geol. Oxford, 1871, p. 185, woodcuts xli, f. 8, 9, xlii, f. 1-5, xliii-liii, lviii, f. 7-9.

Loc. Gt. Oolite ; Enslow Bridge, Eyeford, and Stonesfield, Oxfordshire. [Skull and teeth; Oxford Mus.] 
STENEOSAURUS (continued) -

brevior, J. F. Blake (er Owen, MS.), in Tate and Blake, Yorkshire Lias, 1876 , p. 244 , pl. i, f. 1-3; R. Lydekker, Cat. Foss. Rept. B. M., pt. i, I888, p. II I.

Teleosaurus brevior, R. Owen, Brit. Foss. Rept., vol. iii (1884), p. 140, pl. xvi (Crocodilia).

Mystriosaurus brevior, A. S. Woodward, Geol. Mag. [3] vol. ii, 1885 , p. 499 , and table p. 508.

LOC. U. Lias; Whitby. [Skull ; Brit. Mus.]

brevirostris, R. Owen, Rep. Brit. Assoc., I84I (1842), pp. 82, 190. [Indeterminable.]

chapmani (Buckland, $c x$ lioenig, MS.), R. Lydekker, Cat. Foss. Rept. B. M., pt. i, I888, p. I10.

Teleosaurus chapmanni, W. Buckland (pars), Geol. and Mineral., ed. 2, vol. ii, 1837, p. 35, pl. xxv, f. I ; R. Owen, Rep. Brit. Assoc., I84I (I842), p. 75 ; J. Morris, Cat. Brit. Foss., 1854 , p. 354 ; M. Simpson, Foss. Yorkshire Lias, 1855, p. 14, and ibid., ed 2, I884, p. 6; J. F. Blake, in Tate and Blake, Yorkshire Lias, I876, p. 244.

Steneosaurus?, E. Charlesworth, Mag. Nat. Hist., n.s., vol. i, 1837, p. $53 \mathrm{I}$, woodcut 65 .

Mystriosaurus chapmani, [-nni] J. J. Kaup, in Bronn and Kaup, Gavialartige Reptilien, 1841, p. 27 ; A. S. Woodward, Geol. Mag. [3] vol. ii, 1885, p. 499 , table p. 508.

Mystriosaurus stukelyi var. chapmani, T. C. Winkler, Archiv. Mus. Teyler, vol. iv, art. 1, 1876, p. 132. [Winkler also suggests substitution of varietal name anglicus for chapmani.]

'Allegator,' W. Chapman and T. Wooller, Phil. Trans., 1758, pp. 688,786 , pls. xxii, xxx.

'Fossil Skeleton,' G. Young, Mem. Wemer. Soc., vol. iii, 1819 (1821), p.450, pl. xxii, and Edinb. New Phil. Journ., vol. xiii, i 825 , p. 76 , with plate ('Crocodile').

'Great Whitby Crocodile,' G. Young and I. Bird, Geol. Surv. Yorkshire Coast, ed. 2, 1828, descr. to pl. xvi, f. 1-3. [Skeleton; Whitby Mus.]

LOC. U. Lias; Greens Norton, Saltwick, and Whitby. [Imperfect skeleton ; Brit. Mus.]

dasycephalıs, Seeley. v. Metriorhynchus superciliosum.

? geotfroyi, R. Owen, Brit. Foss. Rept., vol. iii (1884), p. 144, pl. xviii (Crocodilia), f. I.

Macrorhynchus?, E. Koken, Palæont. Abhandl., vol. iii (1887), p. 399.

Loc. Gt. Oolite; Oxford. [Cranium.]

gracilis, Phillips. v. Metriorhynchus gracile.

? lat1ceps, R. Owen, Brit. Foss. Rept., vol. iii (1884), p. 145, pl. xviii (Crocodilia), f. 2.

Macrorhynchus ?, E. Koken, Palæont. Abhandl., vol. iii (1887), p. 399.

Loc. Gt. Oolite ; near Oxford. [Cranium.] 
STMNBOSAURUS (continued) -

latıfrons (Owen), R. Lydekker, Cat. Foss. Rept. B. M., pt. i, 1888, p. II 3 .

Teleosaurus latifrons, R. Owen, Brit. Foss. Rept., vol. iii (1884), p. I4I, pl. xvii (Crocodilia).

LOC. U. Lias; Northamptonshire. ? Gt. Oolite; Northamptonshire. [Skull.]

longirostris, auct. $v$. Steneosaurus megistorhynchus.

megarhinus (Hulke), R. Lydekker, Cat. Foss. Rept. B. M., pt. i, 1888 , p. I 17.

Teleosaurus megarhinus, J. IV. Hulke, Quart. Journ. Geol. Soc., vol. xxvii, 1871, p. 442, pl. xviii ; A. S. Woodward, Geol. Mag. [3] vol. ii, I885, p. 500.

Loc. Kim. Clay; Kimeridge Bay. [Snout; Brit. Mus.]

mogistorhynchus (Deslongchamps), R. Lydekker, Cat. Foss. Rept. B. M., pt. i, I 888 , p. I 16 .

Teleosaurus megistorhynchus, E. E. Deslongchamps, Bull. Soc. Linn. Normandie [2] vol. i, I868, p. 155.

Steneosaurus rostro-major, auct. (pars.)

Steneosaurus longirostris, auct. (pars.)

? Steneosaurus longirostris, J. Phillips, Geol. Oxford, 1871, p. 388, woodcut cxc. [Tooth ; Oxford Mus.]

Loc. U. Inf. Oolite (Gryphite Grit); Leckhampton. [Portion of snout; Brit. Mus.]

oxoniensis, De la Beche, MS. v. Steneosaurus boutilieri. palpebrosus, Phillips. v. Metriorhynchus palpebrosum.

? purbeckensis, J. C. Mansel-Pleydell, Proc. Dorset. Nat. Hist. Field Club, vol. ix, 1888, p. 12.

Loc. Purbeck; Swanage. [Skull ; Dorchester Mus.]

rostro-major, auct. $v$. Steneosaurus megistorhynchus.

rostro-minor (Geoffroy), Owen. $v$. Metriorhynchus palpebrosum.

stephant, J. W. Hulke, in Mansel-Pleydell, Proc. Dorset. Nat. Hist. Field Club, vol. i, 1877 , p. 29, pl. i ; R. Lydekker, Cat. Foss. Rept. B. M., pt. i, I888, p. 114 .

LOc. Cornbrash; Closworth, Dorset. [Portions of skull and mandible ; Brit. Mus.]

temporal1s, R. Owen, Brit. Foss. Rept., vol. iii (1884), p. 145, pl. xix (Crocodilia).

LOC. Great Oolite; Bath. [Skull.]

STEREOSAURUS, H. G. Seeley, Index to Aves, etc., Woodwardian Mus., 1869, p. xviii (name only).

cratynotus, H. G. Seeley, ibid., pp. xviii, 44 (name only).

LOC. Cambridge Greensand. [Vertebræ; Woodwardian Mus.] 
STEREOSAURUS (continued)-

platyomus, H. G. Seeley, ibid., pp. xviii, 43 (name only). T.

LOC. Cambridge Greensand. [Vertebræ; Woodwardian Mus.]

stenomus, H. G. Seeley, ibid., pp. xviii, 43 (name only).

Lóc. Cambridge Greensand. [Vertebræ: Woodwardian Mus.]

STREPTOSPONDYLUS, H. von Meyer. v. Iguanodon.

cuvieri, R. Owen, Rep. Brit. Assoc., 1841 (1842), p. 88 (? non

Brit. Foss. Rept., vol. i (1884), p. 400, pl. xx, Crocodilia).

[? Cetiosaurus.]

LOC. Oolite; Chipping Norton. [Portion of vertebra ; olim

J. Kingdon Coll.] [? non 'Lias ; Whitby.']

major, Owen. $v$. Iguanodon bernissartensis.

recentior, J. W. Hulke (ex Owen), Quart. Journ. Geol. Soc., vol. $x x x, 1874$, p. 519 (name only).

Loc. Wealden. [Vertebra.]

STRONGTLOSTINUS, T. Hawkins, Great Sea Dragons, I 840. =Ichthyosaurus.

SUCHOSAURUs, R. Owen, Rep. Brit. Assoc., 1841 (1842), p. 67. [See also Hylaosaurus armatus.]

cultridens, R. Owen, Rep. Brit. Assoc., I84I (I842), p. 67, and Rept. Weald. and Purb. Form., Suppl. viii (Pal. Soc., 1878), p. 12, pl. iv, f. 5-8 ; J. Morris, Cat. Brit. Foss., I 854 , p. 354 ; R. Lydekker, Cat. Foss. Rept. B. M., pt. i, 1888, p. 90.

Crocodilus cultridens, R. Owen, Odontography (184I), p. 287 , pl. Ixii A, f. Io.

Suchosuturus lavidens, R. Owen, Brit. Foss. Rept., vol. ii (1884), expl. of Crocodilia, pl. v, f. 4 .

' Gavial of Tilgate Forest,' G. A. Mantell, Illustr. Geol. Sussex, 1827, p. 65 , pl. v, f. $5,6,8$.

LOC. Wealden; Cuckfield and Tilgate, Sussex, and I. of Wight. [Teeth; Brit. Mus.]

T.

lavidens, Owen. \%. Suchosaurus cultridens.

SYNGONOSAURUS, H. G. Seeley, Quart. Journ. Geol. Soc., vol. $\mathrm{xxxv}, \mathrm{I} 879$, p. $62 \mathrm{I}$.

macrocercus, H. G. Seeley, ibid., p. 621, woodcuts 6-8; R. Lydekker, Quart. Journ. Geol. Soc., vol. xlv, 1889, p. 4 I, woodcut $\mathbf{I}$.

LOC. Cambridge Greensand. [Vertebræ; Woodwardian MIus.]

TANYSTROPHCUS, H. von Meyer : recorded in error from Keuper of Leicestershire by J. Plant, Rep. Leicester Lit. and Phil. Soc., 1874 , p. 40 (M. Browne, Vert. Animals Leicestershire, 1889, p. 174). 
TrIxOSAURUS, Geofiroy St. Hilaire, Mém. du Musćum, vol. xii, IS25, p. 135 .

asthezodeirus, R. Owen, Rep. Brit. Assoc., I $\delta_{4}$ I $\left(1 \delta_{42}\right)$, p. $\delta$ I, and Brit. Foss. Rept., vol. iii (ISS4), p. I 4 I ; J. Morris, Cat. Brit. Foss., 1854, p. 354 ; J. Phillips, Geol. Oxford, 187 r, p. 3 So.

I.OC. Kim. Clay; Shotover. [Cervical vertebre; Oxford Mus.]

bollensis. Holl. $\because$. Steneosaurus bollensis.

brevidens, Phillips. 2'. Steneosaurus brevidens.

brevior, Owen. $\because$. Steneosaurus brevior.

brevirostris, R. Owen, Palcontology, ed. 2, IS6r, p. 299 , woodcut 103 (I); A. S. Woodward, Geol. Mag. [3] vol. ii, ISS5, p. 50S, table. [? Stencosaurus brevior, S. chapmani, or S. bollensis.]

I.oc. U. Lias; Whitby. [Vertebra.]

cadomensis (Lamouroux), E. Geoffroy St. Hilaire, Mém. du Muséum, vol. xii, ISz5, p. 124, pl. vi, f. I-4. (Crocodilus catomensis, Lamouroux, Ann. Gén. Sci. Phys., vol. iii, 1820, p. 163, name only:) kecorded from the Stonesfield Slate, upon doubtful evidence, by R. Owen, Rep. Brit. Assoc., I $\&_{41}\left(1 \delta_{42}\right)$, p. $\delta_{1}$, and J. Morris, Cat. Brit. Foss., I $\$_{54}$, p. 354 .

chapmani. Buckland. a'. Steneosaurus chapmani and Pelagosaurus brongniarti.

eucephalus. Seeley. ¿. Pelagosaurus brongniarti.

geoffroyi, E. E. Deslongchamps, Bull. Soc. Linn. Normandie [2] vol. i, I868, p. 348; R. Lydekker, Cat. Foss. Rept. B. M., pt. i, ISSS, p. 120.

LOC. Stonesfield Slate. [Portions of head; Brit. Mus.]

ischnoton. E. Charlesworth, Rep. Brit. Assoc., IS54 (1855), Sect., p. So (name only).

Loc. U. Lias; Whitby. [? Unknown fossil in York Mus.]

latifrons, Owen. $\approx$. Steneosaurus latifrons.

megarhinus, Hulke. v. Steneosaurus megarhinus.

megistorhynchus, Deslongchamps. $\because$. Steneosaurus megistorhynchus.

subulidens, J. Phillips, Geol. Oxford, I87 I, p. 194, woodcuts xlii, f. 6-1o, liv, lv, lviii, f. I-3; R. Lydekker, Cat. Foss. Rept. B. M., pt. i, ISSS, p. IzI.

LOC. Stonesfield Slate. [Mandib. symphysis; Oxford Mus.]

TEzER PETON, G. A. Mantell, Quart. Journ. Geol. Soc, vol. viii, IS 52 , p. 100 .

LEPTOPLEURON, R. Owen, Literary Gazette, I85 I, p. 900, and Palzeontology, ed. 2, IS6I, p. 284. 
FEIMTPTON (continued)-

elginense, G. A. Mantell, Quart. Journ. Geol. Soc., vol. viii, 1852, p. 100, pl. iv; P. Duff, Edinb. New. Phil. Journ., vol. lii, 1854 , p. 353 ; J. Morris, Cat. Brit. Foss., 1854 , p. 354 ; T. H. Huxley, Quart. Journ. Geol. Soc., vol. xxiii, 1867 , p. 77, woodcuts A-E ; R. Lydekker, Cat. Foss. Rept. B. M., pt. i, I888, p. 295.

'Skeleton of four-footed reptile,' L. Brickenden, Edinb. New Phil. Journ., vol. lii, 1854, p. 353.

Leptopleuron lacertinum; R. Owen, Literary Gazette, I851, p. 900, and Palcontology, ed. 2, 1861, p. 284. T. (Leptopleuron.)

LOC. Keuper; Lossiemouth, near Elgin. [Skeleton, wanting head; J. Powrie Coll.]

T.

TEMNODONTOSAURUS, Lydekker. \%. Ichthyosaurus platy odon.

T'ERATOSAURUS, Meyer. v. Zanclodon.

TבSTUDO, Linnæus, Syst. Nat., ed. 12, vol. i, 1766, p. 350.

cantabrigiensis, H. G. Seeley, Index to Aves, etc., Woodwardian Mus., 1869, pp. xix, 32 (name only). [? Genus.]

Loc. Cambridge Greensand. [Mandibie; Woodwardian Mus.]

duncani, R. Owen, Rep. Brit. Assoc., I84I (1842), pp. I60, 190. [Name applied to footprints from the New Red Sandstone of Corncockle Muir.]

orbicularis, Linnæus. v. Emys orbicularis.

plana, Koenig. $v$. Lytoloma crassicostatum.

stricklandi, Phillips. v. Protochelys stricklandi.

(remains), Owen and Morris. v. Protochelys stricklandi.

THAIASSEMYS, L. Rütimeyer, Verh. schweiz. Ges. Nat., 1858 (1859), p. 57, and Neue Denkschr. schweiz. Ges. Nat., vol. xxv, art. 2 (1873), p. 27.

Enaliochelys, H. G. Seeley, Index to Aves, etc., Woodwardian Mus., I869, p. 108 (insufficiently described).

hugii, L. Ruitimeyer, Neue Denkschr. schweiz. Ges. Nat., vol. xxv, art. 2 (1873), p. 31, pl. i, pl. vi, f. 4, pl. x, f. I-3; R. Lydekker, Cat. Foss. Rept. B. M., pt. iii, 1889 , p. 148 .

Enaliochelys cheionia, H. G. Seeley, Index to Aves, etc., Woodwardian Mus., 1869, p. 108.

T. (Enaliochely's.)

Loc. Kim. Clay; Devizes and Ely. [Bones; Woodwardian Mus.]

ruetimeyeri, R. Lydekker, Cat. Foss. Rept. B. M., pt. iii, I889, p. I49, woodcut 37.

Eurysternum, R. Lydekker and G. A. Boulenger, Geol. Mag. [3].vol. iv, 1887, p. 274.

Loc. Purbeck; Dorsetshire. [Carapace; Brit. Mus.] 
rzazASSOCrraxys, L. J. Fitzinger, Ann. Wien. Mus., vol. i, 1835 , p. 121 .

crassicostata (Owen), Cope. $\%$. Lytoloma crassicostatum.

eocanica, R. Lydekker, Proc. Geol. Assoc., vol. xi (1889), p. 177, woodcut I, and Cat. Foss. Rept. B. M., pt. iii, 1889, p. 50.

LOC. Bracklesham Beds. [Humerus ; J. B. Ogle Coll.]

planimentum (Owen), Cope. $v$. Lytoloma planimentum:

sp., R. Lydekker, Proc. Geol. Assoc., vol. xi (1889), p. 178, and Cat. Foss. Rept. B. M., pt. iii, 1889 , p. 50.

LOC. London Clay; Sheppey. [Mandib. symphysis; Brit. Mus., no. 38995.]

TIAUmATOSAURUS, H. von Meyer, Neues Jahrb., I84I, p. 176.

Rhomaleosaurus, H. G. Seeley, Quart. Journ. Geol. Soc., vol. $\mathrm{xxx}, \mathrm{I} \delta 74$, p. 448.

arcuatus (Owen), R. Lydekker, Cat. Foss. Rept. B. M., pt. ii, 1889 , p. 163.

Plesiosaurus arcuatus, R. Owen, Rep. Brit. Assoc., I 839 (1840), p. 75, and Trans. Geol. Soc. [2] vol. v (1840), p. 521 , pl. xliv, f. 5 (vertebra); J. Morris, Cat. Brit. Foss., 1854, p. 352.

Plesiosaurus, W. Buckland, Geol. and Mineral., ed. 2, vol. ii, I837, p. 30, pl. xix, f. 3,4 .

Plesiosaurus tritarsostinus, T. Hawkins, Mem. Ichthyosauri, 1834, pl. xxvi, and Sea Dragons, 1840, list of plates, pl. xxvi.

LOC. L. Lias; Bath, Cheltenham, Lyme Regis, and Street. [Dentary and other bones; Brit. Mus.]

carinatus (Cuvier), R. Lydekker, Cat. Foss. Rept. B. M., pt. ii, 1889, p. 168, woodcut 57 .

Plesiosaurus carinatus, Cuvier, Ossem. Fossiles, ed. 2, vol. v, pt. ii, 1824, p. 486 ; H. E. Sauvage, Ann. Sci. Nat. (Zool.) [6] vol. viii, 1879, art. 13, p. 19, pl. xxvi, f. 2.

LOC. Kim. Clay; Shotover, Portland, and Swindon. [Vertebre; Brit. Mus.]

cramptoni (Carte and Baily), R. Lydekker, Cat. Foss. Rept. B. M., pt. ii, I889, p. I6I.

Plesiosaurus cramptoni, A. Carte and W. H. Baily, Rep. Brit. Assoc., I862 (I863), Sect., p. 68, and Journ. Roy. Dublin Soc., vol. iv, I863, p. 160, pls. i, ii ; S. Haughton, Man. Geology, I865, p. 273, woodcut 38; H. G. Seeley, Ann. Mag. Nat. Hist. [3] vol. $\mathrm{xv}, \mathrm{I} 865$, p. 50.

Rhomaleosaurus cramptoni, H. G. Seeley, Quart. Journ. Geol. Soc., vol. $x x x, 1874$, p. 448.

LOC. U. Lias; Kettleness, Kingsthorpe, and Whitby. [Skeleton; Mus. Science and Art, Dublin.] 


\section{TIX AUMATOSAURUS (continued) -}

megacephalus (Stutchbury), R. Lydekker, Cat. Foss. Rept. B. M., pt. ii, 1889 , p. 166.

Plesiosaurus megacephalus, S. Stutchbury, Quart. Journ. Geol. Soc., vol. ii, 1846, p. $4 \mathrm{I} 2$, pl. xviii ; J. Morris, Cat. Brit. Foss., 1854 , p. 352 ; W. J. Sollas, Quart. Journ. Geol. Soc., vol. xxxvii, 1881, p. 472, woodcuts 8-13.

Loc. L. Lias ; Somersetshire. [Skeleton; Bristol Mus.]

philarchus (Seeley), Lydekker. v. Peloneustes philarchus.

propinquus (Blake), R. Lydekker, Cat. Foss. Rept. B. M., pt. ii, 1889 , p. 170.

Plesiosaurus propinquus, J. F. Blake, in Tate and Blake, Yorkshire Lias, 1876, p. 247, pl. ii, f. I.

Plesiosaurus simpsoni, J. F. Blake (errore), ibid. (index of synonyms, p. I).

Plesiosuurus marrocephalus?, A. Carte and W. H. Baily, Journ. Roy. Dublin Soc., vol. iv, I863, p. 169 (table of measurements).

LOC. M. Lias ; Bugbrook. U. Lias ; Crick, Northamptonshire, and Whitby. [Skeleton; Whitby Mus.]

zetlandicus (Phillips), R. Lydekker, Cat. Foss. Rept. B. M.. pt. ii, I889, p. 167 .

Plesiosaumus zetlandicus, J. Phillips, Rep. Yorksh. Phil. Soc., I 852 (I853), p. 7 (name only) (misquoted P. zetlanti on p. 21), and ibid., 1853 (1854), p. 7 ; A. Carte and W. H. Baily, Journ. R. Dublin Soc., vol. iv, I863, p. I69 (table of measurements); J. F. Blake, in Tate and Blake, Yorkshire Lias, I876, p. 249, pl. i, f. 5 ( $P$. zetlandi).

LOC. U. Lias ; Lofthouse. [Skeleton; York Mus.]

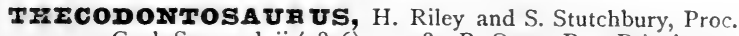
Geol. Soc., vol. ii (1836), p. 398 ; R. Owen, Rep. Brit. Assoc., I 84 I (I 842 ), p. I 53 .

antiquus, $J$. Morris, Cat. Brit. Foss., I843, p. 211 ; R. Owen, Palæontology, ed. 2, 186I, p. 275; R. Lydekker, Cat. Foss. Rept. B. M., pt. i, I888, p. 175.

Thecodontosaurus, H. Riley and S. Stutchbury, Trans. Geol. Soc. [2] vol. v (1840), p. 349, pl. xxix, f. I-3; T. H. Huxley, Quart. Journ. Geol. Soc., vol. xxvi, 1870, p. 43, pl. iii, f. 1, 2, 5-8.

LOC. U. Trias (Dolomitic Conglomerate); Durdham Down, Bristol. [Mandib. ramus; Bristol Mus.] T.

platyodon (Kiley and Stutchbury), T. H. Huxley, Quart. Journ. Geol. Soc., vol. xxvi, 1870, p. 43 ; R. Lydekker, Cat. Foss. Rept. B. M., pt. i, I888, p. 174 , woodcut 30.

Palcosaurus platyodon, H. Riley and S. Stutchbury, Proc. Geol. Soc., vol. ii (1836), p. 398, and Trans. Geol. Soc. [2] vol. v. (I840), p. 352, pl. xxix, f. 5 ; R. Owen, Odontography (184I), p. 267, pl. Ixii A, f. 7 ; J. Morris, Cat. Brit. Foss., 1854, p. 351.

LOC. U. Trias (Dolomitic Conglomerate); Durdham Down, Bristol. [Tooth ; Bristol Mus.] 


\section{TIIECODONTOSAURUS (continued) -}

sp., J. Morris, Cat. Brit. Foss., 1854, p. 354.

'A small tooth from Leamington,' R. I. Murchison and H. E. Strickland, Trans. Geol. Soc. [2] vol. v, 1836 (1840), pl. xxviii, f. 7 A.

LOC. Trias; Leamington. [Tooth ; Geol. Soc. London.]

TrFCospondy vol. xxxviii, I882, p. 457 .

laviesi (Seeley), Lydekker. v. Cœlurus daviesi.

horneri, H. G. Seeley, ibid., p. 457, pl. xix, and ibid., vol. xliv, I 888, p. 79 ; R. Lydekker, Cat. Foss. Rept. B. M., pt. i, I888, p. 153.

LOC. Hastings Sand; Southborough, Kent. [Sacrum; Brit. Mus.]

Trzirosucrus, R. Owen, Rept. Weald. and Purb. Form., Suppl.' ix (Pal. Soc., 1879), p. Io, and Quart. Journ. Geol. Soc,, vol. $x x x v, 1879$, p. I49.

pusillus, R. Owen, ibid., p. Io, pl. ii, f. 24 , pl. iii, f. $3^{-17}$, pl. iv, and ibid., p. 149, pl. ix, and Journ. R. Micros. Soc., 1878, p. 233, pl. xiii ; R. Lydekker, Cat. Foss. Rept. B. M., pt. i, 1888, p. 78.

LOC. M. Purbeck; Durdlestone Bay. [Skeleton and skull; Brit. Mus.]

I.

TrTANOSA URUS, R. Lydekker, Rec. Geol. Surv. India, vol. x, I877, p. 38.

? sp., R. Lydekker, Quart. Journ. Geol. Soc., vol. xliii, I887, p. 157, woodcut ('=Ornithopsis'), and Cat. Foss. Rept. B. M., pt. i, I 888 , p. I 35 .

LOC. Wealden; Brook, I. of Wight. [Caudal vertebra ; Brit. Mus.]

? sp., R. Lydekker, Quart. Journ. Geol. Soc., vol. xliv, I888, pp. 54, 58, and Cat. Foss. Rept. B. M., pt. i, I888, p. I36.

LOC. U. Greensand; I. of Wight. [Caudal vertebra; Brit. Mus.]

TrACizODON, J. Leidy, Proc. Acad. Nat. Sci. Philad., vol. viii, 1856 (1857), p. 72 .

Hadrosaurus, J. Leidy, ibid., I858 (1859), p. 215.

cantabrigiensis, R. Lydekker, Quart. Journ. Geol. Soc., vol. xliv, 1888, p. 47, and Cat. Foss. Kept. B. M., pt. i, 1888, p. 244, woodcut $5 \mathrm{I}$.

Iguanodon mantelli, R. Owen (errore), Rept. Cret. Form., Suppl. ii (Pal. Soc., 1864), p. 30, pl. vii, f. 16, I7.

Hadrosaurus, J. Leidy, Smithsonian Contrib., vol. xiv, art. 6, 1865 , p. 86 ; H. G. Seeley, Quart. Journ. Geol. Soc., vol. xxxv, 1879 , p. 59 I.

LoC. Cambridge Greensand. [Tooth; Brit. Mus.] . 
TRACIYASPIS, H. von Meyer, Neues Jahrb., I 843 , p. 699.

hantoniensis, R. Lydekker, Ann. Mag. Nat. Hist. [6] vol. iii, I 889, p. 54, and Cat. Foss. Rept. B. M., pt. iii, 1889, p. 131. Loc. U. Eocene; Hordwell. [Marginal scute; Brit. Mus.]

TH.1CHYDERMOCHELYS, H. G. Seeley, Index to Aves, etc., Woodwardian Mus., I869, pp. xix, 35 (name only).

phlyctcenus, H. G. Seeley, ibid., pp. xix, 35 (name only).

LOC. Cambridge Greensand. [Fragments of carapace; Woodwardian Mus.]

TrmTostrenum, R. Owen, Rep. Brit. Assoc., I84 I (1842), p. 165 (Tretosternon).

bakewelli (Mantell), J. Morris, Cat. Brit. Foss., I854, p. 354 (Tretosternon); R. Lydekker and G. A. Boulenger, (itul. Mag. [3] vol. iv, I887, p. 273 ; R. Lydekker, Cat. Foss. Rept. B. M., pt. iii, 1889, p. I38, woodcut 33 .

Tretosternon punctatum, R. Owen (errore), Rep. Brit. Assoc., 184I (1842), p. 167.

Trionyx hakewelli, G. A. Mantell, Geol. S. E. England, 1833, p. 255, woodcut.

Trionyx, G. A. Mantell, Illustr. Geol. Sussex, 1827, p. 6r, pl. vi, f. I, 3 .

LOC. Wealden; Tilgate and Cuckfield, Sussex. [Costal scute ; Brit. Mus.]

punctatum, R. Owen, Rep. Brit. Assoc., I84I (1842), p. 165; R. Lydekker and G. A. Boulenger, Geol. Mag. [3] vol. iv, I 884, p. 273 ; R. Lydekker, Cat. Foss. Rept. B. M., pt. i:i, 1889, p. 141. [See also Tretosternum bakerelli.]

LOC. Wealden; I. of Wight. M. Purbeck; Durdlestone Bay;, Swanage. [Portions of shell ; unknown.]

r.

sp., R. Lydekker, Cat. Foss. Rept. B. M., pt. iii, I889, p. I40.

Loc. Wadhurst Clay; Hastings. [Portions of shell; Brit. Mus., no. R. 970.]

TRIONצx, E. Geoffroy St. Hilaire., Ann. du Muséum, vol. xiv, I809, p. I.

Autacochelys, R. Lydekker, Ann. Mag. Nat. Hist. [6] vol, iii, 1889 , p. 53, and Cat. Foss. Rept. B. M., pt. iii, 1889, p. 22.

bakewelli, Mantell. v. Tretosternum bakewelli.

barbara, R. Owen, Rept. London Clay, pt. i (P.al. Soc., 1849), p. 50 , pl. xvi A ; J. Morris, Cat. Brit. Foss., 1854, p. 354 ; R. Lydekker, Cat. Foss. Rept. B. M., pt. iii, 1889, p. 13.

LOC. U. Eocene; Hordwell. [Carapace; Brit. Mus.]

bowerbanki, R. Lydekker, Cat. Foss. Rept. B. M., pt. iii, 1889. p. 19, woodcut 4 .

Trionyx sp., R. Owen, Rept. London Clay, pt. i (Pal. Soc, 


\section{TEION XX bowerbanki (continued)-}

1849), p. 6r, pl. xix D, f. 7, and in F. Dixon, Geol. Sussex, 1850, p. 221, pl. xii, f. 15, and ibid., ed. 2, 1878, p. 266, same pl. and fig. ; J. Miorris, Cat. Brit. Foss., 1854, p. 354. [Right hypoplastron; Brit. Mus.]

LoC. Bracklesham Beds. [Nuchal scute ; Brit. Mus.]

circumsulcatus, R. Owen, Rept. London Clay, pt. i (Pal. Soc., 1849), p. 59, pl. xix B, f. I, 2, 3 ; J. Morris, Cat. Brit. Foss., I 854 , p. 354 ; G. Baur, Ann. Mag. Nat. Hist. [6] vol. iii, 1889 , p. 273.

Aulacochelys circumsulcata, R. Lydekker, Ann. Mag. Nat. Hist. [6] vol. iii, 1889, p. 53, and Cat. Foss. Rept. B. M., pt. iii, 1889 , p. 22.

T. (Aulacochelys.)

LOC. U. Eocene; Hordwell. [Costal scute ; Brit. Mus.]

henrici, R. Owen, Rept. London Clay, pt. i (Pal. Soc., 1849), p. 46, pl. xvi ; J. Morris, Cat. Brit. Foss., 1854, p. 354; R. Lydekker, Cat. Foss. Rept. B. M., pt. iii, 1889, p. I3.

Trionyx marginatus, R. Owen, Rept. London Clay, pt. i (Pal. Soc., I849), p. 55, pl. xix*; J. Morris, Cat. Brit. Foss., 1854, p. 354. [Carapace; Brit. Mus.]

Trionyx, R. Owen, Rep. Brit. Assoc., I 847 (I848), Sect., p. 65. LOC. U. Eocene; Hordwell. [Carapace ; Brit. Mus.]

incrassatus, R. Owen, Rept. London Clay, pt. i (Pal. Soc., 1849), p. 5 I, pls. xvii, xviii, xix ; J. Morris, Cat. Brit. Foss., I 854 , p. 354 ; R. Lydekker, Cat. Foss. Rept. B. M., pt. iii, 1889 , p. I7.

LOC. U. Eocene ; Hordwell (I. of Wight, Owen errore). [Portion of carapace ; Brit. Mus.]

marginatus, Owen. v. Trionyx henrici.

planus, R. Owen, Rept. London Clay, pt. i (Pal. Soc., I849), p. 58, pl. xix C ; J. Morris, Cat. Brit. Foss., 1854, p. 354 ; R. Lydekker, Cat. Foss. Rept. B. M., pt. iii, 1889, p. 18.

LOC. U. Eocene; Barton and Hordwell. [Posterior portion of carapace ; Brit. Mus.]

pustulatus, R. Owen, Rept. London Clay, pt. i (Pal. Soc., 1849), p. 6o, pl. xix B, f. 7, 8, 9 ; J. Morris, Cat. Brit. Foss., 1854 , p. 354 .

LOC. London Clay; Sheppey. [Costal plate.]

rivosus, R. Owen, Rept. London Clay, pt. i (Pal. Soc., I849), p. 56, pl. xviii A ; J. Morris, Cat. Brit. Foss., 1854, p. 354 ; R. Lydekker, Cat. Foss. Rept. B. M., pt. iii, I889, p. 19. [? Immature T.planus.]

LOC. U. Eocene; Hordwell. [Posterior portion of carapace; Brit. Mus.]

sp, R. Owen, Rept. London Clay, pt. i (Pal, Soc., 1849), descr. to pl. xix D, f. 3-5 ; J. Morris, Cat. Brit. Foss., 1854, p. 354.

LOC. U. Eocene; Hordwell. [Cervical vertebra and pubis.] 
Tr2ONYX (continued)-

sp., R. Lydekker, Cat. Foss. Rept. B. M., pt. iii, 1889, p. Ir.

Loc. L. Miocene; Hempstead. [Costal plate; Brit. Mus., no. 36812 a.]

sp., R. Lydekker, ibid., p. 2 I.

LOC. London Clay; Sheppey. [Costal plate; Brit. Mus., no. 33303.]

sp., W. H. Bensted, Geologist, vol. v, 1862, p. 296, woodcut 2. [Referred to by R. Lydekker, Cat. Foss. Rept. B. M., pt. iii, I 889, p. 38, as either Chelone or Lytoloma].

LOC. Chalk; Rochester. [Femur ; Brit. Mus., no. 36254.]

sp., R. Owen, Rep. Brit. Assoc., I84I (1842), p. 168; P. Duff, Geol. Moray, 1842 , p. 65 , pl. v, f. 10. [Chelonian femur from the Rhætic of Linksfield, near Elgin, genus non det.; Brit. Mus.]

TROPIDEmYS, L. Rütimeyer, Neue Denkschr. schweiz. Ces. Nat., vol. xxv (1873), p. 27.

? langi, L. Rütimeyer, ibid., pp. 28-43, pl. vii, f. I, 3, 4, pl. x, f. 5, 6; R. Lydekker, Cat. Foss. Rept. B. M., pt. iii, 1889, p. I 56, woodcut 38 .

LOC. Kim. Clay; Weymouth. [Fragments of shell; Brit. Mus.]

Troprdonorvs, H. Kuhl, Ferussac's Bull. Sci., vol. ii, I824, p. 81 ; F. Boie, Isis (Oken), 1827, p. 518.

natrix (Linnæus), Boie, ibid; E. T. Newton, Geol. Mag. [2] vol. ix, 1882, p. 8, and Vert. Forest Bed (Mern. Geol. Surv., I882), p. II 4 , pl. xvii, f. 4,5 .

Coluber natrix, Linnæus, Syst. Nat., ed. 12, vol. i, 1766, p. 380

LOC. Forest Bed; West Runton and Bacton. [Vertebrce; E. T. Dowson Coll. and Owens College, Manchester.]

varawus, B. Merrem, Tent. Syst. Amphib., I 820 , p. 58.

margariticeps, Gervais. v. Placosaurus margariticeps.

vacTISA UR US, J. W. Hulke, Quart. Journ. Geol. Soc., vol. xxxv, I879, p. 421 .

valdensis, $J$. W. Hulke, ibid., p. 421 , pl. xxi.

LOC. Wealden; I. of Wight. [Vertebræ and ilium; J. W. Hulke Coll.]

VIPERA, J. N. Laurenti, Syn. Rept., 1768, p. 99.

berus (Linnæus), F. M. Daudin, Hist. Nat. Rept., vol. vi, an xi (1803), p. 89, pl. Ixxii.

Pelias berus, B. Merrem, Versuch Syst. Amphib. (Tent. Syst. Amphib.), 1820, p. 148; E. T. Newton, Geol. Mas. [2] vol. ix, I882, p. 8, and Vert. Forest Bed (Mem. Geol. Surv., 1882), p. 115 , pl. xvii, f. 6 .

Coluber berus, Linnæus, Syst. Nat., ed. I2, vol. i, I766, p. 377.

LOC. Forest Bed; West Runton. [Vertebra; Mus. Pract. Cicol.] 
ZAMCIODON, T. Plieninger, Württ. Jahresh., vol. ii, I846, p. 248. Teratosaurus, H. von Meyer, Palæontographica, vol. vii, I86I, p. 258 .

? \$p., R. Lydekker, Cat. Foss. Rept. B. M., pt. i, I888, p. 172.

LOC. Rhætic; Aust Cliff. [Phalangeal bones; Brit. Mus., no. 23812.]

? sp., R. Lydekker, ibid., p. I73, woodcut 28.

LOC. L. Lias; Lyme Regis. [Tooth; Brit, Mus., no. 41352.]

? sp.: Teratosaurus? sp., T. H. Huxley, Quart. Journ. Geol. Soc., vol. xxvi, $1870, \mathrm{p} .46$, pl. iii, f. 11 .

LOC. Keuper ; Warwickshire. [Tooth; Warwick Mus.]

\section{MISCELLANEOUS REPTIIIA.}

'Allegator,' Chapman and Wooller, 1758. \%. Steneosaurus chapmani.

'Axis of Ininosaur from Brook,' H. G. Seeley, Quart. Journ. Geol, Soc., vol. xxxi, 1875, p. 46.1, woodcuts I, 2. [? /:uanodon.] LOC. Wealden; Brook, I. of Wight. [Woodwardian Mus ]

'Axis of (? Theropodous) Dinosaur from the Wealden,' R. Lydekker, Quart. Journ. Geol. Soc., vol. xlv, I889, p. 44, woodcut 2.

L.OC. Wealden; I. of Wight. [Brit; Mus.]

'Bones, nature unknown,' Mantell, I $827 \ldots \%$. Hylæosaurus armatus.

'Cetiosaurus or Pelorosaurus,' Owen, 1884. v. Hoplosaurus armatus.

'Chelonian dentary bone,' A. S. Wroodward, Proc. Geol. Assoc., vol. x (1888), p. 276. ['Ordinal position uncertain,' R. Lydekker, Cat. Foss. Rept. B. M., pt. iii, 1889, p. 232.]

Orthagoriscus, F. Dixon, Geol. Sussex, 1850, descr. to pl. xxxii, f. 3,4 .

LOC. Chalk; Sussex. [Brit. Mus.]

'Chelonian scutes,' C. C. Blake, I863. v. Protochelys stricklandi.

'Coprolites,' W. Buckland, Trans. Geol. Soc. [2] vol. iii '(r829), p. 223, pls. xxviii-xxxi. [Original specimens, recent and fossil, in Mus. Geol. Soc. London.] T. Hawkins, Sea Dragons, 1840, pls. xxix, xxx. [Coprolites from Lias in Brit. Mus.]

'Crocodile,' Mantell, 1827. v. Goniopholis crassidens and Morosaurus brevis.

'Crocodilian jaw,' E. T. Newton, Quart. Journ. Geol. Soc., vol. xxxiv, 1878 , p. 398 , pl. xvi. [Regarded as probably Plesiosaurian by H. G. Seeley, ibid., p. 401.].

Loc. Corallian; Weymouth. [Imperfect mandible; Mus. Pract. Geol.]

'Crocodilian skull,' Hulke, I878. \%. Goniopholis sima. 
'Crocodilus saullii,' R. Owen, Brit. Foss. Rept., index to vol. ii, I834, p. vi.

Crocodilus?, R. Owen, Rept. Cret. Form. (Pal. Soc., 1851), p. 45, pl. xv.

Genus non det., A. S. Woodward, Geol. Mag. [3] vol. ii, 1885, table, p. 508, and Proc. Geol. Assoc., vol. ix (i886), p. 326.

LOC. Wealden; Hastings. [Slab with small bones and scutes ; Brit. Mus.]

'Curved spine,' Metcalfe, i 884. ข. Hyperodapedon gordoni.

'Dermal plates,' J. E. Lee, Ann. Mag. Nat. Hist., vol. xi, 1843, p. 5 , pl. i, f. C. [Probably Dinosaurian.]

LOC. Wealden; I. of Wight. [Specimen lost:]

'Dinosaurian ilium,' Hulke, I874. $v$. Iguanodon bernissartensis.

'Emys de Sheppey,' Cuvier, I824. v. Chrysemys testudiniformis.

'Emys de Sussex,' Cuvier, I824. \%. Hylæochelys belli.

'Fossil head of Tortoise,' Parkinson, I8I I. $v$. Lytoloma planimentum.

'Fossil Monitor of Mæstricht,' Mantell, I822. ข. Mosasaurus sp.

'Fossil Tortoise,' Parkinson, I8I I. v. Lytoloma longiceps.

'Fossil remains of an animal more nearly allied to the Fishes than' any of the other classes of animals,' E. Home, Phil. Trans., 1814 , p. 57 , pls. xvii-xx, and ibid., 1816, p. 318, pls. xiii-xvi. $=$ species of Ichthyosaurus ( $v$. I. platyodon.)

'Fossil skeleton,' Young, 1819. v. Steneosaurus chapmani.

'Fossil from Old Passage,' referred to Trionyx by J. Parkinson, Organic Remains, vol. iii, I8 I I, p. 269, pl. xviii, f. I. = Ceratodus latissimus. [PISCES.]

'Gavial of Tilgate Forest,' Mantell, I827. v. Suchosaurus cultridens.

'Gigantic fossil Saurian,' Owen, I842. $v$. Dinodocus mackesoni.

'Great Whitby Crocodile,' Young and Bird, 1822. v. Steneosaurus chapmani.

'Horn of Saurian,' Mantell, 1849. v. Iguanodon bernissartensis.

'Humerus of crocodile,' Mantell, r827. $v$. Iguanodon mantelli.

'Humerus of unknown animal,' Mantell, 1827. $v$. Iguanodon mantelli.

'Humerus of unknown aquatic animal,' Mantell, I827. v. Iguanodon bernissartensis.

'Lacertian vertebra,' Mantell, I849. $v$. Heterosuchus sp.

'Large animal,' Stukeley, 17 19. v. Plesiosaurus dolichodeirus.

'Large bones from Rhætic of Aust,' W. Sanders, Rep. Brit. Assoc., 1875 (1876), Sect., p. 80. [Probably Dinosaurian.]

Loc. Rhætic ; Aust Cliff. [Limb-bones ; Bristol Mus.]

'Large Reptilian skull from Brook,' Hulke, I871. ข. Iguanodon bernissartensis.

'Large Turtle,' Camper, 1786. v. Chelone hoffmanni. 
'Leathery Turtle,' A. S. Woodward, 1887. v. Psephophorus sp.

'Lizard or Turtle,' G. A. Mantell, Illustr. Geol. Sussex, 1827, pl. viii, f. 5, I5. Chelonian (R. Lydekker, Cat. Foss. Rept. B. M., pt. iii, s889, p. 203).

LOC. Wealden; Cuckfield. [Imperfect limb-bones; Brit. Mus.]

'Marine Turtle,' G. A. Mantell, Illustr. Geol. Sussex, 1827, pl. vii, f. $1,5,6$.

LOC. Wealden; Cuckfield. [Chelonian fragments ; Brit. Mus.]

'Oolithes bathonicæ,' J. Buckman, Quart. Journ. Geol. Soc., vol. xvi, I 860, p. 107, woodcuts I, 2 ; R. Lydekker, Cat. Foss. Rept. B. M., pt. i, 1888, p. 122 .

LOC. Gt. Oolite ; Somersetshire, Wiltshire, and Gloucestershire. [Eggs of reptiles.]

'Plastron from the Wealden,' Lydekker, I889. v. Archæochelys valdensis.

'Plectronites maximus corticeus,' Luidius, 1760. $\%$. Polyptychodon interruptus.

'Plesiosaurus,' Barrett, 1858. v. Plesiosaurus eleutheraxon.

'Pleurodont Eocene Lizard,' Owen, 1850. $v$. Lacerta eocena.

'Repsile or fish,' T. Smith, I846. v. Pachyrhizodus gardneri. [P1SCES.]

'Saurian,' D. Williams, Proc. Geol. Soc., vol. ii (1834), p. I12. = Thecodontosaurus and Palæosaurus.

'Saurian,' Green, 1842. v. Talpa europæus. [MAMmaLia.]

'Saurian animal,' Phillips, 1835. v. Ichthyosaurus acutirostris.

'Saurian bones from Loxwood,' Murchison, 1825. ข. Iguanodon mantelli.

'Saurian remains,' Riley and Stutchbury, 1836. v. Thecodontosaurus antiquus.

'Saurian vertebræ,' R. Owen, Quart. Journ. Geol. Soc., vol. xvi, I86o, p. 492, woodcuts $\mathrm{I}-\mathbf{5}$.

LOC. Rhætic ; Frome, Somersetshire. [Bath Mus.]

'Saurien encore plus extraordinaire,' Cuvier, 1824. $v$. Iguanodon mantelli.

'Scincoid Oolite Lizard,' R. Owen, Rep. Brit. Assoc., I841 (1842), p. 145 .

Loc. Stonesfield Slate. [Femur, etc.]

'Skeleton of four-footed reptile,' Brickenden, 1854. v. Telerpeton elginense.

'Small tooth from Leamington,' Murchison and Strickland. v. Thecodontosaurus sp.

'Swanage Crocodile,' Mantell, I839. v. Goniopholis crassidens.

'Sternal of Emys,' G. A. Mantell, Illustr. Geol. Sussex, 1827, pl. vi, f. 6. Loc. Wealden; Cuckfield. [Left hyoplastron; Brit. Mus.] 
'Sternal apparatus of Iguanodon,' J. W. Hulke, Quart. Journ. Geol. Soc., vol. xli, 1885, p. 473 , pl. xiv. [Variously interpreted by L. Dollo, Revue Quest. Scient., 1885, p. 664; G. Baur, Zool. Anzeiger, vol. viii, 1885, p. 561 ; E. D. Cope, Amer. Nat., I 886, p. 153; H. G. Seeley, Geol. Mag. '[3] vol. iv, 1887 , p. 84, and Proc. Roy. Soc., vol. xliii, 1887, p. 240 ; and R. Lydekker, Quart. Journ. Geol. Soc., vol. xliv, 1888, p. 52.$]$

'Undetermined bone,' G. A. Mantell, Phil. Trans., 1841, p. I48, pl. viii, f. 20. [Brit. Mus.]

LOC. Wealden; Hastings and Tilgate Forest. [S. H. Beckles Coll.]

'Teeth of a peculiar form,' Mantell, I839. $\%$. Pleurocœlus valdensis.

'Teeth of the Hylæosaur,' Owen, 1858. v. Pleurocolus valdensis.

'Thigh bone,' Platt, 1758. ข. Cetiosaurus oxoniensis.

'Tooth allied to Palcosaurus,' Strickland, 1842. ข. Palæosaurus stricklandi.

'Tooth of unknown Saurian,' Wright, 1852. v. Hoplosaurus armatus.

'Testudo remains,' Owen, 1841. v. Protochelys stricklandi.

Trionyx, Mantell, 1827. $v$. Tretosternum bakewelli.

'Tympanic bone of Iguanodon,' Mantell, I 833, and Owen, I841, I 855. v. Morosaurus brevis.

'Undescribed Wealden Vertebra,' Hulke, I870. \%. Ornithopsis hulkei.

'Undetermined bone,' Carter, 1888. $\%$. Hyperodapedon gordoni.

'Vertebra of unknown saurian,' Mantell, 1841. 2 . Morosaurus brevis.

'Very young Iguanodon,' Mantell, 1849. ข. Hypsilophodon foxi.

'Young Iguanodon,' Owen, 1864. v. Camptosaurus valdensis.

\section{C H N I T ES.}

Vide J. Morris, Cat. Brit. Foss., I854, pp. 355, 356, and the following references:-

S. M. Saxby, Phil. Mag. [3] vol. xxix, 1846, p. 310, woodcuts. [Greensand ; I. of Wight.]

J. Cunningham, Proc. Lit. Phil. Soc. Liverpool, vol. iv, 1848, p. 128, woodcuts. [Trias; Storeton and Flaybrick Hill Quarries.]

IV. Lee, Proc. Geol. Polyt. Soc. Yorkshire, vol. i, 1849, p. 409. [Carboniferous.]

E. W. Binney, Quart. Journ. Geol. Soc., vol. xii, 1856, p. 350. ['Chelichnus ingens,' Millstone Grit ; 'Tintwhistle.]

P. B. Brodie, ibial., vo!.xvi, I 860, p. 278. ['Chcirotherium,' U. Keuper; Shrewley and Rowington, near Warwick.]

W. Lister, Geologist, vol. iii, 1860, p. 308, and Rep. Brit. Assoc., I860 (1861), Sect., p. 87. ['Lubyrinthodon and Rhynchosaurws,' Trias; Brewood, North of Wolverhampton.] 
'T,' Geologist, vol. v, 1862, p. 432, woodcuts. [Stigmarian Sandstone.]

G. H. Morton, Proc. Liverpool Geol. Soc., sess.4, I863, p. I7. [Cheirotherium stortonense, Trias ; Storeton, Cheshire.]

W. C. Williamson, Quart. Journ. Geol. Soc., vol. xxiii, I867, p. 56, pl. iii. [Cheirotherian footprint, L. Keuper; Daresbury, Weston Point, Cheshire.]

T. P. Barkas, Coal Meas. Palæont., 1873, p. 53, f. I79, I8o. ['Platytherium psammobatis' (=? Crustacean tracks, fide R. Howse, in litt.).] Ibid., p. 55, f. 181, 182. ['Tridactylosaurus sandersoni.' (三 ? Crustacean tracks, fide R. Howse, in litt.).] [L. Carb. Limest.; Deanhead, Northumberland.]

P. Dudgeon, Proc. Roy. Soc. Edinb., vol. ix, 1876, p. 154 . [' Herpetichnus loxodactylus,' Permian; Locharbriggs Quarry, Dumfriesshire.]

T. H. Huxley, Mem. Geol. Surv. United Kingdom, Monogr. iii, r877, p. 45 , pls. xiv-xvi. [Stagonolepis, Trias; Cummingstone.]

W. J. Sollas, Quart. Journ. Geol. Soc., vol. xxxv, 1879, p. 5 I I, woodcut. [Trias; South Wales.]

T. H. Thomas, Trans. Cardiff Naturalists' Soc., vol. x, 1879, p. 72, pls. i, ii. [Idem.]

G. V. Smith, Rep. Brit. Assoc., 1883 (1884), p. 5 IO. [Penrith Sandstone ; near Penrith.]

C. Ricketts, Proc. Liverpool Geol. Soc., sess. 25, 1886, p. 168. [Rhynchosaurus, Trias; Oxton, Cheshire.]

H. M. Ormerod, Trans. Manchester Geol. Soc., vol. xviii (I886), p. 293. [L. Coal-Meas. ; Tyldesley.]

R. Etheridge, Foss. Brit. Islands, pt. i, I888, p. 375.

'T. P. Barkas, Rep. Brit. Assoc., I889. [Millstone Grit.] 
(301)

\section{AVES.}

Fx, F. Boie, Isis (Oken), I828, p. 329 (Aix).

? sponsa (Linnæus), F. Boie, ibid.

Anas sponsa, Linnæus, Syst. Nat., ed. 12, vol. i, 1766, p. 207 ; [-or] W. Buckland, Phil. Trans., 1822, p. 182 , pl. xxv, f. 28 , 29, and Reliq. Diluv., 1824, p. 35, pl. xi, f. 28, 29 ; [-or] S. Woodward, Syn. Tab., I830, p. 38 ; [-or] J. Morris, Cat. Brit. Foss., 1854, p. 356.

LoC. Cave; Kirkdale. [Coracoid.]

AIAUDA, Linnæus, Syst. Nat., ed. I2, vol. i, 1766, p. 287.

arvensis, Linnæus, ibid., p. 287 ; S. Woodward, Syn. Tab., 1830, p. 38 ; J. Morris, Cat. Brit. Foss., 1854, p. 356.

'Lark,' W. Buckland, Phil. Trans., I 822 , p. 182, pl. xxv, f. 24 , and Reliq. Diluv., I824, p. 35, pl, xi, f. 24.

Loc. Cave ; Kirkdale. [Ulna.]

AICA, Linnæus, Syst. Nat., ed. 12, vol. i, 1766, p. 2 10.

impennis, Linnæus, ibid., p. 2 Io ; R. Owen, Trans. Zool. Soc., vol. v (1864), p. 317, pls. li, lii; S. Laing, Prehist. Remains Caithness, 1866, p. 50, woodcut 57 ; R. Howse, Nat. Hist. Trans. Northumb. and Durham, vol. vii (1880), p. 361 ; S. Grieve, Journ. Linn. Soc. (Zool.), vol. xvi (1883), p. 479, pl. ix, and Trans. Edinb. Nat. Field Club, vol. i (I883), p. 58, and ibid., vol. ii (1888), p. 93, 2 pls., and 'The Great Auk, 4to. Lon:lon, 1885 ( $q$.v., for full literature and localities).

LOC. Pleist. ? and Recent; Cleadon Hills, Durham; Oronsay and Caithness.

torda (Linnæus): recorded by S. Laing, Prehist. Remains Caithness, i866, p. 50, from Kitchen Midden, Caithness.

ArAS, Linnæus, Syst. Nat., ed. 12, vol. i, 1766, p. 194.

boscas, Linnæus, ibid., p. 205 (boschas).

'Wild duck,' H. P. Blackmore, Edinb. New Phil. Journ., n.s., vol. xix, 1864, p. 74 .

LOC. River deposit ; Fisherton, Salisbury. [Egg-shells; Salisbury Mus.] 
AxAS (continued)-

leucopsis, Bechstein. v. Bernicla leucopsis.

mollissima, Linnæus. $v$. Somateria mollissima.

segetum, Gmelin. v. Anser segetum.

sp.: 'Duck,' H. W. Vernon, Phil. Mag., n.s., vol. vii, r830, p. 2 ;

J. F. Blake, Rep. Brit. Assoc., 1873 (1874), Sect., p. 75.

LOC. Pleist.; North Cliff, Yorkshire. [Ulna, tibia, and clavicle; York Mus.]

sp.: 'Humerus and radius of an aquatic bird of the Duck family,' W. Davies, Cat. Pleist. Vert. Coll. Brady, 1874, p. 6r.

LOC. Pleist. ; Ilford. [Brit. Mus.]

sp., E. T. Newton, Geol. Mag. [2] vol. ix, 1882, p. 7, and Vert.

Forest Bed (Mem. Geol. Surv., 1882), p. 113, pl. xvii, f. 2.

LOC. Forest Bed; West Runton. [Metacarpal and coracoid; A. Savin Coll.]

Axser, A. D. Brisson, Ornithologia, vol. vi, I760, p. 26 I (Anserinum), p. 262 (Anser).

? cinereus, B. Meyer, in Meyer and Wolf, Taschenb. deutsch. Vögelk., vol. ii, I810, p. 552.

Anser sp., ( $c f$. Gray Lag Goose), H. P. Blackmore, Edinb. New Phil. Journ., n.s., vol. xix, 1864, p. 74.

Anser ferus, J. F. Stephen, in G. Shaw, Zool., vol. xii, pt. ii, 1824, p. 28 ; J. F. Walker, Ann. Mag. Nat. Hist. [4] vol. ii, I868, p. 388. [Right humerus; J. F. Walker Coll.]

Anser palustris, J. Fleming, Brit. Anim., 1828, p. 126 ; femur and coracoid, doubtfully assigned to this form by J. Evans, Quart. Journ. Geol. Soc., vol. xx, 1864, p. 192. [Salisbury Mus.]

LOC. Fens ; Cambridgeshire. River deposit ; Fisherton, Salisbury, and West of Salisbury. [Bones and egg-shells; Salisbury Mus.]

ferus (Stephen), Walker. $v$. Anser cinereus.

leucopsis (Bechstein), Adams. $v$. Bernicla leucopsis.

palustris (Fleming), Evans. $\%$. Anser cinereus.

? segetum (Gmelin), A. L. Adams, Trans. Roy. Irish Acad., vol. xxvi (Sci.), 1876, p. 229.

Anas segetum, J. F. Gmelin, in Linnæus, Syst. Nat., ed. I3, vol. i, I788, p. 512.

Loc. Cave; Shandon, Waterford (Ireland). [Humerus, coracoid, etc.; Dublin Mus.]

sp., E. T. Newton, Geol. Mag. [2] vol. ix, I882, p. 7, and Vert. Forest Bed (Mem. Geol. Surv., 1882), p. I12, pl. xvii, f. I.

LOC. Forest Bed; East Runton. [Metacarpal ; A. Savin Coll.]

? sp.: '? Goose,' W. Buckland, Reliq. Diluv., r824, p. 27, pl. xiii, f. 9, Io.

LOC. ? Pleist. ; Lawford, near Rugby. [Humerus.] 
Aquila pelagica, Pallas. ข. Haliaetus pelagicus.

ARDEA, Brisson. [Grallator.] v. Miscellaneous Aves.

ARGIIIORNIS, R. Owen, Quart. Journ. Geol. Soc., vol. xxxiv, 1878 , p. 124.

? LithoRnis, J. S. Bowerbank (non Owen), Ann. Mag. Nat. Hist. [2] vol. xiv, 1854 , p. 263.

? Megalornis, H. G. Seeley, Ann. Mag. Nat. Hist. [3] vol. xviii, 1866, p. 110, and Quart. Journ. Geol. Soc., vol. xxx, I874, p. 708 .

longipennis, R. Owen, Quart. Journ. Geol. Soc., vol. xxxiv, 1878, p. 124, pl. vi (limb-bones), and ibid., vol. xxxvi, 1880, p. 23, pl. ii (skull).

? Lithornis emuinus, J. S. Bowerbank, Ann. Mag. Nat. Hist. [2] vol. xiv, I854, p. 263, woodcut. [Shaft of limb-bone ; Brit. Mus.

T. (Lithornis.)

? Megalornis emiinus, H. G. Seeley, Ann. Mag. Nat. Hist. [3] vol. xviii, 1866, p. I10, and Quart. Journ. Geol. Soc., vol. xxx, I874, p. 708, woodcut I. [Shaft of limb-bone; Woodwardian Mus.]

T. (Megalornis.)

'Gigantic bird from London Clay,' J. S. Bowerbank, Rep. Brit. Assoc., 185 I (1852), Sect., p. 55.

LOC. London Clay; Sheppey. [Limb-bones; Brit. Mus.] T.

BaRurCzA, F. Boie, Isis (Oken), I822, pt. i, p. 563.

? leucopsis (Bechstein), W. Macgillivray, Hist. Brit. Birds, vol. iv, 1852, p. 622.

Anas leucopsis, J. M. Bechstein, Ornith. Taschenb., pt. ii, r803, p. 424.

Anser leucopsis?, A. L. Adams, Trans. Roy. Irish Acad., vol. xxvi (Sci.), 1876, p. 229.

Loc. Cave; Shandon. [Coracoids; Dublin Mus.]

BUBO, Cuvier, Règne Anim., vol. i, 1817, p. 331.

maximus, J. Fleming, Brit. Anim., 1828, p. 57 ; E. T. Newton, Geol. Mag. [3] vol. iv, 1887 , p. I46, pl. iv, f. 3-5.

$[=B$. ignavus, Forster, vide R. B. Sharpe, Cat. Striges Brit. Mus., 1875, p. 14.]

Loc. Forest Bed; East Runton. [Tarso-metatarsus; A. Savin Coll.]

CIMOLIORNIS, Owen. v. Ornithocheirus. [ReptILIA.]

diomedeus, Owen. $v$. Ornithocheirus diomedius. [Reptilia.]

COIUnמBA, Linnæus, Syst. Nat., ed. 12, vol. i, 1766, p. 279.

sp., S. Woodward, Syn. Tab., 1830, p. 38 ; J. Morris, Cat. Brit. Foss., 1854, p. 356.

'Pigeon,' W. Buckland, Phil. Trans., 1822, p. 182, pl. xxv, f. 26,27 , and Reliq. Diluv., 1824, p. 35, pl. xi, fo 26, 27.

LOC. Cave ; Kirkdale. [Ulna.] 
COIצ מM BUS, Linnæus, Syst. Nat, ed. 12, vol. i, 1766, p. 220.

septentrionalis, Linnxus, ibid., p. 220 ; A. L. Adams, Trans. Roy. Irish Acad., vol, xxvi (Sci.), 1876, p. 229; E. T. Newton, Geol. Mag. [2] vol. x, 1883, p. 97, pl. iii.

LOC. Cave; Shandon (Ireland). [Husterus, femur, etc.; Dublin Mus.] River Bed; Mundesley. [Limb-bones; Mus. Pract. Geol.]

troile, Linnæus. $v$ Uria troile.

CORvUS, Linnæus, Syst. Nat., ed. 12, vol. i, 1766, p. 155.

corax, Linnæus, ibid., p. 155; S. Woodward, Syn. Tab., 1830, p. 38 ; J. Morris, Cat. Brit. Foss., 1854, p. 356 ; J. A. Smith, Proc. Soc. Antiq. Scotland, n.s., vol. iii, 1881, p. 364 ; A. L. Adams, Trans. Roy. Irish Acad,, vol. xxvi (Sci.), 1876, p. 229.

'Raven,' W. Buckland, Phil. Trans., 1822, p. 182, pl. xxv, f. 19-23, and Reliq. Diluv., 1824, p. 35, pl. xi, f. 19-23.

LOC. Cave; Kirkdale. [Ulna.] Shandon (Ireland). [Imperfect skeleton; Dublin Mus.]

? Pleist.; Dundas Castle, Linlithgowshire. [Femur.]

CYGNשS, J. M. Bechstein, Gemein. Naturg. Vögel Deutschl., vol, iv, pt. i, 1809, p. 814.

bewicki, [-ii] W. Yarrell, Trans. Linn. Soc., vol. xvi, 1833; p. 445 .

Cygnus bewicki (vel olor, vel ferus), A. L. Adams, Trans. Roy. Irish Acad., vol. xxvi (Sci.), 1876, p. 229.

LOC. Cave; Shandon (Ireland). [Humerus and scapula; Dublin Mus.]

ferus, Fleming, v. Cygnus musicus.

musicus, J. M. Bechstein, Gemein. Naturg. Vögel Deutschl, vol. iii, r 809, p. 830 .

Cygnus ferus, J. Fleming, Brit. Anim., I828, p. I26; R. Owen, Quart. Journ. Geol. Soc., vol. xii, I856, p. 2 II, pl. iii, f. I3.

LOC. Pleist. ; Grays. [Portion of tibia; Brit. Mus.]

olor, Gmelin. v. Cygnus bewicki.

DASORNIS, R. Owen, Trans. Zool. Soc., vol. vii (1869), p. 145.

londiniensis, R. Owen, ibid., p. 145, pl. xvi.

LOC. London Clay; Sheppey. [Cranium; Brit. Mus.]

DIOIIEDEA, Linnæus, Syst. Nat., ed. 12, vol. i, 1766, p. 214.

sp., R. Lydekker, Quart. Journ. Geol. Soc., vol. xlii, 1886, p. 366, woodcut 2.

'Wader,' A. and R. Bell, Proc. Geol. Assoc., vol. ii (1872), p. 202.

Loc. Red Crag; Foxhall. [Tarso-metatarsal ; Ipswich Mus.]

White Crag ; Orford. [UIna ; Mus. Pract. Geol.] 
INAIIORNIS, H. G. Seeley, Index to Aves, etc., Woodwardian Mus., I869, pp. xvii, 7 (name only).

Pelargonis, H. G. Seeley, Proc. Camb. Phil. Soc., vol. i (1864), p. 228 (name only) [misprint].

Pelagornis, H. G. Seeley (non E. Lartet, 1857), Ann. Mag. Nat. Hist. [3] vol. xviii, I866, p. I Io.

Paleocolymbus, H. G. Seeley, Proc. Camb. Phil. Soc., vol. i (1864), p. 228 (name only, misprinted P'alcoocolyntus).

barretti, H. G. Seeley, Index to Aves, etc., Woodwardian Mus., I869, p. xvii (name only), and Quart. Journ. Geol. Sor., vol. xxxii, I876, p. 496, pl. xxvi, f. I-I I, I4-27, and pl. xxvii, f. 4, $5,12,19-25$.

Palcocolyntus barretti, H. G. Seeley, Proc. Camb. Phil. Soc., vol. i (1864), p. 228 (name only) [misprint].

Palaocolymbus barretti, H. G. Seeley, Quart. Journ. Geol. Soc., vol. xxxii, 1876, p. 497, note.

Pelagornis barretti, H. G. Seeley, Ann. Mag. Nat. Hist. [3] vol. xviii, 1866, p. I1 10.

'Bird about the size of a woodcock,' R. Owen, Palrontology', ed. 2, I 861, p. 327.

LOC. Cambridge Greensand. [Bones; Woodwardian Mus.] 포.

sedgwick1, H. G. Seeley, Index to Aves, etc., Woodwardian Mi us., 1869, pp. xvii, 8 (name only), and Quart. Journ. Geol. Soc., vol. xxxii, 1876, p. 501, pl. xxvi, f. 12, 13, and pl. xxvii, f. 6-11, I $3-18$.

Pelastornis scdgrwicki, H. G. Seeley, Proc. Camb.Phil. Soc., vol. i, 1864, p. 228 (name only) [misprinted Pelargonis].

LOC. Cambridge Greensand. [Bones; Woodwardian Mus.]

IAICO, Linnæus, Syst. Nat., ed. 12, vol. i, 1766, p. 124.

sp. (cf. peregrimus, but larger), R. Owen, Brit. Foss. Mamm. and Birds, 1846 , p. 558.

Falco peregrimus, Gmelin (ex Brisson), in Linnæus, Syst. Nat., ed. I3, vol. i, I788, p. 272. = F. communis, Gmelin (cx Buffon), ibid., p. 270 (vidc R. B. Sharpe, Cat. Accipitres Brit. Mus., I 874, p. 376).

LOC. Cave ; Berry Head, Torbay. [Scapula, humerus, ulna, and metacarpus.]

GASTORIIS, E. Hébert, Comptes Rendus, vol. xl, I S;5, p. 579.

klaasseni, E. T. Newton, Proc. Zool. Soc., I885, p. 445, and ibid., 1889, p. 220, and Proc. Geol. Assoc., vol. ix (1886), p. 349, and Trans. Zool. Soc., vol. xii (I886), p. 143, pls. xxviii, xxix.

LOC. L. Eocene ('Blue Estuarine Beds') ; Park Hill, Croydon. [Limb-bones ; Mus. Pract. Geol.] 
GII5, J. M. Bechstein, Gemein. Naturg. Vögel. Deutschlands, vol. iii, 1793, p. 60.

cinerea, Naumann : recorded by R. Harkness, Rep. Brit. Assoc., 1870 (1871), p. 150, from Kitchen Midden of Ballycotton, Cork. [= Grus communis, Bechstein.]

XIACYORMIS, R. Owen, Brit. Foss. Mamm. and Birds, is 46 , p. 554 .

toliapicus, R. Owen, ihid., p. 554, wnodcuts 234, 235 ; J. Morris, Cat. Brit. Foss, 1854 , p. 356. [C.enus non det.]

Larustoliapicus, C. Koenig, Icon. Foss. Sect., 1825, pl. xvi, f. 193 (name only).

LOC. London Clay; Sheppey. [Cranium; Brit. Mus.] T.

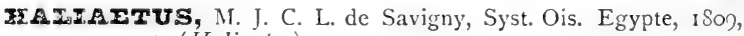
p. 254 (Haliaetus).

pelagfcus (Pallas), H. Woodward, Geol. Mag., vol. vi, I869, p. 387, and Trans. Essex Field Club, vol, iii, 1883, p. 7.

Aquila pelagica, P. S. Pallas, Zoogr. Rosso-Asiat., vol. i, ISI I, p. 343 , pl. xviii.

LOC. ?Pleist. ; Walthamstow. [Humerus; Brit. Mus.]

IAG IPU5, A. D. Brisson, Ornithologia, vol. i, I760, pp. I81, 216.

scoticus (Latham), A. L. Adams, Trans. Roy. Irish Acad., vol. xxvi (Sci.), 1876, p. 229.

Tetrao scoticus, J. Latham, Index Ornith., vol. ii, 1790, p. 641 . LOC. Cave; Shandon, Waterford (Ireland). [Humerus, femur, and coracoid; Dublin Mus.]

Ltorus toliapicus, Koenig. v. Halcyornis toliapicus.

LITIIORYIS, Bowerbank. v. Argillornis.

emuinus, Bowerbank. $v$. Argillornis longipennis.

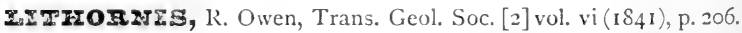

vulturinus, R. Owen, ibill., p. 206, pl. xxi, f. 5, 6, and Cat. Mamm. Aves Mus. Roy. Coll. Surgeons, I845, p. 337, and Brit. Foss. Mamm. and Birds, 1846, p. 549, woodcut 232; J. Morris, Cat. Brit. Foss., I 854 , p. 356.

LOC. London Clay; Sheppey. [Sternum, etc.; Brit. Mus.] $\boldsymbol{T}$.

MACRORNIS, H. G. Seeley, Ann. Mag. Nat. Hist. [3] vol. xviii, 1866, p. 110.

tanaupus, H. G. Seeley, ibil., p. I Io.

LOC. U. Eocene; Hordwell. [Proximal end of tibia; Woodwarảian Mus.] 
IIEGALORN WS, Seeley. \%. Argillornis.

emuinus, Seelcy. v. Argillornis longipennis.

ODONTOPrERyx, R. Owen, Quart. Journ. Geol. Soc., vol. xxix, 1873, p. 511 .

toliapicus, R. Owen, ibid., p. 511 , pls, xvi, xvii.

LOC. London Clay; Sheppey. [Skull ; Brit. Mus.]

T.

ardara, J. Fleming, Phil. Zool, vol. ii, IS22, p. 260 (Oidcmia).

?nigra (Linnæus), R. Walker, Ann. Mag. Nat. Hist, vol. xii, 1863, p. 387 (Oidemia).

Anas nigra, Linnæus, Syst. Nat., ed. 12, vol. i, 1766, p. 196.

LOC. ? Pleist.; Tyrie. [Portion of cranium; St. Andreas L1us.]

[perspicillata]: Anasperspicillatc, Linnæus, Syst. Nat., ed.12, vol. i, 1766, p. 201.

Oidema (surf duck), D. Page, in WV. Turner, Proc. Roy. Soc. Edinb., vol, vii (1870), p. 107, note.

LOC. ? Pleist.; Stratheden. [Skeleton; St. Andrewos .Mus.]

OrNitrom vol, xliii, 1887 , p. 206 .

cluniculus, H. G. Seeley, ibid., p. 205, pl. xii, f. I-5.

[Regarded as probably Reptilian by R. Lydekker, Cat. Foss. Rept. B. M., pt. i, 1888, p. 42.]

LOC. Wealden; Brook, I. of Wight. [Sacrum; Brit. Mus.] $\boldsymbol{x}$.

PALAOCOLYAIBUS, Seeley. \%. Enalionis.

barretti, Seeley. v. Enaliornis barretti.

I'alacolyntus buretti, Seeley. Misprint for Palæocolymbus. $q . \tau$.

PELAGORNIS, Seeley. v. Enaliornis.

barretti, Seeley. v. Enaliornis barretti.

sedgwicki, Seeley. $\%$ Enaliornis sedgwicki.

PALAORNIS, Mantell. \%. Ornithocheirus. [Reptilia.]

clifti, Mantell. \% Ornithocheirus clifti. [REPTILIA.]

PELARGONIS, Seeley. Misprint for Pelagornis, q.v.

PEIPCA ITUS, Linnxus, Syst. Nat., ed. 12, vol. i, 1766, p. 215.

carbo, Linnæus. $v$. Phalacrocorax carbo.

sp., A. Newton, Proc. Zool. Soc., 1868, p. 2, and ibil., I87r, p. 702 ; A. Milne-Edwards, Comptes Rendus, vol. lxvi, 1868, p. 1242, and Ann. Mag. Nat. Hist. [4] vol. ii, 1868, p. 165 .

LOC. ? Prehist.; Feltwell Fen, Norfolk. [Humerus; Woodwardian Mus.] 
PIRDIX, A. D. Brisson, Ornithologia, vol. i, 1760, p. 2 I9.

cinereus, J. Latham, Gen. Syn. Birds, Suppl. i, 1787, p. 290 ; [-a] S. Woodward, Syn. Tab., 1830, p. 38 ; [-a] J. Morris, Cat. Brit. Foss., 1854, p. 356.

Tetrao perdix, Linnæus, Syst. Nat., ed. I2, vol. i, I766, p. 276.

'Partridge,' J. Phillips, Geol. Yorkshire, vol. i, 1829, p. I77.

LOC. Cave; Kirkdale.

Pzamacrocorax, A. D. Brisson, Ornithologia, vol. vi, I76o, p. 5 II.

carbo (Linnreus), S. Laing, Prehist. Remains Caithness, I866, p. 50 ; E. T. Newton, Geol. Mag. [3] vol. iv, I887, p. I47, pl. iv, f. 8.

Pelecanus carbo, Linnæus, Syst. Nat., ed. 12, vol. i, 1766, p. 216. LOC. Forest Bed (freshwater bed); West Runton. [Coracoid; $W$. Barker Coll.] Kitchen Midden; Caithness.

graculus (Linnæus) : recorded by S. Laing, Prehist. Remains Caithness, 1866, p. 50, from Kitchen Midden, Caithness.

Prgmorñs, H. G. Seeley, Ann. Mag. Nat. Hist. [3] vol. xviii, I866, p. Iog (genus only).

Loc. Hempstead Beds. [Portion of coracoid; Woodwardian Mus.]

S COIOPAX, Linnæus, Syst. Nat., ed. 12, vol. i, 1766, p. 242.

? gallinago, Linnæus, ibid,, p. 244.

'Snipe?,' W. Buckland, Reliq. Diluv., 1824, p. 35, pl. xiii, f. I1, 12. LOC. Cave; Kirkdale. [Humerus.]

\$OMATERIA, F. Boie, Isis (Oken), I822, pt. i, p. 564.

mollissima (Linnæus), A. L. Adams, Trans. Roy. Irish Acad., vol. xxvi (Sci.), I 876, p. 229.

Somateria (surf duck), D. Page, Proc. Roy. Soc. Edinb., vol. vii (1870), p. 107, note.

Anas mollissima, Linnæus, Syst. Nat., ed. 12, vol. i, 1766, p. 198. LOC. Cave; Shandon. [Left coracoid; Dublin Mus.] ?Pleist. ; Stratheden. [Skeleton; St. Andrews Mus.]

SPATUTA, F. Boie (non Gray), Isis (Oken), I822, pt. i, p. 564.

clypeata (Linnæus), E. T. Newton, Geol. Mag. [3] vol. iv, 1887, p. 146 , pl. iv, f. 6,7 .

Anas clypeata, Linnæus, Syst. Nat., ed. 12, vol. i, I766, p. 200.

Loc. Forest Bed; West Runton. [Coracoid; W. Barker Coll.]

SI UR2

vulgaris, Linnæus, ibid., p. 290 ; S. Haughton, Proc. Roy. Irish Acad. [2] vol. ii (Sci.), 1876, p. 483.

LOC. Cave ; Knockninny (Ireland). [Skull.] 
SUIA, A. D. Brisson, Ornithologia, vol. vi, I760, p. 497.

basana (Linnæus): recorded by $S$. Laing, Prehist. Remains Caithness, 1866, p. 50, from Kitchen Midden, Caithness.

TETRA O, Linnæus, Syst. Nat., ed. I2, vol. i, I 766, p. 273.

perdix, Linnæus. $v$. Perdix cinerea.

scoticus, Latham. v. Lagopus scoticus.

URIA, A. D. Brisson, Ornithologia, vol. vi, I760, p. 70.

trolle (Linnæus), W. H. Flower, in Crowfoot and Dowson, Proc. Norwich Geol. Soc., vol. i (1878), p. 27.

Colymbus troile, Linnæus, Syst. Nat., ed. 12, vol. i, I765, p. 220. Loc. Norwich Crag ; Aldeby. [Humerus; Norwich Mus.]

\section{MISCELLANEOUS AVES.}

'Ardea? sp.,' G. A. Mantell, Illustr. Geol. Sussex, 1827, p. 82, pl. viii, f. I, 18. [Pterosaurian.]

LOC. Wealden; Tilgate Forest. [Shafts of limb-bones.]

'Avis ex palmipedum ordine?', Koenig, 1825. = Bucklandium. [PISCES.]

'? Bird,' G. A. Mantell, Illustr. Geol. Sussex, 1827, pl. viii, f. 17. $=$ Chelonian (R. Lydekker, Cat. Foss. Rept. B. M., pt. iii, I 889, p. 203).

LOC. Wealden; Cuckfield. [Imperfect femur ; Brit. Mus.]

'Bird about the size of a woodcock,' Owen, 186r. $\%$. Enaliornis barretti.

'Bird remains,' R. Owen, Brit. Foss. Mamm. and Birds, 1846, p. 557 [humerus of 'bird of flight, size of barn owl,' Norwich Crav'; Fitch Coll.] ; ibid., p. 557 [tarso-metatarsal, Eocene, Hordwell ; Oxford Mus. (olim J. W. Flower Coll.)]

'Bird-bone,' Mantell, I827. v. Ornithocheirus clifti. [Reptilia.]

'Bird remains,' J. Prestwich, Quart. Journ. Geol. Soc., vol. x, I854, p. 157 (Woolwich and Reading Beds; Counter Hill). [Olim De la Condamine Coll.]

[Grallator] (cf. Ardeidæ), R. Owen, Brit. Foss. Mamm. and Birds, 1846, p. 556, woodcut 236.

LOC. London Clay; Primrose Hill. [Sternum; Brit. Mus.]

'Ornitholite,' Owen, I84I. v. Ornithocheirus diomedius. [REPTILIA.]

'Wader,' Bell, 1872. ข. Diomedea sp. 
'Remains of Birds,' J. B. P. Dennis, Quart. Journ. Micros. Soc., vol. v 1857, p. 63, pl., and in S. J. Mackie, Geologist, 1864, p. 18, pl. xii, f. 9-I6. [See also J. Phillips, Geol. Oxford, 187I, p. 229.]

LOC. Stonesfield Slate. [=Pterosaurian Bones.]

'Sacrum of a Bird,' R. Owen, Brit. Foss. Mamm. and Birds, 1846, p. 553 , woodcut 233 .

Loc. London Clay; Sheppey. [Brit. Mus.]

[Remainz of the following birds have been recorded from the Peat of the Fenland, by Alfred Bell, in Miller and Skertchley, Fenland, I878, p. 340:-Ardea stellaris, Cygnus musicus, Cygnus olor, Fulica atra, Pelecanus crispus or onocrotalus, Podiceps cristatus, Querquedula creca: the majority probably belong to the Historic Period.] 


\section{$(3 \operatorname{Ir})$ \\ MAMMALIA.}

ACERATHERIUM, Kaup. a'. Rhinoceros.

incisivrm, Kaup. $\%$ Rhinoceros incisivus.

ACFIRODON, R. Owen, Brit. Mesoz. Mamm. (Pal. Soc., i87I), p. 37 .

nanus, R. Owen, ibid., p. 37 , pl. ii, f. 5-7; R. Lydekker, Cat. Foss. Mamm. B. M., pt. v, 1887, p. 276 ; H. F. Osborn, Proc. Acad. Nat. Sci. Philad., I888, p. 299.

LOC. M. Purbeck; Durdlestone Bay. [Mandib. rami; Brit. Mus.]

pusillus, R. Owen, Brit. Mesoz. Mamm. (Pal. Soc., 1871), p. 39, pl. ii, f. 8 ; R. Lydekker, Cat. Foss. Mamm. B. M., pt. v, 1887, p. 277 ; H. F. Osborn, Proc. Acad. Nat. Sci. Plilad., I 888 , p. 299.

LOC. M. Purbeck; Durdlestone Bay. [Left mandib. ramus; Brit. Mus.]

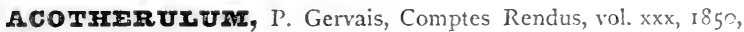
p. 604 .

saturninum, P. Gervais, ibid., p. 604 ; R. Lydekker, Cat. Foss. Mamm. B. M., pt. ii, 1885 , p. I82.

Loc. Headon Beds; Hordwell. Bembridge? Beds; Isle of Wight. [Tecth; Brit. Mus.]

ADAPIS, Cuvier, Ossem. Fossiles, ed. 2, vol. iii, 1822, p. 265.

magna, [-us] H. Filhol, Ann. Sci. Gćol., vol. v, 1874, art. 4, p. 18 ; R. Lydekker, Cat. Foss. Mamm. B. M., pt. i, 1885 , p. 262.

I.OC. Headon Beds; Hordwell. [Portions of jaws; Brit. Mus.]

AIIURUS, F. Cuvier, Hist. Nat. Mamm. (IS25), livr. $5 \mathrm{c}$.

anglicus, W. B. Dawkins, Quart. Journ. Geol. Soc., vol. xliv I888, p. 228, pl. x.

LOC. Red Crag; Fe'ixstowe. [Portion of mandible; York Mus.] 
Aluctherium huxleyi (Lankester), Van Beneden. v. Trichechus huxleyi.

AICES, Hamilton Smith, in Griffith's Animal Kingdom, vol. v, 1827, p. 303 (Alce). [Non Alie, Blumenbach, 1803: ข. p. 332 .

gigantea, Blumenbach. $v$. Cervus giganteus.

latifrons (Johnson), R. Lydekker, Cat. Foss. Mamm. B. M., pt. ii, 1885 , p. 78 ; W. B. Dawkins, Brit. Pleist. Mamm., pt. vi (Pal. Soc., I 887 ), p. I, pl. i.

Cervus latifrons, R. Johnson, Ann. Mag. Nat. Hist. [4] vol. xiii, 1874 , p. 2, pl. i ; E. T. Newton, Geol. Mag. [2] vol. vii, 1880, p. 45 I, and Vert. Forest Bed (Mem. Geol. Surv., 1882), p. 58.

Cervus (Megaceros) latifrons, R. Johnson, Trans. Norfolk and Norwich Nat. Soc., vol. ii (1877), p. 289.

Cirvus booides, R. Johnson (ex Gunn, MS.), Trans. Norfolk and Norwich Nat. Soc., vol. ii (1877), p. 290.

Cervus bovides, E. T. Newton, Geol. Mag. [2] vol. vii, I880, p. 449, and Vert. Forest Bed (Mem. Geol. Survey, 1882), p. 52 .

Loc: Forest Bed; near Cromer, Dogger Bank, Happisburgh. [Base of antler; Colman Coll., Corton.]

machuis, W. Ogilby, Proc. Zool. Soc., 1836, p. 135 ; E. Tindall, Proc. Geol. Polyt. Soc. Yorksh., vol.v (1870), p. Io; J. Young, Proc. Nat. Hist. Soc. Glasgow, vol. ii (1876), p. 176 ; R. Lydekker, Cat. Foss. Mamm. B. M., pt. ii, I885, p. 78 ; W. B. Dawkins, Brit. Pleist. Mamm., pt. vi (Pal. Soc., 1887), p. 3 .

Cervus alces, Linnæus, Syst. Nat., ed. 12, vol. i, 1766, p. 92 ; W. Thompson, Proc. Zool. Soc., I 837, p. 53; R. Howse, Trans. Tyneside Nat. Field Club, vol. v (I861), p. 113, pl.v ; A. L. Adams, Journ. Roy. Geol. Soc. Ireland, vol. iv (1877), p. 248 ('specimen imported').

Cervus alces (Alces machlis), J. A. Smith, Proc. Soc. Antiq. Scotland, vol. ix (1872), p. 279, 7 woodcuts, and ibid., n.s., vol. v (1883), p. 325, woodcut.

Alces palmatus, R. Owen, Geol. Mag., vol. vi, I869, p. 389.

LOC. Pleist. and Prehist.; Bridlington, Carnaby, Chirdon Burn, Durham, Hartlepool, Mainfort near Sedgefield, Walthamstow, Wetton.-Auchtergaven, Airleywight, Brechin, North Berwick, Coldingham, Dunse, Duddingstone Loch, Forfarshire, Greycrook, Hawick, Kinloch, Kirkurd, Marlee, Mertoun, Oakwood, Selkirk, Strath Halladale, Trinity Muir, Whitrig Bog, Williesstruther Loch, Wigtown (Scotland). - Stewardstown, Tyrone (Ireland).

Cave ; Llandebie.

palmatus, Owen. v. Alces machlis. 


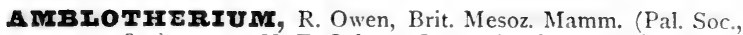
1871), p. 29: H. F. Osborn, Journ. Acad. Nat. Sci. Philad., vol. ix, 1888, p. 199, and Proc. Acad. Nat. Sci. Philad., I888, p. 299.

Peraspalax, R. Owen, Brit. Mesoz. Mamm. (Pal. Soc., I871), p. 40.

Peralestes (Phascolestes), R. Owen (pars), ibid., p. 35 ; H. F. Osborn, Journ. Acad. Nat. Sci. Philad., vol. ix (1888), p. 200, and Proc. Acad. Nat. Sci. Philad., I888, pp. 298, 300 .

? dubius: Phascolestes dubius, R. Owen, Brit. Mesoz. Mamm. (Pal. Soc., 187 I), descr. to pl. i, f. 4 I.

Indet., R. Lydekker, Cat. Foss. Mamm. B. M., pt. v, I887, p. 294 .

LOC. M. Purbeck; Durdlestone Bay. [Mandib. ramus; Brit. Mus.]

mustelula, R. Owen, Brit. Mesoz. Mamm. (Pal. Soc., 187I), p. 3I, pl. ii, f. 2 ; R. Lydekker, Cat. Foss. Mamm. B. M., pt. v, I887, p. 275 ; H. F. Osborn, Proc. Acad. Nat. Sci. Philad., 1888, p. 299.

Peralestes (Phascolestes?) longirostris, R. Owen (pars), Brit. Mesoz. Mamm. (Pal. Soc., 1871), p. 35, pl. ii, f. 4 .

LOC. M. Purbeck; Durdlestone Bay. [Right mandib. ramus; Brit. Mus.]

soricinum, R. Owen, Brit. Mesoz. Mamm. (Pal. Soc., I87I), p. 29, pl. ii, f. I ; R. Lydekker, Cat. Foss. Mamm. B. M., pt. v, 1887 , p. 274 ; H. F. Osborn, Proc. Acad. Nat. Sci. Philad., 1888, p. 299. [? = Stylodon robustus. $]$

LOC. M. Purbeck; Durdlestone Bay. [Right mandib. ramus; Brit. Mus.]

talpoides (Owen), R. Lydekker, Cat. Foss. Mamm. B. M., pt. v, I 887, p. 275.

Peraspalax talpoides, R. Owen, Brit. Mesoz. Mamm. (Pal. Soc., I 87 I), p. 40, pl. ii, f. 9, 9 A ; H. F. Ostorn, Proc. Acad. Nat. Sci. Philad., 1888, p. 299.

T. (Peraspalax.)

LOC. M. Purbeck; Durdlestone Bay. [Left mandib. ramus; Brit. Mus.]

A МPHICETUS, Van Beneden. $\%$. Cetotherium.

AMPHIGONUS, Agassiz. v. Amphitherium.

AMPIIIESTrS, R. Owen, Encyclop. Brit., ed, 8, vol. xvii, I 850, p. I57; H. F. Osborn, Journ. Acad. Nat. Sci. Philad., vol. ix (1888), p. 193, and Proc. Acad. Nat. Sci. Philad., 1888, p. 292.

broderipi, [-ii] R. Owen, Palæontology, ed. 2, I86I, p. 341 ; R. Lydekker, Cat. Foss. Mamm. B. M., pt. v, 1887, p. 271, woodcut $4 \mathrm{I}$.

Thylacotherium prevostii, R. Owen (errore), Trans. Geol. Soc. [2] vol. vi (1841), p. 49, pl. v, f. I. 


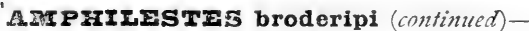

Amphitherium broderipii, R. Owen, Odontogr. (1845), p. 377, pl. xcix, f. 3, and Brit. Foss. Mamm., I846, p. 58, woodcut 19, and Brit. Mesoz. Mamm. (Pal. Soc., 1871), p. 15, pl. i, f. 25 ('? Amphilestes') ; J. Morris, Cat. Brit. Foss., 1854, p. 357 ; J. Phillips, Geol. Oxford, 1871, p. 234, woodcut lxxx. Amplitherium prevostii, R. Owen (errore), Encycl. Brit., ed. 8, vol. xvii, 1859, p. 157, woodcut 80 , and Palreont., ed.2, 1861, p. 340, woodcut I I 2 .

LOC. Stonesfield Slate. [Mandib. ramus; York Mus.]

? sp., R. Lydekker, Cat. Foss. Mamm. B. M., pt. v, 1887, p. 272.

Phascolotherium bucklandi, J. Phillips (errore), Geol. Oxford, I87I, p. 235, woodcut lxxxi.

LOC. Stonesfield Slate. [Mandib. ramus; Oxford Mus.]

AIIPIITHERIUIM, H. D. de Blainville, Comptes Rendus, vol. vii, I838, p. 417 ; H. F. Osborn, Journ. Acad. Nat. Sci. Philad., vol. ix (1888), p. I9I, and Proc. Acad. Nat. Sci. Philad., I888, p. 296.

Thylacotherium, A. Valenciennes (pars), Comptes Rendus, vol. vii, 1838, p. 580 .

Amphigonus, L. Agassiz, in Buckland, Geol. und Mineral. (German ed.), vol. ii, I839, descr. to pl. ii, p. 3, footnote.

broderipi, Owen. v. Amphilestes broderipi.

prevosti, [-ii] H. D. de Blainville (ex Cuvier, MS.), Comptes Rendus, vol. vii, I838, p. 417 ; [-ii] R. Owen, Odontogr. (1845), p. 377, pl. xcix, f. 2, and Brit. Foss. Mamm., 1846, p. 44, woodcuts I6, I7, and Brit. Mesoz. Mamm. (Pal. Soc., 1871), p. 12, pl. i, f. 21 , 22 ; [-ii] J. Morris, Cat. Brit. Foss., I854, p. 357 ; [-ii] J. Phillips, Geol. Oxford, I87I, p. 233, woodcut lxxvii ; R. Lydekker, Cat. Foss. Mamm. B. M., pt. v, I887, p. 273. [See also Amphitylus oweni and Amphilestes broderipi.]

Didelphis prevostii, H. D. de Blainville, Comptes Rendus, vol. vii, I 838, p. 403 .

Thylacotherium prevostiz, A. Valenciennes, Comptes Rendus, vol. vii, 1838 , p. 580 ; R. Owen, Trans. Geol. Soc. [2] vol. vi (I 84 I), p. 49, pl. v, f. 3.

'Didelphe,' Cuvier, Ossem. Foss., vol. v, pt. ii, 1824, p. 349.

'Didelphe,' C. Prevost, Ann. Sci. Nat., vol. iv, 1825, p. 397 , pl. xviii, f. 2. [Mandib. ramus; Brochant Coll.]

'Small Didelphys,' IV. Buckland, Geol. and Mineral., ed. 2, I837, vol. ii, p. I8, pl. ii, f. B.

Loc. Stonesfield Slate. [Mandib. ramus; Oxford Mus.]

T.

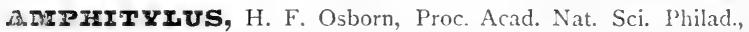
I887, p. 283, and ibir'., I888, p. 294.

oweni, H. F. Osborn, Journ. Acad. Nat. Sci. Philad., vol. ix (I888), p. 228 , woodcut 106 . 
AMPIITYIUS oweni (continued)-

Amphitherium prevostii, R. Owen (errore), Odontogr. (1845), p. 377 , pl. xcix, f. 1, and Brit. Foss. Mamm., I 846, p. 29, woodcut 15, and Brit. Mesoz. Mamm. (Pal. Soc., I871), p. 12, pl. i, f. 23 ; J. Phillips, Geol. Oxford, I871, p. 234, woodcuts lxxviii, lxxix.

Gemus non det., R. Lydekker, Cat. Foss. Mamm. B. M., pt. v, I887, p. 27 I, note I, and p. 273.

LOC. Stonesfield Slate. [Mandib. ramus; Oxford Mus.] IJ

Ascrinorums, P. Gervais, Zool. et Pal. Franç., ed. I, vol. ii (1848-52), Expl. to pl. xxxv, p. 8.

desmaresti, P. Gervais, ibid., p. 8; R. Lydekker, Cat. Foss. Mamm. B. M., pt. iii, 1886, p. 43.

Anchitherium radegondense?, P. Gervais, Geologist, vol. i, I 858 , p. I 53 , woodcuts I -5 .

LOC. U. Eocene; Bembridge and Headon Hill. [Feeth; Brit. Mus.]

Anchitherium radegondense, Gervais. v. Anchilophus desmaresti.

ANOPZOTMERIIM, Cuvier, Ann. du Muséum, vol. iii, I804, p. 370 .

Eurythririum, P. Gervais, Zool. et Pal. Franç., ed. I, vol. ii $(1848-52)$, Expl. to pl. xxxvi, p. 3 .

commune, Cuvier, Ann. du Muséum, vol. iii, I804, p. 469 ; R. Owen, Trans. Geol. Soc. [2] vol. vi (184I), p. 44, pl. iv, f. 5 , and Brit. Foss. Mamm., I846, p. 432, woodcuts I76, I78, and I79, and Odontography (1845), p. 524, pl. cxxxv, f. I, 2 ; J. Morris, Cat. Brit. Foss., I 854 , p. 357 ; R. Lydekker, Cat. Foss. Mamm. B. M., pt. ii., I885, p. 189.

? Eurytherium latipes, P. Gervais, Zool. et Pal. Franç., ed. I, vol. ii (1848-52), Expl. to pl. xxxvi, p. 3.

LOC. U. Eocene; Binstead and Seafield. [Teeth; Brit. Mus.]

lichobunes, Cuvier, MS. \%. Dichodon cervinus.

secundarium, Cuvier, Ossem. Foss., ed. 2, vol. iii, 1822, p. 59, pl. xi, f. 2 , and ibid., vol. v, pt. ii, 1824 , p. 528 ; R. Uwen, Trans. Geol. Soc. [2] vol. vi (1841), p. 44, pl. iv, f. 4, and Brit. Foss. Mamm., 1846, p. 434, woodcut 177 ; J. Morris, Cat. Brit. Foss., I854, p. 357 ; R. Lydekker, Cat. Fuss. Mamm. B. M., pt. ii, 1885 , p. 194.

LOC. U. Eocene; Binstead and Seafield. [Toot/t.]

'ANOPLOTHERIUM,' R. Owen, Rep. Brit. Assoc., I842 (1843), pp. 57, 74. Vide 'Group of bones', Miscellanious MAMMALIA.

ANTHRACOTHzRIUra, Cuvier, Ossem. Fossiles, ed. 2, vol. it, 1822, p. 396.

TAPINodon, H. von Meyer, Neues Jahrb., IS46, P. 471. 
ANTHRACOTHERIUM (continued)-

alsaticum, Cuvier, ibid., ed. 2, vol. iv, 1823, p. 500, and ibid., yol. v, pt. ii, 1824, p. 528 ; R. Lydekker, Cat. Foss. Mamm. B. M., pt. ii, I885, p. 239.

Anthracotherium sp. (cf. alsaticum), R. Lydekker, Geol. Mag. [3] vol. i, 1884, p. 548.

LOC. Hempstead Beds; I. of Wight. [Tooth; Brit. Mus.]

gresslyi (H. von Meyer), R. Lydekker, Geol. Mag. [3] vol. ii, I885, p. 69, woodcuts 5, 6, and Cat. Foss. Mamm. B. M., pt. ii, 1885, p. 244 , woodcuts 35,36 .

Tapinodon gresslyi, H. von Meyer, Neues Jahrb., 1846, p. 47 I.

Hyopotamus gresslyi, L. Rütimeyer, Denkschr. schweiz. Ges. Nat., vol. xix, art. 3 (I862), p. 7 o.

LOC. Headon Beds; Hordwell. Bembridge Beds; I. of Wight. [Skull and mandible; Brit. Mus.]

minus, Cuvier, Ossem. Fossiles, ed. 2, vol. iii, 1822, p. 403, and ibid., vol. v, pt. ii, I 824 , p. 528 ; R. Lydekker, Cat. Foss. Mamm. B. M., pt. ii, 1885, p. 242.

Anthracotherium sp. (cf. minus), R. Lydekker, Geol. Mag. [3] vol. i, I884, p. 548.

LOC. Hempstead Beds; I. of Wight. [Portion of mandib.; Brit. Mus.]

velamus, Cuvier. v. Hyopotamus velaunus.

sp., R. Lydekker, Cat. Foss. Mamm. B. M., pt. ii, 1885, p. 247. LOC. Headon Beds; Hordwell. [Right ramus of mandible; Brit. Mus., no. 29856.]

ARGIIIOTBrgrum, W. Davies, Geol. Mag. [3] vol. i, I884, p. 438.

toliapicum, W. Davies, ibid., p. 438 ; R. Lydekker, Cat. Foss. Mamm. B. M., pt. i, 1885, p. 4I ; W. Branco, Neues Jahrb., I 885 , pt. ii, p. I 45 .

LOC. London Clay; Sheppey. [Imperfect skull; Brit. Mus.]

ARVICOLA, De Lacépède. v. Microtus.

agrestis, Linnæus. v. Microtus agrestis and M. arvalis. amphibius, Linnaus. $\%$ Microtus amphibius.

ervalis, Pallas. v. Microtus arvalis.

glareolus, Schreber. v. Microtus glareolus.

gregalis, Newton. v. Microtus gregalis.

gulielmi, Sanford. v. Myodes torquatus.

intermedius, Newton. " Microtus intermedius.

mivalis, Martins. $v$. Microtus nivalis.

pratensis, Baillon. च. Microtus glareolus.

retticeps, Keyserling and Blasius. $\%$ Microtus ratticeps.

riparia, Yarrell. v. Microtus glareolus. 
Asimus fossilis, Owen. v. Equus caballus.

ATHRODON, Osborn. v. Kurtodon. pusillus, Osborn. $v_{0}$ Kurtodon pusillus.

Axrs, Hamilton Smith, in Griffith's Animal Kingdom, vol.v, I827, p. 312 .

sp., H. Falconer, Palront. Mem., vol. ii, 1868, p. 480 (name only); E. T. Newton, Vert. Forest Bed (Mem. Geol. Surv., I882), p. 64 (has not seen specimen, but suggests it is very doubtful and was probably obtained from the Norwich Crag). [Portion of antler.]

BAIreNA, Linnæus, Syst. Nat, ed. I2, vol. i, 1766, p. 105.

Balaenula, P. J. Van Beneden, Bull. Acad. Roy. Belg. [2] vol. xxxiv, 1872 , p. II.

Balaenotus, P. J. Van Beneden, Bull. Acad. Roy. Belg. [2] vol. xxxiv, 1872 , p. I3.

affinis, R. Owen, Proc. Geol. Soc., vol. iv (I843), p. 285, woodcut ; R. Lydekker, Quart. Journ. Geol. Soc., vol. xlini, 1887 , p. 8, and Cat. Foss. Mamm. B. M., pt. v, 1887, p. 17, woodcut 7 .

Balanodon affinis, R. Owen, Brit. Foss. Mamm., I846, table facing p. xlvi, p. 530, woodcut 22I (Balana); J. Morris, Cat. Brit. Foss., 1854, p. 357.

Loc. Red Crag; Felixstowe and Woodbridge. [Tympanic bone ; cast in Brit. Mus.]

balænopsis (Van Beneden), R. Lydekker, Quart. Journ. Geol. Soc., vol. xliii, I887, p. Io, and Cat. Foss. Mamm. B. M., pt. v, 1887, p. 23.

Balaenula balaenopsis, P. J. Van Beneden, Bull. Acad. Roy. Belg. [2] vol. xxxiv, I872, p. II.

Loc. White Crag; Sudbourn. Red Crag; Boyton and Woodbridge. [Tympanic bone; Ipswich Mus. Allas vertebra; Brit. Mus.]

biscayensis, J. E. Gray, Proc. Zool. Soc.. 1864, p. 201 ; E. T. Newton, Quart. Journ. Geol. Soc., vol. xlii, 1886, p. 319 , pl. xi, f. 5-8; R. Lydekker, Cat. Foss. Mamm. B. M., pt. v, I887, p. 19.

'Cetacean vertebræ,' E. T. Newton, Quart. Journ. Geol. Scc., vol. xlii, 1886, p. 322. [W. Barker Coll.]

Balanoptera, E. T. Newton, Vert. Forest Bed (Mem. Geol. Surv., 1882), p. 108 (pars).

Loc. Forest Bed; Overstrand. [Cervical vertebra; J. Backhouse Coll.]

River deposit; Thames (Wapping). (Also specimens from Eastern Coast and North Sea, Lewes, and Wandsworth, are provisionally referred to this species by Lydekiker). 
IAIFE⿱A (continued)-

boops, Linnæus. $v$. Megaptera boops.

alefineta, Owen. v. Balænoptera definita,

cmarginata, Owen. $v$. Balæenoptera emarginata.

yabosa, Owen. v. Balænoptera emarginata.

zosignis (Van Beneden), R. Lydekker, Cat. Foss. Mamm. B. M., pt. v, 1887, p. 26.

Balaenotus insignis, P. J. Van Beneden, Bull. Acad. Roy. Belg. [2] vol. xxxiv, I872, p. I3.

LOC. Red Crag; Felixstowe and Woodbridge. [Tympanic bones; Brit. Mus.]

meteculus, Linnæus. $\%$ Balænoptera musculus.

roysticetus, Linnæus, Syst. Nat., ed. 12, vol. i, 1766, p. 105 ; R. Lydekker, Cat. Foss. Mamm. B. M., pt. v, I887, p. I7. [See also Balanoptera borealis.]

LOC. River deposit; Deptford and Limehouse. [Probably introduced]. [Scapula and cervical vertebra; Brit. Mus.]

primigenia, [-us] P. J. Van Beneden, Bull, Acad. Roy. Belg. [2] vol. xxxiv, 1872, p. 9 ; R. Lydekker, Quart. Journ. Geol. Soc., vol. xliii, I887, p. 9, woodcut I, pl. ii, f. I, 2, and Cat. Foss. Mamm. B. M., pt. v, 1887 , p. $2 \mathrm{I}$, woodcut 8.

LoC. Red Crag; Woodbridge, Suffolk. [Tympanic bones; Brit. Mus, and $W$. Colchester Coll.]

rostrata, Müller. $v$. Hyperoodon rostratus.

? \$p.y E. Crisp, Rep. Brit. Assoc., I 868 (I869), Sect., p. 6I.

LOC. Chillesford Clay; East Coast, Suffolk. [Skeleton; destroyed during excavation.]

SAEIFODON, R. Owen, Brit. Foss. Mamm., I 846 , p. 536 ; R. Lydekker, Quart. Journ. Geol. Soc, vol. xliii, I887, p. If. affinis, Owen. v. Balrena affinis.

refinitu, Owen. v. Balænoptera definita.

emaryinatı, Owen. $\%$. Balænoptera emarginata.

yibbosc, Owen. $v$. Balænoptera emarginata.

physaloldes, R. Owen, Brit. Foss. Mamm., 1846, pp. 525, 536 , woodcuts 219, 226, 227 ; J. Morris, Cat. Brit. Foss., 1854 , p. 358 ; R. Lydekker, Cat. Foss. Mamm. B. M., pt. v, I887, p. 58.

Eelemnoziphius and Squalodon (?), E. R. Lankester, Quart. Journ. Geol. Scc., vol. xxi, 1865, p. 23I, pl. xi, f. 3, 5, 6, 7.

Loc. Red Crag; Felixstowe. [Portion of tooth; Brit. Mus.] $\mathbf{T}$.

EA I des Ordres, etc., p. xxxvi. [Balenoptera, Van Beneden.] boops (Linnæus), Owen. v. Megaptera boops. 
EATFENOPTERA (continued)-

borealina, P. J. Van Beneden, Bull. Acad. Roy. Belg. [2] vol. 1, 1880, p. 15 ; R. Lydekker, Quart. Journ. Geol. Soc., vol. xliii, 1887 , p. 12, and Cat. Foss. Mamm. B. M., pt. v, 1887, p. 39.

LOC. Red Crag; Woodbridge, Suffolk. [Tympanic and periotic bones; Ipswich Mus. and Brit. Mus.]

borealis, R. P. Lesson, Hist. Nat. Mamm. (Cétacés), I 828 , p. 342 ; R. Lydekker, Cat. Foss. Mamm. B. M., pt. v, 1887 , p. 38 .

Balana mysticetus, G. A. Mantell (errore), Medals of Creation, 1844, vol. ii, p. 825 ; R. Owen (errore), Brit. Foss. Mamm., 1846, p. 543 ; J. Morris (errore), Cat. Brit. Foss., 1854 , p. 357 .

LOC. 'Elephant Bed'; Brighton. [Vertebra and mandib. ramus; Brit. Mus.]

Dredged; North Sea, Norfolk Coast. [Vertebra; Brit. Mus.]

definita (Owen), R. Lydekker, Quart. Journ. Geol. Soc, vol. xliii, 1887, p. II, pl. ii, f. 3 , and Cat. Foss. Mamm. B. M., pt. v, I 887, p. 34 .

Balcena definitu, R. Owen, Proc. Geol. Soc., vol. iv (1843), p. 285.

Balanodon definila, R. Owen, Brit. Foss. Mamm., 1846, table facing p. xlvi and p. 53I, woodcut 222 (Balana); J. Morris, Cat. Brit. Foss., I854, p. 357.

Balenoptera sibbalitina, P. J. Van Beneden, Bull. Acad. Roy. Belg. [2] vol. 1, 1880, p. I 4 .

LOC. Red Crag; Suffolk. [Tympanic bone: R. Coll. Surgeons Mus.]

emarginata (Owen), R. Lydekker, Quart. Journ. Geol. Soc., vol. xliii, 1887, p. 12, and Cat. Foss. Mamm. B. M., pt. v, I887, p. 4 O.

Balana emarginata, R. Owen, Proc. Geol. Soc., vol. iv (1\&43), p. 286.

Balana gibbosa, R. Owen, ibid., p. 286. [Tympanic bone.]

Balcenodon emarginata, R. Owen, Brit. Foss. Mamm., I846, table facing p. xlvi, and p. 533, woodcut 224 (Balana); J. Morris, Cat. Buit. Foss., 1854, p. 357 .

Balcnodon gibbosa, R. Owen, Brit. Foss. Mamm., 1845, table facing p. xlvi, and p. 532, woodcut 223 (Balana), and Palæont., ed. 2, I85i, p. 375, woodcut 135; J. Morris, Cat. Brit. Foss., 1854 , p. 358 .

Balenoftira rostratell, P. J. Van Beneden, Bull. Acad. Roy. Belg. [2] vol. 1, 1880, p. 16.

LOC. Red Crag; Woodbridge. [Tympanic bone; R. Coll. Surgeons Mus.]

garopi (Van Beneden), R. Lydekker, Quart. Journ. Geol. Soc., vol. xliii, 1887, p. 12, and Cat. Fozs. Mamm. B. M., pt. v, 1887, p. 36 (giropi). 
BAIFIOPTYRA garopi (continued)-

Plesincetus garopii, P. J. Van Beneden, Bull. Acad. Roy. Belg. [2] vol. xxxiv, 1872 , p. I 5 .

Balenoptera musculoides, P. J. Van Beneden, ibid. [2] vol. 1, 1880 , p. 15.

LOC. Red Crag; Woodbridge. [Tympanic bone; Brit. Mus.]

? juddi, H. G. Seeley, Quart. Journ. Geol. Soc., vol. xxxvii, I88I, p. 7o9, woodcuts $1-3 ;$ R. Lydekker, Cat. Foss. Mamm. B. M., pt. v, I 887 , p. 4 I.

LOC. U. Eocene (Brockenhurst Beds); Roydon, Hampshire. [Vertebra ; Brit. Mus.]

musculoides, Van Beneden. v. Balænoptera garopi.

? musculus (Linnæus), (?) W. Turner, Proc. Roy. Phys. Soc. Edinb., vol. viii, 1885, p. 336, and Rep. Brit. Assoc., I 889.

Balana musculus, Linnæus, Syst. Nat., ed. 12, vol. i, 1766, p. 106.

Balcenoptera, J. Leitch, Trans. Cumberland Assoc., no. ix, 1885, p. I 7 I.

LOC. ? Pleist.; Airthey, Blair Drummond, and Silloth.

robustu, Lilljeborg. v. Eschrichtius robustus.

rostratella, Van Beneden. $v$. Balænoptera emarginata.

sibbalalina, Van Beneden. v. Balænoptera definita.

sp., E. T. Newton, Quart. Journ. Geol. Soc., vol. xlii, 1886, p. 322.

Balcenoptera?, E. T. Newton, Geol. Mag. [2] vol. viii, I881, p. 316, and Vert. Forest Bed (Mem. Geol. Surv., I 882), p. 108 (pars).

'Two species of whales,' J. Gunn, Geol. Norfolk, I864, p. I9.

Balanoptera, C. Lyell, Antiq. Man, I863, p. 217.

LOC. Forest Bed; Mundesley and Bacton. [Vertebræ; Norwich Mus.]

BALAENOTUS, Van Beneden. v. Balæna.

insignis, Van Beneden. $\%$ Balæna insignis.

$\boldsymbol{H} \boldsymbol{A} \boldsymbol{L} \boldsymbol{A} \boldsymbol{E} \boldsymbol{V} \boldsymbol{U} \boldsymbol{L} \boldsymbol{A}$, Van Beneden. $\%$ Balæena.

balaenopsis, Van Benedon. ข. Balæna balænopsis.

IELEMNOZIPIIIUS, Hux'ey. v. Mesoplodon.

compressus, Huxley. \%. Mesoplodon compressus.

longirostris (Cuvier), Huxley. v. Mesoplodon longirostris.

sp., Lankester. v. Balænodon physaloides.

$\boldsymbol{B E L U} \boldsymbol{U} \boldsymbol{A}$, Gray. v. Delphinapterus.

BISOM, Hamilton Smith, in Griffith's Animal Kingdom, vol.v, I827, p. 373 .

bonasus (Linnæus), var. priscus (Bojanus), R. Lydekker, Cat. Foss. Mamm. B. M., pt. ii, 1885, p. 24 ; E. T. Newton, Geol. Mag. [3] vol. vi, 1889, p. I46.

Bos bonasus, Linnæus, Syst. Nat., ed. 12, vol. i, 1766, p. 99. 
BISON bonasus (continued)-

Urus priscus, L. H. Bojanus, Nova Acta Acad. Cres. Leop.-Carol. vol. xiii (2), 1827, p. 427 ; R. Owen, Rep. Brit. Assoc., 1843 (1844), p. 232.

Bison priscus, R. Owen, Brit. Foss. Mamm., I846, p. 491, woodcuts 205, 207 ; J. Morris, Cat. Brit. Foss., I 854 , p. 358 ; H. Falconer, Palæont. Mem., vol. if, 1868, p. 567 ; IV. 13. Dawkins, Quart. Journ. Geol. Soc, vol. xxxi, 1875, p. 246 ; R. Johnson, Trans. Norfolk and Norwich Nat. Soc., vol, ii (1877), p. 286; M. Browne, Vert. Animals Leicester., 1889, p. 29.

Bos primigenius?, E. T. Newton, Geol. Mag. [2] vol. vii, I880, p. 449, and Vert. Forest Bed (Mem. Geol. Surv., I882), p. 47.

Bison europaus, H. H. Winwood, Proc. Bath Nat. Hist. Club, vol. vi (1886), p. 95.

LOC. Forest Bed; Happisburgh, Mundesley, Overstrand, Pakefield, Runton, Sidestrand, Trimingham. [Horncores; J.J. Colman Coll., and A. Savin Coll.]

Pleist.; Barrington (Camb.), Box (Middle Hill), Cropthorne, Erith, Essex Coast, Folkestone, Freshford, Ilford, Leicester, London, Lincolnshire, Peckham, Stonehouse, Walton-on-the-Naze, Westbury (Wilts), Woolwich.

Caves ; Cresswell, Devon, Kirkdale, Somerset, South Wales, and Windy Knoll.

europaeus, Winwood. $v$. Bison bonasus, var. priscus.

minor, Owen. $v$. Bos taurus, var. longifrons.

priscus, auct. $v$. Bison bonasus and Bos taurus.

BorOdoN, R. Owen, Brit. Mesoz. Mamm. (Pal. Soc., 1871), p. 54. crassidens, R. Owen, ibid., p. 54, pl. iii, f. 5 ; R. Lydekker, Cat. Foss. Mamm. B. M., pt. v, I887, p. 203.

LOC. M. Purbeck; Durdlestone Bay. [Portion of right maxilla ; Brit. Mus.]

T.

BOS, Linnæus, Syst. Nat., ed. 12, vol. i, i 766, p. 98.

bonreste, Linnæus. $v$. Bison bonasus.

brachyceros, Owen. $v$. Bos taurus, var. longifrons.

frontosus, Nilsson. $v$. Bos taurus, var. longifrons.

giganteus, Owen, MS. v. Bos taurus, var. primigenius.

latifrons, Wilde. $v$. Bos taurus, var. longifrons.

longifrons, Owen. $v$. Bos taurus, var. longifrons.

moschatus, Zimmermann. $v$. Ovibos moschatus.

primigenius, Bojanus. $v$. Bos taurus, var. primigenius.

priscus, Bronn. $v$. Bos taurus, var. primigenius.

taurus, Linnæus. $v$. Bos taurus, var. longifrons and var. primigenius. 
BOS (continued) -

taurus, Linnæus, var. longifrons (Owen), R. Lydekker, Cat. Foss. Mamm. B. M., pt. ii, I885, p. 16.

Bos taurus, Linnæus, Syst. Nat., ed. 12, vol. i, 1766, p. 98 ; J. Fleming, Brit. Animals, 1828, p. 24; S. Woodward, Syn. Tab., 1830, p. 39 ; S. Haughton, Proc. Rcy. Irish Acad. [2] vol. ii (Sci.), 1876, p. 483.

Bos longifrons, R. Owen, Rep. Brit. Assoc., I843 (1844), p. 235, and Brit. Foss. Mamm., 1846, p. 508, woodcuts 211, 212; J. A. Smith, Edinburgh New Phil. Journ., vol. liv, 1853, p. 122, pl. ii, and ibid., vol. lvii, 1854, p. 162, and Proc. Soc. Antiq. Scotland, vol. ix (1873), p. 587, pl. 1xi, 3 woodcuts, and ibid., vol. xii (1878), p. 493, woodcuts ; J. Morris, Cat. Brit. Foss., 1854, p. 358; W. R. Wilde, Proc. Roy. Irish Acad., vol. vii (1858), p. 67, woodcuts, and ibid. (1859), p. 210 , woodcut 14; R. Howse, Trans. Tyneside Nat. Field Club, vol. v (I86I), p. 120; G. E. Roberts, Journ. Anthrop. Soc., vol. ii, 1864, p. Ixii; W. B. Dawkins and W. A. Sanford, Brit. Pleist. Mamm. (Pal. Soc., I866), p. xxv; J. Bennie, Trans. Geol. Soc. Glasgow, vol, ii, I867, p. 152 ; T. W. Tew, Proc. Geol. Polyt. Soc. Yorksh., vol. v (1870), p. 55; H. Dumbleton, Trans. Woolhope Nat. Field Club, 1871 (1874), p. 44; G. Rolleston, Journ. Anthrop. Inst., vol. vi (1876), p. 24; R. Johnson, Trans. Norfolk and Norwich Nat. Soc., vol, ii (1877), p. 287 ; A. L. Adams, Proc. Roy. Irish Acad. [2] vol. iii (Sci.), r878, p. 91, and Trans. Roy. Dublin Soc. [2] vol. i (I88I), p. I97, pl. xiii, f. 4 (B. longifrons?); J. E. Harting, Brit. Anim. Extinct, I880, p. 213 ; V. Ball, Trans. Roy. Dublin Soc. [2] vol. iii (1885), p. 343; M. Browne, Vert. Animals Leicester., 1889, p. 30.

Bos frontosus, S. Nilsson, Ofvers. K. Vetensk. Akad. Förh, I847 (1848), p. I16, and Ann. Mag. Nat. Hist. [2] vol. jv, I 849 , p. 349 ; W. R. Wilde, Proc. Roy. Irish Acad., vol. vii (1859), p. 209; S. J. Mackie, Geologist, vol. v, I862, p. 44I, pl. xv; W. D[avies], Geol. Mag., vol. i, 1864, p. 296.

Bos latifrons, W. R. Wilde, Proc. Roy. Irish Acad., vol. vii (1858), p. 67.

Bos, W. Clift, Phil. Trans., 1823, p. 84, pl. viii, f. 1, pl. ix, f. 4, 5; (?) J. A. Smith, Proc. Soc. Antiq. Scotland, vol. ix (1875), p. 587, and ibid., vol. xii (I878), p. 493.

Bison minor, R. Owen, Brit. Foss. Mamm., I846, p. 497, woodcut 206 ; J. Morris, Cat. Brit. Foss., I854, p. 358 .

Bos brachyceros, R. Owen, Brit. Foss. Mamm., I846, p. 509 ('Cat. R. Coll. Surgeons, I830').

'Small ox,'H.Woods, Descr. Foss. Skull Ox, 1839, p. 28, woodcut. Loc. Prehist. and Hist. ; Audley End, Battersea, Bawdsey Bog, Berkshire, Bricklehampton Bank, Berry Head (Devon), Bridgewater, Bracklesham Bay, Clacton, Dilham Canal (Norfolk), Diglis, Greenwich, Happisburgh, Hartlepool, Herne Bay, Jarrow Dock, Lambeth, Langorse, Leicester, Lewes, Monkhill, Newbury, Peckham, Plumstead, 
BOS taurus, var. longifrons (continued) -

Purfleet, Saffron Walden, Swaff ham Fen, Vauxhall, Walthamstow, Walton (Essex).-Blair Drummord, Burrian, Borness, Borgue, Balgone, North Cowford, Cupar Angus, Cill Trölla, Drem, Dowalton Loch, Edinburgh, Glaserow, Harris, Hawick, Inveresk, Inchkeith, Kinleith, Keiss, Kirkwall, Kettleburn, Kinneil, Little, Liddesdale, Newstead, Orkney, North Ronaldshay, Searliff, St. Cyrus, Bay of Skaill, Skara, Lower Stonehouse, Tain, Thrumster, Warburton, Yarhouse (Scotland),-Ballinderry, Callanagh, Dowth, Dunslaughlin (Meath), Lough Gur, Longford, Tyrone (Ireland).

Caves ; ? Kirkdale, Oreston.-Ballynamintra, Knockninny and Shandon (Ireland).

taurus, Linnæus, var. primigenius (Bojanus), R. Lydekker, Cat. Foss. Mamm. B. M., pt. ii, 1885, p. 2.

Bos taurus, Linnæus, Syst. Nat., ed. 12, vol. i, 1766, p. 98.

Bos primigenius, L. H. Bojanus, Nova Acta Acad. Cies. Leop.Carol., vol. xiii (2), 1827, p. 422, pls. xx-xxiv; H. Woods, Descr. Fossil Skull Ox, 1839, p. 20, woodcut and pl. (lower fig.) ; R. Owen, Brit. Foss. Mamm., 1846, p. 498, wondcuts 208-210; P. J. Selby, Proc. Berwick Nat. Club, vol. iii (1850), p. 45 ; H. Denny, Proc. Geol. Polyt. Soc. Yorksh,, vol. iii, 1853 (1859), p. 336 ; J. Morris, Cat. Brit. Foss., I 854 , p. 358; W. Turner, Proc. Roy. Phys. Scc. Edinb., vol. ii (1859), p. 72 ; J. A. Smith, Proc. Roy. Phys. Soc. Edinb., vol. ii (1859), p. II I ; S. J. Mackie, Geologist, I860, p. 28I, woodcut 20 ; R. Howse, 'Trans. Tyneside Nat. Field Club, vol. v (I86I), p. I19; G. Logan, Proc. Roy. Phys. Soc. Edinb., vol. iii ( 8 S6), p. 347 ; J. Bennie, Trans. Geol. Soc. Glasgow, vol. ii, I867, p. 152 ; J. Adams, Wiltshire Archæol. Mag., vol. xi (I 860 ), p. 283, woodcuts 7 and 8 ; C. Moore, Proc. Bath Nat. Hist. Soc., vol. ii (1870), p. 52 ; E. Charlesworth, Journ. Anthrop. Soc., vol. viii (1870), p. ii ; J. A. Smith, Proc. Soc. Antiq. Scotland, vol. ix, 1873, p. 641, woodcuts; G. Busk, Phil. Trans., I873 (1874), p. 521, pl. xlvi, f. II ; J. Carter, Geol. Mag. [2] vol. i, I874, p. 492 ; J. W. Clark, Proc. Camb. Phil. Soc., vol. ii (1874), p. 357 ; G. Rolleston, Journ. Anthrop. Inst., vol. vi (1876), pp. 21, 27 ; R. Johnson, Trans. Norfolk and Norwich Nat. Soc., vol. ii (1877), p. 287 ; Miller and Skertchley, Fenland, 1878 , p. 344, pl.; S. Palmer, Trans. Newbury Dist. Field Cluh, vol. ii (1878), p. 133; J. E. Harting, Brit. Anim. Extinct, 1880, p. 213, woodcuts; J. Leitch, Trans. Cumberland Assoc., no. ix, 1885, p. I7I, pl. ; T. IV. Shore, Papers Hampshire Field Club, no. 3, I889, p. 53, pl. ii ; J. C. Mansel-Pleydell, Proc. Dorset. Nat. Hist. Field Club, vol. $x, 1889$, p. 8I, woodcut; M. Browne, Vert. Animals Leicester., 1889, p. 30.

Tête de bouf,' Cuvier, Ossem. Fossiles, ed. 2, vol. iv, I823, p. 15 x, pl. xi, f. I-4 (foreign). 
BOS taurus, war. primigenius (continued)-

Bos giganteus, W. Davies (ex Owen, MS.), Cat. Pleist. Vert. Coll. Brady, 1874, p. 47.

? Bos priscus, J. Brown, Ann. Mag. Nat. Hist. [2] vol. xx, 1857, p. 398.

Bos urus, J. Fleming, Brit. Animals, 1828, p. 24 ; S. Woodward, Syn. Tab., 1830, p. 39 ; W. B. Dawkins, Quart. Journ. Geol.

Soc., vol. xxii, 1866, p. 391 .

'Fossil Ox,' J. MacEnery, Cavern Researches, I859, pl. Q, f. 6-8.

LOC. Pleist., Prehist., and Hist. ; ? Aymestry, Bath, Broadmead(Folkestone), Beckford, Bielbecks, Bricklehampton Bank, Burwell Fen, Clacton, Crayford, Cropthorne, Dogger Bank (dredged), Fisherton, Grays, Greenwich, Hedingham, Herne Bay, Hurley Bottom, Ilford, Leicester, London, Loxbrook, ? Larkhall (Somerset), ? Lawford, ? Little Comberton, ? Leeds, Lowestoft, Maidstone, Melksham, Moulsham, Newbury, Norfolk, Northampton, Northwich, Oundle, ? Oxford, Peckham, Pilling Moss, Preston, Slade Green, Sunbiggen Tarn, Stroud, Thame, Twizell, ? Thetford, Tisbury, Tyneside Salt Marsh, Walthamstow, Walton-on-the-Naze, West Stour (Dorset).Athol, Ale Water, Belhelvie Moss, Bower, Breckigo, Crofthead, River Clyde, Dunse, Drummond Castle, Dumfriesshire, Fifeshire, Galloway, Glasgow, Hapsburn, Jedburgh, Keiss, Kintradwell, Kircudbrightshire, Linton Loch, Lanark, Maybole, Mertoun, Moulin, Muthill, Newburgh, Skara, Sandwick, Stonehouse, Shaw, Swinton Mill, Whitrig Bog, Whitmuirhall (Scotland).

Caves; Banwell, Bench, Bleadon, Brixham, Caswell Bay, Kent's Hole, Kirkdale, Maidstone (fissure), Oreston, Portland (fissure), Sandford Hill, and Wookey Hole.

urus, Fleming. v. Bos taurus, var. primigenius.

sp., R. Lydekker, Cat. Foss. Mamm. B. M., pt. ii, 1885, p. 24.

LOC. Red Crag ; Felixstowe. [Tooth ; Brit. Mus., no. 43989.]

Bubalus moschatus, Owen. v. Ovibos moschatus.

BURTINOPSIS, Van Beneden. v. Megaptera.

minutus, Van Beneden. v. Megaptera minutus.

similis, Van Beneden. v. Megaptera similis.

CANIS, Linnæus, Syst. Nat., ed. 12, vol. i, 1766, p. 56.

crocuta, Erxleben. v. Hyæna crocuta.

familiaris, Linnæus, Syst. Nat., ed. 12, vol. i, 1766, p. 56; R. Owen, Brit. Foss. Mamm., I846, p. 133; J. Morris, Cat. Brit. Foss., I 854 , p. 358 ; S. Haughton, Proc. Roy. Irish Acad. [2] vol. ii (Sci.), 1876, p. 482. 
CANIS familiaris (continued)-

Canis hibernicus, W. R. Wilde, Proc. Roy. Irish Acad., vol. vii, 1859, p. 194, woodcuts 2, 3 ; (?) A. L. Adams, Trans. Roy. Dublin Soc. [2] vol. i, 1881, p. 205. [See also G. A. Graham, 'Irish Wolf Hound,' I 885 .]

Canis domesticus, var, hibernicus, V. Ball, Trans. Roy. Dublin Soc. [2] vol. iii (1885), p. 340.

'Dog,' H. Denny, Proc. Geol. Polyt. Soc. Yorkshire, vol. iii, 1857 (1859), p. 538.

Canis lupus, H. Woodward (errore), Geol. Mag., vol. vi, I869, p. 387.

LOC. ? Prehist.; Norwich and Walthamstow.--Dunslaughlin.

Caves; England. - Ballynamintra, Knockninny, and Shandon (Ireland).

hibernicus, Wilde. $v$. Canis familiaris.

hycena, S. Woodward. v. Hyæna crocuta.

lagopus, Linnæus, Syst. Nat., ed. 12, vol. i, I766, p. 59; G. Busk, Quart. Journ. Geol. Soc., vol. xxxi, 1875, p. 685, woodcut 2.

LOC. Cave; Cresswell Crags. [Verteorce; Owens College, Manchester.]

Iupus, Linnæus, Syst. Nat., ed. I2, vol. i, I766, p. 50 ; S. Woodward, Syn. Tab., 1830, p. 39; R. Owen, Brit. Foss. Mamm., 1846, p. 123, woodcuts 45-49; J. Morris, Cat. Brit. Foss., 1854 , p. 358 ; W. B. Dawkins, Quart. Journ. Geol. Soc., vol. xxxi, 1875 , p. 252 ; G. Busk, Ouart. Iourn. Geol. Soc., vol. xxxi, 1875 , p. 684 ; A. L. Adams, Trans. Roy. Irish Acad., vol. xxvi, 1876, p. 227, and Proc. Roy. Irish Acad. [2] vol. iii (Sci.), 1878, p. 99, and Sci. Proc. Roy. Dublin Soc., vol. ii, I880, p. 65, pl. i, and Trans. Roy. Dublin Soc. [2] vol. i (18So), p. 205 ; S. Haughton, Proc. Roy. Irish Acad. [2] vol. ii (Sci.), 1876, p. 482 ; J. E. Harting, Pop. Sci. Rev., vol. xvii, I878, p. 53, and Brit. Anim. Extinct, 1880 , p. I15; (?) E. T. Newten, Vert. Forest Bed (Mem. Geol. Surv., 1882), p. 19, pl. iv, f. I; R. Lydekker, Cat. Foss. Mamm. B. M., pt. i, 1885, p. 123 ; V. Ball, Trans. Roy. Dublin Soc. [2] vol. iii (1885), p. 335; J. Simpson, Trans. Edinburgh (Geol. Soc., vol. v (I 887), p. 297.

Canis spelceus, Goldfuss, Nova Acta Acad. Cæs. Leop.-Carol., vol. xi (2), 1823, p. $45 \mathrm{I}$.

'Wolf,' W. Clift, Phil. Trans., I823, p. 85, pl. viii, f. 2, 3, pl. xii, f. Io; W. Buckland, Reliq. Diluv., I824, p. I8, pl. xiii, f. 5, 6 ; J. Rutter, Delin. County Somerset, I 829 , p. I56; J. C. Bellamy, Nat. Hist. S. Devon, I839, p. 439, pl. xv, f. 5 ; J. MacEnery, Cavern Researches, I859, pl. G.

'Loup fossile,' Cuvier, Ossem. Fossiles, vol. iv, I823, p. 46 I.

LOC. Forest Bed; East Coast.

Pleist. ; Bielbecks, Bracklesham, Grays, Murston, Newbury, Slade Green, Thame, Tewkesbury, Windsor, and dredged off Eastern Coast.

Caves; Banwell, Bacon Hole, Bench, Bleadon, Bosco's Hole, Brixham, Cresswell Crags, Crow Hole, Durdlam 
CArIS lupus (continued) -

Down, Deborah's Den, Hutton, Kent's Hole, Kirkdale, Long Hole, Minchin Hole, Oreston, Paviland, Pleasley Vale, Ravenscliff, Sandford Hill, Spritsail Tor, Teesdale, Uphill, Vale of Clwyd, Windy Knoll, Yealm Bridge.Pentland Hills(Scotland). - Ballynamintra, Knockninny, Shandon (Ireland).

primigenius, E. R. Lankester, Ann. Mag. Nat. Hist. [3] vol. xiv, I864, p. 358 , pl. viii, f. II.

Loc. Red Crag ; Suffolk. [Canine tooth; York Mus.]

spelceus, Goldfuss. $v$. Canis lupus.

vulpes, Linnæus, Syst. Nat., ed. 12, vol. i, 1766, p. 59 ; R. Owen, Rep. Brit. Assoc., I842 (1843), p. 70 ; W. B. Dawkins, Quart. Journ. Geol. Soc., vol. xxviii, I872, p. 417 , and Cave Hunting, 1874 , p. 418 ; (i. Busk, Phil. Trans., 1873 (1874), p. 548, and Quart. Journ. Geol. Soc., vol. xxxi, 1875, p. 685; S. Haughton, Proc. Roy. Irish Acad. [2] vol. ii (Sci.), 1376, p. 483 ; A. L. Adams, Trans. Roy. Irish Acad., vol.xxvi (Sci.), 1876, p. 228, and Proc. Roy. Irish Acad. [2] vol. iii (Sci.), I873, p. 89, and Trans. Roy. Dublin Soc. [2] vol. i (1880), p. 205 ; (?) E. T. Newton, Vert. Forest Bed (Mem. Geol. Surv., I882), p. 19, pl. iv, f. 2 ; R. Lydekker, Geol. Mag. [3] vol. i, 1884, p. 443, woodcut I, and Cat. Foss. Mamm. B. M. pt. i, 1885, p. I30, woodcut 19. [See also Phoca sp.]

Vulpes r'ulgaris, S. Woodward, Syn. Tab., I83o, p. 39; R. Owen, Brit. Foss. Mamm., I 846, p. I34, woodcuts 5I-53; J. Morris, Cat. Brit. Foss., 1854 , p. 362 ; A. L. Adams, Sci. Proc. Roy. Dublin Soc., vol. ii, I88o, p. 66.

'Fox,' W. Buckland, Phil. Trans., I822, p. I84, pl. xx, f. 8-14, pl. xxiv, f. 9, I0, 13, and Reliq. Diluv., I824, p. 35, pl. vi, f. 8-14, pl. x, f. 9, IO, I3; W. Clift, Phil. Trans., 1823, p. 82 ; J. Rutter, Delin. County Somerset, I829, p. I57; J. MacEnery,

Cavern Researches, 1859, pl. E, f. I4.

LOC. Red Crag [? Recent]; Boyton(Suffolk). [Skull; Brit. Mus.] Pleist. ; Dartford, Fisherton, Grays, Ilford, Ipswich.

Caves; Bacon Hole, Bench, Bleadon, Bosco's Hole, Banwell, Brixham, Caswell Bay, Crow Hole, Cresswell Crags, Durdham Down, Gower, Hutton, Kent's Hole, Kirkdale, Long Hole, Minchin Hole, Oreston, Paviland, Ravenscliff, Sandford Hill, Spritsail Tor, Teesdale, Uphill, Vale of Clwyd, Wookey Hole, Weston.-Ballynamintra, Knockninny, and Shandon (Ireland).

sp., R. Owen, Quart. Journ. Geol. Soc., vol. xii, I856, p. 227, woodcuts 2I, 22 ('? = C. lupus').

LOC. Red Crag ; Woodbridge. [Tooth and mandib. ramus.]

CAPRA, Linnæus, Syst. Nat., ed. 12, vol. i, 1766, p. 94.

hircus, Linnæus, ibid., p. 94 ; R. Owen, Brit. Foss. Mamm., I846, p. 489, woodcut 204; J. Morris, Cat. Brit. Foss., I 854, p. 358 ; W. R. Wilde, Proc. Roy. Irish Acad., vol. vii, I859, p. 205, wood- 
CAPRA hircus (continued)-

cuts $7,8,9$; C. C. Blake, Geologist, I862, p. 2 I 8 ; IV. 13. Dawkins and W. A. Sanford, Brit. Pleist. Mamm., pt. i (Pal. Soc., I866), p. xxvi ; S. Haughton, Proc. Roy. Irish Azad. [2] vol. ii (Sci.), 1876, p. 483; G. Rolleston, Journ. Anthrop. Inst., vol. vi (1876), pp. 25,27 ; A. L. Adams, Trans. Roy. Dublin Soc. [2] vol. i (1881), p. 198, pl. xiii, r. 2 ; R. Lydekker, Cat. Foss. Mamm. B. M., pt. ii, 1885 , p. 40.

Loc. Prehist. and Hist.; Chippenham, London, Newbury, Walthamstow, Walton-on-the-Naze. - Dunslaughlin, Fermanagh (Ireland).

Caves; Heathery Burn. - Ballynamintra, Knockninny (Ireland).

CAPREOIUS, Hamilton Smith, in Griffith's Animal Kingdom, vol. v, I827, p. 313 .

caprea, J. E. Gray, List of Mamm. B. M., I843, p. 176 (C.capraca), R. Lydekker, Cat. Foss. Mamm. B. M., pt. ii, I885, p. 74.

Cervus capreolus, Linnæus, Syst. Nat., ed. 12, vol. i, I766, p. 94 ; R. Owen, Rep. Brit. Assoc., I843 (1844), p. 236, and Brit. Foss. Mamm., I 846, p. 487, woodcuts 201-203; J. Morris, Cat. Brit. Foss., I 854 , p. 358 ; J. Hardy, Proc. Berwick Nat. Club, vol. iv (1860), p. 206 ; C. C. Blake, Geologist, 1862, p. 218 ; W. B. Dawkins and W. A. Sanford, Brit. Pleist. Mamm., pt. i (Pal. Soc., I 866), p. xxviii ; G. Rolleston, Journ. Anthrop. Inst., vol. vi (1876), pp. 24,27 ; (?) E. T. Newtun, Geol. Mag. [2] vol. vii, 1880, p. 450, and Vert. Forest Bed (Mem. Geol. Surv., I882), p. 52.

Capreolus capreolus, G. Busk, Phil. Trans., I873 (1874), p. 528.

'Roebuck,' H. Denny, Proc. Geol. Polyt. Soc. Yorksh., vol. iv (186I), p. 146.

Cervus rectus, E. T. Newton, Geol. Mag. [3] vol. vi, I889, p. I45, pl. v, f. I. [Frontal; A. Savin Coll.] [Synonym according to W. Davies and W. B. Dawkins in litt.]

LOC. Forest Bed ; Bacton, Bawdsey (dredged), Cromer, Ostend, Sidestrand.

Pleist., Prehist., and Hist.; Coldingham, Fisherton, Grays, Ipswich, London, Newbury, Oxford, Reach Fen (Camb.), Sutton (Essex), Walthamstow.

Caves; Bosco's Hole, Bleadon, Bacon Hole, Brixham, Caldy Island, Heathery Burn, Oreston, Paviland, Pembrokeshire, Spritsail Tor, Teesdale, Vale of Clwyd.

capreolus (Linnæus), Busk. v. Capreolus capræa.

CApRovis, B. H. Hodgson, Journ. Asiatic Soc. Bengal, vol. xri, pt. ii, I847, p. 702 .

savini, [-ii] E. T. Newton, Geol. Mag. [2] vol. vii, I88o, p. 449, and Vert. Forest Bed (Mem. Geol. Surv., I 882), p. 49, pl. x.

Loc. Forest Bed; Overstrand. [Right horn-core and Irontal; A. Savin Coll.] 
CAsтOR, Linnæus, Syst. Nat., ed. 12, vol. i, 1766, p. 78.

europaus, Owen. v. Castor fiber.

fiber, Linnæus, Syst. Nat., ed. 12, vol. i, 1766, p. 78 ; P. Neill, Mem. Werner. Soc., vol. iii, 1819 (1821), p. 207, and Edinburgh Phil. Journ., vol. i, I819, p. I77; S. Woodward, Syn. Tab., 1830, p. 39, and Geol. Norfolk, 1833, p. 46 ; M. Fisher, Zoologist, vol. i, 1843 , p. 348 ; R. Owen, Geol. Mag., vol. vi, I 869, pp. 49, 52 ; J. E. Harting, Brit. Anim. Extinct, I880, p. II, woodcut; R. Lydekker, Cat. Foss. Mamm. B. M., pt. i, 1885, p. 214.

Castor fossilis, Goldfuss, Nova Acta Acad. Cæs. Leop.-Carol., vol. xi (2), 1823, p. 488.

Castor europaus, R. Owen, Brit. Foss. Mamm., I846, p. 190, woodcuts 74, 75, and Quart. Journ. Geol. Soc., vol. iv, 1848, p. 42 ; J. Morris, Cat. Brit. Foss., 1854, p. 358 ; J. Adams, VViltshire Archæol. Mag., vol. xi (1869), p. 282, woodcut 6 ; E. T. Newton, Geol. Mag. [2] vol. viii, I88I, p. 258, and Vert. Forest Bed (Mem. Geol. Surv., I882), p. 78, pl. xii, f. $1-9$.

Castoreuropaus $=C$. fiber, Coues and Allen, N. Amer. Rodentia, I877, p. 434.

Castor, A. Savin, Proc. Norwich Geol. Soc., vol. i (1878), p. 27.

'Beaver,'J. Collett, Phil. Trans., 1757, p. I12 ; T. Pennant, Brit. Zool., vol. i, 1812, p. 6o, note; J. Okes, Trans. Camb. Phil. Soc., vol. i, 1820, p. 175; Wilson, Proc. Berwick Nat. Club, vol. ii (1843), p. 48 ; W. Buckland, Proc. Ashmolean Soc., vol. ii, 1854, p. 21 ; E. Tindall, Proc. Geol. Polyt. Soc. Yorksh., vol. v (1870), p. 9, pl.

LOC. ? Norwich Crag; Sizewell Gap.

Forest Bed ; Bacton, Happisburgh, Kessingland, Mundesley, West Runton.

Pleist., Prehist., and Hist. ; Burwell Fen, Berwick (Middlestot Bog), Chatteris, Christian Malford, Ditton Fen, East Coast (dredged), Ely Fen, Grays, Hilgay, Ilford, Isleham, Lincolnshire, Newbury, Ressondale, Romsey, Walthamstow, Watcham Fen, Wawne (near Beverley, Yorks), Whelpmoor Fen. - Dumfriesshire, Kimmerghame, Kinloch, Linton Lough (Roxburghshire) (Scotland).

Cave; Kent's Hole.

fossilis, Goldfuss. $v$. Castor fiber.

moschatus, Linnæus, $v$. Myogale moschatus.

trogontherium, Cuvier. v. Trogontherium cuvieri.

veterior, E. R. Lankester, Ann. Mag. Nat. Hist. [3] vol. xiv, I 864, p. 355 , pl. viii, f. 5 .

Loc. Red Crag ; Sutton. [Upper premolar; York Mus.] 
cervuxus, H. D. de Blainville, Bull. Soc. Philom. Paris, i $\varepsilon_{1} 6$, p. 74 .

?dicranoceros (Kaup), (?) R. Lydekker, Cat. Foss. Mamm. B. M., pt. ii, I885, p. II7.

Cervus dicranocerus, J. J. Kaup, Archiv. f. Mineral., vol. vi, I 833 , p. 219, pl. iv, f. 6-11.

LOC. Red Crag; Felixstowe. [Antler fragments ; Brit. Mus.]

CERVซS, Linnæus, Syst. Nat., ed. 12, vol. i, 1766, p. 92.

Megaceros, R. Owen, Rep. Brit. Assoc., I843 (1844), p. 237.

Strongyloceros, R. Owen, Brit. Foss. Mamm., 1846, p. 469.

Eucladoceros, H. Falconer, Palæont. Mem., vol. ii, i868, p. 472.

Elaphus, R. Owen, Rep. Brit. Assoc., 1843 (1814), p. 236.

Dicranoceros, R. Owen, Quart. Journ. Geol. Soc., vol. xii, 1856, p. 224 .

alces, Linnæus. \% Alces machlis and Cervus giganteus.

ardeus ("Croizet and Jobert'), H. Falconer, MS. List of Gunn Collection; W. B. Dawkins (fide Falconer?), Quart. Journ. Geol. Soc., vol. xxv, I869, p. 2 Io; E. T. Newton, Vert. Forest Bed (Mem. Geol. Surv., 1882), p. 63 (rejects this species).

barbarus, Gray. ข. Cervus elaphus.

booides, Gunn, MS. v. Alces latifrons.

bovides, Newton. $\%$. Alces latifrons.

browni, W. B. Dawkins, Quart. Journ. Geol. Soc., vol. xxiv, 1868, p. 5 II, and Brit. Pleist. Mamm., pt. vi (Pal. Soc., I887), p. 17, pl. iv ; [-ii] V. Brooke, Nature, vol. xi, I875, p. 2 Io, woodcut I $("=C$. dama $)$; R. Lydekker, Cat. Foss. Mamm. B. M., pt. ii, 1885, p. 89.

Cervus clactonianus, H. Falconer, Palæont. Mem., vol. ii, I868, p. 479.

LOC. Pleist. ; Clacton. [Portions of antlers ; Brit. Mus.]

bucklandi, Owen. v. Rangifer tarandus.

capreolus, Linnæus. $v$. Capreolus caprea.

caræutorum, Laugel, Bull, Soc. Géol. France [2] vol. xix, I862, p. 713; W. B. Dawkins, Quart. Journ. Geol. Soc., vol. xxviii, I872, p. 408, woodcut 3 ; (?) E. T. Newton, Geol. Mag. [2] vol. vii, 1880, p. 450, and Vert. Forest Bed (Mem. Geol. Surv., 1882), p. 53.

Cervus (Megaceros) carmutorum, R.I Johnson, Trans. Norfoik and Norwich Nat. Soc., vol. ii (1877), p. 289.

LOC. Norwich Crag; Aldeby, Covehythe, Easton.

? Forest Bed; Norfolk Coast (dredged).

clactonianus, Falconer. $v$. Cervus browni. 


\section{Carvus (continued) -}

dama, Linnæus, Syst. Nat., ed. 12, vol. i, 1766, p. 93. Not a British fossil. [Doubtfully recorded by R. Owen, Brit. Foss. Mamm., 1846, p. 483, from Paviland Cave and Newbury; also recorded by authors from the Forest Bed, but see E. T. Newton, Vert. Forest Bed (Mem. Geol. Surv., I882), p. 63, who rejects the species; also W. W. Cordeaux, Proc. Cambridge Phil. Soc., vol. iii (1880), p. 348, records it from Newnham.] [See V. Brooke, Nature, vol. xi, I875, p. 210, and W. B. Dawkins, ibid., pp. I 12,226 .]

dawkinsi, E. T. Newton, Geol. Mag. [2] vol. vii, I880, p. 450, and Vert. Forest Bed (Mem. Geol. Surv., I882), p. 54 ; W. B. Dawkins, Brit. Pleist. Mamm., pt. vi (Pal. Soc., 1887), p. 7, pl. ii, f. I. [See also C. fitchi.]

C. verticornis, W. B. Dawkins, Quart. Journ. Geol. Soc., vol. xxviii, I872, p. 406, woodcut I (pars).

LOC. Forest Bed ; Norfolk (near Cromer). [Portion of antler; Mus. Pract. Geol.]

dicranios, Nesti, MS. v. Cervus sedgwicki.

dicranocerus, Owen. v. Cervus suttonensis.

elaphus, Linnæus, Syst. Nat., ed. I2, vol. i, 1766, p. 93 ; R. Owen, Rep. Brit. Assoc., I843 (1844), p. 236 ; J. Morris, Cat. Brit. Foss., 1854 , p. 358 ; W. R. Wilde, Proc. Roy. Irish Acad., vol. vii (1859), pp. 200, 204, woodcut 6; R. Howse, Trans. Tyneside Nat. Field Club, vol. v (I86I), p. II7; W. Davies, Geol. Mag., vol. ii, I865, p. 46 ; R. Q. Crouch, Trans. Roy. Geol. Soc. Cornwall, vol. vii, I85 I, p. I86; W. B. Dawkins and W. A. Sanford, Brit. Pleist. Mamm., pt. i (Pal. Soc., I 886), p. xxvii ; F. Dixon, Geol. Sussex, 1850, p. I8, woodcut 4 , and ed. 2, 1878, p. 22, woodcut 4; G. Rolleston, Journ. Anthrop. Soc., vol. vi, I876, p. 25; A. L. Adams, Trans. Roy. Irish Acad., vol. xxvi (Sci.), 1876, p. 223, and Proc. Roy. Irish Acad. [2] vol. iii (Sci.), 1878, p. 89, and Sci. Proc. Roy. Dublin Soc., vol. ii, I880, p. 82, and Trans. Roy. Dublin Soc. [2] vol. i (I88I), p. I99; D. Robertson, Trans. ('eol. Soc. Glasgow, vol. vi (I879), p. 53, pl. i (C.elephas); (?) J. H. Collins, Trans. Roy. Geol. Soc. Cornwall, vol. x (I88I), p. 98 ; J. A. Smith, Proc. Soc. Antiq. Scotland, n.s., vol. iii, I88I, p. 37, woodcuts I-3; E. T. Newton, Vert. Forest Bed (Mem. Geol. Surv., I882), p. 55 (C. elaphus ?); W. G. Smith, Journ. Anthrop. Inst., vol. xiii (1883), p. 377, woodcut 33; W. Turner, Proc. Roy. Phys. Soc. Edinburgh, vol. viii (1885), p. 334 ; R. Lydekker, Cat. Foss. Mamm. B. M., pt. ii, 1885, p. 94 , woodcut 9; V. Ball, Trans. Roy. Dublin Soc. [2] vol. iii (I885), p. 337 ; J. Leitch, Trans. Cumberland Assoc., no. ix, 1885, p. 17I; M. Browne, Trans. Leicester Lit. and Phil. Soc., n.s., pt. ix, 1888, p. 12, pl. i, and Vert. Animals Leicestershire, 1889 , p. 32.

Cervus (Strongyloceros) elaphus, R. Owen, Brit. Foss. Mamm., I 846 , p. 472 , woodcut I96; G. Busk, Phil. Trans., 1873 (1874), p. 524. 
CFRvus elaphus (continued)-

Cervus elaphus fossilis, S. Haughton, Man. Geology, I865, p. 339, woodcut 63 .

Cervus elaphus, var. fossilis hibernicus, S. Haughton, Ann. Mag. Nat. Hist. [3] vol. xii, I863, p. 444, and Journ. Geol. Soc. Dublin, vol. x, 1864, p. 125, pl. xi.

Cervus strongyloceros, H. Woodward, Geol. Mag., vol. vi, I869, p. 387 , and Trans. Essex Field Club, vol. iii, 1883, p. 7.

Strongyloceros spelaus, R. Owen, Brit. Foss. Mamm., I 846 , p. 469, woodcuts 193 (? and 195), and Palæont., ed. 2, I86I, p. 408 ; J. Morris, Cat. Brit. Foss., I854, p. 36 I.

'Red Deer,' J. MacEnery, Cavern Researches, I859, pl. H, f. 3, pl. K, f. 2-4; B. White, Proc. Perthshire Soc. Nat. Sci, vol. i (1884), p. II7; H. W. Lett, Proc. Belfast Nat. Fieid Club, 1884-5 (1886), p. 378 ; J. Shortt, Proc. Soc. Antiq. London [2] vol. xii, I888 (1889), p. 88 ; W. Turner, Rep. Brit. Assoc., I 889 (associated with Balanoptera).

? 'Stag's head and horns,' R. Baker, Phil. Trans., I785, p. 353.

'Large deer,' W. Buckland, Phil. Trans., I822, p. 233, pl. xxiii, f. 3, 4, and Reliq. Diluv., 1824, p. 263, pl. ix, f. 4 .

'Horn of the stag kind,' J. Hopkins, Phil. Trans., 1733, p. 257 , pl., f. 4.

'Deer's horns,' T. Knowlton, Phil. Trans., 1747, p. I24, pl. i, f. 2.

'Stag of Canada,' C. Leigh, Nat. Hist. Lancashire, I700, Book i, p. 184, pl. v.

'Hart's horn,' W. Borlase, Nat. Hist. Cornwall, 1758, p. 281, pl. xxvii, f. 5 .

LOC. Forest Bed; Norfolk.

Pleist., Prehist., and Hist. ; Alport, Aymestry, Bedford, Brentford, Bielbecks, Brighton, Bracklesham, Bricklehampton, Clacton, Cropthorne, Chatteris, Copford, Carnon, Colchester, Crayford, Diglis, Durham, Edmundsea, Eckington, Fawler, Folkestone, Grays, Greenwich, Grundisburgh, Hammersmith, Huntingdonshire, Hurley Bottom, West Hartlepool, Ilford, Jarrow Dock, Lancashire, Leicester, Lewes, Maidstone, Newbury, No Man's Land Shoal, Northampton, Norwich, Oundle, Oxford, Preston, Pentuan, Rugby, R. Rye (Yorkshire), Saltersford, Selsey, Silloth, Severn Valley, St. Columb (Cornwall), Slade Green, Tewkesbury, Thame, Lower Wick, Walthamstow, Walton (Essex), Wellingboro', Woodbridge, Wooler, Worthing, Yorkshire.-Athelstaneford, Ashkirk, Borgue, Bowden, Cockenzie, Coldingham, Carse of Gowrie, River Cree, Dundas Castle, Drem, Edinburgh, Elphinstone, Eckford, Jedburgh, Kilmaurs, West Kilbride, Kimmerghame, Linton, Maybole, Maxton, Maralin, Meiklewood, Paisley, Rowenstone, Roberton, Shaw, Stirling, Selkirk, Southdean, 


\section{CrzvשS elaphus (continued)-}

Seacliff, Tarbolton, Uphall, Whitrig Bog, Westruther (Scotland).-Ardakillan Lough, Annagossin, Ballymore, Ballinderry Lough, Ballycumber Bridge, Bohoe, Clonfree, Dungarvan, Foxborough, Grosse's Island, Lehid, Toberscanovan (Ireland).

Caves; Bacon Hole, Bleadon, Brixham, Caswell Bay, Kent's Hole, Kirkdale, Long Hole, Maidstone (fissure), Pleasley Vale, Spritsail Tor, Teesdale, Wookey Hole, Vale of Clwyd.-Ballynamintra, Shandon (Ireland).

? etueriarum, J. B. Croizet and J. Jobert, Ossem. Foss. Puy de Dôme, 1828, pls. vii, viii, ix; doubtfully recorded by E. T. Newton, Geol. Mag. [2] vol. vii, 1880, p. 450, and Vert. Forest Bed (Mem. Geol. Surv., I882), p. 55.

LOC. Forest Bed; Cromer. [Fragment of antler; Mus. Pract. Geol.]

euryceros, Hibbert. \%. Cervus giganteus.

falconeri, W. B. Dawkins, Quart. Journ. Geol. Soc., vol. xxiv, 1868, p. 517, pl. xviii, f. 9-12; R. Lydekker, Cat. Foss. Mamm. B. M., pt. ii, I885, p. 91 .

LOC. Red Crag; Suffolk. Norwich Crag; Thorpe. [Portions of antlers; Brit. Mus.]

ftchi, [-ii] E. T. Newton (ex Gunn, MS.), Geol. Mag. [2] vol. vii, I 880, p. 450, and Vert. Forest Bed (Mem. Geol. Surv., I882), p. 56.

Cervus dawkinsi, W. B. Dawkins (errore), Brit. Pleist. Mamm., pt. vi (Pal. Soc., 1887), p. 7, pl. ii, f. 2, pl. iii, f. I. [Antlers; Norwich Mus. and A. Savin Coll.]

LOC. Forest Bed; Bacton. [Antler; R. Fitch Coll.]

riganteus (Blumenbach), S. Woodward, Geol. Norfolk, I833, p. 45; R. Lydekker, Cat. Foss. Mamm. B. M., pt. ii, I885, p. 82.

Alce gigantea, J. F. Blumenbach, Beitr. z. Naturgeschichte (Ist French ed.), vol. ii, I803, p. 407.

Cervus hibernus, A. G. Desmarest, Mammalogie, I822, p. 446. Cervus alces hibernicus, S. Woodward, Syn. Tab., I830, p. 39.

Cervus (Megaceros) hibernicus, R. Johnson, Trans. Norfolk and Norwich Nat. Soc., vol. ii (1877), p. 288; W. R. Wilde, Proc. Roy. Irish Acad., vol. vii (1859), p. 195, woodcuts 4, 5 ; V. Ball, Trans. Roy. Dublin Soc. [2] vol. iii (1885), p. 337, pl. xi.

Cervus megaceros, J. Hart, Descr. Foss. Deer Ireland, I825, pls., and ibid., ed. 2, 1830, and Journ. Geol. Soc. Dublin, vol. i, 1838, p. 2 I ; J. Scouler, Journ. Geol. Soc. Dublin, vol. i, 1838, p. 197 ; G. Gray, New Statist. Account of sicotland, vol. v, 1845, p. 353 ; H. D. Richardson, Facts Nat. Hist. gigantic Irish deer, 1846; R. Ball, Proc. Roy. Irish Acad., vol. v (185I), p. 214 ; G. E. Roberts, Journ. Anthrop. Soc., vol. ii, I864, p. lxiii ; J. B. Jukes, Journ. Geol. Soc. Dublin, vol. x, 1864 , p. 127 , pls. xii-xv (cuts in bone); 
CDRvor giganteus (continued)-

G. Busk, Quart. Journ. Geol. Soc., vol. xxxi, 1875, p. 689; R. I. Moss, Proc. Roy. Irish Acad. [2] vol. ii (Sci.), 1876, p. 552; A. L. Adams, Proc. Roy. Irish Acad. [2] vol. iii (Sci.), I878, p.9r, and Sci. Proc. Roy. Dublin Soc., vol. ii, 1880 , p. 75 , and Trans. Roy, Dublin Soc. [2] vol. i (1881), p. 200, pl. xiv, f. 7, 8; A. Savin, Proc. Norwich Geol. Soc., vol. i (1878), p. 28 ; W. Williams, Sci. Proc. Roy. Dublin Soc. [2] vol. ii, I88o, p. I05; R. J. Ussher and A. L. Adams, Sci. Proc. Roy. Dublin Soc. [2] vol. ii, 1880, p. 234 ; T. R. Jones, Geol. Mag. [2] vol. viii, $188 \mathrm{I}$, pp. 95,480 ; (?) E. T. Newton, Vert. Forest Bed (Mem. Geol. Surv, 1882), p. 58 ; G. H. Kinahan, Trans. Edinburgh Geol. Soc., vol. iv, 1883, p. 343.

Megaceros hibernicus, R. Owen, Rep. Brit. Assoc., 1843 (I844), p. 237, and Brit. Foss. Mamm., 1846, p. 444, woodcuts 182-190, 194, and Quart. Journ. Geol. Soc., vol. iv, 1848, p. 42 ; J. Morris, Cat. Brit. Foss., I 854, p. 360 ; H. Denny, Proc. Geol. Polyt. Soc. Yorksh., vol. iii, 1855 (1859), p. 400 ; J. Hardy, Proc. Berwick Nat. Club, vol. iv (1860), p. 206, pl.ii; R. Howse, Trans. Tyneside Nat. Field Club, vol. v ( I 86I), p. 114, pl. iv ; D. Leslie, Geologist, 1864, p. 165; N. Moore, Ann. Mag. Nat. Hist. [3] vol. xx, 1867, pp. 77, 30I ; G. V. Du Noyer, Journ. Roy. Geol. Soc. Ireland, vol. i, I867, p. 247 ; A. Carte, Journ. Roy. Geol. Soc. Ireland, vol. i, 1867, p. 247 ; J. A. Smith, Proc. Soc. Antiq. Scot., vol. ix, 1873, p. 345 W. Williams, Geol. Mag. [2] vol. viii, I881, p. 354.

Cervus euryceros, S. Hibbert, Edinburgh Journ. Sci., vol. iii, 1825, p. 129, and ibid., n.s., vol. ii, I83o, p. 63 ; [-us] H. Falconer, Palæont. Mem., vol. ii, I868, p. 565.

Megaceros, R. I. Ussher and A. L. Adams, Journ. Roy. Geol. Soc. Ireland, vol. v, I880, p. I70.

'Fossil dama of Ireland,' Hamilton Smith, Griffith's Animal Kingdom, vol. v, 1827, p. 306.

'Fossil Elk from Ireland,' J. Parkinson, Organic Remains, vol. iii, 18ri, p. 314, pl. xx, f. I.

'Irish Elk,' R. E. Grant, in Thompson's Annual, 1838, p. 306, fig.

'Large Horns,' T. Molyneux, Phil. Trans., 1697, p. 489, pl. (Cervus platyceros altissimus).

'Fossil Elk,' [Anon.] Edinburgh Phil. Journ., vol, viii, 1823, p. 198; S. Hibbert, Edinburgh Journ. Sci., vol, iii, 1825, p. I5, pl. ii ; H. R. Oswald, Edinburgh Journ. Sci., vol. iii, 1825, p. 28 ; T. Weaver, Phil. Trans., 1825, p. 429.

'Elk,' J. Kelly, Phil. Trans., 1725, p. 122, pl. ; J. MacEnery, Cavern Researches, i859, pl. I.

'Deer's Horns,' J. Knowlton, Phil. Trans., 1747, p. 125, pl. i, f. 3. LOC. ? Forest Bed ; Cromer.

Pleist., Prehist., and Hist. ; Audley End, Brentford. Burweil Fen, Crowthor, Coldringham, Dogger Bank, Folkestone, Grays, Ilford, Isle of Man, Ipswich, Lancashire, 
Crzvणs giganteus (continued)-

London, Loxbrook, Oxford, Seaton Snook, S. Shields, Ufton (Berks), Walton (Essex).-Crofthead, Mavbole (Scotland).-Axe Bog, Ballebetagh, Ballynamore, Bumlin, Cloggy Bridge, Cavan, Chapelizod, Cloone, Dee River (Meath), Drumsna Bridge, Derryholms River, Dundrum, Fermanagh, Kilnagross, Kilmegan Bog, Kilskear (Meath), Legan Bog, Leighlin, Leitrim, Limerick, Lisduff, Lough Derg, Lough Gur Bog, Monaghan (Shirley Estate, in gypsum), Naul Bog, Rathcannon Bog, Reg Bog, Roscommon, Rooksey, Strokestown, Shannon, Tuam, Waterford (Ireland).

Caves; Bleadon, Brixham, Cresswell Crags, Hutton, Kent's Hole, Kirkdale, Long Hole, Spritsail Tor, Vale of Clwyd, Wookey Hole.-Ballynamintra, Cappagh, Kilowen Hole, Shandon (Ireland).

gunni, A. Bell, Proc. Geol. Assoc., vol. ii, 1872, p. 217 (C.gunni?) [-ii] R. Johnson, Trans. Norfolk and Norwich Nat. Hist. Soc., vol. ii (1877), p. 290; [-ii] E. T. Newton, Geol. Mag. [2] vol. vii, 1880, p. 45I, and Vert. Forest Bed (Mem. Geol. Surv., 1882), p. 57 .

LOC. Forest Bed; Mundesley. [Fragment of antler; Mus. Pract. Geol.]

hibernus, Desmarest. v. Cervus giganteus.

Jatifrons, Johnson. v. Alces latifrons.

matialis, Gunn (non Gervais). ข. Cervus sedgwicki.

megaceros, Hart. v. Cervus giganteus.

platyceros altissimus, Molyneux. v. Cervus giganteus.

polignacus, F. Robert, Ann. Soc. Agric. Sci. du Puy, I829, p. 8o, pl, iii, f. I (C. Dama polignacus); H. Falconer, Palæont. Mem., vol. ii, 1868, p. 479 ; R. Johnson, Trans. Norfolk and Norwich Nat. Soc., vol. ii (1877), p. $289 ;$ E. T. Newton, Geol. Mag. [2] vol. vii, 1880, p. 45I, and Vert. Forest Bed (Mem. Geol. Surv., 1882), p. 59 ; R. Lydekker, Cat. Foss. Mamm. B. M., pt. ii, I885, p. II 5.

LOC. Forest Bed; Mundesley, Norfolk. [Portion of antler; Norivich Mus.]

rectus, Newton. v. Capreolus caprea.

savini, W. B. Dawkins, Proc. Roy, Soc., vol. xxxviii, 1885, p. 345 , and Brit. Pleist. Mamm., pt. vi (Pal. Soc, i 887 ), p. is, pl. iii, f. 2-5; R. Lydekker, Cat. Foss. Mamm. B. M., pt. ii, 1885, p. 91 .

LOC. Forest Bed; Norfolk. [Portions of antlers; A. Savin Coll.]

seagwicki, [-ii] H. Falconer (ex Gunn, MS.), Palreont. Mem., vol. ii, 1868, p. 472, pl. xxxvii, f. I ; [-ii] E. T. Newton, Geol. Mag. [2] vol. vii, 1880, p. 451 ; R. Lydekker, Cat. Foss. Mamm. B. M., pt. ii, 1885 , p. 93.

Ceruus (Encladoceros) sedgwicki, R. Johnson, Trans. Norfolk and Norwich Nat. Soc., vol. ii (1877), p. 288. 
CrRvus sedgwicki (continued)-

Cervus sedgwickii $=C$. dicranios, E. T. Newton, Vert. Forest Bed (Mem. Geol. Surv., 1882), p. 60.

Cervus dicranios, F. Nesti, MS.; [-us] L. Rütimeyer, Abh. schweiz. Pal. Ges., vol. vii, 1881, descript. to pl. i.

Cervus dicranios $=C$. sedgwickii, W. B. Dawkins, Early Man in Britain, 1880 , p. 128 .

? Cerzus martialis, J. Gunn (non Gervais), Geol. Norfolk, I864. p. 19 ; E. T. Newton, Vert. Forest Bed (Mem. Geol. Surv.), 1882, p. 64 (rejects this determination of Gunn's and suggests the specimens are now included in ('. sedgwicki).

LOC. Forest Bed; Bacton, near Cromer, Kessingland. [Antler; Norwich Mus.]

strongyloceros, H. Woodward. v. Cervus elaphus.

suttonensis, W. B. Dawkins, Quart. Journ. Geol. Soc, vol, xxxiv, 1878 , p. 4 II, woodcuts 7-IO; R. Lydekker, Cat. Foss. Mamm. B. M., pt. ii, 1885 , p. 109.

Cernus dicranocerns, R. Owen (non Kaup), Quart. Journ. Geol. Soc., vol. xii, 1856, p. 224, woodcuts 14, 16.

LOC. Red Crag; Felixstowe, Ipswich, Sutton, Tallingstone, Woodbridge. [Portions of antlers; Brit. Mus.]

tarandus, Linnæus. \%. Rangifer tarandus.

tetraceros, W. B. Dawkins (emend. ex Bravard, MS.), Quart. Journ. Geol. Soc., vol. xxxiv, 1878, p. 416, woodcuts $13-17$, and ibid., vol. xxxix, 1983, p. 579; R. Lydekker, Cat. Foss. Mamm. B. M., pt. ii, I885, p. I13.

Cervus tetracroceros, S. J. Mackie (ex Bravard, MS.) Geologist, I86I, p. 466, woodcut.

LOC. ? Norwich Crag; Norfolk. [Fragment of antler; Brit. Mus., no. 33506.]

Forest Bed; Norfolk. [Portions of antler; Mus. Pract. Geol. and J. Backhouse Coll.]

verticornis, W. B. Dawkins, Quart. Journ. Geol. Soc., vol. xxviii, 1872, p. 405, woodcut 2 (non I), and Proc. Roy. Soc., vol. xxxviii, 1885 , p. 345, and Brit. Pleist. Mamm., pt. vi (Pal. Soc., I887), p. 22 , pls. v, vi, vii ; R. Johnson, Trans. Norfolk and Norwich Nat. Soc., vol. ii (1877), p. 289; E. T. Newton, Geol. Mag. [2] vol. vii, 1880, p. 45I, and Vert. Forest Bed (Mem. Geol. Surv., I 882), p. 6I (accepts the larger typespecimen, but refers the smaller one to $C$. divikinsi); R. Lydekker, Cat. Foss. Manım. B. M., pt. ii, 1885, p. 92.

'Large Strongyloceros,' H. Falconer, Palæont. Mem., vol. ii, I868, p. 479 (no. b).

LOC. Red Crag; Trimley, near Felixstowe.

Forest Bed; Cromer, Happisburgh, Hopton, Kessingland. Overstrand, Pakefield, W'est Runton, Sidestrand, Trimmingham. [Portion of antler; Norwich Mus.] 
CrTOzirgrim, J. F. Brandt, Bull. Phys.-Math. Acad. Imp Sci. St. Pétersbourg, vol. i, I 842 (I 843 ), p. 145.

Plesiocetopsis, J. F. Brandt, Mém. Acad. Imp. Sci. St. Pétersbourg, vol. $\mathrm{xx}, \mathrm{I} 873$, p. 143 .

Plesiocetus, P. J. Van Beneden, Bull. Acad. Roy. Belg. [2] vol. xxxiv, 1872, p. 15.

Heterocetus, P. J. Van Beneden, ibid., vol. 1, I880, p. 2 r.

Amphicetus, P. J. Van Beneden, ibid., vol. 1, 1880, p. 20.

? brevifrons, P. J. Van Beneden, Bull. Acad. Roy. Belg. [2] vol. xxxiv, 1872, p. 17; R. Lydekker, Quart. Journ. Geol. Soc., vol. xliii, 1887, p. 12, and Cat. Foss. Mamm. B. M., pt. v, 1887, p. 46.

Heterocetus brevifrons, P. J. Van Beneden, Bull. Acad. Roy. Belg. [2] vol. 1, 1880, p. 22.

Cetotherium (Plesiocetopsis) brevifrons, J. F. Brandt, Mém. Acad. Imp. Sci. St. Pétersbourg, vol. xx, I873, p. 145.

LOC. Red Crag; Woodbridge. [Atlas and axis vertebra; Brit. Mus.]

brialmonti (Van Beneden), R. Lydekker, Quart. Journ. Geol. Soc., vol. xliii, 1887, p. 12, and Cat. Foss. Mamm. B. M., pt. v, 1887, p. 42 .

Plesiocetus brialmontii, P. J. Van Beneden, Bull. Acad. Roy. Belg. [2] vol. 1, I88o, p. I8.

LoC. Red Crag; Woodbridge. [Axis vertebra; Brit. Mus.]

burtini, Van Beneden. v. Cetotherium hupschi.

dubium, P. J. Van Beneden, Bull. Acad. Roy. Belg. [2] vol. xxxiv, 1872 , p. 18 ; R. Lydekker, Quart. Journ. Geol. Soc., vol. xliii, I 887 , p. I2, and Cat. Foss. Mamm. B. M., pt. v, I887, p. 43.

Plesiocetus dubius, P. J. Van Beneden, Bull. Acad. Roy. Belg. [2] vol. 1, 1880, p. I8.

Cetotherium (Plesiocetopsis) dubium, J. F. Brandt, Mém. Acad. Imp. Sci. St. Pétersbourg, vol. xx, 1873, p. 146.

LOC. Red Crag; Woodbridge. [Periotic bones; Brit. Mus.]

? hupschi, [-ii] P. J. Van Beneden, Bull. Acad. Roy. Belg. [2] vol. xxxiv, 1872, p. 17 ; R. Lydekker, Cat. Foss. Mamm. B. M., pt. $v, 1887$, p. 45 .

Plesiocetus hupschii, P. J. Van Beneden, Bull. Acad. Roy. Belg. [2] vol. 1, 188o, p. 19.

Cetotherium (Plesiocetopsis) hupschii, J. F. Brandt, Mém. Acad Imp. Sci. St. Pétersbourg, vol. xx, 1873, p. I44.

Cetotherium hupschi, vel C. burtini, R. Lydekker, Quart. Journ Geol. Soc., vol. xliii, 1887, p. 12.

LOC. Red Crag ; Woodbridge. [Centrum of axis, centrum or caudal, and part of right temporal; Brit. Mus.] 
Carropotamus, Cuvier, Ossem. Fossiles, ed. 2, vol. iii, 1822, p. 260 (Charopotame).

chvieri, Owen. $v$. Chœropotamus gypsorum.

gצpsorum, A. G. Desmarest, Mammalogie, r822, p. 545 ; R. Lydekker, Cat. Foss. Mamm. B. M., pt. ii, 1885, p. 247.

Choropotamus cuvieri, R. Owen, Trans. Geol. Soc. [2]. vol. vi (I84I), p. 4I, pl. iv, f. I, 2, and Brit. Foss, Mamm., I846, p. 4 I3, woodcuts 163,164 ; J. Morris, Cat. Brit. Foss., 1854, p. 358. [Mandib. ramus ; Brit. Mus.]

Charopotamus parisiensis, Cuvier, Ossem. Foss., ed. 2, vol. v, pt. ii, 1824, p. 528.

LOC. U. Eocene ; Binstead and Seafield, I. of Wight. [Mandib. ramus and teeth; Brit. Mus.]

parisiensis, De Blainville. $v$. Chœropotamus gypsorum.

CrrovazIPIrUS, G. L. Duvernoy, Ann. Sci. Nat. (Zool.) [3] vol. xv, I85 I, p. 6I ; R. Lydekker, Cat. Foss. Mamm.'B. M., pt. v, I887, p. 64 .

cuvieri, Lankester. $v$. Choneziphius planirostris.

packardi, E. R. Lankester, Quart. Journ. Geol. Soc., vol. xxvi, 1870, p. 502, pl. xxxiii, f. I-4 ; R. Lydekker, Quart. Journ. Geol. Soc., vol. xliii, I887, p. 14, and Cat. Foss. Mamm. B. M., pt. v, I887, p. 67 (? C. planus).

Loc. Red Crag; Felixstowe. [Rostrum; Ipswich Mus.]

planirostris (Cuvier), E. R. Lankester, Quart. Journ. Ceol. Soc., vol. xxvi, 1870, p. 507, woodcut I ; R. Lydekker, Quart. Journ. Geol. Soc., vol. xliii, I887, p. I4, pl. ii, f. 7, and Cat. Foss. Mamm. B. M., pt. v, I887, p. 64, woodcut 12.

Ziphius planirostris, Cuvier, Ossem. Fossiles, ed. 2, vol. v, pt. i, I823, p. 356.

Ziphius cuvicri, R. Owen, Crag Cetacea (Pal. Soc., 1870), p. 6, woodcut 3 .

Choneziphius cuvieri, E. R. Lankester, Quart. Journ. Geol. Soc., vol. xxvi, 1870, p. 507, woodcut 2 .

LOC. Red Crag; Southwold, Woodbridge. [Cranial rostra; Brit. Mus.]

ז.

planus (Owen), R. Lydekker, Quart. Journ. Geol. Soc., vol. xliii, 1887 , p. 14, and Cat. Foss. Mamm. B. M., pt. v, 1887, p. 67.

Ziphius planus, R. Owen, Crag Cetacea (Pal. Soc., I870), p. 16, pl. ii, f. $\mathrm{I}$.

LOC. Red Crag; Shotley. [Rostrum; Brit. Mus.]

CORXPIJODON, R. Owen, Olontography (I845), p. 607, and Brit. Foss. Mamm., I846, p. 299.

anthracoideum, Gervais. \%. Coryphodon eocanus.

croydonensis, E. T. Newton, l'loc. Geol. Assoc., vol.. viii (1883), p. 254 , pl. iii.

Loc. L. Eocene ; Park Hill, Croyclon. [Ulna; Mus. I'ract. Geol.] 
COEX DODON (continued) -

eocrenug, R. Owen, Brit. Foss. Mamm., 1846, p. 299, woodcuts I03, 104, I07; J. Morris, Cat. Brit. Foss., 1854, p. 358; R. Lydekker, Cat. Foss. Mamm. B. M., pt. iii, I886, p. I78.

Coryphodon, R. Owen, Odontography (1845), p. 607, pl. cxxxv, f. 9. Coryphodon anthracoideum, P. Gervais, Zool. et Pal. Franç., ed. 2 , vol. i (1859), p. 53.

Lophiodon anthracoideum, H. D. de Blainville, Ostéographie, vol. iv, 1846 , p. II 7 .

LOC. London Clay; Dulwich, between Harwich and St. Osyth (dredged), and Sheppey. [Fragment of mandib. ramus; Brit. Mus.]

$\boldsymbol{T}$.

? sp., Owen. v. Palæotherium magnum.

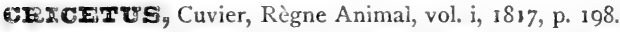

songarus (Pallas), W. A. Sanford, Quart. Journ. Geol. Soc., vol. xxvi, 1870 , p. 128, pl. viii, f. 6, and Proc. Somerset. Nat. Hist. Soc., vol. xv, 1870, p. 56, pl., f. 6.

Mus songarus, P. S. Pallas, Nov. Spec. Glirium, 1778, p. 269.

Loc. Cave ; Hutton, Mendip Hills. [Portions of skull and mandible; Taunton Mus.]

G20\$\$0

fodiens, Lydekker (non Pallas). $v$. Sorex pygmæus.

remifer, Owen, Morris, and Lydekker (non Geoffroy St. Hilaire). v. Sorex vulgaris.

DACRYThrrex M, H. Filhol, Comptes Rendus, vol. Ixxxii, I 876, p. 288.

oซ́num (Owen), R. Lydekker, Cat, Foss. Mamm. B. M., pt. ii, 1885, p. 187.

Dichobune ovinum, R. Owen, Rep. Brit. Assoc., I856 (1857), Sect., p. 72, and Quart. Journ. Geol. Soc., vol. xiii, 1857, p. 254 , pl. viii (D. ovina).

Loc. Headon Beds; Hordwell and I. of Wight. [Portions of mandible; Brit. Mus.]

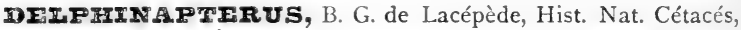
1804, p. xli.

Beluga, J. E. Gray, Spicilegia Zoologica, I828, pt. i, p. 2.

leucas (Pallas), R. Lydekker, Cat. Foss. Mamm. B. M., pt. v, I887, p. 79; E. T. Newton, Geol. Mag. [3] vol. vi, 1889, p. I48, pl. v, f. 3 .

Delphinus leucas, P. S. Pallas, Reise Prov. Kuss. Reichs, vol. iii, 1776, p. 85 .

Loc. Forest Bed; East Runton and Overstrand. [Caudal and lumbar vertebra; $A$. Savin Coll.]

Dredged; North Sea. [Caudal vertebra; Brit. Mus.] 
2EIPXIxU

? delphis, Linnæus, ivid., p. I08; (?) E. T. Newton, Geol. Mag. [2] vol. viii, 188I, p. 317 , and Vert. Forest Bed (Mem. Geol. Surv., 1882), p. 110, pl. vi, f. 2.

Delphinus, W. H. Flower, in Crowfoot and Dowson, Proc. Norwich Geol. Soc., vol. i (1878), p. 27.

LOC. Fluvio-marine Crag; Aldeby. [Vertebra; Norzuch Mus.] Forest Bed; Overstrand. [Vertebra; A. Sazin Coll.]

leucas, Pallas. $v$. Delphinapterus leucas.

melas, Traill. \% Globicephalus melas.

orere, Linnæus. ข. Orca gladiator.

orcoides, Lankester. v. Globicephalus uncidens.

tursio, Bonnaterre. $v$. Tursiops tursio.

uncidens, Lankester. $v$. Globicephalus uncidens.

sp., E. T. Newton, Geol. Mag. [2] vol. viii, 1881, p. 317, and Vert. Forest Bed (Mem. Geol. Surv., 1882), p. II 1, pl. vi, f. 3.

LOC. Forest Bed; Overstrand. [Caudal vertebra; A. Savin Coll.]

IICrOBUars, Cuvier, Ossem. Fossiles, ed, 2, vol. iii, 1822, p. 70 cervinus, Owen. v. Dichodon cervinus.

ovinum, Owen. v. Dacrytherium ovinum.

II IJIODCN, R. Owen, Quart. Journ. Geol. Soc., vol. iv, 1848, p. 36. cervinus (Owen), [-um] P. Gervais, Zool. et Pal. Franç., ed. 2, vol. i (1859), p. 159, pl. xxxv, f. 5 ; R. Lydekker, Cat. Foss. Mamm. B. M., pt. ii, 1885 , p. 165 .

Dichobunes cervinum, R. Owen, Trans. Geol. Soc. [2] vol. vi, I841, p. 45, and Brit. Foss. Mamm., I 846, p. 440, woodcut 181 ; J. Morris, Cat. Brit. Foss., I854, p. 359.

Cf. Moschus, S. P. Pratt, Trans. Geol. Soc. [2] vol. iii $(1832$, p. 452 , woodcut.

Anoplotherium dichobunes, Cuvier, MS., in S. P. Pratt, ibid., p. 453.

LOC. Binstead Beds and Headon Beds; I. of Wight. [Portions of mandible ; Brit. Mus.]

cuspidatus, R. Owen, Quart. Journ. Geol. Soc., vol. iv, 1848 , p. 42 , pl. iv, f. 2-6, and ibid., vol. xiii, 1857, p. 190, pl. iii, and Rep. Brit. Assoc., 1856 (1857), Sect., p. 72, and Palæontology, ed. 2, 1861, p. 368, woodcut 130; J. Morris, Cat. Brit. Foss., 1854 , p. 359 ; T. Wright, Ann. Mag. Nat. Hist. [2] vol. x, 1852, p. 87 , and Proc. Cotteswold Nat. Club, vol. i, 1853, p. 229 ; R. Lydekker, Cat. Foss. Mamm. B. M., pt. ii, 1885 , p. 164, woodcut 19 , and ibid., pt. v, 1887 , p. 333 .

Loc. Headon Beds; Alum Point and Hordwell. [Portions of jaws; Brit. Mus.] 
DICRANOCEROS, Owen. v. Cervus.

DIDIIPYYS, Linnæus, Syst. Nat, ed. 12, vol. i, 1766, p. 7 I (Didelphis).

Peratherium, A. Aymard, Ann, Soc. Agric. Sci. du Puy, vol. xiv, 1850 , p. 81 .

bucklandi, Broderip. $v$. Phascolotherium bucklandi.

colchesteri, R. Owen, Brit. Foss. Mamm., r846, p. 71, woodcut 22 ; J. Morris, Cat. Brit. Foss., I854, p. 359.

'Opossum jaw,' E. Charlesworth, Mag. Nat. Hist., vol. iii, 1839, p. 450 , woodcut 60.

Didelphys?, C. Lyell, Ann. Mag. Nat. Hist, vol. iv, I840, p. 190 ; R. Owen, Ann. Mag. Nat. Hist., vol. iv, 1840, p. 192, woodcuts $2 a, b, c$.

LOc. L. Eocene Sand; Kyson (Kingston), Suffolk. [Teeth ; W. Colchester Coll.]

prevosti, De Blainville. v. Amphitherium prevosti.

\$p., R. Lydekker, Cat. Foss. Mamm. B. M., pt. v, 1887, p. 286.

Loc. Headon Beds; Hordwell. [Portion of mandib. ramus ; Brit. Mus., no. 30350.]

I OPLODON, Gervais. " Mesoplodon.

angulatus, Gervais. ข. Mesoplodon angulatus.

angustus, Gervais. v. Mesoplodon angustus.

compressus, Gervais. v. Mesoplodon compressus.

gibbus, Gervais. v. Mesoplodon gibbus.

longirostris (Cuvier), Capellini. ข. Mesoplodon longirostris.

medilineatus, Gervais. $\%$. Mesoplodon longirostris.

tenuirostris, Gervais. $\%$. Mesoplodon tenuirostris.

IIPIOPUs, W. Kovalevsky, Phil. Trans., I 873 (1874), p. 30.

aymardi, W. Kovalevsky, ibid., p. 30, pls. xxxv-xxxviii (pars); R. Lydekker, Cat. Foss. Mamm. B. M., pt. ii, I885, p. 2 I6.

LOC. Headon Beds; Brockenhurst and Hordwell. [Limbbones ; Brit. Mus.]

ELEPIAS, Linnæus, Syst. Nat., ed. I2, vol. i, 1766, p. 48 ; H. Falconer, Quart. Journ. Geol. Soc., vol. xxi, 1865, p. 253 ; E. D. Cope, Amer. Nat., I889, p. I9I, pls.

STEGODON H. Falconer, Quart. Journ. Geol. Soc., vol, xiii, STEGODON 1857, p. 318. [Originally described as subgenera, LOXODON but ranked as genera by some later writers. The EUElephas genus Loxolon is preoccupied by a genus of Sharks (Müler and Henle, 184I).]

africanus, S. Woodward. v. Elephas meridionalis. 
IIIPIAS (continued)-

axtiquus, H. Falconer, Quart. Journ. Geol. Soc., vol. xiii, 1857, table facing p. 319, and Palreont. Mem., vol. ii, 1868, pp. 177, $25 \mathrm{I}$ (note), pl. ix, f. I-4; W. 13. Dawkins and W. A. Sanford, Brit. Pleist. Mamm., pt. i (Pal. Soc., I866), p. xxxy ; C. Moore, Proc. Bath Nat. Hist. Soc., vol. ii (1870), p. 51 ; A. L. Adams, Brit. Foss. Elephants, pt. i (Pal. Soc., 1877), pp. 1-68, pls. i-v, and ibid., 1881, p. 174, parts of pls. xvixxii ; A. Savin, Proc. Norwich Geological Soc., vol. i (1878), p. 28 ; F. Dixon, Geol. Sussex, ed. 2, 1878, p. xxiii ; E. T. Newton, Geol. Mag. [2] vol. viii, I881, p. 316, and Vert. Forest Bed (Mem. Geol. Surv., 1882), p. 104 ; H. H. Winwood, Proc. Bath Nat. Hist. Club, vol. vi (1886), p. 95; R. Lydekker, Cat. Foss. Mamm. B. M., pt. is, 1886, p. 122, woodcut 26, and ibid., pt. v, 1887, p. 339 ; H. Pohlig, Nova Acta Acad. Caes. Leop.-Carol., vol. lini, 1888, no. I; M. Browne, Vert. Anim. Leicester., 1889 , p. 27.

Elephas (Euelephas) antiquus, R. Johnson, Trans. Norfolk and Norwich Nat. Soc., vol. ii (1877), p. 283.

Elephas (Loxodon) priscus, H. Falconer and P. T. Cautley, Faunat Antiqua Sivalensis, 1846, pt. ii, pl. xiv, f. 6, 7; W. B. Dawkins and W. A. Sanford, Brit. Pleist. Mamm., pt. i (Pal. Soc., 1866), p. xxxiv. [Portion of tooth; Brit. Mus.]

Elephas (antiquus) trogontheriz, H. Pohlig, Sitz. niederrhein. Ges., 4 Feb. 1884, p. 32, and ibia., 9 Nov. 1885, p. 287.

Elephas primigenius, F. Dixon (errore), Geol. Sussex, I850, p. 16, woodcuts 1, 2, and ibid., ed. 2, 1878, pp. xxiii, 21, woodcuts I, 2.

Elephas meridionalis?, H. Falconer and P. T. Cautley (errore), Fauna Ant. Sival., 1846, pl. xiii A, f. 5, pl. xiv, f. I, 2, pl. xiv A, f. I-I3.

LOC. Norwich Crag; Easton, Suffolk, Thorpe.

Forest Bed; Bacton, Cromer, Happisburgh, West Runton.

Pleist.; Abingdon, Aylesford, Barrington, Barrow-onSoar, Barnstaple, Bedford, Brentford, Bracklesham, Camberwell, Cambridge, Canterbury, Coppenhall, Chatham, Clacton, Culham, Dogger Bank, Grays, Harwich, Huntingdonshire, Hurley Bottom, Ilford, Kent, Larkhall, Lawford, Lexden, London, Maidstone, Oundle, Oxford, Peckham, Peterborough, Saffron Watden, Slade Green, Southwold, St. Mary Stoke, Walton, Whittlesea, Woodborough. [Teeth; Brit. Mus.]

Caves; Bacon Hole, Bleadon, Crow Hole, Durdham Down, Hutton, Kirkdale, Long Hole, Minchin Hole, Ravenscliff, Spritsail Tor, Vale of Clwyd.

asiaticus, S. Woodward. $\%$ Elephas primigenius.

meridionalis, F. Nesti, Nuovo Giorn. Letter., vol. xi, 1325 , pp. 195, 211, pl. i; O. Fisher, Geol. Mag. [2] vol. iv; 1877 , p. 527, and Quart, Journ. Geol. Soc, vol, xliv, 1885, p. 8is, 
EIEPYAS meridionaliz (continued)-

woodcuts 2, 3 ; A. Savin, Proc. Norwich Geol. Soc., vol. i (1878), pp. 27, 28; W. B. Dawkins, Quart. Journ. Geol. Soc., vol. xxxvi, 1880, p. 395 ; A. L. Adams, Brit. Foss. Elephants, pt. iii (Pal. Soc., I881), pp. I8I-229, parts of pls. xvi-xxvi; E. T. Newton, Geol. Mag. [2] vol. viii, I 881, p. 316, and Vert. Forest Bed (Míem. Geol. Surv., 1882), p. 105; R. Lydekker, Cat. Foss. Mamm. B. M., pt. iv, I886, p. I07; J. C. ManselPleydell, Proc. Dorset. Nat. Hist. Field Club, vol. x, I889, p. I.

Elephas (Loxodon) meridionalis, H. Falconer, Palæont. Mem., vol. ii, 1868, pp. 104, 204, pl. viii ; R. Johnson, Trans. Norfolk and Norwich Nat. Soc., vol. ii (1877), p. 283.

Elephas antiquus?, A. L. Adams (errore), Brit. Foss. Elephants, pt. iii (Pal. Soc., I 88I), pl. xxvi, f. 2, 4.

Elephas africanus, S. Woodward (errore), Syn. Tab., 1830, p. 39, and Geol. Norfolk, 1833: p. 46.

'Fossil elephant's tooth,' J. Parkinson, Organic Remains, vol. iii, I811, p. 344, pl. xx, f. 6.

LOC. Red Crag; Fakenham, Felixstowe, Trimley.

Norwich Crag; Easton Cliff, Horstead, Thorpe.

? Pliocene; Dewlish (Dorset).

Forest Bed; Bacton, Cromer, Essex Coast (dredged), Happisburgh, Mundesley, West Runton.

Pleist. ; Clacton.

prinigenius, I. F. Blumenbach,* Handb. der Naturgeschichte (Ist French ed.), vol. ii, I803, p. 407; S. Woodward, Syn. 'Tab., I830, p. 39 (E. primogenius), and Geol. Norfolk, I833, p. 50 ; E. Chas lesworth, Mag. Nat. Hist., vol. iii, 1839, p. 347, woodcut 40 ; R. Owen, Brit. Foss. Mamm., I846, p. 217 , woodcuts $86,87,90-95$; H. Falconer and 1'. T. Cautley, Fauna Ant. Siv., pt. i, 1845, pl. i, f. I, pt. ii, 1846, pl. xiii A, f. 3 ; J. Morris, Cat. Brit. Foss., I 854 , p. 359 ; H. Denny, Proc. Geol. Polyt. Soc. Yorksh., voi. iii, I8j3 (Is59), p. 336 ; A. Brady, Rep. Brit. Assoc., 1859 (1860), Sect., p. I00 ; E. Brenan and A. Carte, Journ. Roy. Dublin Soc., vol. ii, 1860, pp. 344, 351, pl. x; H. Woodward, Geol. Mag., vol. i, r864, p. 24I, woodcut, and ibid, vol. v, I868, p. 540, pls. xxii, xxiii; W. Brockbank, Proc. Lit. and Phil. Soc. Manchester, vol. iv, 1865, p. 46; H. Falconer, Palæont. Mem., vol. ii, 1868, p. I6o; C. Moore, Proc. Bath Nat. Hist. Soc., vol. ii (1870), p. 51 ; J. Phillips, Geol. Oxford, I87I, p. 511; G. Busk, Quart. Journ. Geol. Soc., vol. xxviii, 1872 , p. 469, and ibid., vol. xxxi, 1875, p. 691, and Phil. Trans., 1873 (1874), p. 517 ; W. Davies, Cat. Pleist. Vert. Coll. Brady, I874, pp. 2, 3, and Geol. Mag. [2] vol. v, 1878 , p. 443, pl. xii ; R. W. Cheadle, Proc. W. London Sci. Assoc., vol. i (1876), p. 79; A. L. Adams, Trans. Roy. Irish Acad., vol. xxvi (Sci.), 1876, p. 212 , and Proc. Roy. Irish

* Owen $(\mathbf{r} 8,5)$ and Bronn $(\mathbf{r} 848)$ both quote Voigt's Magazin f. d. Neueste l'hys. Naturg., vol. v, pt. I, 1788, pp. I6 and 127, as being the first mention of this specific name. It does not, however, occur at these references, 'Elephant knochen' being recorded on p. 18, from Switzerland. 
BIIPIAS primigenius (contimuet)-

Acad. [2] vol. iii (Sci.), r878, p. 93, and Brit. Foss. Elephants, pt. ii (1'al. Soc., 1879), pp. 69-146, pls. vi-xv, woodcuts, and ibit., IS81, pp. 147-174, pls. xvi-xxii, xxvi (purs), and Sci. Proc. Roy. Dublin Soc., vol. ii, r880, p. 68, pl. ii ; W. B. Dawkins, Quart. Journ. Geol. Soc, vol. xxxv, 1879, p. I $38 ;$ H. Walker, Trans. Epping Forest and Essex [icicld Club, vol. i (1880), p. 27 ; E. T. Newton, Geol. May. |2! vol. viii, 188I, p. 31 5, and Vert. Forest Bed (Mem. Geol. Surv., 1882), p. 106; J. Gunn, Geol. Mag. [2] vol. x, 1883, p. 456, and ibid. [3] vol. i, r884, p. 47 ; A. T. Metcalfe, Journ. Derby. Archæol. Soc., I 885, pl. vi ; R. Owen, Quart. Journ. Gevi. Soc., vol. xli, 1885 , p. 32 , woodcuts 1,2 ; V. Ball, 'Trans. Roy. Dublin Soc. [2] vol. iii (1885), p. 344 ; H. H. Winwood, Proc. Bath Nat. Hist. Club, vol. vi (1886), p. 95; R. Lydekker, Cat. Foss. Mamm. B. M., pt. iv, 1886, p. 175, woodcut 3o ; J. A. Brown, Proc. Geol. Assoc., vol. x (1888), p. 36I (with flint implements); M. Browne, Vert. Anim. Leicester., 1889, p. 26. [See also Elephas antiquus.]

Elephas (Euelephas) primigenius, H. Falconer, Palæont. Mem., vol. ii, I868, p. I 58 ; R. Johnson, Trans. Norfolk and Norwich Nat. Soc., vol. ii (1877), p. 284.

Elephas, P. O. Hutchinson, Trans. Devon. Assoc., vol. iii, I869, p. I43, pl.; H. Falconer, Palæont. Mem., vol. 1868, p. 566.

'Elephant,' J. Luff kin, Phil. Trans., 170r, p. 924 ; J. Morton, Nat. Hist. Northamptonshire, 1712 , p. 252, pl. xi, f. 4; H. Baker, Phil. Trans., 1745, p. 331, pl. i, f. 7 ; E. Jacob, Phil. Trans., 1754, pt. ii, p. 626; R. Bald, Mem. Werner. Soc., vol. iv, I 822 , p. 58, pl. iv ; W. Buckland, Phil. Trans., I 822 , p. 182, pl. xxi, f. I, 2 , and Reliq. Diluv., 1824 , p. 262, pl. vii, f. I, $2 ;$ H. T. De la Beche, Trans. Geol. Soc. $\{2]$ vol. i (1824), p. 42 I ; J. Layton, Edinburgh Journ. Sci., vol. vi, I827, p. 199, pl. iv, f. I, 2 (tusk); G. Young and J. Bird, Geol. Surv. Yorkshire Coast, ed. 2, I828, pl. xvii, f. I ; J. Scouler, Edinburgh Journ. Nat. Geogr. Sci., vol. ii, 1830, p. 4 ; J. C. Bellamy, Nat. Hist. S. Devon, 1839 , p. 438, pl. xi, f. 1, 2 ; J. Brown, Mag. Nat. Hist., vol. viii, 1835, p. 354, and Ann. Mag. Nat. Hist. [2] vol. xx, 1857, p. 396; J. MacEnery', Cavern Researches, I859, pl. M, f. I-3; J. Gray, Geol. Mag., vol. i, 1864, p. 46 ; R. Harkness, Geol. Mag, vol. vii, I870, p. 253 .

'Mammoth,' R.'Dadd, Mag. Nat. Hist., vol. iv, I831, p. 240, woodcut 49; A. Carte, Nat. Hist. Rev., vol. vi (1859), p. 501 ; A. L. Adams, Journ. Roy. Geol. Soc. Ireland, vol. iv, 1877 , p. 247 ; W. B. Dawkins, Quart. Journ. Geol. Soc., vol. xxxi, 1879, p. 138, and Trans. Manchester Geol. Soc., vol. xv, 1878, p. 55 ; H. H. Howorth, Geol. Mag. [2] vol. vii, I 880 , vol. viii, 1881 (passim), and Mammoth and Flood, 1858; J. Prestwich, Geol. Mag. [3] vol. vi, 1889, p. I13.

Elephas asiaticus, S. Woodward (errore), Syn. Tab., 1830, p. 39, and Geol. Norfolk, 1833, p. 46. 
IIIFEAS pximigenius (continued)-

'Fossil Elephant's tooth,' J. Parkinson, Organic Remains, vol. iii, 1811, p. 345 , pl. xx, f. 8.

'Large teeth,' F. Nevile, Phil. Trans., I 7 15, p. 367 ; T. Molyneux, Phil. Trans., 1715, p. 370.

LOC. Forest Bed; near Cromer, Mundesley, and Sidestrand.

Chalk Debris ; Horstead.

Pleist.; Atwick, Aylesford, Avon Valley (Warwick), Alton, Arundel, Abingdon, Acton, Bagley Wood, Betchworth, Barnstaple, Brandsburton, Bognor, Bickley (Worcester), Bracklesham, Barnwood, Beckford, Brentford, Ballingdon, Bath, Broughton Giffard, Battersea, Bostal, Barnwell, Brighton, Bridport, Box, Bielbecks, Bromwich Hill, Burton, Bowden parva, Camps Hill, Chedzoy, Canterbury, Clapton, Chatham, Chippenham, Clacton, Crayford, Church Cliff (Lyme Regis), Cambridge, Charmouth, Culham, Dogger Bank, Dorking, Droitwich, Dorchester, Dudley, Elton, Erith, Eastnor, Eckington, Fawler, Foxham, Fenny Stratford, Fladbury, Fisherton, Freshford, Flint, Folkestone, Freshwater, Green Street, Grays, Gloucester, Happisborough, Holyhead, Hinton, Harswell, Hartlip, Harwich, Hoxton, Heddingham, Hoxne, Huntingdonshire, Harborough, llford, Ipswich, Isle of Dogs, Icklingham, 1. of Wight, Knoll Bank, Kew, Kettering, Kingsland, Kings Newnham, Larkhall, Loxbrook, Lyncombe, Leeds, Lawford, Littlehampton, Lambridge, Leicester, Lyme Regis, Lamarsh, Lewisham, Middleton, Maidstone, Maidenhead, Malvern, Milford Hill, Murston, Morefield, Millbank, Medway River, Northampton, Newington, Northwich, Overton, Oxford, Orford, Otterham, Oundle, Peterborough, Peasmarsh, Peckham, Rugby, Rodborough, near Kochester, St. Audries, Slade Green, Salisbury, Selsey, Stratford-on-Avon, Southwold, Southall, Sandlin, Sittingbourne, Stour Valley, Stroud, Sheppey, Spalding, Sidmouth, Shoreham (Kent), Strood, Stowe Valley, Tewkesbury, Thetford, Thame, Trentham, Tooting, Turnham Green, Tiverton, Walton (Essex), Weston, Worthing, Walton, Wellesborne, Wallingford, Witham, Whitstable, Walthamstow, Yarnton, Yarmouth, Yeovil.-Airdrie, Bishopsbridge, Clifton Hall, Kilmaurs, Kilmarnock, Kinloch, Kimmerghame (Scotland).-Ballyrudder, Corncastle (Antrim), Galway Bay, Dungarvan, Maghery (Cavan), Tully-dolly (Ireland).

Caves ; Bench, Bleadon, Boxhill, Brixham, Coygau, Cresswelt Crags, Derbyshire, Durdham Down, Hutton, Kent's Hole, Kirkdale, Maidstone (fissure), Laugharne, Longr Hole, Oreston, Paviland, Portland, Sandford Hill, Spritsail Tor, Vale of Clwyd, Wells, Wookey Hole, Yealm Bridge.-Shandon (Ireland).

priscas, Falconer and Cautley. 2\%. Elephas antiquus.

trogontherie, Pohlig. v. Elephas antiquus 


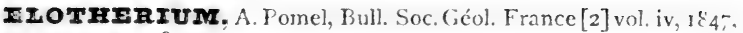
p. 1083 .

Entelodon, A. Aymard, Ann. Soc. Agric. Sci. du Puy, vol. xii, 1846, p. 240.

aymiardi, Pomel. v. Elotherium magnum.

magnum, A. Pomel, Bull. Soc. Géol. France [2] vol. iv, 1847, p. 108 3 : R. Lydekker, Cat. Foss. Mamm. B. M., pt. ii, 1885, p. 249.

Entclodon magnum. A. Aymard, Ann. Soc. Agric. Sci. du l uy, vol. xii, 1846, p. 240.

Elotherium aymardi, A. Pomel, Catal. Méthod., 1853, p. 89.

I.OC. Hempstead Beds; 1. of Wight. [Tooth; Brit. Mus.]

ENTELODON, Aymard. 2\%. Elotherium.

magnum, Aymard. ¿. Elotherium magnum.

IQUU: Linnaus, Syst. Nat., ed. I2, vol. i, I766, p. 100.

Hippidion, R. Uwen, Phil. Trans., I 869 (I870), p. 572 (subgenus).

adamiticus, Schlotheim. \% Equus caballus.

brevirostris, Kaup. v. Eyuus caballus.

caballus, Linnxus, Syn. Nat., ed. 12, vol. i, 1766, p. 100 ; S. Woodward, Syn. Tab., 1830, p. 39, and Geol. Norfolk 1833 , p. 45 ; C. C. Blake, Geologist, 1862, p. 218 ; C. Mone, Proc. Bath Nat. Hist. Soc., vol. ii (1870), p. 52 ; G. Busk, Phil. Trans., 1873 (1874), p. 519, woodcut; A. L. Adams, Trans. Roy. Irish Acad., vol. xxvi (Sci.), 1876, p. 215, and Proc. Roy. Irish Acad. [2] vol. iii, I878, p. 90, and Sci. Proc. Roy. Dublin Soc., vol, ii, 1880, p. 71; A. Savin, Proc. Norwich Geol. Soc, vol. i (1878), p. 28 ; R. Lydekker, Cat. Foss. Mamm. B. M., pt. iii, 1886, p. 73; M. Browne, Vert. Anim. Leicester, 1889, p. 28.

Equus caballus-fossilis, E. T. Newton, Geol. Mag. [2] vol. vii, 1880 , p. 448, and Vert. Forest Bed (Mem. Geol. Surv., I88z), p. 30, pl. vii, f. I-8; H. H. Winwood, Proc. Bath Nit. Hist. Club, vol. vi (1886), p. 95.

Equus cabullus ferus, V. Ball, Trans. Roy. Dublin Soc. [2] vol, iii (1885), p. 336.

Equus adamiticus, E. F. von Schlotheim, Petrefactenkunde, 1820 , p. 11.

Equus brevirostris, J. J. Kaup, Neues Jahrb., 1833, p. 518, pl. vii.

Equus fossilis, $H$. von Meyer, Palxologica, 1832, p. 79 ; R. Owen, Brit. Foss. Mamm., I846, p. 383 , woodcuts 143 , 145-148 ; J. Morris, Cat. Brit. Foss., 1854, p. 359 ; J. Hogt, Trans. Tyneside Nat. Field Club, vol. vi, $1864, P .28$ (E. fossilis?); W. B. Dawkins and W. A. Sanford, liti. Pleist. Mamm., pt. i (Pal. Soc., 1866), p. xxix.

? Equus plicidens, R. Owen, Rep. Brit. Assoc, $1843(1844$, p. 23I, and Brit. Foss. Mamm., 1846, p. 392, woodctits 152-156; J. Morris, Cat. Brit. Foss.. 1854 P. 359; K John-wr. 'Trans. Norfolk and Norwich Nat. Soc., vol. in (1877), F. 2is. ['Teeth from Oreston.]

Equas spelows, R. Owen, Phil, Trans, I869 (1870), p. 544. 
IQTS caballus (continued) -

Asinus fossilis, R. Owen, Brit. Foss. Mamm., 1846, p. 396, woodcuts $157,158$.

'Cheval fossile,' Cuvier, Ossem. Fossiles, ed. 2, vol. ii, pt. i, I822, p. 109.

Equus, A. Carte, Nat. Hist. Rev., vol. vi (1859), p. 502 ; G. Busk, Quart. Journ. Geol. Soc., vol. xxviii, I872, p. 465 ; J. Simpson, Trans. Edinb. Geol. Soc., vol. v, 1887, p. 296.

'Horse,' W. Buckland, Phil. Trans., 1822, p. 182, pl. xxi, f. 7 , pl. xxiv, f. I, and Reliq. Diluv., 1824, p. 262, pl. vii, f. 7, pl. x, f. I ; W. Clift, Phil. Trans., I823, p. 86 , pl. x, f. 6 ; J. C. Bellamy, Nat. Hist. S. Devon, 1839 , p. 438 , pl. xii, f. 3, 4 ; J. MacEnery, Cavern Researches, 1859 , pl. K, f. 6, pl. Q, f. 9-12 ; W. B. Dawkins, Quart. Journ. Geol. Soc., vol. xxxiii, 1877, p. 599 .

LOC. ? Norwich Crag; Thorpe.

Forest Bed; Cromer, Happisburgh, Kessingland, Mundesley, and West Runton.

Pleist. and Prehist.; Acton, Audley End, Applecake Hill, Brentford, Bielbecks, Bonchurch, Bracklesham, Brighton, Bath, Box, Camps Hill, Chatham, Crayford, Clacton, Dogger Bank, Erith, Freshford, Fisherton, Fladbury, Felixstowe, Grays, Godmanchester, Greenwich, Hartlip, High Lugwardine, Hessle, Ilford, Iffley, Ipswich, Kyson, Larkhall, Leicester, Little Comberton, London, Loxbrook, Maidstone, Murston, Newbury, Norton (Tyneside), Otterham, Oundle, Oxford, Peterborough, Saffron Walden, Slade Green, Stockton, Sutton, Sittingbourne, Thetford, Walthamstow, Westonsuper-Mare, Walton-on-the-Naze, Windsor.-Pentland Hills (Scotland).-Antrim, Broughshane, Downpatrick, Lough Erne, Newry (Ireland).

Caves; Bleadon, Brixham, Caswell Bay, Coygau, Cresswell Crags, Durdham Down, Heathery Burn, Hutton, Kent's Hole, Kirkdale, Long Hole, Laugharne, Maidstone (fissure), Oreston, Paviland, Pleasley Vale, Portland (fissure), Ravenscliff, Sanford Hill, Spritsail Tor, Teesdale, Uphill, Vale of Clwyd, Wookey Hole.Ballintoy, Ballynamintra, Shandon (Ireland).

cabcallus-fossilis, Newton. ข. Equus caballus.

fossilis, Meyer. v. Equus caballus.

grexcilis, Kaup. v. Hipparion gracile.

plicidens, Owen. $v$. Equus caballus and Equus sp.

spelocus, Owen. $v$. Equus caballus.

stenonis, I. Cocchi, Mem. Soc. Ital. Sci. Nat., vol. ii, 1867, art. 7, p. 18 ; E. T. Newton, Vert. Forest Bed (Mem. Geol. Surv., I 882), p. 35, pl. vii, f. 9, 10 ; R. Lydekker, Cat. Foss.

Mamm. B. M., pt. iii, 1886, p. 69.

LOC. Norwich Crag; Thorpe.

Forest Bed; Cromer, Happisburgh, Sidestrand. 
खQUUS (continued)-

sp. ('larger than E. caballus or E. stenonis'), R. Lydekker, Cat.

Foss. Mamm. B. M., pt. iii, '1886, p. 89.

Equns cf. plicidens, R. Owen, Quart. Journ. Geol. Soc., vol. xii, 1856, p. 223, woodcut 12 .

Loc. Norwich Crag; Norfolk.

Red Crag; Bawdsey. [Teeth; Brit. Mus., no. 2704, etc.]

IRINACEUS, Linnæus, Syst. Nat., ed. I2, vol. i, I766, p. 75.

europæus, Linnæus, ibid., p. 75 ; A. L. Adams, Trans. Roy. Dublin Soc. [2] vol. i (188I), p. 206.

Loc. Cave; Ballynamintra (Ireland). [Tibia; Dublin Mus.]

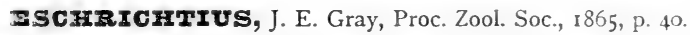

robustus, J. E. Gray, ibid., p. 40; W. Lilljeborg, Nova Acta Reg. Soc. Sci. Upsala [3] vol. vi, I868, fasc. 2, mem. I, p. 16, pls. i-viii ; W. H. Flower, Ann. Mag. Nat. Hist. $[+]$ vol. ix, 1872, p. 440, and Trans. Roy. Geol. Soc. Conwall, vol. ix (1875), p. 117 .

Balanoptera robusta, W. Lilljeborg, Förh. Skand. Naturf. (1860, Copenhagen), I86I, p. 602, and Upsala Univ. Aarsk., 1862, Naturvet., p. 39.

Loc. Pleist.: Petuan, Cornwall. [Portions of skeleton; Penzance Mus.]

FSTHON $\mathrm{XX}$, Leidy. v. Platychœrops richardsoni.

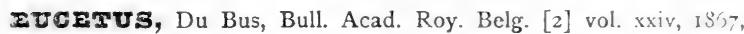
p. 572 .

amblyodon, Du Bus, ibid., p. 572 ; R. Lydekker, Quart. Journ. Geol. Soc., vol. xliii, 1887 , p. 13, woodcut 2, and Cat. Fuss. Mamm. B. M., pt. v, 1887 , p. 54 , woodcut II.

Loc. Red Crag; Felixstowe and Woodbridge. [Teeth; Brit. Mus.]

s.

WUCLADOCEROS, Falconer. v. Cervus.

ZUUELEPHAS, Falconer. v. Elephas.

primigenius, Blumenbach. $v$. Elephas primigenius.

EURYTHERIUM, P. Gervais. v. Anoplotherium.

latipes, P. Gervais. $v$. Anoplotherium commune.

FEIIS, Linnæus, Syst. Nat., ed. I2, vol. i, 1766, p. 60.

antiqua, Cuvier. v. Felis pardus.

brevirostris, Croizet and Jobert, Oss. Foss. Puy-de-Dôme, vol. i, 1828, p. 200 ; R. Laing, Rep. Brit. Assoc., 1889.

LOC. Cave; Cresswell Crags. [Jaws; R. Laing Coll.] 
EIIIS (continued)-

caffra, A. G. Desmarest, Mammalogie, 1822 (Suppl.), p. 540 (F. cafra); W. A. Sanford, Proc. Somerset. Nat. Hist. Soc., vol. xiv, 1869, p. 144, pl. xxiv, f. 6, 7 (F. caffer); W. B. Dawkins and W. A. Sanford, Brit. Pleist. Mamm., pt. iv (Pal. Soc., I872), p. 182, pl. xxiv, f. 6 (F. caffer).

Loc. Caves; Bleadon, Mendip Hills. [Right mandib. ramus; Taunton Mus.]

catus, Linnæus, Syst. Nat., ed. 12, vol. i, 1766, p. 62 ; R. Owen, Brit. Foss. Mamm., 1846, p. 172, woodcut 67 ; J. Morris, Cat. Brit. Foss., I854, p. 359 ; W. A. Sanford, Proc. Somerset. Nat. Hist. Soc., vol. xiv, 1869, p. 147 , pl. xxiv, f. 8, 9 ; W. B. Dawkins and W. A. Sanford, Brit. Pleist. Mamm., pt. iv (Pal. Soc., 1872), p. I83, pl. xxiv, f. 8, 9; R. Lydekker, Cat. Foss. Mamm. B. M., pt. i, I885, p. 64.

Felis catus ferus, W. B. Dawkins, Quart. Journ. Geol. Soc., vol. xxxiii, 1877 , p. 596.

'Cat,' J. MacEnery, Cavern Researches, 1859, pl. E, f. 18.

ג.oC. Pleist.; Grays. [Mandib. ramus; Oxford Mus.]

Caves; Bleadon, Cresswell Crags, Kent's Hole, Long Hole, Ravenscliff, Teesdale, ? Uphill, Vale of Clywd.

Ieo, Linnæus, Syst. Nat., ed. 12, vol. i, 1766, p. 60; J. Morris, Cat. Brit. Foss., I854, p. 359 ; R. Lydekker, Cat. Foss. Mamm. B. M., pt. i, 1885, p. 50.

Felis spelcea, Goldfuss, Die Umgebungen von Muggendorf, 1810 , p. 277 , pl.v, and Nova Acta Acad. Cres. Leop.-Carol., vol. x, 182 1, p. 489, pl. xlv ; R. Owen, Rep. Brit. Assoc., I84.2 (1843), p. $7 \mathbf{1}$, and Brit. Foss. Mamm., I846, p. I6I, woodcuts $63-65$, and Phil. Trans., 1859, p. 317, pl. xii, f. I ; J. Morris, Cat. Brit. Foss., 1854 , p. 359 ; W. B. Dawkins and W. A. Sanford, Brit. Pleist. Mamm., pt. i (Pal. Soc., I866), p. r, pls. i-v, and ibid., pt. ii, 1868, pp. 29-124, pls. vi-xix, and ibid., pt. iii, I869, pp. 125-171, pls. xx-xxii B ; H. Falconer, Palæont. Mem., vol. ii, I 868 , p. 455 , pl. xxxv, f. I I ; W. B. Dawkins, Pop. Sci. Rev., vol. viii, 1869 , p. 150; W. A. Sanford, Proc. Somerset. Nat. Hist. Soc., vol. xiv, 1869, p. 103, pls. i-xxiii ; G. Busk, Phil. Trans., I873 (1874), p. 528, pl. xlv, f. 4-8; W. B. Dawkins, Quart. Journ. Geol. Soc., vol, xxxiii, I877, p. 595.

'Lion,' W. Salmond, Phil. Mag., n.s., vol. vi, 1829, p. 231. [Sce also Vernon, Phil. Mag., n.s., vol. vii, 1830 , p. 2.]

'Tiger,' J. MacEnery, Cavern Researches, 1859, pl. c, f. I, pl. E, f. I, 2 (5-7 ?).

LOC. Pleist.; Barnwell, Bielbecks, Brentford, Crayford, Clacton, Fisherton, Hartlip, Hurley Bottom, llford, Ipswich London, Loxbrooke, Otterham, Oxford, Sittingbourne, Slade Green.

Caves; Banwell, Bleadon, Brixham, Cresswell Crags, Durdham Down, Hutton, Kent's Hole, Kirkdale, Kirby Moorside, Long Hole, North Hill 'Tor, Ravensclift, Sandford Hill, Spritsail Tor, Vale of Clwyd, Wookey Hole. 
IㄹIIS (continued)-

leopardus, Lyell. v. Felis pardoides.

Iyax, Linnæus, Syst. Nat, ed. 12, vol. i, 1766, p. 62 ; W. H. Ransom, Rep. Brit. Assoc., I 866 (1867), Sect., p. 66 ; W. B. Dawkins and W. A. Sanford, Brit. Pleist. Mamm., pt. iii (Pal. Soc., 1869), pp. 172-176, pl, xxiii.

'Lynx,' W. Davies, Geol. Mag. [2] vol, vii, I880, p. 346, pl. xi.

Loc. Caves; Teesdale, Yew Tree Cave, and Pleasley Vale (Derbyshire).

pardoides, R. Owen, Brit. Foss. Mamm, 1846, p. 169, woodcut 66, and Quart. Journ. Geol. Soc., vol. xii, 1856, p. 226, woodcut 19; J. Morris, Cat. Brit. Foss., 1854, p. 359.

Felis leopardus, C. Lyell, Ann. Nat. Hist., vol. iv, 1840, p. I86, woodcut $\mathrm{I}$.

Loc. Red Crag ; Newbourne, Suffolk. [Tooth ; Ipswich Mus.]

pardus, Linnæus, Syst. Nat., ed. 12, vol. i, 1766, p. 6r ; W. A. Sanford, Proc. Somerset. Nat. Hist. Soc., vol. xiv, 1869 , p. 142, pl. xxiv, f. I-5 ; W. B. Dawkins, Quart. Journ. Geol. Soc., vol, xxxiii, I877, p. 595 (ectocuneiform).

Felis (Leopardus) pardus, W. B. Dawkins and W. A. Sanford, Brit. Pleist. Mamm., pt. iv (Pal. Soc., 1872), p. 177, pl. xxiv ( $F$. pardus antiqua on plate).

Felis antiqua (Cuvier), W. A. Sanford, Rep. Brit. Assoc., I864 (1865), Sect., p. 69; W. B. Dawkins and W. A. Sanford, Brit. Pleist. Mamm., pt. i (Pal. Soc, 1866), p. xxi. [Teeth; Taunton Mus.]

Loc. Caves ; Banwell, Bleadon or Hutton, and Cresswell Crags. spelæa, Goldfuss. $v$. Felis leo.

? sp., E. T. Newton, Vert. Forest Bed (Mem. Geol. Surv, 1882), p. 23 ; R. Lydekker, Cat. Foss. Mamm. B. M., pt. i, 1885, p. 68.

LOC. Forest Bed; Kessingland. [Humerus; Brit. Mus., no. M. 1625. Portion of fibula ; Mus. Pract. Geol.]

GAzraIA, H. D. de Blainville, Bull. Soc. Philom. Paris, 1816, p. 75. anglica, E. T. Newton, Quart. Journ. Geol. Soc., vol. xl, I884, p. 280, pl. xiv; R. Lydekker, Cat. Foss. Mamm. B. M., pt. ii, 1885 , p. 52 .

LOC. Norwich Crag; Thorpe, near Norwich. [Right irontal and horn-core; Mus. Pract. Geol.]

GALESTES. A supposed Purbeck Mammal, quoted in geological text-books; the name not existing in zoological literature.

GבOR צCrus, J. K. W. Illiger, Prodr. Syst, Mamm, x 8 r, p. 87. sp., Owen. v. Siphneus sp.

GLOBICEPS, Flower. v. Globicephalus, 
GIOBICEPIAIUS, * R. P. Lesson, Nouv. Tab. Règne Anima? (Mammifères), 1842, p. 200 (Globicephala); J. E. Gray, Zool. 'Erebus' and 'Terror,' 1846, p. 32.

Globiceps, W. H. Flower, Proc. Zool. Soc., I883, p. 508.

elas (Traill), R. Lydekker, Cat. Foss. Mamm. B. M., pt. v, 1887, p. $8 \mathrm{I}$.

Delphinus melas, T. S. Traill, Nicholson's Journal, vol. xxii, 1809 , p. $8 \mathrm{r}$, pl. iii.

LOC. Pleist.; Barking (Essex), Eastern Coast. [Imperfect cranium and vertebre, Brit. Mus.]

azcidens (Lankester), R. Lydekker, Quart. Journ. Geol. Soc., vol. xliii, 1887, p. 16, pl. ii, f. 11, and Cat. Foss. Mamm. B. M., pt. v, 1887 , p. 8I, woodcut 17 .

Delfhinus (Phoccna) uncidens, E. R. Lankester, Ann. Mag. Nat. Hist. [3] vol. xiv, 1864, p. 356, pl. viii, f. 12, 13.

Delphinus (Phocana) orcoides, E. R. Lankester, ibid., p. 356, pl. viii, f. 14-18. [Teeth; olim Whincopp Coll.]

ı.oc. White Crag; Orford.

Red Crag ; Birtley River, Felixstowe, Sutton, and Woodbridge. [Teeth; York Mus.]

sp., R. Lydekker, Cat. Foss. Mamm. B. M., pt. v, 1887, p. 83.

LOC. Norwich Crag; [unknown.] [Centra of lumbarvertebræ; Brit. Mus., nos. 33504-05.]

sp., W. Turner, Proc. Roy. Phys. Soc. Edinb., vol. viii (1885), p. 336.

Balcenoptera, J. Leitch, Trans. Cumberland Assoc., no. ix, 1885 , p. 172 .

LOC. Pleist. ; Silloth. [Vertebra.]

CULO, G. C. C. Storr, Prodr. meth. Mamm., 1780, p. 34, table A.

Iuscus (Linnæus), W. B. Dawkins and W. A. Sanford, Brit. Pleist. Mamm., pt. i (Pal. Soc., 1866), p. xxi ; W. B. Dawkins, Quart. Journ. Geol. Soc., vol. xxvii, 1871, p. 406 ; G. Busk, Quart. Journ. Geol. Soc., vol. xxxi, I875, p. 687, woodcut 4; E. T. Newton, Geol. Mag. [2] vol. vii, 1880, p. 424, pl. xv, and Vert. Forest Bed (Mem. Geol. Surv., 1882), p. 17, pl. vi, f. I.

Ursus luscus, Linnæus, Syst. Nat., ed. 12, vol. i, 1766, p. 71.

Mustela gulo, Linnæus, ibid., p. 67.

'Glutton,' J. C. Bellamy, Nat. Hist. S. Devon, I839, pp. 89, 94, 102 ; W. Pengelly, Trans. Devon. Assoc., vol. iv, 1871, pp. 98, I02, and Geol. Mag. [2] vol. vii, 1880, p. 480; W. B. Dawkins, Cave Hunting, I874, p. 287, woodcut 82, and Early Man in Britain, 1880 , p. 100 , woodcut 21 .

LOC. Forest Bed; near Mundesley. [Portion of mandible; R. Fitch Coll., Norwich.]

Caves; Banwell, Bleadon, Cresswell Crags, Vale of Clwyd, Yealm Bridge.

* The reason for adopting this name instead of Globiceps is given by 7V. H. Flower in Proc. Zool. Soc., 1884, p. 418. 
MAITTHERIUME, J. J. Kaup, Neues Jahrb., 1838 , p. 319 ( $/ \mathrm{Hal}$. therium).

canhami, W. H. Flower, Quart. Journ. Geol. Soc., vol. xxx, 1874 , p. I, pl. i; R. Lydekker, Cat. Foss. Mamm. B. M., pt. v, I 887, p. $\%$.

Loc. Red Crag; Foxhall. [Abraded cranium; Ipswich Mus.]

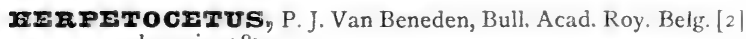
vol. xxxiv, 1872, p. 20.

scaldiensis, P. J. Van Beneden, ibid., p. 20; R. Lydekker, Quart. Journ. Geol. Soc., vol. xliii, I887, p. I3, and Cat. Foss. Mamm. B. M., pt. v, 1887 , p. 47.

Loc. Red Crag; Felixstowe. [Tympanic bone; Mus. Prait. Geol.]

IIETEROCETUS, Van Beneden. v. Cetotherium.

brevifrons, Van Beneden. v. Cetotherium brevifrons.

gyrparioN, J. de Christol, Ann. Sci. Indust. Midi France, vol. i, I832, pp. 180, 181 .

Hippotherium, J. J. Kaup, Neues Jahrb., 1833, p. 32\%

? gracile (Kaup), A. Gaudry, Anim. Foss. Mont Léberon, 1873, p. 32; R. Lydekker, Quart. Journ. Geol. Soc., vol. xlii, 1886, p. 366, and Cat. Foss. Mamm. B. M., pt. iii. I886, p. 51 .

Equus (Hippotherium) gracilis, J. J. Kaup, Neues Jahrb., 1833, p. 327.

Loc. Red Crag; Suffolk. [Teeth; Brit. Mus.]

IIIPPIDION, Owen. $v$ Equus.

IIIP POPOTAIXUS, Linnæus, Syst. Nat., ed. I2, vol. i, 1766, p. 101 .

amphibius, Linnæus, Syst. Nat., ed. 12, vol. i, 1766, p. 10I; R. Lydekker, Cat. Foss. Mamm. B. M., pt. ii, 1885, p. 277.

Hippopotamus antiquus, A. G. Desmarest, Mammalogie, 1822, p. 388.

Hippopotamus major, R. Owen, Rep. Brit. Assoc., 1843 (1844), p. 223 , and Brit. Foss. Mamm., 1846, p. 399, woodcuts 159-162, and Palæontology, ed. 2, 186r, p. 399, woodcuts 153, 154; J. Morris, Cat. Brit. Foss., 1854, p. 359; H. Denny, Proc. Geol. Polyt. Soc. Yorksh., vol. iii, 1853 (1859), p. 336 ; W. R. Dawkins and W. A. Sanford, Brit. Pleist. Mamm. pt. i (Pal. Soc., I866), p. xxviii; H. Falconer, Palæont. Mem., vol. ii, I868, p. 564; G. W. Ormerod, Trans. Devon. Assoc., vol. iii, 1869, p. 79; W. Pengelly, Trans. Devon. Assoc., vol. iii, I869, p. 483 ; G. Busk, Quart. Journ. Geol. Soc., vol. xxviii, 1872 , p. 467 , pl. xxix ; K. Johnson, Trans. Norfolk and Norwich Nat. Soc., vol. ii (1877), p. 285 ; E. T. Newton, Geol. Mag. [2] vol. vii, 1880, p. 448, anid Vert. Forest Bed (Mem. Geol. Surv., 1882), p. 42 ; P. Lake, Geol. Mag. [3] vol. ii, 1885, p. 318. 


\section{IIPPOPOTAMUS amphibius (continued)-}

Hippopotamus, W. K. Trimmer, Phil. Trans., 1813, p. 131, pl. ix, f. I, 3, pl.x; W. Buckland, Phil. Trans., I822, p. 182, pl. xxi, f. 8-10, and Reliq. Diluv., I824, p. 263 , pl. vii, f. 8-10, pl. xxii, f. 5 ; S. Woodward. Syn. Tab., 1830, p. 39, and Geol. Norfolk, 1833 , p. 46 ; T. Thompson, Geol. Mag., vol. vi, 1869 , p. 206 ; A. L. Adams, Journ. R. Geol. Soc. Ireland, vol. iv, 1877, p. 247. [The supposed fossil skull of Hippopotamus from a Lancashire Peat-bog, noticed by C. Leigh, Nat. Hisi. Lancashire, I700, p. 185, pl. vi, f. 4 , must be regarded as a buried recent specimen.]

LOC. Forest Bed; Bacton, Cromer, Happisburgh.

Pleist.; Alconbury, Acton, Bedford, Burfield, Brentford, Barrington, Cold Higham?, Cropthorne, Chelmsford, Camden Town (London), Evesham, Folkestone, Grays, Greenwich, Huntingdonshire, Ilford, Lavenham, Leeds, Motcombe, Overton, Peckham, Walton. - ? Antrim (Ireland).

Caves ; Durdham Down, Kent's Hole, Kirkdale, Ravenscliff.

antiquus, Desmarest. $v$. Hippopotamus amphibius.

major, Owen. v. Hippopotamus amphibius.

II IPPTHERIUM, Kaup. v. Hipparion.

ser Oro, Linnæus, Syst. Nat., ed. 12, vol. i, r766, p. 28.

sapiens, ibid., p. 28 ; T. H. Huxley, Geologist, 1862 , p. 202, pl. xi, woodcut I (Trent Valley, Heathery Burn Cave, Nore River -Queen's Co., Blackwater River-Armagh); C. C. Blake, Geologist, 1862, p. 205, woodcuts (East Ham, Glamorgan, Mewslade, Montrose, Nether Urquhart, Plymouth, Sennan -Cornwall), and Journ. Anthrop. Soc., vol. ii ( I864), p. cclxxiii (Kent's Hole); W. B. Dawkins, Early Man in Britain, 8vo. London, 1874; G. Busk, Journ. Anthrop. Inst., vol. iii (1874), p. 392, pl. xxiv (Victoria Cave): G. Rolleston, Journ. Anthrop. Inst., vol. vi (1876), p. 31, pl. xix (Cissbury); S. Parry, Geol. Mag. [2] vol. iii, 1876, p. 528 (Voryd, near Rhyl) ; T. M. Reade, Geol. Mag. [2] vol. x, 1883, p. 547 (Birkdale, Southport); H. Prigg, Journ. Anthrop. Ins'., vol. xiv (1884), p. 51, pl. v (Bury St. Edmunds) ; R. Owen, Proc. Roy. Soc., vol. xxxvi (1883), p. 136, and 8vo. London, 1884, 32 pp., pls. ; T. V. Holmes, Trans. Essex Field Club, vol. iv (1885), p. I35; A. H. W. Ingram, Geol. Mag. [3] vol. ii, 1885, p. 307 (Great Orme); R. N. Worth, Trans. Plymouth Inst., vol. x (1888), p. Io, 2 pls., woodcuts, and Trans. Roy. Geol. Soc, Cornwall, vol. xi (1888), p. 105, pl. (Castledown Cave, Devon). [These are various bones, mostly Prehistoric and Historic; but a human humerus from the true cave-earth of Kent's Hole, now in the British Museum, has been determined by W. Davies.]

7 tomocetus villersi (Du Bus), Lydekker. v. Physeterula dubusi. 
IIOPxocmru 3 , P. Gervais, Zool. et Pal. Franç., ed. I, vol. i, $1848-52$, p. I6I.

borgerhoutensis, 1) 1 13us, Bull. Acad. Rny. Belg. [2] vol. xxxiv, I872, p. 502; R. Lydekler, (Quart. Journ. Genl. Soc., vol. xhii, I887, p. If ('orgehoutensis), and Cat. Foss. Mamm. B. M., pt. v, 1887, p. 61 .

Loc. Red Crag; Suffolk. [Te'cth; Brit. Ifus.]

crassidens, P. Gervais, Zool. et Pal. Franç, ed. I, vol. i, I $8_{4} 8-52$, p. I6I; R. Owen, ()uart. Journ. Geol. Soc., vol. xii, 1856, p. 228; R. Lydekker, Quar. Journ. Gen. Soc., vol. xliii, I887, p. I4, and Cat. Foss. Mamm. B. M., pt. v, I887, p. 60.

Loc. Red Crag; Suffolk. [Teeth; Brit. Mus.]

curvidens, P. Gervais, Zonl. et Pdl. Franç., ed. I, vol. i, I848-52, p. I6r; R. Lydekker, (2uart. Journ. Geol. Soc., vol. xliii, I887, p. I4, and Cat. Foss. Mamm. B. M., pt. v, I887, p. 62. Loc. Red Crag; Felixstowe. [Teeth; Brit. Mus.]

IXY Fis A, E. A. W. Zimmermann, Specimen Zool. Geogr., 1777, p. 365 .

antiquu, Lankester. $v$. Hyæna striata.

arvernensis, Croizet and Jobert. $\%$. Hyæna striata.

crocuta (Erxleben), W. B. Dawkins, Quart. Journ. Geol. Soc., vol. xxxiii, 1877, p. 596; R. Lydekker, Cat. Foss. Mamm. B. M., pt. i, 1885, p. 69.

Canis crocuta, J. C. P. Erxleben, Syst. Regn. Anim., I777, p. 578.

Hyana crocuta, var. spelae, E. T. Newton, Geol. Mag. [2] vol. x, I 883 , p. 433 , pl. $x$.

Hyana maculata, E. A. W. Zimmermann, Spec. Zool. Geogr., 1777, p. 470.

Hyana fossilis, J. C. Bellamy, Nat. Hist. S. Devonshire, IS39, p. 439 , pl. xv, f. 6, pl. xvi, f. 3 .

Hycena spelcea, Goldfuss, Nova Acta Acad. Cas. Leop.-Carol., vol. ix (2), 1823, p. 456 ; R. Owen, Brit. Foss. Mamm., I 846 , p. 138, woodcuts 54, 55, 57-61 ; J. Morris, Cat. Brit. Foss., 1854, p. 359 ; W. 13. Uawkins, Nat. Hist. Rev., n.s., vol. v, I 865 , p. 80, woodcuts $1-6$; H. Hicks, Geol. Mag., vol. iv, I867, p. 307 ; G. Busk, Phil. Trans., 1873 (1874), p. 529, pl. xlv, f. I-3.

Hycena gigintea, F. Holl, Handb. Petref., pt. i, I829, p. 36.

Canis hycent, S. Woodward, Syn. Tab., 1830, p. 39.

Hycena, W. Buckland, Phil. Trans., I 822, p. 182, pls. xvii-xx, xxiv, and Ann. Phil., vol. xx, 1822, p. 140, pl. xv, f. 2.3 , and Reliq. Diluv., 1824, p. 17, pls. iii-vi, xii, xiii ; W. Clift, Phil. Trans., I 823 , p. 86 , pl. x, f. 7 , pl. xi, f. 8 , pl. xii, f. 9 ; [Anon] Phil. Mag., n.s., vol. ii, 1827, p. 73; G. Young and J. Bird, Geol. Surv. Yorkshire Coast, ed. 2, 1828, pl. xvii, f. 3, 6, I7; J. MacEnery, Cavern Researches, I859, pl. B, pl. c, f. 3 , pl. M, f. 8,9 . 
IFY PENA crocuta (continued)-

'Wolf,' W. Buckland, Phil. Trans., I822, p. 232, pl. xx, f. I5-18.

LOC. Forest Bed; Corton Cliff.

Pleist.; Aymestry, Brentford, Dogger Bank, Erith, Grays, Lawford (near Rugby), Walton (Essex), Weston, Yarmouth.

Caves; Banwell, Bench, Bacon Hole, Brixham, Bleadon, Burrington Combe, Caswell Bay, Coygau, Cresswell Crags, Durdham Down, Hutton, Kent's Hole, Kirkdale, Long Hole, Laugharne, Maidstone (fissure), Minchin Hole, Oreston, Paviland, Ravenscliff, Sandford Hill, Spritsail Tor, Uphill, Vale of Clwyd, Wookey Hole, Yealm Bridge.

[For bones gnawed by this $H_{y^{\prime}}$ ena, see especially Buckland and MacEnery.]

fossilis, Bellamy. v. Hyæna crocuta.

gigantea, Holl. $v$. Hyæna crocuta.

maculatı, Zimmermann. $\%$ Hyæna crocuta.

spelcea, Goldfuss. \%. Hyæna crocuta.

striata, E. A. W. Zimmermann, Spec. Zool. Geogr., I777, p. 366 ; R. Lydekker, Quart. Journ. Geol. Soc., vol. xlii, I886, p. 364 , woodcut I, and Cat. Foss. Mamm. B. M., pt. v, I887, p. 315, woodcut 49.

Hycena arvernensis, Croizet and Jobert, Ossem. Foss. Puy-deDôme, I 828 , p. 180, pls. ii-iv.

Hyana antiqua, E. R. Lankester, Ann. Mag. Nat. Hist. [3] vol. xiii, 1864, p. 56, pl. viii, f. 5, 6, and ibia. [3] vol. xiv, I864, p. 358 , pl. viii, f. 7, 8, and Quart. Journ. Geol. Soc., vol. xxvi, 1870, p. 511 , pl. xxxiii, f. 5, 6; R. Lydekker, Cat. Foss. Mamm. H. M., pt. i, I885, p. 87 . [Upper premolar; Brit. Mus.]

LOC. Red Crag; Felixstowe and Trimley-St.-Mary. [Upper seitorial molar; Ipsavich Mus.]

IrYARARCTOS, H. Falconer and P. T. Cautley, in R. Owen, Odontography (1845), p. 505.

sp., WV. H. Flower, (2uart. Journ. Geol. Soc., vol. xxxiii, I877, p. 534, woodcut (cf. H. sivalensis, Falconer and Cautley); R. Lydekker, Palieont. Indica [10] vol. ii, I884, pp. 227, 228, and Cat. Foss. Mamm. B. Mi., pt. i, I885, p. I55.

LOC. Red Crag; Suffolk. [Upper molars; York Mus. and Ipswich Mus.]

Ix ZrENODON, M. de Laizer and de Parieu, Comptes Rendus, vol. vii, 1838 , p. 442 .

leptorhynchus, Dawkins. \%. Hyrenodon minor. 
IIY FINODON (continued)-

minor, P. Gervais, Zool. et Pal. Franç., ed. I, rol. i, I 8+8-52, n. I29; R. Lydekker, Geol. Mag. [3] vol. i, 1884, p. 444, and Cat. Foss. Mamm. B. M., pt. i, I885, p. 25.

Hyanodon, [B.] Hastings, Bull. Soc. Géol. France [2] vol. ix, 1852, p. 198, and E. B. Tawney and H. Keeping, Quart. Journ. Geol. Soc., vol. xxxix, 1883, p. 571 .

Hycenodon leptorlynchus, W. B. Dawkins (errorc), Quart. Journ. Geol. Soc., vol, xxxvi, I880, p. 383.

Loc. Headon Beds; Hordwell. [Mandible; Brit. Mus.]

sp., R. Lydekker, Cat. Foss. Mamm. B. M., pt. i, I885, pp. 38, 40. LCC. Headon Beds; Hordwell. [Limb-bones; Brit. Mus., nus. 29869, 29726, 30132.]

IrOPOTAmUs, R. Owen, () wart. Journ. Geol. Soc., vol. iv, r 848 . p. I03; W. Kovaleviky, Phil. Trans., I873 (1874), p. 30, pls. xxxy-xl.

bovinus, R. Owen, Quart. Journ. Geol. Soc., vol, iv, 1848, p. 103, pls. vii, viii ; j. Morris, Cat. Brit. Foss., I 854 , p. 359 ; R. Lydekker, Geol. Mag. [3] vol, i, I 884 , p. 547, and Cat. Foss. Mamm. B. M., pt. ii, I885, p. 22 I, woodcuts 29, 30.

Ancodus howinus, H. Filhol, Ann. Sci. Gćol., vol. xii (I882), p. 189 .

Hycpotamus vectionus, R. Owen (errore), Quart. Journ. Geol. Soc, vol. iv, I848, pl. vii, f. 6, 7. [Upper molar; Brit. Mus.]

LOC. Hempstead Beds ; I. of Wight. [Upper molar ; Brit. Mus.]

gresslyi, Rütimeye:. $\quad$. Anthracotherium gresslyi.

porcinus, P. Gervais, Zool. et Pal. Franc., ed. I, vol. ii, I848-52, Expl. to pl. xxxi, p. I ; R. Lydekker, Geol. Mag. [3] vol. i, I884, p. 547, and Cat. Foss. Mamm. B. M., pt. ii, 1885 , p. 228 , woodcuts $3 \mathrm{I}, 32$.

Hyopotemus, W. Kovalevsky, Phil. Trans., 1873 (1874), p. I9, pl. xxxy, f. 2 , pl. xxxvi, f. 3 .

Loc. Hempstead Beds; I. of Wight. [Portions of jaws; Brit. Llus.]

vectianus, Owen. $\%$. Hyopotamus velaunus.

velaunus (Cuvier), R. Lydekker, Genl. Mar. [3] vol. i, I884, p. 547, and Cat. Foss. Mamm. B. M., pt. ii, 1885, p. 218.

Anthracotherium velaunum, Cuvier, in $\mathrm{H}$. von Meyer, Palæologica, 1832, p. 82.

Hyopotamus aectianus, R. Owen, Quart. Journ. Geol. Soc, vol. iv, $18+8$, p. 103 , pl. viii; J. Mlorris, Cat. Brit. Foss., IS $5+$, p. 359. [See also H. bovinus.] T. (Hyopolimus.)

Ancodus arelaumus, A. Pomel, Catal. Méthod., I853, p. 91.

LOC. Hempstead Beds; 1. of Wight. [Portions of jazc's; Brit. Hus.] 
IYYPROODON, B. r. L. de Lacépède, Hist. Nat. Cétacés, Table des Ordres, etc., I804, p. xliv.

butzkopf, De Lacépìde. \%. Hyperoodon rostratus.

rostratus (Müller), R. Lydekker, Quart. Journ. Geol. Soc., vol. xliii, I887, p. I4, pl. ii, f. 6 (cf. H. rostratus), and Cat. Foss. Mamm. B. M., pt. v, 1887, p. 63.

Balcena rostrata, O. F. Müller, Zool. Dan. Prod., I776, p. 7.

Hyperoodon butzkopf, De Lacépède, Hist. Nat. Cétacés, I804, p. 319.

Hyperoudon butzkoff, D. Embleton, Trans. Tyneside Nat. Field Club, vol. iv (1858), p. 50.

LOC. Red Crag; Suffolk. [Periotic bone; Ipswich Mus.]

? Pleist. ; Bed of Tyne, and East Ham Marshes, Essex.

HYPSIPRYMNOPSIS, Dawkins. v. Microlestes.

vhcticus, Dawkins. $v$. Microlestes rhæticus.

IrYRACOTHERI $\mathbf{v} \mathbf{M}$, R. Owen, Trans. Geol. Soc. [2] vol. vi, pt. i (I 84 I), p. 203 .

Pliolorhus, R. Owen, Quart.Journ. Geol. Soc., vol. xiv, I 857, p. 54.

Orohippes, O. C. Marsh, Amer. Journ. Sci. [3] vol. iv, I872, p. 207.

cuntculus, R. Owen, Ann. Mag. Nat. Hist., vol. viii, I84 I (I842), p. I, woodcuts 2, 4, 5, and Rep. Brit. Assoc., 1843 (1844), p. 227 , and Brit. Foss. Mamm., I 846, p.424, woodcuts I 70, I 7 I ; J. Morris, Cat. Brit. Foss., I854, p. 359 ; R. Lydekker, Cat. Foss. Mamm. R. M., pt. iii, I886, p. I2.

Macacus eocrmus, R. Owen, Brit. Foss. Mamm., i846, p. 5, woodcut 1, and Odontography (1845), p. 443, pl. cxvi, f. 3 . [Referred to Hyracotherium cuniculus by R. Owen, Ann. Mag. Nat. Hist. [3] vol. x, 1862 , p. 240.7

'Quadrumanous Animal,' S. V. Wood, Mag. Nat. Hist., n.s., vol. iii, 1839, p. 444, woodcut 57 .

Macacus, R. Owen, Mag. Nat. Hist., n.s., vol. iii, 1839, p. 446, woodcuts $58 \mathrm{~A}, \mathrm{~B}, \mathrm{C}$, and Ann. Nat. Hist., vol. iv, I840, p. I9I, woodcut 1 ; C. Lyell, Ann. Mag. Nat. Hist., vol. iv, I840, p. I90.

Loc. L. Eocene Sand; Kyson (Kingston), Suffolk. [Portion of jaw ; Ipswich Mus.]

leporinum, R. Owen, Trans. Geol. Soc. [2] vol. vi (184I), pp. 203, 2 I I, pl. xxi, f. I-4, and Ann. Mag. Nat. Hist., vol. viii, I 84 I ( 842 ), p. I, woodcuts I, 3, and Brit. Foss. Mamm., I 846 , p. 4 I9, woodcuts I65-I69, and Geol. Mag., vol. ii, I865, p. 339 , pl. x, f. 2 ; J. Morris, Cat. Brit. Foss., 1854, p. 359 ; R. Lydekker, Cat. Foss. Mamm. B. M., pt. iii, I886, p. Io.

Pliolophus i'ulpiceps, R. Owen, Quart. Journ. Geol. Soc., vol. xiv, I 858 , p. 54 , pls. ii-iv, and Palieontology, ed. 2, 1861, p. 358 , woodcuts 121, 123, 124, and Geol. Mag., vol. ii, 1865, p. 339 , pl. $x$, f. 4 . [Head and other portions of skeleton; slim R. Bull Coll., I pswich (fragments in Brit. Mus.).] T. (Pliolophus.) Loc. London Clay; Harwich, Herne Bay (Studd Hill), and Sheppey.

Red Crag; Suffolk (derived fossil). [Skull; Brit. Mus.] $\mathbf{T}$. 
İOGIA, J. E. Gray, Zool. 'Erebus' and 'Terror,' 1846, p. 22.

breviceps (Blainville), J. E. Gray, ibid.; W. H. Flower, Cat. Osteol. Dent. Vert. Mus. R. Coll. Surgeons, pt. ii, I884, p. 555 ; doubtfully recorded by R. Lydekker, Cat. Foss. Mamm. B. M., pt. v, 1887, p. 62.

Physcter breaiceps, H. D. de Blainville, Ann. Anat. Physiol., vol. ii, 1838, p. 337 .

LOC. Ked Crag; Suffulk. [Vericbra; Brit. Mus.]

IR URTODON, H. F. Osborn, Amer. Nat., is $88_{-}^{-}$, p. 1020, and Journ. Acad. Nat. Sci. Philad., vol. ix (1888), p. 208, and Proc. Acad. Nat. Sci. Philad., I888, p. 300. [Name preoccupied by Curtodus, Sauvage, I867: v. p. I2.] $[?=$ Stylodon. $]$

Athrodon, H. F. Osborn (non Sauvage), Proc. Acad. Nat. Sci. Philad., I887, p. 290.

pustllus, H. F. Osborn, Proc. Acad. Nat. Sci. Philad., 1887, p. 290, woodcut (Athrodon), and Journ. Acad. Nat. Sci. Philad., vol. ix (1888), p. 209, woodcut 4.

Stylodon pusillus, R. Owen (errore, fide Osborn), Brit. Mesoz. Mamm. (Pal. Soc., 187I), p. 45, pl. ii, f. I 4.

Genus non det., R. Lydekter, Cat. Foss. Mamm. B. M., pt. v, 1887, p. 291.

LOC. M. Purbeck; Durdlestone Bay. [Maxilla; Brit. Mus.]

$\mathbf{T}$

IA GOMrYs, Cuvier, Tabl. Elém. d'Hist. Nat., 1798, p. 132.

pusillus (Pallas), W. A. Sanford, Quart. Journ. Geol. Soc., vol. xxvi, 1870, p. 126; R. Lydekker; Cat. Foss. Mamm. B. MI, pt. i, I885, p. 255 .

Lepus pusillus, P. S. Pallas, Nov. Comm. Petrop,, vol. xiii, I769, p. 531, pl. xiv.

Lagomys spelcus, R. Owen, Brit. Foss. Mamm., I 846, p. 2I3, woodcuts 82-84, and Proc. Roy. Suc., vol. xxii, 1874 , p. 365 ; J. Morris, Cat. Brit. Foss., I 554 , p. 359 ; C. Busk, I'hil. Irans., 1873 (1874), p. 548, pl. xivi, t. 12, I3. [Skull; Brit. Mus.]

Lagomus, J. MacEnery, Cavern Researches, 1859, pl. E, f. 1 I. Loc. Caves; Bleadon, Brixham, and Kent's Hole.

spelceus, Owen. v. Lagomys pusillus.

LEOPAIDUS. $\%$. Felis pardus.

LEM MUS, Tiedemann. \%. Myodes.

norvegicus, Desmarest. v. Myodes lemmus.

torquatus, Desmarest. v. Myodes torquatus.

LEITOCLADUS, Owen. \%. Peramus.

dubius, Owen. v. Peramus dubius. 
IヌPUS, Linneus, Syst. Nat., ed. 12, vol. i, I766, p. 77.

cuniculus, Linnæus, ibit., p. 77 ; S. Woodward, Syn. Tab., I830, p. 39 (L. cunniculus); R. Owen, Brit. Foss. Mamm., 1846, p. 212, wondcut 81 ; J. Morris, Cat. Brit. Foss., 1854, p. 359 ; W. A. Sanford, guirt. Journ. Geol. Soc., vol. xxvi, I870, p. 128; S. Haughton, Sci. Proc. Roy. Irish Acad. [2] vol. ii, 1876 , p. 483 : A. L. Adams, Trans. Roy. Dublin Soc. [2] vol. i (18SI), p. 206; R. Lydekker, Cat. Foss. Mamm. B. MI. pt. i, I 885 , p. 261 .

'Rabbit, $\bullet \mathbb{W}$. Buckland, Phil. Trans., I 822. p. I 82, pl. xxiv, f. I4-I 8 , pl. xxv, f. 10, and Reliq. Diluv., I824, p. I9, pl. x, f. 14-18, pl. xi, f. ıо ; J. MacEnery, Car ern Researches, I859, pl. E, f. 8. Loc. Caves ; Brixham, Kent's Hole, Kirkdale, and Long Hole. - Knockninny, Shandon (Ireland).

diluvianus, H. von Mever, Palaeologica, I832, p. 6I ; IV. A. Sanford, Quart. Journ. Geol. Soc., vol. xxvi, 1870, p. I26, pl. viii, f. 5 , and Proc. Somerset. Nat. Hist. Soc., vol. xv, I 870 , p. 53, Fl., f. 5.

LOC. Cave; Somersetshire. [Skull; Taunton .1/his.]

europreus, Pallas. \%. Lepus timidus.

hibernicus, Sanford. ¿'. Lepus variabilis.

pusillus, Pallas. \%. Lagomys pusillus.

timidus, Linnaeus, Syst. Nat., ed. I2, vol. i, I766, p. 77; S. Woodward, Syn. Tab., I830, p. 39 ; R. Owen, Brit. Foss. Mamm., I846, p: 210, woodcut So; J. Morris, Cat. Brit. Foss., I854, p. 360 ; IV. A. Sanfurd, Quart. Journ. Geol. Soc., vol. xxvi, 1870, p. I27; R. Lydekker, Cat. Foss. Mamm. B. II., pt. i, I 885 , p. 259.

Lefus e'uropecus, P. S. Pallas, Nov. Spec. Glirium, 1778, p. 30.

'Hare, or very larse rabbit,' W. Buckland, Reliq. Diluv., I824, pp. I9, 267 , pl. xiii, f. 8 .

'Hare', J. MacEnery, Cavern Researches, 1859 , pl. E, f. 9.

LOC. Pleist.; Fisherton.

Caves; Hrixham, Durdham Down, Kent's Hole, Kirkdale, Lon: Hole, Spritsail Tor.

variabilis, I'. S. Pallas, Nov. Spec. Glirium, I778, p. I ; A. L. Adams, Trans. Roy. Irish Acad.. vol. xxvi (Sci.), I876, p. 228 (L. requiabilis?), and Sci. Proc. Roy. Irish Acad. [2] vol. iii, I 878 , p. 99, and Trans. Roy. Dublin Soc. [2] vol. i (1881), p. 205 (L. adriabilis?) ; S. Haughton, Sci. Proc. Roy. Irish Acad. [2] vol. ii, 1876 , p. 483 .

Lipus hibernicus?, IV. A. Sanford, Quart. Journ. Geol. Soc., vol. xxvi, 1870, p. I27.

LOC. Caves; Mendip Hills.-Knockninny and Shandon (Ireland).

IOPHIODON, Cuvier, Ossem. Fossiles, ed. 2, vol. ii, pt. i, I\$22, p. 176

"uthmeqideum, Gervais. \% Coryphodon cocinus. 
IOPIIODON (continued)-

minimus, [-um] J. B. Fischer, Syn. Mammalium, I829, p. 413 ; R. Owen, Brit. Foss. Mamm., 1846, p. 3II, woodcut IOS, and in F. Dixon, Geol. Sussex, ed. 2, 1878, p. 266, pl. xi [12], f. 27 ; J. Morris, Cat. Brit. Foss., I854, p. 360 ; R. Lydekker, Cat. Foss. Mamm. B. M., pt. iii, 1886, p. Io.

Pachynolophus minimus, P. Gervais, Zool. et Pal. Franç., ed. 2, 1859 , p. 126.

'Très-petite espèce de Lophiodon,' Cuvier, Ossem. Fossiles, ed. 2, vol. ii, pt. i, IS22, p. 194.

Loc. M. Eocene ; Bracklesham. [Tectin; Brit. Luus.]

sp., Owen. v. Palæotherium magnum.

LOXODON, Falconer. $\approx$ Elephas.

meridionalis (Nesti), Falconer. $\%$ Elephas meridionalis.

IUTrA, J. C. P. Erxleben (ex Ray), Syst. Regn. Anim., 1777, P. 445.

vulgaris, J. C. P. Erxleben, Syst. Regn. Anim., 1777, p. 448;

R. Owen, Brit. Foss. Mlamm., I 846 , p. II 9 , woodcuts $+3,44$;

J. Morris, Cat. Brit. Foss., I 854 , p. 360 ; C. C. Biake, Geologist, 1862, p. 217 ; R. Lydekker, Cat. Foss. Mamm. B. M., pt. i, ISS5, p. I9I ; E. T. Newton Geol. Mag. [3] vol. iv, I887, p. I 45, pl. iv, f. I, 2 .

Mustela lutra, Linnæus, Syst. Nat., ed. 12, vol. i, I766, p. 66. LOC. Norwich Crag; Aldeborough and Southwold. [llindib. ramus and hamerus.]

Forest Bed; East Runton. [Mandib. ramus; A. Siniın Coll.]

Pleist. and Prelist; Grays, Ipswich, Lincolnshire Fens, and Watcham Fen.

Caves; Banwell, Bleadon, Durdham Down, Heathery Burn, Kent's Hole, Long Hole, and Teesdale.

sp., Miller and Skertchley. $\%$. Mustela martes.

IXCAON, J. Brookes, in Griffith's Acimal Kingdom, vo!. v, IS27, p. $15 \mathrm{I}$.

anglicus, R. Lydekker, Geol. Mag. [3] vol. i, I884, p. 443, and Cat. Foss. Mamm. B. M., pt. i, I885, p. 122.

'Hyænoid wolf,' H. Falconer, Palicont. Mem., vol. ii, I868, p. 462, pl. xxxvi, f. I, 2 .

LOC. Caves; Gower, Spritsail Tor. [Mandib. ramus; Brit. MLus.]

MACACUS, B. G. E. de Lacépède, Mém. de l'Inst., vol. iii, ISor, p. 490 (Maraca) (non Cuvier and Geoffroy St. Hiliare, 1795).

eocremus, Owen. $\%$. Hyracotherium cuniculus.

? pliocenus, R. Owen, Brit. Foss. Mamm., IS46, p. xlvi, woodcut ; J. Morris, Cat. Brit. Foss., 1854, p. 360 ; R. Lydekker, Cat. Foss. Mamm. B. MI., pt. i, I 885 , p. 4.

Loc. Pleist. ; Grays. [Upper molar 2; Hrit. Mus.] 
IMACHFrRODUS, J. J. Kaup, Descr. Ossem. Foss. Mus, Darmstadt, 1833, pt. ii, p. 24 (Machairodus); H. Falconer, Palæont. Mem., vol. ii, I868, p. 459.

cultridens (Cuvier), J. J. Kaup, ibid., p. 24, pl. i, f. 5 ; R. Lydekker, Cat. Foss. Mamm. B. M., pt. v, I887, p. 31 Io.

Ursus cultridens, Cuvier, Ossem. Fossiles, ed. 2, vol. ii, 1824, p. 516.

Macharodus, E. R. Lankester, Geol. Mag., vol. vi, I869, p. 440, and E. T. Newton, Geol. Mag. [2] vol. vii, 1880, p. I 53, and Vert. Forest Bed (Mem. Geol. Surv., 1882), p. 21 , pl. iv, f. 5 ; J. Backhouse and R. Lydekker, Quart. Journ. Geol. Soc., vol. xlii, I886, p. 309, pl. x. [See also Macharodus latidens.]

LOC. Forest Bed; Kessingland. [Mandib. ramus; J. Backhouse Coll. $]$

latidens, R. Owen, Brit. Foss. Mamm., I 846, p. I79, woodcuts 69, 70 ; J. Morris, Cat. Brit. Foss., I854, p. 360 ; W. Pengelly, Trans. Devon. Assoc., vol. iii, I 869, p. 483, and ibid., vol. v, I872, p. I65, and ibid., vol. vii, I875, p. 247, and Geol. Mag., vol. viii, I 871 , p. 42, and Rep. Brit. Assoc., I 872 (1873), Sect., p. II9; W. B. Dawkins and W. A. Sanford, Brit. Pleist. Mamm., pt. iv (Pal. Soc., 1872), p. 184, pl. xxv, woodcuts I-3; C. Lyell, Antiq. Man., ed. 4, I873, p. 105, woodcuts 9, 10 ; W. B. Dawkins, Quart. Journ. Geol. Soc., vol. xxxiii, 1877 , pp. 590, 594, woodcut 3; R. Johnson, Trans. Norfolk and Norwich Nat. Soc., vol. ii (I877), p. 29I ; [non A. Savin, Proc. Norwich Geol. Soc., vol. i (1878), p. 28: see E. T. Newton, Vert. Forest Bed (Mem. Geol. Surv., I882), p. 21]; R. Lydekker, Cat. Foss. Mamm. B. M., pt. i, I885, p. 44.

Ursus cultridens, J. MacEnery (errore), Cavern Researches, I859, pl. F.

LOC. Caves; Cresswell Crags and Kent's Hole. [Canine tooth from Kent's Hole; Mus. R. Coll. Surgeons.]

meganthereon (Croizet and Jobert), wrongly quoted as Brish by J. Morris, Cat. Brit. Foss., 1854, p. 360.

MI I ITES, Nilsson. $\%$. Mustela.

sylvatica, Nilsson. v. Mustela martes.

IIASTODON, Cuvier, Ann. du Muséum, vol. viii, ISo6, p. 270; E. D. Cope, Amer. Nat., I889, p. I9I.

TRILOPHODON T. Falconer, Quart. Journ. Geol. Soc,

TETRALOPHODON $\}$ vol. xiii, I857, p. 313 (as subgenera).

(lngustidens, Owen (non Cuvier). v. Mastodon arvernensis.

arvernensis, Croizet and Jobert, Ossem. Fos`. Puy-de-Dôme, I828, p. I 38 ; R. I.ydekler, ( uart. Joum. Geol. Soc., vol. xlii, I 886 , p. 36 , and Cat. Foss. Manm. B. M., pt. iv, I886, p. 52.

3.ritodon (\%emblophodon) aremensis, H. Falconer, Quart. Journ. Geril. Soc, vol. xiii, 1857, p. 360. pl. xii, f. I-4 ; R. Johnsun, Trans. Nurfolk and Norwich Nat. Soc., vol. ii (1877), p.282. 
IMASTODON arvernensis (continued)-

Mustodon angustidens, R. Owen, Rep. Brit. Assoc., I843 (1844), p. 220 , and Brit. Foss. Mamm., I846, p. 27 I, woodcuts 96-10I, and Quart. Journ. Gecl. Soc., vol, xii, I856, p. 223 ; J. Morris, Cat. Brit. Foss., 1854 , p. 360.

Mastodon gigantens, S. Woodward, Mag. Nat. Hist., vol. ix, I 836, p. $15 \mathrm{I}$, woodcuts 22, 23.

Mastodon latidens, S. Woodward (emore), ibid., p. I $5 \mathrm{I}$.

Mastodon longirostris, R. Owen (errore), in C. Lyell, Mag. Nat. Hist., n.s., vol. iii, I $S_{39}$, p. 317

Mastodon, R. Bakewell, Mag. Nat. Hist., vol. ix, I836, p. 39 ; H. Alexander, Mag. Nat. Hist., n.s., vol. iii, I839, p. 466.

'Fossil Tooth,' W. Smith, Strata ident., 1816, introd., pl. i.

Loc. Norwich Crag; Bramerton, Easton Cliff, ? Horstead, Norwich, Postwick, Sizewell Gap, Thorpe, and Whitlingham.

Red Crag; Felixstowe, Southwold, and Woodbridge. [Teetil; Brit. ./us., Ipsivich.Mus., etc.]

borscmi, I. Hays, Trans. Amer. Phil. Soc. [2] vol. iv, 1834 , p. 334 ; E. R. Lankester, Geol. Mag., vol. vi, IS69, p. 355; R. Lydekker, Quart. Journ. Geol. Soc., vol. xlii, 1886, p. 365, and Cat. Foss. Mamm. B. N., pt. iv, ise6, p. 25.

LoC Red Crag; Sutton, Swattham, and Woodbridge. [Tooth; rork Mus.]

giganteus, S. Woodward. \%'. Mastodon arvernensis.

latidens, S. Woodward, Syn. Tab., I 30 , p. 39 : recorded in error from Pleistocene of Horstead. [See also $1 \%$. aremensis.]

longirostris, J. J. Kaup, O-sem. Fuss. Mus. Darmstadt, pt. iv, 1835 , p. 65, pls. xvi-xviii ; R. Lvdekker, Quart. Journ. Geol. Soc., vol. xlii, 1886, p. 365 , and Cat. Foss. Mamm. B. M., pt. iv, 1886, p. 61. [See also .1/. arvernensis.]

'Trilophodont Mastodon,' E. R. Lankester, Quart. Journ. Geol. Soc., vol. xxri, I870, p. 507 , pl. xxxiv, f. I-t.

LOC. Red Crag; Sutton and Woodbridye. [Teeth; Brit. Mus.]

sp., R. Lydekker, Cat. Foss. Mamm. B. M., pt. iv, I886, p. 78.

l.oC. Red Crag; Woodbridge. [Tooth; Brit. MIus., no. 46922.]

MEGACEROS, Owen. \%. Cervus.

hibernicus, Owen. \%. Cervus giganteus.

IMEGA P TraA, J. E. Cray, Zool. 'Erebus' and 'Terror', Is f', p. 16.

Burtinopsis, P. J. Van Beneden, Bull. Acad. Roy. Lelg. [2] vol. xxxiv, 1872, p. I9.

? afinis, P. J. Van Beneden, Bull. Acad. Roy. Belg. [2] rol. l, ISSC, p. I3; (?) R. Lydekker, () art. Journ. (ieol. Soc., Vol. xliii, i 887 , p. II, pl. ii, f. 4, and Cat. Foss. Mamm 1. M., pt. 1, ISS7, p. 32. Loc. White Crag; Sudbourn. ? Red Crag; Woodbridge. [Tympanic bone; Mus. Pract. Geol.] 
IMEAPTrRA (continued)-

boops (Linnæus), Van Beneden and I'. Gervais, Ostéogr. Cétacés (1870), p. I20.

Balana boops, Linnæus, Syst. Nat., ed. 12, vol. i, 1766, p. Io5.

Balcenoptere boops, R. Owen, Brit. Foss. Mamm., 18+6, table facing p. xlvi ; J. Morris, Cat. Brit. Foss., I 854 , p. 358.

LOC. Pleist. ; Bridgewater.

minuta (Van Beneden), R. Lydekker, Quart. Journ. Geol. Soc., vol. xliii, 1887, p. II, pl. ii, f. 5, 5 a, and Cat. Foss. Mamn. B. M., pt. v, 1887 , p. 33 .

Burtinopsis minutus, P. J. Van Beneden, Bull. Acad. Roy. Belg. [2] vol. l, I 880 , p. I7.

Loc. White Crag; Suffolk. [Tympanic bone; Ipswich Ifus.]

? similis (Van Beneden), R. Lydekker, Quart. Journ. Geol. Soc., vol. xliii, I887, p. II, and Cat. Foss. Mamm. B. M., pt. v, 1887 , p. 33 .

Burtinopsis similis, P. J. Van Beneden, Bull. Acad. Roy. Belg. [2] vol. xxxiv, I872, p. I9.

Loc. Red Crag; Woodbridge. [Perio'ic bone; Brit. Mius.]

MEבgs, G. C. C. Storr, Prodr. meth. Mamm., 1780, p. 34 (Miles), and table $\mathrm{A}$.

tasus, P. Boddaert, Elenchus Animalium, vol. i, I785, p. 80 ; R. Owen, Brit. Foss. Mamm., 1846, p. Io9, woodcut 37; J. Norris, Cat. Brit. Foss., 1854 , p. 360 ; C. C. Blake, Geologist, 1862, p. 217 ; A. L. Adams, Trans. Roy. Dublin Soc. [2] vol. i (1881), p. 204 (Miles tatus); R. Lydekker, Cat. Foss. Mamm. b. M., pt. i, I885, p. 189.

Ursus meles, Linnæus, Syst. Nat., ed. 12, vol. i, i766, p. 70.

'Badger,' J. NacEnery, Cavern Researches, I859, pl. E, f. I3.

LOC. Pleist. ; Grovehurst (Kent) and Newbury (Berkshire).

Caves; Banwell, Berry Head, Bacon Hole, Bench, Durdham Down, Heathery Burn, Kent's Hole, Long Hole, Ravenscliff, Spritsail Tor, Uphill, Vale of Clwyd, Wookey Hole.-Shandon (Ireland).

MrSOPTODON, P. Gervais, Ann. Sci. Nat. (Zool.) [3] vol. xiv, 1850, p. 16; WV. H. Flower, Trans. Zool. Soc., vol. viii (1871), p. 208 (for full synonymy).

Dloplodon, P. Gervais, Zool. et Pal. Franç., ed. I, vol. ii, I $848-52$, Expl. to pl. xl, p. 4 .

Beleinoziphius, T. H. Huxley, Quart. Journ. Geol. Soc., vol. $\mathrm{xx}, \mathrm{I} 864$, p. 388.

angulatus (Owen), R. Lydekker, Cat. Foss. Mamm. B. M., pt.v, 1887, p. 73.

Ziphius angulutus, R. Owen, Crag Cetacea (Pal. Soc., I870), p. 20, pl. iv, f. 1, 2, woodcut 9.

Dioplodin angulatus, Van Beneden and P. Gervais, Osteographie des Cétacés (? 1872), p. 422.

LOC. Red Crag ; Sulfolk. [Rostrum; Brit. Mus.] 
MrSOPXODON (continucd)-

angustus (Owen), R. Lydekker, Cat. Foss. Mamm. B. M., pt. v, 1870 , p. 72 .

Ziphius angustus, R. Owen, Crag Cetacea (Pal. Soc., 1870), p. I9, pl. iii, f. I, 2.

Dioplodon ansus'us, Van Beneden and P. Gervais, Ostéographie des Cétacés ? I 872), p. 422.

Loc. Red Crag; Felixstowe and IVoodbridge. [Rostrum; Brit. Mus.]

compressus (Huxley), R. Lydekker, Quart. Journ. Geol. Soc., vol. xliii, I887, p. I5, and Cat. Foss. Mamm. B. M., pt. v, 1887, p. 73 .

Belemnoziphizs compressus, T. H. Huxley, Quart. Journ. Geol. Soc., vol. xx, I864. p. 393, pl. xix.

T. (Belemnoziphius.)

? Ziphius compressus, R. Owen, Crag Cetacea (Pal. Soc., I870), p. 25 , pl. v, f. 3. [Rostrum ; Brit. Mus.]

Dioplodon compressus, Van Beneden and P. Gervais, Ostéographie des Cétacés (? I 872), P. $\$ 22$.

LOC. Red Crag; near Ipswich. [Rostrum.]

floweri, W. H. Flower (ex H. Canham, MS., I884), Cat. Osteol. Dent. Vert. Mus. R. Coll. Surgeons, 1884, p. 562, no. 2915.

Loc. Red Crag ; Trimley. [Rostrum; Ipswich MIus.]

gibbus (Owen), R. Lydekker, Cat. Foss. Mamm. B. M., pt. v, 1887, p. 7 I.

Ziphius gibbus, R. Owen, Crag Cetacea (Pal. Soc., 1870), p. I7, pl. ii, f. 2, pl. iii, f. 3 .

Dioplodon gibbus, Van Beneden and P. Gervais, Ostéographie des Cétacés (? I 872), p. 421 ; G. Capellini, Mem. R. Accad. Sci. Istit. Bologna [4] vol. vi, 1885, p. 295.

LOC. Red Crag; Felixstowe and Shotley. [Rostrum; Brit. Mus.]

longirostris (Cuvier), R. Lydekker, Ouart. Journ. Geol. Soc., vol. xliii, 1887, p. I 5, pl. ii, f. S, and Cat. Foss. Hamm. B. MI., pt. v, I887, p. 68, woodcut 14 .

Ziphius longirostris, Cuvier, Ossem. Fossiles, ed. 2, vol. v, pt. i, 1823, p. 357 ; Du Bus, Bull. Acad. Roy. Belg. [2] vol. xxiv, 1867 , p. 570.

Belemnoziphius longirostris, T. H. Huxley, Quart. Journ. Geol. Soc., vol. xx, I864, p. 393.

Ziphius medilineatus, R. Owen, Crag Cetacea (Pal. Soc., I870), p. 22, pl. iv, f. 3, woodcut Io. [Purtion of rostrum; Brit. Mus.]

Dioplodon medilineatus, Van Beneden and P. Gervais, Ostéographie des Cétarés (? 1872 ), p. 422.

Dioplodon longirostris, G. Capellini, $\mathrm{Mcm}$. R. Accad. Sci. Istit. Bologna [4] vol. vi, 1885, p. 294.

LOC. Red Crag; Falkenham, Felixstowe, Sutton, and IToudbridge. [Rostra; Brit. Mus.] 


\section{MESOPIOI ON (continued) -}

tenuirostris (Owen), R. Lydekker, Cat. Foss. Mamm. B. M., pt. v, I 887, p. 7 I.

Ziphius tenuirostris, R. Owen, Crag Cetacea (Pal. Soc., I870), p. 24 , pl. v, f. I, 2.

Dioplodon tenuirostris, Van Beneden and P. Gervais, Ostéographie des Cétacés (? 1872), p. 422; G. Capellini, Mem. R. Accad. Sci. Istit. Bologna [4] vol. vi, I885, p. 296.

Loc. Red Crag; Felixstowe. [Rostrum; Brit. Mus.]

IMICR OCrICERU, S, S. V. Wood, Ann. Mag. Nat. Hist., vol. xiv, I $8+4$, P. 350 .

erinaceus, S. V. Wood, ibid., p. 350, and London Geol. Journ., no. I, I 846 , p. 5 , pl. ii, f. I-3; J. Morris, Cat. Brit. Foss., I854, p. 360 ; R. Lydekker, Quart. Journ. Geol. Soc., vol. xli, 1885 , p. 529, woodcut, and Cat. Foss. Mamm. B. M., pt. v, 1887 , p. 303, woodcut 45.

LOC. U. Eocene; Hordwell. [Portions of skull and mandible; Brit. Mus.]

\section{T.}

MICROIISTIS, T. Plieninger, Württ. Jahresh., vol.iii, I 847, p. 164. HyPSIPRYMNOPSIS, W. B. Dawkins, Quart. Journ. Geol. Soc., vol. $\mathrm{xx}, \mathrm{i} 86+$, p. 406.

moorei, R. Owen, Brit. Mesoz. Mamm. (Pal. Soc., 1871), p. 6, pl. i, f. I-13 ; R. Lydekker, Cat. Foss. Mamm. B. M., pt. v, I 87 I, p. 203.

Loc. Rhrtic Fissure; Frome. [Teeth ; Bath Mus.]

rhæticus (Dawkins), R. Owen, Brit. Mesoz. Mamm. (Pal. Soc., I $87 \mathrm{I})$, p. 8 , pl. i, f. I6.

Hypsiprymnopsis rhuticus, W. B. Dawkins, Quart. Journ. Geol. Soc., vol. xx, I 864, p. +II, woodcut 3. $\boldsymbol{x}$. (Hypsiprymnopsis.)

LOC. Rhetic; Watchet, Somersetshire. [Tooth; Oxford MIus.]

MICROTUS, F. and P. Schrank, Fauna Boica, vol, i, I798, p. 72.

ARvicola, De Lacépède (? I799), Mlém. de l'Institut, vol. iii, 1801, p. 490.

agrestis (Linnzus), F. Lataste, Le Naturaliste, I883, p. 349, and Actes Soc. Linn. Bordeaux, vol. xxxviii, 1884, p. 36.

Arvicola agrestis, R. Owen, Brit. Foss. Mamm., 1846, p. 206, woodcut 77; J. Morris, Cat. Brit. Foss., 1854, p. 357 ; W. A. Sanford, Quart. Journ. Geol. Soc., vol. xxvi, I870, p. 124 ; H. P. Blackmore and E. R. Alston, Proc, Zool. Soc., 1874, p. 467; R. Lydekker, Cat. Foss. Mamm. B. M., pt. i, 1885, p. 232. [See also Microtus arvalis.]

[Mus agrestis, Linnæus, Fauna Suecica, ed. alt., I76I, p. II].

Mus gregurizes, Linnæus, Syst. Nat., ed. 12, vol, i, 1766, p. 84.

?'Water rat,' W. Buckland, Phil. Trans., I822, p. 182, pl. xxv,

f. II, and Reliq. Diluv., I824, p. I8, pl. xi, f. I I.

Loc. Pleist.; Crayford and Grays.

Caves; near Bath Kent's Hole, and Kirkdale. 
amphibius (Linneus), F. and P. Schrank, Fauna Boica, vol. i, I798, p. 72.

Arvicolic amplibius, [-a] R. Owen, Brit. Foss. Mamm., r $\$_{4} 6$, p. 20I, woodcut 76 ; [-a] J. Morris, Cat. Brit. Foss., 1854, p. 357 ; [-a] C. C. Blake, Geologist, I862, p. 218 ; H. P. Blackmore and E. R. Alston, Proc. Zool. Soc., I874, p. 462, woodcut I ; R. Johnson, Trans. Norfolk and Norwich Nat. Soc., vol. ii (1877), p. 291 ; [-a] A. Savin, Proc. Norwich Geol. Soc., vol. i (1878), p. 27; [-a] WV. W. Stoddart, Proc. Bristol Nat. Soc., vol. ii (1879), p. 142, pl.; E. 'T. Newton, Geol. Mag. [2] vol. viii, I881, p. 258 (A. amphibia?), and Vert. Forest Bed (Mem. Geol. Surv., 1882), p. 87 ( $A$. amphibius ?); R. Lydekker, Cat. Foss. Mamm. B. M., pt. i, I885; p. 230. [See also Microtus intermedius.]

Mus amphibius, Linnæus, Syst. Nat., ed. 12, vol. i, 1766, p. 82. 'Water rat,' W. Buckland, Phil. Trans., 1822, p. 235, pl. xxv, f. 12, and Reliq. Diluv., 1824, p. 18, pl. xi, f. 12.

Loc. ? Forest Bed; Bacton, Ostend, West Runton.

Pleist. ; Crayford, Erith, Grays, Newbury, and Sutton.

Caves; Berry Head, Bacon Hole, Bosco's Hole, Crow Hole, Durdham Down, Heathery Burn, Kent's Hole, Kirkdale, Long Hole, Minchin Hole, Oreston, Paviland, Ravenscliff, Spritsail Tor, and Wookey Hole.

arvalis (Pallas), F. Lataste, Actes Soc. Linn. Bordeaux, vol. xxxviii, I 884 , p. 36.

Arvicola arzalis, H. P. Blackmore and E. R. Alston, Proc. Zool. Soc., I 874 , p. 468, woodcut 3 ; E. T. Newton, Geol. Mag. [2] vol. viii, I88I, p. 258, and Vert. Forest Bed (Mem. Geol. Surv., 1882), p. 88, pl. xiv, f. 2-5 (also var. (?. A. nivalis), p. 90, pl. xiv, f. 7).

Arvicola agrestis, A. Savin and R. Johnson (errore), Trans. Norfolk and Norwich Nat. Soc., vol. i (1878), p. 27; R. Johnson, ibid., vol. iii (1877), p. 292.

.Mus amalis, P. S. Pallas, Nov. Spec. Glirium, 1778, p. 78.

LOC. Forest Bed; West Runton.

Cave ; Fissure near Frome.

glareolus (Schreber), F. Lataste, Actes Soc. Linn. Bordeaux, vol, xxxviii, I 884, p. 36.

Mus glareolus, J. C. D. von Schreber, Säugeth., vol. iii (1774), p. 680 .

Arvicola glareolus, W. A. Sanford, Quart. Journ. Geol. Soc., vol. xxvi, I87o, p. 124; H. P. Blackmore and E. R. Alston, Proc. Zool. Soc., 1874, p. 46I ; E. T. Newton, Vert. Forest Bed (Mem. Geol. Surv., I 882), p. 82, pl. xiv, f. I ; R. Lydekker, Cat. Foss. Mamm. B. M., pt. i, 1885, p. 233.

Arvicola (Evotomys) glarcola, E. T. Newton, Geol. Mag. [2] vol. viii, 1881, p. 258 . 


\section{MICROPUS glareolus (continued) -}

Arvicola pratensis, F. Cuvier (ex Baillon), Hist. Nit. Mamm., vol. iv, pt. lxviii (1834), tab. gén., p. 4 ; R. Owen, Brit. Foss. Mamm., I 846 , p. 208, woodcut 78 ; J. Morris, Cat. Brit. Foss., I854, p. 357.

Arvicola riparia, IV. Yarrell, Proc. Zool. Soc., 1832, p. Io9.

L.OC. Forest Bed; West Runton, Norfolk.

Caves; near Bristol, Brixham, Hutton, Kent's Hole, Wookey Hole.

gregalis (Pallas): Arrichle gregalis, J. Woldrich, Sitzungsb. k. Akad. Wiss. Wien, vol. lxxxii, I880, p. 25 , pl. ii, f. I2;

E. T. Newton, Vert. Forest Bed (Mem. Geol. Surv., I882), p. 90, p!. xiv, f. 6.

Mus gregalis, P. S. Pallas, Nov. Spec. Glirium, I778, p. 238.

LOC. Forest Bed; West Runton.

intermedius : Arvicoli (Eiotomys) intermedia, E. T. Newton, Geol. Mag. [2] vol. viii, I88 I, p. 258, and Vert. Forest Bed (Mem. Geol. Surv., I882), p. 83 , pl. xiii, f. I-I 3 .

'Arricola, intermediate between $A$. amplibizus and $A$. arvalis,'

R. Owen, Brit. Foss. Mamm., I846, p. 205.

Aricola amphibius, H. P. Blackmore and E. R. Alston (errore),

Proc. Zool. Soc., IS74, p. 463 (and var. terrestris, p. 463).

LOC. Norwich Crag; Bramerton, Dunwich, Thorpe, and Wangford. [Jaws; Reeve Coll.]

Weybourn Crag; East Runton and Trimingham.

Forest Bed; Bacton, Kessingland, Ostend, East and West Runton. [Jaws; Mus. Pract. Geol. and A. Savin Coll.]

nivalis (Martins), F. Lataste, Le Naturaliste, 1883 , p. 349, and Actes Soc. Linn. Bordeaux, vol. xxxviii, I884, p. 23.

Arvicola nivalis, C. Martins, Comptes Rendus, vol. xv, I842, p. 805 ; H. P. Blackmore and E. R. Alston, Proc. Zool. Soc., I 874 , p. 466, woodcut 2 (? British). [See also M. arvalis.]

LOC. River Deposit; Fisherton, near Salisbury. [Manilib. ramus; Salisbury Hus.]

ratticeps: Aritiola ratticeps, A. von Keyserling and $\mathrm{J}, \mathrm{H}$. Blasius, Mém. présentés Acad. Imp. Sri. St. Pétérsbourg, vol. iv, livr. 3, 4, I841, p. 319 ; W. A. Sanford, Quart. Journ. Geol. Soc., vol. xxvi, 1870, p. 125, pl. viii, f. I, and Proc. Somerset. Nat. Hist. Soc., vol. xw, 1870, p. 54, pl., f. I; H. P. Blackmore and E. R. Alston, Proc. Zool. Soc., 1874 , p. 464 , woodcut 2.

LOC. River Deposit; Fisherton. [Portions of skeletons; Salisbury Mus.]

Caves; Somersetshire. [Slutl and mandible; Taunton Mus.]

MIOLOPIUS, Owen. v. Platychœrops.

planiceps, Owen. \%. Platychœrops richardsoni. 
IMONODON, Linnæus, Syst. Nat., ed. 12, vol. i, 1766, p. 105.

monoceros, Linneus, ibid., p. I05; J. Parkinson, Introduction, I 822 , p. 275 ('London clay'); (?) S. Woodward, Syn. Tab., 1830 , p. 38 ; R. Owen, Rep. Brit. Assoc., I842 (I843), p. 72 , and Brit. Foss. Mamm., 1846, p. 52 I, woodcut $21 \%$; J. Morris, C.it. Brit. Foss., I 854 , p. 360 ; E. T. Newton, Geol. Mag. [2] vol. viii, I88I, p. 316, and ibid. [3] vol. vi, I889, p. I48, and Vert. Forest Bed (Mem. Geol. Surv, I882), p. Io9, pl. ix, f. 2.

Monndon, R. E. Grant, in Thompson's Annual, 1839, p. 269 ('London Clay').

'Narwhal,' J. Parkinson, Organic Remains, vol, iii, I8 I I, p. 309 (portion of tusk, olim Enniskillen Coll.); C. Lyell, Antiq. Nian, 1863, p. 217.

I.OC. Forest Bed ; near Mundesley and Overstrand. Pleist. ; ? Lewes, Sprowston, near Norwich.

IMUS, Linnieus, Syst. Nat., ed. I2, vol. i, I766, p. 79.

agjestis, Linnzus. \%. Microtus agrestis.

amphibils, Linnæus. $v$. Microtus amphibius.

aspalax, Pallas. $\%$. Siphneus aspalax.

citellus, Linneus. $\%$ Spermophilus sp.

? decumanus, P. S. Pallas, Nov. Spec. Glirium, 1778, p. 91; S. Woodward, Syn. Tab., I830, p. 39.

'Water-rat,' W. Buckland, Phil. Trans., I822, p. 234, pl. xxv, f. I, and Reliq. Diluv., I824, p. 265 , pl. xi, f. I.

I.OC. Kirkdale Cave. [Mandib. ramus.]

glrveolus, Schreber. \%. Microtus glareolus.

gregalis, Pallas. $\%$. Microtus gregalis.

gregarius, Linnæus. $v$. Microtus agrestis.

lemmus, Linnæus. v. Myodes lemmus.

musculus, Linnæus, Syst. Nat., ed. 12, vol. i, 1766, p. 83; S. Woodward, Syn. Tab., I830, p. 39 ; R. Owen, Brit. Foss. Mamm., I846, p. 209, woodcut 79 (M. musculus?); J. Morris, Cat. Brit. Foss., I854, p. 360 ; W. B. Dawkins, Quart. Journ. Geol. Soc., vol. xxv, 1869, p. 198; R. Bell, Proc. Geol. Assoc., vol. ii (1871), p. 217 ; E. T. Newton, Vert. Forest bed (Mem. Geol. Surv., I882), p. 93 ('insufficient evidence').

'Mouse,' W. Buckland, Phil. Trans., I 822, p. 235, pl. xxv', f. 7, 8, 9, and Reliq. Diluv., I824, p. 265, pl. xi, f. 7, 8, 9.

LOC. ? Forest Bed; Norfolk.

Pleist. ; Copford.

Caves; Durdham Down, Kirkdale, Kent's Hole.

pygmere, Pallas. \% Sorex pygmaus.

songu'us, Pallas. \%. Cricetus songarus.

sjlvaticus, Linneus, Syst. Nat., ed. 12, vol. i, 1766, p. S4; E. 'T. Newton, Geol. Mag. [2] vi.. viii, I08I, p. 250 ', and Vert. Forest Bed (Mem. Geol. Surv, i 882), p. 93, pl. xiv, f. II. LOC. Forest Bed; West Runton. [Portions of mandible; A. Savin Coll. and Mus. Pract. Geol.] 
MUs TIIA, Linnæus, Syst. Nat., ed. 12, vol. i, I766, p.-66. Putorius, Cuvier, Régne Animal, vol. i, I817, p. I4I. MARTeS, S. Nilsson, Skand. Fauna, vol. i, I820, p. 4 I.

erminea, Linnzeus, Syst. Nat., ed. 12, vol. i, 1766, p. 68 ; IV. B. Dawkins and W. A. Sanford, Brit. Pleist. Mamm., pt. i (Pal. Soc., I 866), p. xxii ; R. Lydekker, Cat. Foss. Mamm. B. M., pt. i, 1885, p. I So. [See also $M$. putorius.]

Putorius ermineus, R. Owen, Brit. Foss. Mamm., I846, p. I I6, woodcuts 40-42 ; J. Morris, Cat. Brit. Foss., I854, p. $36 \mathrm{r}$.

Putorius vilgaris, R. Owen, Rep. Brit. Assoc., I842 (1843), p. 70.

'Weasel,' W. Buckland, Phil. Trans., I 822, p. 185, pl. xx, f. 28, 29 , and Reliq. Diluv., 1824, pp. 262, 278, pl. vi, f. 28,29 , pl. xxiii, f. I I-I 3 ; J. MacEnery, Cavern Researches, I859, pl. E, f. I7. LOC. Pleist.; Plymouth (raised beach).

Caves; Bacon Hole, Berry Head, Kent's Hole, Kirkdale, ? Oreston.

gulo, Linnæus. $\%$. Gulo luscus.

lutre, Linnzus. \%. Lutra vulgaris.

martes, Linnæus, Syst. Nat., ed. 12, vol. i, 1766, p. 67 ; W. B. Dawkins and IV. A. Sanford, Brit. Pleist. Mamm., pt. i (Pal. Soc., 1866), p. xxi.

Martes sylratica, S. Nilsson, Skand. Fauna, vol. i, 1820, p. 41; E. T. Newton, Geol. Mag. [2] vol. vii, I880, p. I54, and Vert. Forest Bed (Mem. Geol. Surv., I882), p. 25, pl. iv, f. 3 . Martes sylvestris, A. L. Adams, Trans. Roy. Dublin Soc. [2] vol. i (I88I), p. 205.

Lutra, Niller and Skertchley (errore), Fenland, I878, p. 505.

Loc. Forest Bed; West Runton. [Mandib ramus; Mus. Pract. Geol.]

Caves; Bleadon, Long Hole, Ravenscliff, and Spritsail Tor.-Shandon (Ireland).

putorius, Linnæus, Syst. Nat., ed. 12, vol. i, 1766, p. 67 ; W. B. Dawkins and WV. A. Sanford, Brit. Pleist. Mamm., pt. i (Pal. Soc., 1866), p. xxii ; R. Lydekker, Cat. Foss. Mamm. B. M., pt. i, I 885 , p. 179.

Putorius vulgaris, R. Owen, Brit. Foss. Mamm., I846, p. I12, woodcuts 38,39 .

LOC. ? White Crag; Orford, Suffolk. [Mandib. ramus; Brit.Mus.] Pleist.; Raised Beach, Plymouth. [? Mustela erminea.]

Caves; Bacon Hole; Berry Head, Brixham, Long Hole, and Spritsail Tor.

vulgaris, J. C. P. Erxleben, Syst. Regn. Animal., 1777, p. 47I ; S. Woodward, Syn. Tab., 1830, p. 39; R. Lydekker, Cat. Foss. Mamm. B. M., pt. i, I 885 , p. 179.

LOC. Caves; Brixham and Kirkdale.

sp., R. Lydekker, Cat. Foss. Mamm. B. M., pt. i, I885, p. I83.

Loc. Pleist.; Suffolk. [Lower pm. 3, m. I ; Brit. Mus., no. $18232-34$.] 
Iry ODIS, P. S. Pallas, Zoogr. Rosso-Asiat, vol. i, ISII, p. I73.

LemmUS, F. Tiedemann, Zoologie, vol. i, I SoS, p. 473.

lemmus (Linneus), H. P. Blackmore and E. R. Alston, Proc. Zool. Soc., 1874, p. 470.

Mus lemmus, Linnæus, Syst. Nat., ed. 12, vol. i, 1766, p. 80.

Lemmus norvegicus, A. G. Desmarest, Mammalogic, pt. ii, 1822, p. 287.

Lemmus norvegicus, var., W. A. Sanford, Quart. Journ. Geol. Soc., vol. xxvi, 1870, p. $125, \mathrm{pl}$. viii, f. 3 , and Proc. Somerset. Nat. Hist. Soc., vol. xv, 1870 , p. 55 , pl., f. 3.

LOC. Cave; Somersetshire. [Mandib. ramus; Taunton Mrus.]

torquatus (Desmarest), H. P. Blackmore and E. R. Alston, Proc, Zool. Soc., 1874, p. 469.

Lemmus torquatus, A. G. Desmarest, Mammalogie, pt. ii, 1822 p. 289.

Lemmus torquatus, var, W. A. Sanford, Quart. Journ. Geol. Soc., vol, xxvi, 1870 , p. 125, pl. viii, f. 4 , and Proc. Somerset. Nat. Hist. Soc., vol. xv, 1870 , p. 55 , pl., f. 4 .

Arvicola gulielmi, W. A. Sanford, ibid., f. 2, and ibid., f. 3 (assigned to Arvicola ambiguus, Pomel, by R. Lydekker, Cat. Foss. Mamm. B. M., pt. i, I885, p. 231).

LOC. Cave; Wookey Hole.

River Deposit; Fisherton, near Salisbury. [Portion of skull, with teeth; Salisbury Mus.]

MYOGAIZ, Cuvier, Tab. de Classif. Mamm, appended to 'Leçons d'Anat. Comp.', vol. i, 1800 (Mygale).

Paleospalax, R. Owen, Brit. Foss. Mamm., i846, p. 25.

moschata (Linnæus), J. Gunn, Geology of Norfolk, I864, p. 19) (Mygale); W. B. Dawkins, Quart. Journ. Geol. Soc., vol. xxi, 1869, p. 210 (Mystrale); E. T. Newton, Geol. Mag. [2] vol. viii, 1881, p. 259, and Vert. Forest Bed (Mem. Geol. Surv., 1882), p. 98, pl. xvi, f. 1-Io ; R. Lydekker, Cat. Foss. Mamm. B. M., pt. i, I885, p. I6.

Castor moschatus, Linnzeus, Syst. Nat., ed. 12, vol. i, 1766, p. 79.

Sorex moschatus, W. B. Dawkins and W. A. Sanford, Brit. Pleist. Mamm., pt. i (Pal. Soc., I 866), p. xxiv; W. B. Dawkins, Quart. Journ. Geol. Soc., vol. xxviii, 1872, p. 417, and Early Man in Britain, 1880, p. 128.

Talpa magna, A. Pomel, Archiv. Sci. Phys. Nat., vol. ix, I848, p. 160.

Palcospalax magnus, R. Owen, Brit. Foss. Mamm., I846, p. 25 , woodcuts 12, 13 ; J. Morris, Cat. Brit. Foss., 1854, p. 360 ; R. Johnson, Trans. Norfolk and Norwich Nat. Soc, vol. ii (1877), p. 292 ; A. Savin, Proc. Norwich Geol. Soc., vol. i(1878), p. 27. [Mandib. ramus; Brit. Mus.] . (Palacospalax.) ?'Rodenta,' C. Green, Hist. Bacton, 1842, p. 59 (pars).

Loc. Forest Bed; near Bacton, Ostend, and West Runton. 


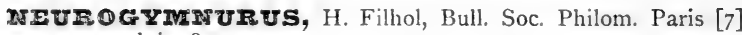
vol. i, I 877, p. 53.

major, R. Lydekker, Cat. Foss. Mamm. B. M., pt. v, 1887, p. 302. LOC. U. Eocene ; Hordwell. [Mandib. rami ; Brit. Mus.]

min or, H. Filhol, Bull. Soc. Sci. Phys. Nat. Toulouse, vol. v, I884, p. 468, pl. ii, f. I3-15 ; R. Lydekker, Cat. Foss. Mamm. B. M, pt. v, I 887, p. 302 .

Loc. Headon Beds; Headon Hill. [Mandib. ramus; Brit. Mus.]

Ore CA, J. E. Gray (ex Rondelet), Zool. 'Erebus' and 'Terror,' 1846, p. 33 .

? citoniensis, G. Capellini, Mem. R. Accad. Sci. Istit. Bologna [4] vol. iv, I883, p. 670: R. Lydekker, Quart. Journ. Geol. Soc., vol. xliii, 1887, p. 16, pl. ii, f. 9, 10, and Cat. Foss. Mamm. B. M., pt. v, I887, p. 80.

LOC. Red Crag; Woodbridge. [Tooth; Ipswich Mus.Periotic; Mus. Pract. Geol.]

gladiator, J. E. Gray, Zool. 'Erebus' and 'Terror,' 1846, p. 33 ; W. H. Flower, Cat. Osteol. Dent. Vert. Mus. Roy. Coll. Surgeons, 1884, p. 572.

Delphinus orca, Linnæus, Syst. Nat., ed. 12, vol. i, 1766, p. 108 ; D. Embleton, Trans. Tyneside Nat. Field Club, vol. iv (1858), p. 50.

LOC. River Deposit; Bed of Tyne.

OROHIPUS, Marsh. v. Hyracotherium.

OVIzos, H. D. de Blainville, Bull. Soc. Philom. Paris, 1816, p. 76.

moschatus (Zimmermann), E. Lartet, Quart. Journ. Geol. Soc., vol. xxi, 1865 , p. 474 ; C. Moore, Proc. Bath Nat. Hist. Soc., vol. ii (1870), p. 52 ; W. B. Dawkins, Brit. Pleist. Mamm., pt. v (Pal. Soc., I872), p. I, pls. i-v, and Quart. Journ. Geol. Soc, vol, xxxix, 1883, p. 575, woodcut, and ibid., vol. xli, I885, p. 242, woodcut ; W. Davies, Geol. Mag. [2] vol. vi, I879, p. 246 ; C. Reid, Trans, Norfolk and Norwich Nat. Soc., vol. iii (I884), p. 632 (skull); R. Lydekker, Cat. Foss. Mamm. B. M., pt. ii, 1885, p. 38.

bios moschatus, E. A. W. von Zimmermann, Geogr. Geschichte, vol. ii, I780, p. 86.

Bubalus moschatus, R. Owen. Quart. Journ. Geol. Soc., vol. xii, I 856 , p. I24, woodcuts I-3.

LOC. Forest Bed; Trimingham.

Pleist.; Barnwood, Crayford, Dogger Bank (drenged), Freshford, Green Street (Bromley), Maidenhead.

OVIS, Linnæus, Syst. Nat, ed. I2, vol. i, I766, p. 97.

? aries, Linnaeus, ibid., p. 97.

Ovis aries, var. o polycerata, Linnæus, ibid., p. 97. 
OvIs ? aries (continued) -

Ovis polycoratus, W. R. Wilde, Proc. Roy. Irish Acad., vol. vii, I859, p. 207, woodcuts IO, II.

Ovis aries?, S. Haughton, Proc. Roy. Irish Acad. [2] vol. ii, I876, p. 483 .

LOC. Pleist.; Ballinderry, Dunslaughlin (Ireland). Caves; Knockninny (Ireland). [Probably historic.]

? sp., R. Lydekker, Cat. Foss. Mamm. B. M., pt. ii, I885, p. 40.

LOC. Cave; Kent's Hole. [Radius and ulna ; Brit. Mus., no. I6758.]

Pachynolophus minimus, Gervais. v. Lophiodon minimus.

PAGormys, J. E. Gray, Proc. Zool. Soc., I864, p. 31. foetidus (Fabricius), Turner. $v$. Phoca hispida.

PALAOBALANA, Seeley. v. Palæocetus. sedgwickii, Seeley. v. Palæocetus sedgwicki.

PAגFFOCETUS, H. G. Seeley, Geol. Mag., vol. ii, I865, p. 54 ; J. E. Gray, Geol. Mag., vol. ii, 1865, p. 57.

Palæobalena, H. G. Seeley, Proc. Camb. Plil. Soc., vol. i (I864), p. 228 (name only).

sedgwicki, H. G. Seeley, Geol. Mag., vol. ii, I865, p. 54, pl. iii; W. H. Flower, Nature, vol. xxix (I883), p. I70; R. Lydekker, Cat. Foss. Mamm. B. M., pt. v, I887, p. 31 .

Palcobalcna sedgwicki, H. G. Seeley, Proc. Camb. Phil. Soc., vol. i (1864), p. 228 (name only).

'Cetaceous Animal,' R. Owen, Palieont., ed. 2, I861, p. 355.

Loc. Boulder Clay; Ely ('derived from Kimeridgian'). [Cervical vertebræ; Woodwardian Mus.]

PALAOELPIIS, Du Bus. v. Physodon. firsiformis, Du Bus. v. Physodon fusiformis. grandis, Du Bus. \%. Physodon grandis.

PALAOSPALAX, Owen. v. Myogale. magmus, Owen. v. Myogale moschatus.

PAIfFOThIRIU MM, Cuvier, Ann. du Muséum, vol. iii, I 804, p. 289.

Plagiolophus, A. Pomel, Bull. Soc. Géol. France [2] vol. iv, 1847, p. 586.

PAloplotherium, R. Owen, Quart. Journ. Geol. Soc, vol. iv, I 848 (read I847), p. I7.

annectens (Owen), R. Lydekker, Cat. Foss. Mamm. B. M. pt. iii, 1886, p. 34, woodcut 6 (annectans).

Paloplotherium annectens, R. Owen, Quart. Journ. Geol. Soc., vol. iv, I 848 , p. 28 , pl. iii, f. I-4, and Geol. Mag., vol. vii, 1870 , p. 143; J. Morris, Cat. Brit. Foss., 1854, p. 361.

3i (Palopletherium.)

Pligiolophus annectens, A. Pomel, Catal. Méthod., 1853, p. 83. Loc. Headon Beds; Binstead, I. of Wight, and Hordwell.

[Skull; Brit. Mus.] 
PAIFETHERUIM (continued)-

crassum, Cuvier, Ann. du Muséum, vol. vi, 1805, p. 348; R. Owen, Trans. Geol. Soc. [2] vol. vi (1841), p. 44, pl. iv, f. 7, and Brit. Foss. Mamm., 1846, p. 322, woodcuts II3, II 5 , I16, and Odontography (1845), p. 599, pl. cxxxv, f. 6; J. Morris, Cat. Brit. Foss., 1854, p. 360 ; R. Lydekker, Cat. Foss. Mamm. B. M., pt. iii, 1886, p. 25.

LOC. Headon Beds; Bembridge, Hordwell, and Headon Hill. [Teeth; Brit. Mus.]

curtum, Cuvier, quoted as British, without authority, by $\mathrm{J}$. Morris, Cat. Brit. Foss., 1854, p. 360.

magnum, Cuvier, Ann. du Muséum, vol. iii, I804, p. 367, and Ossem. Fossiles, vol. iii, I823, p. I 57 ; R. Owen, Odontography (1845), p. 599, pl. cxxxv, f. 4, 5, and Brit. Foss. Mamm., 1846, p. 316, woodcuts 109, 110 ; J. Morris, Cat. Brit. Foss., 1854 , p. 360 ; T. H. Huxley, Geol. Mag., vol. vii, 1870, p. 153, pl. vi ; R. Lydekker, Cat. Foss. Mamm. B. M., pt. iii, 1886, p. 16.

Lophiodon, or Palantherium, or Coryphodon, R. Owen, Brit. Foss. Mamm., I 846, p. 309, woodcut I06; R. Lydekker, Cat. Foss. Mamm. B. M., pt. iii, 1886, p. 19. [Phalangeal bone; Brit. Mus.]

LOC. U. Eocene; Bembridge, Hempstead, Hordwell, and Seafield. [Teeth; Brit. Mus.]

medium, Cuvier, Ann. du Muséum, vol. iii, 1804, p. 289; R. Owen, Trans. Geol. Soc. [2] vol. vi (1841), p. 44, pl. iv, f. 6, and Brit. Foss. Mamm., 1846, p. 319, woodcuts II1, II 2, I 14 ; J. Morris, Cat. Brit. Foss., I854, p. 36 I ; R. Lydekker, Cat. Foss. Mamm. B. M., pt. iii, 1886, p. 20.

LoC. U. Eocene; Bembridge and Binstead, I. of Wight, and Hordwell. [Teeth and jaws; Brit. Mus.]

T.

minus, Cuvier, Ann. du Muséum, vol. iii, I804, p. 47I; R. Owen, Brit. Foss. Mamm., 1846, p. 323, woodcut I17; J. Morris, Cat. Brit. Foss., 1854 , p. 361 ; R. Lydekker, Cat. Foss. Mamm. B. M., pt. iii, 1886, p. 38.

Paloplotherium minus, P. Gervais, Zool. et Pal. Franç., ed. I, vol. iv, 1848-1852, p. 63, pls. xiii, xiv.

Plagiolophus minor, A. Pomel, Bull. Soc. Géol. France [2] vol. iv, 1847, p. 586.

LOC. U. Eocene; Bembridge and Seafield, I. of Wight, and Hordwell. [Portions of skull and mandible, etc.]

PALOPLOTIIERIUM, Owen. \%. Palæotherium.

annectens, Owen. $v$. Palæotherium annectens.

minus, Gervais. v. Palæotherium minus.

PERAIzSTIS, R. Owen, Brit. Mesoz. Mamm. (Pal. Soc., iS7i), p. 33 ; H. F. Osborn, Proc. Acad. Nat. Sci. Philad., I 888, p. 298. [? Spalacotherium.] 
PפRAISTES (continued) -

longirostris, R. Owen, Brit. Mesoz. Mamm. (Pal. Soc., r871), p. 33, pl. ii, f. 3 (non f. 4) ; R. Lydekker, Cat. Foss. Manm. B. M., pt. v, 1887, p. 294. [See also Amblotherium mustelula.]

LOC. M. Purbeck; Durdlestone Bay. [Right maxilla; Brit. Mus.]

? sp., R. Owen, Brit. Mesoz. Mamm. (Pal. Soc., I871), p. 36, pl. i, f. 40 (called Phascolestes dubius in explan. to plate).

Spalacotherium tricuspidens, R. Owen, Quart. Journ. Geol. Soc., vol. x, 1854, p. 43I, woodcut 12.

'Genus indet.,' R. Lydekker, Cat. Foss. Mamm. B. M., pt. v, I 887, p. 294.

Loc. M. Purbeck; Durdlestone Bay. [Mandib. ramus; Brit. Mus.]

PgRAmUS, R. Owen, Brit. Mesoz. Mamm. (Pal. Soc., 1871), p. 41 ; H. F. Osborn, Proc. Acad. Nat. Sci. Philad., 1888, p. 295.

Leptocladus, R. Owen, Brit. Mesoz. Mamm. (Pal. Soc., 1871), p. 53.

tenuirostris, R. Owen, ibid., p. 4I, pl. ii, f. Io (? f. II-13); R. Lydekker, Cat. Foss. Manm. B. M., pt. v, I887, p. 277.

? Peramus tenuirostris (minor?), R. Owen, Brit. Mesoz. Mamm. (Pal. Soc., I87I), p. 44, pl. ii, f. I3.

LOC. M. Purbeck; Durdlestone Bay. [Mandib. ramus; Brit. Mus.]

T.

dubius (Owen), H. F. Osborn, Proc. Acad. Nat. Sci. Philad., I888, p. 295, woodcut I $b$.

Leptocladus dubius, R. Owen, Brit. Mesoz. Mamm. (I'al. Soc., 1871), p. 53, pl. iii, f. 4 ; R. Lydekker, Cat. Foss. Mamm. B. M., pt. v, I887, p. 292 . T. (Leptocladus.)

LOC. M. Purbeck; Durdlestone Bay. [Mandib. ramus; Brit. Mus.]

minor (Owen), H. F. Osborn, Proc. Acad. Nat. Sci. Philad., I 888, p. 295, woodcut I $a$.

Spalacotherium minus, R. Owen, Brit. Mesoz. Mamm. (Pal. Soc., 187I), p. 28, pl. i, f. 39 ; R. Lydekker, Cat. Foss. Mamm. B. M., pt. v, 1887 , p. 293.

Loc. M. Purbeck; Durdlestone Bay. [Mandib. ramus; Brit. Mus.]

PERASPALAX, Owen. v. Amblotherium.

talpoides, Owen. \%. Amblotherium talpoides.

I'ERATHERIUM, Aymard. v. Didelphys.

I'HASCOLESTES, Owen. \%. Amblotherium.

dubius, Owen. $v$. Amblotherium dubium and Peralestes sp.

longirostris, Owen. $v$. Amblotherium mustelula. 
EIASCOIOTHzrIUM, R. Owen, Proc. Geol. Soc., vol. iii (1838), p. 9, and Trans. Geol. Soc. [2] vol. vi (1841), pp. 57, 58, and Palæontology, ed. 2, I861, p. 341, woodcut II 3; H. F. Osborn, Proc. Acad. Nat. Sci. Philad., 1888, p. 293. [See also W. Ogilby, Proc. Geol. Soc., vol. iii (1838), p. 2 I ; H. D. de Blainville, Comptes Rendus, vol. vii, 1838, p. 402 ; A. Valenciennes, Comptes Rendus, vol. vii, 1838, p. 572.]

THyLACOTHERIUM, A. Valenciennes, ibid., p. 580 (pars).

bucklandi (Broderip), R. Owen, Trans. Geol. Soc. [2] vol, vi (1841), p. 58, pl. vi, f. 2, and Odontography (1845), descr. to pl. xcix, f. 4, and Brit. Foss. Mamm., 1846, p. 6r, woodcut 20, and Brit. Mesoz. Mamm. (Pal. Soc., 1871), p. 16, pl. i, f. 26 ; J. Morris, Cat. Brit. Foss., I854, p. $36 \mathbf{I}$; J. Phillips, Geol. Oxford, 1871, p. 236, woodcut lxxxii [J. Parker Coll.]; R. Lydekker, Cat. Foss. Mamm. B. M., pt. v, I887, p. 270, woodcut 40. [See also Amphilestes sp.]

Didelphys bucklandi, W. J. Broderip, Zool. Journ., vol. iii, I828, p. 408, pl. xi; W. Buckland, Geol. and Mineral., ed. 2, vol. ii, 1837 , pl. ii, f. A.

Thylacotherium bucklandii, A. Valenciennes, Comptes Rendus, vol. vii, 1838, p. 580.

LOC. Stonesfield Slate. [Mandib. ramus; Brit. Mus.]

T.

? sp., 'Femur and humerus of small mammal,' H. G. Seeley, Quart.Journ. Geol. Soc., vol. xxxv, I879, p. 456, woodcuts I, 2.

LOC. Stonesfield Slate. [Femur and humerus; Brit. Mus.]

PIOCA, Linnæus, Syst. Nat., ed. 12, vol. i, 1766, p. 55.

barbata, O. Fabricius, in O. F. Müller, Zool. Dan. Prodr., 17\%6, p. viii ; E. T. Newton, Geol. Mag. [3] vol. vi, I889, p. 147, pl. v, f. 2 [Phoca (Erignathus) barbata].

LOC. Forest Bed; Overstrand. [Humerus; A. Savin Coll.]

foctida, Fabricius. $v$. Phoca hispida.

? groenlandica, O. Fabricius, in O. F. Müller, Zool. Dan. Prodr., 1776, p. viii ; R. Walker, Ann. Mag. Nat. Hist. [3] vol. xii, 1863 , p. 382.

LOC. 'Pliocene' [Pleist.]; Fife. [Skeleton; Edinburgh Mus.]

? hispida, J. C. D. von Schreber, Säugethiere, vol. iii, I778, p. 312, pl. Ixxxvi ; W. H. Flower, Cat. Osteol. Dent. Vert. Mus. Roy. Coll. Surgeons, 1884, p. 207 [for synonymy].

Phoca foetida, O. Fabricius, in O. F. Müller, Zool. Dan. Prodr., 1776, p. viii (not described, and withdrawn by author (Skriv. Nat. Selsk., vol. i (2), 1791, p. 74) in favour of P. hispida).

Pagonys fatidus?, W. Turner, Proc. Roy. Soc. Edinb., vol. vii (1870), p. I14, woodcut.

LOC. Pleist.; Grangemouth and Puggiston. [Portions of two skielitons.] 
PIOCA (continued) -

? vitulina, Linnæus, Syst. Nat., ed. I2,vol. i, 1766, p. 56; (?) D. Page, Advanced Text-book Geology, 1859, p. 307 ; Allman, Proc. Roy. Soc. Edinburgh, vol. iv (1859), pp. 99, I90.

LOC. Pleist.; Stratheden (Fifeshire), Tyree, Kirkcaldy, and Portobello.

spp. : recorded by D. Page, in W. Turner, Proc. Roy. Soc. Edinb., vol. vii (1870), p. 107, from Pleistocene of Dunbar, Errol, Garbridge, Invernetty, Seafield, and Stratheden.

sp.: 'Seal,' Knox, Mem. Wernerian Soc., vol. v, I825, p. 572.

LOC. Pleist.; Camelon.

sp., E. T. Newton, Vert. Forest Bed (Mem. Geol. Surv., I882), p. 29, pl. v, f. I, 2.

Canis vulpes, A. Savin (errore), Proc. Norwich Geol. Soc., vol. i, 1878, p. 27.

LOC. Forest Bed; West Runton and Wood Hill. [Left radius; Mus. Pract. Geol.-Tooth; A. Savin Coll.]

ProCFrna, Cuvier, Règne Animal, vol. i, 1817, p. 279.

communis, R. P. Lesson, Man. de Mammalogie, 1827, p. 413 ; E. T. Newton, Geol. Mag. [3] vol. vi, I889, p. I49, pl. v, f. 4 . Loc. Forest Bed; Sidestrand. [Caudal verlebra; $A$. Sarin Coll.]

crassidens, Owen. ข. Pseudorca crassidens.

PIIYSETrE, Linnæus, Syst. Nat., ed. I2, vol. i, I766, p. 107.

breviceps, Blainville. $v$. Kogia breviceps.

? macrocephalus, Linnæus, Syst. Nat., ed. I2, vol. i, I766, p. I07; R. Owen, Brit. Foss. Mamm., I846, p. 524, woodcut 217 ; J. Morris, Cat. Brit. Foss., I854, p. 36I ; E. T. Newton, Quart. Journ. Geol. Soc., vol. xlii, 1886, p. 316, pl. xi, f. I-4. LOC. Forest Bed; Sidestrand. [Tooth; Mus. Pract. Geol.] Pleist.; Essex. [Teeth.]

PHysatra UגA, P. J. Van Beneden, Bull. Acad. Roy. Belcr. [2] vol. xliv, 1877, p. 85 I.

dubusi, [-ii] P. J. Van Beneden, Bull. Acad. Roy. Belg. [2] vol. xliv, 1877 , p. 851 ; R. Lydekker, Cat. Foss. Mamm. B. M., pt. v, I887, p. 54 .

Homocetus villersi, R. Lydekker (errore), Quart. Journ. Geol. Soc., vol. xliii, 1887, p. 14.

LOC. Red Crag; Woodbridge. [Tooth; Brit. Mus.]

T.

Priysodos, P. Gervais, Bull. Soc. Géol. France [2] vol. xxix, 1872, p. IOI.

PAlæodelphis, Du Bus, Bull. Acad. Roy. Belg. [2] vol, xxxiv; 1872 , p. 503. 
PIXYSODON (continued)-

? fusiformis (Du Bus), (?) R. Lydekker, Quart. Journ. Geol. Soc., vol. xliii, 1887, p. 14, and Cat. Foss. Mamm. B. M., pt. v, 1887 , p. 59.

Palcodelphis fusiformis, Du Bus, Bull. Acad. Roy. Belg. [2] vol. xxxiv, I872, p. 506.

LOC. Red Crag; Woodbridge. [Teeth; Brit. Mus.]

grandis (Du Bus), R. Lydekker, Quart. Journ. Geol. Soc., vol. xliii, I887, p. I4, and Cat. Foss. Mamm. B. M., pt. v, I 887 , p. 59 .

Palaodelphis grandis, Du Bus, Bull. Acad. Roy. Belg. [2] vol. xxxiv, 1872 , p. 503 .

LOC. Red Crag; Felixstowe, Woodbridge. [Teeth; Brit.Mus.]

PIAGIAUIAX, H. Falconer, Quart. Journ. Geol. Soc., vol. xiii, I857, p. 261, and ibid., vol. xviii, 1862, p. 348, and Encycl. Brit., ed. 8, vol. xvii, I859, p. 161.

Plioprion, E. D. Cope, Amer. Nat, vol. xviii, i884, p. 69 I.

becklesi, [-ii] H. Falconer, Quart. Journ. Geol. Soc., vol. xiii, I 857 , p. 262, woodcuts $1-5,7^{-13}$ (non 14 ), and Palæont. Men., vol. ii, I868, p. 4I6, pl. xxxiii (non pl. xxxiv,f. I) ; [-ii] R. Owen, Palieont., ed. 2, I86I, p. 353, woodcut II9, and Brit. Mesoz. Mamm. (Pal. Soc., I871), p.77, pl. iv, f. 10, II, I3, I4 (? f. I2, I 5), woodcuts 8, 23 ; R. Lydekker, Cat. Foss. Mamm. B. M., pt. v, 1887, p. I97, woodcut 31. [See also P. medius.] LOC. M. Purbeck; Durdlestone Bay. [Mandib. ramus; Brit. Mus.]

falconeri, R. Owen, Brit. Mesoz. Mamm. (Pal. Soc., I871), p. 84, pl. iv, f. 16; R. Lydekker, Cat. Foss. Mamm. B. M., pt. v, I 887 , p. 198.

LOC. M. Purbeck ; Swanage. [Mandib. ramus ; Brit. Mus.]

medius, R. Owen, Brit. Mesoz. Mamm. (Pal. Soc., I87i), p. 85, woodcuts 7, 12; R. Lydekker, Cat. Foss. Mamm. B. M., pt. v, 1887, p. 198.

Plagiaulax becklesii, H. Falconer (errore), Quart. Journ. Geol. Soc., vol. xiii, 1857, p. 280, woodcut 14, and Palæont. Mem., vol. ii, 1868, pl. xxxiv, f. I.

LOC. M. Purbeck; Durdlestone Bay. [Mandib. ramus; Brit. Mus.]

minor, H. Falconer, Quart. Journ. Geol. Soc., vol. xiii, I857, pp. 264, 281, woodcut 15 , and Palæont. Mem., vol. ii, I868, p. 4Io, pl. xxxiv, f. 2-6; R. Owen, Palæont., ed. 2, 186I, p. 354, woodcut 120, and Brit. Mesoz. Mamm. (Pal. Soc., I 87 I), p. 75 , pl. iv, f. 9, woodcut 6 ; R. Lydekker, Cat. Foss. Mamm. B. M., pt. v, 1887 , p. 198.

Plioprion minor, E. D. Cope, Amer. Nat., vol. xviii, 1884, p. 691 .

Loc. M. Purbeck; Swanage. [Mandib. ramus; Brit. Mus.] 
PLAGIOLOPHUS, Pomel. v. Palæotherium. annectens, Pomel. v. Palæotherium annectens. minor, Pomel. v. Palæotherium minus.

PIATYCy arops, E. Charlesworth, Rep. Brit. Assoc., 1854 (1855), Sect., p. 80.

Miolophus, R. Owen, Geol. Mag., vol. ii, 1865, p. 339.

richardsoni, [-ii] E. Charlesworth, Rep. Brit. Assoc., 1854 (1 855 , Sect., p. 80 ; H. Woodward, Geol. Mag., vol. iii, I866, p. 48; R. Lydekker, Cat. Foss. Mamm. B. M., pt. v, 1887, p. 4.

Miolophus planiceps, R. Owen, Geol. Mag., vol. ii, 1865, p. 339, pl. $x$, f. I, 3 .

T. (Miolophus.)

Platychorops $=$ Esthonyx, R. Lydekker, Geol. Mag. [3] vol. ii, I 885, p. 360 .

LOC. London Clay; Herne Bay. [Palatal portion of skull; York Mus.]

T.

PLESIOCETOPSIS, Brandt. v. Cetotherium.

brevifrons, Brandt. $\%$. Cetotherium brevifrons.

dubius, Brandt. v. Cetotherium dubium.

hupschi, Brandt. v. Cetotherium hupschi.

PLESIOCETUS, Van Beneden. v. Cetotherium:

brialmonti, Van Beneden. $v$. Cetotherium brialmonti.

Aubius, Van Beneden. $v$. Cetotherium dubium.

garopi, Van Beneden. $v$. Balænoptera garopi.

hupschi, Van Beneden. $\%$. Cetotherium hupschi.

PLIOLOPHUS, Owen. ข. Hyracotherium.

vulpiceps, Owen. v. Hyracotherium leporinum.

PLIOPRION, Cope. v. Plagiaulax.

minor, Cope. v. Plagiaulax minor.

PSIUDORCA, J. T. Reinhardt, Oversigt K. Danske Vid. Selsk. Forh., I862, p. I 5 I.

crassidens (Owen), W. H. Flower, Cat. Osteol. Dent. Vert. Mus. Roy. Coll. Surgeons, 1884, p. 573.

Phocana crassidens, R. Owen, Brit. Foss. Mamm., 1846, p. 516 , woodcuts 213, 214, 216; J. Morris, Cat. Brit. Foss., I 854, p. 361.

LOC. Prehist. ; Fen near Stamford (Lincolnshire). [Skeleton ; Mus. Stamford Institution.]

PTrRODON, H. D. de Blainville, Ann. Franç. et Etrans. dinnt. et Physiol., vol. iii, 1839, p. 23.

dasyuroides, $H$. D. de Blainville, ibid., p. 23 ; R. Lydekker, Geol. Mag. [3] vol. i, 1884 , p. 444, and Cat. Foss. Mamm. B. M., pt. i, 1885 , p. 33.

LOC. Bembridge Limest.; I. of Wight. [Teeth; Brit. ITus.] T. 
PUTORIUS, Cuvier. v. Mustela.

ermineus, Owen. v. Mustela ermineus.

vulgaris, Owen. v. Mustela putorius.

IANGIFER, Hamilton Smith, in Griffith's Animal Kingdom, vol. v, 1827, p. 304.

tarandus (Linnæus), R. Lydekker, Cat. Foss. Mamm. B. M., pt. ii, 1885, p. 79 ; J. Simpson, Trans. Edinb. Geol. Soc., vol. v (1887), p. 295, pls. vii and viii, f. I-3.

Cervus tarandus, Linnæus, Syst. Nat., ed. 12, vol. i, 1766, p. 93 ; R. Owen, Brit. Foss. Mamm., I846, p. 479, woodcuts 197I99; T. Oldham, Journ. Geol. Soc. Dublin, vol. iii, I849, p. 252 ; J. Morris, Cat. Brit. Foss., I854, p. 358 ; W. R. Wilde, Proc. Roy. Irish Acad., vol. vii, 1859, p. 200 ; S. J. Mackie, Geologist, I861, p. 467, pl. xviii (C. tarandus?); S. Haughton, Man. Geology, I865, p. 339, woodcut 64 ; A. Carte, Journ. Geol. Soc. Dublin, vol. x, 1864, p. I03, pl. vii ; H. Falconer, Palæont. Mem., vol. ii, I868, p. 568 ; J. A. Smith, Proc. Soc. Antiq. Scotland, vol. viii, 187r, p. 186, woodcuts, and ibid., n.s., vol. i, 1879, p. 36I ; G. Busk, Phil. Trans., 1873 (1874), p. 526, and Quart. Journ. Geol. Soc., vol. xxxi, 1875, p. 689 ; J. Young, Proc. Nat. Hist. Soc. Glasgow, vol. ii (1875), p. 5 ; W. B. Dawkins, Quart. Journ. Geol. Soc., vol. xxxi, 1875, p. 248 ; R. J. Moss, Proc. Roy. Irish Acad. [2] vol. ii (Sci.), 1876, p. 547 ; A. L. Adams, 'Trans. Roy. Irish Acad., vol. xxvi (Sci.), 1876, p. 217 , and Proc. Roy. Irish Acad. [2] vol. iii (Sci.), I878, p. 92, and Sci. Proc. Roy. Dublin Soc., vol. ii, I880, p. 76, pl. i, f. I ; J. E. Harting, Brit. Anim. Extinct, i 880 , p. 6I, woodcuts ; E. T. Newton, Vert. Forest Bed (Mem. Geol. Surv., I882), p. 64 (rejects this sp. from Forest Bed); V. Ball, Trans. Roy. Dublin Soc. [2] vol. iii (1885), p. 338; H. H. Winwood, Proc. Bath Nat. Hist. Club, vol. vi (1886), p. 95 ; M. Browne, Vert. Anim. Leicester., I889, p. 34.

Cervus (Rangifer) tarandus, A. Carte, Nat. Hist. Rev., vol. vi (1859), p. 502.

'Reindeer,' J. Scouler, Edinburgh New Phil. Journ., vol. lii, 1852 , p. I 35 ; C. Moore, Proc. Bath Nat. Hist. Soc., vol. ii (1870), p. 52.

'Deeply grooved bones,' Neill, Edinburgh Phil. Journ., vol. i, 1819, p. 183.

Cervus bucklandi, R. Owen, Brit. Foss. Mamm., r846, p. 485, woodcut 200 ; J. Morris, Cat. Brit. Foss., I854, p. 358. [Base of antler; Oxford Mus.]

'Smaller species of Deer of Kirkdale,' W. Buckland, Reliq. Diluv., 1824, pp. 19, 264, pl. ix, f. 5 .

IOC. Pleist. and Prehist.; Beckford, Brentford, Bedford, Bemerton, Bilney Moor, Culham, Camps Hill, Dogger Bank (dredged), Eckington, Folkestone, Fawler, Freshfield, Fladbury, Greenwich, Harrowdean(Northampton), Kew, Larkhall, Leicester, London, Lawford, Loxbrook, 
RANGIFRR tarandus (continued)-

Murston, Otterham, Oxford, Sittingbourne, Stroud, Stonehouse, ? Tewkesbury, Windsor--Carluke, River Clyde, Croftaurie, Craigton, Cill Trölla, Kilmaurs, Keiss, Marlee, Pentland Hills, Raesgill, Ronsay, Shaws, Tain, Yarhouse (Scotland).-Ashbourne, Ballybetagh, Ballyguiry, Clare, Curragh, Lough Gur, Meath (Ireland).

Caves; Anstie's, Banwell, Bacon Hole, Bench, Bosco's Hole, Berry Head, Bleadon, Brixham, Cresswell Crags, Caswell Bay, Coygau, Durdham Down, Hutton, Kent's Hole, Kirkdale, Long Hole, Laugharne, Maidstone (fissure), Minchin Hole, Oreston, Paviland, Pleasley Vale, Spritsail Tor, Uphill, Vale of Clwyd, Windy Knoll, Wookey Hole. - ? Ballynamintra and Shandon (Ireland).

RIIINOCEroS, Linnæus, Syst. Nat., ed. I2, vol. i, I766, p. 104. ACeratherium, J. J. Kaup, Isis (Oken), 1832, p. 904.

antiquitatis, J. F. Blumenbach, Handb. Naturgeschichte, ist French ed., vol. ii, 1803, p. 408; H. Falconer, Palxont. Mem., vol.ii, 1868, p. 399; R. Lydekker, Cat. Foss. Mamm. B. M., pt. iii, 1886, p. 92.

Rhinoceros tichorhinus, G. Fischer de Waldheim, Zoognosia tab. syn. illustr., ed. 3, vol. iii, 1814 , p. 304 ( $\kappa$. ticheorhinus); R. Owen, Brit. Foss. Mamm., I846, p. 325, woodcuts 120I30 ; J. Morris, Cat. Brit. Foss., 1854, p. 36 I : W. B. Dawkins, Nat. Hist. Rev., n.s., vol. iii, 1863 , p. 525, pls. i-iii, and Quart. Journ. Geol. Soc., vol. xxiii, 1867, p. 226; H. Falconer, Palæont. Mem., vol. ii, I868, p. 399 ; C. Moore, Proc. Bath Nat. Hist. Soc., vol. ii (1870), p. 53; G. Busk, Phil. Trans., 1873 (1874), p. 518, pl. xliv, and Quart. Journ. Geol. Soc., vol. xxxi, 1875, p. 690; E. Clarke, Proc. W. London Sci. Assoc., vol. i (i 876 ), p. 98 (radius ? R. tichorhinus); R. Johnson, Trans. Norfolk and Norwich Nat. Soc., vol. ii (1877), p. 284; R. Pennington, Trans. Manchester Geol. Soc., vol. xv (1878), p. 51 ; H. H. Winwood, Proc. Bath Nat. Hist. Club, vol. vi (1886), p. 95.

'Strange bones,' W. Somner, News from Chartham, 4to. London, 1669, and Phil. Trans., 1701, p. 882, pl. [Brit. Mus.]

'Rhinoceros,' W. Buckland, Phil. Trans., 1822, p. 182, pl. xxi, f. 3-6, and Reliq. Diluv., 1824, p. 262, pl. vii, f. 3-6; J. C. Bellamy, Nat. Hist. S. Devon, 1839, p. 438 , pl. xi, f. 3 ; J. MacEnery, Cavern Researches, 1859 , pl. L, pl. M, f. 4, 5, pl. P, pl. Q, f. $1-5$.

LOC. Pleist.; Applecake Hill, Aymestry, Batheaston, Barnston, Barnwood, Beckford, Bedford, ? Brentford, Brighton, Bromwich Hill, Burfield, Camps Hill, Canterbury, Chartham, Chatham, Clacton, Clapham, Culham, Cold Higham, Dogger Bank, Doward Wond, Erith, Eckington, Easton Cliff, Freshford, Fisherton, Flad. 


\section{RFINOCrROS antiquitatis (continued)-}

bury, Fleets Bank, Folkestone, Harborough, Harrowdean (Northampton), Greenwich, Hammersmith, Hartlip, Hurley Bottom, Ipswich, Ilford, Lambridge, Larkhall, Lexden, Loxbrook, London, Lawford, Maidstone, Murston, Morefield, Newington, Oxford, Peckham, Peterborough, Peltridge Common, Plymouth, Regent's Park, Rugby, Sittingbourne, Stonehouse, Stroud, Thame, Tewkesbury, E. Wickham, Wellingborough, ? Walton (Essex), Wilton Jail, Woodstock Road Station, Worcester, Wyre.

Caves; Berry Head, Caswell Bay, Cresswell Crags, Durdham Down, Kent's Hole, Kirkdale, Laugharne, Long Hole, Maidstone (fissure), Oreston, Pleasley Vale, Paviland, Peak Cavern, Sandford Hill, Spritsail Tor, Vale of Clwyd, Wirksworth, Wookey Hole, Yealm Bridge.

etruscus, H. Falconer, Quart. Journ. Geol. Soc., vol. xv, I859, p. 602 ; J. Gunn, Geol. Norfolk, I864, p. I9 ; H. Falconer, Palæont. Mem., vol. ii, I868, pp. 345,355 , pl. xxii, f. I-3, 5 ; W. B. Dawkins, Quart. Journ. Geol. Soc., vol. xxiv, I868, p. 207, pls. vii, viii, and ibid., vol. xxviii, I872, p. 4II, and Cave Hunting, I874, pp. 4I8, 419, 424; R. Johnson, Proc. Norfolk and Norwich Nat. Soc., vol. ii (1877), p. 285 ; A. Savin, Proc. Norwich Geol. Soc., vol. i (1878), p. 28 ; E. T. Newton, Geol. Mag. [2] vol. vii, 1880, p. 448, and Vert. Forest Bed (Mem. Geol. Surv., I 882), p. 38, pl. viii, f. I-7 ; R. Lydekker, Cat. Foss. Mamm. B. M., pt. iii, I886, p. I19. LOC. Forest Bed ; Bacton, Cromer, Happisburgh, Kessingland, Mundesley, Pakefield, and West Runton. [Teeth; Mus. Pract. Geol.]

hemitochus, Falconer. v. Rhinoceros leptorhinus.

incisivus (Kaup), R. Lydekker, Cat. Foss. Mamm. B. M., pt. iii, I886, p. 145. [See also R. schleiermacheri.]

Aceratherium incisizum, J. J. Kaup, Isis (Oken), 1832, p. 904.

LOC. Red Crag; Felixstowe and Woodbridge. [Teeth; Brit. Mus.]

Ieptorhinus, R. Owen (non Cuvier), Brit. Foss. Mamm., I 846 , p. 356, woodcuts 131-140 ; J. Morris, Cat. Erit. Foss., I 854 , p. 361 ; W. Davies, Geol. Mag., vol. ii, I865, p. 334 ; W. B. 1)awkins, Proc. Roy. Soc., vol. xv, I866, p. I06; W. Davies, Cat. Pleist. Vert. Coll. Brady, 1874, p. 29, plate; H. Woodward, Geol. Mag. [2] vol. i, 1874, p. 398, pl. xv ; R. Johnson, Trans. Norfolk and Norwich Nat. Soc., vol. ii (1877), p. 2S5; A. Savin, Proc. Norwich Geol. Soc., vol. i (1878), p. 28; W. B. Dawkins, Quart. Journ. Geol. Soc., vol. xxiii, 1867, p. 213, pl. x, and ibid., vol. xxxvi, I880, p. 398; H. Woodward, Trans. Essex Field Club, vol. iii, 1883, p. 17, woodcut 8 ; R. Lydekker, Cat. Foss. Mamm. B. M., pt. iii, 1886, p. Ior, woodcut 13. [See also R. megarhinus.] 
RINOCEROS leptorhinus (continued)-

Rhinoceros hemitcechus, H. Falconer (pars), Quart. Journ. Geol. Soc., vol. xvi, 1860, p. 488, and Palæont. Mem., vol. ii, 1868, pp. 311, 35I, pls. xv-xvii, pl. xviii, f. 5, pls. xix-xxi, xxiii, xxiv, pl. xxv, f. 1-4.

LOC. Pleist.; Barrington, Bielbecks, Brentford, Crayford, Clacton, Grays, Ilford, Lawford, Lexden, Maidenhead, Northampton, Peckham, Thame, Walton-on-the-Naze. [Cranium; Brit. Mus.]

Caves; Bacon Hole, Brixham, Coygau, Crayley Rock, Crow Hole, Durdham Down, Long Hole, Minchin Hole, Kirkdale, Ravenscliff, Vale of Clwyd, Wookey Hole.

megarhinus, J. de Christol, Ann. Sci. Nat. [2] vol. iv, 1835, p. 76 ; J. Gunn, Geol. Norfolk, 1864, p. 19; W. B. Dawkins, Nat. Hist. Rev., n.s., vol. v, 1865, p. 399, woodcuts I-15, and Quart. Journ. Geol. Soc., vol. xxiii, 1867, p. 214, and Early Man in Britain, 1880 , p. 140, woodcuts 29, 30 ; (?) E. T. Newton, Vert. Forest Bed (Mem. Geol. Surv., 1882), p. 40, pl. ix, f. 1; R. Lydekker, Cat. Foss. Mamm. B. M., pt. iii, 1886, p. 114, woodcut 14.

Rhinoceros merckii, G. F. Jäger, in J. J. Kaup, Akten der Urwelt, $184 \mathrm{I}$, p. 3 ; to replace $R$. kirchorgensis, but withdrawn in the 'Beiträge,' ed. 1, pt. i, 1854, p. vii, as being identical with $R$. leptorhinus, Cuvier.

Rhinoceros leptorhinus, G. Busk (errore), Quart. Journ. Geol. Soc., vol. xxvi, 1870, p. 457, woodcuts 1-4.

Rhinoceros leptorhinus (R. megarhinus), H. Falconer, Palæont. Mem., vol. ii, 1868, pls, xxx-xxxii.

LOC. ? Forest Bed; Cromer.

Pleist.; Crayford, Grays, Ilford, Norfolk, Walton-on-theNaze.

Caves; Oreston, Gower.

merclici, Jäger. $v$. Rhinoceros megarhinus.

? schleiermacheri, R. Owen, Quart. Journ. Geol. Soc., vol. xii, I 856, p. 217, woodcuts I-7. Doubtfuliy referred by Lydekker to $R$. incisizus (Kaup): see Cat. Foss. Mamm. B. M., pt. iii, 1886, p. 149.

LOC. Red Crag; Suffolk. [Teeth.]

tichorhinus, Fischer. $v$. Rhinoceros antiquitatis.

IRINOIOPIUS, A. G. Desmarest, Nouv. Ilict. d'Hist. Nat. vol. v, 1803, p. II 1, and vol. xix, 1803, p. 383 .

ferrum-equinum (Schreber), R. Owen, Brit. Foss. Mamm., 1846 , p. I5, woodcut 6 ; J. Morris, Cat. Brit. Foss., 1854 , p. 361 ; R. Lydekker, Cat. Foss. Mamm. B. MI., pt. i, 1885, p. II. Vespertilio ferrum-equinum, J. C. D. von Schreber, Saugethiere, vol. i, 1775, p. 174.

Loc. Cave; Kent's Hole. [Portions of skull and mandible, Brit. Mus.] 
Saiga tarbarica, Pallas [? tartarica, Linnæus], H. Woodward, Geol. Mag., vol. vi, 1869, p. 67 ; probably fragment of Capra (E. T. Newton, Quart. Journ. Geol. Soc., vol. xl, I884, p. 290).

SCIUR US, Linnæus, Syst. Nat., ed. I2, vol. i, I766, p. 86.

? vulgaris, Linnæus, ibid., p. 86 ; (?) E. T. Newton, Geol. Mag. [2] vol. viii, 1881, p. 259, and Vert. Forest Bed (Mem. Geol. Surv., 1882), p. 92, pl. xiv, f. 12; R. Lydekker, Cat. Foss. Mamm. B. M., pt. i, 1885. p. 209.

Loc. Forest Bed; Ostend, Norfolk. [Humerus; Brit. Mus.]

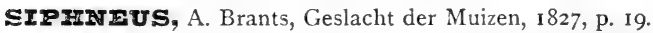

sp. (cf. aspalax, Pallas), A. Brants, ibid., p. 20.

Mus aspalax, P. S. Pallas, Nov. Spec. Glirium, 1778, p. I65, pl.x. Georychus aspalax, R. Owen (errore), Proc. Roy. Soc., vol. xxii, I 874, p. 365 .

Loc. River Deposit; near Salisbury.

SOZIX, Linnæus, Syst. Nat., ed. 12, vol. i, I766, p. 73.

fodiens, Owen (errore). v. Sorex pygmæus.

moschatus (Linnæus), Dawkins. $v$. Myogale moschata.

? pygmreus, P. S. Pallas, Zoogr. Rosso-Asiat., vol. i, I831, p. I34; E. T. Newton, Vert. Forest Bed (Mem. Geol. Surv., I882), p. 97 , pl. xv, f. II, I2.

? Sorex fodiens, R. Owen (errore), Brit. Foss. Mamm., 1846, p. 28, woodcut 14, no. I ; J. Morris (errore), Cat. Brit. Foss., I 854 , p. 361 .

? Crossopus fodiens, R. Lydekker (errore), Cat. Foss. Mamm. B. M., pt. i, 1885 , p. 17 .

LOC. Forest Bed; Ostend and West Runton. [Mandib. ramus; Brit. Hus.]

remifer, Owen (errore). $v$. Sorex vulgaris.

vulgaris (Linnæus, Mus. Adolp. Frid., I754, p. Io); H. Nathusius, Wiegmann's Archiv, vol. iv, I 838, p. 46 ; R. Owen, Brit. Foss. Mamm., 1846, p. 28 ; J. Moris, Cat. Brit. Foss., I854, p. 361 ; E. T. Newton, Vert. Forest Bed (Mem. Geol. Surv., I 882), p. 97 , pl. xv, f. 5-10.

? Sorex remifer, R. Owen (errore), Brit. Foss. Mamm., I846, p. 28, woodcut I4, no. 3; J. Morris (errore), Cat. Brit. Foss., 1854, p. 36r. [Mandib. ramus; Brit. Mus.]

? Crossopus remifer, R. Lydekker (errore), Cat. Foss. Mamm.

B. M., pt. i, 1885 , p. 17 .

LOC. Forest Bed; Ostend and West Runton. [Mandib. ramus; Mus. Pract. Geol.].

Caves; Kent's Hole and Teesdale.

? sp., R. Lydekker, Cat. Foss. Mamm. B. M., pt. i, I885, p. 16.

Loc. Pleist.; Grays, Essex. [Portions of mandib.; Brit. Mus., no. 28079.$]$ 
SPAIACODON, E. Charlesworth, Ann. Mag. Nat. Hist., vol. xiv, 1844 , p. 350, and London Geol. Journ., 1846, p. 6.

sp., E. Charlesworth, ibid., 1846, p. 6, pl. ii, f. 5 ; J. Morris, Cat. Brit. Foss., I854, p. 361 .

LOC. U. Eocene; Hordwell. [Mandib. ramus; Oxford Mus. (olim J. W. Flower Coll.)]

$\mathbf{T}$.

SPAIACOTfigziUT, R. Owen, Quart. Journ. Geol. Soc., vol. $x, 1854$, p. 426.

brodiei, J. Morris, Cat. Brit. Foss., 1854, p. 36r ; misprint for Macellodus brodiei. [REPTILIA.]

minus, Owen. $v$. Peramus minor.

tricuspidens, R. Owen, Quart. Journ. Geol. Soc., vol. x, I854, p. 426, woodcuts 9-I I (non I2), and Palæontology, ed. 2, I86I, p. 350, woodcut 117 , and Brit. Mesoz. Mamm. (Pal. Soc., I 87 I), p. 21, pl. i, f. 32-j8 ; R. Lydekker, Cat. Foss. Mamm. B. M., pt. v, I887, p. 292, woodcut 43. [See also Peralestes sp.] LOC. M. Purbeck; Durdlestone Bay. [Portion of mandib. ramus; Brit. Mus.]

r.

SPgraxopyruts, F. Cuvier, Mém. Mus. Paris, vol. ix, isz2, p. 293 (Spermophile).

? altaicus, E. Eversmann, Addenda ad Pallasii Zoogr. RossoAsiat., fasc. ii, I84I, p. 3 ; E. T. Newton, Geol. Mag. [2] vol. ix, 1882, p. 51, pi. ii.

Spermophilus eversmanni, J. F. Brandt, Bull. Sci. Acad. Imp. St. Pétersbourg, vol, ix, no. I95, 184I (1842), p. 43.

LOC. Arctic Freshwater Bed; Mundesley. [Teeth and bones; Mus. Pract. Geol.]

erythrogenoides, H. Falconer, Palæont. Mem., vol. ii, is68, p. 453, pl. xxxv, f. I-3; W. A. Sanford, Quart. Journ. Geol. Soc., vol. xxvi, 1870, p. I28; R. Lydekker, Cat. Foss. Mamm. B. M., pt. i, I 885, p. 212.

Spermophilus sp. (cf. citillus), W. B. Dawkins and IV. A. Sanford, Brit. Pleist. Mamm., pt. i (Pal. Soc., 1866), p. xxxviii.

Spermophilus, R. Owen, Proc, Roy. Soc., vol. xxii, 1874, p. 365; R.W. Cheadle, Proc. W. London Sci. Assoc., vol.i(1876), p. 7 i.

LOC. Pleist. ; Erith. River Deposit ; Fisherton.

Cave ; ? Bleadon, ? Sandford Hill.

SQUAIODON, J. P. S. de Grateloup, Actes Acad. Bordeaux, is fo, p. 208 .

antwerpiensis, P. J. Van Beneden and P. Gervais, Ostéogr. Cétacés (? 1872), p. 438 ; E. R. Lankester, Quart. Journ. Geol. Soc., vol. xxi, 1865, p. $23 \mathrm{I}$, pl. xi, f. 4 , and ibid., vol, xxvi, I870, p. 5 I2. [Hoplocetus, pars.]

Squalodon sp., W. H. Flower, Cat. Osteol. Dent. Vert. Mus. R. Coll. Surgeons, I 884, p. 566. [Three molars; I pswich Mus.]

LOC. Red Crag; Felixstowe and Woodbridge. [1ecth; Woodwardian Mus., York Mus., etc.]

sp., Lankester. $v$. Balienodon physaloides. 
STEGODON, Falconer. v. Elephas.

STERPOGNATHUS, E. Charlesworth, Rep. Brit. Assoc., $18 \not 4$ (1855), Sect., p. 80.

ooliticus, E. Charlesworth, ibid., p. 80; R. Owen, Rep. Brit. Assoc., 1856 (1857), Sect., p. 73, and Quart. Journ. Geol. Soc., vol. xiii, 1857, p. I, pl. i, and Palæontology, ed. 2, I 86 I, p. 345, woodcuts 11 5, I16, and Brit. Mesoz. Mamm. (Pal. Soc., 1871), p. 18, pl. i, f. 27-30; J. Phillips, Geol. Oxford, 1871, p. 236, woodcut lxxxiii.

Loc. Stonesfield Slate. [Portion of jaw, with teeth; Mus. Pract. Geol.]

$\mathbf{T}$.

STRONGYLOCEROS, Owen. v, Cervus,

spelcers, Owen. v. Cervus elaphus.

STYIODON, R. Owen, Geol. Mag., vol, iii, 1866, p. 199.

pusillus, R. Owen, ibid., p. 199, pl. x, f. 1, 2, and Brit. Mesoz. Mamm. (Pal. Soc., 1871), p. 45, pl. ii, f. 15-19 (non f. I4), pl. iii, f. 2, 3 ; R. Lydekker, Cat. Foss. Mamm. B. M., pt. v, i887, p. 290 ; H. F. Osborn, Proc. Acad. Nat. Sci. Philad., 1 888, p. 299. [See also Kurtodon pusillus.]

Loc. M. Purbeck; Durdlestone Bay. [Mandib. ramus.]

zobustus, R. Owen, Brit. Mesoz. Mamm. (Pal. Soc., I 871), p. 52, pl. iii, f. I; R. Lydekker, Cat. Foss. Mamm. B. M., pt. v, 1887 , p. 290 ; H. F. Osborn, Proc. Acad. Nat. Sci. Philad., I 888, p. 299.

I.OC. M. Purbeck; Durdlestone Bay. [Mandib. ramus; Brit. Mus.]

Sข $\mathbf{5 , ~ L i n n æ u s , ~ S y s t . ~ N a t . , ~ e d . ~ 1 2 , ~ v o l . ~ i , ~ 1 7 6 6 , ~ p . ~} 102$; R. Lydekker, Palæont. Indica [10] vol. iii, 1884, p. 49.

antiquus, J. J. Kaup, Ossem. Foss. Mus. Darmstadt, pt. ii, I833, p. 8, pl. iii ; R. Owen, Quart. Journ. Geol. Soc, vol. xii, 1856, p. 223, woodcut II.

Sus sp. [allied to S. erymanthius], R. Lydekker, Cat. Foss. Mamm. B. M., pt. ii, 1885, p. 268, and ibid., pt. v, 1887, p. 337, and Quart. Journ. Geol. Soc., vol. xlii, 1886, p. 366. [Tooth; Ipswich Mus.]

LOC. Red Crag ; Ramsholt and Sutton. [Tooth.]

palæochœrus, J. J. Kaup, Ossem. F sss. Mus. Darmstadt, pt. ii, 1833, p. II, pl. ix ; R. Owen, Quart. Journ. Geol. Soc., vol. xii, 1856, p. 223 , woodcut 1o ; R. Lydekker, Cat. Foss. Namm. B. M., pt. ii, 1885 , p. 273 .

LOC. Red Crag; Suffolk. [Teeth.]

palustris, Rütimeyer. \%. Sus scrofa.

scrofa, Linnzus, Syst. Nat., ed. I2, vol. i, 1766, p. 102 ; R. Owen, Rep. Brit. Assoc., 1843 (1844), p. 228, and Brit. Foss. Mamm., 1846, p. 426, woodcuts 172-174; M. Fisher, Zoologist, vol. i, 1843 , p. 348 ; J. Morris, Cat. Brit. Foss., 
SUS scrofa (continued)-

I 854 , p. 362 ; W. R. Wilde, Proc. Roy. Irish Acad., vol. vii, I859, p. 208, woodcuts 12, I3 ; R. Howse, Trans. Tyneside Nat. Field Club, vol.v (1S6I), p. II2; C. C. Blake, Geologrist, I862, p. 2 I 8 ; S. Haughton, Proc. Roy. Irish Acad. [2] vol. ii (Sci.), 1876, p. 483 ; A. L. Adams, l'roc. Roy. Irish Acad. [2] vol. iii (Sci.), ı878, p. 90, and Sci. Proc. Roy. J)ublin Soc., vol. ii, I880, p. 74 ; J. E. Harting, Brit. Anim. Extinct, I880, p. 77 ; E. T. Newton, Geol. Mag. [2] vol. vii, I880, p. 449, and Vert. Forest Bed (Mem. Geol. Surv., I882), p. 43, pl. vi, f. 4 , pl. ix, f. 3 ; R. Lydekker, Cat. Foss. Mamm. B. MI., pt. ii, I 885 , p. 260 ; M. Browne, Vert. Animals Leicester., I S8\%, p. 35 (also $S$. palustris, ibid., p. 36).

Sus scrofa-ferox, C. Moore, Proc. Bath Nat. Hist. Soc., vol, ii (1870), p. 52 ; G. Rolleston, Journ. Anthrop. Inst., vol. vi, I876, p. 22 ; H. Woodward, Trans. Essex Field Club, vol. iii, 1883 , p. 7 .

Sus scrofa fertus, V. Ball, Trans. Roy. Dublin Soc. [2] vol. iii ( $\left.18 S_{5}\right)$, p. 339 ; H. H. Winwood, Proc. Bath Nat. Hist. Club, vol. vi (I886), p. 95.

'Hog,' W. Buckland, Phil. Trans., 1822, p. 235, pl. xxr', f. 30-33, and Reliq. Diluv., I 824, p. 59, pl. xi, f. 30-33; C. Lyell, Ann. Nat. Hist., vol. iv, I840, p. I88, woodcut 2.

'Boar,' J. C. Bellamy, Nat. Hist. S. Devon, I839, p. 438, pl. xiv, f. 7-9.

LOC. Forest Bed; Happisburgh, Kessingland, Sidestrand.

Pleist. and Prehist. ; Beckford, Bath, Burwell Fen. Brentford, Brighton, Burnt Fen, Clacton, Colchester, Durham, Defford, Fisherton, Freshfield, Grays, Hammersmith, Ilminster, Lambeth, Larkhall, Leicester, Croft (Lincolnshire), Maidstone, Newbury, North Bridge, Ouse River (Newport Pagnell), South Shields, Walthamstow, Wooler. - Ballycumber Bridge, Ballingderry Lough, Dunslaughlin, Lough Gur (Ireland).

Caves; Abinger, Arnside Knott, Bacon Hole, Bleadon, Bosco's Hole, Crow Hole, Heathery Burn, Hutton, Kent's Hole, Long Hole, Minchin Hole, Oreston, Paviland, Portland (fissure), Ravenscliff, Spritsail Tor, Teesdale, Uphill, Vale of Clwyd, Yealm Bridge.Ballynamintra, Knockninny (Ireland).

scrofu-ferox (ferus), auct. \% Sus scrofa.

sp., Lydekker. $v$. Sus antiquus.

TAIPA, Linnæus, Syst. Nat., ed. 12, vol. i, 1766, p. 73.

europæa, Linnaus, ibid., p. 73 ; E. T. Newton, Gicol. Mag. [2] vol. viii, $188 \mathrm{r}$, p. 259 , and Vert. Forest Bed (Mem. (ieol. Surv., 1882), p. 95, pl. xv, f. I-t; R. Lydekker, Cat. Foss. Mamm. B. M., pt. i, I 885 , p. 15.

Talpa vulgaris, R. Owen, Brit. Foss. Mamm., 1846, p. 19, woodcut 8 ; J. Morris, Cat. Brit. Foss., 1854 , p. 362. 
IAIPA europæa (continued)-

'Sauriar,' C. Green, Hist. Bacton, 1842 , p. 66, pl. [Skeleton ; Norwich Mus.]

LOC. Forest Bed; Bacton, Ostend, IVest Runton. Cave; Teesdale.

magna, Pomel. v. Myogale moschata.

vulgaris, Owen. $v$. Talpa europæa.

TAPINODON, Meyer. \%. Anthracotherium.

gresslyi, Meyer. $v$. Anthracotherium gresslyi.

TAPIR U5, Cuvier, Tabl. Elément. Hist. Nat., 1798, p. I52.

priscus, J. J. Kaup, Oss. Foss. Mus. Darmstadt, pt. ii, I833, p. 4 ; R. Owen, Quart. Journ. Geol. Soc., vol. xii, 1856, p. 222, woodcuts 8,9 .

Tapimus elegans, R. Lydekker (? non Pomel), Cat. Foss. Mamm. B. M., pt. iii, I886, p. 3 .

LOC. Red Crag; Woodbridge, Sutton. [Teeth.]

TETRALOPHODON, Falconer. v. Mastodon.

THERIDOMYs, A. J. L. Jourdan, Comptes Rendus, vol. v, I837, p. 483 .

? aquatilis, A. Aymard, Ann. Soc. Agric. Sci. du Puy, vol. xiv, I 850, p. 82 ; R. Lydekker, Geol. Mag. [3] vol. i, I 884, p. 445, and Cat. Foss. Mamm. B. M., pt. i, 1885, p. 237.

Loc. Headon Beds; Hordwell and Headon Hill. [Mandib. rami; Brit. Mus.]

TIIILACOTIIERIUH, Prevost. \%. Amphitherium and Phascolotherium.

bucklandi, Valenciennes. $\%$. Phascolotherium bucklandi.

prevosti, Valenciennes. \%. Amphilestes broderipi and Amphitherium prevosti.

TIIACANTHODON, Owen. $\because$ Triconodon.

serrula, Owen. $\%$. Triconodon mordax.

TRICIIECODON, Lankester. $\%$ Trichechus.

huxleyi, Lankester. $v$. Trichechus huxleyi.

TRICHrCrivg, Linnæus, Syst. Nat., ed. 12, vol. i, I766, p. 49.

Trichecodon, E. R. Lankester, Quart. Journ. Geol. Soc., vol. xxi, 1865, p. 226.

huxleyi, E. R. Lankester, Trans. Linn. Soc. [2] Zool., vol. ii, I882, p. 213 , pl. xxii, f. I-7 ; E. T. Newton, Vert. Forest Bed (Mem. Geol. Surv., 1882), p. 26, pl. v, f. 3 ; R. Lydekker, Cat. Foss. Mamm. B. M., pt. i, I885, p. 197. 


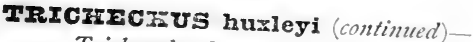
Trichecodon huxleyi, E. R. Lankester, Quart. Journ. Geol. Soc.,
vol. xxi, I 865 , p. 226, pl. x, f. I-3, 5, 6, pl. xi, f. I ; E. T.
Newton, Geol. Mag. [2] vol. vii IsSo, p. 154. Newton, Geol. Mag. [2] vol. vii, 1880, p. 154

Alactherium huxlcyi, P. J. Van Beneden, Osse Trichecodon.) d'Anvers, pt. i, 1877, pp. 47, 53. LOC. Red Crag ; Essex, and Feli tusk; Brit. Mus.] [Portion of

Forest Bed; Cromer, between Cromer and Sidestrand.

rosmarus, Linnecus, Syst. Nat., ed. I2, vol. i, I766, p. 49 ; IV. Southwell, Science Gossip, vol. xiii, 1877, pp. 3, I7S; Cint. Foss. Mamm. B. M., pt. i, I88 , p. 97 ; R. Lydekker, LOC, Dredged; Dogger Bank, 1885 , p. 197.

Brit. Muss.] Peat ; near Lly. [Skull; Cambridge Anat. Mus.]

TrICONODON, R. Owen, Encyclop. Brit., ed. 8, vol. xvii, i 859 ,
p. I6I. Triacanthodon, R. Owen, Brit. Mesoz. Mamm. (Pal. Soc., ferox, R. Owen, Brit. Mesoz. Mamm. (Pal. Soc., I871), p. 64,
pl. iii, f. II-13, I7-19, pl. iv, f. I; R. Lydekker, Cat. Foss.
Mamm. B. M., pt. v, I887, p. 260. Mamm. B. M., pt. v, I887, p. 260. Triconodon mordax, R. Owen (errore), Brit. Mesoz. Mamm. (Pal.
Soc., $187 \mathrm{I}$ ), p. 64, pl. iii, f. 8 .

? Tricnnodon occisor, R. Owen, ibid., p. 69, pl. iv, f. 2. [Mandible ; Brit. Mus.]

LOC. M. Parbeck; Durdlestone Bay. [Mandib. ramus; Brit.
Mus.] major, R. Owen, Brit. Mesoz. Mamm. (Pal. Soc., IS7 1), o. 7o,
pl. iv, f. 3 ; R. Lydekker, Cat. Foss. Mamm. B. M., pt. v,
I 887 , p. 262 .

LOC. M. Purbeck; Durdlestone Bay. [Mandib. ramus; Brit.
Mus.]

mordax, R. Owen, Encyclop. Brit., ed. 8, vol. xvii, I859, p. 161, woodcut 86, and Palarontology, I860, p. 318, woodcut 9i, (?9, 10, non f. 8) Mamm. (Ial. Soc., 1871), p. 58, pl. iii, f. 7 pt. v, I 887, p. 258 , woodcuts 38 , Cat. Foss. Mamm. B. M.,

? Triconodon mordit. (?), E. Willett, Q [Sce also T. ferex:] vol. xxxvii, 1881, p. 378, woodcut. [Mandib. ramus; Moc.,
Pract. Geol.]

? Triacanthodon scrula, R. Owen, Brit. Mesoz. Mamm. (Pal. Soc., 1871 ), p. 72 , pl. iv, f. 7,8 ; O. Thomas, Phil. Trans., I 887 , pt. B, p. 448, pl. xxvii, f. Io. [Mandib. ramus, etc.;
Brit. Mus.] LOC. M. Purbeck; I) urdlestone Bay. [Mandib. ramtis; Brit.
Mus.] 
TRICONODON (continued)-

occisor, Owen. $\%$. Triconodon ferox.

sp., R. Owen, Brit. Mesoz. Mamm. (Pal. Soc., I87I), pp. 62, 63, pl. iii, f. 1 5, I6, 20, 2 I, pl. iv, f. 5, 6; R. Lydekker, Cat. Foss. Mamm. B. M., pt. v, 1887, p. 263 .

LOC. M. Purbeck; Durdlestone Bay. [Mandib. rami; Brit. Mus.]

TRILOPHODON, Falconer. v. Mastodon.

TrOGONTHgRIUM, G. Fischer de Waldheim, Mém. Soc. Imp. Nat. Moscou, vol. ii, 1809, p. 250.

cuvieri, Cuvier (? ex Fischer, MS.), Ossem. Fossiles, ed. 2, vol. v, pt. i, I823, p. 59; R. Owen, Brit. Foss. Mamm., I846, p. I84, woodcuts $7 \mathrm{I}-73$, and Geol. Mag., vol. vi, I869, p. 49, pl. iii, woodcuts I-4; J. Morris, Cat. Brit. Foss., I854, p. 362 ; E. T. Newton, Geol. Mag. [2] vol. viii, I88I, p. 256, and Vert. Forest Bed (Mem. Geol. Surv., I S82), p. 65, pl. xi, f. I-20 ; R. Lydekker, Cat. Foss. Mamm. B. M., pt. i, I 885 , p. 216.

Trogontherium, G. Fischer de Waldheim, Mém. Soc. Imp. Nat. IIoscou, vol. ii, I809, p. 250 ; A. Savin, Proc. Norwich Geol. Soc., vol. i (1878), p. 27.

Castor trogontherium, Cuvier, Ossem. Fossiles, ed. I, vol. v, pt. i, I 823 , p. 59.

Loc. Norwich Crag; Thorpe. [Portion of femur; Brit. Muss.] Forest Bed; Bacton, Cromer, Kessingland, Mundesley, W. Runton, Walker's Cliff. [Various bones and tecth; Brit. Lus. and Mus. Pract. Geol.]

TURSIOPS, P. Gervais, Hist. Nat. Mamm., vol. ii, I855, p. 323.

? tursio (Bonnaterre), R. Lydekker, Cat. Foss. Mamm. B. M., pt.v, I 887, p. 84 .

Delphimus tursio, Bonnaterre, Encycl. Méthod. (Cétologie), 1789, p. 21.

Loc. Pleist. ; Grays, Essex. [Lumbar vertebra; Brit. Mus.]

sp., R. Lydekker, Cat. Foss. Mamm. B. M., pt. v, I887, p. 84 .

LOC. White Crag; Ramsholt. [Caudal vertebra; Brit. Nus, no. 35042 a. $]$

URS US, Linnzeus, Syst. Nat., ed. 12, vol. i, 1766, p. 69 ; H. Falconer, Palæont. Mem., vol. ii, I868, p. 466.

arctoiders, Blumenbach. \%. Ursus spelreus.

arctos, Linnzus, Syst. Nat., ed. 12 , vol. i, 1766, p. 69 ; R. Owen, Rep. Brit. Assoc., I 842 (1843), p. 69, and Brit. Foss. Mamm., I 846, p. 77, woodcut 24, ant in R. Ball, Proc. Roy. Irish Acad., vol. iv (I 849), p. 416 ; J. Morris, Cat. Brit. Foss., IS 54 , p. 362 ; W. R. Wilde, Proc. Roy. Irish Acad., vol. vii, 1859 , 
URSUS arctos (continued) -

p. I92, woodcut I ; A. Carte, Nat. Hist. Rev., vol. vi (1859), p. 502 ; E. Brenan and A. Carte, Journ. Roy. Dublin Soc., vol. ii, 1860, p. 34t, pls. xi, xii ; H. Denny, Proc. Geol. Polyt. Soc. Yorksn., vol. iv (I864), p. 349, pl. x, f. 2 ; W. B. Dawkins and II. A. Sanford, Brit. Pleist. Mamm., pt. i (Pal. Soc., 1866), p. xxiii ; (i. Busk, Phil. Trans., I873 (1874), p. 532 , pl. xlvi, f. 3-5 (? 8, Io), and pl. xlvii, f. I6-18: A. L. Adams, Journ. Roy. (jeol. Suc. Ireland, vol. iv, I877, p. 246 ; J. A. Smith, Proc. Soc. Antiq. Scotland, n.s., vol. i, 1879, p. 360 , woodcuts 2, 3; J. E. Harting, Brit. Anim. Extinct, 1880, p. II, woodcuts ; R. Lydekker, Cat. Foss. Mamm. B. M., pt. i, ISS5, p. 173 .

LOC. Pleist. and Prehist.; Crayford, Grays, ? Murston, Manea Fen (Camb.).-Dumfriesshire (Scotland).-Ballymahon, Kilrathmurray, Westmeath (Ireland).

Caves; Bleadon, Brixham, Deborah's Den, Durdham Down, Hutton, Kent's Hole, Kirkdale, Llandebie, Oreston, Paviland, Sandford Hill, Spritsail Tor, Wookey Hole.

? arvernensis, J. B. Croizet and A. C. G. Jobert, Ossem. Foss. Puy-de-Dôme, I828, p. I88 ; E. R. Lankester, Ann. Mag. Nat. Hist. [3] vol. xir, I864, p. 358 , pl. viii, f. I-4 ; J. Gunn, Geol. Norfolk, I864, p. 19; A. Savin, P'roc. Norwich Geol. Soc., vol. i (1878), p. 28 ; W. B. J)awkins, Quart. Journ. Geol. Soc., vol. xxviii, I872, p. 417 , and Cave Hunting, I874, p. 4 IS (recorded from Forest Bed); E. 'I. Newton, Vert. Forest Bed (Mem. Geol. Surv., is8z), pp. 5-16 ('no description of a specimen of $U$. arvernensis from these deposits has ever been published, and if such a specimen is in existence its resting place is not known').

LOC. Red Crag; Sutton. [Teeth.]

cultridens, Cuvier. $\%$. Machærodus cultridens and M. latidens.

? etruscus, Cuvier, Ossem. Foss., ed. 2, vol. iv, I823, p. 3 So. Doubtfully recorded from Forest Bed by J. Prestwich, () uart. Journ. Geol. Soc, vol. xxvii, I871, p. 466: see also E. T. Newton, Vert. Forest Bed (Mem. Geol. Surv., I88z), pp. 5,6 .

ferox-fossilis, Newton. $\%$ Ursus horribilis. ferox-spelceus, Adams. \%'. Ursus spelacus.

fossilis, Goldfuss. \%. Ursus horribilis.

horribilis, Ord, in Guthrie's Geography, and American ed., vol. ii, 1815, pp. 291, 299; R. Lydekker, Cat. Foss. Mamm. B. M., pt. i, 1885 , p. 166.

Ursus fossilis, Goldfuss, Nova Acta Acad. Cres. Leop.-Carol., vol. $x(2), 1821$, p. 26 I.

Ursus fossilis sive ferox, A. I. Adams, Proc. Roy. Irish Acad. [2] vol. iii (Sci.), 1878 , p. 94. 
URSUS horribilis (continued) -

Ursus fossilis vel ferox-fossilis, A. L. Adams, Sci. Proc. Roy. Dublin Soc., vol. ii, is8o, p. 49, pl. iv, f. 1-3, 8, 9.

Ursus planafrons, H. Denny, Proc. Geol. Polyt. Soc. Yorkshire, vol. iv (I 864 ), p. 347, pl. x, f. I (see also Adams, Sci. Proc. Roy. Dublin Suc. [2] vol. ii, I878, pp. 52, 53). [Skull; Leeds Mus.]

Ursus priscus, Goldfuss, Nova Acta Acad. Cres. Leop.-Carol., vol. xi (2), 1823, p. 468 ; R. Owen, Brit. Foss. Mamm., I846, pp. 82, 106, woodcuts 25. 35; J. Morris, Cat. Brit. Foss., I854, p. 362 ; G. Busk, Phil. Trans., I873 (I874), p. 532, pl. xlvi, f. $2,6,7$, pl. xlvii, f. I3; IV. B. Dawkins, Quart. Journ. Geol. Soc., vol. xxxi, I875, p. 25 I, woodcut I ; R. Johnson, Trans. Norfolk and Norwich Nat. Soc., vol. ii (1877), p. $29 \mathrm{I}$.

Ursus ferox $=U$. priscus, G. Busk, Quart. Journ. Geol. Soc., vol. xxiii, I867, p. 342.

Ursus ferox, G. Busk, Quart. Journ. Geol. Soc., vol. xxxi, I875, p. 684; W. B. Dawkins, Quart. Journ. Geol. Soc., vol, xxxi, I 875 , p. 250 , woodcuts 1,$3 ;$ A. L. Adams, Trans. Roy. Irish Acad., vol. xxvi (Sci.), I876, p. 225.

? Ursus feror? ?, U. arctes?, W. B. Dawkins, Quart. Journ. Geol. Soc., vol. xxxiii, I 877 , p. 598.

Ursus ferox-fossilis, E. T. Newton, Vert. Forest Bed (Mem. Geol. Surv., I 882 ), p. 5, pl. i, f. 5, pl. iii, f. Io.

Lrsus (ferox) horriuilis, V. Ball, Irans. Roy. Dublin Soc. [2] vol. iii (1885), p. 334 .

Ursus maritimus, A. Carte (errore), Journ. Geol. Soc. Dublin, vol. x, I864, p. 114 ; A. L. Adams, Journ. Roy. Geol. Soc. Ireland, vol. iv, 1877 , p. 247 (fails to perceive the resemblance and refers the bones to $U$. spelatis $=U$. ferox); A. L. Adams, Proc. Roy. Irish Acad. [2] vol. iii (Sci.), 1878, p. 94.

Loc. ? Forest Bed; Mundesley.

Pleist.; ? Copford, Grays, ? Ilford, Ipswich, Kew Bridge, ? Murston, Stonehouse, ? Tewkesbury, Woodbridge, ? Walton, ? Whitstable. - Ballinamore, Clonbourne, Kilrathmurray, King's Co., Longford, Lough Gur, WVaterford, Westmeath (Ireland).

Caves; Brixham, ? Deborah's Den, Gower, Kent's Hole, Kirkdale, Minchin Hole, Oreston, Spritsail Tor, Vale of Clwyd, Wookey Hole, Windy Knoll.-Ballynamintra, Kildare, Shandon (Ireland).

luscus, Linnzeus. \%. Gulo luscus.

maritimus, Carte (errore). $v$. Ursus horribilis.

meles, Linnæus. $\%$. Meles taxus.

planafions, Denny. ¿. Ursus horribilis. priscus, Goldfuss. $\because$. Ursus horribilis. 
Ursus (continued) -

spelæus, J. C. Rosenmüller, Oss. Fossil. Anim., 1794, p. I8, pl.; J. C. Bellamy, Nat. Hist. S. Devon, 1839, p. 439, pl. xvi, f. 2 ; W. Buckland, Phil. Trans., I 822, p. $184, \mathrm{pl}$. xx, f. I, and Reliq. Diluv., 1824, p. 261, pl. vi, f. i ; R. Owen, Brit. Foss. Mamm., I846, p. 86, woodcuts $29,30,32,34-36$; J. Mlorris, Cat. Brit. Foss., 1854 , p. 362 ; A. Carte, Nat. Hist. Rev, vol. vi (1859), p. 501 ; J. MacEnery, Cavern Researches, I 859, pl. D, f. 2-6, pl. E, f. 3, 4 ; H. Falconer, Palaent. Mem., vol. ii, 1868 , p. 469; G. Busk, Phil. Trans., 1873 (1874), p. 532, pl. xlvi, f. I, 7, pl. xlvii, f. I, 6, II (U. spelaus?); W. B. Dawkins, Quart. Journ. Geol. Soc., vol. xxxi, 1875 , p. 251 , woodcut 2 , and Proc. Lit. Phil. Soc. Manchester, vol. xiv, I875, p. 6(" = Bison'), and ibid., vol. xv, 1876, p. 150 $("=U$. ferox-fossilis" ; J. Plant, I'roc. Lit. Phil. Soc. Manchester, vol. xv, I876, p. 107; R. Johnson, Trans. Norfolk and Norwich Nat. Soc., vol. ii (1877), p. 290 ; E. T. Newton, Geol. Mlag. [2] vol. vii, 1880, p. 153, and Vert. Forest Bed (Mem. Geol. Surv., I882), p. 5, pls. i, ii, iii ; R. Lydekker, Cat. Foss. Mamm. B. M., pt. i, IS\&5, p. 159.

? Cf. Ursus spelaus, G. R. Waterhouse, in J. Brown, Quart. Journ. Geol. Sor., vol. viii, 1852, p. 188, woodcut 2.

Ursus ferox-spelaus, A. L. Adams, Trans. Roy. Dublin Soc. [2] vol. i (1881), p. 201 , pl. xiv, f. I-5.

Ursus arctoideus, J. MacEnery, Cavern Researches, 1859, pl. D, f. I ; S. J. Mackie, Geologist, I 863, p. 333.

'Fossil different from the white bear,' J. Hunter, Phil. Trans., I794, p. 4 I7, pl. xix, f. 2 (foreign).

'Bear,' J. Rutter, Delin. County Somerset, 1829, p. 155.

LOC. Forest Bed; Bacton, East Coast.

Pleist. ; ? Bedford, ? Murston, ? Windsor. [Copford, Grays, Whitstable (Owen,? U. spelaus).]

Caves; Bosco's Hole, Bleadon, Banwell, Bacon Hole, Burrington Combe, Brixham, Crow Hole, ? Deborah's Den, Durdham Down, Hutton, Kent's Hole, Kirkdale, Kirby Moorside, Long Hole, Minchin Hole, Paviland, Ravenscliff, Sandford Hill, Spritsail Tor, Vale of Clwyd, Windy Knoll,Wookey Hole, Yealm Bridge.-? Shandon (Ireland).

sp.: Ursus, C. Lyell, Ann. Nat. Hist., vol. iv, i $\$ 40$, p. $1 \$ 8$.

'Ursus smaller than U. spelaus,' R. Owen, Brit. Foss. Mamm. 1846 , p. 106.

LOC. Red Crag; Newbourne. [Crown of molar; dim IV. Colchester Coll.]

sp., W. Davies, Cat. Pleist. Vert. Coll. Brady, I874, p. 2. [P'rovisionally referred to $U$. homibilis by $R$. Lydekker, Cat. Foss. Mamm. B. M., pt. i, I8S5, p. 171.]

LOC. Pleist.; Ilford. [Portions of skeleton; Brit. Mus.]

Urus priscus, Bojanus, $\approx$. Bison bonasus. 
VESPERTIII0, Linnæus, Syst. Nat., ed. I2, vol. i, I766, p. 46. fermm-equinum, Schreber. $\%$ Rhinolophus.

noctula, Schreber. $v$. Vesperugo noctula.

sp., Morris. v. Insectivorous Bat. [Miscellaneous Mammalia.]

VESPER UGO, A. von Keyserling and J. H. Blasius, Wirbelthiere Europa's, vol. i, I840, pp. xiv, 45.

? noctula (Schreber), Keyserling and Blasius, ibid., p. 46 ; W. H. Flower, Cat. Osteol. Dent. Vert. Mus. Roy. Coll. Surgeons, I 884 , p. 658 .

I'espertilio noctula, J. C. D. von Schreber, Säugethiere, vol. i, I775, p. I66, pl. lii ; R. Owen, Brit. Foss. Mamm., I846, p. II, woodcut 5 ; J. Morris, Cat. Brit. Foss., I 854 , p. 362.

LOC. Caves; Banwell, Burrington Combe, Hutton.

VIVERRA, Linnæus, Syst. Nat., ed. I2, vol. i, I766, p. 63.

hastingsiæ, W. Davies, Geol. Mag. [3] vol. i, I884, p. 433, pl. xv ; R. Lydekker, Cat. Foss. Mamm. B. M., pt. i, I885, p. IOO, woodcuts II, I2.

Loc. Headon Beds; Hordwell. [Cranium and mandible; Brit. Mus.]

Fulpes vulgaris, auct. v. Canis vulpes.

XIPHODON, Cuvier, Ossem. Fossiles, ed. 2, vol. iii, I822, p. 62.

platyceps, W. H. Flower, Proc. Zool. Soc., I876, p. 3, pl. i, and Cat. Osteol. Dent. Vert. Mus. Roy. Coll. Surgeons, I 884, p. 335. LOC. Red Crag; Woodbridge. [Cranium; Mus. Roy. Coll. Surgeons.]

ZEUGIODON, R. Owen, Proc. Feol. Soc., vol. iii (1839), p. 24, and Trans. Geol. Soc. [2] vol. vi (I84I), p. 70.

wanklyni, H. G. Seeley, Quart. Journ. Geol. Soc., vol. xxxii, I 876, p. 428 .

'Zeuglonon,' H. G. Seeley, Rep. Brit. Assoc., I872 (1873), Sect., p. I22 (title only).

I.oc. Barton Clay; Barton. [Imperfect skull ; olim A. Wanklyn Coll.]

ZIPYIUS, Cuvier, Ossem. Foss., ed. 2, vol. v, pt. i, 1823, p. 352.

amgulatus, Owen. $v$. Mesoplodon angulatus.

amgustus, Owen. $v$. Mesoplodon angustus.

compressus, Owen. \% Mesoplodon compressus.

curieri, Owen. \%. Choneziphius planirostris.

gibbus, Owen. \% Mesoplodon gibbus.

lomgirostis, Cuvier and Du Bus. \% Mesoplodon longirostris.

medilineutus, Owen. v. Mesoplodon longirostris.

plamirostris, Cuvier. $\%$. Choneziphius planirostris.

plamus, Owen. $\%$ Choneziphius planus.

tenuirostris, Owen. \% Mesoplodon tenuirostris. 


\section{MISCELLANEOUS MAMMALIA.}

- Carnassial tonth of a Carnivore, allied to Hyanodon and Ptcrodon,' R. Owen, Quart. Journ. Geol. Soc., vol. xii, is 56, p. 230, woodcut 20.

LOC. Red Crag; Woodbridge.

'Carnivore,' W. Davie', Geol. Mag. [3] vol. i, I \$84, p. 437; R. Lydekker, Cat. Foss. Namm. B. M., pt. i, 1885, p. 106.

LOC. Headon Beds; Hordwell. [Imperfect cranium; Brit. Mus., no. 36791.]

'Castoroid Rodent,' R. Lydekker, Cat. Foss. Mamm. 13. M., pt. i, 188 ;, p. 221, woodcut 30 ; 'Beaver,' J. Brown, Quart. Journ. Geol. Soc., vol. viii, 1852 , p. 188 , woodcut 3 .

LOC. Pleist. ; Copford. [Tooth ; Brit. Mus.]

Cetacean remains are recorded by:-R. Bald, Edinb. Phil. Journ., vol. i, I8I9, p. 393, and Keddock, Edinb. Phil. Journ., vol. xi, 1824 , p. 415 ("Whale'; Airthey, near Stirling); W. Richardson, Mag. Nat. Hist., vol. iii, 1839, p. 99 ("Whale'; Herne Bay) ; H. IV. Crosskey, Proc. Phil. Soc. Glasgow, vol. v, I863 (I854), p. 243 ('Cetacean'; Banks of R. Irvine); Hardy, Proc. Lit. Phil. Soc. Manchester, vol. v, I 866, p. 32 ('Cetacean vertebra'; near 'Twisley, Yorkshire); J. F. Stewart, Trans. Geol. Soc. Glasgow, vol. vi (I879), p. 49 ("Whale'; Stirling); R. Lydekker, Quart. Journ. Geol. Soc., vol. xliii, I 887 , p. I6, and Cat. Foss. Mamm. B. MI., pt. v, I 887, p. 84 ('Cetacean periotics and tympanics' from White Crag, Beccles Old Abbey, and Red Crag, Woodbridge).

'Cetacean vertebre,' Newton, 1886. \%'. Balæena biscayensis.

'Cetaceous Animal,' Owen, I86I. \% Palæocetus sedgwicki.

'Dama, fossil, of Ireland,' Hamilton Smith, 1827. \%. Cervus giganteus.

'Deeply grooved bones,' Neill, I 8 I9. $\%$. Rangifer tarandus.

'Deer's horns,' Knowlton, 1747. \%. Cervus elaphus and C. giganteus.

'Didelphe,' Cuvier, I824. \%. Amphitherium prevosti.

'Didelphys, small,' Buckland, i837. \%. Amphitherium prevosti.

'Elk,' auct. ж'. Cervus giganteus.

'Femur and humerus of small mammal,' Sceley, $1879 . \quad \%$ Phascolotherium sp.

'Fossil tooth,' W. Smith, 18ı6. \%. Mastodon arvernensis.

'Group of bones,' C. Green, Hist. Bacton, I $\$ 42$, p. 59, pl. The remains noticed by Green are referred to by $R$. Owen in the Rep. Brit. Assoc., $1842(18+3)$, p. 57 , as resembling Anoplotherimm, though subsequently (p. 74), regarded as 'partly Roebuck and other Animals.' [See also E. Charlesworth, London Geol. Journ., pt. i, I846, p. 25.]

'Hart's horn,' Borlase, 1758. v. Cervus elaphus.

' Horns of antelope,' J. Collet, Phil. Trans., I757, p. I12, and S. Woodward, Syn. Tab., 1830, p. 39 (Pleist. ; Newbury). = undetermined Cervus. 
'Horn of the stag kind,' Hopkins, I733. v. Cervus elaphus.

'Hyænoid wolf,' Falconer, i868. $v$. Lycaon anglicus.

'Insectivorous bat,' R. Owen, Ann. Mag. Nat. Hist., vol. iv, I840, p. I94, woodcut 3 ; 'Cheiropterous (?) insectivore,' R. Owen, Brit. Foss. Mamm., I846, p. I7, woodcut 7 ; Vespertilio sp., J. Morris, Cat. Brit. Foss., I854, p. 362.

LOC. L. Eocene Sand; Kyson (Kingston), Suffolk. [Teeth ; Ipswich Mus.]

'Large deer,' Buckland, I822. v. Cervus elaphus.

'Large horns,' Molyneux, 1697. v. Cervus giganteus.

'Large Strongyloceros,' Falconer, i868. v. Cervus verticornis.

'Large teeth,' Nevile and Molyneux, I7 I5. $\%$ Elephas primigenius.

'Moschus,' Pratt, 1835. v. Dichodon cervinus.

'Opossum jaw,' Charlesworth, I839. $v$. Didelphys colchesteri.

'Quadrumanous Animal,' S. V. Wood, I839. v. Hyracotherium cuniculus.

'Stag of Canada,' Leigh, 1700. ข. Cervus elaphus.

'Stag's head,' Baker, 1785. v. Cervus elaphus.

'Strange bones,' Somner, 1699. \%. Rhinoceros antiquitatis.

'Tiger,' MacEnery, I859. v. Felis leo.

'Whales, two species of,' Gunn, 1864. v. Balænoptera sp. 


\section{ADDENDA ET CORRIGENDA.}

Page 2, etc. The locality Wardie, near Edinburgh, is often quoted as Newhaven or New Haven in the earlier memoirs.

" 7, etc. Though lepis is a feminine Greek word, the universal custom of regarding it as masculine when employed in scientific names is followed in this work.

9. The fragment of dentition of Aetobatis figured in R. Damon, Geol. Weymouth, ed. 2, I880, Suppl., pl. xii, f. I, is evidently an Eocene fossil ; it is now in the British Museum.

14. The undescribed type of Asteracanthus stutchburyi is in the Bristol Museum; it is of doubtful age.

"17. Bery. microcephalus and B. radiuns are first named in Agassiz, Poiss. Foss., Feuill., 1835, p. 55.

"40. Since the earlier sheets were printed the negotiations for the purchase of the E. IV. Binney Collection by the Manchester Museum have failed. The types of Hoplopygus binneyi and Strepsodus sauroides are therefore not yet preserved in a public collection.

"49. Add Ctranacantryus dubius, J. W. Davis, Trans. Roy. Dublin Soc. [2] vol. i (1883), p. 340, pl. xliv, f. 7. LOC. L. Carb. Limest.; Armagh. [Spine ; Brit. Mus.]

" 65, line 6, dele Passage Beds.

" 67. The type of Dittodus divergens is now in the British Museum.

"74. Figures of Elonichthy's caudalis, egertoni, oblongus, and semistriatus are also given by J. IVard, Trans. N. Staffs. Inst. Mining Engin., vol. x, 1889, pl. iv, f. I, 2, pl. v, f. 4-6.

"So, line 4, for J. W. Salter read F. M'Coy.

" 82. The type of Ganacrodus hastula is now in the British Muscum.

"10I. Mr. Edward Wilson is unable to recognise the type of Hybodus laviusculus in the Bristol Museum, and considers that the spine named H. leptodus is probably a Rhatic fossil.

"1 130, line 28 , for $\mathrm{J}$. W. Salter read F. $\mathrm{II}^{6} \mathrm{Coy}$.

"132, line $1 \mathrm{I}$, for $18+3$ read 1837 .

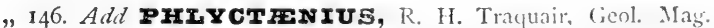
[3] vol. vii, 1890, p. 21, with the single british species, P. anglicus, to be described and figured, ibid., February 1890. LOC. L. Old Red Sandst.; Herefordshire. [Imperfect shield; Edinburgh Mus.] 
Page I66, line 30, for vol. xii, 1856, p. I00, read vol. xvii, I86r, p. I66.

" I 88 , line 4 , before J. E. Lee insert E. R. Lankester, Quart. Journ. Geol. Soc., vol. xxiv, 1868, p. 547.

"202. The various Labyrinthodonts from Warwick described by Owen in the Trans. Geol. Soc. [2] vol. vi, are also noticed in the Rep. Brit. Assoc., I84I (1842), p. I8I.

" 270. Add PuEsrosa ureus platydeirus, R. Owen, Cat. Foss. Rept. Mus. Roy. Coll. Surgeons, I 854, p. 59. LOC. L. Lias; Lyme Regis. [Vertebra; Mus. Roy. Coll. Surgeons.]

"330, line 27, add S. Laing, Prehist. Remains Caithness, I866, p. 50, woodcut 58 (antlers from Keiss).

"333. The following reference is omitted because it relates to a tooth from the Red Crag which must be regarded as specifically indeterminable:-Megaceros hibernicus, R. Owen, Quart. Journ. Geol. Soc., vol. xii, I856, p. 226, woodcut i8.

Among the Pleistocene Mammalia, mere lists of occurrences not verified by specialists are ignored; and it has been necessary to omit many records, from their being noted without scientific precision under English names. The term 'Prehistoric' is employed in the sense defined by Professor Boyd Dawkins. 




\section{PLEASE DO NOT REMOVE}

CARDS OR SLIPS FROM THIS POCKET

\section{UNIVERSITY OF TORONTO LIBRARY}

P\&A Sci. 
